This document is downloaded from DR-NTU (https://dr.ntu.edu.sg) Nanyang Technological University, Singapore.

Viral diagnostics and analysis of host response to viral infection using microarray technology

Chen, Hui

2014

https://hdl.handle.net/10356/61660

https://doi.org/10.32657/10356/61660 

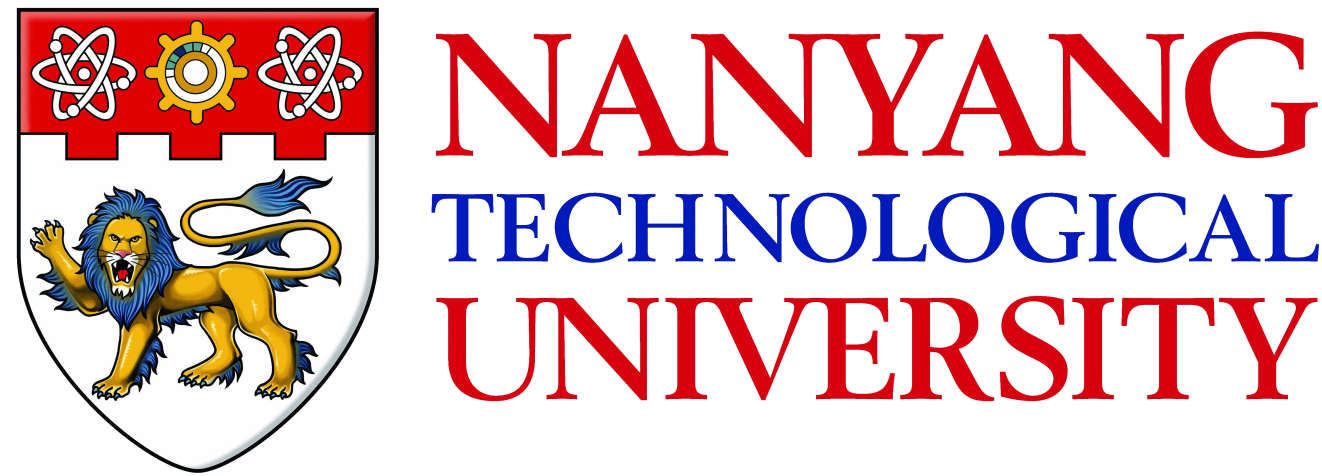

VIRAL DIAGNOSTICS AND ANALYSIS OF HOST RESPONSE TO VIRAL INFECTION USING MICROARRAY TECHNOLOGY

\section{CHEN HUI}

SCHOOL OF BIOLOGICAL SCIENCES 


\title{
VIRAL DIAGNOSTICS AND ANALYSIS OF HOST RESPONSE TO VIRAL INFECTION USING MICROARRAY TECHNOLOGY
}

\section{CHEN HUI}

School of Biological Sciences

\author{
A thesis submitted to the Nanyang Technological University \\ in fulfillment of the requirement for the degree of \\ Doctor of Philosophy
}




\section{ACKNOWLEDGEMENTS}

I would like to give my most sincere thanks to my supervisor Associate Professor Richard J. Sugrue for supporting my bioinformatics studies in his laboratory, as well as invaluable guidance and patience during my Ph.D period. Special thanks also go to Assistant Professor Tan Boon-Huan for giving me an opportunity to participate in her project and offering continuous guidance, patience and encouragement in the processing of my paper writing. I would also like to thank Professor Li Jinming for providing suggestions in the field of bioinformatics and assisting me in my $\mathrm{Ph} . \mathrm{D}$ application process.

I wish to also express my heartfelt gratitude to Assistant Professor Tang Kai and Associate Professor Mu Yuguang for their help in my Ph.D period. Thanks are also given to my lab mates: Richard Sutejo, Debbie Ko Huiling, Muhammad Raihan Jumat, Myint Zu Myaing, Laxmi Ravi Iyer, Loo Liat Hui for their assistance and friendship. Besides, I appreciate the assistance from lab mates in DSO National Laboratories: Yeo Su Yin Dawn, Dr. Ayi Teck Choon, Kuah Li Fang and Chan Ka Wei.

I would like to thank Nanyang Technological University for providing me with a research scholarship.

Lastly, I would like to express my thanks to my parents, grandparents, my sisters and brothers, as well as my friends for their love and accompany forever. 


\section{TABLE OF CONTENTS}

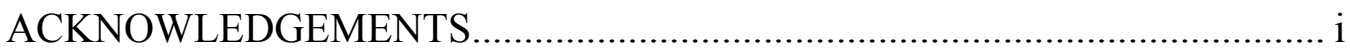

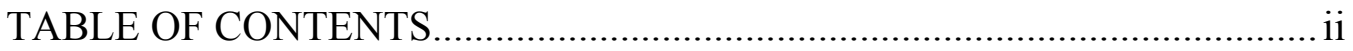

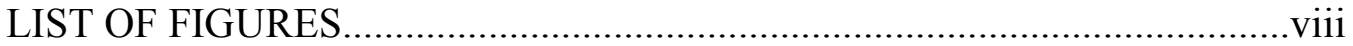

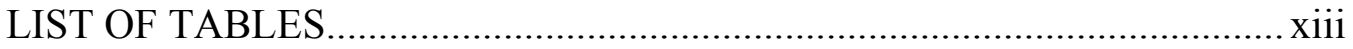

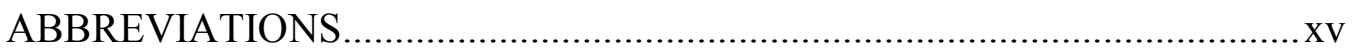

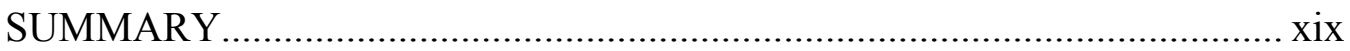

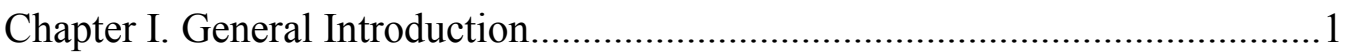

1.1 Outline of virus replication cycle.................................................... 1

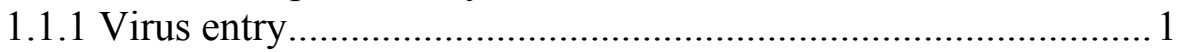

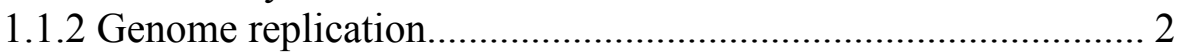

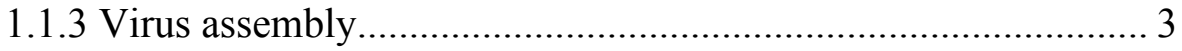

1.2 Host responses to viruses and viral strategies to counter host responses 3

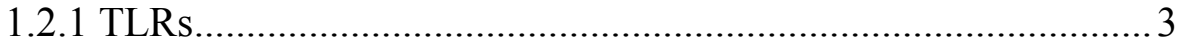

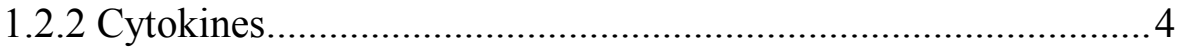

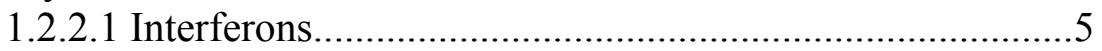

1.2.2.2 Pro-inflammatory cytokines......................................... 5

1.2.2.3 Chemokines........................................................ 6

1.2.3 Cell apoptosis...................................................................... 7

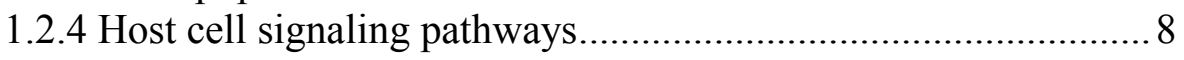

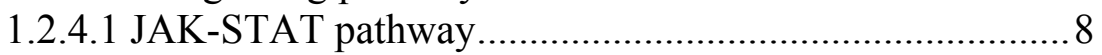

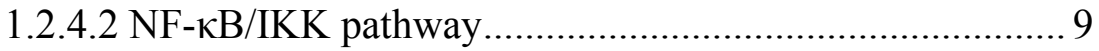

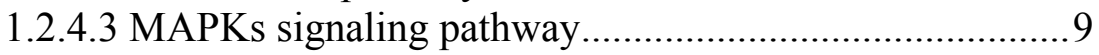

1.3 Traditional strategies in virology studies........................................ 10

1.3.1 Traditional strategies of virus identification............................10

1.3.2 Traditional strategies of host gene expression investigation

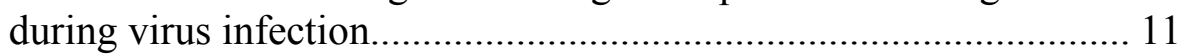

1.4 Design of DNA microarray technology........................................... 12

1.4.1 Different applications of DNA microarray...............................12

1.4.2 Application of microarray in virus identification...................... 13

1.4.3 Application of microarray in host gene expression investigation

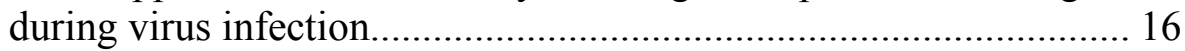

1.5 Bioinformatics tools involved in virology researches....................... 17

1.5.1 Bioinformatics pre-processing for microarray data................... 18

1.5.2 Bioinformatics software for virus identification....................... 18

1.5.3 Bioinformatics software for host gene expression during virus

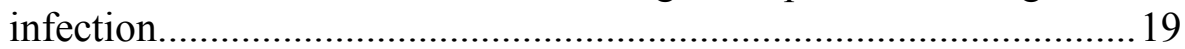

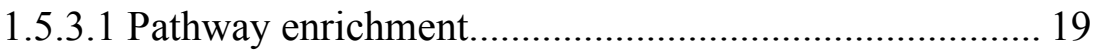

1.5.3.2 Clustering analysis.....................................................20

1.5.3.3 Regulatory network discovery ...................................21

1.5.3.4 Transcription factor binding prediction.........................2 21

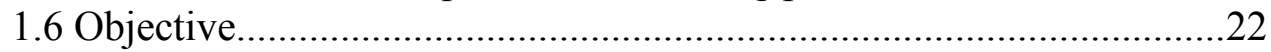

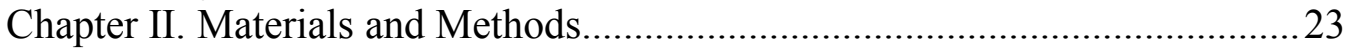

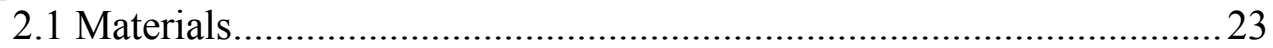

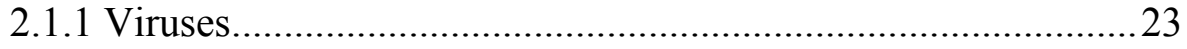

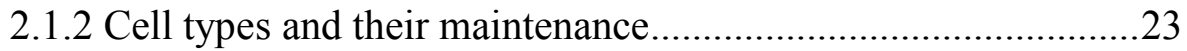

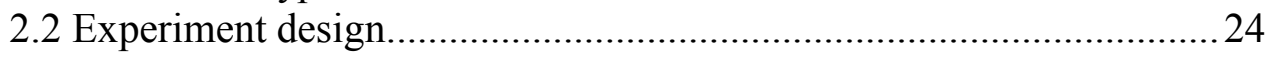

2.2.1. Experiment workflow for pathogen detection.........................24

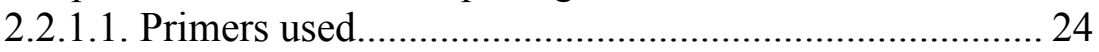




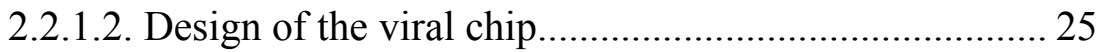

2.2.1.3. Fabrication............................................................... 25

2.2.1.4. Extraction of viral nucleic acids, amplification, and labelling............................................................................ 25

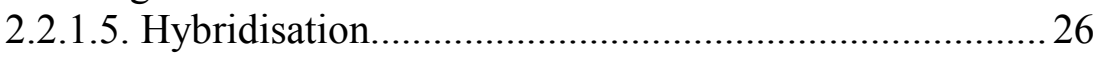

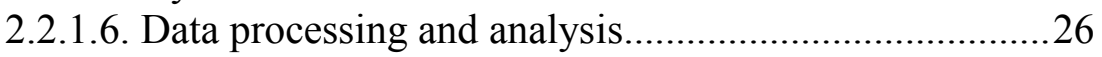

2.2.2. Experiment workflow for RSV infections.............................26

2.2.3. Experiment workflow for influenza A viruses infections........ 28

2.2.4. Experiment workflow for poxviruses infections..................... 29

2.2.5. Quantitative Real-time quantitative PCR (qPCR)...................31

2.3 Bioinformatics analysis of microarray data.......................................32

2.3.1 Gene expression profiles....................................................... 32

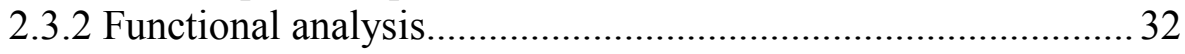

2.3.3 Core analysis...................................................................... 33

Chapter III. A Bioinformatics Approach to Detect Virus using Microarray

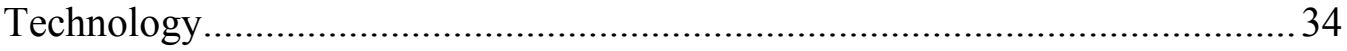

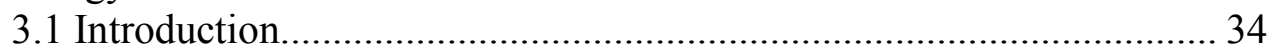

3.1.1 Background of virus identification based on microarray

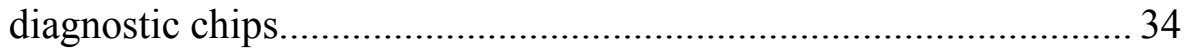

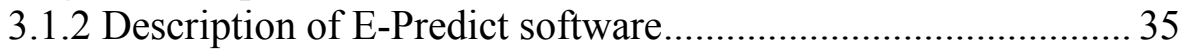

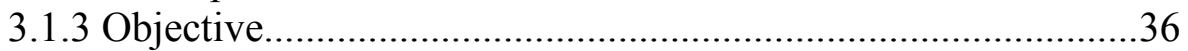

3.2 Experiment workflow of pathogen detection..................................... 37

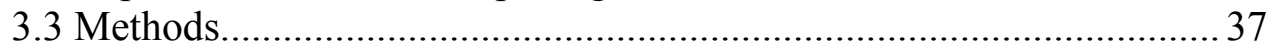

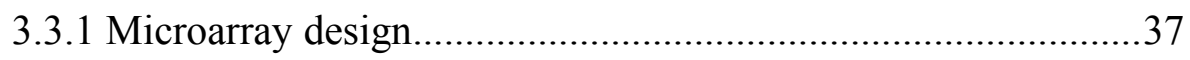

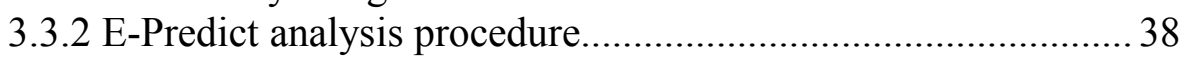

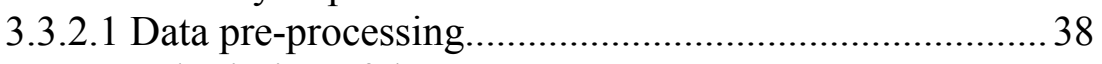

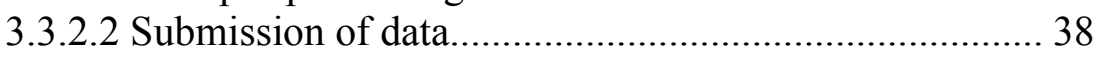

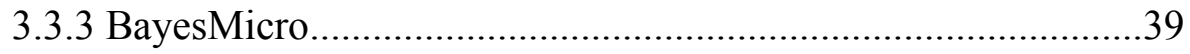

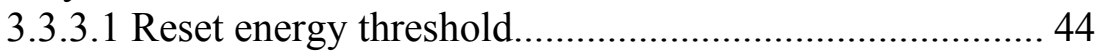

3.3.3.2 Initial data transformation/normalization...................... 44

3.3.3.3 Bayesian model selection.............................................. 46

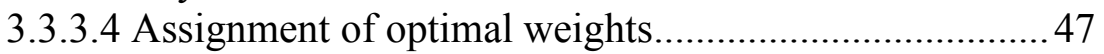

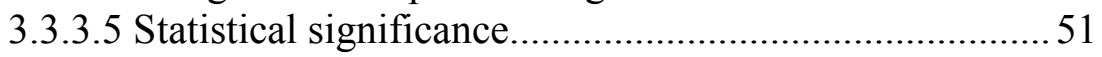

3.3.3.6 Differential detection within virus families....................51

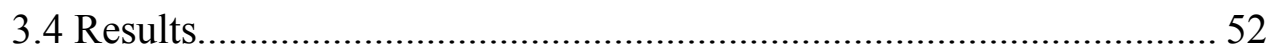

3.4.1 Comparison of different parameters in E-Predict and

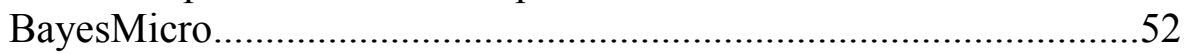

3.4.2 Validation of E-Predict and BayesMicro................................ 54

3.4.3 Global detection at virus species level.................................... 55

3.4.3.1 E-Predict and BayesMicro both indicated good predictive

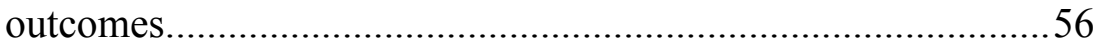

3.4.3.2 BayesMicro performed better than E-Predict.................56

3.4.4 Differential detection within virus families................................56

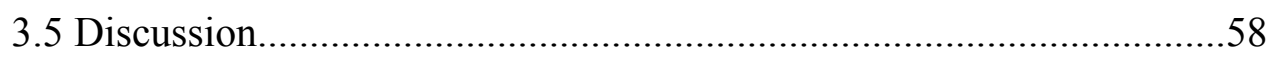

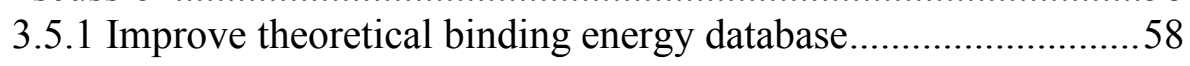

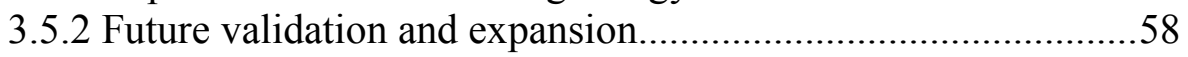

3.5.3 Comparison with E-Predict.................................................. 59

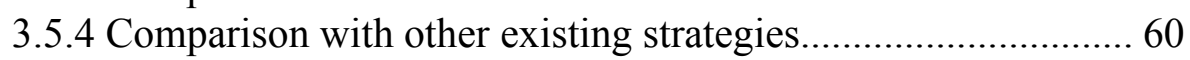

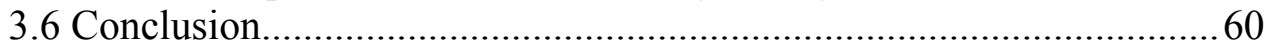

Chapter IV. Respiratory Syncytial Virus.......................................................62 


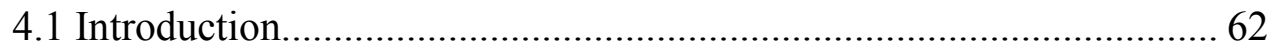

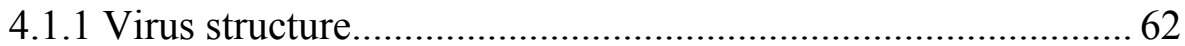

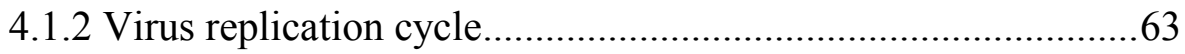

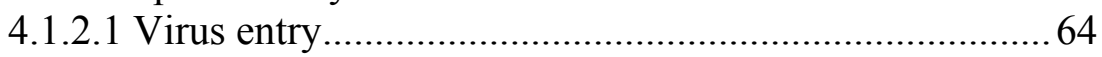

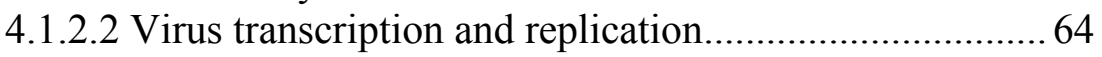

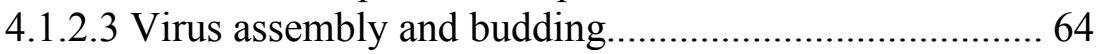

4.1.3 Virus-Host interactions............................................................66

4.1.3.1 Actin cytoskeleton and Rho GTPases.............................66 66

4.1.3.2 Immune response to RSV infection...............................67

4.1.3.3 Cytokine response to RSV infection............................. 68

4.1.3.4 Delayed programmed cell death to facilitate virus

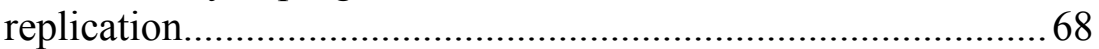

4.1.3.5 NS1/ NS2 - viral antagonists of the host antiviral

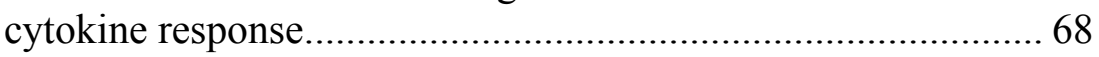

4.1.3.6 The role of $\mathrm{G}$ protein in immune evasion.......................70

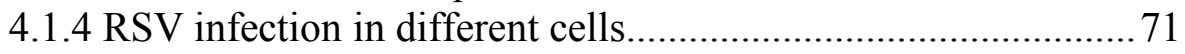

4.1.4.1 RSV infection in Hep2 cells........................................ 71

4.1.4.2 RSV infection in macrophages................................... 71

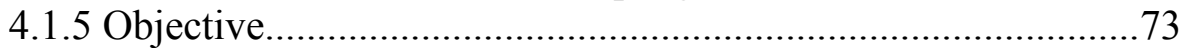

4.3.1 Global profiling of gene expression........................................ 73

4.3.1.1 Heat maps of global gene expression........................... 73

4.3.1.2 Distribution of differentially expressed probe sets.........75

4.3.1.3 Functional classification.............................................. 78

4.3.1.5 Core analysis in macrophages.................................... 86

4.3.2 Functional groups related to host response............................. 88

4.3.2.1 RSV infected Hep2 cells.......................................... 91

4.3.2.1.1 Immune response.............................................91

4.3.2.1.2 Cell death........................................................93

4.3.2.1.3 Cholesterol biosynthesis..................................... 94

4.3.2.1.4 Genes with remarkable regulations..................... 95

4.3.2.2 RSV infected macrophages...................................... 96

4.3.2.2.1 Immune response...........................................96

4.3.2.2.2 Cell death....................................................... 98

4.3.2.2.3 Genes with remarkable regulations.................... 99

4.3.3 Regulations of gene expression in canonical pathways.......... 100

4.3.3.1 Interferon signaling............................................... 100

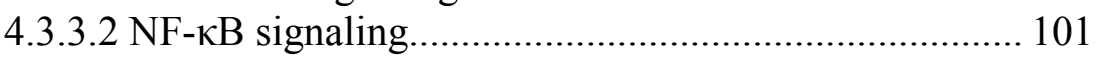

4.3.3.3 Toll-like receptor signaling...................................... 102

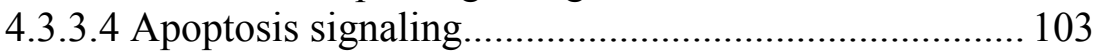

4.3.3.5 Cell cycle: G1/S checkpoint regulation....................... 103

4.3.3.6 Cell cycle: G2/M DNA damage checkpoint regulation104

4.3.3.7 Antigen presentation pathway...................................... 104

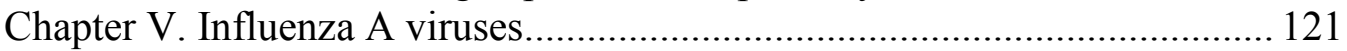

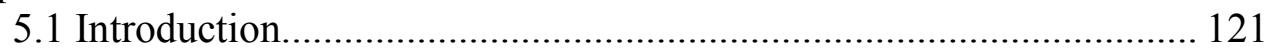

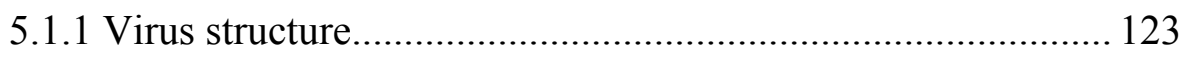

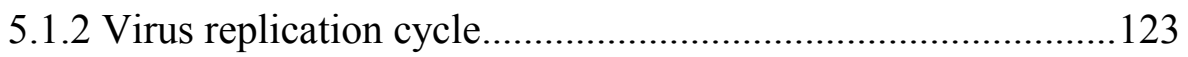

5.1.2.1 Entry of virus into the host cell................................ 124

5.1.2.2 Entry of vRNPs into the nucleus............................... 125

5.1.2.3 Transcription and replication of viral genome............. 126

5.1.2.4 Export of vRNPs from the nucleus.............................127 
5.1.2.5 Assembly and budding of virus.................................. 127

5.1.3 Viral-Host interactions....................................................... 128

5.1.3.1 Activation of the innate type I IFN system................. 130

5.1.3.2 Type I IFN stimulated gene products.......................... 130

5.1.3.2.1 Myxovirus Resistance (Mx)........................... 130

5.1.3.2.2 The 2' - 5' oligoadenylate synthetase and

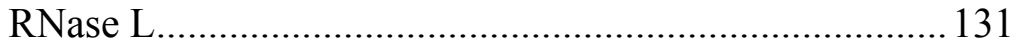

5.1.3.2.3 Viperin (RSAD2) ...........................................131

5.1.3.2.4 The protein kinase R.......................................131

5.1.3.3 Viral strategies to counteract to the innate immune responses

5.1.3.3.1 NS1 - a viral antagonist of the innate immune response

5.1.3.3.2 The role of PB1-F2 ........................................ 133

5.1.4 Influenza A viruses in different hosts..................................134

5.1.4.1 Human................................................................... 134

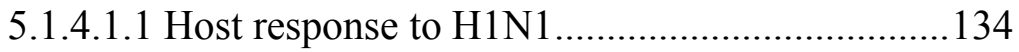

5.1.4.1.2 Host response to $\mathrm{H} 3 \mathrm{~N} 2 \ldots \ldots \ldots \ldots \ldots \ldots \ldots \ldots \ldots \ldots \ldots . . . . .134$

5.1.4.1.3 Host response to Pandemic 2009 H1N1 Swine

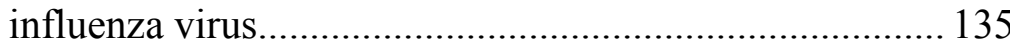

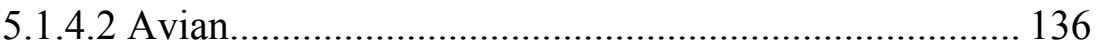

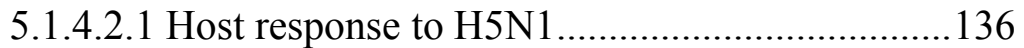

5.1.4.2.2 Host response to H9N2 ...................................137

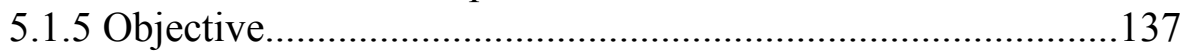

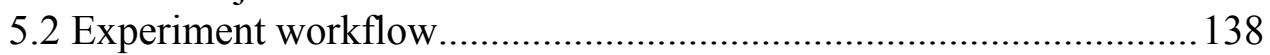

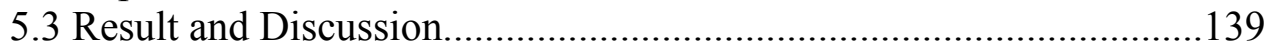

5.3.1 Host gene expression in A549 cells.............................................139

5.3.1.1 Global profiling of gene expression......................................... 139

5.3.1.1.1 Heat maps of global gene expression...................................... 139

5.3.1.1.2 Distribution of differentially expressed probe sets 140

5.3.1.1.3 Functional classification.................................... 140

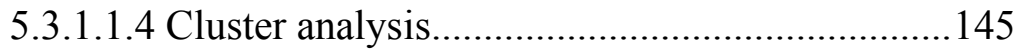

5.3.1.2 Functional groups related to host response................. 157

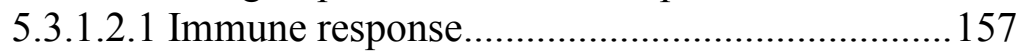

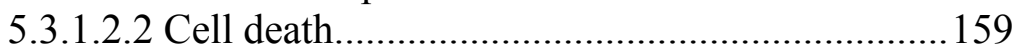

5.3.1.2.3 Genes with remarkable regulations.................. 160

5.3.1.3 Regulations of gene expression in canonical pathways 163

5.3.1.3.1 Interferon signaling pathway........................... 170

5.3.1.3.2 NF- $\kappa \mathrm{B}$ activation by viruses............................ 170

5.3.1.3.3 Role of MAPK signaling in pathogenesis in influenza................................................................... 172

5.3.1.3.4 Role of PI3K/Akt signaling in pathogenesis in influenza virus............................................................. 173

5.3.1.3.5 Role of Wnt/GSK-3 $\beta$ signaling in pathogenesis in

influenza virus.............................................................. 174

5.3.1.3.6 Cell cycle: G1/S and G2/M checkpoints regulation 174

5.3.2 Host gene expression in CEF cells........................................ 176

5.3.2.1 Global profiling of gene expression........................... 176

5.3.2.1.1 Heat maps of global gene expression................ 176

5.3.2.1.2 Distribution of differentially expressed probe sets 176 
5.3.2.1.3 Functional classification....................................179

5.3.2.1.4 Cluster analysis...............................................179

5.3.2.2 Functional groups related to host response................. 185

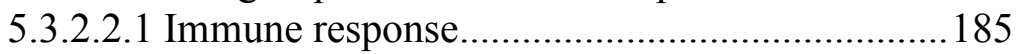

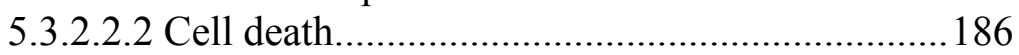

5.3.2.2.3 Genes with remarkable regulations................... 186

5.3.3 Host gene expression in MDCK cells.................................... 188

5.3.3.1 Global profiling of gene expression............................ 188

5.3.3.1.1 Heat maps of global gene expression................ 188

5.3.3.1.2 Distribution of differentially expressed probe sets 188

5.3.3.1.3 Functional classification................................... 189

5.3.3.2 Functional groups related to host response................. 189

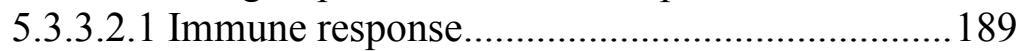

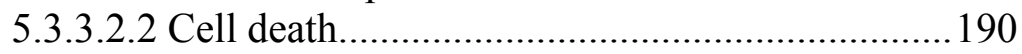

5.3.3.2.3 Genes with remarkable regulations................... 190

5.3.4 Host gene expression in macrophages...................................200

5.3.4.1 Global profiling of gene expression...........................200

5.3.4.1.1 Heat maps of global gene expression................200

5.3.4.1.2 Distribution of differential expressed probe sets200

5.3.4.1.3 Functional classification.................................... 202

5.3.4.1.4 Core analysis in macrophages......................... 204

5.3.4.2. Regulations of gene expression in canonical pathways 215

5.3.4.2.1 Interferon signaling...................................... 215

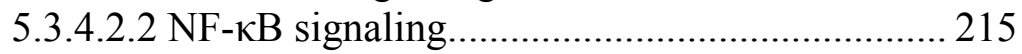

5.3.4.2.3 Toll-like receptor signaling............................. 215

5.3.4.2.4 Antigen presentation pathway......................... 216

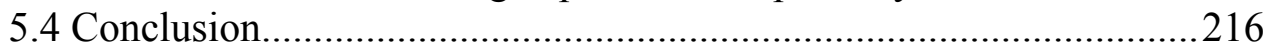

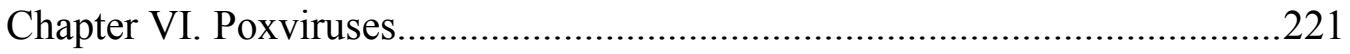

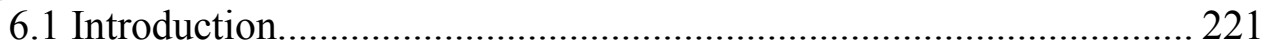

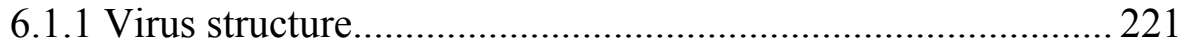

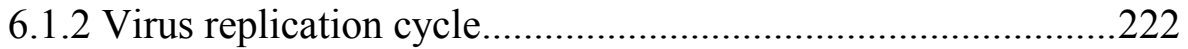

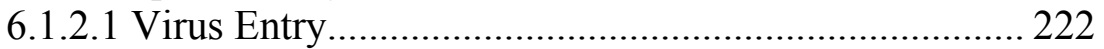

6.2.1.2 DNA release from the core to cytoplasm.................... 222

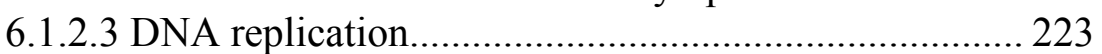

6.1.2.4 Virus assembly..................................................... 223

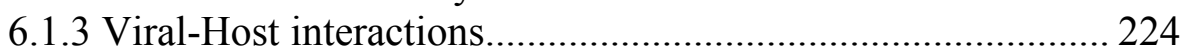

6.1.3.1 Blockade of interferon response................................224

6.1.3.2 Suppression of cytokine signaling............................225

6.1.3.3 Inhibition of TNF-induced responses........................226

6.1.3.4 T-cell evasion by repression of MHC I expression......227

6.1.3.5 Blockade of host cell apoptosis................................. 227

6.1.4 Poxviruses infections in different host cells...........................228

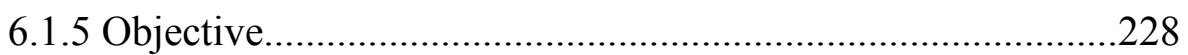

6.3.1 Global Profiling of gene expression...................................... 230

6.3.1.1 Heat maps of global gene expression......................... 230

6.3.1.2 Distribution of differentially expressed probe sets.......231

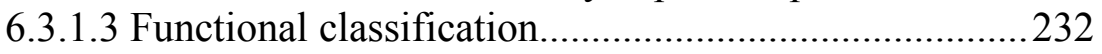

6.3.1.4 Cluster analysis in A549 cells................................... 245

6.3.1.5 Core analysis in mouse RAW cells............................ 254 
6.3.1.6 Selection of common genes modulated by infections of different poxviruses in mouse RAW cells...............................256

6.3.2 Regulations of gene expression in canonical pathways.......... 273

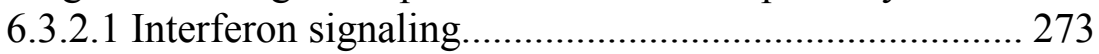

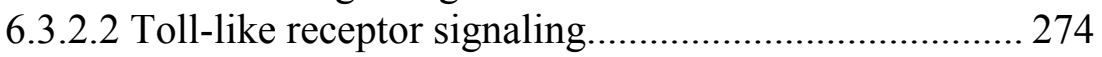

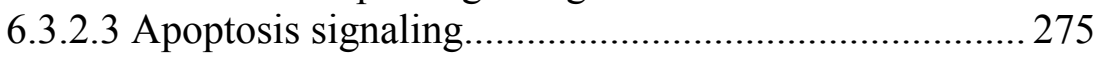

6.3.2.4 Cell cycle: G1/S checkpoint regulation......................276

6.3.2.5 Antigen presentation pathway................................. 277

Chapter VII. Conclusion and Future Work................................................... 281

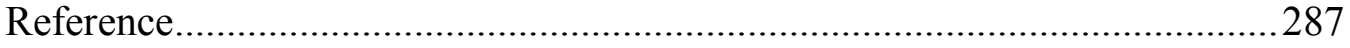

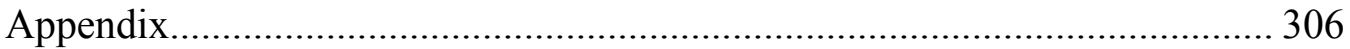

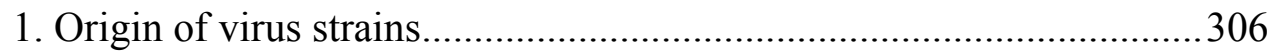

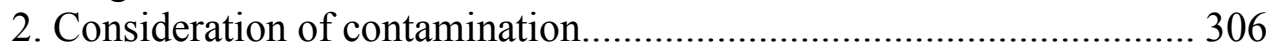

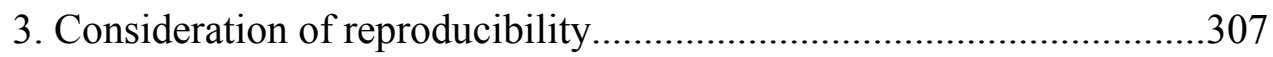

4. Consideration of results that a gene transcript showed down-regulated expression at a time point but less down-regulated expression at a later time point

5. The productivity of the infection of RSV during the whole investigated infection time course for each of the cell types.

6. Relationship between the time of the analyses and different steps of virus replication cycle in RSV study.

7. Selection of multiplicity of infection in our influenza viruses study... 312

8. The infectivity of each of the viruses for each of the cell types in our influenza viruses study.

9. Selection of multiplicity of infection in our poxviruses study.....

10. Correlation between results from microarray study and the stage of virus infection in our poxviruses study.....

11. Perl script for data pre-processing before E-Predict analysis.

12. Perl script for functional classification.

13. Perl script for venndiagram 


\section{LIST OF FIGURES}

Figure 1.1 Entry pathway of an enveloped animal virus....................2

Figure 1.2 Toll-like receptors recognize nucleic acids.......................4

Figure 1.3 Apoptosis signaling pathway.................................. 7

Figure 1.4 The IFN receptors and Jak-STAT signaling..................... 8

Figure 1.5 MAP kinase pathways in mammals............................. 10

Figure 1.6 Strategy to identify viruses based on microarray technology ........14

Figure 3.1 The experiment workflow which was performed in DSO national

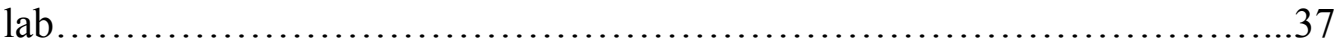

Figure 3.2 User interface of E-Predict.................................4 41

Figure 3.3 Final result generated from E-Predict...........................42

Figure 3.4 An overview of the BayesMicro strategy......................43

Figure 3.5 A normal distribution curve....................................49

Figure 4.1 The structure organization of RSV genome.....................6 63

Figure 4.2 Overview of RSV replication cycle............................65

Figure 4.3 RSV biding and triggering of cellular responses...................69

Figure 4.4 Microarray experimental workflow during RSV infection......... 74

Figure 4.5 Temporal changes in the host cell transcriptome in Hep2 cells infected with RSV ................................................. 76

Figure 4.6 Temporal changes in the host cell transcriptome in macrophages infected with RSV ................................................... 77

Figure 4.7 Overview of distributions of differentially expressed probe sets into different biological functions in Hep2 cells infected with RSV ..............82

Figure 4.8 Overview of distributions of differentially expressed genes into different biological functions in macrophages infected with RSV ...........84 Figure 4.9 Clustering analysis of temporal gene expression profiles in RSVinfected Hep2 cells.....................................................85

Figure 4.10 Summary of top functional groups enriched in differentially expressed genes in RSV-infected with macrophages at 4 hpi................89 Figure 4.11 Top 20 (A) biological functions and (B) canonical pathways significantly enriched in differentially expressed genes from RSV-infected

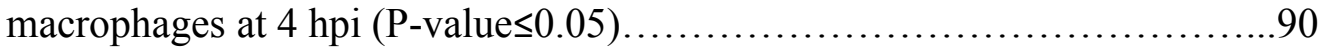

Figure 4.12 Summary of top functional groups enriched in differentially expressed genes in RSV-infected with macrophages at 24 hpi................91 Figure 4.13 Top 20 (A) biological functions and (B) canonical pathways significantly enriched in differentially expressed genes from RSV-infected

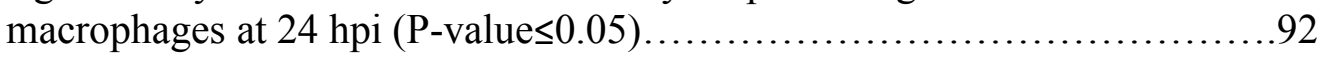

Figure 4.14 Expression of probe sets involved in immune response in RSVinfected Hep2 cells.....................................................110

Figure 4.15 Expression of probe sets involved in cell death in RSV-infected Hep2 cells......................................................112

Figure 4.16 Expression of probe sets involved in cholesterol biosynthesis in RSV-infected Hep2 cells............................................ 114 Figure 4.17 The probe sets with topmost expression in RSV-infected Hep2 cells................................................................. 116 Figure 4.18 Expression of genes involved in immune response in RSV-infected macrophages...................................................... 118 Figure 4.19 Expression of genes involved in cell death in RSV-infected macrophages......................................................119 
Figure 4.20 The genes with topmost expression in RSV-infected macrophage ........................................................... 120

Figure 5.1 Classification of IAV based on HA........................... 124

Figure 5.2 IAV genome structure..................................... 124

Figure 5.3 Replication cycle of IAV ..................................... 126

Figure 5.4 Cap-snatching transcription mechanism.......................128

Figure 5.5 Strategy of the newly assembled IAV RNPs exporting from the nucleus to the cytoplasm............................................. 129

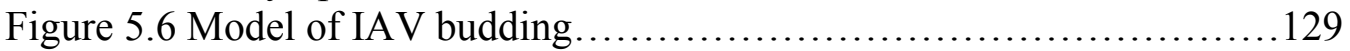

Figure 5.7 The origin of pandemic H1N1 2009 virus (pH1N1)....................135

Figure 5.8 Microarray experiment workflow during influenza A strains infections............................................................139

Figure 5.9 Temporal changes in the host cell transcriptome in A549 cells infected by four influenza virus strains................................. 142

Figure 5.10 Overview of distributions of differentially expressed probe sets into different biological functions in A549 cells infected with influenza A viruses........................................................ 144

Figure 5.11 Clustering analysis of temporal gene expression profiles in H1N1infected A549 cells.................................................150

Figure 5.12 Clustering analysis of temporal gene expression profiles in $\mathrm{pH} 1 \mathrm{~N} 1-$ infected A549 cells....................................................152

Figure 5.13 Clustering analysis of temporal gene expression profiles in H5N2/F118-infected A549 cells...........................................154 Figure 5.14 Clustering analysis of temporal gene expression profiles in H9N2infected A549 cells........................................................156 Figure 5.15 Expression of probe sets involved in immune response in influenza A viruses-infected A549 cells........................................ 166

Figure 5.16 Expression of probe sets involved in cell death in influenza A viruses-infected A549 cells...........................................168

Figure 5.17 The probe sets with topmost expression changes in influenza A viruses-infected A549 cells..........................................169

Figure 5.18 Two virus supportive functions of the $\mathrm{IKK} / \mathrm{NF}-\kappa \mathrm{B}$ signaling module in influenza virus infected cells...................................171

Figure 5.19 Temporal changes in the host cell transcriptome in CEF cells infected by four influenza virus strains...............................178

Figure 5.20 Overview of distributions of differentially expressed probe sets into different biological functions in CEF cells infected with influenza A viruses.183 Figure 5.21 Clustering analysis of temporal gene expression profiles in H1N1infected CEF cells...................................................181

Figure 5.22 Clustering analysis of temporal gene expression profiles in H5N2/F118-infected CEF cells.....................................181

Figure 5.23 Clustering analysis of temporal gene expression profiles in H9N2infected CEF cells.....................................................185 Figure 5.24 Expression of probe sets involved in immune response in influenza A viruses-infected CEF cells.

Figure 5.25 Expression of probe sets involved in cell death in influenza A viruses-infected CEF cells............................................192 Figure 5.26 The probe sets with topmost expression changes in influenza A viruses-infected CEF cells. 
Figure 5.27 Temporal changes in the host cell transcriptome in MDCK cells infected by four influenza virus strains.................................. 194 Figure 5.28 Overview of distributions of differentially expressed probe sets into different biological functions in MDCK cells infected with influenza A viruses

Figure 5.29 The expression of probe sets involved in immune response in influenza A viruses-infected MDCK cells.

Figure 5.30 The expression of probe sets involved in cell death in influenza A viruses-infected MDCK cells

Figure 5.31 The probe sets with topmost expression changes in influenza A viruses-infected MDCK cells

Figure 5.32 Temporal changes in the host cell transcriptome in macrophages infected with influenza A strains.........................................201

Figure 5.33 Overview of distributions of differentially expressed genes into different biological functions in macrophages infected with influenza A strains

Figure 5.34 Summary of top functional groups enriched in differentially expressed genes in H1N1-infected with macrophages at 2 hpi..............205

Figure 5.35 Top 20 (A) biological functions and (B) canonical pathways significantly enriched in differentially expressed genes from H1N1-infected

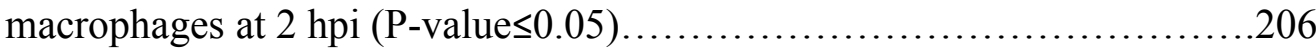

Figure 5.36 Summary of top functional groups enriched in differentially expressed genes in H1N1-infected with macrophages at 24 hpi..............207 Figure 5.37 Top 20 (A) biological functions and (B) canonical pathways significantly enriched in differentially expressed genes from H1N1-infected macrophages at 24 hpi (P-value $\leq 0.05)$. 208

Figure 5.38 Summary of top functional groups enriched in differentially expressed genes in H5N2/F118-infected with macrophages at 2 hpi..........209 Figure 5.39 Top 20 (A) biological functions and (B) canonical pathways significantly enriched in differentially expressed genes from H5N2/F118infected macrophages at $2 \mathrm{hpi}(\mathrm{P}$-value $\leq 0.05)$. 210

Figure 5.40 Summary of top functional groups enriched in differentially expressed genes in H5N2/F118-infected with macrophages at 24 hpi............211 Figure 5.41 Top 20 (A) biological functions and (B) canonical pathways significantly enriched in differentially expressed genes from H5N2/F118-

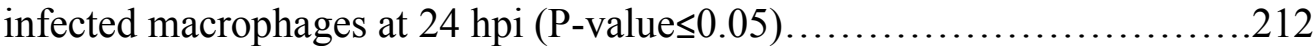
Figure 5.42 Summary of top functional groups enriched in differentially expressed genes in H5N3-infected with macrophages at 24 hpi..............213 Figure 5.43 Top 20 (A) biological functions and (B) canonical pathways significantly enriched in differentially expressed genes from H5N3-infected

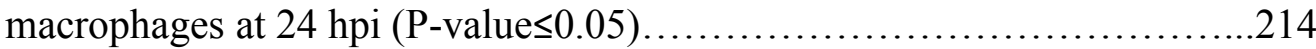

Figure 6.1 Interplay between IFN-signaling pathways and vaccinia virus proteins.........................................................226

Figure 6.2 Microarray experimental workflow during different types of poxviruses infections.............................................230 Figure 6.3 Temporal changes in the host cell transcriptome in A549 cells infected by two types of poxviruses....................................235 Figure 6.4 Temporal changes in the host cell transcriptome in RAW cells infected by three types of poxviruses..................................236 
Figure 6.5 Overview of distributions of differentially expressed probe sets into different biological functions in A549 cells infected with cowpox virus.....238 Figure 6.6 Overview of distributions of differentially expressed probe sets into different biological functions in A549 cells infected with lister virus.........240 Figure 6.7 Overview of distributions of differentially expressed probe sets into different biological functions in RAW cells infected with three types of poxviruses at 2 hpi............................................. 242

Figure 6.8 Overview of distributions of differentially expressed probe sets into different biological functions in RAW cells infected with three types of

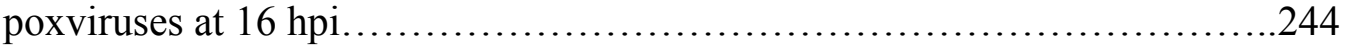

Figure 6.9 Clustering analysis of temporal gene expression profiles in cowpox virus-infected A549 cells.................................................248

Figure 6.10 Clustering analysis of temporal gene expression profiles in lister virus-infected A549 cells..................................................249

Figure 6.11 Clustering analysis of temporal gene expression profiles in cowpox virus-infected A549 cells..............................................251

Figure 6.12 Clustering analysis of temporal gene expression profiles in lister virus-infected A549 cells............................................254

Figure 6.13 Summary of top functional networks of differentially expressed genes in cowpox virus-infected mouse RAW cells at 2 hpi.................259 Figure 6.14 Top 20 (A) biological functions and (B) canonical pathways significantly enriched in differentially expressed genes from cowpox virusinfected mouse RAW cells at 2 hpi (P-value $\leq 0.05) \ldots \ldots \ldots \ldots \ldots \ldots \ldots \ldots . . . . . . . .260$

Figure 6.15 Summary of top functional networks of differentially expressed genes in cowpox virus-infected mouse RAW cells at 16 hpi.................261 Figure 6.16 Top 20 (A) biological functions and (B) canonical pathways significantly enriched in differentially expressed genes from cowpox virusinfected mouse RAW cells at 16 hpi $(\mathrm{P}$-value $\leq 0.05) \ldots \ldots \ldots \ldots \ldots \ldots \ldots . \ldots 262$ Figure 6.17 Summary of top functional networks of differentially expressed genes in lister virus-infected mouse RAW cells at 2 hpi.....................263 Figure 6.18 Top 20 (A) biological functions and (B) canonical pathways significantly enriched in differentially expressed genes from lister virusinfected mouse RAW cells at 2 hpi (P-value $\leq 0.05) \ldots \ldots \ldots \ldots \ldots \ldots \ldots . \ldots . \ldots . \ldots . \ldots 264$ Figure 6.19 Summary of top functional networks of differentially expressed genes in lister virus-infected mouse RAW cells at 16 hpi...................265 Figure 6.20 Top 20 (A) biological functions and (B) canonical pathways significantly enriched in differentially expressed genes from lister virus-

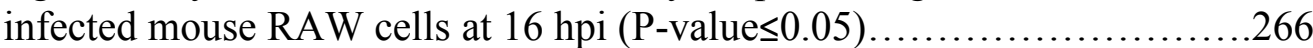

Figure 6.21 Summary of top functional networks of differentially expressed genes in ectromelia virus-infected mouse RAW cells at 2 hpi...............267 Figure 6.22 Top 20 (A) biological functions and (B) canonical pathways significantly enriched in differentially expressed genes from ectromelia virus-

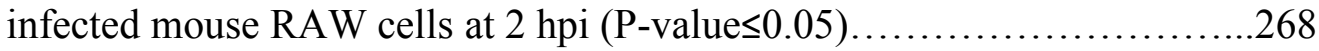

Figure 6.23 Summary of top functional networks of differentially expressed genes in ectromelia virus-infected mouse RAW cells at 16 hpi...............269 Figure 6.24 Top 20 (A) biological functions and (B) canonical pathways significantly enriched in differentially expressed genes from ectromelia virusinfected mouse RAW cells at 16 hpi (P-value $\leq 0.05) \ldots \ldots \ldots \ldots \ldots \ldots \ldots . . . \ldots 270$

Figure 6.25 Venn diagram of differentially up-regulated probe sets between three poxviruses infections. 
Figure 6.26 Venn diagram of differentially down-regulated probe sets between three poxviruses infections............................................. 272 


\section{LIST OF TABLES}

Table 1.1 Advantages and disadvantages of different strategies which are applied in genomic investigation during virus infection................... 12

Table 1.2 Different types of microarray chips in virus detection................14

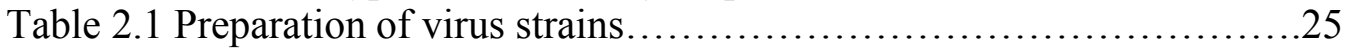

Table 2.2 Design of experiments in RSV infected host cells..................29

Table 2.3 Design of experiments in influenza viruses infected host cells........30

Table 2.4 Design of experiments in influenza viruses infected mouse macrophages..........................................................30

Table 2.5 Design of experiments in poxviruses infected host cells.............31

Table 3.1 Theoretical binding energy profiles.............................. 36

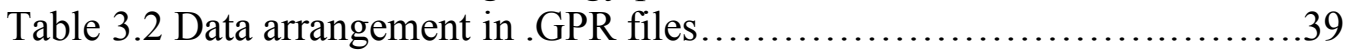

Table 3.3 Final .VDAR files generated from the .GPR files...................40

Table 3.4 Comparison of detection results analyzed based on different thresholds.............................................................43

Table 3.5 The theoretical binding energy profile (A) is rearranged by probes

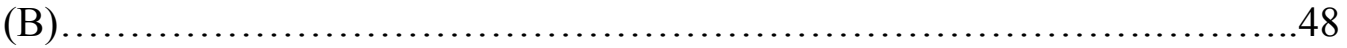

Table 3.6 Calculation of the weights for each theoretical binding energy value...............................................................49

Table 3.7 Transformation of the matrix of theoretical binding energy .........50 Table 3.8 Comparison of different parameters used in E-Predict and BayesMicro..................................................... 54

Table 3.9 Summary of results from E-Predict and BayesMicro...............54

Table 3.10 Virus detection in samples infected with Yellow fever virus........55

Table 3.11 Virus detection in samples infected with Japanese encephalitis virus............................................................... 55

Table 3.12 Virus detection in samples infected with Influenza B virus.........56

Table 3.13 Virus detection in samples infected with influenza A virus (H1N1) ..........................................................5 57

Table 3.14 Virus detection in samples infected with vaccinia virus............59

Table 4.1 Differentially expressed probe sets in Hep2 cells infected with

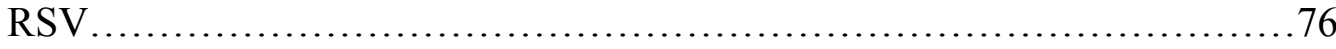

Table 4.2 Differentially expressed genes in macrophages infected with RSV..77 Table 4.3 Summary of functional groups, canonical pathways and transcription factors enriched based on differentially expressed genes in Hep2 cells infected with RSV .........................................................86

Table 5.1 Differentially expressed probe sets in A549 cells infected with influenza A viruses at 10 hpi...

Table 5.2 Summary of functional groups, canonical pathways and transcription factors enriched based on differentially expressed genes in A549 cells infected with $\mathrm{H} 1 \mathrm{~N} 1$.

Table 5.3 Summary of functional groups, canonical pathways and transcription factors enriched based on differentially expressed genes in A549 cells infected with $\mathrm{pH} 1 \mathrm{~N} 1$ 153

Table 5.4 Summary of functional groups, canonical pathways and transcription factors enriched based on differentially expressed genes in A549 cells infected with H5N2/F118. 
Table 5.5 Summary of functional groups, canonical pathways and transcription factors enriched based on differentially expressed genes in A549 cells infected with H9N2 156

Table 5.6 Differentially expressed probe sets in CEF cells infected with influenza A viruses at 10 hpi....

Table 5.7 Summary of functional groups enriched based on differentially expressed genes in CEF cells infected with H1N1 .......................... 184

Table 5.8 Summary of functional groups enriched based on differentially expressed genes in CEF cells infected with H5N2/F118 .184

Table 5.9 Summary of functional groups enriched based on differentially expressed genes in CEF cells infected with H9N2 ......................... 184

Table 5.10 Differentially expressed probe sets in MDCK cells infected with

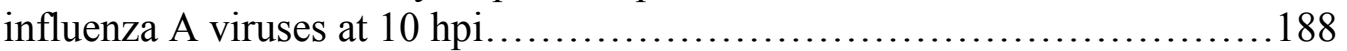

Table 5.11 Differentially expressed genes in macrophages infected with influenza A strains......................................................201

Table 6.1 Differentially expressed probe sets in A549 cells infected with poxviruses at different time points....................................234

Table 6.2 Differentially expressed probe sets in RAW cells infected with poxviruses at different time points.....................................235

Table 6.3 Summary of functional groups, canonical pathways, transcription factors and microRNAs enriched based on up-regulated genes in A549 cells infected with cowpox virus.............................................248

Table 6.4 Summary of functional groups, canonical pathways, transcription factors and chromosome locations enriched based on up-regulated genes in A549 cells infected with lister virus. 250

Table 6.5 Summary of functional groups, canonical pathways, transcription factors, chromosome locations and microRNAs enriched based on downregulated genes in A549 cells infected with cowpox virus .252

Table 6.6 Summary of functional groups, canonical pathways, transcription factors and microRNAs enriched based on down-regulated genes in A549 cells infected with lister virus. .253 


\section{ABBREVIATIONS}

AIDS

AP1

ATCC

AVA

BCL10/9L

BIRC3

BTG1

$\mathrm{CDC}$

CDF

CEF

$\mathrm{CH} 25 \mathrm{H}$

CRISPR

CRM1

DF

EEV

EGF

eIF $2 \alpha$

ELISA

EM

ER

F

FPPS

G

GAGs

GO

HA

HIV

HMM

HP

HPAI

HRSV

HS
Acquired Immunodeficiency Syndrome

Activator Protein-1

American Type Culture Collection

Agri-Food and Veterinary Authority of Singapore

B-cell CLL/lymphoma 10/9-like

Baculoviral IAP repeat-containing protein 3

B-cell Translocation Gene 1

Centers for Disease Control and Prevention

Cumulative Distribution Function

Chick Embryo Fibroblast

Cholesterol 25-hydroxylase

Clusters of Regularly Interspaced Short Palindromic Repeats

Chromosome Region Maintenance protein 1

Dengue Fever

Extracellular Enveloped Virions

Early Growth Response

Eukaryotic Translation Initiation Factor 2 alpha subunit

Enzyme-linked Immunosorbent assay

Eectron Microscope

Endoplasmic Reticulum

Fusion Protein

Farnesyl Diphosphate Synthase

Glyco-protein

Glycosaminoglycans

Gene Ontology

Haemagglutinin

Human immunodeficiency virus

Hidden Markov Model

Heptad Repeat

High Pathogenic Avian Influenza Virus

Human Respiratory Syncytial Virus

Heparin Sulphate 


\begin{tabular}{|c|c|}
\hline IAP & Inhibitor of Apoptosis \\
\hline IAV & Influenza A viruses \\
\hline IBs & Inclusion Bodies \\
\hline IFA & Immunofluorescence Assay \\
\hline IFAR & IFN- $\alpha$ receptor \\
\hline IFN & Interferon \\
\hline IFRG & Interferon Responsive Gene \\
\hline IKK & IкB Kinase \\
\hline IMV & Mature Virions \\
\hline IPA & Ingenuity Pathways Analysis \\
\hline IPS & IFN $\beta$ Promoter Stimulator \\
\hline IRAK & Interleukin-1 Receptor-associated Kinase \\
\hline IRF & Interferon Regulatory Factor \\
\hline ISG & IFN Stimulated Gene \\
\hline ISRE & Interferon Sensitive Response Elements \\
\hline IV & Immature Virion \\
\hline IVNS1ABP & Influenza Virus NS1A Binding Protein \\
\hline $\mathrm{I} \kappa \mathrm{B}$ & Inhibitor of NF-Kb \\
\hline JAK & Janus-family Protein Tyrosine Kinases \\
\hline KEGG & Kyoto Encyclopedia of Genes and Genomes \\
\hline KEREN & Kernel Embedding of Regulatory Networks \\
\hline KL & Kullback-Leibler \\
\hline $\mathrm{L}$ & Large Protein \\
\hline LPAI & Low Pathogenic Avian Influenza Virus \\
\hline LPS & Lipopolysaccharide \\
\hline LRTI & Lower Respiratory Tract Infections \\
\hline M & Matrix Protein \\
\hline MAPK & Mitogen-activated Protein Kinase \\
\hline MDA & Microbial Detection Array \\
\hline MDA5 & Melanoma Differentiation Associated Gene 5 \\
\hline MDCK & Madin Darby Canine Kidney \\
\hline $\mathrm{MHC}$ & Major Histocompatibility Complex \\
\hline MOI & Muiltiplicity Of Infection \\
\hline
\end{tabular}




\begin{tabular}{|c|c|}
\hline MT & Microtubule \\
\hline Mx & Myxovirus Resistance \\
\hline $\mathrm{N}$ & Nucleo-protein \\
\hline NA & Neuraminidase \\
\hline NES & Nuclear Export Signal \\
\hline$N F-\kappa B$ & Nuclear factor kappa B \\
\hline NGS & Next Generation Sequencing \\
\hline NLRs & $\begin{array}{l}\text { Nucleotide-binding Domain and Leucine-rich-repeat- } \\
\text { containing Proteins }\end{array}$ \\
\hline NLSs & Nuclear Localization Signals \\
\hline NO & Nitric Oxide \\
\hline NP & Nucleoprotein \\
\hline NPC & Nuclear Pore Complex \\
\hline NS1 & Non-structural Protein 1 \\
\hline NS2 & Non-structural Protein 2 \\
\hline OAS & Oligoadenylate Synthetases \\
\hline ORF & Open Reading Frame \\
\hline $\mathrm{P}$ & Phosphor-protein \\
\hline PA & Polymerase Acidic \\
\hline PB1 & Polymerase Basic 1 \\
\hline PB2 & Polymerase Basic 2 \\
\hline PI3K & Phosphatidylinositoe-3-Kinase \\
\hline PKB & Protein Kinase B \\
\hline PKR & Protein kinase $\mathrm{R}$ \\
\hline PRR & Pathogen Recognition Receptor \\
\hline RT-PCR & Reverse Transcription - Polymerase Chain Reaction \\
\hline RIG-I & RNA Helicases Retinoic Acid Inducible Gene-I \\
\hline RNP & Ribonucleoprotein \\
\hline RSAD2 & Viperin \\
\hline RSV & Respiratory Syncytial Virus \\
\hline SARS & Severe Acute Respiratory Syndrome \\
\hline SAGE & Serial Analysis of Gene Expression \\
\hline SH & Small Hydrophobic Protein \\
\hline
\end{tabular}


Systemic Lupus Erythematosus

SOCS

Suppressor of Cytokine Signalling

STAT

Signal Transducers and Activators of Transcription

TBK1

TANK-binding kinase 1

TF

Transcriptional Factor

TLR

Toll-like Receptor

TNF

Tumor Necrosis Factor

TRIM25

Tripartite Motif 25

VFs

Virus Filaments

vRNA

viral genome RNA

vRNP

viral ribonucleoprotein

WHO

World Health Organization

WV

Wrapped Virion

XAF1

XIAP associated Factor-1

ZBP1

Z-DNA-binding Protein 1

ZNF

Zinc Finger 


\section{SUMMARY}

Viruses are major factors of human infectious diseases. They not only threaten the public health but also cause economic loss in the worldwide. During the past decades, more and more cases of virus infections in human beings have been reported. Thus, establishment of an accurate pathogen diagnostic system is urgent for clinical virus identification. Moreover, deep understanding of the transcriptomic profiles in typical virus-infected cells is also imperative for vaccination, drug design as well as other medical treatment in the future.

Microarray as an advanced technology has been well developed and extensively applied in last decades. In the field of virology studies, microarray technique has been widely applied in the pathogen diagnosis and transcriptomic examination of host cells upon pathogen invasion. With the application of this advanced technique, a large scale of data is generated at one time, which cause the high demand of bioinformatics tools in data interpretation.

In one project, DNA microarrays were applied in pathogen detection. To interpret the data from diagnostic microarray chips, a novel computational software, BayesMicro, was designed and established. The virus identification results from BayesMicro were then compared with the results from previously published software called E-Predict. And the final comparison demonstrated that BayesMicro was capable of generating positive predictions in all 12 tested arrays, indicating that this software performed effectively and could be applied in virus detection as an alternative tool.

In other projects, global gene expression profiles were examined for comprehensive understanding of the host-virus interactions in different host cells under different viruses infections using microarray system. The preprocessing of the microarray data was performed under GeneSpring GX 11.0 software. Other softwares such as Expander 5.0 and Ingenuity Pathway Analysis were applied to perform further functional annotation. The final results demonstrated that different global gene expression profiles were observed in different host cells infected with different viruses. Genes in key biological groups and canonical pathways that have been reported previously showed differential regulations at their expression levels. In addition to these well- 
known cellular processes, other interesting networks were also significantly representative in our data. 


\section{Chapter I. General Introduction}

Viruses are major factors of human infectious diseases. With more and more cases of virus infections in human beings reported in last decades, viruses have become threats not only to public health but also to the worldwide economy. For example, respiratory syncytial virus (RSV) and influenza viruses are important causes of respiratory tract infections. The former contributed to 34 million cases of lower respiratory tract infections (LRTI) in children $<5$ years of age and led to 66,000 to 199,000 mortalities majorly in children and elderly patients in 2005 [1]. The latter commonly cause 3 to 5 million clinical infections and 250,000 to 500,000 fatal cases annually [2]; Dengue fever (DF) caused by one serotype of dengue virus is one of the most common widespread vector borne diseases, with 100 million cases reported annually [3]; Human immunodeficiency virus (HIV) is a kind of virus that enables to cause acquired immunodeficiency syndrome (AIDS), in which humans would get progressive failure of their immune system. It has been estimated that 86,500 people in the UK were infected with this virus only according to 2009 statistics. In terms of these data, it is urgent and necessary to establish an accurate pathogen diagnostic system. Moreover, deep understanding of the host-virus interactions in different viruses-infected cells is also imperative for vaccination, drug design as well as other medical treatment in the future.

\subsection{Outline of virus replication cycle}

All viruses share the same basic replication cycle, but the time involved depends on a number of factors, including the size and genetic complexity of the virus itself as well as the nature of the host cells [5].

\subsubsection{Virus entry}

Viruses first recognize and bind to cells that they infect. Virus-coded proteins on the surface of the virion bind to specific proteins, carbohydrate or lipid on the cell surface. Structural surface proteins of many viruses bind to carbohydrate residues found in surface glycoproteins and glycolipids that are widely distributed on many cell types. 
Once they locate on the cell surface, some enveloped animal viruses fuse their lipid envelopes directly with the plasma membrane of the cell, releasing the viral capsid and genome into the host cell. Some other animal viruses are directly taken up into the cytoplasm in vesicles formed at the plasma membrane. These vesicles then release the virion or its genome into the cytoplasm, and the genome may be further transported into the nucleus [Figure $1.1]$.

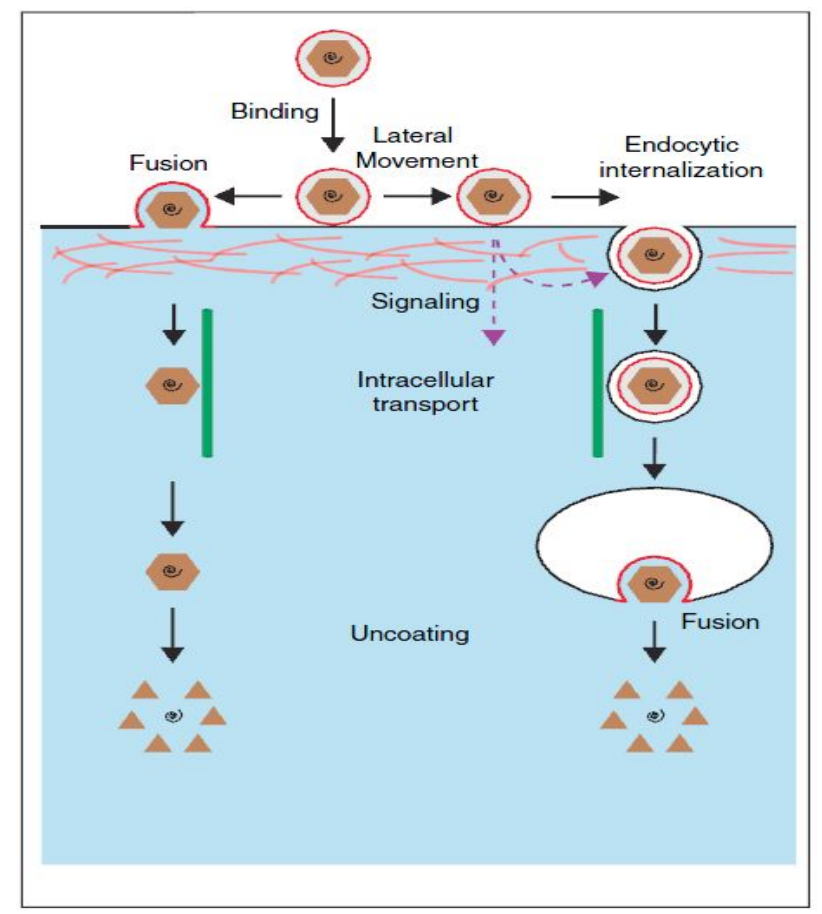

Figure 1.1 Entry pathway of an enveloped animal virus (adapted from Schmidt $\mathrm{F}$ et al., 2012)[311]. Enveloped animal viruses enter host cells in a stepwise process: viral particles bind to attachment factors in the host cell plasma membrane and may undergo lateral movement. Virions may employ two entry routes: fusion at the plasma membrane or endocytosis. The latter may be triggered by virus-receptor interactions, followed by vesicular transport. Maturation of endosomes provides the cues for membrane fusion. Capsid release, whether at the plasma membrane or from an intracellular vesicle, is followed by intracellular transport of capsids and genome uncoating at the site of viral replication.

\subsubsection{Genome replication}

Once delivered into the appropriate compartment of the cell, the viral genomes express some early proteins which enable to promote the replication of viral genome. All RNA viruses should synthesize an RNA-dependent RNA polymerase to replicate their genomes. In this process, a variety of cellular proteins also assist the RNA polymerase to complete the replication of viral genomes. 
DNA viruses always produce early proteins that enable to induce the production of a batch of cellular enzymes, and these enzymes are able to assist virus in its genome replication. Thousands of copies of genome can be synthesized in one cell, and these genomes can be treated as a template for synthesis of viral mRNAs or further genome replication.

\subsubsection{Virus assembly}

Stuctrural proteins always participate in packing the viral genomes and assembling the capsid in infected host cells. The simplest virus capsids consist of one protein that forms either a closed shell or a helical tube within which the viral genoms is packed. Large and complex viruses may have numerous capsids proteins. Enveloped viruses code glycoproteins that are inserted into lipid membranes to directly form a viral envelop, and this process is always called budding. Once the progeny virions are formed, they leave the cell and infect new host cells, in which another replication cycle is initiated.

\subsection{Host responses to viruses and viral strategies to counter host responses}

To stay healthy and survive, organisms must defend themselves against invading pathogens, including viruses. Upon the virus invasion, host intrinsic cellular defense system is initiated rapidly. A variety of cellular proteins are involved in this antiviral process: toll-like receptors (TLRs) are responsible for detecting invading pathogens; cytokines, including interferons, are secreted to inhibit virus replication; a self-destruction process called apoptosis is induced to reduce the spread of virus infection.

\subsubsection{TLRs}

TLRs are a class of membrane-spanning receptors that consist of an extracellular leucine-rich repeat domain, a transmembrane-spanning domain and a cytoplasmic Toll-interleukin-1 receptor-resistance domain. In recent years, TLR family has been discovered and characterized as an important member in pattern-recognition receptors (PRRs), which detect pathogen-associated molecular patterns (PAMPs) on pathogen invading. After the recognition, the anti-viral signaling pathways are activated, promoting the production of proinflammatory cytokines and type I interferons (IFNs) [6]. 
Members of the TLR family are involved in responses to viral infection. Among all TLR members, TLR1, TLR2, TLR4, TLR5, TLR6, and TLR11 are located on plasma membrane, while TLR3, TLR7, TLR8, TLR9, and probably TLR13 of mice are expressed intracellularly within the endoplasmic reticulum, endosomes, multivesicular bodies, and lysosomes [387]. TLR3 senses dsRNA and some RNA viruses; TLR9 interacts with DNA containing unmethylated CpG motifs; TLR7 and TLR8 close resemble with TLR9 and sense ssRNA from the viral genomes [Figure 1.2]; TLR2 and TLR4 sense viral infection through recognition of protein components of the viral particle [7].

TLR3 induces the TLR domain-containing adaptor protein to trigger IFN $\beta$ signaling pathway, which in turn stimulate TANK-binding kinase 1 (TBK1) and IKKe. Induction of TBK1 and IKKe finally result in phosphorylation of interferon regulatory factor (IRF)-3, as well as activation of

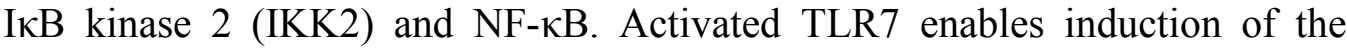
adaptor protein MyD88 and subsequently leads to the phosphorylation of IRF7 [8].

To interrupt the recognition by TLRs, some viruses have found to encode different viral proteins in order to target different factors that play critical roles in signaling transduction. Subsequently, these encoded viral proteins are able to inhibit TLR-mediated signaling as well as further host immune response [9].

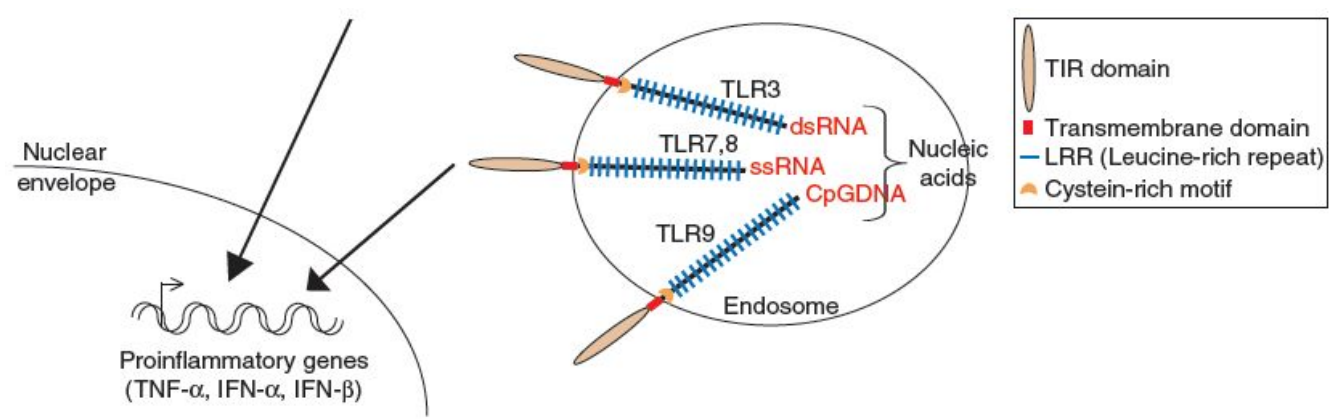

Figure 1.2 Toll-like receptors recognize nucleic acids (adapted from Galiana-Arnoux D et al., 2006) [7].

\subsubsection{Cytokines}

Cytokines are small secreted proteins used for intercellular signaling and communication. Cytokines not only participate in the innate immune respone 
but also mediate the communication between the innate and adaptive immune systems. There are different types of cytokines: interferons (IFNs), proinflammatory cytokines and chemokines.

\subsubsection{Interferons}

As a family of secreted cytokines, IFNs function in inhibiting virus replication and limiting viral spread via direct antiviral and indirect immunoregulatory activities at the very beginning of viral invasion. There are three main types of IFN: type I IFNs, IFN- $\alpha / \beta$, signal through IFNAR1/IFNAR2; type II IFN, IFN- $\gamma$, signals through IFNGR1/IFNGR2; type III IFNs, IFN- $\lambda 1 / 2 / 3$ referred to IL-29/28a/28b, signals though IL-28R [10]. These IFNs transduct signals through Jak-STAT signaling pathway, subsequently leading to the activation of downstream transcription factors and induction of IFN-stimulated genes (ISGs).

IFN- $\alpha / \beta$ are always produced upon viral infection. Initially, recognition of viral components by TLRs and retinoic inducible gene I (RIG-I) activates the transcription factor NF- $\kappa \mathrm{B}$ which is essential for IFN- $\beta$ expression. IRF3 as another transcription factor that is activated via RIG-I and TLR-3 mediated signal transduction pathways also plays a critical role in expressional induction of IFN- $\beta$ and activation of several ISGs. Upon the binding of secreted IFN- $\beta$ to its receptor, this initial response is further amplified. IFNAR as a receptor is coupled to the JAK that phosphorylate STAT1/2, which in turn initiate transcription of ISGs [11]. OAS and PKR are downstream interferon stimulated genes which play key roles in further antiviral activities.

Due to the prominently antiviral functions of IFNs, diverse mechanisms have been developed and employed to impede their activities so as to counterract the host defenses. These strategies include inhibition of IFN production, competition for binding to IFN receptors, interference with the JAK/STAT signaling pathway and suppression of ISGs at their expression level $[12,13]$.

\subsubsection{Pro-inflammatory cytokines}

Other cytokines that are rapidly produced following virus infection include tumor necrosis factor (TNF) and interleukins. TNF is a pro- 
inflammatory cytokine that is involved in the activation of the innate immune responses to viral infection and the control of cell apoptosis, survival as well as differentiation. Two surface receptors of TNF are TNFR1 that is expressed in most cell types and TNFR2 that is expressed in immune and endothelial cells. TNF expression is triggered by multiple PRRs such as TLRs upon recognition of viral infection. Among different TLRs, TLR2 and TLR4 are able to detect viral particles, while TLR3 and TLR7/8 recognize dsRNA and uridine-rich sequences of ssRNA respectively. Additionally, nucleic acids produced by virus can be also recognized by RIG-I and melanoma differentiation associated gene 5 (MDA5/IFIH1). Induction of TNFs results in activation of NF- $\kappa B$, a key transcription factor for proinflammatory.

IKK plays a role on phosphorylation and subsequent degradation of $\mathrm{I} \kappa \mathrm{B}$

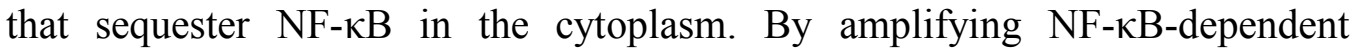
expression of anti-apoptotic molecules and activation of the MAPK signaling, TNF was also proved to mediate cell apoptosis [11]. In terms of different signaling pathways, viruses have evolved strategies to deal with them [14].

Interleukins are a group of cytokines that function primarily on the differentiation and activation of immune cells. For example, IL-1 $\alpha$ and IL-1 $\beta$ from IL-1 family are proinflammatory cytokines that mediate a variety of host responses including increasing acute-phase signaling, trafficking immune cells to the site of primary infection, activating epithelial cell and secondary cytokine production $[13,15]$.

\subsubsection{Chemokines}

Chemokines are the largest family of cytokines consisting of four types (CXC, CC, C, and CX3C) based on the spacing of their first two cysteine residues. Cytokines serve to recruit immune cells such as lymphocytes and antigen-presenting cells to the site of infection, and these recruited immune cells will function to limit virus replication and stimulate the adaptive immune respone [13]. For example, CCL5 that is responsible for the recruitment of CD8 $\mathrm{T}$ cells to the lung has been proved to participate in not only the classical IFN- $\gamma$ dominant Th1 responses but also Th2 response during RSV infection in mice [16]. Evidences have been also provided that certain viral proteins interrupt the host defense system through interfering with MHC functions [17]. 


\subsubsection{Cell apoptosis}

Many host cells respond to a virus infection by inducing a selfdestruction process called apoptosis or programmed cell death. This can be an effective host defense system, becaused the premature death of virus-infected cells reduces the spread of the infection wihin the orgranism [18]. Viruses also have evolved some stratigies to delay apoptosis of the host cell until their replication cycle is completed [17].

Apoptosis signaling generally triggers two major pathways, the intrinsic and extrinsic pathway. The former one is controlled by permeabilization of the mitochondrial membrane and release of cytochrome c. Cytochrome c enables to form a complex with Apaf-1 and procaspase-9, and this complex subsequently activates downstream caspases. Members from Bcl-2 family have been proved to be involved in the control of this pathway. The latter pathway is activated by the TNF family through binding to the domains of the death receptors including Fas, DR4 (Trail-R1), TNFRI, and TNFRII, which in turn lead to caspase activation [19] [Figure 1.3].

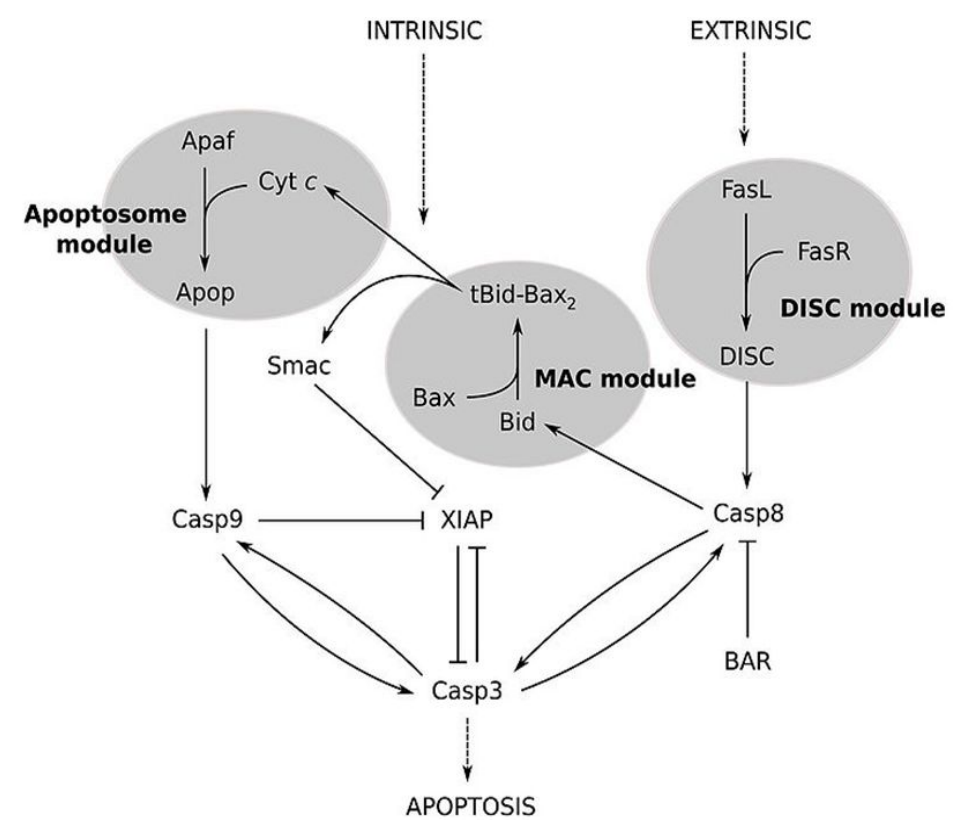

Figure 1.1 Apoptosis signaling pathway. Initially, the extrinsic stimuli such as TNF and Fas ligand stimulate death receptor trimerization, and then caspase 8 is activated after being recruited to the cytoplasmic domain of the death receptor. Activation of caspase 8 leads to activation of either caspase 3 or the BH3-only protein Bid. In the case of Bid activation, Bid is translocated to the mitochondria in order to activate Bak, subsequently the mitochondrial membrane is permeabilized and the pro-apoptotic factors are released. Eventually, the downstream caspases are activated to fulfill the final cell death. Intrinsic stimuli activate BH3only proteins that activate $\mathrm{Bak}$ and $\mathrm{Bax}$, or repress anti-apoptotic $\mathrm{Bcl}-2$ proteins, thereby regulating apoptosis at the mitochondria. 


\subsubsection{Host cell signaling pathways}

\subsubsection{JAK-STAT pathway}

Once IFN $\alpha / \beta$ are secreted by an infected cell, these cytokines will bind to the ubiquitously expressed IFNAR. Signaling through this receptor leads to activation of the JAK-STAT pathway and expression of interferon production, amplifying the response to viral infection. The JAK-STAT pathway demonstrates the activation of STATs protein by JAK proteins [Figure 1.4]. JAK proteins are activated by interferons and interleukins. After the binding into the receptors, the JAK proteins phosphorylate the STAT1 proteins (pSTAT1), turning them into activated form. pSTAT1 proteins dimerize and translocate to the nucleus [20]. However, Pauli EK et al (2008) proposed that phosphorylation of STATs in the IFN $\beta$ signaling might be regulated by some cellular factors, such as proteins of SOCS family [21]. These SOCSs have been proposed to have high affinity for JAK and STAT proteins and therefore inhibit the transmission of IFN $\alpha / \beta$ signaling. Validation experiment indicates that SOCS-3 is partly responsible for the inhibitory activity via NF- $\kappa \mathrm{B}$-dependent induction [22].

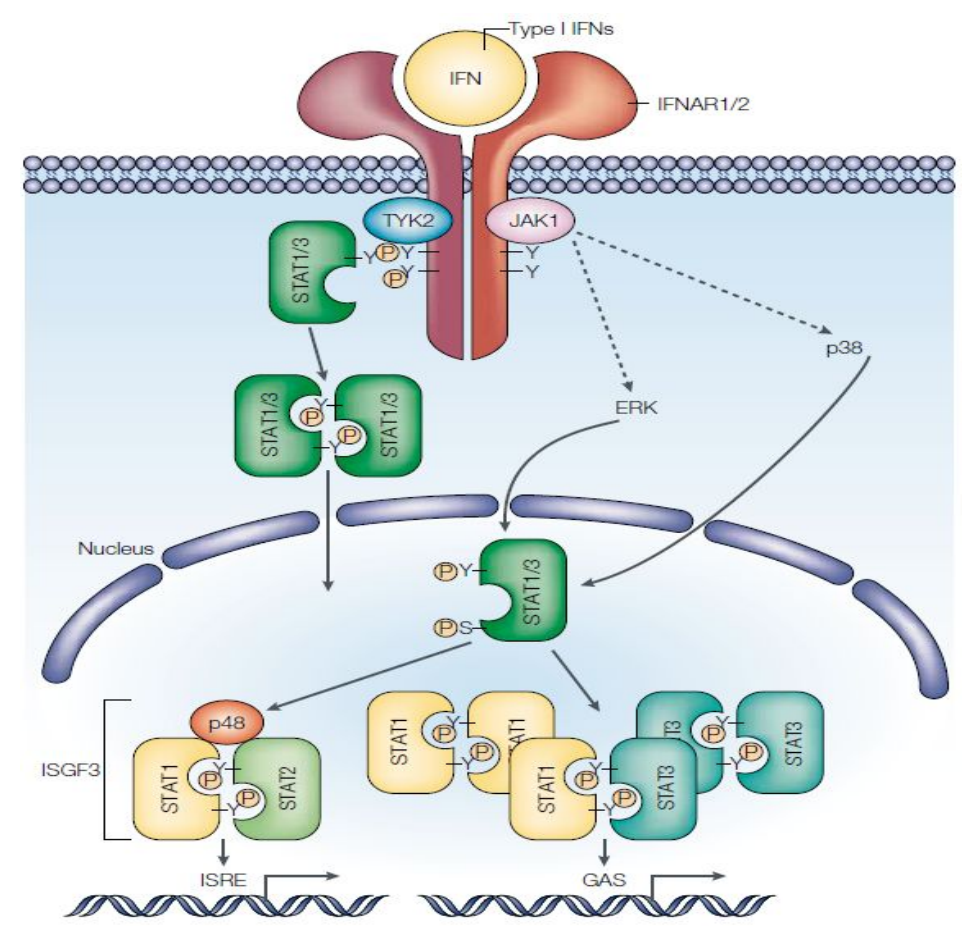

Figure 1.4 The IFN receptors and Jak-STAT signaling (adapted from Katze MG et al., 2002) [10]. 


\subsubsection{NF-кB/IKK pathway}

The NF- $\kappa \mathrm{B}$ family plays a central role in the cellular inflammation response through controlling a network of gene expression. It is commonly activated upon virus infection and its activation leads to the expression of an array of cytokine and chemokine genes, including IFN $\beta$ [23]. Apart from its function as regulator of the expression of inflammatory molecules, NF- $\kappa \mathrm{B}$ also regulates mechanisms of controlled cell apoptosis in several cell types.

The mechanism of NF- $\kappa \mathrm{B}$ activation depends on the activation of the inhibitor of IKK complex. The IKK complex consists of at least IKK1/IKKa, $\mathrm{IKK} 2 / \mathrm{IKKb}$ and NEMO/IKKg. Amon these isozyme, IKK2/IKKb is most important for the activation of the canonical NF- $\kappa \mathrm{B}$ pathway through

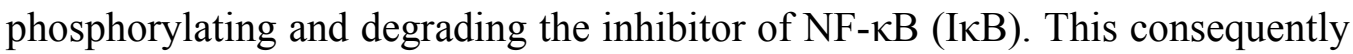
leads to the release and translocation of NF- $\mathrm{\kappa B}$ factors p65 and p50 dimers that migrate to the nucleus to exert its biological functions.

\subsubsection{MAPKs signaling pathway}

MAP kinases play an essential role in cellular responses such as proliferation, differentiation, immune response and cell death. Three main families of MAPKs in mammals all have their own activators, inactivators, substrates and scaffolds, and all these factors form a fine signaling network in reaction to different stimulations from extracellular or intracellular. Three concrete pathways are MAPK/ERK, SAPK/JNK and p38 MAPK. MAPK signaling promotes cell survival by a dual phosphorylation event on threonine and tyrosine residues. The upstream MAPKK regulates these 4 enzyme activities. Two MAPKK (MKK3/6, MKK4/7) are responsible for activation of p38 and JNK respectively. These enzymes are involved in apoptosis and cytokine expression, and can be activated by environmental stress conditions. The upstream Raf controls the phosphorylation of MAPK/ERK kinase $(\mathrm{MEK}) 1 / 2$, which regulates the activation of ERK1/2 that plays a regulatory role in cell proliferation and differentiation. Lastly, enzyme ERK5 is activated by MEK5 [Figure 1.5] [24]. 


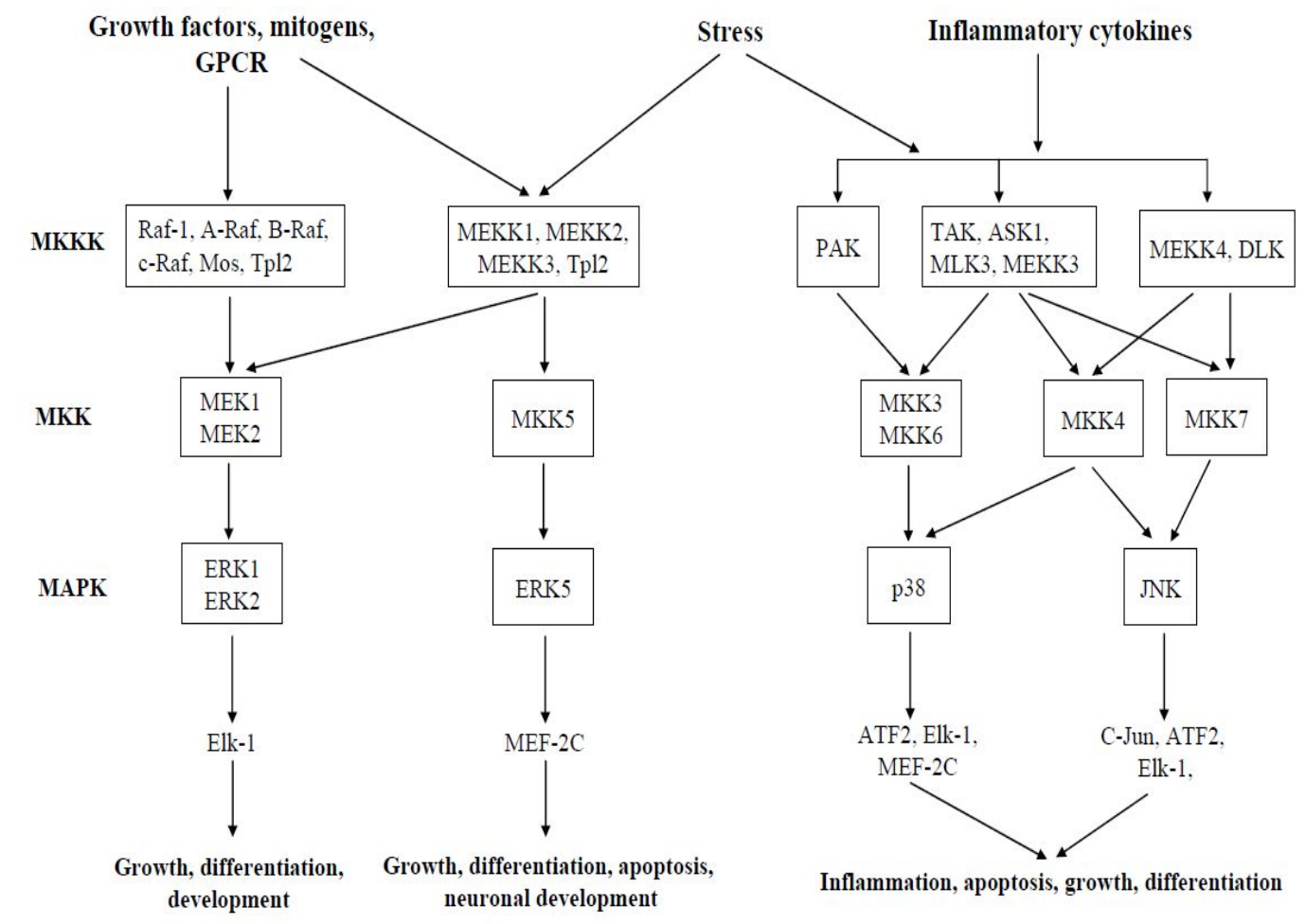

Figure 1.5 MAP kinase pathways in mammals. MAPKs are activated by dual phosphorylation on Thr and Tyr caused by specific MKKs, which are in turn activated by MKKKs (adapted from Zhang Y et al., 2005) [24].

\subsection{Traditional strategies in virology studies}

\subsubsection{Traditional strategies of virus identification}

In tradition, various techniques such as tissue culture, immunofluorescence assay (IFA), enzyme-linked immunosorbent assay (ELISA), electron microscope (EM) and reverse transcription-polymerase chain reaction (RT-PCR) are applied in pathogen detection.

IFA is a technique used with a fluorescence microscope. This technique specifies the antibodies carrying fluoresecent dyes to target their corresponding antigens, and therefore allows visualisation of the distribution of the target molecules. EM is another technique that is based on antibodies. Antigens from samples are attached to a surface and specifically bind the corresponding antibodies, and substances are able to react with the enzymes that are linked to the antibodies, thereby detectable signals are produced. Both these two traditional techniques have been widely applied in pathogen detection [25] and the major limitations of them lie in the signal measurement: photobleaching in IFA and complex enzyme activity in ELISA. 
Another technique called RT-PCR is also applied in pathogen detection [26]. This technique is based on the polymerase chain reaction, and it is able to not only detect the pathogen sequence but also quantify the pathogen sequence in the sample. The limitation of this technique is that it heavily relied on further pathogen sequencing. EM is a type of microscope that has great resolving power and can reveal the structure of small objects. The diadvantage of this technique is that it is very expensive to build and maintain, and the operation and analysis heavily relies on highly-trained technicians.

Currently, advanced methods such as microarray have been widely applied in pathogen studies, mainly because of the following advantages: (1) small volumes of probes and samples required; (2) a large amount of information generated within one experiment; (3) broad-based and co-infectious detection. Although it is treated as a promising technology, challenges still have been posed to users. These challenges majorly lie in reproducibility, hybridization efficiency, highly-trained technicians and professional manpower for data analysis. In addition, sophisticated machine for image processing and rigorous bioinformatics software for effective data mining are also required [27].

\subsubsection{Traditional strategies of host gene expression investigation during virus infection}

In order to examine host genomic expression profiles following virus infection, techniques including RT-PCR, northern blot, RNase protection assays and serial analysis of gene expression (SAGE) have been employed in quantifying gene expression for a long period [28-31]. In the meanwhile, other methods such as ELISA and western blot have been also widely applied in protein expression quantification since a long time ago [32, 33]. Although relatively accurate quantification could be generated using these techniques, they are limited to quantify multiple genes or proteins together at one time (Table 1.1).

In the past decades, several advanced technologies have been pioneered and applied to investigate the gene or protein expression in the field of virology. These technologies including microarray, mass spectrometry and next generation sequencing (NGS) enable to quantify tens of thousands of genes or proteins in parallel. However, the common disadvantages of these technologies 
are the demand of highly-trained technicians and rigorous bioinformatics software for accurate data interpretation.

Table 1.1 Advantages and disadvantages of different strategies which are applied in genomic investigation during virus infection.

\begin{tabular}{|c|c|c|c|}
\hline Strategies & Target & Advantages & Disadvantages \\
\hline $\begin{array}{l}\text { Northern } \\
\text { blot }\end{array}$ & $\begin{array}{c}\text { gene } \\
\text { expression }\end{array}$ & high specificity & $\begin{array}{l}\text { not sensitive \& dangerous } \\
\text { chemicals }\end{array}$ \\
\hline RNase & gene & very senstive & highly-trained technicians needed \\
\hline SAGE & $\begin{array}{c}\text { gene } \\
\text { expression }\end{array}$ & $\begin{array}{c}\text { accurate } \& \text { do not need the } \\
\text { sequence }\end{array}$ & expensive \& low-scale studies \\
\hline RT-PCR & gene & fast \& sensitive & rely on further sequencing \\
\hline ELISA & $\begin{array}{c}\text { protein } \\
\text { expression }\end{array}$ & affordable & complex enzyme activity \\
\hline $\begin{array}{l}\text { Western } \\
\text { Blot }\end{array}$ & $\begin{array}{c}\text { protein } \\
\text { expression }\end{array}$ & Sensitive & long time $\&$ require antibodies \\
\hline \multirow{2}{*}{ Microarray } & $\begin{array}{c}\text { gene } \\
\text { expression }\end{array}$ & $\begin{array}{c}\text { detect expression of multiple } \\
\text { genes/proteins in an }\end{array}$ & expensive \\
\hline & $\begin{array}{c}\text { protein } \\
\text { expression }\end{array}$ & $\begin{array}{l}\text { Small volumes of probes and } \\
\text { sample required }\end{array}$ & highly-trained technicians needed \\
\hline \multirow{2}{*}{$\begin{array}{c}\text { Mass } \\
\text { Spectrum }\end{array}$} & \multirow{2}{*}{$\begin{array}{c}\text { protein } \\
\text { expression }\end{array}$} & $\begin{array}{c}\text { detect expression of multiple } \\
\text { proteins in an experiment }\end{array}$ & expensive \\
\hline & & $\begin{array}{l}\text { Small volumes of probes and } \\
\text { sample required }\end{array}$ & highly-trained technicians needed \\
\hline \multirow{2}{*}{ NGS } & \multirow{2}{*}{$\begin{array}{c}\text { gene } \\
\text { expression }\end{array}$} & \multirow{2}{*}{$\begin{array}{l}\text { detect expression of multiple } \\
\text { genes in an experiment }\end{array}$} & expensive \\
\hline & & & highly-trained technicians needed \\
\hline
\end{tabular}

\subsection{Design of DNA microarray technology}

A microarray chip is an orderly arrangement of a rectangular grid of "spots". Thousands of short DNA fragments (probes) are printed onto a small solid matrix in order to examine cDNA representing mRNA (targets). The basic theory of the microarray experiments lies in the hybridization by base pairing between probes and targets. Dependent on this special structural design of microarray, thousands of different probes are able to be immobilized into a single array for parallel investigation $[34,35]$.

\subsubsection{Different applications of DNA microarray}

There are a number of applications of microarray technology in past years. In the area of phylogenomics in pathogens, comparative genomics can identify genetic factors responsible for transmission, evolution, virulence, and even antimicrobial resistance among different pathogen phenotypes [36]. 
Another application is to study host-microbe interaction, and this transcriptome analysis of intracellular pathogens in in-vitro and in-vivo models at different infection time points offers better understanding of the molecular biology and life cycle of pathogens [37, 38].

The microarray platform can also be exploited to array protein markers and glycans. This platform offers a high throughput screening for patient to test for antibodies to a wide array of proteins. These proteins serve as immunoreactive antigens as in the case of Severe Acute Respiratory Syndrome (SARS) virus and can be used as serological markers in epidemiological study [39]. In addition, the array can be used as therapeutic proteins for vaccine development as in the case of smallpox vaccine [40]. In another example, the development of a glycan array allows the study on the receptor affinity of different influenza A virus subtypes and the information is extrapolated to understand the host specificity [41].

Another exploitation of the platform is the array of a library of small molecules, which could potentially be drug candidates. These are then screened with a panel of target proteins from pathogens such as the anthrax lethal factor [42].

In the field of virology, the most popular applications of microarray technique could be majorly classified into two repects: microbial or medical diagnosis $[43,44]$ and transcriptomic examination [45].

\subsubsection{Application of microarray in virus identification}

During the last decade, the broad-based virus detection using the microarray DNA chip was developed (Figure 1.6) and different types of microarray chips designed for different detection purposes were pioneered (Table 1.2).

The broad-based virus detection using the microarray DNA chip was pioneered by the DeRisi group [27, 48]. In this chip, long oligonucleotides at 70 mers were designed and each virus was represented by the top 5 highly conserved oligonucleotides and the corresponding reverse complement oligonucleotides. These long oligonucleotides were arrayed for a total of 1000 viruses and represent all known viruses at that time the virus pathogen chip was designed in year 2003. These viruses cause diseases in human, animals, 
agricultural and are also found in the environments. Subsequently other viral chips were developed based on a similar platform. For example, the 40-mer microarray chip was developed by Wong et al (2007) with the aiming of detecting a small group of RNA viruses [49]. Another group in Taiwan reported the use of 70-mer microarray chip in a similar design to DeRisi's group [50].

Table 1.2 Different types of microarray chips in virus detection. The chip from DeRisi group can be used to detect around 1,000 viruses; Greene Chip is applied in identifying a variety of micro-organisms; Commercial microarray chips like TessArae performs well in microbial detection and genetic testing [27, 46, 47].

\begin{tabular}{|c|c|c|}
\hline DeRisi group & Greene Chip & TessArae \\
\hline 70 & 0-mer oligonucleotides & $5-\mathrm{m}$ \\
\hline $\begin{array}{l}\text { each virus was } \\
\text { represented by the top } 5 \\
\text { highly conserved } \\
\text { oligonucleotides and the } \\
\text { corresponding reverse } \\
\text { complement } \\
\text { oligonucleotides }\end{array}$ & $\begin{array}{l}\text { oligonucleotide probes } \\
\text { from one conserved } \\
\text { target and two variable } \\
\text { targets are taken into } \\
\text { consideration for the } \\
\text { design }\end{array}$ & $\begin{array}{l}\text { Eight probes are designed for } \\
\text { each nucleotide pair; four } \\
\text { each for the forward and } \\
\text { reverse strands with each set } \\
\text { of four tiled together as a } \\
\text { probe set. The probes vary } \\
\text { only at the center with an A, } \\
\text { G, C or T at that position }\end{array}$ \\
\hline $\begin{array}{l}\text { detect about 1,000 viruses } \\
\text { which cause diseases in } \\
\text { human, animals, } \\
\text { agricultural and are also } \\
\text { found in the environments }\end{array}$ & $\begin{array}{l}\text { detect a variety of } \\
\text { micro-organisms such } \\
\text { as viruses, bacteria, } \\
\text { parasites, fungal }\end{array}$ & $\begin{array}{l}\text { Microbial detection } \\
\text { genetic testing }\end{array}$ \\
\hline
\end{tabular}

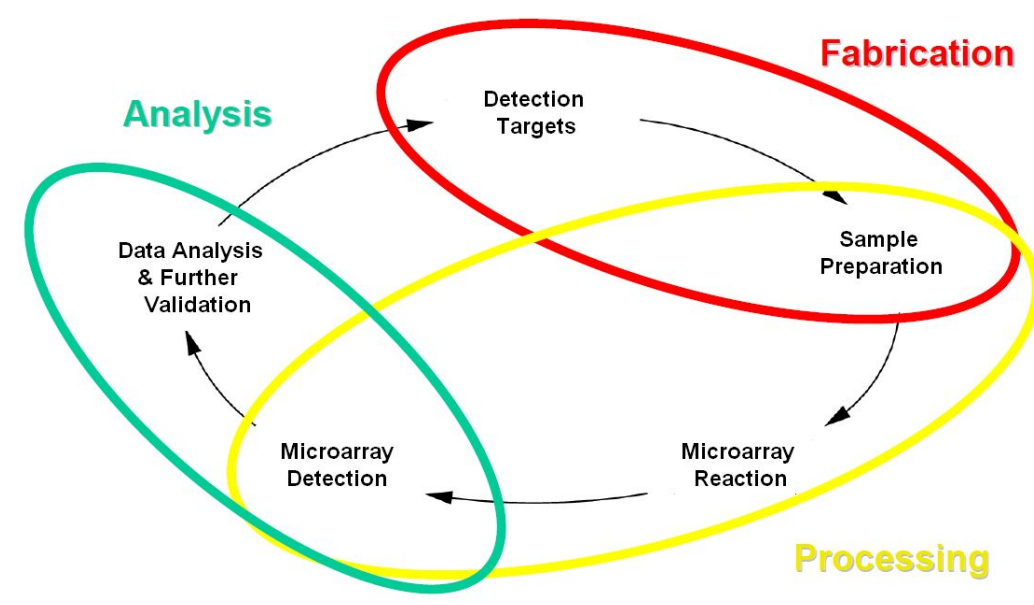

Figure 1.6 Strategy to identify viruses based on microarray technology. With regard to different detection purposes, array design and samples preparation should be considered carefully. Microarray reaction, detection, and data analysis are fulfilled step by step. Finally, methods such as PCR, IFA, and ELISA are applied for further validation. 
The GreeneChip is another viral microarray chip that is not commercially available, but has been incorporated as part of the novel pathogen discovery program led by Dr Lipkin's group in Columbia University, New York [46]. In this case, the GreeneChip were arrayed with 60-mer oligonucleotides, and these were selected from a variety of micro-organisms (viruses, bacteria, parasites, fungal). The selection of probe sets for viruses were more logical compared to Wang et al $(2002 ; 2003)$ [27, 48]. Oligonucleotide probes from one conserved target usually coding the enzyme genes and two variable targets usually coding the structural genes are taken into consideration for the design.

In some pathogen chips, simultaneous detection and partial characterization in further reveals the exact sequence information hence providing preliminary and confirmatory identification. For example the resequencing chip developed by Lin et al., 2006 was so successful that it was taken over commercially and marketed under TessArae (http://www.tessarae.com). It can provide identification and partial sequencing of important virus genes and targets a range of respiratory viruses in the Centers for Disease Control and Prevention (CDC) list of category A, B and C agents. It is also able to perform whole genome sequencing of a comprehensive list of haemorrhagic fever viruses.

There are other types of microarray chips which provide partial characterization such as the chip used for subtyping influenza viruses [47]. In this chip, specific 21-mer oliognucleotides representing different subtypes of influenza viruses were arrayed on glass slides, and the genetic materials from samples were allowed to hybridize to the oligonucleotides.

The clusters of regularly interspaced short palindromic repeats (CRISPR)/Cas (CRISPR associated proteins) system that functions on defending against invading nucleic acids is broadly distributed in microbial immunity. The CRISPR spacer sequences have been proved to be unique within individual microbe genomes and may even be unique within different cell types. Accordingly, CRISPR spacer-based microarrays were designed by Snyder JC et al (2010) to identify unknown virus[51].

Another system called Pan-viral microarray targets to virus genus specific detection [52]. In that chip, 628 63-mer genus specific oligonucleotides 
were designed as probes to screen 32 human pathogenic RNA virus genera from 14 virus families.

Recently, another pan-microbial detection array (MDA) that targets to all known viruses and bacteria has been also established [53, 54]. Probes specific to different virus families were designed for all sequenced viral and bacterial genomes, segments, as well as plasmids. Some sequence variations were tolerated during the probe design process so that detection of divergent species with homology became possible.

\subsubsection{Application of microarray in host gene expression investigation during virus infection}

When the microarray technique is applied in monitoring gene expression (mRNA abundance), the genes which play critical roles on inner cellular function are majorly investigated. Since these genes are transcribed from genomic DNA, their expression profile also refers to as transcriptome. The genomic transcription from DNA to mRNA is the initial step during the process of protein synthesis, thus differences in gene expression might indicate specific cellular responses in reaction to intra or extra interferences. Comprehensive understanding of the detailed gene expression in different situations is essential to analyze the biological functions of its encoded protein. Moreover, changes of the whole gene expression profiles can provide insight into cellular regulatory mechanisms and underlying biological signaling pathways [55].

It has been well known that viruses are not able to propagate independently in their host cells. In every infection cycle, viruses must enter into their host cell for replication and transcription. In this process, many host cell functions are required to assist them to complete the multiple steps of their life cycle. And these cellular functions could be divided into several aspects: cellular translation and translocation, sorting machinery to target glycoproteins to the proper cellular destination, a lipid-synthesizing machinery for virus lipid envelope and the cellular nucleus that is necessary for DNA viruses (except poxviruses) replication [56].

Besides, viruses have also evolved the mechanisms to avoid the negative influence from the host antiviral actions. For instance, expression of molecules in immune response signaling pathways have been suppressed in order to make 
sure their replication happen without interference from the inflammation responses; cytoskeleton and cellular signal transduction pathways have been manipulated to benefit their easily entry and following cellular travel; new strategies have been also evolved to prevent host cell apoptosis to guarantee their smooth replication and budding [56].

In view of these complex interactions, microarray technology has been widely applied in defining the global transcriptional activities in virusesinfected host cells to facilitate virology research as well as further antiviral drug discovery[57, 58]. To date, many groups have reported the applications of microarray in the investigation of gene expression profile in viruses-infected host cells. For instance, Katze MG et al (2008) applied microarray technique to examine the global gene expression profiling in various viral infected models, aiming to explore viral pathogenesis and host-pathogen interactions at a systems-level [59]; microarray technique was also involved in identifying differentially expressed genes and pathways in cattle infected with Alcelaphine herpesvirus 1 [60] as well as in human retinal pigment epithelium infected with West Nile virus [61]; genomic host responses upon laryngotracheitis virus vaccine infection were also checked in chicken embryo lung cells based on a microarray platform ; Other applications of microarray technique in studying the transcriptome under virus infection have been also extensively published [63-66].

\subsection{Bioinformatics tools involved in virology researches}

In recent years, bioinformatics tools and databases that interpret genomic, proteomic, and other functional information have become more and more indispensable for virology studies. The applications of these bioinformatics analysis in virology researches include open reading frame (ORF) identification, gene prediction, homology searching, sequence alignment, pattern/motif/epitope recognition, short tandem repeats, transmembrane domains as well as secondary and tertiary structural studies [67].

With the dramatic development of techniques such as microarray, mass spectrometry (MS)-based proteomics and NGS, mRNA and protein abundances could be surveyed in a routine, rapid and high-intensity [68-70]. Due to largescale of quantification at one time, these advanced techniques have been now 
widely applied in virology studies $[71,72]$. At the meanwhile, more and more bioinformatic tools have been also pioneered in order to extract useful information in their abundant data [35, 73-75].

\subsubsection{Bioinformatics pre-processing for microarray data}

Sophisticated bioinformatics processing is required before abstracting the meaningful data from microarray experiments. This procedure commonly include: background correction, data truncation, outlier correction, averaging, quality control and normalization. A variety of statistical considerations and algorithms are involved in this procedure to retrieve the accurate observed signal intensities from the microarray chips [76].

\subsubsection{Bioinformatics software for virus identification}

While many virus detection microarray chips have been described, there are only a couple of algorithms published for analyzing data from these diagnostic chips [77]. The algorithm for E-Predict [78], DetectiV [79], and VIPR/ VIPR HMM $[80,81]$ are published and their software are available to the public. Other algorithm such as GreeneLAMP [46], PDA [49], PhyloDetect [82] and CLiMax [53] are available but not the software. These different computation strategies are established in aim to target different microarray diagnostic chips based on different statistical hypothesis.

E-predict is the first computation strategy raised together with the corresponding 70-mer oligonucleotides microarray chip by DeRisi group, and treated as their default computation strategy. It is a computational strategy used for virus species identification based on microarray experimental hybridization intensity pattern (http://derisilab.ucsf.edu/epredict/). Using this strategy, a library of theoretical binding energy profiles that represent different virus species with known genomic sequences, was computed and then compared with the experimental hybridization intensity pattern. The final detection result is reached through calculating Uncentered Pearson correlations between the theoretical binding energy profiles and the experimental hybridization intensity patterns.

DetectiV is an R-based method for significant testing. It integrates many $\mathrm{R}$ packages including limma, marray, affy together to achieve the virus 
detection goal and meanwhile offered an additional visualized maps [83, 84].

VIPR, as a probabilistic algorithm, applies Bayesian inference in capitalizing on empirical training data so as to optimize virus detection sensitivity. And VIPR HMM implements the analysis by incorporating a hidden Markov model (HMM) into existing VIPR in order to detect the recombinant genomes.

PDA software fulfills the pathogen detection based on two steps: a modified Kullback-Leibler Divergence (KL) is imported to evaluate the hybridization intensity of probes in each pathogen r-signature; AndersonDarling test is performed to complete further statistical analysis.

GreeneLAMP centers the log-transformed intensity, with QFAST algorithm is applied to compute the tail probability (P-value) for each virus. And these P-values for positive probes associated with the virus are finally used to calculate the tail probability.

In PhyloDetect, it is assumed that the probes in microarray yield a hybridization signal with a matching microbial sequence but no signal with a non-matching one. On the basis of this probe-microbe match matrix, the microbial detection outcome is fulfilled by three steps: grouping of nondistinguishable microbes, arrangement of microbial groups in a hierarchical tree and computation of likelihood.

CLiMax is based on a biophysical model of probe-target hybridization, and a greedy algorithm is applied to find a local maximum for the likelihood.

\subsubsection{Bioinformatics software for host gene expression during virus infection}

Genomic interpretation of the microarray data generally focus on the following directions: enrichment of functional pathways and processes, clustering of gene expression profiles, discovery of regulatory networks as well as prediction of transcription factor binding $[85,86]$.

\subsubsection{Pathway enrichment}

Through analyzing biochemical pathways and biological processes, it will be easy to understand how these molecules interact with each other, as well as their functional roles at the systems level. In virology studies, more and more 
evidences have been provided that molecules in pathways associated with immune response, apoptosis and antigen representing show un-normal expression. Accordingly, investigations on dysregulation of these specific pathways or processes during virus infection may be beneficial to understand the host-viral interaction mechanisms, and therefore potential antiviral interventions and vaccine targets could be pioneered [67].

A commonly used pathway database is Kyoto Encyclopedia of Genes and Genomes (KEGG), which includes information of metabolic pathways and regulatory pathways [87]. Another pathway database called HumanCyc, which is under BioCyc system, and this database contains the metabolic pathways which are only specific to human [88]. As to the bioinformatics software which enables to enrich the significantly regulated pathways are involved GeneSpring, Gene Set Enrichment Analysis and Ingenuity Pathway Analysis [89].

\subsubsection{Clustering analysis}

In the traditional analysis of microarray data, individual differentially expressed gene is not able to provide the complete picture of the gene regulations. Noise and variation in microarray expression data may influence the expression performance of individual gene. Thus the employing of clustering analysis on studies of these expression data makes it easy to detect a group of functionally related genes with coordinated expression profiles. These groups of genes with similar expression trend are assumed to have related biological functions and correspond to some critical cellular processes and pathways $[90,91]$.

Different clustering algorithms generate different results even when given the same expression dataset. For biologist, it is difficult to choose an appropriate clustering method. Till now, a diverse set of clustering algorithms have been proposed by different groups. These are five traditional methods: kmeans clustering, the hierarchical clustering based on average linkage or complete linkage, and the methods due to McQuitty and Ward. Besides, some more graph-based approaches such as k-clique communities, WGCNA, NNN, CAST, and CLICK have been established. Finally, two other approaches called self-organizing maps and QT Clust have been also applied in microarray data analysis recently [92]. 


\subsubsection{Regulatory network discovery}

In biological cells, mRNA and proteins interact with each other with various degrees of specificity. These molecules and their interactions form different gene regulatory networks. Discovery of these gene regulatory networks will help us understand the inner signal transduction system in the host cells. In virus infected host cells, examining the regulation status of different networks will be absolutely beneficial for the understanding of virus invasion and host antiviral mechanisms [91].

Traditional methods which are applied in constructing gene regulatory networks from microarray data include linear models, Bayesian networks and Boolean networks [93]. In these years, Ruan J et al (2010) have proposed a new and robust method called gene co-expression networks, and Zare $\mathrm{H}$ et al (2011) also has built up a new algorithm called Kernel Embedding of Regulatory Networks, which is based on the gene regulation association [94].

Recently, more and more findings have proven that both virus and host encode microRNAs, which in turn participate in the intricate host-virus interactions. Thus the regulatory networks of microRNA-mRNA interactions during the pathogen infections will draw more and more attentions in virology researches [95-97].

\subsubsection{Transcription factor binding prediction}

In fundamental biological processes, transcriptional regulation affects the translation level of proteins by regulating cellular mRNA levels. When it comes to higher eukaryotes, the complexity of them primarily resides in the sophisticated regulatory networks [98, 99]. Accordingly, discovering the potential binding motif using bioinformatics knowledge will be beneficial for the deep understanding of inner cellular function.

Several classic databases of transcriptional factor elements and their binding sites have been established and updated these years. Among them, two representative databases are TRANSFAC and JASPAR [98]. Software which have been reported to enrich the potential transcriptional factor and their binding sites include oPOSSUM human single site analysis package [100], PRIMA [101] and STOP [102]. 


\subsection{Objective}

As we know, pathogens such as influenza viruses, RSV and poxviruses have become greatest threats to human health. And in the past decades, scientists have paid more and more attentions on virus researches, not only aiming to improve the virus diagnostic system but also making efforts to investigate the host-virus interactions.

Advanced high-throughput technologies such as microarray have shown superiority when compared to other traditional methods. With its application, multiple bioinformatic databases and tools become more and more imperative to accurately interpret these microarray data. In our studies, we exert efforts to solve the popular issues in virology researches with the help of microarray technology, and in this process, relevant bioinformatic approaches have been pioneered or employed to achieve the data analysis.

In the project regarding to pathogen diagnostic chips, a new computation strategy, called BayesMicro, is described. This software is designed based on a novel Bayesian metric and implemented in a Perl environment. It is used to not only detect the virus from the global species level but also provide further differential virus detection within virus families.

In other projects, standard microarray chips (Affymetrix) are involved in investigating the global transcriptomic features in different cell lines after infections of different viruses. The pre-processing and further interpretation of microarray data were performed under the software including GeneSpring GX 11.0, Expander 5.0 and Ingenuity Pathway Analysis. The examination results demonstrate that different global gene expression profiles are detected in different virus infected different cells. The pathways which have been mentioned in previous reports also illustrate differential regulation in our experiment. In addition to these published pathways, genes in some other pathways have also been enriched to show differently transcriptional changes during the virus infection. 


\section{Chapter II. Materials and Methods}

\subsection{Materials}

\subsubsection{Viruses}

RSV A2

$\mathrm{A} / \mathrm{WS} / 33$

A/Singapore/478/2009

A/Duck/Malaysia/01

A/Duck/Malaysis/F118/08/2004

A/Duck/Malaysia/F59/04/1998

A/Duck/Malaysia/F189/07/2004

A/Duck/Malaysia/F119/3/1997

A/umbrellacockatoo/Singapore/F47/12/92

A/fairybluebird/Singapore/F92/09/94

Cowpox virus Brighton strain ATCC VR302

Vaccinia virus Lister strain ATCC 1549

Ectromelia virus Moscow strain VR1374

ATCC
ATCC
DSO National Laboratories
AVA
AVA
AVA
AVA
AVA
AVA
AVA
ATCC
ATCC
ATCC

ATCC

ATCC

DSO National Laboratories

AVA

AVA

AVA

AVA

AVA

ATCC

ATCC

\subsubsection{Cell types and their maintenance}

Hep2 cells were derived from a human epidermoid cancerous cell line. It was abtained from American Type Culture Collection (ATCC). They were maintained in 10\% Dulbecco's Modified Eagle's medium (DMEM) (Invitrogen) containing $10 \%$ fetal bovine serum (FBS) and 1\% penicillin/streptomycin (Glibco) at $37^{\circ} \mathrm{C}$ in $5 \% \mathrm{CO} 2$ and antibiotics.

Human alveolar basal epithelial (A549, ECACC 86012804) and MadinDarby canine kidney (MDCK, ECACC 84121903) cells were obtained from European Collection of Cell Cultures. Chick embryo fibroblasts (CEF) were prepared from 8 to 10 day-old chick embryos. After ethanol cleaning, the eggs were processed using forceps to isolate the intact body part. The intact body part was then made into single cell suspension. They were maintained in $10 \%$ Dulbecco's Modified Eagle's medium (DMEM) (Invitrogen) containing 10\% fetal bovine serum (FBS) and $1 \%$ penicillin/streptomycin (Glibco) at $37^{\circ} \mathrm{C}$ in $5 \% \mathrm{CO} 2$ and antibiotics.

Murine lung CD11b + cells were prepared from 6-8 weeks old special pathogenfree (SPF) female Balb/c mice. The lungs were digested with

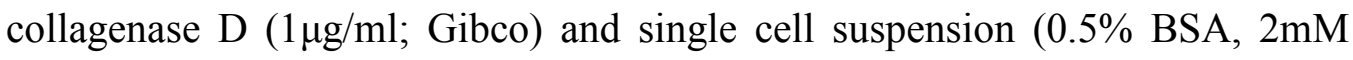
EDTA, in 1XPBS) obtained was passed through a $30 \mu \mathrm{m}$ filter. CD11b + cells were purified using CD11b microbeads and a LS positive selection column 
(Miltenyi Biotec). The cells were eluted from the microbeads and cultured in L929 cell conditioned $(30 \% \mathrm{v} / \mathrm{v})$ medium for 3 days at $37^{\circ} \mathrm{C}$ in $5 \% \mathrm{CO}_{2}$.

RAW 264.7 (RAW) cells (Mouse leukaemic monocyte macrophage cell line) were purchased from ATCC (TIB-71) and maintained in 10\% Minimum Eagle's Media (MEM media) (D1152 media, Sigma) containing 10\% FBS and $1 \%$ penicillin/streptomycin (Glibco) at $37{ }^{\circ} \mathrm{C}$ in $5 \% \mathrm{CO}_{2}$.

Chick embryo fibroblasts (CEF) were prepared from 8 to 10 day-old chick embryos and maintained in DMEM containing 10\% FBS and pen/strep [394].

\subsection{Experiment design}

\subsubsection{Experiment workflow for pathogen detection}

Nucleic acids were isolated from virus-infected cell cultures (Qiagen). Their amplification consists of three steps: random-primed reverse transcription, second strand cDNA synthesis and PCR. The PCR products ran on the agarose gel electrophoresis and amino-allyl (aa)-dUTP were incorporated. The aadUTP-incorporated DNAs were fragmented and purified using the PCR purification kit (Qiagen). After that, the amino-allyl group was then labeled with Cy3-fluorescent dye (GE Healthcare). The printed microarray slides were UC cross-linked and blocked first, and the labeled PCR products were subsequently hybridized overnight to the microarray chip. The hybridization signal intensities were retrieved using Genepix software, and subsequently reformatted and submitted into E-Predict and BayesMicro software for further analysis [27][48][78].

For all the virus infections, they were generally infected with a MOI $>1$, and infection was carried out at $37^{\circ} \mathrm{C}$ in the presence of $\mathrm{CO}_{2}$ [Table 2.1].

\subsubsection{Primers used}

\begin{tabular}{|l|l|c|}
\hline Primer name & Primer sequence (5' to 3') & Size (bp) \\
\hline Primer A & GTT TCC CAG TCA CGA TCN NNN NNN NN & 26 \\
\hline Primer B & GTT TCC CAG TCA CGA TC & 17 \\
\hline
\end{tabular}


Table 2.1 Preparatino of virus strains.

\begin{tabular}{|c|c|c|c|c|}
\hline Virus & Strains & Origins & Propagation & DNA/RNA extraction method \\
\hline Chikungunya virus & Oka & & Propagated in C6/36 cells & Rneasy (Qiagen) \\
\hline Coronavirus & OC43 & ATCC VR-1558 & & Rneasy (Qiagen) \\
\hline Cowpox VIRUS & Brighton & ATCC VR-302 & Passaged in BHX cells & DNA mini (Qiagen) \\
\hline Influenza A virus & H1N1 & & Clinical sample isolated and propagated in eggs $^{*}$ & Rneasy (Qiagen) \\
\hline Influenza A virus & H3N2 & ATCC VR810 & Propagated in eggs ${ }^{*}$ & Rneasy (Qiagen) \\
\hline Influenza B virus & Lee & ATCC VR101 & Propagated in eggs ${ }^{\star}$ & Rneasy (Qiagen) \\
\hline Japanese encephalitis virus & Nakayama & & Propogated in Vero cells & Rneasy (Qiagen) \\
\hline Measles virus & Edmonton & ATCC VR-24 & & Rneasy (Qiagen) \\
\hline Mouse poxvirus & Moscow & ATCC VR 1374 & Passaged in Vero E6 cells & DNA mini (Qiagen) \\
\hline Rhinovirus & Type 14 & ATCC VR-1059 & & Rneasy (Qiagen) \\
\hline Vaccinia virus & Lister & & Propagated in Vero E6 & DNA mini (Qiagen) \\
\hline Yelllow fever virus & 17D & & Propogated in Vero E6 & Rneasy (Qiagen) \\
\hline
\end{tabular}

*9-11 day old embroynated chicken eggs

\subsubsection{Design of the viral chip}

The complete list of viral oligonucleotide sequences represented on the microarray viral chip by Wang et al., 2003 was downloaded from http://derisilab.ucsf.edu/virochip. Oligonucleotides belonging to plant viruses were removed from the list, and only human or zoonotic viruses were synthesized. Each virus was represented by the top 5 highly conserved oligonucleotides and the corresponding reverse complement oligonucleotides. These oligonucleotides were 70 mer in length. In addition, we also randomly selected 10 oligonucleotides from 10 human genes as controls for microarray scanning. In total, 6233 oligonucleotides were synthesized.

\subsubsection{Fabrication}

The oligonucleotides were suspended in $3 \mathrm{XSSC}$ at a concentration of 50 pmol/ul and printed on glass slides (Full moon biosystem).

\subsubsection{Extraction of viral nucleic acids, amplification, and labelling}

Nucleic acids were extracted from infected cell culture with either the DNA minikit or RNeasy kit respectively (QIagen, CA, USA), following manufacturers' instructions. $2 \mu \mathrm{g}$ of nucleic acids were reverse transcribed with Superscript II or III using primer A (Table 1). Primer A possesses a stretch of 9 random bases at the 3' end which allows it to randomly prime any nucleic acid sequences belonging to the template, and a non-random 5' tag. After reverse 
transcription, a second strand cDNA synthesis was carried out with Sequenase (US Biochemicals, USA). PCR amplification of the ensuing double-stranded template was then carried out with primer B, which targets the non-random tag on primer A. The resultant PCR product was then amplified a second round with primer $\mathrm{B}$, in the presence of aminoallyl-dUTP andthe amplicons, containing aminoallyl group was then purified, and labelled with Cy3-dyes. The dye ratio was monitored, and we only proceed with hybridisation when the dye ratio lies between 30 and 60 .

\subsubsection{Hybridisation}

Microarray hybridisation was performed. All arrays were imaged using a 4000B Axon scanner with the GenePix software Version 4 (Foster City, CA). The microarray data were converted to a colour visualisation in which the Cy-3 intensity of each viral-specific sequences hybridised onto the chip was imaged. Generally, the microarray was imaged at $500 \mathrm{pmt}$, and only at $400 \mathrm{pmt}$ if the background is high.

\subsubsection{Data processing and analysis}

The image was mapped to a .gal file containing the oligonucleotide identification, the spots aligned manually or by default, and normalised to subtract the background signals. The final output data from GenPix software were extracted into a .gpr file. The .gpr file required a Perl program to further process the useful data within the .gpr file into a .vdar files before submission to the E-predict and BayesMicro for analysis.

\subsubsection{Experiment workflow for RSV infections}

RSV A2 strains were infected in two types of cell lines (Hep2 and mouse macrophages) [Table 2.1]. Two types of Affymetrix GeneChips including GeneChip HG-U133 Plus 2.0 Array and GeneChip Mouse 4302.0 Array were applied in our experiment to examine the genome-wide gene expression in human Hep2 cells and mouse macrophages at different post infection time points.

The RSV A2 strain was was prepared using Hep2 cell culture. RSV particles were recovered from tissue culture media by centrifugation at 
$150,000 \mathrm{~g}$ for $2 \mathrm{hr}$ at $4^{\circ} \mathrm{C}$, after which the virus was gently and uniformly resuspended in an equal volume of fresh DMEM with $2 \%$ FCS and stored at $80^{\circ} \mathrm{C}$. A Hep 2 cell microplaque assay was applied to confirm the infectivity of the resulting inoculum. During the time of infection, the virus was thawed and spun at $1200 \mathrm{rpm}$ for 10 minutes and the cells were infected with RSV A2 at an $\mathrm{MOI}=5$ using DMEM (Invitrogen) with $2 \% \mathrm{FBS}$ at $33^{\circ} \mathrm{C}$ in $5 \% \mathrm{CO} 2$.

Investigation of transcriptome from 4hpi to 15hpi in RSV-infected Hep2 cells allow to observe the formation of progeny virus, but is prior to the cell damage that occurs later in the replication cycle that could cause indirect changes in the host cell expression profile. Besides, the virus infectivity recovered from macrophages indicated virus titres of $2 \times 10^{1} \mathrm{pfu} / \mathrm{ml}$ (similar between $2.5 \mathrm{hpi}$ and 20hpi), and only sporadic stained cells were detected using the tissue culture supernatant of RSV-infected macrophages. Thus, RSV infection in macrophages might result in the formation of virus antigen and the production of inclusion bodies, however, the efficient infectious virus particle production did not occur [156][364].

The genome-wide gene expression profiles in Hep2 cells and mouse macrophages were examined using the GeneChip Mouse Genome 4302.0 Array (Affymetrix, USA) and GeneChip Human Genome HG U133 Plus 2.0 Array (Affymetrix, USA) high density microarray systems. Different infected cells were harvested at $4^{\circ} \mathrm{C}$ using RNAlater (Ambion) in PBS buffer, and total RNA was extracted using RNeasy minikit (Qiagen). Double-stranded cDNA was synthesized from $3 \mu \mathrm{g}$ of total cRNA with the GeneChip One-Cycle cDNA synthesis kit (Invitrogen, Affymetrix), followed by synthesis of biotin-labelled cRNA using the GeneChip IVT labeling kit (Affymetrix). After cRNA fragmentation, $15 \mu \mathrm{g}$ of labeled cRNA was hybridized to Mouse Genome 430 2.0 Array (Affymetrix, USA) and GeneChip Human Genome HG U133 Plus 2.0 Array (Affymetrix, USA). After hybridization, these chips were then washed and stained using the GeneChip Fluidics Station 450 (Affymetrix). Finally, GeneChip scanner 3000 (Affymetrix) was employed in scanning the chips, and quality control and data acquisition were performed according to the standard protocols available from Affymetrix. Normalization using a global scaling strategy to a target intensity of 500 was first performed using GCOS (v1.1, Affymetrix) before generating the .CHP files [156][364]. 


\subsubsection{Experiment workflow for influenza A viruses infections}

Eight influenza A virus strains with five subtypes (H1N1/WS, H9N2, H5N3, H5N2/F59, H5N2/F189, H5N2/F118, H5N3, H7N1 and pH1N1/478) from egg propagated were infected in three types of cell lines including A549, Chick Embryo Fibroblast (CEF) and Madin Darby Canine Kidney (MDCK) cells [Table 2.2]. RNA quantification and qPCR analysis suggested that infection of H5N3 contributed to the highest vRNA levels in A549 cells, followed by $\mathrm{H} 5 \mathrm{~N} 2 / \mathrm{F} 118, \mathrm{H} 1 \mathrm{~N} 1 / \mathrm{WS}$ and H9N2. In CEF cells, infection of H5N3 also caused the highest vRNA levels, while infection of other viruses resulted in similar vRNA levels. The higher vRNA levels were detected in H1N1/WS and H5N3 infected MDCK cells when compared to H5N2/F118 and H9N2 infected MDCK cells, and pH1N1/478 replicated least efficiently [365].

Three types of Affymetrix GeneChips including GeneChip HG-U133 Plus 2.0 Array, Chicken Genome Array and Canine Genome 2.0 Array were applied in our experiment to examine the genome-wide gene expression in human, chicken and canine at different post infection time points. Another three subtypes of influenza A viruses were also infected in mouse macrophages, and GeneChip Mouse 430 2.0 Array was applied to examine the genome-wide gene expression profiles in mouse macrophages at different post infection time points [Table 2.3].

All virus stocks were prepared in 9 to 11-day-old embryonated chicken eggs, and the infectivity was confirmed based on standard overlay plaque assay. Virus infections in A549, MDCK and CEFs were carried out in Dulbecco's Modified Eagle's medium (DMEM) (Invitrogen) with 2\% FBS and pen/ strep at $37 \mathrm{uC}$ in $5 \% \mathrm{CO} 2$. Virus was allowed to absorb to the cell monolayer for $1 \mathrm{hr}$ at $37^{\circ} \mathrm{C}$, and virus infections at an $\mathrm{MOI}=4$ in all cells were carried out in Dulbecco's Modified Eagle's medium (DMEM) (Invitrogen) with 2\% FBS and pen/ strep at $37^{\circ} \mathrm{C}$ in $5 \% \mathrm{CO} 2$.

The cells either mock-infected or virus-infected at specific infection time points were harvested in RNAlater (Ambion, USA) and PBS buffer (1:1), aliquoted, pelleted and stored at $-80^{\circ} \mathrm{C}$. Total RNA was extracted using the RNeasy MiniKit (Qiagen, USA) and quantified using the Nanodrop ND-1000 Spectrophotometer (Thermo Fischer Scientific, USA). Double-stranded cDNA was synthesized from $3 \mu \mathrm{g}$ of total cRNA with the GeneChip One-Cycle cDNA 
synthesis kit (Affymetrix, USA), followed by synthesis of biotin-labelled cRNA using the GeneChip IVT labelling kit (Affymetrix, USA). After cRNA fragmentation, $15 \mu \mathrm{g}$ of biotin-labelled cRNA was hybridized to the GeneChip Canine Genome 2.0 Array (Affymetrix, USA), the GeneChip Chicken Genome Array (Affymetrix, USA), Genechip Human Genome HG U133 Plus 2.0 Array (Affymetrix, USA) and GeneChip Mouse 4302.0 Array (Affymetrix, USA), seperately. The arrays were then washed and stained using the Hybridization, Wash and Stain Kit (Affymetrix, USA) and the GeneChip Fluidic Station 450 (Affymetrix, USA), respectively. Finally, GeneChip scanner 3000 (Affymetrix, USA) was applied in scanning the arrays, and Affymetrix .CHP files were generated from GeneChip Operating Software (GCOS) version 5.0 after appropriate normalization [365].

Table 2.2 Design of experiments in RSV infected host cells. RSV A2 strains were infected in Hep2 cells and mouse macrophages, and host cell gene expression profiles were detected at different post infection hours.

\begin{tabular}{|l|l|l|l|l|l|}
\hline Name of strain & Subtype & Abbreviation & Origin & Cell type & Post infected time points \\
\hline RSV A2 & A & RSV & Human & Hep2 & 4hpi, 8hpi, 12hpi, 15hpi \\
\hline RSV A2 & A & RSV & Human & Macrophage & 4hpi, 24hpi \\
\hline
\end{tabular}

There are two antigenic subgroups of HRSV, calles A and B, which exhibit aa sequence identity ranging from $96 \%(\mathrm{~N})$ to $53 \%(\mathrm{G})$, and which are approximately $50 \%$ or $5 \%$ related antigenically in the $\mathrm{F}$ or $\mathrm{G}$ protein, respectively, with the overall differene in reciprocal crossneutralization being up to four-fold [389].

\subsubsection{Experiment workflow for poxviruses infections}

Three subtypes of Poxviruses were infected in two types of cell lines (A549 and mouse RAW cells) [Table 2.4]. Two types of Affymetrix GeneChips including GeneChip HG-U133 Plus 2.0 Array and GeneChip Mouse 4302.0 Array were applied in our experiment to examine the genome-wide gene expression in human lung epithelial cell line, A549 cells and mouse RAW cells at different post infection time points.

All poxviruses stocks were prepared using VERO cell culture, and the infectivity was assessed based on overlay plaque assay. Virus was allowed to absorb to the cell monolayer for $1 \mathrm{hr}$ at $37^{\circ} \mathrm{C}$, and virus infections in A549 and RAW were carried out in Dulbecco's Modified Eagle's medium (DMEM) (Invitrogen) with $2 \% \mathrm{FBS}$ and $1 \%$ pen/ strep at $37^{\circ} \mathrm{C}$ in $5 \% \mathrm{CO} 2$. 
A549 and RAW were either mock-infected or virus-infected at specifc time points at an $\mathrm{MOI}=10$. Double stranded cDNA was synthesized from $3 \mu \mathrm{g}$ of total RNA with the GeneChip One-Cycle cDNA synthesis kit (Affymetrix), followed by synthesis of biotin-labelled cRNA using the GeneChip IVT labelling kit (Affymetrix). After cRNA purification and fragmentation, $15 \mu \mathrm{g}$ of biotin-labelled cRNA was hybridized to the GeneChip HG-U133 Plus 2.0 Array or the GeneChip Mouse 4302.0 Array. The arrays were washed and stained using the Hybridization, Wash and Stain kit and the GeneChip Fluidics Station 450 (Affymetrix) according to standard Affymetrix protocols. Finally, the arrays were scanned using the GeneChip scanner 300 (Affymetrix) and .CHP files were generated from GeneChip Operating Software (GCOS) after proper normalization.

Table 2.3 Design of experiments in influenza viruses infected host cells. Six subtypes influenza viruses were infected in three type cell lines with different combinations, and host cell gene expression profiles were detected at different post infection hours in each combination.

\begin{tabular}{|c|c|c|c|c|c|}
\hline Name of strain & Subtype & Abbreviation & Origin & Cell type & Post infected time points \\
\hline ANS/33 & H1N1 & H1N1 & Human & A549, CEF, MDCK & 2hpi, 4hpi, 6hpi, ${ }^{*} 8 \mathrm{hpi}, 10 \mathrm{hpi}$ \\
\hline A/Singapore/478/2009 & H1N1 & pH1N1 & Human & A549 & 2hpi, 4hpi, 6hpi, 8hpi, 10hpi \\
\hline A/Duck/Malaysia/01 & H9N2 & H9N2 & Avian & A549, CEF, MDCK & 2hpi, 4hpi, 6hpi, 8hpi, 10hpi \\
\hline A/Duck/Malaysis/F118/08/2004 & H5N2 & H5N2(F118) & Avian & A549, CEF, MDCK & 2hpi, 4hpi, 6hpi, 8hpi, 10hpi \\
\hline A/Duck/Malaysia/F59/04/1998 & $\mathrm{H} 5 \mathrm{~N} 2$ & H5N2(F59) & Avian & A549, CEF, MDCK & 10hpi \\
\hline A/Duck/Malaysia/F189/07/2004 & $\mathrm{H} 5 \mathrm{~N} 2$ & H5N2(F189) & Avian & A549, CEF, MDCK & 10hpi \\
\hline A/Duck/Malaysia/F119/3/1997 & H5N3 & H5N3 & Avian & A549, CEF, MDCK & 10hpi \\
\hline Alfairybluebird/Singapore/F92/09/94 & $\mathrm{H} 7 \mathrm{~N} 1$ & H7N1 & Avian & A549, CEF, MDCK & 10hpi \\
\hline
\end{tabular}

A549 represents "Human lung adenocarcinoma epithelial cell"; CEF represents "Chicken embryo fibroblast cell"; MDCK represents "Madin-darby canine kidney cell". * represents that H1N1 infection at 8 hpi was only investigated in CEF cells.

Table 2.4 Design of experiments in influenza viruses infected mouse macrophages. Six subtypes influenza viruses were infected in mouse macrophages, and host cell gene expression profiles were detected at different post infection hours.

\begin{tabular}{|l|l|l|l|l|l|}
\hline Name of strain & Subtype & Abbreviation & Origin & Cell type & Post infected time points \\
\hline ANW/33 & H1N1 & H1N1 & Human & Macrophage & 2hpi, 24hpi \\
\hline A/Duck/Malaysis/F118/08/2004 & H5N2 & H5N2(F118) & Avian & Macrophage & 2hpi, 24hpi \\
\hline A/Duck/Malaysia/F119/3/1997 & H5N3 & H5N3 & Avian & Macrophage & 24hpi \\
\hline
\end{tabular}


Table 2.5 Design of experiments in poxviruses infected host cells. Three subtypes of Poxviruses were infected in A549 cells, and mouse RAW cells with different post infection time points, and host cell gene expression profiles were detected at different post infection hours.

\begin{tabular}{|c|c|c|c|c|c|}
\hline Name of strain & Subtype & Abbreviation & Origin & Cell type & Post infected time points \\
\hline Brighton strain & Cowpox virus & Cowpox virus & Cow & A549 & $2 \mathrm{hpi}, 4 \mathrm{hpi}, 6 \mathrm{hpi}, 8 \mathrm{hpi}, 10 \mathrm{hpi}$ \\
\hline Lister strain & Vaccinia virus & Lister virus & N/A & A549 & $2 \mathrm{hpi,} 4 \mathrm{hpi,} 6 \mathrm{hpi}, 8 \mathrm{hpi}, 10 \mathrm{hpi}$ \\
\hline Brighton strain & Cowpox virus & Cowpox virus & Cow & Mouse Raw & $2 \mathrm{hpi}, 16 \mathrm{hpi}$ \\
\hline Lister strain & Vaccinia virus & Lister virus & N/A & Mouse Raw & $2 \mathrm{hpi,} \mathrm{16hpi}$ \\
\hline Moscow strain & Ectromelia virus & Ectromelia virus & Mouse & Mouse Raw & $2 \mathrm{hpi}, 16 \mathrm{hpi}$ \\
\hline
\end{tabular}

A549 represents "Human lung adenocarcinoma epithelial cell".

Species can be classified by pock morphologies and by ceiling temperature for growth on the chorioallantoic membrane of embryonated chicken eggs. Ecological niche and host range are useful in some cased, but in others can be misleading. Restriction enzyme polymorphisms of the terminal regions of viral DNA outsides of the core of common genes also aids the classigication process [389].

\subsubsection{Quantitative Real-time quantitative PCR (qPCR)}

Total RNA was extracted from cells at $4 \mathrm{uC}$ using the RNeasy kit (Qiagen, USA) and reverse-transcribed using Superscript II (Invitrogen, USA) according to manufacturer's instructions. Primers for cell-specific genes were designed using the Probefinder software (http://qpcr.probefinder.com/organism.jsp) from the Universal Probe Library (UPL) Design Center (Roche). Quantitative Real-time PCR (qPCR) was carried out with the iCycler System (BioRad) following the protocol previously described. The sequences of the elongation factors (EF) EEF1A1 (H. sapiens) (Genebank Accession Number NM_001402), EEF1A1 (G. gallus) (Genebank Accession Number NM_204157) and EEF1A2 (C. lupus familiaris) (Genebank Accession Number NM_531877) were used as the reference genes since their expression were validated as being "not significantly changed" throughout all observed time points in the microarray analyses (P-value,0.05). Both absolute and relative quantification analysis were done using comparative $\mathrm{Ct}$ (DDCt method). Standard curves for $M$ and EF were generated and the number of copies of $\mathrm{M}$ for each virus was calculated relative to 104 copies of corresponding cell line's EF gene. Relative fold-change of the host virus gene expression were calculated with respect to the mock-infected cells and normalized with the corresponding cell line's EF gene. Primers and probes used for the virus $\mathrm{M}$ and host EF gene are shown in Table S4. The statistical analysis was performed on single and paired samples as appropriate by applying the student t-test using a significance cutoff of P-value, 0.05 . 


\subsection{Bioinformatics analysis of microarray data}

\subsubsection{Gene expression profiles}

GeneSpring GX (version 11) was employed to analyze the data derived from different virus strains infected different cell lines. Normalization was performed using the RMA method, among which (a) per chip normalization to the 50th percentile and (b) per gene normalization where virus-infected samples were normalized to mock infected Samples. Genes were selected for statistical analysis according to the following criteria: (i) only genes that were flagged as present in all three replicates (mock- or virus-infected), and (ii) a fold change $\geqslant$ 2 (up- or down-regulated) between virus- and mock-infected samples in all triplicate microarray experiments. Finally, statistical analysis based on the Benjamini \& Hochberg False Discovery method or one-way analysis of variance (ANOVA)was performed with a P-value cutoff of $\leq 0.05$ to determine significantly expressed genes during virus infection. For data from RSV and influenza A strains infected macrophages, a gene-level analysis in GeneSpring GX (version 11) was applied with signals from probes summarized into gene level. After that, the same normalization and statistical analysis mentioned above were performed step by step by step [156, 364-365].

\subsubsection{Functional analysis}

Probe sets were clustered by similar expression patterns and analyzed for enrichment of gene ontology (GO) terms and transcriptional factor (TF) binding sites using the Expander (version 5.2) software package [103, 104]. Those probe sets that significantly expressed at least at one time point were uploaded into the software with their corresponding expression fold changes. The enrichment of particular GO terms or TF binding sites within clusters was done by using the TANGO and PRIMA algorithms, respectively, within the Expander package, using a P-value threshold of 0.05. Eventually, the lists of GO terms and TFs were significantly enriched with the corresponding gene probe sets, and the number of genes within each functioned GO term or regulated by TFs along with the p-value are reported. 


\subsubsection{Core analysis}

The key networks, biological functions and canonical pathways were investigated by core analysis using Ingenuity Pathways Analysis (IPA; Ingenuity Systems http://www.ingenuity.com). Those differentially expressed probe sets/genes with known gene-probe ID numbers and corresponding expression fold changes were uploaded into the software. IPA uses a righttailed Fisher's exact test to calculate P-value for different functional groups, with P-value cut-off of $\leq 0.05$. 


\section{Chapter III. A Bioinformatics Approach to Detect Virus using Microarray Technology}

\subsection{Introduction}

\subsubsection{Background of virus identification based on microarray diagnostic chips}

Infectious diseases caused by bacterial and viral pathogens have posed a growing threat to public health and economy. With the recent outbreak of pandemics including Severe Acute Respiratory Syndrome and Swine Influenza A (H1N1), viruses researches have been of grave concern of World Health Organization (WHO). And studies on pathogens identification will be beneficial for the future clinical diagnostics and drug discoveries [105-107].

In past decades, different viral diagnostic techniques have developed rapidly. Among them, DNA microarray as an advanced technique has been widely initiated and applied in broad-based viral diagnosis [42, 108-111]. Various of diagnostic chips such as ViroChip from DeRisi group have been subsequently designed and pioneered [77].

However, the application of microarray technology in viral diagnosis has also posed a challenge to users due to the difficulty in the data interpretation (Wang et al., 2003)[27]. In the process of generating the detection result based on observed hybridization intensity pattern, strong and precise software are necessary and essential for the data analysis. In terms of this demand, several computational strategies such as E-Predict [78], DetectiV [79], PDA [49] and PhyloDetect [82] have been reported for analyzing pathogen diagnostic microarray data. However, only some of these published strategies are built into software which are available to the public. Moreover, these published strategies have been developed based on different hypothesis and algorithms which are dependent on different chips.

In our project, we fabricated the diagnostic microarray chip from Wang et al (2003), and this group also developed E-Predicted software in order to interpret the data retrived from this kind of chip [27]. On this basis, E-Predict was employed to analyze our microarray data. Although the results generated from E-Predict indicated that this software was able to predict the positive outcomes in most of our sample, the negative outcome still existed. Detailed 
investigation provided clues that there are two major weaknesses lying in EPredict: one is regarding to the choices of normalization and scoring functions; the other is the least separation of virus species within the same virus family [77]

To improve the sensitivity of virus detection, a web-accessible novel software called BayesMicro was designed as an alternative analysis tool. In this new strategy, we employed a new calculation formula in which the non-linear relationship between experimental hybridization intensity signals and theoretical binding energy values was taken into consideration. At the meanwhile, we applied an optimized transformation formula in which the outliers with extremely high hybridization intensity signals were treated separately. Besides, a new algorithm was proposed for differential detection on different virus species within the same virus family. Consequently, the virus detection results generated from BayesMicro showed that it was able to report all the positive outcomes in our 12 test arrays, suggesting the effectivity of this novel software.

\subsubsection{Description of E-Predict software}

E-Predict (http://derisilab.ucsf.edu/epredict/) is the first computation strategy raised together with the corresponding 70-mer oligonucleotides microarray chip by DeRisi group, and it is treated as the default computation strategy to interpret the data retrieved from this ViroChip. Since we fabricated the same diagnostic microarray chip, E-Predict was also employed to analyze our microarray data.

Before calculation of E-Predict, a library of theoretical binding energy profiles, representing species with known genomic viruses sequences until July 2004, was computed by ArrayOligoSelector software [112]. As shown in Table 3.1, all these probe sequences representing different viruses constituted a theoretical binding energy matrix collectively, in which the rows represented different virus species and columns contained different probes.

The evidence provided by Bozdech et al (2003) has proved that the experimental hybridization intensity pattern was closely relevant to the theoretical binding energy profile, especially when the theoretical binding energy values were more significant than $-30(\mathrm{kcal} / \mathrm{mol})$ [112]. Thus, E-Predict 
applied several similarity metrics such as Pearson correlation to score the correlation between these theoretical binding energy profiles and the experimental hybridization intensity pattern [78]. In this process, only the theoretical binding energy values with high-level significance (more significant than $-30(\mathrm{kcal} / \mathrm{mol}))$ were considered for the score calculation in order to avoid the false positive results caused by cross-hybridization. In the final detection list, the candidate virus with the higher correlation score presented its higher possibility to be the positive prediction.

Table 3.1 Theoretical binding energy profiles. The rows represent different virus species, and columns contain different probe IDs as well as their corresponding theoretical binding energy values when theoretically hybridize with the specific virus.

\begin{tabular}{|c|c|c|c|c|}
\hline \multirow[b]{2}{*}{ Virus species } & \multicolumn{2}{|c|}{ Probe No.1 } & \multicolumn{2}{|c|}{ Probe No.2 } \\
\hline & Probe_ID & $\begin{array}{c}\text { Energy } \\
\text { (kcal/mol) }\end{array}$ & Probe_ID & \begin{tabular}{|c|} 
Energy \\
(kcal/mol
\end{tabular} \\
\hline 11809 | Moloney murine sarcoma virus | Retroviridae & 9626962 1_rc & -109.4 & 9626953 2_rc & -67.6 \\
\hline 11663 Ovine lentivirus | Retroviridae & VirusP70_10147 & -16.4 & $9629258 \quad 45$ & -38.3 \\
\hline $12465 \mid$ Barley yellow mosaic virus | Potyviridae & 21427654_285_rc & -43 & 1580806593 & -100.9 \\
\hline 10862 Xanthomonas phage Cf1c | Inoviridae & 9626133280 rc & -117.1 & $9790346 \_172$ rc & -19.2 \\
\hline 12466 Barley mild mosaic virus $\mid$ Potyviridae & $21450043 \_159$ & -13.9 & 19749338 224_rc & -108.3 \\
\hline 11665 Equine infectious anemia virus | Retroviridae & 962653099 & -91.6 & $9627277 \quad 240$ & -12.3 \\
\hline 10867 | Enterobacteria phage lke | Inoviridae & 2042857198 & -17.9 & 17426217 142_rc & -19.4 \\
\hline 10868 Enterobacteria phage If1 | Inoviridae & 17426217 142_rc & -22 & 9630747113 rc & -95.9 \\
\hline 10869 Enterobacteria phage l2-2 Inoviridae & 2042857198 & -17.9 & 17426217 142_rc & -15.5 \\
\hline
\end{tabular}

...... indicates that there are more virus species as well as more corresponding probes and theoretical binding energy values existed.

\subsubsection{Objective}

In our previous researches, we applied E-Predict to analyze our microarray data, however, the detection result were not sensitive to generate positive predictions in all our microarray samples. Thus, proposing another alternative computation strategy is imperative to enhance the reliability of the virus detection results. In this chapter, we aim to describe and evaluate a new software, BayesMicro, in aim to identify virus based on pathogen diagnostic microarray chips.

BayesMicro (http://microarray.sbs.ntu.edu.sg/bayes/entry.html) as a web-accessible software is designed based on a novel Bayesian metric and fulfilled in a Perl language environment. This software not only introduces a new strategy for global detection at the virus species level, with new transformation and scoring 
formulas proposed, but also initiates a second algorithm for differential detection within the virus families.

\subsection{Experiment workflow of pathogen detection}
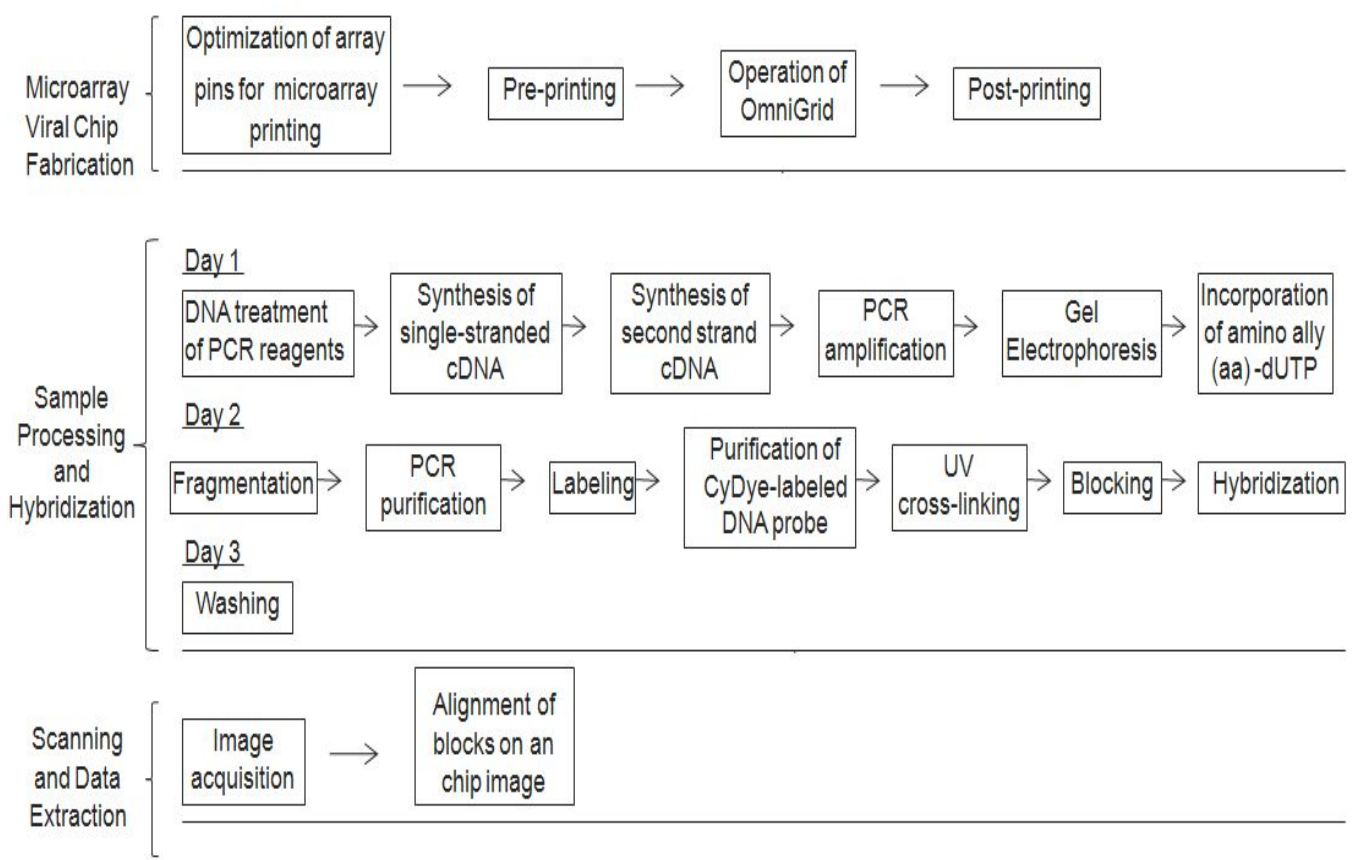

Figure 3.1 The experiment workflow which was performed in DSO national lab. The experiment workflow which was performed in DSO national lab. The experiment procedure can be divided into three parts: (1) Microarray viral chip fabrication; (2) Sample processing and hybridization; (3) Scanning and data extraction.

\subsection{Methods}

\subsubsection{Microarray design}

The complete list of viral oligonucleotide sequences represented on the microarray viral chip was downloaded from DeRisi's website (http://derisilab.ucsf.edu/virochip) (Wang et al., 2003)[27]. Each virus was represented by top 5 highly conserved oligonucleotides and corresponding 5 reverse complement oligonucleotides. In addition, 10 random oligonucleotides from 10 human genes were selected as controls. In total, 6233 70-mer oligonucleotides that target to around 600 viruses were synthesized (Sigma) and spotted onto microarray glass slides (Full Moon Biosystems) using Omnigrid microarrayer (GeneMachines). 


\subsubsection{E-Predict analysis procedure}

The Genepix results files .GPR contained the raw experimental hybridization intensity value. Before submitted to E-Predict, the .GPR files needed to be pre-processed in order to generate E-Predict standard input files .VDAR for further analysis.

\subsubsection{Data pre-processing}

To convert the .GPR files into the .VDAR files, description rows and columns needed to be deleted first, with only the columns including ProbeID, F532Mean, F532Mean-B532, F532Median, F532Median-B532 and Flags arrayed in order. After that, the flag values corresponding to each row (each spot in microarray chip) in .GPR files were examined for quality control. If the flag value equaled to -50 or -100 , which meant that the spot on the array was in bad quality or absent, then all the experimental hybridization intensity value corresponding to this spot were reset to 0 (including F532Mean / F532MeanB532 / F532Median / F532Median-B532).

Subsequently, the .GPR files were only composed of rows containing experimental hybridization intensity values (each spot on the microarray chip is corresponding to one probe in the .GPR files) (Table 3.2). Lastly, the duplicate experimental hybridization intensity values representing the same probe were averaged, and the .GPR files were split into four separate .vdar files as shown in Table 3.3. In this pre-processing procedure, all transformations of data format were fulfilled by our own Perl scripts in Appendix 1.

\subsubsection{Submission of data}

The .VDAR files were submitted to E-Predict, and appropriate parameters were selected (following the default setting) (Figure 3.2). After calculation, virus detection result was listed in an output file, in which top suspected virus families were ranked in order. The scores and P-values corresponding to each virus were shown in the result file, and the probes contributing mostly to the final score of each listed virus were also ranked in order (Figure 3.3). 


\subsubsection{BayesMicro}

The set of theoretical binding energy profiles was employed in BayesMicro for comparison with the matrix of experimental hybridization intensity patterns. Distance scores of each candidate virus were calculated and the virus with the most significant distance score was treated as the positive prediction.

In BayesMicro, the computing procedure for global detection at the virus species level consisted of an initial data transformation, Bayesian model selection, as well as assignment of optimal weights. And the distance scores and P-values were calculated for each candidate virus, with 0.05 set as the threshold P-value in known virus detection. In extreme cases that viruses within the same family showed close distance scores, differential detection within the virus family was introduced as an option to improve the reliability of the detection (Figure 3.4).

Table 3.2 Data arrangement in .GPR files. The .gpr files only contain experimental hybridization intensity values from F532Mean/F532Mean-B532/F532Median/F532MedianB532. Each row corresponds to one probe in microarray chip.

\begin{tabular}{|c|c|c|c|c|}
\hline \multirow{2}{*}{ Probe_ID } & \multicolumn{4}{|c|}{ Experimental hybridization intensitie values } \\
\hline & F532 Mean & F532 Mean - B532 & F532 Median & F532 Median - B532 \\
\hline $10120197 \_13$ & 0 & 0 & 0 & 0 \\
\hline 10120197 13_rc & 398 & 307 & 394 & 303 \\
\hline 1012019955 & 0 & 0 & 0 & 0 \\
\hline 10120199 5_rc & 148.5 & 65.5 & 145 & 62 \\
\hline $10120211 \_14$ & 0 & 0 & 0 & 0 \\
\hline 10120211_14_rc & 0 & 0 & 0 & 0 \\
\hline 10120225 & 279 & 175 & 278.5 & 174.5 \\
\hline 1012022512 rc & 0 & 0 & 0 & 0 \\
\hline 101202256 & 126 & 48 & 107 & 29 \\
\hline
\end{tabular}

...... indicates that there are more probes as well as corresponding experimental hybridization intensity values existed. 
Table 3.3 Final .VDAR files generated from the .GPR files. (A), (B), (C), (D) are corresponding to the experimental hybridization intensity coming from F532Mean/ F532MeanB532/ F532Median/ F532Median-B532 respectively.

A

\begin{tabular}{|c|c|c|c|c|c|}
\hline \multicolumn{6}{|c|}{ F532Mean } \\
\hline & Probe No.1 & Probe No.2 & Probe No.3 & Probe No.4 & Probe No.5 \\
\hline Probe_ID & $8486125 \_53$ & 8486125_53_rc & $8486125 \_68$ & 8486129_20 & 8486129_20_rc \\
\hline Hybridization intensity & 211 & 369 & 0 & 239 & 4158 \\
\hline
\end{tabular}

$\mathrm{B}$

\begin{tabular}{|c|c|c|c|c|c|}
\hline \multicolumn{7}{|c|}{ F532Mean-B532 } \\
\hline & Probe No.1 & Probe No.2 & Probe No.3 & Probe No.4 & Probe No.5 \\
\hline Probe_ID & $8486125 \_53$ & $8486125 \_53 \_r C$ & $8486125 \_68$ & $8486129 \_20$ & $8486129 \_20 \_$rC \\
Hybridiza... \\
\hline
\end{tabular}

$\mathrm{C}$

\begin{tabular}{|c|c|c|c|c|c|c|}
\hline \multicolumn{7}{|c|}{ F532Median } \\
\hline Probe_ID & Probe No.1 & Probe No.2 & Probe No.3 & Probe No.4 & Probe No.5 & \\
\hline Hybridization intensity & $8486125 \_53$ & $8486125 \_53 \_$rC & $8486125 \_68$ & $8486129 \_20$ & $8486129 \_20 \_$rC \\
\hline
\end{tabular}

$\mathrm{D}$

\begin{tabular}{|c|c|c|c|c|c|}
\hline \multicolumn{7}{|c|}{ F532Median-B532 } \\
\hline & Probe No.1 & Probe No.2 & Probe No.3 & Probe No.4 & Probe No.5 \\
\hline Probe_ID & $8486125 \_53$ & $8486125 \_53 \_r c$ & $8486125 \_68$ & $8486129 \_20$ & $8486129 \_20 \_$rC \\
\hline Hybridiz.... \\
\hline
\end{tabular}

..... indicates that there are more probes as well as corresponding experimental hybridization intensity values existed. 


\section{E-Predict...}

\section{Submit}

Dataset to analyze

Arrays to analyze All

User weights MV_72worst_medRaw500_badYdens_Goatpox_Sheeppox_Lumpy

Array normalizaion Sum $\checkmark$

E matrix 2004-07-22

E matrix normalization Quadratic $\checkmark$

E matrix energy filter $\sqrt{30} \mathrm{~V}$

Number of iterations $1 \checkmark$

Distance Metric Pearson Uncentered

Vector Length All oligos

Output sort by Distance $\checkmark$

Output $10 \vee$ top families per array

Output $3 \vee$ top genomes per family

Output $5 \vee$ top oligos per genome

Generate E-Cluster using None $\checkmark$

Redirect output to file

\section{Submit}

Figure 3.2 User interface of E-Predict. Different normalization methods, similarity metrics and other parameters are offered (adapted from http://derisilab.ucsf.edu/epredict/). 


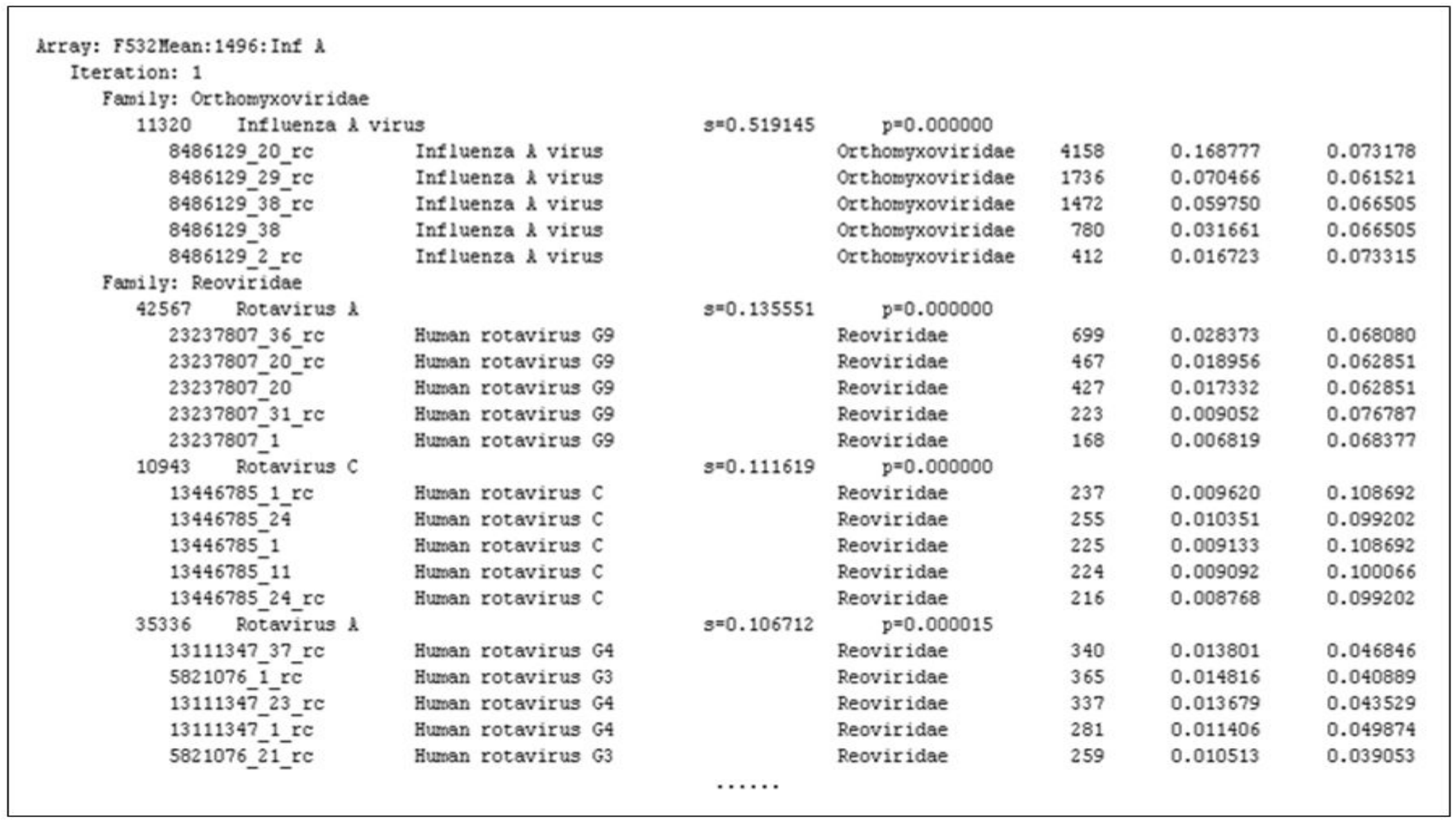

Figure 3.3 Final result generated from E-Predict. In this list, influenza A virus ranked top with the highest score. The experimental hybridization intensities from probe 8486129_20rc, 8486129_29_rc and 8486129_38_rc contribute mostly to identify influenza A virus. .....indicates that more viruses with corresponding top 5 probe sets with highest hybridization intensities were detected. 


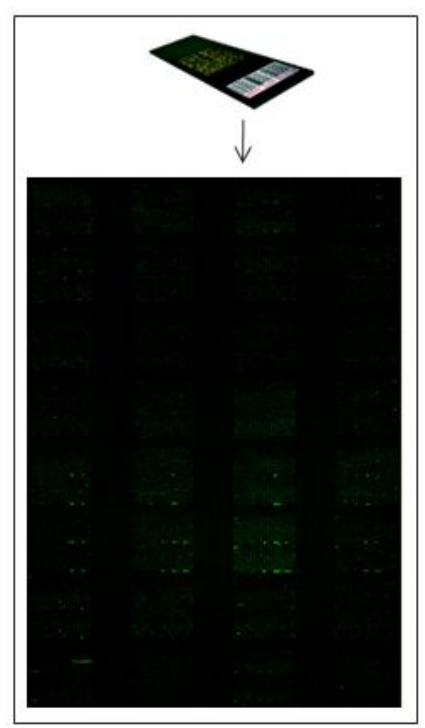

a microarray chip after image scanning in our datasets

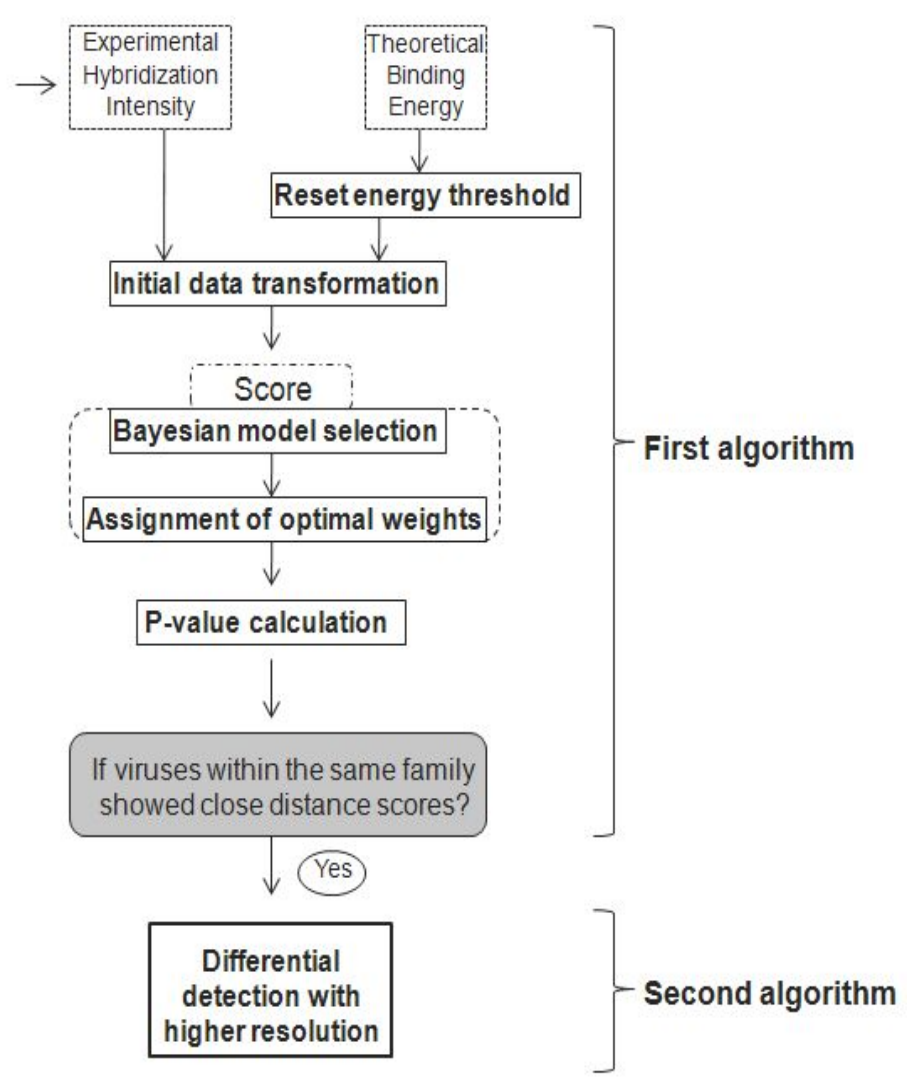

Figure 3.4 An overview of the BayesMicro strategy. Cultured or clinical samples were hybridized to a pathogen diagnostic chip. The experimental hybridization intensity pattern retrieved from microarray chip was compared with a set of theoretical binding energy profiles that was introduced from E-Predict software. Firstly, these two groups of data were transformed into the same scale. Secondly, the distance score and P-values were calculated for each candidate virus on the basis of a Bayesian model with optimal weights assigned. Lastly, the result list was investigated carefully. If the distance scores of viruses within the same family ranked top with close distance scores in this process, a further detection within virus family would be considered to validate the previous virus species identification result.

Table 3.4 Comparison of detection results analyzed based on different thresholds.

\begin{tabular}{|c|c|c|c|c|c|c|}
\hline \multicolumn{7}{|c|}{ Human coronavirus $\mathrm{OC} 43$} \\
\hline \multirow{2}{*}{$\begin{array}{l}\text { Threshold } \\
\text { (kcal/mol) }\end{array}$} & \multicolumn{3}{|c|}{ E_Predict } & \multicolumn{3}{|l|}{ BayesMicro } \\
\hline & Virus species & \begin{tabular}{|c|} 
Similarity \\
Score
\end{tabular} & |Distance| & Virus species & \begin{tabular}{|c|} 
Distance \\
Score \\
\end{tabular} & |Distance \\
\hline \multirow{2}{*}{-30} & Human coronavirus $\mathrm{OC} 43$ & 0.1837 & \multirow{2}{*}{0.0230} & Avian adeno-associated virus ATCC VR-865 & 0.2418 & \\
\hline & Bovine coronavirus & 0.1607 & & Human coronavirus $\mathrm{OC} 43$ & 0.2679 & \\
\hline \multirow{2}{*}{-40} & Human coronavirus $\mathrm{OC} 43$ & 0.1843 & \multirow{2}{*}{0.0236} & Human coronavirus $\mathrm{OC} 43$ & 0.2679 & \multirow{2}{*}{0.0259} \\
\hline & Bovine coronavirus & 0.1607 & & Murine adenovirus $\mathrm{A}$ & 0.2938 & \\
\hline \multirow{2}{*}{-60} & Human coronavirus $\mathrm{OC} 43$ & 0.1891 & \multirow{2}{*}{0.0265} & Human coronavirus $\mathrm{OC} 43$ & 0.2679 & \multirow{2}{*}{0.0484} \\
\hline & Bovine coronavirus & 0.1626 & & Ground squirrel hepatitis virus & 0.3163 & \\
\hline \multirow{2}{*}{-70} & Human coronavirus $\mathrm{OC} 43$ & 0.1969 & \multirow{2}{*}{0.0292} & Human coronavirus $\mathrm{OC} 43$ & 0.2679 & \multirow{2}{*}{0.1272} \\
\hline & Bovine coronavirus & 0.1677 & & Bovine coronavirus & 0.3951 & \\
\hline
\end{tabular}

Bold represents positive virus; Italic repesents significant result and corresponding threshold. 


\subsubsection{Reset energy threshold}

It has been indicated that high cross-hybridization is majorly present in the region where the binding energy is less significant than $-30(\mathrm{kcal} / \mathrm{mol})$ [112]. Thus E-Predict took this information into consideration, and set $-30(\mathrm{kcal} / \mathrm{mol})$ as its default energy threshold in order to avoid the negative effects from crosshybridization.

In our study, evaluation based on different thresholds were performed, and the detection results based on threshold $-70(\mathrm{kcal} / \mathrm{mol})$ indicated to be most significant. In one typical example shown in Table 3.4, Human coronavirus OC43 ranked $1^{\text {st }}$ based on threshold $-70(\mathrm{kcal} / \mathrm{mol})$, while this positive virus only ranked $4^{\text {th }}$ based on threshold $-30(\mathrm{kcal} / \mathrm{mol})$ using BayesMicro software. Although the positive virus showed the topmost based on threshold -40 and -60 $(\mathrm{kcal} / \mathrm{mol})$, distances of scores between the $1^{\text {st }}$ and $2^{\text {nd }}$ viruses showed smaller compared to the distance of scores based on threshold $-70(\mathrm{kcal} / \mathrm{mol})$. Moreover, the comparison of the detection results using E-Predict software also indicated that the result based on new threshold $-70(\mathrm{kcal} / \mathrm{mol})$ was also most significant. Taken together, the virus detection became more sensitive if we adopt the new energy threshold $-70(\mathrm{kcal} / \mathrm{mol})$ in our cultured samples. Consequently, this default threshold of the energy was raised to $-70(\mathrm{kcal} / \mathrm{mol})$ for our known virus detection in BayesMicro. Under this new threshold -70 ( $\mathrm{kcal} / \mathrm{mol})$, more crosshybridization would be ignored, and therefore the virus detection sensitivity would be increased. In the event of unknown virus detection, the threshold could be set with less stringent value.

\subsubsection{Initial data transformation/normalization}

Before calculating the distance scores between the theoretical energy value profiles and experimental hybridization intensity pattern, it was observed that these two groups of data located in different scales: the value of theoretical binding energy profiles varied from 0 to 100 while the value of experimental hybridization intensity varied from 0 to 2,000 (even 5,000). The magnitudes between two groups of data caused the overall comparison to be difficult, so that transforming them into the same level was necessary. In our study, we practiced our data with different transformation formulas, such 


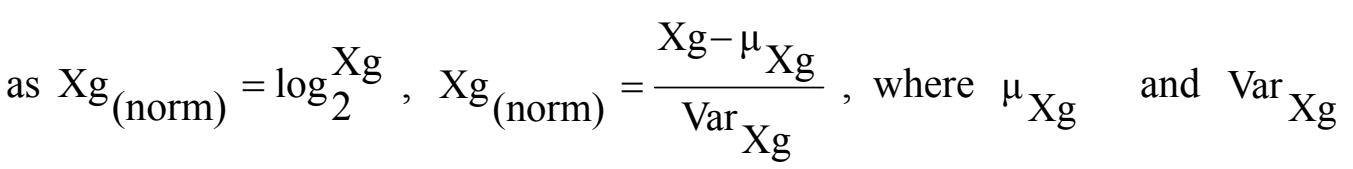
represent the mean and variance of $\mathrm{Xg}$ respectively, and the formulas applied in E-Predict $\mathrm{Xg}_{(\text {norm })}=\frac{\mathrm{Xg}}{\sum \mathrm{Xg}}$ and $\mathrm{Xg}_{(\text {norm })}=\frac{\mathrm{Xg}^{2}}{\sum \mathrm{Xg}^{2}}$, where $\sum \mathrm{Xg}$ and $\sum \mathrm{Xg}^{2}$ represent the sum and quadratic sum of $\mathrm{Xg}$ separately. Eventually, the formula (1) performed best, and therefore was adopted in our new strategy:

$$
\mathrm{Xg}_{(\text {norm })}=\frac{\mathrm{Xg}-\mu_{\mathrm{Xg}}}{\max \left(\operatorname{abs}\left(\mathrm{Xg}-\mu_{\mathrm{Xg}}\right)\right)}
$$

where $\mathrm{Xg}$ and $\mathrm{Xg}_{\text {(norm) }}$ are the values before or after the transformation of each theoretical binding energy value or experimental hybridization intensity value respectively.

After transformation using this formula (1), all the data in both two groups could be reset to a zero mean sequence, with the maximum absolute value of each sequence reset as 1 . Subsequently, these two groups of data enabled to be used for straightforward comparison regardless of the amplitude of change.

When formula (1) was applied in pre-processing the data, it did not perform well as we expected in the experimental hybridization intensity values. Investigation suggested that the instability was originated from the outliers with high value in experimental hybridization intensity value. These outliers are most likely to be given rise by systematic instability or experimental errors on microarray platform [113]. Tracing back to the above equation (1), existence of outliers with high value enabled to increase the value of $\max (\operatorname{abs}(\mathrm{Xg}-\mu \mathrm{g}))$, and therefore compressed other intensity value too low to reflect its original significance. In order to avoid the negative impact from unexpected outliers with high value, the set of experimental hybridization intensities was preprocess by a package called outliers for R. After that, the right outliers with high value were reset as 1 directly, and the remaining data participated in 
transformation. Thus, the final transformation formula used in our strategy is as follows (2):

$$
\begin{cases}\mathrm{Xg}(\text { norm })=1, & \text { (Outliers with high value) } \\ \mathrm{Xg}_{(\mathrm{norm})}=\frac{\mathrm{Xg}-\mu_{\mathrm{Xg}}}{\max \left(\operatorname{abs}\left(\mathrm{Xg}-\mu_{\mathrm{Xg}}\right)\right)}, & \text { (Remaining data) }\end{cases}
$$

\subsubsection{Bayesian model selection}

In BayesMicro, the scoring formula was originated from BayesGen deduced by Nguyen et al (2009) [114]. In BayesGen, the distances between different genes in different conditions were calculated as a ratio between two alternative hypotheses, so that functionally related genes that had similar expression trends in different conditions could be identified.

In BayesMicro, we considered this formula from another perspective. The purpose of BayesMicro was to calculate the distance between the experimental hybridization intensity pattern and different theoretical binding energy profiles representing different viruses. Finally, the candidate virus with smallest distance between these two conditions was treated as the positive prediction. And the full Bayesian formula is as follows (3-5):

$$
\mathrm{d}(\mathrm{i}, \mathrm{j})=\prod_{\mathrm{k}=1}^{\mathrm{n}} \frac{\mathrm{p}\left(\mathrm{Xi}^{\mathrm{k}}\right) \mathrm{p}\left(\mathrm{Xj}^{\mathrm{k}}\right)}{\mathrm{p}\left(\mathrm{Xi}^{\mathrm{k}}, \mathrm{Xj}^{\mathrm{k}}\right)} \propto \sum_{\mathrm{k}=1}^{\mathrm{n}} \log \mathrm{p}\left(\mathrm{Xi}^{\mathrm{k}}\right)+\log \mathrm{p}\left(\mathrm{Xj}^{\mathrm{k}}\right)-\log \mathrm{p}\left(\mathrm{Xi}^{\mathrm{k}}, \mathrm{Xj}^{\mathrm{k}}\right)
$$

Where

$$
\begin{gathered}
\log \left(\mathrm{Xi}^{\mathrm{k}}\right) \propto-\log \left[\left(\mathrm{Xi}^{\mathrm{k}}-\mathrm{m}\right)^{2}+\frac{\mathrm{v}^{\mathrm{k}}}{2}\right], \log \mathrm{p}\left(\mathrm{Xj}^{\mathrm{k}}\right) \propto-\log \left[\left(\mathrm{Xj}^{\mathrm{k}}-\mathrm{m}\right)^{2}+\frac{\mathrm{v}^{\mathrm{k}}}{2}\right] \\
\log \mathrm{p}\left(\mathrm{Xi}^{\mathrm{k}}, \mathrm{Xj}^{\mathrm{k}}\right) \propto-\frac{3}{2} \log \left[\sigma^{\mathrm{k}}+\frac{2 \sigma^{\mathrm{k}}}{\sigma^{\mathrm{k}}+v^{\mathrm{k}}}\left(\mu^{\mathrm{k}}-\mathrm{m}^{\mathrm{k}}\right)^{2}+\frac{\mathrm{v}^{\mathrm{k}}}{2}\right]
\end{gathered}
$$

And $\mathrm{m}^{\mathrm{k}}, \mathrm{v}^{\mathrm{k}}, \mu^{\mathrm{k}}$ and $\sigma^{\mathrm{k}}$ are the $\mathrm{k}^{\mathrm{th}}$ component of the data global mean and variance, and the two conditions local mean and variance respectively.

Local mean $\mu^{\mathrm{k}}$ and variance $\sigma^{\mathrm{k}}$ could be generated from $\mathrm{Xi}$ (the $\mathrm{i}^{\text {th }}$ theoretical binding energy) and $\mathrm{Xj}$ (the $\mathrm{j}^{\text {th }}$ experimental hybridization intensity). The global parameters' setting was based on the global condition. After investigation, the global condition contained three elements: (1) experimental 
hybridization intensity of the calculated probe; (2) theoretical binding energy coming from the binding between the calculated probe and the candidate virus; (3) theoretical binding energy coming from the binding between the calculated probe and other viruses. For instance, if we calculated the distance between the candidate Moloney murine sarcoma virus and the positive virus based on 9626962_1_rc, we considered the theoretical binding energy $-109.4(\mathrm{kcal} / \mathrm{mol})$ and the experimental hybridization intensity of this probe in microarray chip as the local parameters. And the energy such as $-84.4(\mathrm{kcal} / \mathrm{mol}),-84.1(\mathrm{kcal} / \mathrm{mol})$, -54.4 ( $\mathrm{kcal} / \mathrm{mol})$, which obtained from the theoretical binding between 9626962_1_rc and other viruses, were treated as other parameters in the global condition [Table 3.5B].

\subsubsection{Assignment of optimal weights}

Weight is the assignment of a quota to a particular segment of the population as a special favor or concession in a proportion. Given matching weights, biased estimates would be obtained for different theoretical binding energy value, and therefore sensitivity of virus detection results would be improved $[115,116]$. In the previous formula (7), we assumed that each probe contributed the same to the final distance, however, in reality they did not. As shown in Table 3.6, 18071213_1713_rc showed a more significant theoretical binding energy value -121 ( $\mathrm{kcal} / \mathrm{mol})$ with Sinorhizobium meliloti phage PBC5 compared to $-40.5(\mathrm{kcal} / \mathrm{mol})$ with Bacteriophage phi CTX, which suggested that more nucleotides in Sinorhizobium meliloti phage PBC5 could be aligned to this probe theoretically. And Sinorhizobium meliloti phage PBC5 subsequently had higher probabilities to be responsible for the high hybridization intensity value of probe 18071213_1713_rc detected in microarray chip. Hence, different weights should be assigned to different theoretical binding energy value before final distance score calculation.

We assumed that the probe Pi had a sequence of theoretical binding energy value $\left(\mathrm{P}_{\mathrm{i}}^{\mathrm{k}}\right.$ representing the $\mathrm{k}^{\text {th }}$ energy of probe $\mathrm{Pi}$ ) corresponding to different viruses. These theoretical binding energy values followed normal distribution, and the weights were calculated with cumulative distribution function (CDF) (Figure 3.5). From the above assumption, the weight of $\mathrm{Pi}^{\mathrm{k}}$ increased when $\mathrm{Pi}^{\mathrm{k}}$ was closer to the right tail of the normal distribution curve. 
Assignment of optimal weight for each probe was listed in Table 3.6. CDF calculation formula of normal distribution is as follows:

$$
\Phi\left(P i^{k}\right)=\frac{1}{\sqrt{2 \pi \int_{-\infty}^{P i^{k}} e^{-t^{2} / 2} d t}}=\frac{1}{2}\left[1+e r f\left(\frac{P i^{k}-\mu}{\sigma \sqrt{2}}\right)\right]
$$

Where $\mu, \sigma$ equal the mean and standard deviation of the probe Pi.

Apply (6) into (3), (7) was obtained:

$$
\mathrm{d}(\mathrm{i}, \mathrm{j})=\prod_{\mathrm{k}=1}^{\mathrm{n}} \frac{\mathrm{p}\left(\mathrm{Xi}^{\mathrm{k}}\right) \mathrm{p}\left(\mathrm{Xj}^{\mathrm{k}}\right)}{\mathrm{p}\left(\mathrm{Xi}^{\mathrm{k}}, \mathrm{Xj}^{\mathrm{k}}\right)} \propto \sum_{\mathrm{k}=1}^{\mathrm{n}}\left[\operatorname{logo}\left(\mathrm{Xi}^{\mathrm{k}}\right)+\log \mathrm{p}\left(\mathrm{Xj}^{\mathrm{k}}\right)-\log \left(\mathrm{Xi}^{\mathrm{k}}, \mathrm{Xj}^{\mathrm{k}}\right)\right] * \Phi\left(P i^{k}\right)
$$

Table 3.5 The theoretical binding energy profile (A) is rearranged by probes (B).

\begin{tabular}{|c|c|c|c|c|}
\hline \multirow[b]{2}{*}{ Virus species } & \multicolumn{2}{|c|}{ Probe No.1 } & \multicolumn{2}{|c|}{ Probe No.2 } \\
\hline & Probe_ID & $\begin{array}{c}\text { Energy } \\
\text { (kcal/mol) }\end{array}$ & Probe_ID & $\begin{array}{c}\text { Energy } \\
(\mathrm{kcal} / \mathrm{mol})\end{array}$ \\
\hline 11809 |Moloney murine sarcoma virus | Retroviridae & $9626962 \_1$ rc & -109.4 & $9626953 \_$_rc & -67.6 \\
\hline $11663 \mid$ Ovine lentivirus | Retroviridae & VirusP70_10147 & -16.4 & $9629258 \_45$ & -38.3 \\
\hline $12465 \mid$ Barley yellow mosaic virus | Potyviridae & 21427654285 rc & -43 & 1580806593 & -100.9 \\
\hline 10862 Xanthomonas phage Cf1c | Inoviridae & $9626133 \_280$ rc & -117.1 & $9790346 \_172$ rc & -19.2 \\
\hline 12466 | Barley mild mosaic virus | Potyviridae & $21450043 \quad 159$ & -13.9 & 19749338224 rc & -108.3 \\
\hline 11665 Equine infectious anemia virus | Retroviridae & $9626530 \quad 99$ & -91.6 & $9627277 \_240$ & -12.3 \\
\hline 10867 |Enterobacteria phage lke | Inoviridae & 20428571998 & -17.9 & 17426217 142_rc & -19.4 \\
\hline 10868|Enterobacteria phage If1 | Inoviridae & 17426217 142_rc & -22 & 9630747113 rc & -95.9 \\
\hline 10869 |Enterobacteria phage 12-2 Inoviridae & 2042857198 & -17.9 & $17426217142 \mathrm{rc}$ & -15.5 \\
\hline
\end{tabular}

A

\begin{tabular}{|c|c|c|}
\hline Probe_ID & Virus species & $\begin{array}{c}\text { Energy } \\
\text { (kcal/mol) }\end{array}$ \\
\hline \multirow{11}{*}{ 9626962_1_rc } & 11809 | Moloney murine sarcoma virus |Retroviridae & -109.4 \\
\hline & $11819 \mid$ Spleen focus-forming virus | Retroviridae & -84.4 \\
\hline & $11830 \mid$ Murine osteosarcoma virus | Retroviridae & -81.4 \\
\hline & $11840 \mid$ Gibbon ape leukemia virus $\mid$ Retroviridae & -54.4 \\
\hline & \begin{tabular}{|l|l|l|}
44561 & Murine type C retrovirus & Retroviridae \\
\end{tabular} & -84.7 \\
\hline & \begin{tabular}{|l|l|l|}
11768 & Feline leukemia virus & Retroviridae \\
\end{tabular} & -30.2 \\
\hline & \begin{tabular}{|l|l|l|l|}
11786 & Murine leukemia virus & Retroviridae \\
\end{tabular} & -67.5 \\
\hline & 11788 Abelson murine leukemia virus $\mid$ Retroviridae & -67.5 \\
\hline & \begin{tabular}{|l|l|l|}
11938 & Rauscher murine leukemia virus & Retroviridae \\
\end{tabular} & -75 \\
\hline & \begin{tabular}{|l|l|l|}
11795 & Friend murine leukemia virus & Retroviridae \\
\end{tabular} & -80.3 \\
\hline & \begin{tabular}{|l|l|l|}
11802 & Murine sarcoma virus & Retroviridae \\
\end{tabular} & -109.4 \\
\hline \multirow{5}{*}{$9625875 \_4939$} & $10320 \mid$ Bovine herpesvirus $1 \mid$ Herpesviridae & -41.9 \\
\hline & \begin{tabular}{|l|l|l|}
10325 & Cercopithecine herpesvirus 1 Herpesviridae \\
\end{tabular} & -45.3 \\
\hline & 10326 | Equine herpesvirus 1 | Herpesviridae & -32.3 \\
\hline & $10335 \mid$ Human herpesvirus 3 Herpesviridae & -125.2 \\
\hline & $10397 \mid$ Tupaia herpesvirus | Herpesviridae & -32.7 \\
\hline
\end{tabular}

B

Bold represents candidate virus and the corresponding theoretical binding energy; Italic representsother viruses and their corresponding theoretical binding energy. ...... indicates that there are more probes as well as corresponding experimental hybridization intensity values existed. 
Table 3.6 Calculation of the weights for each theoretical binding energy value.

\begin{tabular}{|c|c|c|c|}
\hline Probe_ID & Virus species & \begin{tabular}{|c|} 
Energy \\
$(-\mathrm{kcal} / \mathrm{mol})$
\end{tabular} & Weights \\
\hline \multirow{2}{*}{ 18071213_1713_rc } & Bacteriophage phi CTX|Myoviridae & 40.5 & 0.158616 \\
\hline & Sinorhizobium meliloti phage PBC5 |NA & 121 & 0.841384 \\
\hline \multirow{3}{*}{ 20066014_8 } & Grapevine fanleaf virus satellite RNA|NA & 61.8 & 0.47685 \\
\hline & Rift Valley fever virus | Bunyaviridae & 17.4 & 0.116034 \\
\hline & Arabis mosaic virus satellite RNA|NA & 113 & 0.894933 \\
\hline \multirow{7}{*}{$9626510 \_77$} & Invertebrate iridescent virus 6 | Iridoviridae & 19.2 & 0.404081 \\
\hline & Macroptilium yellow mosaic virus | NA & 13.9 & 0.326376 \\
\hline & Yam mosaic virus | Potyviridae & 15.8 & 0.353557 \\
\hline & Apple latent spherical virus | Sequiviridae & 13.9 & 0.326376 \\
\hline & Acholeplasma phage L2|Plasmaviridae & 87.9 & 0.992807 \\
\hline & Melanoplus sanguinipes entomopoxvirus | Poxviridae & 15.1 & 0.343431 \\
\hline & Tobacco leaf curl Yunnan virus associated DNA 1 | Nanoviridae & 12.1 & 0.301468 \\
\hline \multirow{3}{*}{ 9626818_223_rc } & Cryphonectria hypovirus | Hypoviridae & 28.5 & 0.241017 \\
\hline & Cryphonectria hypovirus 1 | Hypoviridae & 107.2 & 0.921462 \\
\hline & Mycobacterium phage L5 | Siphoviridae & 28.2 & 0.23847 \\
\hline
\end{tabular}

Bold represents assignment of weights to each probe. ...... indicates that there are more theoretical binding energy values and corresponding weights coming from the theoretical hybridization between different probes and different viruses.

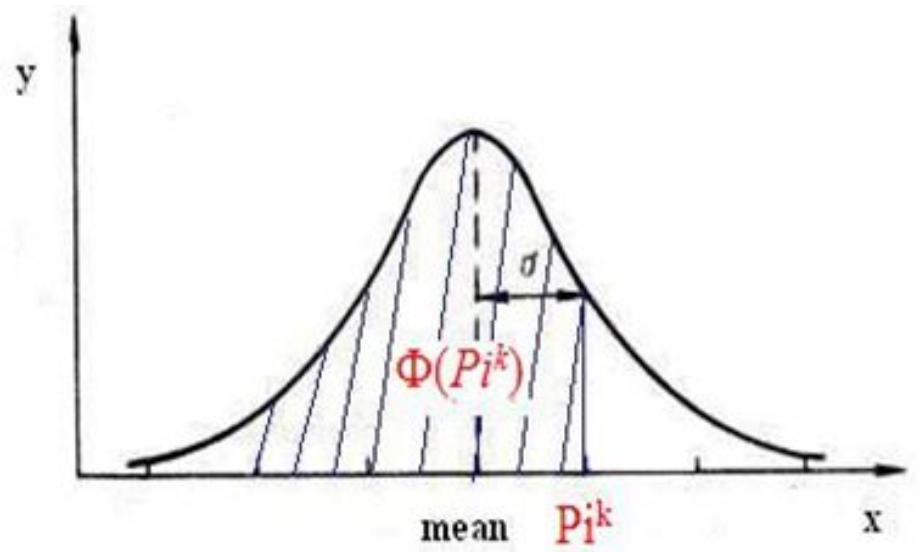

Figure 3.5 A normal distribution curve. We assume that the sequence of theoretical binding energy of each probe follows normal distribution, and $\operatorname{CDF}\left(\Phi\left(\mathrm{Pi}^{\mathrm{k}}\right)\right)$ of individual energy value $\left(\mathrm{Pi}^{\mathrm{k}}\right)$ represents its relative weight. 
Table 3.7 Transformation of the matrix of theoretical binding energy. The original matrix of theoretical binding energy (A) was transformed into another format, with each probe only representing a specific virus (B).The new matrix of theoretical binding energy was sorted by different virus families $(\mathrm{C})$.

A

\begin{tabular}{|c|c|c|}
\hline Probe_ID & Virus species & $\begin{array}{c}\text { Energy } \\
\text { (kcal/mol) }\end{array}$ \\
\hline \multirow[t]{6}{*}{8486125 53_rc } & \begin{tabular}{|l|l|l|}
11320 & Influenza A virus & Orthomyxoviridae \\
\end{tabular} & -75.2 \\
\hline & $157924 \mid$ Streptococcus mitis phage SM1 | NA & -14.3 \\
\hline & $51677 \mid$ Xestia c-nigrum granulovirus | Baculoviridae & -16 \\
\hline & \begin{tabular}{|l|l|l|}
85106 & Adeno-associated virus $1 \mid$ Parvoviridae \\
\end{tabular} & -14.9 \\
\hline & 165803 | Cypovirus $1 \mid$ Reoviridae & -16.4 \\
\hline & \begin{tabular}{|l|l|l|}
39443 & Carnation Italian ringspot virus & Tombusviridae \\
\end{tabular} & -14.9 \\
\hline \multirow[t]{5}{*}{$8486125 \_61$} & \begin{tabular}{|l|l|l|}
66266 & Rice grassy stunt virus & NA \\
\end{tabular} & -13.1 \\
\hline & $11320 \mid$ Influenza A virus | Orthomyxoviridae & -93.5 \\
\hline & 43141 | Watermelon silver mottle virus | Bunyaviridae & -14.8 \\
\hline & \begin{tabular}{|l|l|l|}
10821 & Maize streak virus |NA \\
\end{tabular} & -15 \\
\hline & 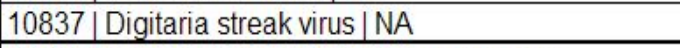 & -15 \\
\hline \multirow[t]{4}{*}{$8486150 \_76$} & \begin{tabular}{|l|l|l|l|}
39116 & Leishmania RNA virus 2-1 & Totiviridae \\
\end{tabular} & -11.7 \\
\hline & \begin{tabular}{|l|l|l|}
11520 & Influenza B virus & Orthomyxoviridae \\
\end{tabular} & -94.9 \\
\hline & \begin{tabular}{|l|l|l|}
75747 & Fusarium poae virus $1 \mid$ Partitiviridae \\
\end{tabular} & -13 \\
\hline & 98832 Influenza B virus Orthomyxoviridae & -51.9 \\
\hline \multirow[t]{4}{*}{32530120} & \begin{tabular}{|l|l|l|l|}
223287 & Mungbean yellow mosaic India virus & NA \\
\end{tabular} & -18 \\
\hline & 69263 | Cowpea golden mosaic virus |NA & -18 \\
\hline & 164038 Soybean yellow mosaic virus $\mid$ NA & -15.9 \\
\hline & 11555 Influenza C virus Orthomyxoviridae & -95.2 \\
\hline
\end{tabular}

B

\begin{tabular}{|c|c|c|}
\hline Probe_ID & Virus species & $\begin{array}{c}\text { Energy } \\
\text { (kcal/mol) }\end{array}$ \\
\hline 8486125_53_rc & 11320 | Influenza A virus | Orthomyxoviridae & 75.2 \\
\hline $8486125 \quad 61$ & 11320 Influenza A virus Orthomyxoviridae & 93.5 \\
\hline $8486150 \quad 76$ & 11520 Influenza B virus Orthomyxoviridae & 94.9 \\
\hline $325301 \_20$ & 11555 Influenza C virus Orthomyxoviridae & 95.2 \\
\hline
\end{tabular}

$\mathrm{C}$

\begin{tabular}{|c|c|c|c|c|c|}
\hline Virus family & Virus species & Probe No.1 & Probe No.2 & Probe No.3 & Probe No.4 \\
\hline \multirow{3}{*}{ Orthomyxoviridae } & Influenza A virus & $8486125 \_53$ & 8486125_53_rc & $8486125 \_61$ & 8486125_61_rc \\
\hline & \begin{tabular}{|l|} 
Influenza $B$ virus \\
\end{tabular} & $8486150 \quad 76$ & 848615076 rc & $8486152 \_19$ & 8486152 19 rc \\
\hline & Influenza C virus & 32530120 & $32530120 \mathrm{rc}$ & 32530124 & 32530124 rc \\
\hline \multirow{4}{*}{ Astroviridae } & Avian nephritis virus & \begin{tabular}{|l|}
$20514394 \_152$ \\
\end{tabular} & 20514394_152_rc & \begin{tabular}{|l|}
$20514394 \_160$ \\
\end{tabular} & 20514394_160_rc \\
\hline & Human astrovirus & 9630726_114 & 9630726_114_rc & 9630726_147 & 9630726_147_rc \\
\hline & Ovine astrovirus & $9635572 \_149$ & 9635572 149_rc & $9635572 \_204$ & 9635572 204_rc \\
\hline & Turkey astrovirus & $9635576 \quad 163$ & $9635576163 \mathrm{rc}$ & $9635576 \quad 172$ & 9635576 172_rc \\
\hline
\end{tabular}

Bold represents positive virus and the corresponding probes; Italic represents negative positive viruses and the corresponding probes. ..... indicates that there are more theoretical binding energy values and corresponding weights coming from the theoretical hybridization between different probes and different viruses. 


\subsubsection{Statistical significance}

A total of 38 independent microarrays consisting of hybridizations including either virus infected samples or mock-infected samples were all treated as the training datasets, and the distance scores were calculated one by one using the scoring formula (7). In our study, it was assumed that any given virus was present in only a small fraction in all the samples, thereby the empirical distributions were representatives of the true negative distance scores. Parameters of the null distributions were estimated as the mean and standard deviation of the observation, and the P-values corresponding to different distance scores were calculated based on these two parameters. The similar assumption was also made by others [49, 78]. For known virus detection, the cutoff for the P-value is set at 0.05 , indicating that P-value less than 0.05 signifies a positive prediction. However, the cutoff can be less stringent in the case for unknown virus detection depending on the performance of the hybridization.

\subsubsection{Differential detection within virus families}

Generally, global virus detection at virus species level with previous scoring method is sufficient to reach the positive predictions, however, false positives may still occur in some occasions. These false positives are always given rise by the conserved sequences of viruses within the same family. The similarity of these conserved sequences may lead to similar theoretical binding energy profiles [50], and therefore the similar distance scores. Consequently, another algorithm was proposed to identify the positive virus within a specific virus family.

Before the calculation, the theoretical binding energy profiles needed to be transformed into another format. In the original matrix of theoretical binding energy profiles, each probe targeted to more than one virus with different theoretical binding energy values considered (Table 3.7A). However, each probe was treated to be unique to one virus which was corresponding to the highest theoretical binding energy value (Table 3.7B). Finally, all virus genus represented by their specific probes were sorted based on their virus families (Table 3.7C). After the transformation of the matrix of theoretical binding energy profiles, viruses within the interested virus family were scored and the 
one with most significant score was treated as the positive prediction. When it came to score the specific virus Gi like Influenza A virus, two groups of data needed to be extracted out. One group contained experimental hybridization intensities from the probes which were identical to the predicted virus Gi (Influenza $A$ virus), the other group was composed of experimental hybridization intensities from the probes which were identical to all other viruses in the same family (Gn, $\mathrm{n}=1$ to $\mathrm{j}$, and $\mathrm{n} \neq \mathrm{i}$ ) (Influenza $B$ virus and Influenza $C$ virus) (Table 3.7C). Finally, we assumed that these two groups of data followed normal distribution and did one-tail t-test.

$$
\begin{array}{cc}
\mathrm{H}_{0}: \mu_{0} \succ \mu_{1}, \quad \mathrm{H}_{1}: \mu_{0} \leq \mu_{1} \\
\mathrm{X}=\frac{\mu_{0}-\mu_{1}}{\sqrt{\frac{\sigma_{0}^{2}}{\mathrm{n}_{0}}+\sqrt{\frac{\sigma_{1}^{2}}{\mathrm{n}_{1}}}} \sim \mathrm{N}(0,1)} \quad \Phi(\mathrm{X})=\frac{1}{\sqrt{2 \pi}} \mathrm{e}^{-\frac{1}{2} \mathrm{X}^{2}}
\end{array}
$$

Where $\mu_{0}, \sigma_{0}$ equal the mean and standard deviation of experimental hybridization intensities of Gi (candidate virus), and $\mu_{1}, \sigma_{1}$ represent the mean and standard deviation of experimental hybridization intensities of Gn (all other viruses in the same family, excluding the candidate virus).

\subsection{Results}

\subsubsection{Comparison of different parameters in E-Predict and BayesMicro}

We applied the same chip fabrication system from DeRisi's group to validate 12 cultured samples, and the observed hybridization intensity pattern was analyzed using E-Predict software. In terms of the detection results, we observed that E-Predict was not sensitive enough to obtain the positive prediction in all our samples.

These false positive predictions maybe due to its weak algorithm especially lying in the normalization and scoring functions, which has also been proposed by other group [77]. It is well known that the outliers with high value are easily generated by systematic instability or experimental errors in high through-put system like microarray [113]. However, the normalization function in E-Predict software did not take these outliers with high experimental 
hybridization values into consideration so that it was easy to lead to serious compression of other experimental hybridization values in this process. When the over-compressed experimental hybridization values participated into further calculation, their corresponding result was not significant.

As to the scoring function, traditional correlation methods such as uncentered Pearson correlation and Euclidean distance were employed to describe the similarity between the experimental hybridization intensity pattern and the theoretical binding energy profiles. However, these metrics assume normal distribution of the data and linear association between these two groups of data, and it may not be the case for high-throughput expression data [117$120]$.

Accordingly, the software BayesMicro was pioneered as an alternative virus detection strategy in order to improve the result sensitivity in our study. In BayesMicro, new transformation formula was involved with considering the outliers with high experimental hybridization intensity values. Besides, the distances between the experimental hybridization intensity pattern and the theoretical binding energy profiles were defined based on a Bayesian model, which explained the inherent nonlinearity in high-throughput expression data more effectively than E-Predict. This model was able to handle the weakness in E-Predict by measuring the nonlinear relationship between two groups of data more precisely. Furthermore, involvement of stringent theoretical binding energy threshold and assignment of optimal weighs enhanced the detection accuracy of BayesMicro.

It has been also revealed that E-Predict provided the best separation in between family comparisons and the least separation within families [77]. To solve this issue, differential detection within virus family as another optional method was also designed for further separation of viruses within the same family (Table 3.8). 
Table 3.8 Comparison of different parameters used in E-Predict and BayesMicro.

\begin{tabular}{|c|c|c|}
\hline Parameters & E-Predict & BayesMicro \\
\hline Original data & \multicolumn{2}{|c|}{ Theoretical binding energy value } \\
\cline { 2 - 4 } & \multicolumn{2}{|c|}{ Experimental hybridization intensities } \\
\hline Energy threshold (kcal/mol) & 30 & 70 \\
\hline Transformation formula & $\mathrm{Xg}_{(\mathrm{norm})}=\frac{\mathrm{Xg}}{\sum \mathrm{Xg}} \quad \mathrm{Xg}_{(\mathrm{norm})}=\frac{\mathrm{Xg}^{2}}{\sum \mathrm{Xg}^{2}}$ & $\mathrm{Xg}_{(\mathrm{norm})}=\frac{\mathrm{Xg}-\mu_{\mathrm{Xg}}}{\max \left(\mathrm{abs}\left(\mathrm{Xg}-\mu_{\mathrm{Xg}}\right)\right)}$ \\
\hline Score calculation & Uncentered pearson correlation (default) $^{\text {Bayesian algorithm }}$ \\
\hline Assignment of weights & $\mathrm{X}$ & $\sqrt{ }$ \\
\hline Detection further at genus level & $\mathrm{X}$ & $\sqrt{ }$ \\
\hline
\end{tabular}

\subsubsection{Validation of E-Predict and BayesMicro}

Based on our 12 culture samples, the analysis was performed using EPredict and BayesMicro, and the virus detection results were summarized in Table 3.9. The results list suggested that E-Predict was able to generate good predictive outcomes in majority of the samples. However, the false positives still occurred in some cases.

When it came to BayesMicro, it was capable to generate good predictive outcomes in all cultured samples, either with topmost ranking or topmost 10 ranking. In summary, comparison of the virus detection results between these two software demonstrated that BayesMicro software performed better than EPredict software. The clinical samples infected with influenza A virus were also accessible, with the good predictive outcomes with topmost ranking generated based on both BayesMicro and E-predict software.

Table 3.9 Summary of results from E-Predict and BayesMicro.

\begin{tabular}{|c|c|c|c|}
\hline Sample Virus & Strains / Species & E-Preidict & BayesMicro \\
\hline Yellow fever virus & 17D & $\sqrt{ }$ (top) & $\sqrt{ }$ (top) \\
\hline Japanese encephalitis virus & Nakayama & $\sqrt{ }$ (top) & $\sqrt{ }$ (top) \\
\hline Influenza A virus & H3N2 & $\sqrt{ }$ (top) & $\sqrt{ }$ (top) \\
\hline Coronavirus & OC43 & $\sqrt{ }$ (top) & $\sqrt{ }$ (top) \\
\hline Chikungunya virus & Oka & $\sqrt{ }$ (top) & $\sqrt{ }$ (top) \\
\hline Influenza B virus & Lee & $\sqrt{ }$ & $\sqrt{ }$ (top) \\
\hline Influenza A virus & H1N1 & $\sqrt{ }$ & $\sqrt{\text { (top) }}$ \\
\hline Rhinovirus & Type 14 & $\sqrt{ }$ & $\sqrt{ }$ \\
\hline Mouse poxvirus & Moscow & $\sqrt{ }$ & $\sqrt{ }$ \\
\hline Vaccinia virus & Lister & X & $\sqrt{ }$ \\
\hline Cowpox virus & Brighton & $\sqrt{ }$ (top) & $\sqrt{ }$ \\
\hline Measles virus & Edmonton & $\sqrt{ }$ (top) & $\sqrt{ }$ \\
\hline
\end{tabular}

$\sqrt{ }$ (top) represents positive virus ranked $1^{\text {st }}, \sqrt{ }$ represents positive virus ranked in top 10 , and $\mathrm{X}$ erpresetns positive virus was not able to be detected in top 10 . 


\subsubsection{Global detection at virus species level}

In general, the virus detection results from both E-Predict and BayesMicro showed similar. And in most cases, both these software could identify the positive viruses with the topmost ranking based on either similarity/distance score or P-value. These situations happened in samples infected with yellow fever virus and Japanese encephalitis virus. Nevertheless, BayesMicro enabled to generate better predictive outcomes than E-Predict in some specific cases. For example, E-Predict reported false positive results while BayesMicro reported positive results in vaccinia virus infected samples; In other samples infected with influenza A virus (H1N1) and influenza B virus, EPredict reported the positive virus with ranking only in top 10 list while BayesMicro reported the positive virus with topmost ranking.

Table 3.10 Virus detection in samples infected with Yellow fever virus.

\begin{tabular}{|l|c|c|l|c|c|}
\hline \multicolumn{3}{|c|}{ Yellow fever virus } \\
\hline \multicolumn{2}{|c|}{ E_Predict } & \multicolumn{3}{c|}{ BayesMicro } \\
\hline \multicolumn{1}{|c|}{ Virus species } & Similarity Score & P-value & \multicolumn{1}{c|}{ Virus species } & Distance Score & P-value \\
\hline Yellow fever virus & 0.725751 & 0.000024 & Yellow fever virus & -0.9897 & 0.0019 \\
\hline Yokose virus & 0.364157 & 0.000212 & Goose parvovirus & 0.7222 & 0.0687 \\
\hline Alkhurma virus & 0.134815 & 0.001421 & Avian adeno-associated virus & 0.8095 & 0.0783 \\
\hline Human rhinovirus B & 0.036516 & 0.017790 & Hepatitis E virus & 0.8904 & 0.088 \\
\hline Human papillomavirus type 16 & 0.033311 & 0.030380 & Adeno-associated virus 3 & 0.8927 & 0.0883 \\
\hline Human papillomavirus type 45 & 0.031912 & 0.018666 & Adeno-associated virus 3B & 0.8927 & 0.0883 \\
\hline Human papillomavirus candHPV85 & 0.031321 & 0.056718 & Adeno-associated virus 4 & 0.9156 & 0.0912 \\
\hline Vaccinia virus & 0.030069 & 0.002261 & Adeno-associated virus 6 & 0.9311 & 0.0932 \\
\hline Variola virus & 0.030044 & 0.001849 & Adeno-associated virus 1 & 0.9357 & 0.0938 \\
\hline Rotavirus A & 0.029861 & 0.000980 & Influenza B virus & 0.9460 & 0.0951 \\
\hline
\end{tabular}

Bold represetns positive virus in the culture sample.

Table 3.11 Virus detection in samples infected with Japanese encephalitis virus.

\begin{tabular}{|l|c|c|l|c|c|}
\hline \multicolumn{7}{|c|}{ Japanese encephalitis virus } \\
\hline \multicolumn{1}{|c|}{ E_Predict } & \multicolumn{2}{c|}{ BayesMicro } \\
\hline Virus species & Similarity Score & P-value & \multicolumn{1}{c|}{ Virus species } & Distance Score & P-value \\
\hline Japanese encephalitis virus & 0.284751 & 0.000040 & Japanese encephalitis virus & 0.1182 & 0.0242 \\
\hline Langat virus & 0.131216 & 0.001382 & Goose parvovirus & 0.7327 & 0.0698 \\
\hline Tick-borne encephalitis virus & 0.099022 & 0.002316 & Avian adeno-associated virus & 0.8125 & 0.0787 \\
\hline Human herpesvirus 1 & 0.088655 & 0.022517 & Adeno-associated virus 3 & 0.8382 & 0.0817 \\
\hline Human rhinovirus B & 0.085275 & 0.002020 & Adeno-associated virus 3B & 0.8382 & 0.0817 \\
\hline Lake Victoria marburgvirus & 0.082579 & 0.000002 & Hepatitis E virus & 0.8423 & 0.0821 \\
\hline Human papillomavirus type 35 & 0.081080 & 0.000859 & Influenza B virus & 0.8451 & 0.0825 \\
\hline Human papillomavirus type 31 & 0.079259 & 0.001120 & Adeno-associated virus 4 & 0.8728 & 0.0858 \\
\hline Human papillomavirus type 83 & 0.072836 & 0.003245 & Adeno-associated virus 1 & 0.8762 & 0.0862 \\
\hline Variola virus & 0.068256 & 0.000138 & Adeno-associated virus 6 & 0.8950 & 0.0886 \\
\hline
\end{tabular}

Bold represents positive virus in the culture sample. 
Table 3.12 Virus detection in samples infected with Influenza B virus.

\begin{tabular}{|l|c|c|l|c|c|}
\hline \multicolumn{7}{|c|}{ Influenza B virus } \\
\hline \multicolumn{7}{|c|}{ E-Predict } & \multicolumn{2}{c|}{ BayesMicro } \\
\hline \multicolumn{1}{|c|}{ Virus species } & Similarity Score & P-value & \multicolumn{1}{c|}{ Virus species } & Distance Score & P-value \\
\hline Human herpesvirus 7 & 0.204744 & 0.006595 & Influenza B virus & 0.3280 & 0.0358 \\
\hline Human papillomavirus type 29 & 0.187067 & 0.000161 & Swinepox virus & 0.5622 & 0.0534 \\
\hline Influenza B virus & 0.153455 & 0.000013 & Rotavirus A & 0.6983 & 0.0662 \\
\hline Rotavirus A & 0.087591 & 0.000031 & Goose parvovirus & 0.7340 & 0.0700 \\
\hline Human rhinovirus B & 0.082278 & 0.002224 & Avian adeno-associated virus & 0.8129 & 0.0787 \\
\hline Swinepox virus & 0.076707 & 0.000005 & Adeno-associated virus 3 & 0.8361 & 0.0814 \\
\hline Monkeypox virus & 0.069625 & 0.000159 & Adeno-associated virus 3B & 0.8361 & 0.0814 \\
\hline Vaccinia virus & 0.066680 & 0.000193 & Hepatitis E virus & 0.8363 & 0.0814 \\
\hline O'nyong-nyong virus & 0.043176 & 0.002038 & Adeno-associated virus 4 & 0.8576 & 0.0840 \\
\hline Igbo Ora virus & 0.052584 & 0.001241 & Adeno-associated virus 6 & 0.8742 & 0.0860 \\
\hline
\end{tabular}

Bold represents positive virus in experimental sample.

\subsubsection{E-Predict and BayesMicro both indicated good predictive outcomes}

For virus detections in cultured samples such as samples infected with yellow fever virus and Japanese encephalitis virus, both E-Predict and BayesMicro were able to generate the positive prediction with topmost ranking (Table 3.10-3.11).

\subsubsection{BayesMicro performed better than E-Predict}

Apart from generating similar positive predictions in some cases, BayesMicro performed better than E-Predict in some other cases. Taken the sample infected with influenza B virus as an example, BayesMicro could highlight the positive virus with topmost ranking, while E-Predict could only identify the positive virus with $3^{\text {rd }}$ ranking in the result list (Table 3.12).

\subsubsection{Differential detection within virus families}

In general, global detection at virus species level using E-Predict and BayesMicro was able to generate good predictive outcomes in majority of microarray samples. However, sometimes false positive viruses that belonged to the same virus family as the positive virus still turned up in the result list. Thus differential detection within virus families was necessary to enhance the reliability of the detection result.

In cultured sample with infection of influenza A virus (H1N1), E-predict was not able to identify the positive virus in the top 10 list while BayesMicro was able to (Table 3.13A). In the result list from BayesMicro, influenza B virus and influenza $\mathrm{C}$ virus from the same virus family showed similar distance scores as influenza A virus $(\mathrm{H} 1 \mathrm{~N} 1)$. Therefore, differential detection using 
BayesMicro was involved for further validation with higher resoluation and the result suggested that influenza A virus (H1N1) was scored as more significant when compared to false positive influenza $\mathrm{B}$ virus and influenza $\mathrm{C}$ virus (Table $3.13 \mathrm{~B})$.

In another cultured sample infected with vaccinia virus, the good predictive outcome was able to be generated by BayesMicro but not E-Predict (Table 3.14A). Careful evaluation of the BayesMicro result list demonstrated that several poxviruses such as ectromelia virus, cowpox virus and monkeypox virus occupied the top 6 positions with close distance scores. Accordingly, differential detection up within virus families was employed again for further investigation, and the ranking of positive vaccinia virus was consequently improved to $2^{\text {nd }}$ position (Table $3.14 \mathrm{~B}$ ).

Taken together, the method for differential detection within virus families performed effective in our experiment data. And BayesMicro software showed better ability to identify the positive prediction than E-Predict software.

Table 3.13 Virus detection in samples infected with influenza A virus (H1N1). (A) Result from E-Predict and BayesMicro up to virus species level. (B) Result from BayesMicro up to virus genus level.

A

\begin{tabular}{|l|c|c|l|c|c|}
\hline \multicolumn{5}{|c|}{ Influenza A virus (H1N1) } \\
\hline \multicolumn{2}{|c|}{ E-Predict } \\
\multicolumn{1}{|c|}{ Virus Name } & Similarity Score & P-value & \multicolumn{1}{c|}{ Virus Name } & Distance Score & P-value \\
\hline Human papillomavirus type 31 & 0.134401 & 0.000252 & Influenza B virus & 0.5537 & 0.0526 \\
\hline Human papillomavirus type 35 & 0.132109 & 0.000224 & Cercopithecine herpesvirus 1 & 0.6022 & 0.0569 \\
\hline Human papillomavirus type 84 & 0.104200 & 0.001941 & Influenza C virus & 0.7254 & 0.0691 \\
\hline Human rhinovirus A & 0.103603 & 0.030498 & Influenza A virus & 0.7428 & 0.0709 \\
\hline Human herpesvirus 5 & 0.094070 & 0.023582 & Goose parvovirus & 0.7483 & 0.0715 \\
\hline Western equine encephalomyelitis virus & 0.080259 & 0.000044 & Rotavirus B & 0.7576 & 0.0725 \\
\hline Rotavirus A & 0.079643 & 0.000034 & Adeno-associated virus 3 & 0.7628 & 0.0731 \\
\hline Variola virus & 0.079222 & 0.000081 & Adeno-associated virus 3B & 0.7628 & 0.0731 \\
\hline Vaccinia virus & 0.078441 & 0.000110 & Hepatitis E virus & 0.7680 & 0.0736 \\
\hline Swinepox virus & 0.077090 & 0.000005 & Porcine adenovirus A & 0.7720 & 0.0741 \\
\hline
\end{tabular}

B

\begin{tabular}{|l|c|}
\hline \multicolumn{1}{|c|}{ Virus species } & Score \\
\hline Influenza A virus & 0.3938 \\
\hline Influenza B virus & 0.3986 \\
\hline Influenza $C$ virus & NA \\
\hline
\end{tabular}

Bold represents positive virus in the sample; Italic represents false positive viruses in the sample. NA means that the hybridization intensities of the candidate virus are lower than the background hybridization intensities within its virus family. 


\subsection{Discussion}

\subsubsection{Improve theoretical binding energy database}

The existing library of theoretical energy profiles was created in July 2004 with only 1,229 viral genomes available [78]. And until November 2012, there were 4314 reference sequences for 3027 viral genomes accessible in viral genome database. According to this information, ArrayOligoSelector will be employed to calculate the theoretical binding energy profiles of other viruses which were not included in viral genome database of July 2004. Consequently, we can broaden the spectrum of virus detection.

In addition, a couple of viruses may co-infect the same sample. In this case, the global experimental hybridization intensity pattern would become an overlapping pattern, and this overlapping pattern with more noisy background will make the virus identification more difficult. To improve the virus detection in these co-infected samples, a database which contains the overlapping theoretical binding energy profiles from any of two or more viruses may be constructed.

\subsubsection{Future validation and expansion}

Our results showed that BayesMicro could generate good predictive outcomes in most of our experiments. When compared to E-Predict, sometimes it could perform as well as E-Predict while sometimes BayesMicro could generate more sensitive detection results. Although the evaluation of BayesMicro showed effective, the test datasets were still limited in amount. Actually, we did quite a lot experiments, however, some of these experiment were performed based on the same batch of sample and I only repsented and summarized the typical results in my result table. In the future, more cultured as well as clinical samples would be involved in further evaluating the performance of BayesMicro. 
Table 3.14 Virus detection in samples infected with vaccinia virus. (A) Result from EPredict and BayesMicro up to virus species level. (B) Result from BayesMicro up to virus genus level.

A

\begin{tabular}{|c|c|c|c|c|c|}
\hline \multicolumn{6}{|c|}{ Vaccinia virus } \\
\hline \multicolumn{3}{|c|}{ E-Predict } & \multicolumn{3}{|c|}{ BayesMicro } \\
\hline Virus Name & Similarity Score & P-value & Virus Name & Distance Score & P-value \\
\hline Ectromelia virus & 0.271197 & 0.000001 & Ectromelia virus & 0.1757 & 0.0270 \\
\hline Monkeypox virus & 0.267852 & 0.000001 & Cowpox virus & 0.2695 & 0.0322 \\
\hline Cowpox virus & 0.267486 & 0.000001 & Monkeypox virus & 0.3131 & 0.0348 \\
\hline Human papillomavirus type 1a & 0.224844 & 0.000007 & Vaccinia virus & 0.3616 & 0.0380 \\
\hline Bunyamwera virus & 0.095440 & 0.000003 & Variola virus & 0.4067 & 0.0411 \\
\hline Human herpesvirus 7 & 0.094832 & 0.027603 & Camelpox virus & 0.4198 & 0.0420 \\
\hline TTV-like mini virus & 0.093180 & 0.000161 & Goose parvovirus & 0.7274 & 0.0693 \\
\hline Human rhinovirus $\mathrm{A}$ & 0.066377 & 0.060127 & Avian adeno-associated virus $A$ & 0.8111 & 0.0785 \\
\hline Human rhinovirus $\mathrm{B}$ & 0.063706 & 0.004539 & Hepatitis E virus & 0.8555 & 0.0837 \\
\hline Human rhinovirus sp. & 0.056507 & 0.000604 & Adeno-associated virus 3 & 0.0842 & 0.0843 \\
\hline
\end{tabular}

B

\begin{tabular}{|l|c|}
\hline \multicolumn{1}{|c|}{ Virus species } & Score \\
\hline Monkeypox virus & 0.3557 \\
\hline Vaccinia virus & 0.3582 \\
\hline Ectromelia virus & 0.3588 \\
\hline Cowpox virus & 0.3707 \\
\hline Variola virus & 0.3754 \\
\hline Camelpox virus & 0.3917 \\
\hline Goatpox virus & NA \\
\hline Fowlpox virus & NA \\
\hline Lumpy skin disease virus & NA \\
\hline Molluscum contagiosum virus & NA \\
\hline Sheeppox virus & NA \\
\hline Swinepox virus & NA \\
\hline Yaba-like disease virus & NA \\
\hline
\end{tabular}

Bold represents positive virus in the sample; Italic represents false positive viruses in the sample. NA means that the hybridization intensities of the candidate virus are lower than the background hybridization intensities within its virus family.

\subsubsection{Comparison with E-Predict}

In our study, a viral diagnostic chip was fabricated based on the design from Wang et al (2003) [27], and we used the E-Predict as the default software to analyze microarray data. However, we were not able to generate good predictive diagnostic outcomes in all our viral samples. These false positive results may be due to the internal weakness of the software which is no physical justification of their choices of normalization and scoring functions [77]. Thus a new software called BayesMicro was developed in our study. In the BayesMicro, several aspects were improved: (1) the transformation formula was optimized for accurate comparison between two groups of data, and 
outliers with high value were filtered out in order to prevent their negative influence; (2) the distance calculation formula based on Bayesian model was employed to interpret the inherent non-linear relationship between experimental hybridization and theoretical binding energy; (3) the assignment of weights to each theoretical binding value was performed in order to enhance the accuracy of the outcome; (4) P-values could be calculated based on a batch training datasets composing of 38 independent microarrays, and the P-value less than 0.05 is treated as a significant detection; (5) a second algorithm used to differential detection within virus families were proposed for further separation.

In addition, E-Predict software is only available as a CGI script for Unix/Linux platforms and the installment has to be performed by a professional computation user. In contrast, BayesMicro requires no setup and configuration. A website is established under an apache server which can be easily accessed through http://microarray.sbs.ntu.edu.sg/bayes/entry.html.

\subsubsection{Comparison with other existing strategies}

We compared the statistical methods of BayesMicro with other existing algorithms for virus detection based on pathogen diagnostic microarray chips. Notably, these algorithms are all different from our BayesMicro in which a Bayes formula is employed as the final scoring function. This function is applied to calculate the distance scores between the examined experimental hybridization intensity pattern and the profiles of theoretical binding energy which represents different viruses.

\subsection{Conclusion}

Overall, we developed a novel software called BayesMicro. In this software, a new algorithm for virus detection based on virus species level was proposed and it was proved to be different from any other existing systems. In this new algorithm, an optimized transformation formula and a new scoring formula were employed to investigate the relationship between theoretical binding energy profiles and experimental hybridization intensity pattern. Moreover, the weights were also assigned to each theoretical binding value in order to enhance the accuracy of the outcome. 
Furthermore, another algorithm was also established for differential detection on virus species within the same virus family. Thus, the implementation of BayesMicro software provided the possibility to further separate the viruses within the same family following the initial virus species detection on the same viral chip. To date, although some microarray diagnostic chips have been developed to identify the viruses within the same family, the application of these chips has been limited to virus-species. On the basis of this new algorithm, BayesMicro can be easily applied to separate the viruses within the same family following the initial virus species detection on the same viral chip.

Application of BayesMicro and E-Predict to a dataset of cultured samples suggested the superior performance of BayesMicro when compared to E-Predict, with BayesMicro generating 12 positive predictions out of 12 samples but E-Predict generating 10 positive predictions out of 12 samples. These data suggested that BayesMicro can be applied in virus detection based on diagnostic microarray chip as a reliable analysis tool.

Finally, this software can be easily accessed via the internet (http://microarray.sbs.ntu.edu.sg/bayes/entry.html) and its application easy to use, including users without computation background. 


\section{Chapter IV. Respiratory Syncytial Virus}

\subsection{Introduction}

Human respiratory syncytial virus (HRSV), which causes infection of the lungs and breathing passages, is a major cause of lower respiratory tract infections in infancy and childhood. It has been reported by WHO that RSV results in around 64 million infections and 160,000 deaths in the worldwide annually. And the risk for severe illness or even fatality always lie in the children with underlying problems such as bronchopulmonary dysplasia as well as patients with compromised immune systems. Moreover, RSV, as a significant pathogen in bone marrow transplant recipients, is one of the most widespread nosocomial infections [121]. Till now, more effective antiviral drugs and a vaccine for protection of the general population from infection of RSV are still lacking [122].

\subsubsection{Virus structure}

RSV that belongs to the subfamily Pneumovirinae under Paramyxoviridae family, is an enveloped virus with a $15.2 \mathrm{kbp}$, negative sense, single-stranded RNA genome. The 10 genes encode 11 proteins including non-structural protein 1 (NS1), non-structural protein 2 (NS2), nucleo- $(\mathrm{N})$ protein, phosphor- $(\mathrm{P})$ protein, matrix $(\mathrm{M})$ protein, small hydrophobic $(\mathrm{SH})$ protein, glyco- $(\mathrm{G})$ protein, fusion (F) protein, M2-1 and M2-2, and the large (L) protein arrayed orderly from 3' to 5' direction [Figure 4.1]. Based on their specific functions, these viral proteins can be grouped into several categories. For instance, G, F, M and SH proteins are functional associated with the viral envelop: $G$ protein functions on virus attachment; F protein promotes virus penetration and benefits host cells fusion; $\mathrm{M}$ protein is responsible for virion morphogenesis and traffics between the cytoplasm and the nucleus; SH protein plays an essential role in viral infectivity. Other five proteins including N, P, M2-1, M2-2, and L protein participate in synthesis of RNA and formation of the ribonucleocapsid structure: $\mathrm{N}$ protein associates with genomic RNA forming the nucleocapsid; M2 protein is the second matrix protein required for transcription; L protein encodes RNA polymerase; P protein is a co-factor for L protein. The two accessory proteins, 
NS1 and NS2, are involved in the inhibition of the $\alpha / \beta$ interferon (IFN) activity in the host response $[123,124]$.

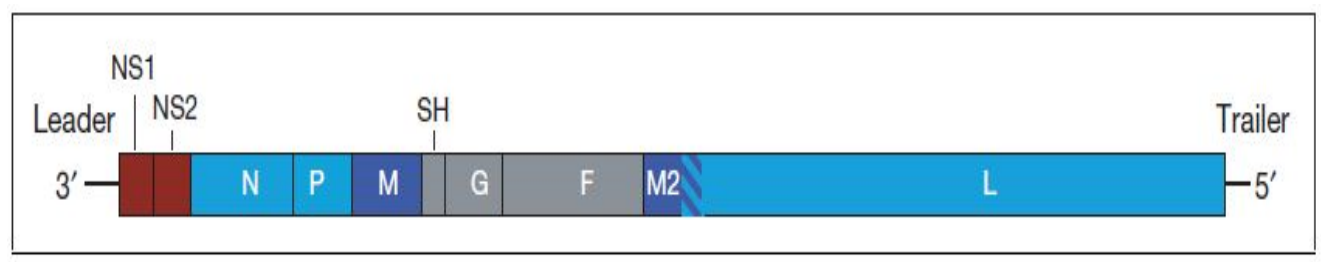

Figure 4.1 The structure organization of RSV genome (adapted from Sugrue RJ, 2006)[125].

Among these proteins, three membrane proteins including F, G and SH protein are embedded in the lipid membrane derived from the host cell. All these three transmembrane glycoproteins are incorporated into virions. F protein is initially translated into F0, an inactive precursor, which is then endoproteolytically cleaved into two disulfide linked subunits, F1 and F2. Subsequently, the highly conserved hydrophobic N-terminus of F1 protein is exposed to mediate the fusion of host cells. G protein consisting of 298 amino acids has been reported to interact with heparin/heparin sulfate. Function of the third small $\mathrm{SH}$ protein is still unclear from previous researches. Several isoforms including $\mathrm{SH} 0, \mathrm{SHg}, \mathrm{SHp}, \mathrm{SHt}$ have been detected during virus infection [126].

The mature and infectious RSV particle comprises a ribonucleoprotein (RNP) complex which is consisted of viral genome RNA (vRNA), N, P and L protein. The lipid envelop derived from host cell surrounds the RNP. An additional $\mathrm{M}$ protein locates between the virus envelope and the RNP in order to regulate virus transcriptional activity [127].

There are two major structural features for RSV observed by electron microscopy: the virus filaments (VFs) and the inclusion bodies (IBs). VFs form on the surface of the infected cells where the virus structural proteins interact to form mature virus particles, while IBs exist in the infected cells where the virus polymerase-associated proteins and virus-specific RNA accumulate.

\subsubsection{Virus replication cycle}

In general, RSV replication cycle is composed by virus entry, RNP complex activity, virus assembly and release [Figure 4.2]. 


\subsubsection{Virus entry}

RSV attachment to cells primarily occurs through the interaction between $G$ protein and cell surface glycosaminoglycans (GAGs), in particular heparin sulphate (HS). Though sulfate modification is essential for the ability of GAGs to mediate RSV infection, only definite sulfate groups are required [128]. And although $\mathrm{G}$ protein helps in attachment of the virus to the cell surface, it has been proved that it is dispensible for the cell attachment. When virus attaches to the cell, F protein initiates a membrane fusion process at neutural $\mathrm{PH}$. In this process, heptad repeat (HP) regions from $\mathrm{F}$ protein interact with the homotrimer to form a triple coiled-coiled structure, and this structure enables to draw the virus envelope and cell membrane close to benefit further membrane fusion. After the lipids derived from virus envelope and cell membrane connect together, RNP complex is transferred into the cytoplasm of the host cell, and further virus transcription and replication are initialted.

\subsubsection{Virus transcription and replication}

After RNP complex is released to the cytoplasm of target cell, the large polymerase L protein initiates viral transcription and replication [129]. With the assistance of viral RNA- dependent polymerase, the negative stranded genome is transcribed into 5'-capped and 3'-polyadenylated mRNAs, and this transcription stated in a sequential manner from the 3 ' end of the viral genome. In the end, ten mRNAs are then subsequently translated into virious viral proteins.

At the meanwhile, anti-genomic RNA is generated as a template and more vRNAs are subsequently synthesized by the virus polymerase.

\subsubsection{Virus assembly and budding}

The final stage of the replication cycle is virus particles assembly and release from the cell. The newly synthesized RNP and RNP-associated protein accumulate in inclusion bodies and target to specific sites like lipid rafts at the cell. The virus glycoproteins are also targeted to specific sits through the secretory pathway. The RNP complex is formed by the interaction between the vRNA and $\mathrm{N}$ protein, followed by $\mathrm{P}$ and $\mathrm{L}$ protein. M protein functions on the 
association of the viral glycoproteins with RNPs. After that, the RNP complex is packed with other proteins to form new infectious virus particles [130].

The final stage of virus maturation is the release of the virus particles from the host cell. The virions mature in clusters at the apical surface in a filamentous form associated with caveolin-1, and extend from the plasma membrane [131]. In this process, various host cell factors, especially those related to cytoskeleton, are thought to be involved. Recent studies unvealed that cells infected with viruses lacking the F glycoprotein decreased the amounts of $\mathrm{G}$ and $\mathrm{SH}$ proteins in released virions and cells infected with viruses lacking the $\mathrm{G}$ protein decreased the amounts of $\mathrm{SH}$ protein in released virions. Thus, these findings indicated that $\mathrm{F}$ and $\mathrm{G}$ glycoproteins play a crucial role in $\mathrm{HRSV}$ particle assembly [132].

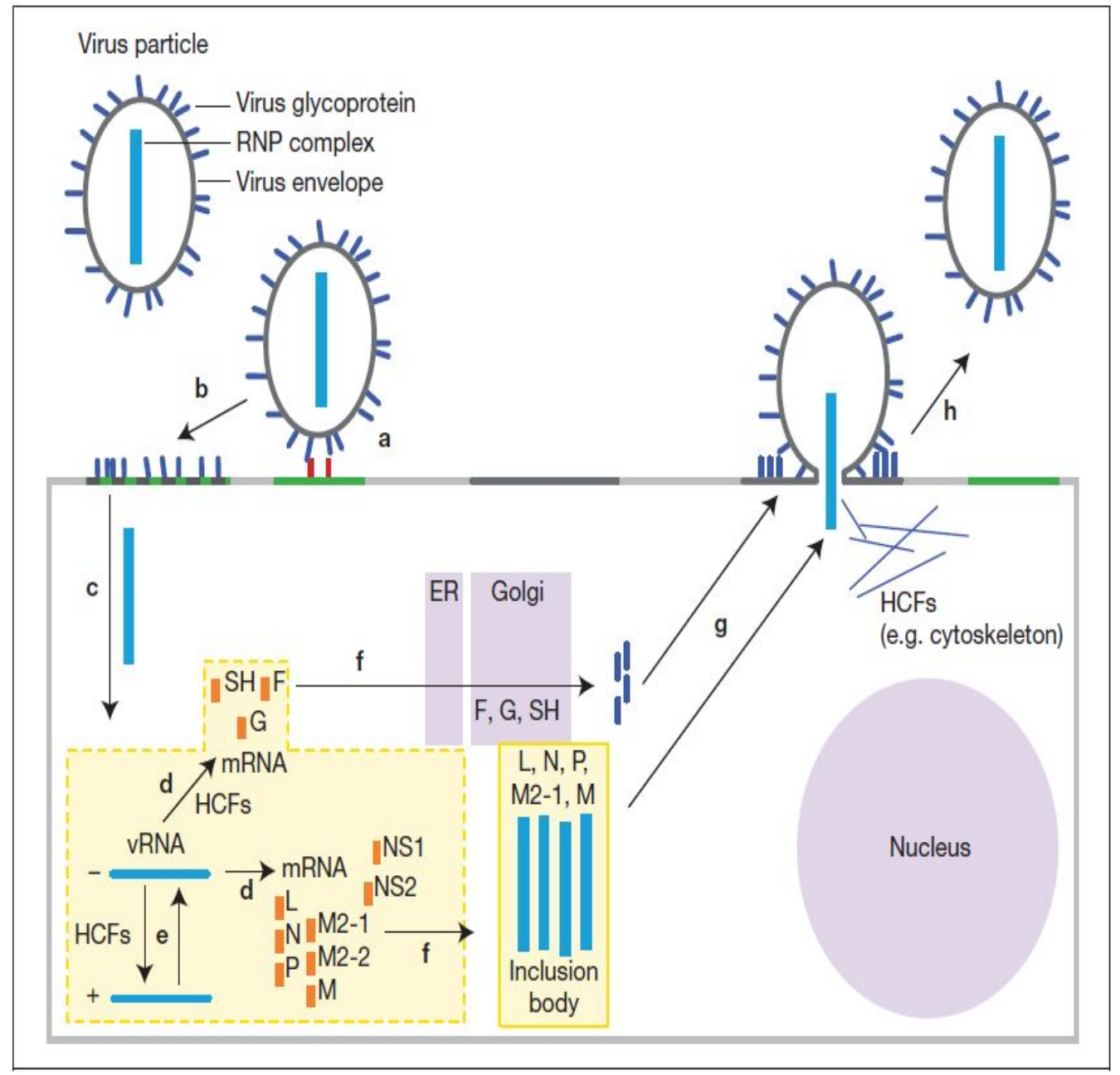

Figure 4.2 Overview of RSV replication cycle (adapted from Sugrue RJ, 2006)[125]. 


\subsubsection{Virus-Host interactions}

In the RSV-infected host cells, a variety of host genes related to different cellular functions such as protein metabolism, cytoskeleton structure, cell growth and inflammation is altered at their expression level. Simultaneously, RSV also has evolved a series of activities to overcome these immune defenses. The detailed virus-host interactions include (but not limited to): a batch of innate immune response and adaptive immune response has been observed to be initiated to conteract the virus invasion via pattern recognition receptors; these host immune response to RSV infection has been reported to be modulated via expression of $\mathrm{G}$ proteins; the activities of IFNs as well as cytokines have been also revealed to be impaired to weaken the host antiviral activities; some mechanisms have been also initiated to delayed programmed cell death and arrested cell cycle to facilitate robust viral titers during RSV infection; altered composition of lipid raft membranes have been also observed to benefit virus assembly and budding [123].

\subsubsection{Actin cytoskeleton and Rho GTPases}

The cytoskeletal framework in the cell is responsible for the trafficking of cellular proteins. It is majorly composed of actin, microtubules, intermediate filaments and other elements. Among them, actin microfilaments are in a dynamic state characterized by rapid cycles of polymerization/depolymerization in response to varying stimuli, and the microtubules are highly ordered with an intrinsic polarity.

Previous studies have proved that viruses use different elements of the cytoskeleton to facilitate entry, replication, transport as well as viral release. Detailed investigation has provided evidences that microtubules play a dominant role in the production of infectious virus, while actin filaments exert a greater effect on virual budding [133].

It has been revealed that $\mathrm{F}$ protein from RSV interacts with a cellular Rho GTPase, RhoA. And this GTPase signaling is closely relevant to many cellular functions associated with RSV pathogenesis, and these functions include organization of actin cytoskeleton and expression of proinflammatory cytokines. Gower TL et al (2001) did a series of experiments and found that the 
expression of RhoA was increased and downstream signaling of RhoA was activated in RSV-infected Hep2 cells [134].

Another Rho GTPase Cdc42 palys a role in regulating cytoskeleton especially filopodia formation, and it has been shown to direct various physiological processes such as cell morphology, migration, and cell cycle progression. Recent study proved that it was required for RSV internalization and infection in A549 cells [135].

\subsubsection{Immune response to $\mathrm{RSV}$ infection}

RSV infection triggers Toll-like receptors' (TLRs) and pathogen recognition receptors' (PRRs) conserved pathogen-associated molecular patterns recognition, which in turn initiates activation of a series of immune response related molecules and pathways through NF- $\kappa B$. For instance, studies in RSV-infected A549 cells have revealed the up-regulation of TLR4 at its expression level. Signaling through TLR4 can activate TNF receptor-associated factor and the adaptor protein MyD88, which in turn activate IKKe/TANKbinding kinase-1 and IL-1 receptor-associated kinase-4, thereby initiating a diverse group of transcription factors including IRF3, IRF7, NF- $\mathrm{B}$, JNK, p38 MAPK, activator protein-1 (AP1) [Figure 4.3].

TLR3, another member in TLR family, also show up-regulated expressions in reaction to RSV infection. Signaling through TLR3 also activate the downstream IKK 8 /TANK-binding kinase-1, which in turn induces IRF3, IRF7 and NF-кB [131]. Furthermore, virus infection sensed by TLRs is also able to activate downstream inflammatory chemokine and cytokine expression through NF-kB induction. And these chemokines and cytokines can take direct or indirect responsibility to virus infection and replication.

STAT proteins belonging to signal transducers are responsible for inducing transcription factors once receive the signal from either type I, type II or type III IFNs. Two mechanisms exist to antagonize the JAK-STAT signaling pathway: one is RSV NS protein mediated STAT2 proteosome degradation, and the other is negative modulation of type I IFN expression by SOCS family members, especially SOCS1 and SOCS3 [136, 137]. 


\subsubsection{Cytokine response to RSV infection}

Cytokines are small cell-signaling molecules that regulate immunity, inflammation and hematopoiesis. A wide range of cytokines have been published to be produced by numerous cell types during RSV infection. Some of these cytokines mediate proinflammatory reaction to activate and recruit immune cells, while others suppress the proinflammatory state. A set of important cytokines that are secreted upon viral infection are type I IFNs. However, several studies suggest that RSV induced type I IFNs in a poor way, which was majorly due to RSV NS protein suppression [139]. Other expressed cytokines mediated by NF- $\kappa$ B include RANTES, MCP, IL-9, TNF- $\alpha$, IL-6, IL-1, CX3CL1. Among these cytokines, several genetic markers that predict severe RSV pathology have been identified in humans, such as RANTES and TNF- $\alpha$ [140].

\subsubsection{Delayed programmed cell death to facilitate virus replication}

More and more evidence have been accumulated to prove that RSV has the ability to postpone programmed cell death of epithelial cells. It was revealed that expression of anti-apoptosis IEX-1L gene was highly up-regulated in RSVinfected respiratory epithelial cells. Since IEX-1L was reported to protect cells from apoptosis induced by TNF- $\alpha$, elevation of IEX-1L expression may suggest that RSV infection potentially protect epithelial cells from TNF- $\alpha$-induced [141]. The delay of apoptosis was also proved by Thomas KW et al (2001) [142]. In their paper, they concluded that RSV inhibited apoptosis through a phosphatidylinositol 3-kinase-dependent pathway. And this programmed cell death delayed phenomenon was believed to facilitate virus replication.

\subsubsection{NS1/ NS2 - viral antagonists of the host antiviral cytokine response}

RSV has been shown to produce proteins, nonstructural NS1 and NS2 proteins, to inhibit cellular innate immunity represented by IFN and its productions. Spann KM et al (2004) reported that these two proteins functioned independently as well as coordinately to achieve the full inhibitory effect on IFN- $\alpha$ and IFN- $\beta$ in A549 cells or macrophages [143]. To examine the concrete regulation network, more and more studies were focused on NS1 and NS2 with more and more details evidence provided to support this antiviral function of 
these two proteins. Ling $\mathrm{Z}$ et al (2009) proposed that expression of NS2 inhibited IFN transcription through either RIG-I or TLR3 pathways [144]. Moreover, it was also mentioned that NS2 inhibited RIG-I-mediated IFN promoter activation through its binding to the N-terminal CARD of RIG-I so as to inhibit its interaction with the downstream component MAVS. Other results indicated that NS2 decreased STAT2 levels and NS1 also degraded STAT2 during RSV infection, thereby modulating interferon-dependent gene expression [136, 145]. Recent experiment also proved that these two nonstructural proteins decreased the expression level of TRAF3, which is a strategic integrator of multiple IFN-inducing signals [146]. In addition, relative researches on NS1/NS2 also mentioned that they might retard premature apoptosis to facilitate virus growth in an NF- $\kappa \mathrm{B}$-dependent, IFN-independent way [147].

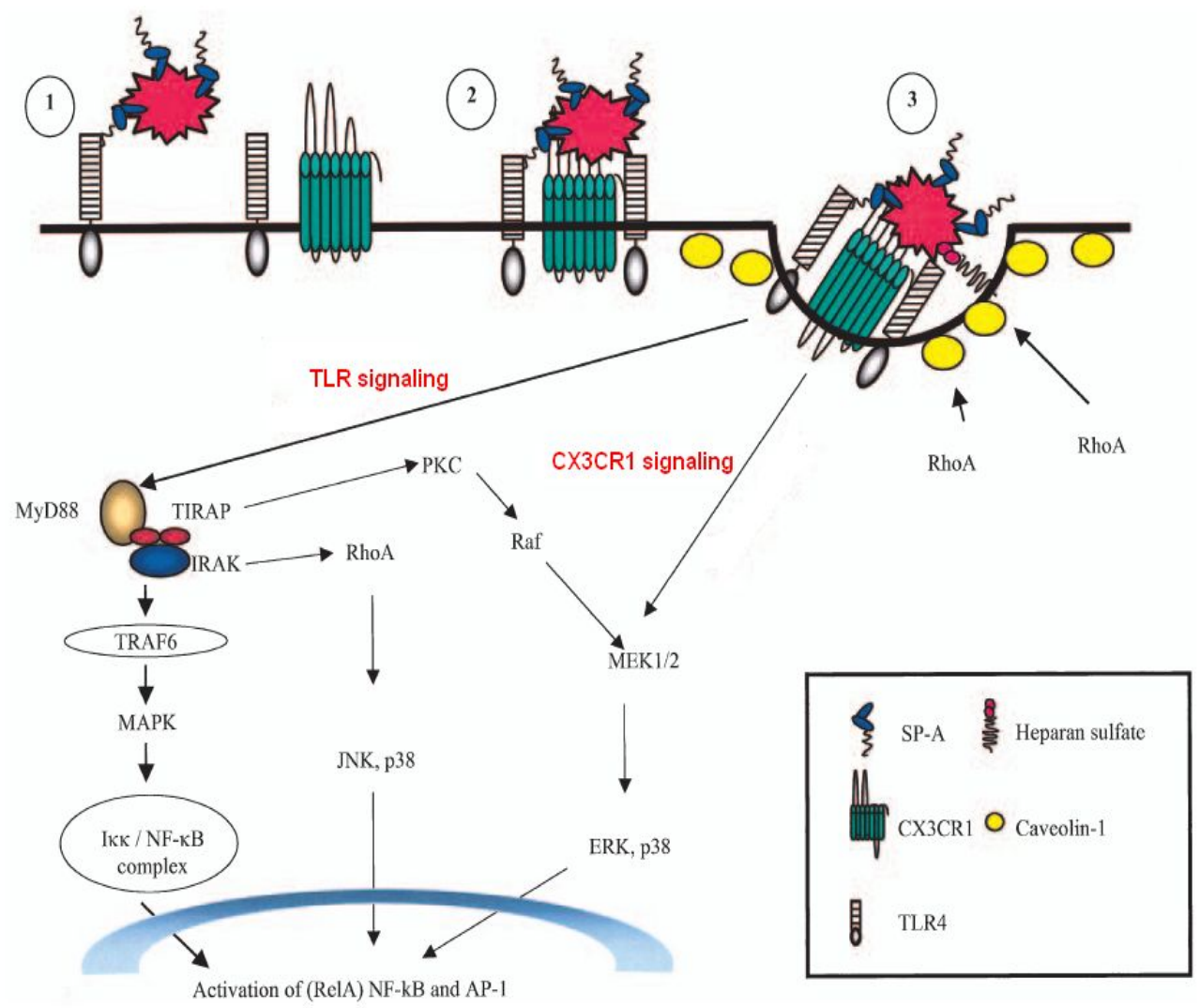

Figure 4.3 RSV biding and triggering of cellular responses (adapted from Harris $\mathrm{J}$ et al., 2003)[138]. 


\subsubsection{The role of $G$ protein in immune evasion}

To our knowledge, RSV infection induces expression of several notable chemokines, including IL-8, RANTES, MCP-1, MIP- $1 \alpha / \beta$ and IP-10. And RSV infection also triggers the proinflammatory mediator release like TNF- $\alpha$ that possesses antiviral activities. In this regard, G protein plays an essential role in modifying innate and adaptive immune responses at the expression levels of cytokines and chemokines. Accumulating evidences have proved that $\mathrm{F}$ and $\mathrm{G}$ proteins from RSV interact with the TLR pathway and modify negative regulation of SOCS protein on cytokine and chemokine expression. The centralconserved cysteine-rich region of $\mathrm{G}$ protein contains a $\mathrm{CX} 3 \mathrm{C}$ chemokine motif at amino acid positions 182-186, and this motif targets to interact with CX3CR1, CX3CL1 receptor. It has been reported that this CX3CR1 mimicry facilitated RSV infection and altered CX3CL1 chemotaxis of human and mouse leukocytes, and this $\mathrm{G}$ protein mediated immune response modulation was likely important in facilitating RSV replication [148]. Based on these findings, new vaccination strategy which sought to induce antibodies that block $G$ protein CX3C-CX3CR1 interaction has been put on the agenda for further RSV vaccine researches [149].

\subsubsection{Disease pathogenesis}

Investigations have indicated that the attack rate of RSV approaches $70 \%$ in the first year of life. The statistical data from US suggests that lower respiratory tract disease (bronchiolitis) develops in $20 \%$ to $30 \%$ of infected children, which result in around 120,000 hospitalizations yearly. RSV can also cause severe lower respiratory illness and lead to respiratory failure with 70$100 \%$ mortality rates in adults [150].

A variety of host factors, particularly cytokines and chemokines, have been reported to be responsible for the RSV disease pathogenesis. Expression of these factors aim to limit virus replication, however, over-expression or extra production of these immune moleculors may exacerbate the inflammatory response so as to promote airway damage and pathogenesis in the process of virus clearance $[151,152]$. Evidence has been provided to indicate that CCL5 (RANTES) resulted in exacerbation of allergic airway inflammation following RSV infection [153]. 
The precise communication of innate and adaptive immune response is important in defining the magnitude of adaptive immunity. Inefficient inflammatory responses triggered by RSV infection may result in the inappropriate induction of T-cell responses. The T1-type responses characterized by the expression of IFN- $\gamma$, IL- 2 and IL-12 are required for the efficient virus clearance, while the Th2-type responses characterized by the expression of IL-4, IL-10 and IL-13 are almost ineffective but able to contribute to allergic diseases and asthma $[131,154]$. And it was published that intrinsic antigenic properties of $G$ protein enables to promote $\mathrm{Th} 2$ responses and eosinophilia [150].

\subsubsection{RSV infection in different cells}

\subsubsection{RSV infection in Hep2 cells}

Ternette $\mathrm{N}$ et al (2011) applied label-free quantitative mass spectrometry to test the proteomics profiling of RSV-infected Hep2 cells and analyzed the data using IPA software [155]. Analysis result demonstrated that mRNA levels of IFIT3 and XRN2 were increased during RSV infection. Moreover, "PI3K/Akt signaling”, "mTOR signaling”, "protein ubiquitination pathway" and "RNA signaling" were enriched as significant pathways. Other experiments majorly focused on uncovering the interaction of lipid raft and RSV in Hep2 cells, and cholesterol depletion studies indicated that membrane cholesterol was required for virus filament formation. In addition, lipid raft microdomains played an important role in RSV maturation process, but dynamics of host-cell interactions and pathway cross-talk associated with RSV-mediated lipid raft microdomain modifications was poorly understood $[127,156]$.

\subsubsection{RSV infection in macrophages}

Macrophages are cells produced by the differentiation of monocytes in tissues. Macrophages function in recognizing, engulfing and destroying many potential pathogens in the innate immune system. Besides these pathogens, macrophages also enable to recognize syngeneic tumor cells, virus-infected cells as well as normal cells undergoing programmed cell death. Besides, macrophages also function in acquired immune response. Upon phagocyosis, macrophages degrade proteins and present antigens on major histocompatibility 
complex (MHC) molecules, where $\mathrm{T}$ cells can recognize the substance as "foreign". Accordingly, pathogens have developed multiple methods to evade the attacks from macrophages.

Macrophages are subjected to classical (Th1) or alternative (Th2) activation, depending on the types of cytokines that they are exposed to. In the first case, macrophages are activated by inflammatory stimuli, such as IFN- $\gamma$ (IFN- $\alpha$ ), in combination with TLR activation by microbial stimuli, such as lipopolysaccharide (LPS). After that, the intracellular pathogens are killed and inflammantory cytokines that amplify Th1 immune responses are secrete through the production of iNOS, which generates nitric oxide (NO) that can damage cells. In the second case, after exposure to Th2 cytokines such as IL-4, IL-10, or IL-13, macrophages produce polyamines and proline so as to induce proliferation and collagen production. This kind of macrophages produces arginase-1 which competes with iNOS for arginine to produce L-ornithine and urea. Moreover, murine alternative-activated macrophages were reported to express "markers" including found in inflammatory zone 1, Ym1, mannose receptor and others, which did not express by classical-activated macrophages $[157,158]$.

Macrophages play a pivotal role in host lung defense mechanisms. As the first line of defense in acute RSV infection, macrophages are recruited in large numbers to the site of infection. Regarding the alterbative activation of macrophages, TLR4 signaling is necessary for expression of PPAR $\gamma$ and IFN- $\beta$ is responsible for expression of IL-4, IL-13, IL-4R $\alpha$, and IL-10 in response to RSV infection. This induction is initiated prior to the development of the adaptive immune response [158]. To fight with these innate and adaptive immune responses coming from macrophage activation, RSV develops distinct mechanisms to impair macrophage IFN- $\alpha / \beta$ - and IFN- $\gamma$-stimulated transcription $[159,160]$. Furthermore, the activities of caspase- 3 and caspase- 9 were reduced and eliminated seperately, while expression of anti-apoptotic proteins such as Bcl-2, Bcl-X and XIAP were enhanced in RSV-infected mouse macrophages. This phenomenon implied that the intrinsic apoptotic pathway was subverted in mouse macrophages after infection of RSV [161]. 


\subsubsection{Objective}

To our knowledge, RSV is the most common cause of acute bronchiolitis, particularly in infants and young children. The pathogenesis of RSV bronchiolitis is involved in a combination of direct cytopathic effects induced by viral replication and the resulting host response of production of proinflammatory cytokines. Otherwise, the detailed interactions between virus and host factors are not quite clearly understood. Moreover, macrophages as "gatekeepers" function to initiate the innate and adaptive immune responses at the first time but it is surprising that some studies demonstrated that RSV infection severely diminished the phagocytic ability of macrophages [162]. Thus, to sort out the internal response of Hep2 cells and macrophages in reaction to RSV infection is meaningful and pressing.

Accordingly, RSV A2 strain was designed to infect Hep2 cells and pulmonary macrophages at specific time points. All these global gene expression profiles were monitored using microarray platform, and expression of interested genes were validated by techniques such as cytokine assays $[156,364]$. Different types of software were employed into further analysis. The aims of this research are as following:

(1). Investigate the host-virus interactions in RSV infected Hep2 cells.

(2). Investigate the host-virus interactions in RSV infected mouse macrophages.

(3). Establish the mechanisms which have been involved in antagonizing the immune response in different host cells upon RSV infection.

\subsection{Experiment workflow}

\subsection{Result and Discussion}

\subsubsection{Global profiling of gene expression}

\subsubsection{Heat maps of global gene expression}

The global transcriptional profile of RSV-infected Hep2 cells illustrated that more and more expression changes occurred with the infection time increasing [Figure 4.5]. It was also observed that there were a larger number of probe sets showing down-regulated expression when compared to those showing up-regulated expression. 
In macrophages infected with RSV, a few more expression changes were also detected at later time point ( $24 \mathrm{hpi}$ ). However, the numbers of genes with up-regulated expression were higher than the numbers of those with downregulated expression in RSV-infected macrophages, which was different from the case detected in RSV-infected Hep2 cells. These observations might indicate the activation of a big scale of host antiviral genes in macrophages upon RSV infection [Figure 4.6].

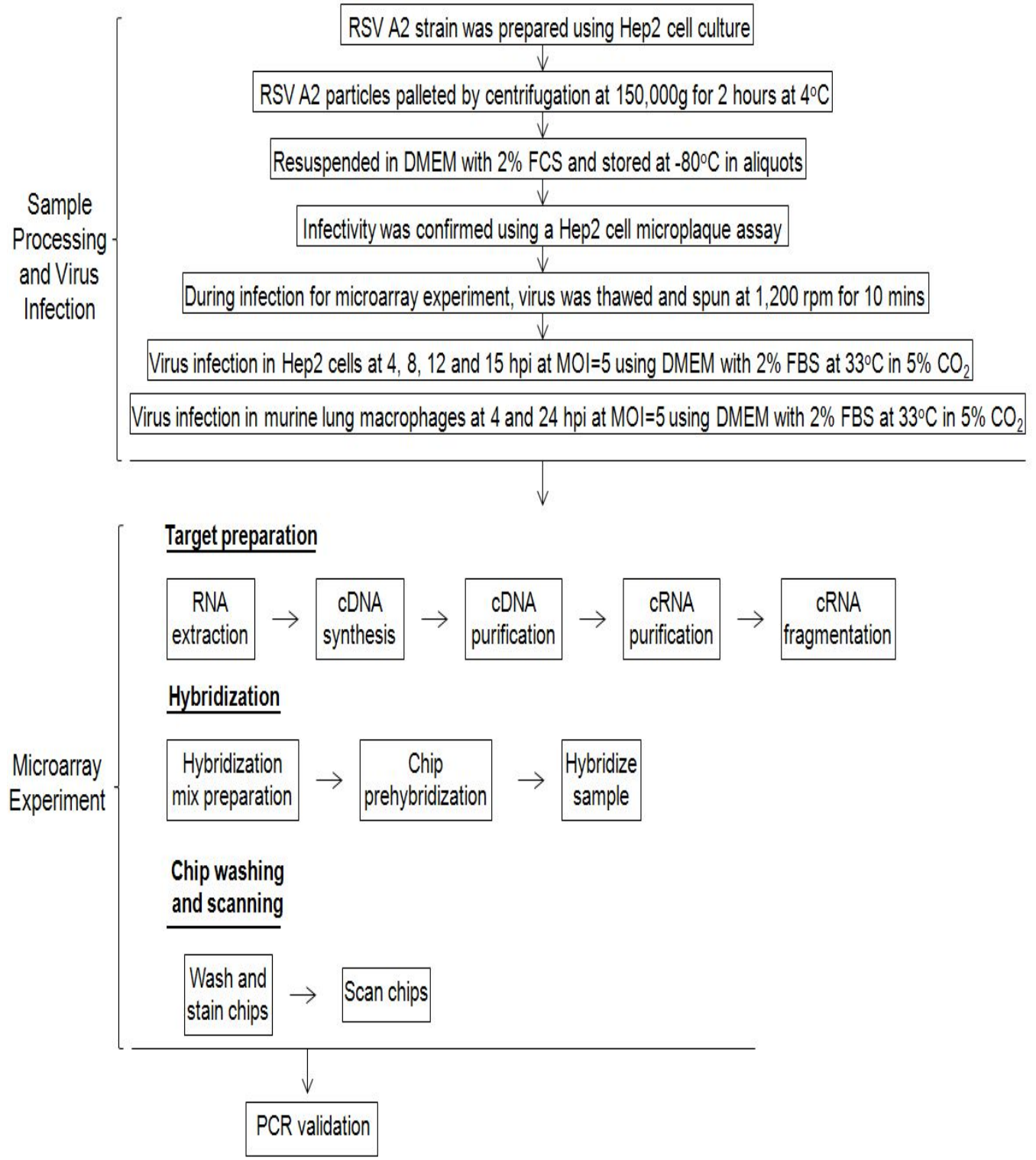

Figure 4.4 Microarray experimental workflow during RSV infection. 


\subsubsection{Distribution of differentially expressed probe sets}

As shown in Table 4.1, almost half of the probe sets with up-regulated expression showed their expression at $\geq 3-\mathrm{FC}$, while less than one fourth of the probe sets with down-regulated expression showed their expression at $\geq 3$-FC across the whole infection period. This observation suggested that up-regulated probe sets showed in a relative strong fold regulation than down-regulated ones at the global level.

In three late infection time points $(8,12$ and $15 \mathrm{hpi})$, the percentages of up-regulated probe sets showed similar and higher than the one from 4 hpi. This observation might implicate a strong and sustained antiviral response from 8 to 15 hpi. The percentages of down-regulated probe sets showed a gradual increase across the whole infection period, indicating the inhibition of more and more cell activities following RSV infection in Hep2 cells.

With regards to macrophages infected with RSV, a large scale of genes was observed with differential expression from 4 hpi, suggesting an early and timely host cell reaction in response to RSV infection [Table 4.2]. The genes showing up-regulated expression were almost twice as many as those showing down-regulated expression, and some of these up-regulated genes even showed outstanding fold regulation at $\geq 5-\mathrm{FC}$. 


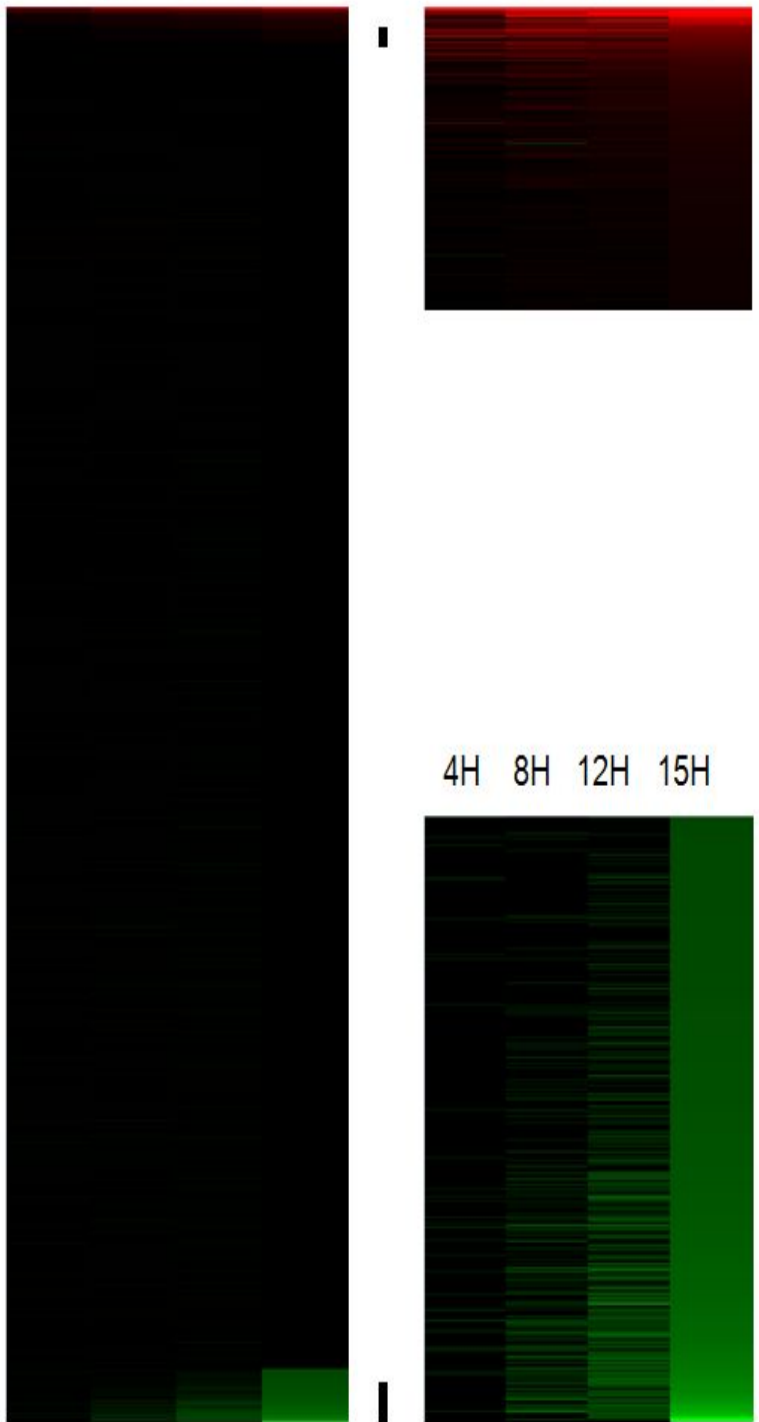

Figure 4.5 Temporal changes in the host cell transcriptome in Hep2 cells infected with RSV. The global host gene expression profiles were retrieved from microarray analysis with different time points examined. The probe sets showing $\geq 2$ fold change (FC) up- or downregulated in expression are indicated (P-value $\leq 0.05)$. Expression profiles of up-regulated (red), down-regulated (green) and no significant change (black) are shown.

Table 4.1 Differentially expressed probe sets in Hep2 cells infected with RSV.

\begin{tabular}{|c|c|c|c|c|c|c|}
\hline \multirow{2}{*}{ Hep2 cells } & \multicolumn{2}{|c|}{ Probe sets $(\geq 2-F C)$} & \multicolumn{2}{|c|}{ Probe sets $(\geq 3-F C)$} & \multicolumn{2}{|c|}{ Probe sets $(\geq 5-\mathrm{FC})$} \\
\hline & Up-regulated & Down-regulated & Up-regulated & Down-regulated & Up-regulated & Down-regulated \\
\hline RSV 4H & $1.46 \%$ & $0.59 \%$ & $0.77 \%$ & $0.09 \%$ & $0.39 \%$ & $0.03 \%$ \\
\hline RSV $8 \mathrm{H}$ & $2.34 \%$ & $1.24 \%$ & $1.11 \%$ & $0.24 \%$ & $0.48 \%$ & $0.03 \%$ \\
\hline RSV 12H & $2.36 \%$ & $2.44 \%$ & $1.05 \%$ & $0.61 \%$ & $0.45 \%$ & $0.12 \%$ \\
\hline RSV 15H & $2.43 \%$ & $3.78 \%$ & $1.16 \%$ & $0.92 \%$ & $0.51 \%$ & $0.16 \%$ \\
\hline
\end{tabular}

The global host gene expression profiles were retrieved from microarray analysis with different time points examined. The ratios of differentially expressed probe sets $(\mathrm{P}$-value $\leq 0.05)$ up- or down-regulated with different fold changes $(\geq 2-F C, \geq 3-F C$ and $\geq 5-F C)$ in relative to their corresponding "expressing probe sets" are represented in percentage. The expressing probe sets refer to probe sets detected in the mock-infected Hep2 cells. 

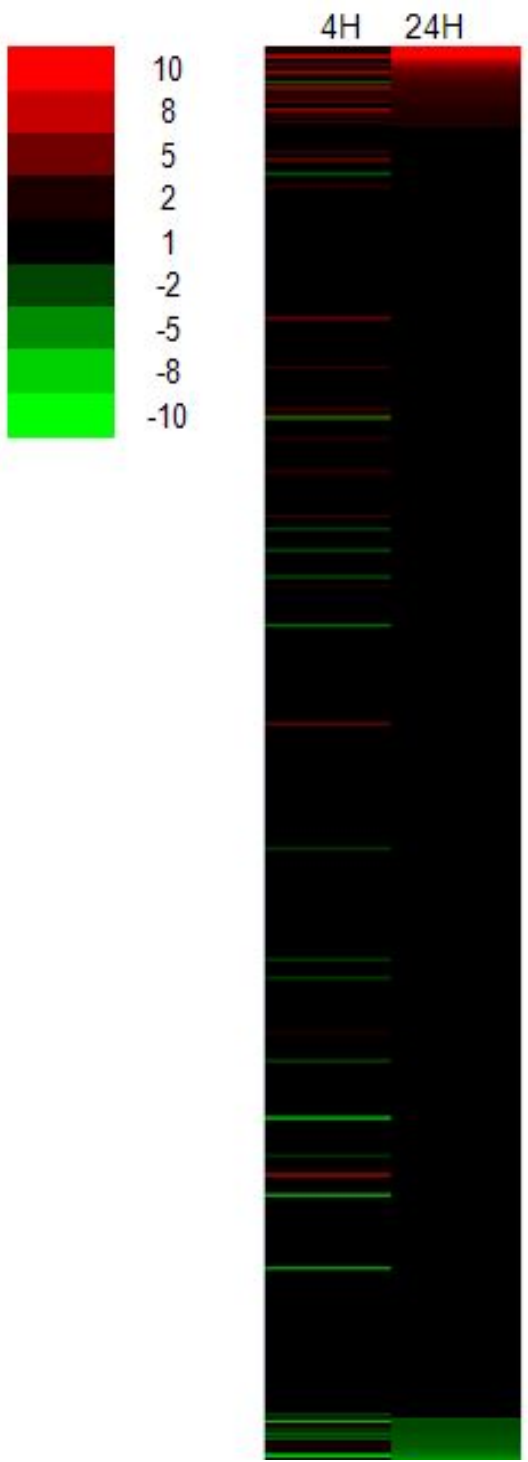

Figure 4.6 Temporal changes in the host cell transcriptome in macrophages infected with RSV. The global host gene expression profiles were retrieved from microarray analysis with different time points examined. The genes showing $\geq 2$ fold change (FC) up- or down-regulated in expression are indicated (P-value $\leq 0.05)$. Expression profiles of up-regulated (red), downregulated (green) and no significant change (black) are shown.

Table 4.2 Differentially expressed genes in macrophages infected with RSV.

\begin{tabular}{|c|c|c|c|c|c|c|}
\hline \multirow{2}{*}{ Macrophages } & \multicolumn{2}{|c|}{ Genes $(\geq 2 \cdot F C)$} & \multicolumn{2}{|c|}{ Genes ( $\geq 3-\mathrm{FC}$ ) } & \multicolumn{2}{|c|}{ Genes ( $\geq 5-\mathrm{FC})$} \\
\hline & Up-regulated & Down-regulated & Up-regulated & Down-regulated & Up-regulated & Down-regulated \\
\hline RSV 4 hpi & $6.38 \%$ & $3.45 \%$ & $2.86 \%$ & $0.74 \%$ & $1.17 \%$ & $0.13 \%$ \\
\hline RSV 24 hpi & $8.51 \%$ & $3.99 \%$ & $3.92 \%$ & $1.12 \%$ & $1.87 \%$ & $0.22 \%$ \\
\hline
\end{tabular}

The global host gene expression profiles were retrieved from microarray analysis with different time points examined. The ratios of differentially expressed genes (P-value $\leq 0.05)$ up- or downregulated with different fold changes $(\geq 2-F C, \geq 3-F C$ and $\geq 5-F C)$ in relative to their corresponding "expressing genes" are represented in percentage. The expressing genes refer to genes detected in the mock-infected macrophages. 


\subsubsection{Functional classification}

In terms of biological functions from GO and KEGG database, differentially expressed probe sets or genes were classified into interested functional families at different infection time points. As shown in Figure 4.7, up-regulated probe sets in RSV-infected Hep2 cells majorly located in the groups such as "Immune Response", "DNA Binding”, "Signal Transduction", "Transcription Factor", "Cell Growth" and particularly "RNA binding" at $\geq 2$ FC. It was surprising that expression of some probe sets that are functional associated with "Immune Response", "RNA Binding" and "Signal Transduction" showed strong elevation with fold regulation even $\geq 10-\mathrm{FC}$, and the number of these probe sets showed a bit higher at 8 hpi than 4, 12 and 15 hpi. These observations might implicate that the strong and sustained host antiviral response across the whole investigated infection period, with the strongest state occurred around $8 \mathrm{hpi}$. And some of genes with up-regulated expression were classified into biological grouping such as "Cell Growth", and this phenomenom might indicate that some mechanisms were exerted to promote the process of host cell cycle in order to speed up viral replication.

Down-regulated probe sets in RSV-infected Hep2 cells were majorly classified into functional groups such as "RNA Binding", "DNA Binding", "Signal Transduction" and "Transcription Factor", and the numbers of downregulated probe sets in each family increased with the infection time increasing. When compared to the up-regulated probe sets, only several down-regulated probes sets could be detected with high-level fold regulation $\geq 5$-FC at 12 and 15 hpi.

When it turns to RSV-infected macrophages, functional groups including "Cytokine", "Antiviral", "RNA Binding", "Cell Death", "Signal Transduction" and "DNA Transcription Factor" were majorly enriched in the up-regulated probe sets at either 4 or 24 hpi [Figure 4.8]. At $\geq 10$-FC, a few genes encoding cytokines were even detected at both examined time points, which implied the early and sustained activation of antiviral host response during RSV infection in macrophages. A small batch of genes belonging to "Cell Death" also showed stimulated expression at both 4 and 24hpi, and a couple of them also showed up-regulated expression with quite prominent fold 
changes. This observation might suggest that the cell apoptosis was initiated strongly from a quites early infectioin stage.

Only a small number of genes showed down-regulated expression with low fold regulation at both time points. And these genes majorly belonged to "Signal Transduction", "RNA Binding", "Kinase", "Cell Cycle" and "Cell Growth".

Genes with faintly down-regulated expression were identified both in RSV-infected Hep2 cells and RSV-macrophage. This observation might imply the interference of cell signaling as well as metabolism by RSV infection.

\subsubsection{Cluster analysis in Hep2 cells}

To our knowledge, genes with similar temporal expression trends might have related biological functions and possibly correspond to some critical cellular processes and pathways [91]. So the aim of this cluster analysis is to classify probe sets with similar expression profiles into common biological groups, which is beneficial for further functional analysis. The Hep2 cells were infected with RSV, and the corresponding transcriptomes were analyzed at 4, 8, 12, and 15 hpi. Probe sets at more than one time point of infection with $\geq 2-F C$ (P-value $\leq 0.05)$ were clustered into similar gene expression profiles using the Expander version 5.0 software [Figure 4.9]. Using the same software, the data were further analyzed into genes relating to different functional groups or canonical pathways, and enriched transcription factor were also identified. All enriched functional groups, canonical pathways and transcriptional factors are displayed in Table 4.3.

Pathway analysis revealed that differentially up-regulated genes shared common pathways including "MAPK signaling”, "Jak-STAT signaling pathway", "B-cell receptor signaling pathway", "Toll-like signaling pathway", "Steroid biosynthesis", "Metabolic pathways", "Focal adhesion" and "Pathways in cancer". Activation of these immune response and steroid synthesis related pathways implied the host antiviral activities and alterations in the composition of the lipid raft membranes during RSV infection in Hep2 cells.

Functional groups such as "Immune system process", "Response to stimulus", "Lipid metabolic process" and "Lipid biosynthetic process" were significantly enriched in these genes with the increased expression trends. Over- 
representation of these functional groups was consistent with the significant enrichment of key pathways mentioned above, providing more evidences that the host antiviral responses and membrane activities were triggered in RSV infected Hep2 cells.

A batch of transcription factors such as SRF, CAC-binding protein, ISRE, Sp1, UF1H3BETA and Bach2 were identified to be enriched in the upregulated genes. SRF, as a member of the MADS box superfamily of transcription factors, regulates the activity of many immediate-early genes and thereby participates in cell cycle and apoptosis regulation [163]. In our study, SRF gene showed a significant expression elevation (around 3-FC) from 8 hpi to $15 \mathrm{hpi}$, which was consistent with its enrichment by downstream upregulated factors and could implicate its potential regulation on cell cycle. Besides, SRF is also the downstream target of many pathways such as MAPK pathway ternary complex factors. Previous finding showed that "MAPK signaling" pathway was enriched by the up-regulated genes, and this finding was concordant with the up-regulation of SRF in its expression. ISRE is an interferon stimulation response element which binds to IRF3 to present in the ISG15 gene promoter and activate its transcriptional activity. Enrichment of this transcription factor in up-regulated genes potentially suggested the partly activation of interferon signaling pathway.

In the meanwhile, the pathway called "Cytokine-cytokine receptor interaction" was enriched in the down-regulated genes, which could be due to the RSV combating the host immune regulation. Another pathway that was also enriched by the down-regulated genes was "ECM-receptor interaction". ECM was found to participate in the regulation of cytoskeleton and apoptosis [164, 165], thus enrichment of this pathway may reveal the negative modulation in these aspects under RSV infection in Hep2 cells. Other pathways such as "Apoptosis" and "Small cell lung cancer" were also enriched in the downregulated genes.

Genes with decreased expression were significantly grouped into functions such as "Nitrogen compound metabolic process", "Cellular biosynthetic process", "Transition metal ion binding" and "Regulation of macromolecule biosynthetic process" with high numbers included for each group. Besides, other functions belonging to a diverse range of cellular 
functions were also enriched based on other genes with down-regulated expression, suggesting the impairment of cell activities from a wide range.

In addition to these pathways and functions, potential transcription factors such as ETF, E2F, c-Myc:Max, TATA, STATx, NF-kappaB_(p65) and Evi-1were also over-represented by the down-regulated genes. E2F and cMyc:Max are important regulator and transcription factor in apoptosis signaling pathway, which was correlated with the enrichment of "Apoptosis" pathway and indicated the inhibition of the apoptosis signal transduction in Hep2 cells after RSV infection. This observation was consistent with previous finding that RSV delayed programmed cell death to facilitate virus replication in human airway epithelial cells [131].
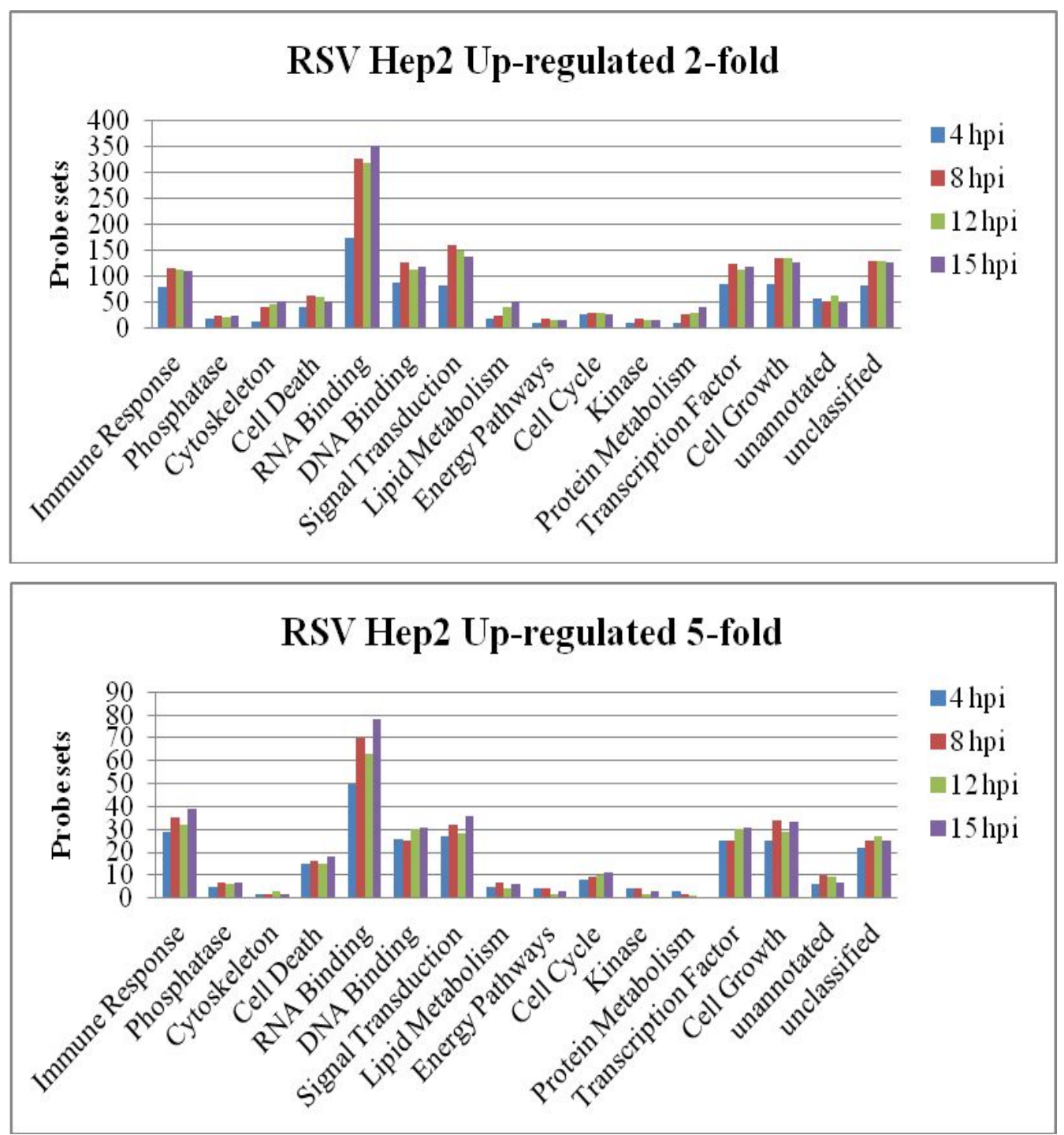

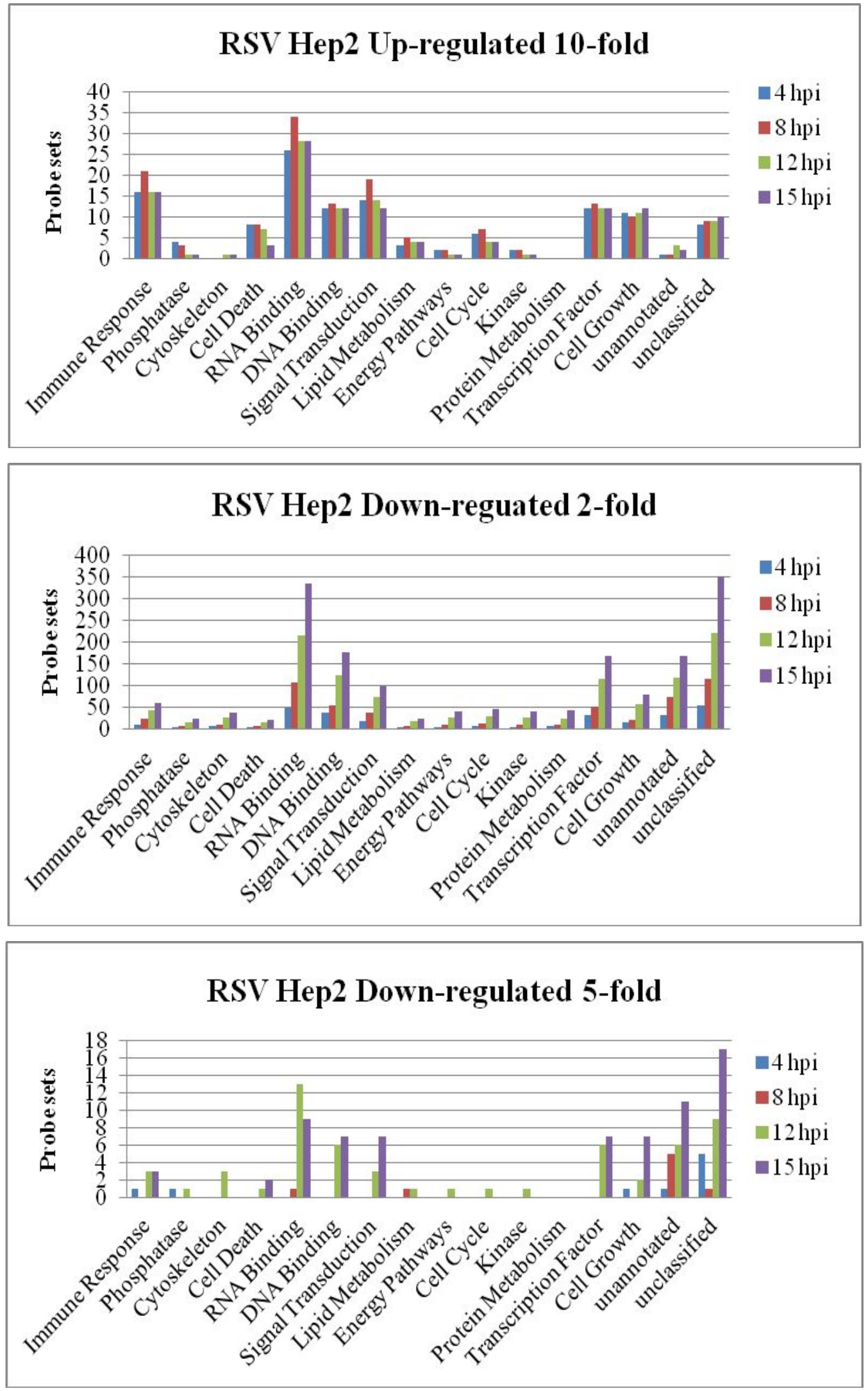

Figure 4.7 Overview of distributions of differentially expressed probe sets into different biological functions in Hep2 cells infected with RSV. The numbers of probe sets in the different functional families, including non-annotated and unclassified groups, showing up- or down-regulated with different fold changes $(\geq 2-F C, \geq 5-F C$ and $\geq 10-F C)$ in gene expression are presented. 

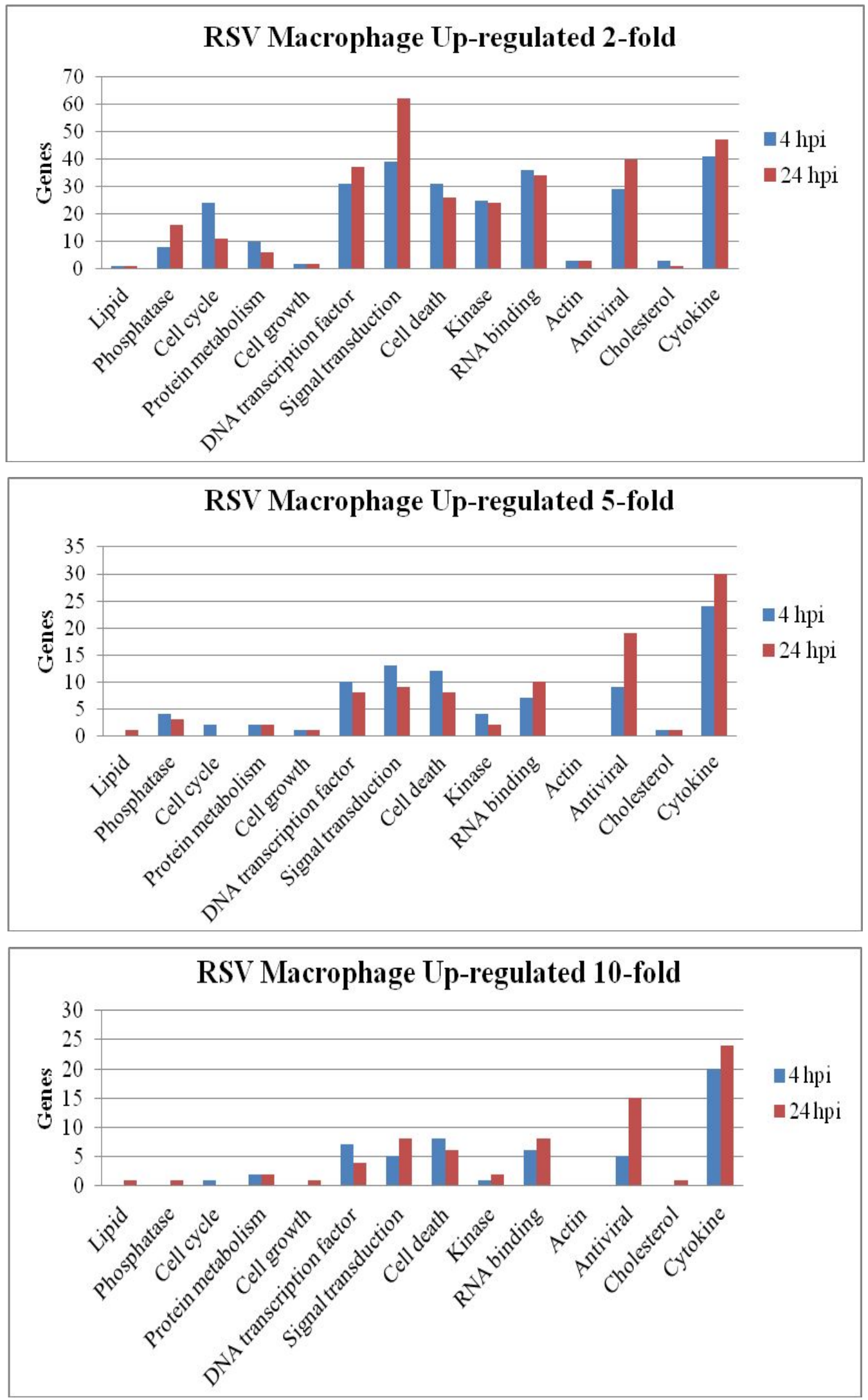

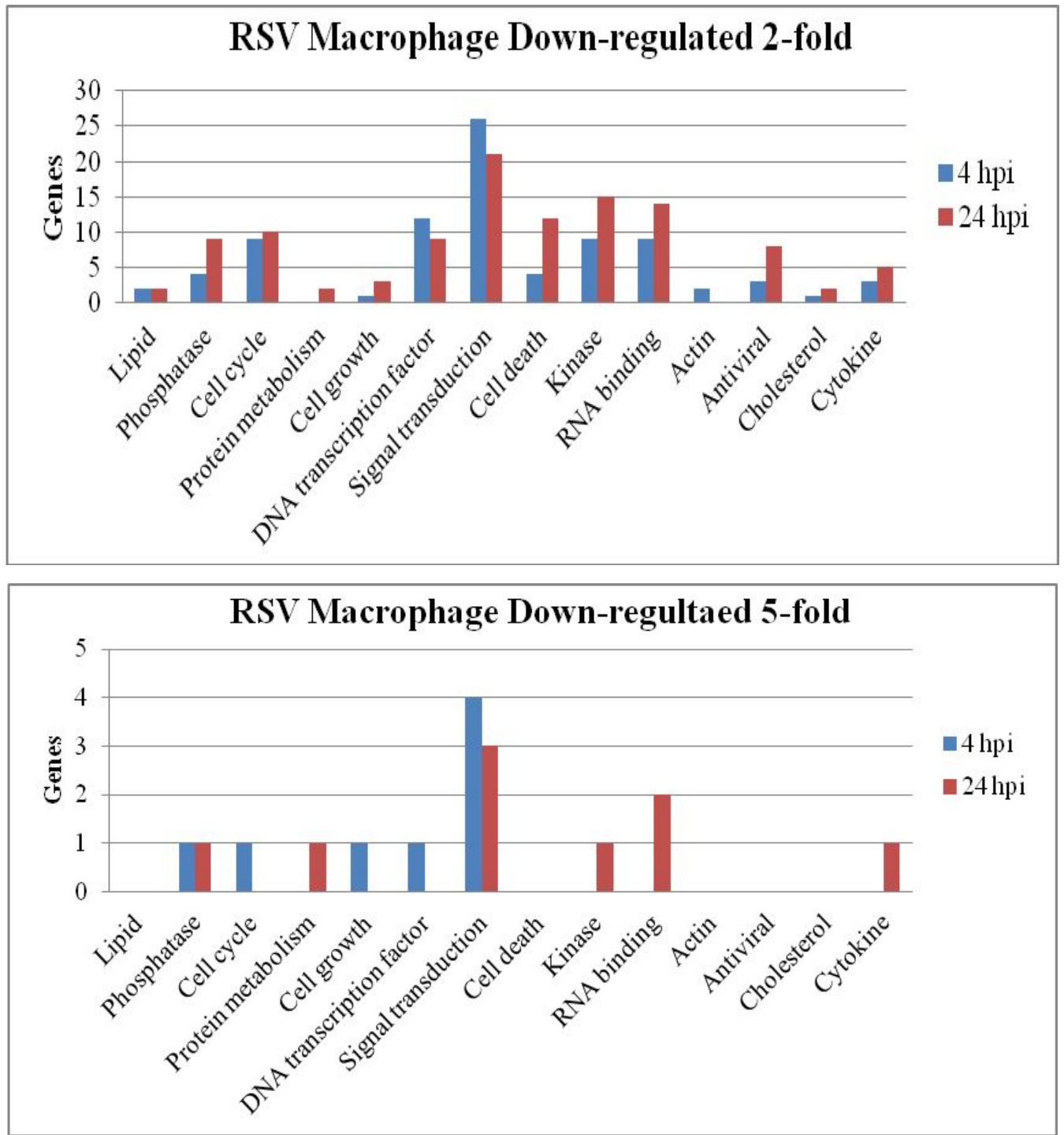

Figure 4.8 Overview of distributions of differentially expressed genes into different biological functions in macrophages infected with RSV. The numbers of genes in the different functional families, showing up-regulated or down-regulated with different fold changes ( $\geq 2$-FC, $\geq 5$-FC and $\geq 10$-FC) in gene expression are presented. 

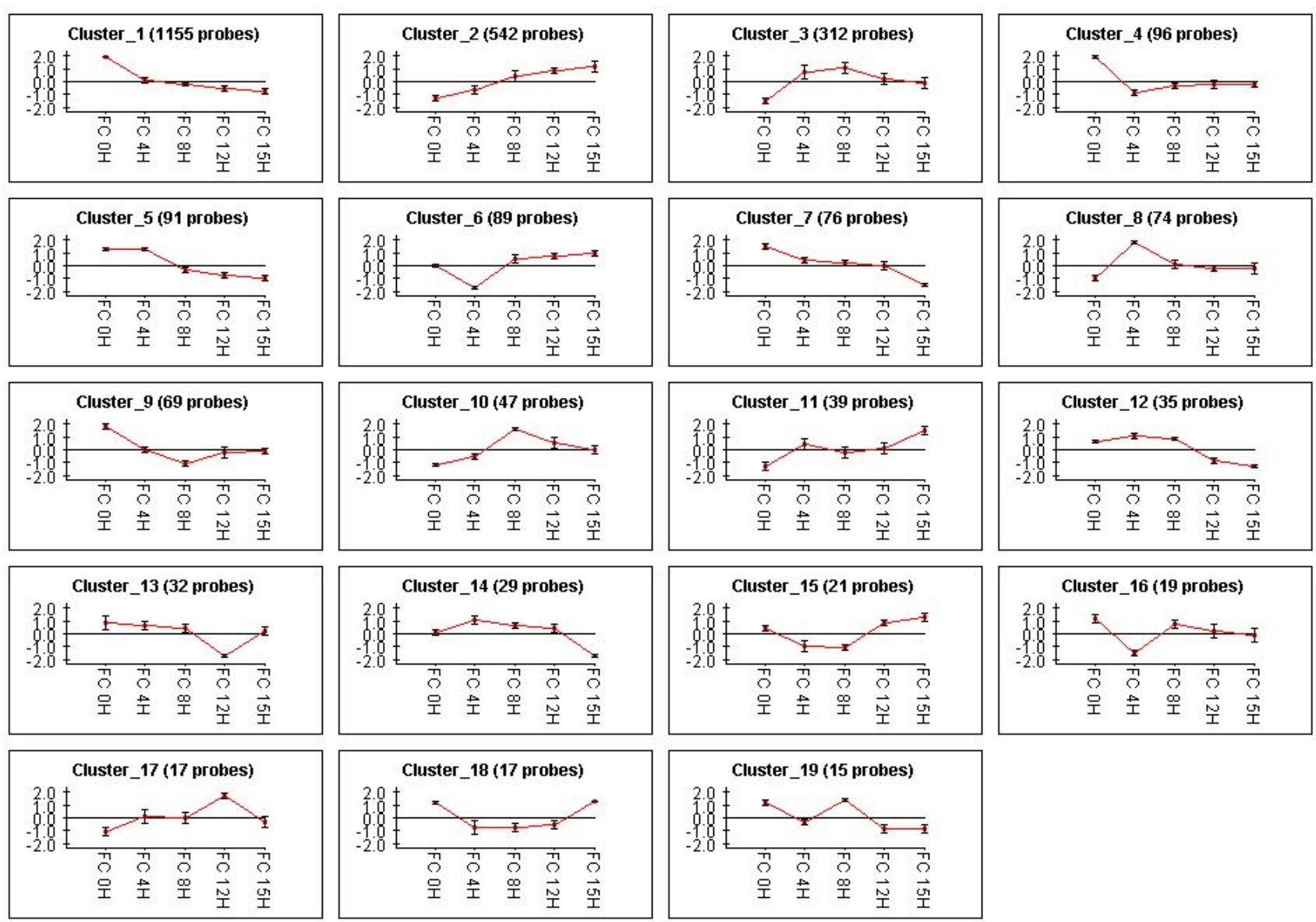

Figure 4.9 Clustering analysis of temporal gene expression profiles in RSV-infected Hep2 cells. Hep2 cells were infected with RSV at 4 , 8 , 12 and 15 hpi. Probe sets showing $\geq 2$-fold changes up- or down-regulated (P-value $\leq 0.05$ ) at least over one time point were analyzed with Expander 5 software. $X$-axis represents post-infection in hours $(\mathrm{H})$, and $\mathrm{Y}$-axis means normalized expression changes of probe sets. 
Table 4.3 Summary of functional groups, canonical pathways and transcription factors enriched based on differentially expressed genes in Hep2 cells infected with RSV.

\begin{tabular}{|c|c|}
\hline Cluster NNo. & Enriched Transcription Factors \\
\hline Cluster 1 & ETF:333 | E2F-434 | c-Myc:Max:199 | E2F-1:179 \\
\hline Cluster 2 & SRF:35 | CAC-binding_protein:108 | ISRE:52 | NF-kappaB_(p65):35 | Sp1:200 | UF1H3BETA:162 | Bach2:52 \\
\hline Cluster 3 & TATA:27 | STATx:32 | NF-kappaB_(p65):21 \\
\hline Cluster 8 & NF-kappaB_(p65):10 \\
\hline Cluster 9 & Evi-1:10 \\
\hline Cluster №. & Enriched Canonical Pathways \\
\hline \multirow{3}{*}{ Cluster 2} & Focal adhesion:20 | Pahtways in cancer:21 | MAPK signaling pathway:17 | Jak-STAT signaling pathway:13 | B cell receptor signaling pathway:9 \\
\hline & Toll-like receptor signaling pathway:10 | Steroid biosynthesis:5 | Hypertrophic cardiomyopathy (HCM):8 | Leukocyte transendothelial migration:9 \\
\hline & Renal cell carcinoma:7 \\
\hline Cluster 3 & Apoptosis:9 | Pathways in cancer:14 | Small cell lung cancer:8 | ECM-receptor interaction:7 | Cytokine-cytokine receptor interaction:10 \\
\hline Cluster 6 & Steroid biosynthesis:3 | Metabolic pathways:12 \\
\hline Cluster 8 & Cytokine-cytokine receptor interaction:6 \\
\hline Cluster №. & Enriched Gene Ontology Terms \\
\hline \multirow[t]{4}{*}{ Cluster 1} & Nitrogen compound metabolic process - G0:0006807:186 | Cellular biosynthetic process - G0:0044249:162 | Gene expression - G0:0010467:142 \\
\hline & Cellular biopolymer biosynthetic process - G0:0034961:128 | Nucleic acid binding - G0:0003676:162 | Transition metal ion binding - G0:0046914:131 \\
\hline & Regulation of macromolecule biosynthetic process - G0:0010556:139 | DNA metabolic process - G0:0006259:34 | Cell cycle - G0:0007049:41 \\
\hline & DNA replication - G0:0006260:19 | Cell cycle checkpoint - G0:0000075:10 \\
\hline \multirow[t]{10}{*}{ Cluster 2} & Immune system process - G0:0002376:47 | Response to stimulus - G0:0050896:89 | Positive regulation of cellular process - G0:0048522:49 \\
\hline & Response to wounding - G0:0009611::28 | Negative regulation of biological process - G0:0048519:51 | Anatomical structure development - G0:0048856:63 \\
\hline & Response to external stimulus - G0:0009605:34 | Developmental process - 60:0032502:75 | Organ development - G0:0048513:48 \\
\hline & Multi-organism process - G0:0051704:27 | Regulation of biological quality - G0:0065008:39 | Regulation of developmental process - G0:0050793:28 \\
\hline & Regulation of cell prolfferation - G0:0042127:26 | Cell death - G0:0008219:25 | Receptor binding - G0:0005102:30 |Actin binding - G0:0003779:18 \\
\hline & Positive regulation of cell proliferation - G0:0008284:17 | Steroid metabolic process - G0:0008202:14 | Regulation of cellular metabolic process - G0:0031323:72 \\
\hline & Regulation of apoptosis - G0:0042981:24 | Cell activation - G0:0001775:14 | Response to virus - G0:0008615:10 | Cell proliferation - G0:0008283:17 \\
\hline & Actin cytoskeleton organization - G0:0030036:13 | Regulation of protein metabolic process - G0:0051246:18 | Biological achesion - G0:0022610:24 \\
\hline & Sterol biosynthetic process - G0:0016126:6 | Transferase activity, transferring phosphorus-containing groups - G0:0016772:30 | Enzyme binding - G00019899:15 \\
\hline & Lipid bioxynthetic process - G0:0008610:15 | Lipid metabolic process - G0:0006629:26 \\
\hline \multirow[t]{8}{*}{ Cluster 3} & Immune system process - G0:0002376:30 | Regulation of cell proliferation - G0:0042127:24 | Response to stimulus - G0:0050896:53 \\
\hline & Negative regulation of biological process- G0:0048519:36 | Regulation of developmental process - G0:0050793:24 | Response to stress - G0:0006950:34 \\
\hline & Response to wounding - G0:0009611:19 | Regulation of signal transduction - G0:0009966: 22| Defense response - G0:0006952:20 \\
\hline & Cell death - G0:0008219:19 | Multicellular organismal development - G0:0007275:42 | Organ development - G0:0048513:28 \\
\hline & Postive regulation of biological process - G0:0048518:28 | Tissue development - G0:0009888:17 | Regulation of immune system process - G0:0002682 \\
\hline & Anatomical structural morphogenesis - G0:0009653:20 | Regulation of multicellular organismal process - G0:0051239:17 \\
\hline & Regulation of myeloid cell differentiation - G0:0045637:6 | Anotomical structural formation involved in morphogenesis - G0:0048646:10 \\
\hline & Response to molecule of bacterial origin - G0:0002237:4 \\
\hline Cluster 4 & Regulation of nucleobase, nucleoside, nucleotide and nucleic acid metabolic process - G0:0019219:21 \\
\hline Cluster 6 & Sterol biosynthetic process - G0:0016126:5 | Lipid metabolic process - G0:0006629:13 | Lipid biosynthetic process - G0:0008610:8 \\
\hline \multirow[t]{3}{*}{ Cluster 8} & Immune system process - G0:0002376:12 | Inflammatory response - G0:0006954:8 | Response to stress - G0:0006950:13 | locomotion - G00040011:7 \\
\hline & Positive regulation of biological process - G0:0048518:12 | Chemokine acticity - G0:0008009:4 | Cell death - G0:0008219:8 \\
\hline & Multi-organism process - G0:0051704:8 | Postive regulation of nitrogen compound metabolic process - G0:0051173:7 | Regulation of apoptosis - G0:0042981:8 \\
\hline \multirow[t]{2}{*}{ Cluster 10} & Regulation of developmental process - G0:0050793:8 | Protein kinase cascade - G0:0007243:6 | Regulation of phosphorylation - G0:0042325:6 \\
\hline & Cell death - G0:0008219:7 \\
\hline Cluster 12 & Regulation of macromolecule biosynthetic process - G0:0010556:13 | Nucleic acid binding - g0:0003676:13 \\
\hline Cluster17 & Cell death - G0:0008219:5 \\
\hline
\end{tabular}

Differentially expressed genes were significantly categorized into different GO terms and pathways. Different transcription factors that can potentially be involved in the regulation of gene expressions are shown for each cluster under Expander 5 software analysis (P-value $\leq 0.05$ ). Each functional group, canonical pathway or transcription factor is followed by the number of corresponding genes.

\subsubsection{Core analysis in macrophages}

Since only two time points were examined in RSV-infected macrophages, it was not reliable to do cluster analysis based on these data. In 
order to identify the significant regulation of key functional groups, core analysis by IPA was performed to analyze the differentially expressed genes in macrophages [Figure 4.10-4.13].

At the early stage of RSV infection, it was noted that many key functional networks and pathways were highlighted. For example, "Lipid Metabolism", “Cell Death", "Cell Cycle”, "DNA Replication”, "Cell-to-cell Signaling" networks were over-represented with high score; "Cancer", "Inflammatory Response", "Immunological Response", "Cellular Growth and Proliferation”, “Cellular Development”, “Cell Death”, "Hematological System Development and Function”, “Tissue Morphology", "Cell-mediated Immune Response" and "Organismal Survival" were ranked as top functions with hundreds of differentially expressed genes included for each; "Activation of IRF by Cytosolic Pattern Recognition Receptors", "Role of Pattern Recognition Receptors in Recognition Bacteria and Viruses", "IL-10 Signaling”, "TNFR2 Signaling" and "CD40 Signaling" were identified as top five canonical pathways with high-level significance. These findings suggested that cell regulations associated with immune response, antigen presentation, cell cycle and cell death had been initiated from a very early infection stage.

Notably, the top networks and functions identified at 24 hpi were similar as those identified at $4 \mathrm{hpi}$, however, more molecules belonging to these functions were detected at 24 hpi than 4 hpi. This phenomenon might provide evidences that growing number of factors participated in cell regulation activities to combat with RSV's invasion with the infection time increasing. Besides, the essential canonical pathways unique to RSV infection at $24 \mathrm{hpi}$ were "Altered T Cell and B Cell Signaling in Rheumatoid Arthritis", "Dendritic Cell Maturation", "Communication between Innate and Adaptive Immune Cells". These pathways were all related to acquired immune response, indicating the strong adaptive immune response at late infection phase in RSVinfected macrophages. Besides, pathways related to cholesterol biosynthesis and lipid metabolism were more highly represented based on genes with downregulated expression at $24 \mathrm{hpi}$ when compared to $4 \mathrm{hpi}$, indicating that virus infection might induce changes in pathways playing a role in cellular metabolism ny $24 \mathrm{hpi}$. 


\subsubsection{Functional groups related to host response}

During RSV infection, many host genes with critical functions have been reported to be differentially expressed in order to fight with the virus invasion or benefit the virus replication and assembly. For example, antiviral genes such as cytokines that are responsible for local immune response might be induced at their expression level after RSV infection in order to fight against virus infection [166]; altered expression of cell death related genes could function on delaying programmed cell death in order to serve for better virus replication [167]; genes involved in lipid raft composition have been also proved to deviate from their regular expression after RSV interruption [156]. Accordingly, expression of genes referring to interesting functions was investigated in order to evaluate the host cell-virus interactions in both infected Hep2 cells and mouse macrophages [Figure 4.14-4.20]. 
1 Lipid Metabolism, Small Molecule Biochemistry, Drug Metabolism

2 Cell Death, Genetic Disorder, Inflammatory Disease

3 Cell Cycle, DNA Replication, Recombination, and Repair, Cancer

4 Connective Tissue Disorders, Organismal Injury and Abnormalities, Cardiovascular System Development and Function

5 Cell-To-Cell Signaling and Interaction, Cellular Growth and Proliferation, Nervous System Development and Function

Top Bio Functions

Diseases and Disorders

\begin{tabular}{lcc} 
Name & p-value & \# Molecules \\
\hline Cancer & $1.65 \mathrm{E}-30-2.31 \mathrm{E}-08$ & 431 \\
Inflammatory Disease & $9.38 \mathrm{E}-24-2.34 \mathrm{E}-08$ & 353 \\
Immunological Disease & $4.56 \mathrm{E}-23-7.69 \mathrm{E}-09$ & 320 \\
Inflammatory Response & $4.58 \mathrm{E}-23-2.07 \mathrm{E}-08$ & 232 \\
Dermatological Diseases and Conditions & $2.98 \mathrm{E}-17-1.76 \mathrm{E}-08$ & 150
\end{tabular}

Molecular and Cellular Functions

\begin{tabular}{lcc} 
Name & p-value & \# Molecules \\
\hline Cellular Growth and Proliferation & $1.82 \mathrm{E}-33-1.89 \mathrm{E}-08$ & 382 \\
Cellular Development & $7.63 \mathrm{E}-32-2.38 \mathrm{E}-08$ & 339 \\
Cell Death & $1.87 \mathrm{E}-31-2.33 \mathrm{E}-08$ & 384 \\
Cellular Function and Maintenance & $1.66 \mathrm{E}-25-2.08 \mathrm{E}-08$ & 149 \\
Cell Cycle & $3.22 \mathrm{E}-23-2.34 \mathrm{E}-08$ & 201
\end{tabular}

Physiological System Development and Function

\begin{tabular}{lcc} 
Name & p-value & \# Molecules \\
\hline Hematological System Development and Function & $2.07 \mathrm{E}-30-2.33 \mathrm{E}-08$ & 262 \\
Tissue Morphology & $2.07 \mathrm{E}-30-1.35 \mathrm{E}-08$ & 184 \\
Hematopoiesis & $1.80 \mathrm{E}-26-2.33 \mathrm{E}-08$ & 172 \\
Cell-mediated Immune Response & $3.28 \mathrm{E}-23-4.92 \mathrm{E}-09$ & 111 \\
Organismal Survival & $1.02 \mathrm{E}-22-3.12 \mathrm{E}-20$ & 188 \\
& & \\
& & \\
Top Canonical Pathways & & Ratio \\
\hline & & $26 / 73(0.356)$ \\
Name & p-value & $25 / 87(0.287)$ \\
\hline Activation of IRF by Cytosolic Pattern Recognition Receptors & $4.97 \mathrm{E}-15$ & $23 / 78(0.295)$ \\
Role of Pattern Recognition Receptors in Recognition of Bacteria & $8.74 \mathrm{E}-12$ & $15 / 33(0.455)$ \\
and Viruses & & $22 / 70(0.314)$ \\
IL-10 Signaling & $1.7 \mathrm{E}-11$ & $2.22 \mathrm{E}-11$ \\
TNFR2 Signaling & $2.27 \mathrm{E}-11$ &
\end{tabular}

Figure 4.10 Summary of top functional groups enriched in differentially expressed genes in RSV-infected with macrophages at $\mathbf{4} \mathbf{~ h p i}$. Differentially expressed genes detected at $\mathbf{4}$ hpi were significantly categorized into different networks, functions and pathways under the analysis of Ingenuity Pathway Analysis (IPA) version 2012 (P-value $\leq 0.05)$. 
A

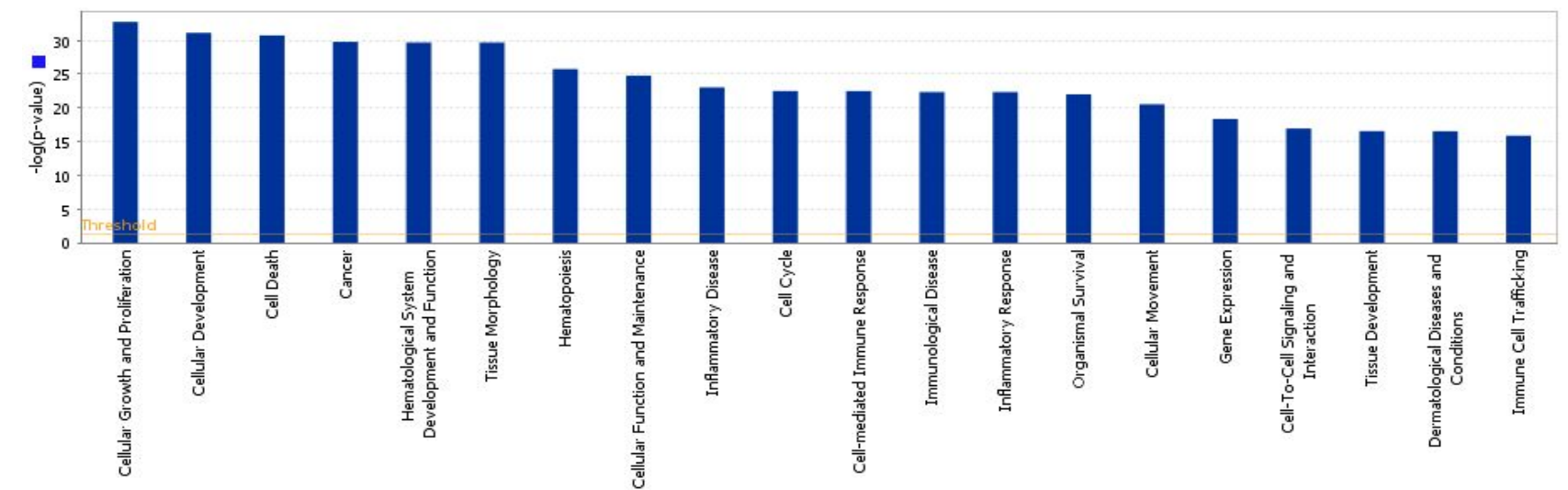

B

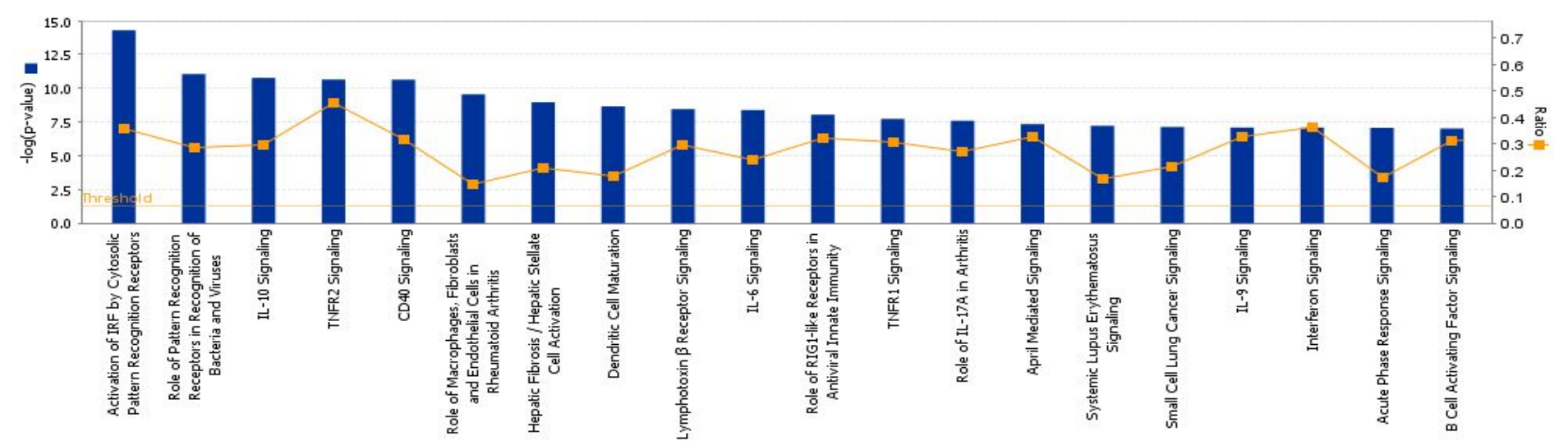

Figure 4.11 Top 20 (A) biological functions and (B) canonical pathways significantly enriched in differentially expressed genes from RSV-infected macrophages at 4 hpi (P-value $\leq \mathbf{0 . 0 5}$ ). Threshold was set at $\mathrm{p}$-value $=0.05$ and indicated as $-\log$ (p-value) on the $\mathrm{Y}$-axis and the $\mathrm{X}$-axis shows the terms of each biological function or canonical pathway. The orange boxes indicate the ratio (Ratio) of the number of genes with differential expression changes and the total number of genes in the respective canonical pathway. 


\begin{tabular}{lc} 
ID Associated Network Functions & Score \\
\hline 1 Cell Signaling, Molecular Transport, Nucleic Acid Metabolism & 40 \\
2 Gene Expression, Neurological Disease, Organismal Injury and Abnormalities & 38 \\
3 Amino Acid Metabolism, Molecular Transport, Small Molecule Biochemistry & 36 \\
4 Genetic Disorder, Inflammatory Disease, Inflammatory Response & 35 \\
5 Lipid Metabolism, Molecular Transport, Small Molecule Biochemistry & 32 \\
Top Bio Functions &
\end{tabular}

\begin{tabular}{|c|c|c|}
\hline Name & $\mathrm{p}$-value & \# Molecules \\
\hline Inflammatory Response & $5.50 \mathrm{E}-35-7.60 \mathrm{E}-09$ & 299 \\
\hline Immunological Disease & $1.98 \mathrm{E}-31-5.27 \mathrm{E}-09$ & 438 \\
\hline Cancer & $5.76 \mathrm{E}-30-7.31 \mathrm{E}-09$ & 511 \\
\hline Skeletal and Muscular Disorders & $4.38 \mathrm{E}-28-6.70 \mathrm{E}-09$ & 459 \\
\hline Inflammatory Disease & $1.94 \mathrm{E}-27-6.70 \mathrm{E}-09$ & 476 \\
\hline \multicolumn{3}{|l|}{ Molecular and Cellular Functions } \\
\hline Name & p-value & \# Molecules \\
\hline Cellular Development & $2.71 \mathrm{E}-31-7.95 \mathrm{E}-09$ & 406 \\
\hline Cellular Movement & $8.54 \mathrm{E}-27-8.10 \mathrm{E}-09$ & 272 \\
\hline Cellular Function and Maintenance & $2.29 \mathrm{E}-26-6.71 \mathrm{E}-09$ & 149 \\
\hline Cell Death & $5.34 \mathrm{E}-25-6.65 \mathrm{E}-09$ & 434 \\
\hline Cellular Growth and Proliferation & $3.90 \mathrm{E}-24-7.82 \mathrm{E}-09$ & 447 \\
\hline \multicolumn{3}{|l|}{ Physiological System Development and Function } \\
\hline Name & p-value & \# Molecules \\
\hline Hematological System Development and Function & $5.51 \mathrm{E}-29-7.60 \mathrm{E}-09$ & 332 \\
\hline Organismal Survival & $5.92 \mathrm{E}-29-2.22 \mathrm{E}-12$ & 229 \\
\hline Hematopoiesis & $1.68 \mathrm{E}-28-8.10 \mathrm{E}-09$ & 209 \\
\hline Cell-mediated Immune Response & $2.29 \mathrm{E}-26-6.67 \mathrm{E}-09$ & 158 \\
\hline Tissue Morphology & $1.93 \mathrm{E}-25-3.53 \mathrm{E}-09$ & 207 \\
\hline \multicolumn{3}{|l|}{ Top Canonical Pathways } \\
\hline Name & p-value & Ratio \\
\hline Altered T Cell and B Cell Signaling in Rheumatoid Arthritis & $8.26 \mathrm{E}-14$ & $32 / 92(0.348)$ \\
\hline Dendritic Cell Maturation & 1.15E-13 & $45 / 188(0.239)$ \\
\hline Communication between Innate and Adaptive Immune Cells & $5.1 \mathrm{E}-13$ & $32 / 109(0.294)$ \\
\hline Type I Diabetes Mellitus Signaling & $9.01 \mathrm{E}-13$ & $35 / 121(0.289)$ \\
\hline Role of Pattern Recognition Receptors in Recognition of & $1.24 \mathrm{E}-12$ & $29 / 87(0.333)$ \\
\hline
\end{tabular}

Role of Pattern Recognition Receptors in Recognition of

Figure 4.12 Summary of top functional groups enriched in differentially expressed genes in RSV-infected with macrophages at $\mathbf{2 4} \mathbf{~ h p i}$. Differentially expressed genes detected at different time points were significantly categorized into different networks, functions and pathways under the analysis of Ingenuity Pathway Analysis (IPA) version 2012 (P-value $\leq 0.05$ ).

\subsubsection{RSV infected Hep2 cells}

\subsection{Immune response}

After RSV infection, the host cells will initiate a series of defense response to counteract the virus invasion. Previous researches have revealed that direct RSV infection induced chemokine secretion [168]. In our study, chemokines such as CCL2, CCL5 (RANTES), CCXL2/3/10/11, IL8 (CXCL8) also showed up-regulation at their expression level. Among these chemokines, the upregulated fold regulation of CCL5 and CXCL10/11 showed ascending, while the up-regulated fold regulation of CCL2 and CXCL2/3 showed descending with the infection time increasing. In addition, the 
A

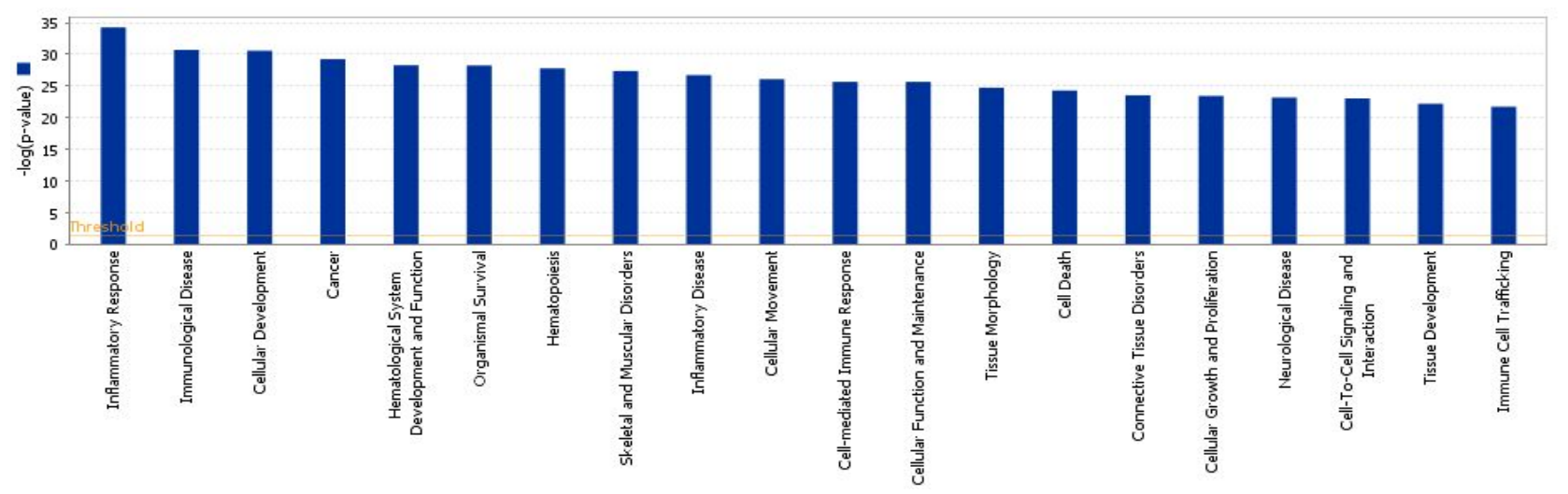

B

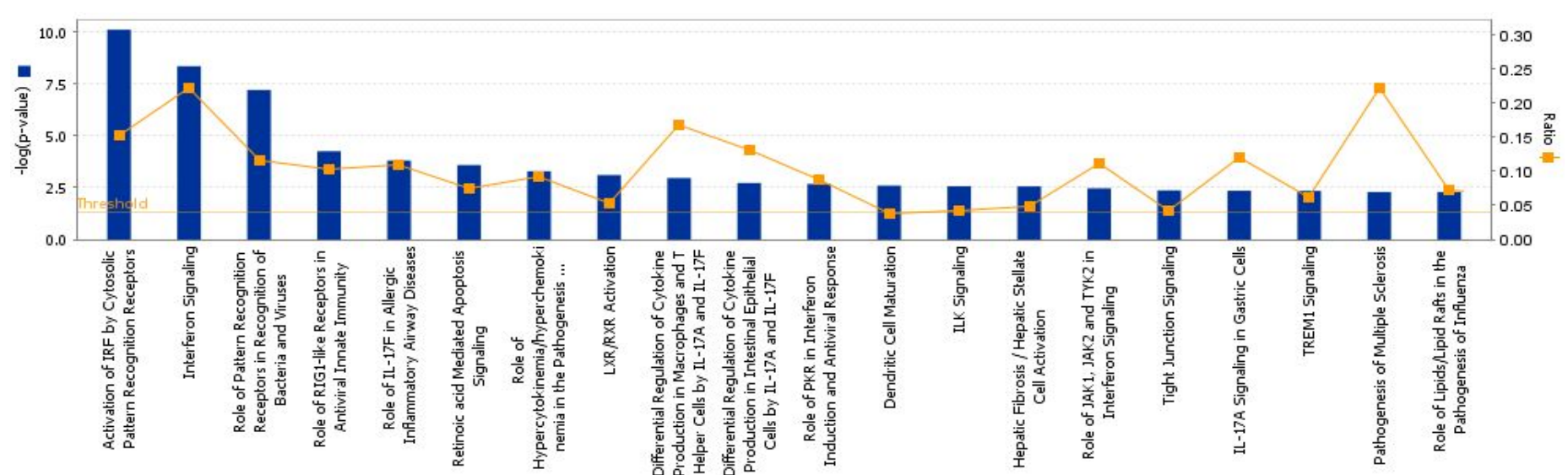

Figure 4.13 Top 20 (A) biological functions and (B) canonical pathways significantly enriched in differentially expressed genes from RSV-infected macrophages at 24 hpi (P-value $\leq \mathbf{0 . 0 5})$. Threshold was set at $\mathrm{p}$-value $=0.05$ and indicated as $-\log (\mathrm{p}$-value) on the $\mathrm{Y}$-axis and the $\mathrm{X}$-axis shows the terms of each biological function or canonical pathway. The orange boxes indicate the ratio (Ratio) of the number of genes with differential expression changes and the total number of genes in the respective canonical pathway. 
expression of IL8 (CXCL8) showed sustained and extraordinary induction across the whole infection period. These observations might demonstrate that these chemokines are under the control of different types of molecular mechanisms when they exert their antiviral function as proinflammatory mediators. Another cytokine, IL6, was also up-regulated with a quite high fold change at its expression level, which implied the strong activation of immune response.

Although no significant expression changes of IFN- $\alpha$ and IFN- $\beta$ were detected, the receptors including IFNAR2 and IFNGR1 showed up-regulated expression with low fold regulation at early time points (4 hpi and $8 \mathrm{hpi}$ ). It might indicate the trigger of IFN type I and II signaling pathways at the beginning of RSV infection, however the activation was not able to last for a long time. IL28A, belonging to IFN type III family, plays a role in both innate and adaptive immune response [169]. Its elevated gene expression at the late stage of RSV infection might be related to the adaptive immune response in infected Hep2 cells.

Besides these cytokines and IFNs, a batch of interferon stimulated genes including NFKB1/1 (NF-אB), NIKBIB/Z, IGS20, IFI44, OAS3, OASL, GBP1/2 were also identified with up-regulated gene expression in RSV infected Hep2 cells. Other antiviral genes including DDX58 (RIG-I), AREG, CD83, EDN1, EMR3, F3, KLF6, LPIN1, LYN, PBEF1, PDCD1LG1, PLAUR, SOCS2, TNIP1, IRAK2 also showed up-regulation in their expression.

\subsection{Cell death}

A group of apoptosis-related genes displayed differential expression during the RSV infection in Hep2 cells, and expression regulations of these genes might be due to the host-virus interactions.

Members in the FOS family dimerise with JUN to form the AP-1, which promotes transcription of a diverse range of genes involved in cellular processes such as proliferation and apoptosis [170]. Thus, increasing up-regulation of FOS and JUN genes at their expression level across the whole infection period might function on apoptosis signaling in infected Hep2 cells.

XIAP associated factor-1 (XAF1) antagonizes the activity of XIAP that inhibit activation of caspases during apoptosis. This factor was detected with 
up-regulated expression from 4 hpi to 12 hpi, which enhanced the hypothesis that the programmed apoptosis was induced from early infection stage.

BIRC3 belongs to the inhibitor of apoptosis (IAP) gene family which encodes proteins that negatively regulate cell death. Another anti-apoptotic molecule, TNFAIP3, has been reported to inhibit NF- $\mathrm{kB}$ activation and TNFmediated apoptosis, in further to limit inflammation. Both these two factors were strongly up-regulated at their expression level, implicating the potential regulation of attenuating apoptosis in Hep2 cells.

Among the few genes down-regulated with faint fold changes, Tp53 is a key regulator that is able to initiate programmed cell death. Down-regulation of its expression might be associated with the delayed programmed cell death, a feature which resulted in more robust viral titers [131].

Collectively, apoptosis of the Hep2 cells was controlled in two opposite directions following RSV infection. On one hand, some evidences proved that the cell death was induced during the virus infection, particularly at early infection stage. On the other hand, some phenomenon implied that virus might take some actions to delay the host apoptosis so as to benefit its replication across the whole infection stage.

\subsection{Cholesterol biosynthesis}

Lipid raft are micro-domains of the plasma membrane. As a main component of lipid rafts, cholesterol is essential for the formation of membrane raft [171]. Lipid rafts serve as platforms for plasma membrane assembly and budding of enveloped viruses. Several research groups have proposed that RSV proteins located in raft domains and purified RSV virion particles contained raft associated cellular proteins. Recent research even recovered that intact plasma membrane rafts were required for release of infectious progeny RSV particles during RSV infection in human lung epithelial cells [172].

To verify the importance and necessity of the lipid rafts during RSV infection in Hep2 cells, we selected an array of differentially expressed genes in this respect for proof of principle. The genes under investigation included HMGCR, HMGCS1, DHCR7, CYP51A1, FDFT1, IDI1, MVK, SQLE, SC4MOL, APOL6, FN1, ITGA2 and etc. Some of them such as DHCR7, CYP51A1, FDFT1, IDI1 and SC4MOL are involved in synthesis of cholesterol. 
Others may exert different functions: APOL6 affects the movement of lipids or allow the binding of lipids to organelles; HMGCR encodes a rate-limiting enzyme for cholesterol synthesis and its expression level is coordinated by the level of free-cholesterol in a negative loop. Notably, these genes were significantly up-regulated especially during the late infection stage, indicating the increased requirement for free-cholesterol as well as the active movement of lipids during virus infection.

Another three genes that are also associated with cell membrane are FN1 and ITGA2/5. FN1 encodes fibronectin, which is a glycoprotein present in a soluble dimeric form in plasma but a dimeric or multimeric form at the cell surface. This glycoprotein is involved in cell adhesion and migration processes. ITGA2 and ITGA5 are integrins that play role as fibronectin receptors. In our RSV-infected Hep2 cells, altered expressions of fibronectin and its receptors might be also associated with the RSV infection. However, detailed interactions and functions retain to be determined in the future.

\subsection{Genes with remarkable regulations}

In addition to these well-known genes, there existed other genes differentially expressed with topmost fold change in RSV-infected Hep2 cells. Among them, the up-regulated genes included LCN2, PTX3, HKR1, ERG1/4, DUSP6 and so on, while the down-regulated genes were ZNF230, ZIC2, HEY2, AHRR and so on.

Among the up-regulated genes, LCN2 is of importance in the innate immune response to bacterial infection. Upon encountering invading bacteria, TLRs stimulate the secretion of this protein that is capable of limiting bacterial growth by sequestering iron-containing siderophores. Unusual expression elevation of this gene potentially indicated that this gene might have a possibility to be involved in the immune response to virus infection.

Another molecule that is also associated with immune response was PTX3. This gene is rapidly produced and released by cells such as mononuclear phagocytes, dendritic cells, fibroblasts and endothelial cells in response to primary inflammatory signals. And it is also known to bind to dying cells during inflammatory reactions so as to clear the apoptotic cells. Thus, strong 
up-regulation of this gene in its expression might indirectly represent the initiation of inflammatory response in Hep2 cells after RSV infection.

HKR1 encoding zinc finger protein and EGR1/4 as a member of the early growth response (EGF) family of zinc finger transcription factors both showed quite significant regulation in their expression. Relevant studies have pointed out that the compounds called AT-2 which inactivate retroviruses through targeting their zinc finger-containing $\mathrm{NC}$ protein could also inactivate RSV accompanied by a significant modification of M2-1 [173]. This finding might indicate that zinc fingers as a group of small protein structure motifs play functions during our RSV infection.

Dusp6 belongs to the dual specificity protein phosphatase subfamily. This family takes responsibility to negatively regulate members in the mitogenactivated protein (MAP) kinase superfamily, which function on cellular proliferation and differentiation. Since p38 MAPK activation was proved to be required for RSV induction of human bronchial epithelial membrane permeability [174], the up-regulated expression of Dusp6 was possible an antiviral action in Hep2 cells.

A few genes encoding zinc finger proteins such as ZNF230 and ZIC2 were detected with decreased expression. These two molecules function as transcription regulators so that inhibition of their expression could implicate the suppression of some specific transcriptional regulation activities.

\subsubsection{RSV infected macrophages}

\subsection{Immune response}

Upon viral infection, macrophages receive chemical signals IFN- $\gamma$ or IL4/13, and then increase their production of MHC II molecules which prepare them for presenting antigens. Activated macrophages produce and secreted TNFs, which help cause inflammation through the production of IL-1, IL-6, $\mathrm{TNF} \alpha$ and activate the other cells of the immune system. Original signal chemicals such as IFN- $\gamma$ (Ifng), IFN- $\gamma$ receptor (Ifngr1) and IL4/13 didn't show any significant expression changes during both time points under RSV infection. However, TNFs, which are supposed to be secreted by activated macrophages, showed up-regulated expression with quite high fold change after RSV infection at both 4 and 24 hpi (especially 4 hpi) [Figure 4.18]. 
Downstream cytokine productions including IL1a, IL1b, IL6 displayed similar expression patterns as TNFs. Other chemokines with differential gene expression included CCL2/3/4/5/7, CCRL2, CX3CR1 and CXCL1/2/3/5/9/10. Careful observations indicated that these chemokines also showed up-regulated expression after RSV infection. Expression levels of these key molecules involved in macrophage activation suggested that macrophages were activated well after RSV infection. Besides, most of these factors showed higher expression levels at 4 hpi than 24 hpi in RSV infected macrophages, indicating that macrophage activation was initiated strongly at a very early stage.

Antigen presentation is a process in which some phagocytes "present" parts of engulfed materials to other cells of the immune system on their cell surface. In activated macrophages, IFN- $\gamma$ usually increases production of MHC II molecules and prepares them for presenting antigens, MHC I molecules thereafter mediate destruction of host cells displaying that antigen [175]. In RSV-infected macrophages, a group of MHC class I molecules such as H2-Q7, H2-Q8, H2-T22, showed up-regulated expressions. At the meantime, another batch of MHC class II molecules such as H2-Aa/b1, H2-DMa/b1, H2-Ea/b1 was detected with down-regulated expression. It was possible that RSV had evolved methods to evade macrophages antigen presentation through repressing the expression levels of these MHC II molecules.

In the macrophages, the primary signal for activation is IFN- $\gamma$ from Th1 type CD4 T cells. The secondary signal is CD40L on the T cell which binds CD40 on the macrophage cell surface. As a result, the macrophage expresses more CD40 and TNF receptors on its surface which help stimulate the activation [176]. A large increase in expression of CD40 was detected from 8.6FC (4 hpi) to 80.9-FC (24 hpi) in RSV-infected macrophages, providing more evidences for the macrophages activation. However, the increasing expression trend of CD40 is different from the previous decreasing expression trend of TNFs and cytokines, and the detailed reason remains to be investigated in the future.

Phagocytosis is the process of engulfing particles such as cell debris, bacteria, and dead tissue cells. In the macrophage phagocytosis process, receptors on the surface of macrophages such as toll-like receptors bind to the "invader" to initiate the phagocytosis [177]. Consequently, high-level up- 
regulation of TLR2 and TLR3 at their expression level were detected after RSV infection, which might implicate strong activation of macrophage phagocytosis in RSV-infected macrophages. DDX60 was proved to be a novel antiviral factor promoting RIG-I (DDX58) -like receptor-mediated signaling and was dispensable for TLR3-mediated signaling by Miyashita M et al (2011) [178]. Its up-regulated expression with high fold regulation in RSV-infected macrophages was consistent with the gene expression trends of DDX58 and TLR3, indicating strong RIG-I-mediated antiviral signaling after RSV infection in macrophages.

Previous studies have proved that RSV had an ability to impair IFN Type I signaling pathway by distinct mechanisms in macrophages [159]. However, IFN Type I and associated stimulated genes exhibited in an elevated expression with quite high fold regulation. Among them, IFN- $\alpha$ and IFN- $\beta$ showed higher fold change at 4 hpi than 24 hpi while other IFN regulatory and stimulated genes including GBP1/2/6, IRF7, ISG20, MX1, OASL1/2, RSAD2, IFI44 showed higher fold change at 24 hpi than 4 hpi. These observations suggested that IFN pathway was strongly activated in RSV-infected macrophages.

\subsection{Cell death}

Various regulations of cell apoptotic activities were also observed in RSV-infected macrophages, with a batch of apoptotic factors such as CFLAR, CASP4, TRAF1/2, MYC, PDCD4, XAF1 differentially expressed [Figure 4.19]. XAF1 is capable of regulating apoptosis by abrogating the anti-apoptotic activities of XIAP. Expression of it showed up-regulated with 5.34-FC at 4 hpi and 33.08-FC at $24 \mathrm{hpi}$, indicating robust apoptosis activities especially at late infection stage following RSV infection.

A heterodimeric complex formed by TRAF1 and TRAF2 is necessary for TNF $\alpha$-mediated activation of MAPK8/JNK and NF-kB. Moreover, this complex also mediates the anti-apoptotic signals from TNF receptors through interacting with IAPs [179]. Up-regulation of these two factors at their expression level during RSV infection might indicate the inhibitory regulation on apoptotic signaling from an opposite direction. 


\subsection{Genes with remarkable regulations}

Besides these well-investigated genes, quite a few other genes were also observed with a stimulated expression in RSV-infected macrophages [Figure 4.20].

Several immune related genes including ZBP1, LCN2 and Serpinb2 were strongly stimulated at their expression levels after RSV infection. Z-DNAbinding protein 1 (ZBP1) has been proved to function as a DNA sensor that activated the innate immune system [180]. Other studies also have identified ZBP1 as being essential for IRF3 and IFN- $\beta$ activation in a JAK/STATdependent manner, therefore inhibiting huuman cytomegalovirus replication [181]. LCN2 is always secreted by Toll-like receptors upon encountering invading bacteria in order to limit bacterial growth by sequestrating the ironladen siderophore [182]. Serpinb2 was proved to be induced during many inflammatory processes and infections and play a physiological role in suppression of Th1-promoting cytokine up-regulated proteins [183]. Strong upregulation of these genes in their expression during RSV infection might suggest a strong immune response.

Genes of the Schlafen (Slfn) family have been uncovered to participate in T cell development [184], cell cycle arrest [185] and generation of IFN $\alpha$ induced growth inhibitory responses [186]. Sohn W et al (2007) reported that CpG-DNA and LPS triggered slfn-2 gene expression by activating NF- $\kappa \mathrm{B}$ and AP-1 pathways in macrophages [187]. In our study, slfn-1/2/3/4/5/8 all showed strong up-regulation at their expression level in macrophages after RSV infection. This could suggest that Slfn genes potentially participated in some critical functions. However, the detailed roles of Slfn family in macrophages are still under investigation.

A few transporter and transmembrane genes were also ranked topmost among the up-regulated genes. For RSV infection, expression of TAP1 and TAP2 showed significant up-regulation only at 24 hpi. RTP4, TMEM171, MS4A4C and MS4A6C showed increasing up-regulation while FLRT3 and TREM1 showed a deceasing up-regulation at their expression levels across the RSV infection period. Furthermore, TREM1 signaling pathway has been reported to amplify the signal from TLRs, NAIP, CIITA and HET-E to produce various chemokines and cytokines [188]. Thus, significant up-regulation of 
TREM1 at its expression level following RSV infection at 4 hpi may potentially contribute to the timely development of the cytokine storm in corresponding infected macrophages.

GPR84 is one of the $\mathrm{G}$ protein-coupled receptors which is largely restricted to macrophage populations and granulocytes. Lattin JE et al (2008) has pointed out that expression of GPR 84 could be highly regulated by LPS via TLR4 in mouse macrophages [189]. However, TLR4 didn't show any differential expression change in infected macrophages. Hence, some other unclear stimuli might be responsible for the remarked up-regulation of GPR84 at its expression level during RSV infection at both 4 and 24 hpi.

\subsubsection{Regulations of gene expression in canonical pathways}

\subsubsection{Interferon signaling}

In RSV-infected Hep2 cells, it was notable that STAT1 and IFN $\alpha / \beta$ only showed faintly up-regulated expression changes at 12 hpi and 15 hpi, separately. Among the downstream genes, IRF showed up-regulated expression with 7.0FC at 2 hpi, and OAS1 and TAP1 showed up-regulated expression at the late stage of infection. These observations may suggest that the IFN signaling pathway and its downstream productions were not strongly activated or induced upon RSV infection in Hep2 cells. SOCS1, as a member of SOCS family, has been proved to block type I and II IFN signaling through JAK-STAT pathway [190]. Subsequently, strong up-regulation of SOCS1 in its gene expression from 8 hpi to 15 hpi could be partly responsible for the status of IFN signaling pathway.

In the case of RSV-infected mouse macrophages, IFN signaling pathway, especially type I, behaved in an activated state at both infection time points, which was different from the situation happened in Hep2 cells. At 24 hpi, expression of ISGs such as IFIT1, IFIT3 and MX1 showed up-regulation with 838.7-FC, 316.8-FC and 146.1-FC respectively. Altogether, these data provided evidences that IFN type I signaling pathway was strongly activated and productions of downstream ISGs were strongly promoted in mouse macrophages after infection of RSV. 


\subsubsection{NF-кB signaling}

$\mathrm{NF}-\kappa \mathrm{B}$ is a major transcription factor which regulates expression of the genes responsible for both the innate and adaptive immune response. Upon virus infection, its activation will result in expression of an array of cytokine and chemokine genes. Besides its function on inflammation response, NF- $\mathrm{BB}$ signaling pathway also plays a vital role in cell proliferation and apoptosis. The canonical mechanism of NF- $\kappa \mathrm{B}$ activation includes $\mathrm{IKK} 2 / \mathrm{IKKb}$ mediated inhibitor of NF- $\kappa \mathrm{B}(\mathrm{I} \kappa \mathrm{B})$ phosphorylation and degradation. This action consequently leads to the release and translocation of NF- $\kappa \mathrm{B}$ factors $\mathrm{p} 65$ and p50 dimers which migrate to the nucleus to exert biological functions.

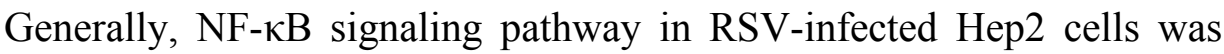
not obviously activated. No expression change of IKK complex and NF- $\mathrm{BB}$ factors p65 was observed across the whole infection period. And some of the downstream cytokines and chemokines only showed faint up-regulation at their expression levels. These observations suggested that NF- $\kappa \mathrm{B}$ signaling pathway was not significantly triggered in Hep2 cells upon RSV infection.

$\mathrm{A} 20$, as a NF- $\mathrm{KB}$ inhibitor, is involved in the feedback suppression of NF- $\kappa \mathrm{B}$ activation induced by TNF $\alpha$ [191]. Quite significant up-regulation of A20 in its expression during the whole RSV infection stage may be responsible to terminate the activation of NF- $\mathrm{kB}$. No expression change was detected for TNF $\alpha$ may imply that there exists alternative mechanism on the A20 induction.

Compared to the gene expression performances of NF- $\mathrm{KB}$ signaling pathway in RSV-infected Hep2 cells, more genes showed up-regulated expression in RSV-infected mouse macrophages. Gene expression of A20 and TNF $\alpha$ both exhibited more significant up-regulation at 4 hpi than 24hpi, which suggested that the activation and inhibition of this pathway were initiated from an early infection stage. In contrary to A20 and TNFa, CD40 showed upregulated expression with higher fold change at 24 hpi. Since CD40 has been proved to mediate NF- $\mathrm{BB}$ activation and cytokine secretion in human colonic fibroblasts [192], expression induction of this gene may enhance activation NF$\kappa \mathrm{B}$ signaling pathway in another way during later infection stage in RSVinfected macrophages. 


\subsubsection{Toll-like receptor signaling}

TLRs are a type of PRRs which recognize pathogen-associated molecules. Activation of TLR signaling causes the transcriptional activation of pro-inflammatory cytokines and chemokines, which subsequently play an essential role in immune response. TLR7/TLR8 can mediate the activation of IRF7 through unique interaction between TLR domain-containing cytosolic adapters such as myeloid differentiation primary-response protein-88 (MyD88) [193]. Independent of MyD88, TLR3 is also able to exert its function by interacting with TRIF, which in turn to activats a complex of IKKe, TRAF3, and TBK1 that phosphorylates IRF3 and IRF7. Activation of IRF3 leads to the expression induction of CD40, CD80, and CD86, while activation of IRF7 promotes the induction of IFN $\alpha$ and IFN $\beta$ gene expression [194].

Many studies have revealed the detailed function of TLRs upon RSV infection. For example, Marchant D et al (2010) reported that TLR4-mediated activation of p38 MAPK was a determinant of RSV entry and tropism [195]; TLR2 and TLR6 activations were critical for controlling RSV viral replication [196]; TLR4 signaling pathway played a predominant role in mediating LPSinduced-IL-6 production of RSV infected epithelial cells [197]; TLR4 expression was increased in infants with RSV bronchiolitis, and F protein of RSV binded to TLR4 and CD14 in order to initiate innate immunity response [198].

Investigation on RSV-infected Hep2 cells suggested that faint upregulation of TLR4 at its expression level was detected at $8 \mathrm{hpi}$, and the expression of downstream p38 MAP2K3 was induced at 8 hpi but repressed at the late infection stage. Remarkable up-regulation of IL6 at its expression level might provide evidences for the expression induction of TLR4 as well as its signaling mediation. In addition, it has been reported that TLR3, TLR7 and TLR8 can also mediate the activation of IRF3 and IRF7 to trigger IFN induction. The expression of TLR3, TLR7 and TLR8 showed no expression change in Hep2 cells, and this might be consistent with the previous observation that interferon signaling pathway showed almost inactivated in RSV-infected Hep2 cells.

In macrophages infected with RSV, extensive activation of toll-like receptors was revealed. Gene expression levels of TLR2, CD14, IRAK as well 
as $\mathrm{NF}-\kappa \mathrm{B}$ were induced at early infection time point while gene expression levels of TLR2, TLR5, TLR3, MYD88, PKR, and NF- $\kappa B$ were stimulated at late infection time point. High-level stimulation of TLR3 at its expression level resulted in strong activation of IFN pathway as well as accumuation of its downstream productions as described before.

\subsubsection{Apoptosis signaling}

Apoptosis is the process of programmed cell death happened in multicellular organisms. All apoptosis signaling pathways converge on a common machinery of cell destruction that is activated by caspases through cleaving proteins at aspartate residues [199]. Several reports have been published to prove that RSV infection induced or inhibited apoptosis in different cell lines with different impact factors [142, 161, 200, 201].

When we investigated the performance of apoptosis signaling pathway in RSV-infected Hep2 cells, we found that there were few genes showing significant expression changes. Baculoviral IAP repeat-containing protein 3 (BIRC3) which functions in providing instructions for making IAPs show highlevel up-regulation at its expression level. IAPs that belong to inhibitor of apoptosis family have been shown to be involved in suppressing the host cell death response to viral infection. Thus, Up-regulated expression of BIRC3 may suggest the anti-apoptosis actions were taken by RSV in order to benefit the viral replication.

In mouse macrophages, RSV infection led to faint up-regulation of caspase 8 , caspase 12 , caspase 3 and caspase 7 at their expression level in apoptosis signaling pathway at $24 \mathrm{hpi}$. TNF, mainly secreted by macrophages, is able to induce apoptotic cell death. This factor showed up-regulated expression with 41.9-FC and 13.4-FC separately at 4 and 24 hpi, which might be responsible for the activation of caspases at $24 \mathrm{hpi}$ mentioned above.

\subsubsection{Cell cycle: G1/S checkpoint regulation}

Cell cycle arrest is controled by interactions of a group of important proteins. An increase in phosphorylation of His-3 protein denotes the cell cycle arrest in $\mathrm{G} 2 / \mathrm{M}$ phase while an increase in dephosphorylation of $\mathrm{Rb}$ protein represents the cell cycle arrest in G0/G1 phase. Gibbs JD et al (2009) has 
claimed that RSV infection induced G2/M cell cycle arrest in human bronchial epithelial cells and G0/G1 cell cycle arrest in A549 cells through TNF- $\beta$ in order to enhance its replication [202].

In our RSV-infected Hep2 cells, only a small batch of genes in G1/S checkpoint regulation pathway was detected with differential expression over the whole infection process. At $8 \mathrm{hpi}$, down-regulated expression of E2F and up-regulated expression of CDK 4/6 and cyclin D were both detected, which may be generated from the fighting of two opposite-direction regulations. At two later time points, E2F was consistently down-regulated but no more expression changes were detected for CDK 4/6 and cyclin D. In addition, p21 as a potent cyclin-dependent kinase inhibitor was detected with an increasing expression elevation over the whole infection period, which may suggest the arrest regulation of cell cycle in a gradual way.

In macrophages, a few up-regulated genes were detected at 4 hpi during RSV infection. These genes not only included the ones that activate the cell cycle G1/S checkpoint such as CDK 4/6, c-Myc and cyclin E but also the ones that induce the cell cycle arrest including TGF- $\beta$, smad3 and $\mathrm{p} 21$. Less significant gene expression cases were detected at 24 hpi after RSV infection in mouse macrophages.

\subsubsection{Cell cycle: G2/M DNA damage checkpoint regulation}

Similar to G1/S checkpoint regulation pathway, few gene expression changes were detected in G2/M DNA damage checkpoint regulation pathway in RSV-infected Hep2 cells. And most of significant gene expression regulations detected at 12 hpi showed quite faint fold changes.

A batch of genes was significantly expressed in RSV-infected macrophages at 4 hpi. However, some of these genes inhibit of cell cycle arrest while others of them facilitate cell cycle arrest. Thus, no apparent conclusion could be reached based on these observations.

\subsubsection{Antigen presentation pathway}

Two types of MHC groups are involved in antigen presentation processes. MAH class I participate in the intracellular antigens' presentation which are produced mainly by viruses replicating within a host cell, while MHC 
class II attend extracellular antigens' presentation coming from exogenous pathogens. Macrophages express low level of MHC class II, but the expression can be up-regulated by microbial products and macrophages can therefore present antigens from the microbe to $\mathrm{CD}^{+} \mathrm{T}$ lymphocytes. Besides, antigens from can elicit an MHC-I-dependent response that results in the proliferation of $\mathrm{CD}^{+}$cytotoxic T lymphocytes [203].

In our experiment, RSV infection resulted in up-regulated expression of MHC I- $\alpha$ and down-regulated expression of MHC II- $\alpha / \beta$ in macrophages at 24 hpi. Expression induction of MHC class I may be due to the fighting with the exogenous RSV, while expression repression of MHC class II may imply that the inner RSV replication was inhibited in macrophages or RSV evolved some mechanism to inhibit the activation of MHC class II. And down-regulated expression of genes involved in MHC class II pathway in RSV infected mice was also observed in another study [375].

\subsection{Conclusion}

Till now, there has been no report published to describe the host response towards RSV infection in Hep2 cells and murine lung macrophages. In this chapter, we made efforts to investigate the global gene expression using microarray system in order to examine the host response in RSV-infected Hep2 cells and murine lung macrophages. Moreover, parallel comparison of genomic expression profiles between Hep2 cells and mouse lung macrophages upon RSV infection was beneficial for us to understand the pathogenesis deeply.

Global evaluation indicated that the numbers of differentially expressed probe sets/genes were increasing with the infection time increasing in RSVinfected Hep2 cells and macrophages. However, among these differentially expressed probe sets/genes, most of the probe sets showed down-regulated expression in Hep2 cells while most of genes showed up-regulated expression in macrophages.

As the results generated from functional classification, it was noted that "DNA Binding", "Immune Response" and "Signal Transduction" were overrepresented and shared by those probe sets/genes with up-regulated expression from both infected host cells. However, the genes with down-regulated expression from infected macrophages were associated with "Cell Cycle", "Cell 
Death", "Kinase" and "Signal Transduction", and these functional groups were not consistent with the over-represented ones based on probe sets with downregulated expression from Hep2 cells.

Besides these functional groups, pathways such as "MAPK signaling", "Jak-STAT signaling pathway" and "B-cell receptor signaling pathway" and potential transcription factors such as SRF, ISRE, Sp1 were enriched based on genes with up-regulated expression in RSV-infected Hep2 cells. At the meanwhile, pathways such as "Cytokine-cytokine receptor interaction" and "Apoptosis" and transcription factors such as ETF, E2F, TATA, NFkappaB_(p65) were enriched based on genes with down-regulated expression in RSV-infected Hep2 cells. In RSV-infected macrophages, a batch of functional networks such as "Inflammatory Response" and "Immunological Disease" and pathways such as "Role of pattern recognition receptors in recognition of bacteria" and "Communication between innate and adaptive immune cells" were significantly enriched in differentially expressed genes, and enrichment of these functions and pathways implicated the strong macrophage activation and immune response signaling.

Through detailed examination on specific genes, a batch of key genes was highlighted. In Hep2 cells, induction of secretion of chemokines such as CCL2, CCL5 and IL8 was observed, genes related to apoptosis such as XAF1 and genes related to cholesterol synthesis such as HMGCR all showed differential expression. And these observations partly overlapped with the results reported by another group of researchers [371]. During their investigation of proteome in RSV infected A549 cells, it has been reported that a group of proteins involved in protein biosynthesis and modification, cellular stress responses, cytoskeleton and structure showed differential expression. In macrophages, expression of genes associated with antigen presentation and cell apoptosis were also altered in our data. Recently, another study in our lab tried to uncover the detailed function of hydroxymethyglutaryl coenzyme A reductase inhibitor lovastatin as an anti-inflammatory drug. And the results revealed the increase of virus antigen and inclusion bodies, as well as the correlated increase of cytokines in their gene expression at from 2 to $16 \mathrm{hpi}$ in RSV-infected RAW cells. Besides, mature F and G glycoproteins were not able to be detected on the surface of the infected RAW cells. In general, these 
observations were consistent with the findings in our study. Furthermore, it was also observed that lovastatin treatment in RAW cells indeed significantly reduce the levels of pro-inflammatory cytokines, which partly explain the phenomenon that lovastatin treatment is effective on protecting mice from lethal RSV infection [391].

Analysis on gene expression performances in canonical pathways including "Interferon signaling", "NF- $\kappa \mathrm{B}$ signaling" and "Toll-like receptor signaling" suggested that only a small batch of genes showed differential expression with faint fold changes in RSV-infected Hep2 cells, indicating poor activation of these pathways.

However, investigation of gene expression performances in these immune response pathways suggested that a wider range of genes were detected with significant expression changes in RSV-infected macrophages, suggesting the well initiation of inflammatory defense.

As described before, the transcriptomic profiles were investigated from 4hpi to 15hpi in RSV-infected Hep2 cells. From 8hpi, it was observed that the virus filaments and inclusion bodies were formed with an increasing numbers, and the quite a lot of virus filaments accumulated at 15hpi. Data from microarray study indicated that the productions of cytokines and chemokines were increased quite early (from 4hpi), indicating that the innate response was activated at the first beginning of virus replication cycle, at least before the observation of obvious virus filaments and inclusion bodies. Compared to the response detected at $4 \mathrm{hpi}$, stronger induction of pro-inflammatory response has been observed from $8 \mathrm{hpi}$ and sustained over the late infection stage. This phenomenon might indicate that the antiviral actions were fully taken to prevent the further assembly and release in host cells. At the meanwhile, several mechanisms including inhibiting the expression of type I IFN, delaying host apoptosis and alteration of lipid raft have also been exerted by RSV in order to interfere with the activated immune defense and benefit the further viral replication [156].

In our RSV-infected macrophages, strong immune response was observed from the early infection stage (4hpi) to the late infection stage (24hpi). At the meanwhile, similar vRNA levels at between 2.5 and 20 hpi were also detected. Thus, it might be assumed that sustained activation of genes involved 
in innate and acquired immune response was responsible for the low respiratory tract infection. In addition, other data also indicated that RSV infection results in the formation of virus antigen and the production of inclusion bodies, efficient infectious virus particle production does not occur [364]. 


\begin{tabular}{|c|c|}
\hline Probe ID & GeneBank \\
\hline 205239_at & NM_001657 \\
\hline 203140_at & NM_001706 \\
\hline 210538 _s_at & U37546 \\
\hline 202094_at & AA648913 \\
\hline 203944_x_at & NM_007049 \\
\hline 211256_x_at & U90̄142 \\
\hline 215493_x_at & AL121936 \\
\hline 216598 s_at & S69738 \\
\hline 1405_i_at & M21121 \\
\hline 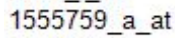 & AF043341 \\
\hline 204655_at & NM_002985 \\
\hline 204440_at & NM_-004233 \\
\hline 155615̄_at & Alo77 7660 \\
\hline 204533_āt & NM_001565 \\
\hline 210163_at & AF030514 \\
\hline 211122 _s_at & AF002985 \\
\hline 209774_x_at & M57731 \\
\hline 207850_at & NM_002090 \\
\hline 218943_s_at & NM_014314 \\
\hline 242961_x_at & Al 304317 \\
\hline 222793_at & AK023661 \\
\hline 218995_s_at & NM_001955 \\
\hline 222802_at & J05008 \\
\hline 1564630 at & $\mathrm{BC} 036851$ \\
\hline 1561042_at & AF086249 \\
\hline 204363_at & NM_001993 \\
\hline $155909 \overline{4}$ at & AK $\overline{095307}$ \\
\hline 1566509_s_at & AK095315 \\
\hline 209864_at & AB045118 \\
\hline 231577_s_at & AW014593 \\
\hline 202269_x_at & BC002666 \\
\hline 202270_at & NM_002053 \\
\hline 242907_at & BF509371 \\
\hline 214453_s_at & NM_006417 \\
\hline 214059_at & BE $\overline{0} 49439$ \\
\hline 208375_at & NM_024013 \\
\hline 207964_x_at & NM_021068 \\
\hline 225661_at & AA $\overline{8} 11138$ \\
\hline 225669_at & AA133989 \\
\hline
\end{tabular}

Gene Title

amphiregulin (schwannoma-derived growth factor)

B-cell CLL/ymphoma 6 (zinc finger protein 51)

baculoviral IAP repeat-containing 3

baculoviral IAP repeat-containing 5 (survivin)

butyrophilin, subfamily 2 , member A1

butyrophilin, subfamily 2 , member $A 1$

butyrophilin, subfamily 2 , member $\mathrm{A} 1$

chemokine (C-C motif) ligand 2

chemokine (C-C motif) ligand 5

chemokine ( $\mathrm{C}-\mathrm{C}$ motif) ligand 5

chemokine ( $\mathrm{C}-\mathrm{C}$ motif) ligand 5

CD83 antigen (activated B lymphocytes, immunoglobulin superfamily)

T-cell immunomodulatory protein

ceell if

The

chemokine (C-X-C motif) ligand 11

chemokine (C-X-C motif) ligand 11

chemokine (C-X-C motif) ligand 2

chemokine (C-X-C motif) ligand 3

DEAD (Asp-Glu-Ala-Asp) box polypeptide 58

DEAD (Asp-Glu-Ala-Asp) box polypeptide 58

DEAD (Asp-Glu-Ala-Asp) box polypeptide 58

endothelin 1

endothelin 1

endothelin 1

Egf-like module containing, mucin-like, hormone receptor-like 3

coagulation factor III (thromboplastin, tissue factor)

F-box protein 9

\section{F-box protein 9}

frequently rearanged in advanced T-cell lymphomas 2

frong

guanylate binding protein 1 , interferon-inducible, $67 \mathrm{kDa}$

guanylate binding protein 1, interferon-

guanylate binding protein 1 , interferon-inducible, $67 \mathrm{kD}$

guanylate binding protein 2 , interferon-inducible

interferon-Induced protein 44

interferon-induced protein 44

interferon, alpha

interferon, alpha 4

interferon (alpha, beta and omega) receptor 1

interferon (alpha, beta and omega) receptor 1

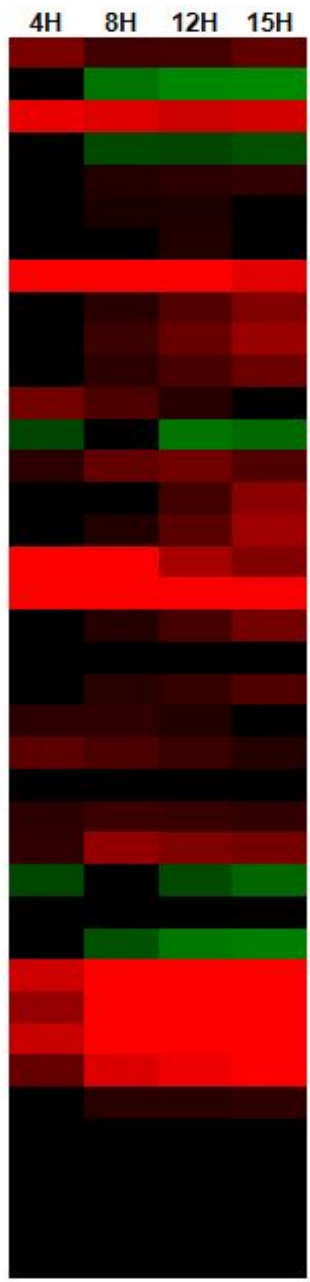




\begin{tabular}{|c|c|c|}
\hline $\begin{array}{l}\text { Probe ID } \\
204786 \text { s_at }\end{array}$ & $\begin{array}{l}\text { GeneBank } \\
\text { L41944 }\end{array}$ & $\begin{array}{l}\text { Gene Title } \\
\text { interferon (alpha, beta and omega) receptor } 2\end{array}$ \\
\hline 227125 at & BF526978 & Interferon (alpha, beta and omega) receptor 2 \\
\hline 208173_at & NM_002176 & interferon, beta 1 , fibroblast \\
\hline 202727_s_at & NM_000416 & interferon gamma receptor 1 \\
\hline 211676 s_at & AF056979 & interferon gamma receptor 1 \\
\hline 201642_at & NM_005534 & interferon gamma receptor 2 (interferon gamma transducer 1$)$ \\
\hline 1552915 at & NM_172138 & interleukin 28A (interferon, lambda 2) \\
\hline 1552609_s_at & NM_172139 & interleukin $28 \mathrm{~A}$ (interferon, lambda 2) \\
\hline 205207_at & NM_000600 & interleukin 6 (interferon, beta 2) \\
\hline $202859^{-}$x_at & NM_000584 & interleukin 8 \\
\hline 211506 _s_at & AF $\overline{0} 43337$ & interleukin 8 \\
\hline 231779_at & Al246590 & interleukin-1 receptor-associated kinase 2 \\
\hline 204698_at & NM_002201 & interferon stimulated exonuclease gene $20 \mathrm{kDa}$ \\
\hline 33304_àt & U889964 & interferon stimulated exonuclease gene $20 \mathrm{kDa}$ \\
\hline 208961 s_at & AB017493 & Kruppel-like factor 6 \\
\hline 224606 at & BG250721 & Kruppel-like factor 6 \\
\hline 1555832 s_at & BU683415 & Kruppel-like factor 6 \\
\hline 210754_s_at & M79321 & v-yes-1 Yamaguchi sarcoma viral related oncogene homolog \\
\hline 202625_at & Al356412 & v-yes-1 Yamaguchi sarcoma viral related oncogene homolog \\
\hline 202626 s at & NM 002350 & v-yes-1 Yamaguchi sarcoma viral related oncogene homolog \\
\hline 209239 at & M55643 & nuclear factor of kappa light polypeptide gene enhancer in B-cells 1 ( $p 105)$ \\
\hline 207535 s_at & NM_002502 & nuclear factor of kappa light polypeptide gene enhancer in B-cells 2 (p49/p 100) \\
\hline 228388_at & AA 044140 & nuclear factor of kappa light polypeptide gene enhancer in B-cells inhibitor, beta \\
\hline 223217 s_at & BE646573 & nuclear factor of kappa light polypeptide gene enhancer in B-cells inhibitor, zeta \\
\hline 223218 s_at & AB037925 & nuclear factor of kappa light polypeptide gene enhancer in B-cells inhibitor, zeta \\
\hline 202869_at & NM_016816 & $2^{\prime}, 5^{\prime}$-oligoadenylate synthetase $1,40 / 46 \mathrm{kDa}$ \\
\hline 205552_s_at & NM_002534 & $2,5^{\prime}$-oligoadenylate synthetase $1,40 / 46 \mathrm{kDa}$ \\
\hline 218400 at & NM_006187 & 2 -5'-oligoadenylate synthetase $3,100 \mathrm{kDa}$ \\
\hline 232666_at & $\mathrm{R} 1 \overline{34} 58$ & 2 '-5'-oligoadenylate synthetase $3,100 \mathrm{kDa}$ \\
\hline 205660 at & NM_003733 & $2^{\prime}-5^{\prime}$-oligoadenylate synthetase-like \\
\hline 210797_s_at & AF $\overline{063612}$ & $2 '-5$ '-oligoadenylate synthetase-like \\
\hline 217739 -s_at & NM_005746 & pre-B-cell colony enhancing factor 1 \\
\hline 243296_at & AA $\overline{8} 73350$ & Pre-B-cell colony enhancing factor 1 \\
\hline $155516 \overline{7}$ _s_at & BC020691 & pre-B-cell colony enhancing factor 1 \\
\hline 212151_at & BF967998 & Pre-B-cell leukemia transcription factor 1 \\
\hline 239210_at & AW074143 & Pre-B-cell leukemia transcription factor 1 \\
\hline 212148_at & AL049381 & Pre-B-cell leukemia transcription factor 1 \\
\hline
\end{tabular}

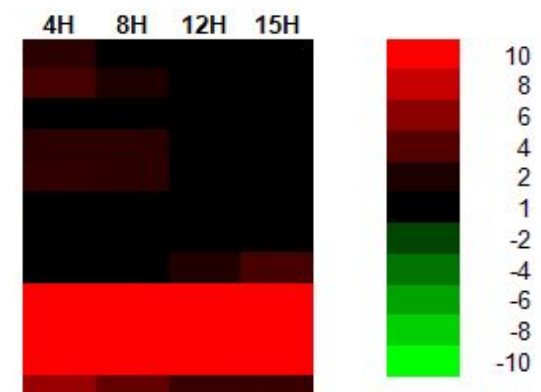

Figure 4.14 Expression of probe sets involved in immune response in RSV-infected Hep2 cells. RSV was infected with Hep2 cells, and the expression of probe sets associated with immune response was examined by microarray analysis at 4, 8, 12 and 15 hpi as shown. The data is represented by heat map analysis showing up-regulated (red), down-regulated (green) or no changes (black) in expression, and the FC range is indicated (P-value $\leq 0.05$ ). 


\begin{tabular}{|c|c|}
\hline $\begin{array}{l}\text { Probe ID } \\
217911 \text { s_at }\end{array}$ & $\begin{array}{l}\text { GeneBank } \\
\text { NM_004281 }\end{array}$ \\
\hline 215037 s at & U72398 \\
\hline 206665 s_at & NM_001191 \\
\hline 212312 at & AL117381 \\
\hline 210538 _s_at & U37546 \\
\hline 206133_at & Al43782 \\
\hline 228617_at & CO439482 \\
\hline 242234 at & Gl439853 \\
\hline $211851 \times x$ at & AF005068 \\
\hline 204531_s_at & NM_007295 \\
\hline 202284_s_at & NM_000389 \\
\hline 208485_x_at & NM_-003879 \\
\hline 209939_x_at & AF005775 \\
\hline 210564_x_at & AF009619 \\
\hline 211316 x_at & AF009616 \\
\hline 211317 s_at & AF041461 \\
\hline 211862 x_at & AF015451 \\
\hline 210563_x_at & U97075 \\
\hline 209508_x_at & AF005774 \\
\hline 203890 s_at & BF686824 \\
\hline 203891_s_at & NM_001348 \\
\hline 233912_x_at & AK021525 \\
\hline 1553928_at & NM_153702 \\
\hline 201324_āt & NM_001423 \\
\hline 201325 s at & NM_001423 \\
\hline 235745_at & AV7̄04183 \\
\hline 204780 s_at & AA164751 \\
\hline 216252_x_at & Z70519 \\
\hline 215719_x_at & X83493 \\
\hline 204781_s_at & NM_000043 \\
\hline 209189 at & BC̄004490 \\
\hline 205409_at & NM_005253 \\
\hline 218880 at & N36̄ 08 \\
\hline 218881_s_at & NM_024530 \\
\hline 225262_at & Al $6 \overline{7} 0862$ \\
\hline 228188_at & Al860150 \\
\hline 204235 s_at & AF200715 \\
\hline 204237_at & NM_016315 \\
\hline $215913 \mathrm{~s}$ at & AK $\overline{02} 3668$ \\
\hline
\end{tabular}

BCL2-like 1

BCL2-like 1

baculoviral IAP repeat-containing 3

$\mathrm{XIAP}$ associated factor- 1

XIAP associated factor- 1

XIAP associated factor- 1

breast cancer 1 early onset

breast cancer 1 early onset

cyclin-dependent kinase inhibitor $1 A$ ( $p 21$, Cip1)

CASP8 and FADD-like apoptosis regulato

CASP8 and FADD-like apoptosis regulato

CASP8 and FADD-like apoptosis regulator

CASP8 and FADD-like apoptosis regulator

CASP8 and FADD-like apoptosis regulator

CASP8 and FADD-like apoptosis regulator

CASP8 and FADD-like apoptosis regulator

CASP8 and FADD-like apoptosis regulato

death-associated protein kinase 3

death-associated protein kinase 3

ELMO domain containing 2

ELMO domain containing 2

epithelial membrane protein 1

epithelial membrane protein 1

endoplasmic reticulum to nucleus signalling 1

Fas (TNF receptor superfamily, member 6 )

Fas (TNF receptor superfamily, member 6 )

Fas (TNF receptor superfamily, member

Fas (TNF receptor superfamily, member

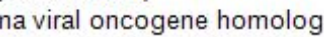

FOS-like antigen 2

FOS like antigen 2

FOS-like antigen 2

FOS-like antigen 2

FOS-like antigen 2

GULP, engulfment adaptor PTB domain containing 1

GULP, engulfment adaptor PTB domain containing 1

GULP, engulfment adaptor PTB domain containing 1

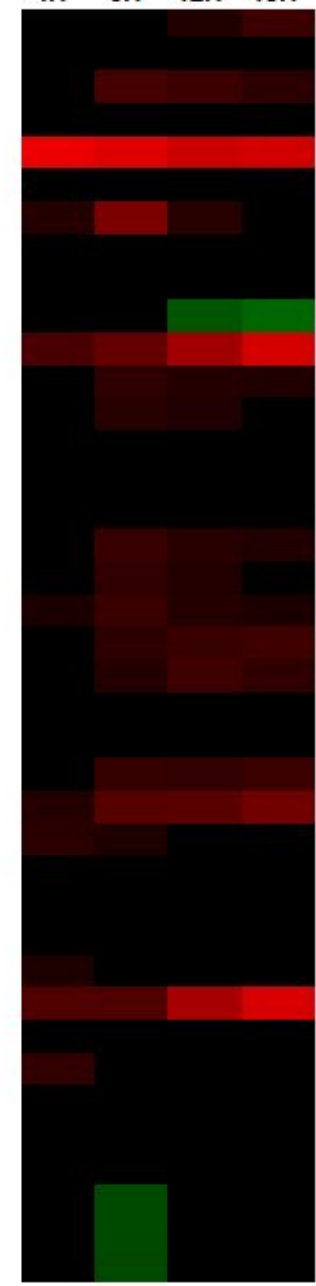




\begin{tabular}{|c|c|}
\hline Probe ID & GeneBank \\
\hline 223837_at & BC001103 \\
\hline 201631_s_at & NM_003897 \\
\hline 204549_at & NM_014002 \\
\hline 36004_at & AF $\overline{074382}$ \\
\hline 209929_s_at & AF091453 \\
\hline 201466_s_at & NM_002228 \\
\hline 201464_x_at & BG491844 \\
\hline 214057_at & H71805 \\
\hline 200796_s_at & BF594446 \\
\hline 200797_s_at & Al275690 \\
\hline 214056_at & BF981280 \\
\hline 200798_x_at & NM_021960 \\
\hline 202014_at & NM_014330 \\
\hline 37028_at & U83981 \\
\hline 203372_s_at & AB004903 \\
\hline 203373_at & NM_003877 \\
\hline 202694_at & AW183478 \\
\hline 241410_at & Al949081 \\
\hline 202693_s_at & AW194730 \\
\hline 202695_s_at & NM_004760 \\
\hline 201207_at & NM_021137 \\
\hline 202510_s_at & NM_006291 \\
\hline 202643_s_at & Al738896 \\
\hline 202644_s_at & NM_006290 \\
\hline 206025_s_at & AW188198 \\
\hline 206026_s_at & NM_007115 \\
\hline 210260 _s_at & BC 005352 \\
\hline 208296_x_at & NM_014350 \\
\hline 227420_at & BF $\overline{3} 38045$ \\
\hline 1552648_a_at & NM_003844 \\
\hline 241371_at & AW $\overline{4} 51259$ \\
\hline 231775_at & W65310 \\
\hline 218856_at & NM_016629 \\
\hline 214581_x_at & BE568134 \\
\hline 201746_at & NM_000546 \\
\hline 226502_at & BG4777315 \\
\hline
\end{tabular}

Gene Title

GULP, engulfment adaptor PTB domain containing 1

mmediate early response 3

nhibitor of kappa light polypeptide gene enhancer in B-cells, kinase epsilon inhibitor of kappa light polypeptide gene enhancer in B-cells, kinase gamma inhibitor of kappa light polypeptide gene enhancer in B-cells, kinase gamma v-jun sarcoma virus 17 oncogene homolog (avian)

v-jun sarcoma virus 17 oncogene homolog (avian)

Myeloid cell leukemia sequence 1 (BCL2-related)

myeloid cell leukemia sequence 1(BCL2-related)

myeloid celleukemia sequence 1 (BCL2-related)

Myeloid celleukemi sequence 1 (BCL2-related)

myelid celleukenia

protein phosphatase 1, regulatory (inhibitor) subunit 15A

protein phosphatase 1, regulatory (inhibitor) subunit 15A

protein phosphatase 1, regulatory (inh

suppressor of cytokine signaling 2

suppressor of cytokine signaling 2

serine/threonine kinase 17a (apoptosis-inducing)

Serine/threonine kinase 17a (apoptosis-inducing)

serine/threonine kinase 17a (apoptosis-inducing)

serine/threonine kinase 17a (apoptosis-inducing)

tumor necrosis factor, alpha-induced protein 1 (endothelial)

tumor necrosis factor, alpha-induced protein 2

tumor necrosis factor, alpha-induced protein 3

tumor necrosis factor, alpha-induced protein 3

tumor necrosis factor, alpha-induced protein 6

umor necrosis factor, alpha-induced protein 6

umor necrosis factor, alpha-induced protein 8

umor necrosis factor, alpha-induced protein 8

tumor necrosis factor, alpha-induced protein 8 -like 1

tumor necrosis factor receptor superfamily, member $10 \mathrm{a}$

Tumor necrosis factor receptor superfamily, member $10 \mathrm{a}$

tumor necrosis factor receptor superfamily, member $10 \mathrm{a}$

tumor necrosis factor receptor superfamily, member 21

tumor necrosis factor receptor superfamily member 21

tumor protein p53 (Li-Fraumeni syndrome)

ELMO domain containing 2

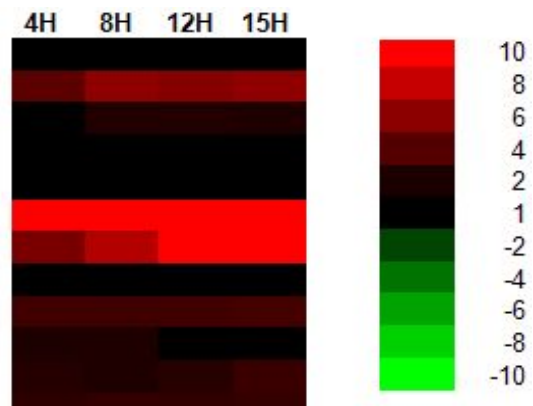

Figure 4.15 Expression of probe sets involved in cell death in RSV-infected Hep2 cells. RSV was infected with Hep2 cells, and the expression of probe sets associated with cell death was examined by microarray analysis at 4, 8,12 and 15 hpi as shown. The data is represented by heat map analysis showing up-regulated (red), down-regulated (green) or no changes (black) in expression, and the FC range is indicated ( $\mathrm{P}$-value $\leq 0.05$ ). 


\begin{tabular}{|c|c|}
\hline $\begin{array}{l}\text { Probe ID } \\
208636 \text { at }\end{array}$ & $\begin{array}{l}\text { GeneBank } \\
\text { Al082078 }\end{array}$ \\
\hline $208637 \times$ at & BC003576 \\
\hline 211160 x_at & M95178 \\
\hline 237401_at & BF062666 \\
\hline 241869_at & AW026509 \\
\hline 219716_at & NM_030641 \\
\hline 216607_s_at & U 40053 \\
\hline 202314_at & NM_000786 \\
\hline 201790 s_at & AW150953 \\
\hline 201791_s_at & NM_001360 \\
\hline 201790_s_at & AW150953 \\
\hline 201791 s a & NM_ 001360 \\
\hline 239358_at & $\mathrm{BE} \overline{6} 45256$ \\
\hline 210950_s_at & BC003573 \\
\hline 241954 at & AA757900 \\
\hline 208647_at & AA872727 \\
\hline 238666 at & BF438300 \\
\hline 243658 at & AA873729 \\
\hline 1558199_at & W73431 \\
\hline $210495 \bar{x}$ at & AF130095 \\
\hline 212464 s_at & X02761 \\
\hline 214701_s_at & AJ276395 \\
\hline 214702_at & AJ276395 \\
\hline 216442 x_at & AK026737 \\
\hline 235629_at & Al333596 \\
\hline $211719^{-} \mathrm{x}$ at & BC005858 \\
\hline 202539_s_at & AL518627 \\
\hline 202540_s_at & NM_000859 \\
\hline 202540 s a & NM_000859 \\
\hline 202539 s at & AL518627 \\
\hline 205822_s_at & NM_002130 \\
\hline $221750^{-}$at & BG035985 \\
\hline $220081 \times$ at & NM_016371 \\
\hline $204615 \times$ a at & NM_004508 \\
\hline $208881 x$ at & $\mathrm{BC} 005247$ \\
\hline $242065 \times$ at & BG477984 \\
\hline 230198 at & AA166617 \\
\hline $242255^{-}$at & R49102 \\
\hline 205032_at & NM_002203 \\
\hline
\end{tabular}

actinin, alpha 1

Actinin, alpha 1

apolipoprotein L, 6

cytochrome P450, family 51 , subfamily A, polypeptide 1

cytochrome $\mathrm{P} 450$, family 51 , subfamily $A$, polypeptide 1

7-dehydrocholesterol reductase

7-dehydrocholesterol reductase

7-dehydrocholesterol reductase

7-dehydrocholesterol reductase

Farnesyl-diphosphate farnesyltransferase

farnesyl-diphosphate farnesyltransferase 1

Farnesyl-diphosphate farnesyltransferase 1

farnesyl-diphosphate farnesyltransferase 1

Farnesyl-diphosphate farnesyltransferase 1

Farnesyl-diphosphate farnesyltransferase 1

fibronectin 1

fibronectin 1

fibronectin 1

fibronectin 1

fibronectin 1

fibronectin 1

Fibronectin 1

fibronectin 1

3-hydroxy-3-methylglutaryl-Coenzyme A reductase

3-hydroxy-3-methylglutaryl-Coenzyme A reductase

3-hydroxy-3-methylglutaryl-Coenzyme A reductase

3-hydroxy-3-methylglutaryl-Coenzyme A reductase

3-hydroxy-3-methylglutaryl-Coenzyme A synthase 1 (soluble)

3-hydroxy-3-methylglutaryl-Coenzyme A synthase 1 (soluble)

hydroxysteroid (17-beta) dehydrogenase 7

isopentenyl-diphosphate delta isomerase 1

isopentenyl-diphosphate delta isomerase 1

isopentenyl-diphosphate delta isomerase 1

Isopentenyl-diphosphate delta isome

sopentenyl-diphosphate delta isomerase

integrin, alpha 2 (CD49B, alpha 2 subunit of VLA-2 receptor)

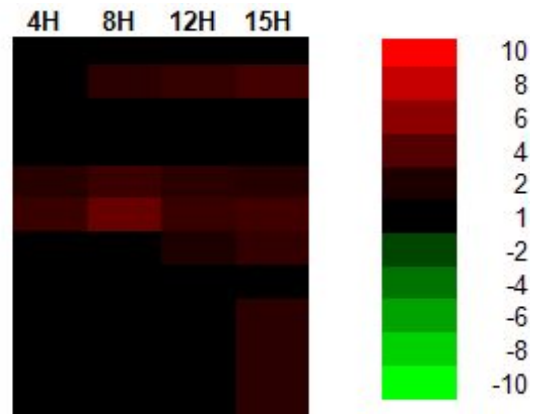




\begin{tabular}{|c|c|c|}
\hline Probe ID & GeneBank & Gene Title \\
\hline 227314_at & & Integrin, alpha 2 (CD49B, alpha 2 subunit of VLA-2 receptor) \\
\hline 237728_at & Al733222 & Integrin, alpha 2 (CD49B, alpha 2 subunit of VLA-2 receptor) \\
\hline $201389^{-}$at & NM 002205 & integrin, alpha 5 (fibronectin receptor, alpha polypeptide) \\
\hline 201124_at & AL 048423 & integrin, beta 5 \\
\hline 201125_s_at & NM_002213 & integrin, beta 5 \\
\hline 214020 _x_at & BE138575 & Integrin, beta 5 \\
\hline 214021_x_at & Al335208 & Integrin, beta 5 \\
\hline 1559203 _s_at & BC029545 & v-Ki-ras2 Kirsten rat sarcoma viral oncogene homolog \\
\hline $1559204 \times$ at & $\mathrm{BC} 029545$ & v-Ki-ras2 Kirsten rat sarcoma viral oncogene homolog \\
\hline 204009 s at & W80678 & v-Ki-ras2 Kirsten rat sarcoma viral oncogene homolog \\
\hline 204010 s_at & NM_004985 & v-Ki-ras2 Kirsten rat sarcoma viral oncogene homolog \\
\hline 214352_s_at & BF 673699 & v-Ki-ras2 Kirsten rat sarcoma viral oncogene homolog \\
\hline 1561654 at & AF085978 & LIM domain kinase 2 \\
\hline 202193 at & NM 005569 & LIM domain kinase 2 \\
\hline 210582_s_at & AL117466 & LIM domain kinase 2 \\
\hline 217475 s_at & AC002073 & LIM domain kinase 2 \\
\hline 204056_s_at & NM_000431 & mevalonate kinase (mevalonic aciduria) \\
\hline 215649 s at & AF 217536 & mevalonate kinase (mevalonic aciduria) \\
\hline 236686 at & AU150926 & mevalonate kinase (mevalonic aciduria) \\
\hline 36907_at & M88468 & mevalonate kinase (mevalonic aciduria) \\
\hline $21192 \overline{6}$ _s_at & Al827941 & myosin, heavy polypeptide 9 , non-muscle \\
\hline 209279_s_at & BC000245 & NAD $(P)$ dependent steroid dehydrogenase-like \\
\hline 215093 at & U82671 & NAD $(P)$ dependent steroid dehydrogenase-like \\
\hline 216755 at & AK024945 & oxysterol binding protein-like 10 \\
\hline 241693_at & BF246603 & Oxysterol binding protein-like $1 \mathrm{~A}$ \\
\hline 202743 at & BE622627 & phosphoinositide-3-kinase, regulatory subunit 3 ( $p 55$, gamma) \\
\hline 211580 s_at & AF028785 & phosphoinositide-3-kinase, regulatory subunit 3 ( $p 55$, gamma) \\
\hline 201087_at & NM_002859 & paxillin \\
\hline $211823 \mathrm{~s}$ at & D86862 & paxillin \\
\hline 227332_at & BF511170 & Paxillin \\
\hline 209146_at & AV704962 & sterol-C4-methyl oxidase-like \\
\hline 243543_at & BF508288 & Sterol-C4-methyl oxidase-like \\
\hline 209218_at & AF098865 & squalene epoxidase \\
\hline 213562 s_at & BF979497 & squalene epoxidase \\
\hline 1557352_at & BU754109 & Squalene epoxidase \\
\hline 213577 at & AA639705 & squalene epoxidase \\
\hline $218806 \mathrm{~s}$ at & AF118887 & vav 3 oncogene \\
\hline 218807 at & NM 006113 & vav 3 oncogene \\
\hline 224221 _s_at & AF118886 & vav 3 oncogene \\
\hline $204165^{-}$at & NM 003931 & WAS protein family, member 1 \\
\hline
\end{tabular}

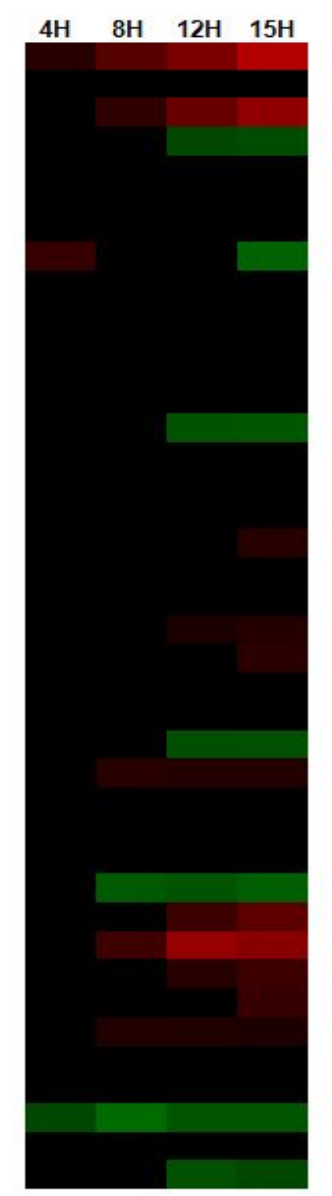

Figure 4.16 Expression of probe sets involved in cholesterol biosynthesis in RSV-infected Hep2 cells. RSV was infected with Hep2 cells, and the expression of probe sets associated with cholesterol biosynthesis was examined by microarray analysis at $4,8,12$ and 15 hpi as shown. The data is represented by heat map analysis showing upregulated (red), down-regulated (green) or no changes (black) in expression, and the FC range is indicated (P-value $\leq 0.05$ ). 


\begin{tabular}{|c|c|}
\hline $\begin{array}{l}\text { Probe ID } \\
\text { 1559077_at }\end{array}$ & $\begin{array}{l}\text { GeneBank } \\
\text { AL833204 }\end{array}$ \\
\hline 229354_at & AB 033060 \\
\hline 206516_at & NM_000479 \\
\hline 1560318_at & AL $\overline{83} 3445$ \\
\hline 205780_at & NM_001197 \\
\hline 204677_at & NM_001795 \\
\hline 213006_at & AV 655640 \\
\hline 212675_s_at & AB011154 \\
\hline 212677_s_at & BG530481 \\
\hline 209101_at & M92934 \\
\hline 202902_s_at & NM_004079 \\
\hline 205765_at & NM_000777 \\
\hline 214234_s_at & $\times 90579$ \\
\hline 215143_at & AL049437 \\
\hline 208893_s_at & BC005047 \\
\hline 201693_s_at & AV733950 \\
\hline 201694_s_at & NM_001964 \\
\hline 227404_s_at & Al45̄9194 \\
\hline 207768_at & NM_001965 \\
\hline 215162_at & AB020691 \\
\hline 219743_at & NM_012259 \\
\hline 1569979 _at & $\mathrm{BC} \overline{0} 10024$ \\
\hline 1569980 x_at & BC010024 \\
\hline 219865_at & NM_014179 \\
\hline 218637_at & NM_018439 \\
\hline 244703_x_at & AA 444166 \\
\hline 1553111]_a at & NM_152903 \\
\hline 208567__s_āt & NM_002244 \\
\hline 1562966_at & BC017424 \\
\hline 230166_at & $\mathrm{BG} 026236$ \\
\hline 207517_at & NM_018891 \\
\hline 212531_at & NM_005564 \\
\hline 236181_at & R38704 \\
\hline 230725_at & AA973100 \\
\hline 240167_at & Al031657 \\
\hline 242260_at & BG283790 \\
\hline 230298_at & Al692906 \\
\hline 207761_s_at & NM_014033 \\
\hline 204475_at & NM_002421 \\
\hline
\end{tabular}

Gene Title

(NESH) binding protein

aryl-hydrocarbon receptor repressor

anti-Mullerian hormone

Rho GTPase activating protein 29

$B C L 2$-interacting killer (apoptosis-inducing)

cadherin 5, type 2 (vascular endothelium)

CCAAT/enhancer binding protein (C/EBP), delta

centrosomal protein $68 \mathrm{kDa}$

centrosomal protein $68 \mathrm{kDa}$

connective tissue growth factor

cathepsin $\mathrm{S}$

cytochrome $\mathrm{P} 450$, family 3 , subfamily A, polypeptide 5

cytochrome P450, family 3 , subfamily A, polypeptide 5

dpy-19-like 2 pseudogene 2 (C. elegans)

dual specificity phosphatase 6

early growth response 1

early growth response 1

Early growth response 1

early growth response 4

GTPase activating Rap/RanGAP domain-like 1

hairy/enhancer-of-split related with YRPW motif 2

GLI-Kruppel family member HKR1

GLI-Kruppel family member HKR1

hypo 157

hypothetical LOC29092

importin 9

kelch repeat and BTB (POZ) domain containing 6

potassium inwardly-rectifying channel, subfamily J, member 12

KIAA1217

KIAA1958

laminin, gamma 2

Hypothetical protein LOC100132181

hypothetical protein LOC100133781

hypothetical protein LOC152742

Matrin 3

metallo-beta-lactamase domain containing 2

methyltransferase like $7 \mathrm{~A}$

matrix metallopeptidase 1 (interstitial collagenase)

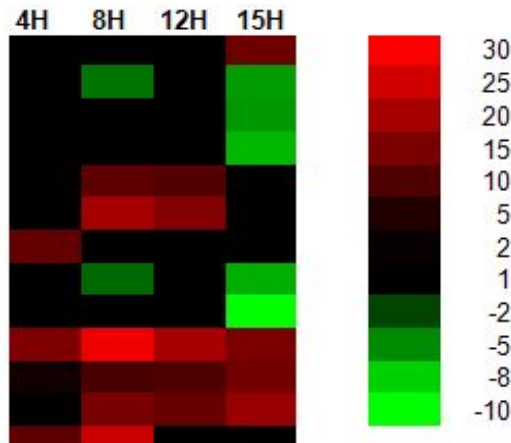




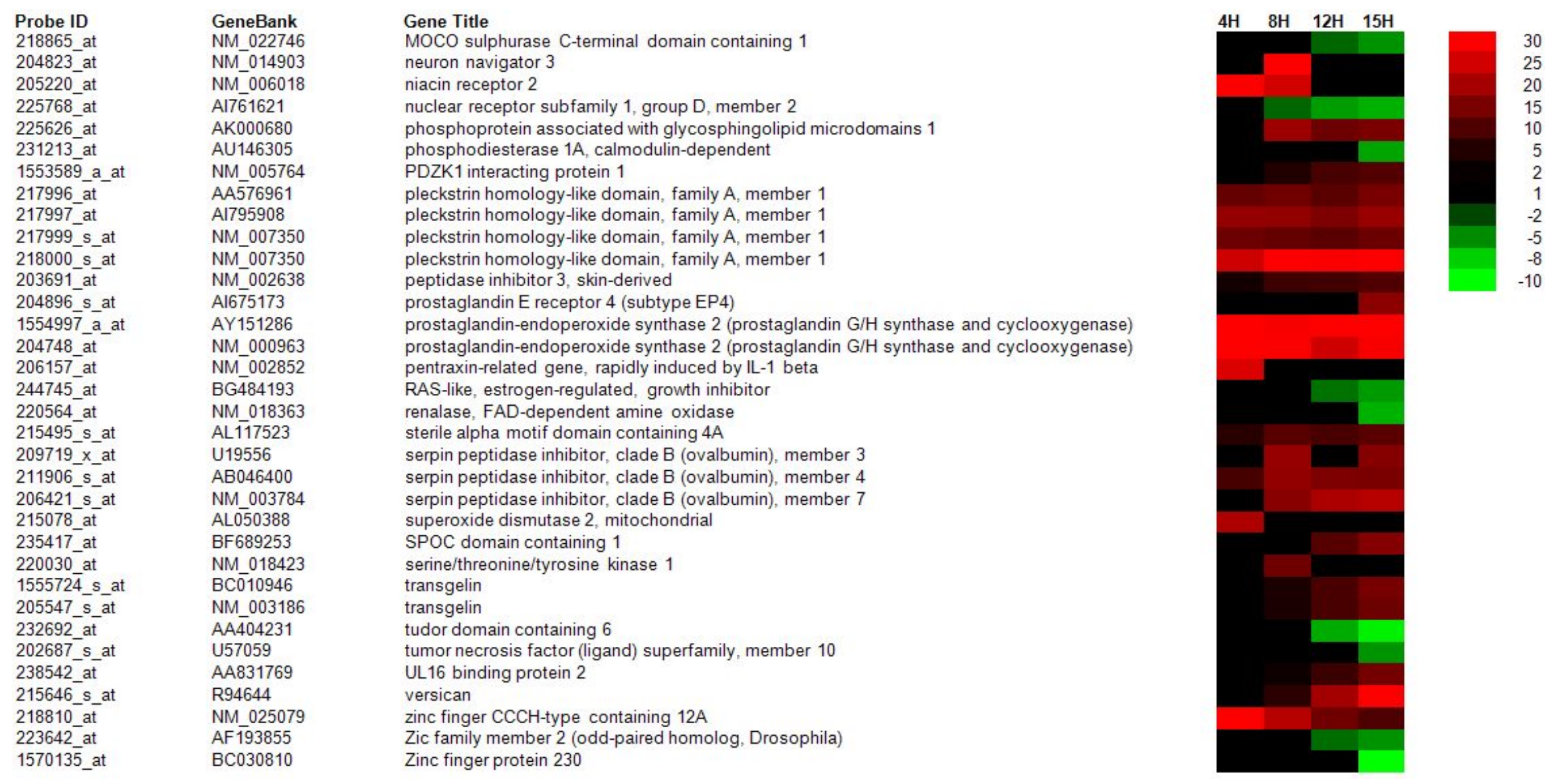

Figure 4.17 The probe sets with topmost expression in RSV-infected Hep2 cells. RSV was infected with Hep2 cells, and the expression of probe sets with top differential expression was examined by microarray analysis at $4,8,12$ and 15 hpi as shown. The data is represented by heat map analysis showing up-regulated (red), down-regulated (green) or no changes (black) in expression, and the FC range is ndicated (P-value $\leq 0.05)$. 
B-cell leukemia/lymphoma 3

chemokine ( $\mathrm{C}-\mathrm{C}$ motif) ligand 2

chemokine ( $\mathrm{C}-\mathrm{C}$ motif) ligand 3

chemokine ( $\mathrm{C}-\mathrm{C}$ motif) ligand 4

chemokine (C-C motif) ligand 5

chemokine ( $\mathrm{C}-\mathrm{C}$ motif) ligand 7

chemokine (C-C motif) receptor-like 2

CD40 antigen

chemokine (C-X3-C) receptor 1

chemokine (C-X-C motif) ligand 1

chemokine ( $\mathrm{C}-\mathrm{X}-\mathrm{C}$ motif) ligand 10

chemokine (C-X-C motif) ligand 2

chemokine (C-X-C motif) ligand 3

chemokine (C-X-C motif) ligand 5

chemokine (C-X-C motif) ligand 9

DEAD (Asp-Glu-Ala-Asp) box polypeptide 58

DEAD (Asp-Glu-Ala-Asp) box polypeptide 60

DEXH (Asp-Glu-X-His) box polypeptide 58

guanylate binding protein 4///RIKEN cDNA 5830443L24 gene///predicted gene, EG634650

guanylate binding protein 1

guanylate binding protein 2

guanylate binding protein 3

guanylate binding protein 6

GTPase, very large interferon inducible 1

histocompatibility 28

histocompatibility 2 , class II antigen $\mathrm{A}$, alpha

histocompatibility 2 , class II antigen A, beta 1

histocompatibility 2 , class II, locus $\mathrm{DMa}$

histocompatibility 2, class II, locus Mb2///histocompatibility 2, class II, locus Mb1

histocompatibility 2, class II, locus Mb2///histocompatibility 2, class II, locus Mb1

histocompatibility 2, class II antigen E alpha

histocompatibility 2, class II antigen E beta

histocompatibility $2, \mathrm{Q}$ region locus 7

histocompatibility $2, \mathrm{Q}$ region locus 8

histocompatibility $2 \mathrm{~T}-22 / \mathrm{T}-10 / \mathrm{T}-17 / \mathrm{T}-9$

histocompatibility $2, \mathrm{~T}-24$

interferon activated gene 202B

interferon, alpha-inducible protein 27 like $2 \mathrm{~A}$

interferon-induced protein 35

interferon-induced protein 44

interferon qamma inducible protein 47

interferon induced with helicase $\mathrm{C}$ domain 1

interferon-induced protein with tetratricopeptide repeats 1

interferon-induced protein with tetratricopeptide repeats 2

interferon-induced protein with tetratricopeptide repeats 3

interferon alpha $2 / 7 / 12 / 6 / 13 / 4 / 9 / 5 / 1 / B / 14$

interferon beta 1 , fibroblast

interferon gamma

interferon gamma receptor 1

interferon-related developmental regulator 1

interferon alpha responsive gene

interferon gamma induced GTPase

interferon inducible GTPase 1

interleukin 10

interleukin 12a

interleukin $12 \mathrm{~b}$

interleukin 13

interleukin 13 receptor, alpha 1

interleukin 13 receptor, alpha 2

interleukin 15

interleukin 15 receptor, alpha chain

interleukin 18 binding protein

interleukin 1 alpha

interleukin 1 beta

interleukin 1 receptor antagonist

interleukin 21 receptor

interleukin 4

interleukin 4 induced 1

interleukin 4 receptor, alpha

interleukin 6

interleukin 7 
Gene Title

interferon regulatory factor 1

interferon regulatory factor 7

interferon regulatory factor 8

interferon regulatory factor 9

immunity-related GTPase family $\mathrm{M}$ member 1

interferon-stimulated protein

similar to Interferon-activatable protein 205 (IFI-205) (D3 protein)

interferon activated gene 205

macrophage activation 2 like

myxovirus (influenza virus) resistance 1

myxovirus (influenza virus) resistance 2

similar to NFIL3/E4BP4 transcription factor///nuclear factor, interleukin 3, regulated similar to NFIL3/E4BP4 transcription factor///nuclear factor, interleukin 3, regulated nuclear factor of kappa light polypeptide gene enhancer in B-cells 1, p105

$2 '-5$ ' oligoadenylate synthetase $1 \mathrm{~A}$

$2 '-5$ ' oligoadenylate synthetase 2

$2 '-5$ ' oligoadenylate synthetase 3

$2 '-5$ ' oligoadenylate synthetase-like 1

$2 '-5$ ' oligoadenylate synthetase-like 2

oxidized low density lipoprotein (lectin-like) receptor 1

oncostatin M

ring finger protein 125

ring finger protein $19 \mathrm{~B}$

radical $S$-adenosyl methionine domain containing 2

serine (or cysteine) peptidase inhibitor, clade A, member $3 \mathrm{G}$

Sp110 nuclear body protein

transporter 1, ATP-binding cassette, sub-family B (MDR/TAP)

transporter 2, ATP-binding cassette, sub-family B (MDR/TAP)

T-cell specific GTPase 2//IT-cell specific GTPase

toll-like receptor 1

toll-like receptor 12

toll-like receptor 13

toll-like receptor 2

toll-like receptor 3

toll-like receptor 4, mRNA (cDNA clone MGC:35879 IMAGE:3493732)///toll-like receptor 4

toll-like receptor 5

toll-like receptor 6

toll-like receptor 7

toll-like receptor 8

toll-like receptor 9

tumor necrosis factor

tumor necrosis factor, alpha-induced protein 3

tumor necrosis factor alpha induced protein 6

tumor necrosis factor receptor superfamily, member 9

tumor necrosis factor (ligand) superfamily, member 10

tumor necrosis factor (ligand) superfamily, member 14

tumor necrosis factor (ligand) superfamily, member 4

tumor necrosis factor (ligand) superfamily, member 8

tumor necrosis factor (ligand) superfamily, member 9

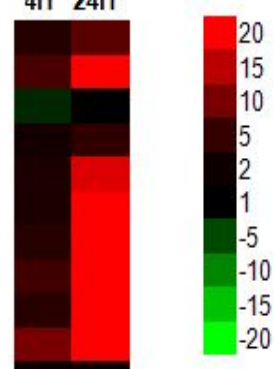

Figure 4.18 Expression of genes involved in immune response in RSV-infected macrophages. RSV was infected with macrophages, and the expression of genes associated with immune response was examined by microarray analysis at 4 and $24 \mathrm{hpi}$ as shown. The data is represented by heat map analysis showing up-regulated (red), down-regulated (green) or no changes (black) in expression, and the FC range is indicated ( $\mathrm{P}$-value $\leq 0.05$ ). 


\section{Gene Title}

apoptosıs-assocláted tyrosıne kinase

baculoviral IAP repeat-containing 2

baculoviral IAP repeat-containing 3

baculoviral IAP repeat-containing 5

caspase 1

caspase 12

caspase 3

caspase 4, apoptosis-related cysteine peptidase

Caspase 6, mRNA (cDNA clone MGC.5873 IMAGE:3491126)///caspase 6

caspase 7

caspase 8

cyclin-dependent kinase inhibitor $1 \mathrm{~A}(\mathrm{P} 21)$

cyclin-dependent kinase inhibitor $2 \mathrm{~A}$

cyclin-dependent kinase inhibitor $2 \mathrm{C}$

CASP8 and FADD-like apoptosis regulator

cysteine-serine-rich nuclear protein 1

Fas death domain-associated protein

DNA-damage inducible transcript 3

DNA-damage-inducible transcript 4

Inhibin beta-A (Inhba), mRNA///inhibin beta-A

Janus kinase 2

myelocytomatosis oncogene

programmed cell death 4

receptor(TNFRSF)-interacting serine-threonine kinase 2

receptor-interacting serine-threonine kinase 3

tumor necrosis factor (ligand) superfamily, member 10

TNF receptor-associated factor $1 / / / \mathrm{TNF}$ receptor-associated factor 1 (Traf1), mRNA

TNF receptor-associated factor 2

TNF receptor-associated factor 3

TNF receptor-associated factor 5

XIAP associated factor 1

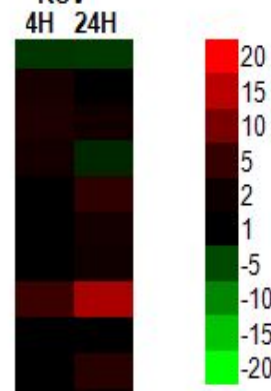

Figure 4.19 Expression of genes involved in cell death in RSV-infected macrophages. RSV was infected with macrophages, and the expression of genes associated with cell death was examined by microarray analysis at 4 and 24 hpi as shown. The data is represented by heat map analysis showing up-regulated (red), down-regulated (green) or no changes (black) in expression, and the FC range is indicated (P-value $\leq 0.05)$. 
bactericidal/permeability-increasing protein-like 2

cholesterol 25-hydroxylase

C-type lectin domain family 4 , member $e$

cytidine monophosphate (UMP-CMP) kinase 2, mitochondrial

calponin 1

cystatin $\mathrm{F}$ (leukocystatin)

DEXH (Asp-Glu-X-His) box polypeptide 58

ectonucleotide pyrophosphatase/phosphodiesterase 4

fibronectin leucine rich transmembrane protein 3

formyl peptide receptor 2

Growth arrest and DNA-damage-inducible 45 beta, mRNA

GTP binding protein (gene overexpressed in skeletal muscle)

gap junction protein, alpha 1

G protein-coupled receptor 84

hect domain and RLD 5

immunoresponsive gene 1

lipocalin 2

macrophage activation 2 like

membrane-spanning 4-domains, subfamily $\mathrm{A}$, member $4 \mathrm{C}$

membrane-spanning 4-domains, subfamily $\mathrm{A}$, member $6 \mathrm{C}$

myeloid differentiation primary response gene 116

nuclear receptor subfamily 4 , group $A$, member 1

nuclear receptor subfamily 4 , group A, member 2

placenta-specific 8

proteasome (prosome, macropain) subunit, beta type 10

receptor transporter protein 4

serine (or cysteine) peptidase inhibitor, clade B, member 2

schlafen 1

schlafen 2

schlafen $4 / / /$ schlafen 3

schlafen $4 / / /$ schlafen 3

schlafen 5

schlafen 8

transporter 1, ATP-binding cassette, sub-family B (MDRTAP)

transporter 2, ATP-binding cassette, sub-family B (MDR/TAP)

transmembrane protein 171

triggering receptor expressed on myeloid cells 1

ubiquitin-conjugating enzyme E2L 6

similar to ubiquitin specific protease UBP43///ubiquitin specific peptidase 18

similar to ubiquitin specific protease UBP43///ubiquitin specific peptidase 18

Z-DNA binding protein 1

Figure 4.20 The genes with topmost expression in RSV-infected macrophages. RSV was infected with macrophages, and the expression of genes with top differential expression was examined by microarray analysis at 4 and 24 hpi as shown. The data is represented by heat map analysis showing up-regulated (red), down-regulated (green) or no changes (black) in expression, and the FC range is indicated (P-value $\leq 0.05)$ 


\section{Chapter V. Influenza A viruses}

\subsection{Introduction}

Influenza A viruses (IAV) are classified by the surface glycoproteins hemagglutinin (HA) and neuraminidase (NA). To date, almost all combinations between 16 subtypes of HA and 9 subtypes of NA have been found [Figure 5.1]. All known subtypes are isolated from wild birds, and 3 subtypes have been found that circulated in humans. Numerous subtypes have also been identified in poultry. Among these subtypes, H5 and H7 viruses may cause outbreaks of highly pathogenic avian influenza (HPAI) virus, which can be generated from low pathogenic avian influenza (LPAI) virus through the shift of amino acids at the HA cleavage site.

In humans, seasonal IAV H3N2 annually contributes to millions of human infections worldwide, which have caused severe health and economic concerns [204]. Pandemic IAV that spread from human to human efficiently have also posed serious threats to public health [205]. In 1957 and1968, two overwhelming pandemics, caused by H2N2 (Asian flu) and H3N2 (Hong Kong flu) caused excess mortality, especially in infants, the elderly and persons with chronic diseases [376]. The outbreak of H1N1 (also called 'Spanish influenza') in 1918 is the best-known example of a pandemic, with around 50 million individuals died [206]. In 2009, a novel strain of influenza A (H1N1) was recognized to have caused outbreaks of serious respiratory illness in the worldwide [207].

In 1997, an outbreak of HPAI H5N1 in poultry occurred, with 18 people infected and 6 died. This record provided the first evidence that an avian influenza virus is able to infect humans and finally cause fatality. In the following years, this virus also caused major outbreaks in southeast Asia, with the mortality rate of human cases reaching around 50\%. By October 2006, the WHO declared that 256 cases from 10 countries in Asia and Africa were reported [208]. The latest data from WHO website has pointed out that H5N1 has resulted in 641 cases, of which 380 were lethal [367].

The H6N1 subtype was isolated with seven H5N1-like segments. The high homology between the genes from H9N2, H6N1 and H5N1 allows their internal gene exchange and therefore provide a potential source of new 
pathogenic influenza virus strains. Experiment performed by Hoffmann E et al (2000) revealed that A/teal/Hong Kong/W312/97 (H6N1) virus behaved more like the less pathogenic H5N1 group initially but more like the highly pathogenic H5N1 group by the third passage after rapidly adapted to mice [209]. Investigation suggested that the initial lower pathogenicity might be due to the missing of the highly cleavable HA.

Several outbreaks of $\mathrm{H} 7$ viruses (LPAI and HPAI) in poultry have been reported to result in transmission to humans. In 1996, a H7N7 virus (LPAI) was transmitted from ducks to a 43-year-old woman. In 2003, a large outbreak of H7N7 virus (HPAI) resulted in the infections of 89 humans, with 1 died. In 2004, an outbreak of H7N3 virus (HPAI) caused mild influenza-like illness in two individuals. In 2006, an outbreak of H7N3 virus (LPAI) led to a case of conjunctivitis in a poultry worker. In 2007, a low pathogenic H7N2 virus contributed to infections of several people [210, 211].

On 31 March 2013, the Chinese National Health and Family Planning Commission announced the occurrence of three human infections with H7N9 subtype influenza viruses. By 25 Apr 2013, 112 human cases have been confirmed, including 22 deaths [212]. Detailed sequence analysis suggested that this virus was reassorted from previous avian viruses that circulate in birds and ducks. H7 influenza viruses are originated from 3 major lineages, the Eurasian (EA), American, and equine lineages, and six internal genes are derived from avian influenza A (H9N2) viruses. Furthermore, this H7N9 virus has been detected in poultry and environmental samples obtained in wet markets, implying that H7N9 virus-infected poultry might be one of the sources of human infections [213].

It is striking that some of these human H7N9 viruses have evolved mutations in order to facilitate avian viruses to adapt into mammalian hosts. Based on the three H7N9 samples identified in the first three patients who presented with fever, cough, and dyspnea, Gao and colleagues (2013) reported that substitution Q226L at the 210-loop in HA protein was found in two of the three samples [214], another substitution T160A at the 150-loop in HA protein and a deletion of five amino acids in the NA stalk region were commonly found in all three samples [215]. 
Recently, another subtype of HA identified in bats is significantly divergent from all know influenza A viruses [366]. This HA of bat virus was estimated to have diverged at roughly the same time as the known subtypes of HA and was designated as H17.

\subsubsection{Virus structure}

IAV, as negative sense, single-stranded and segmented RNA viruses, belong to the Orthomyxoviridae family. IAV have eight segments that encode 11 or 12 viral proteins: the nuclear export protein NS2/NEP, the non-structural protein NS1, the matrix protein M1, the ion channel protein M2, the haemagglutinin (HA), the neuraminidase (NA), the nucleoprotein (NP), the polymerase acidic (PA), the polymerase basic 1 (PB1) and the polymerase basic 2 (PB2). Besides, a few novel proteins have been also identified recently, for example, two proteins called M42 and PA-X are both encoded by segment 3 [369] [388], and another two proteins, PB1-N40 and PB1-F2, are both encoded by segment 2 .

The viral envelop is made up of a lipid bilayer, which derives from host cell's plasma membrane. This bilayer contains both cholesterol-enriched lipid rafts and non-raft lipids, with three viral transmembrane proteins HA, NA and M2 included. Among these three transmembrane proteins, HA mediates the binding of virus to host cells and the entry of viral genome, while NA cleaves sugars that bind the mature viral particles so as to release progeny virus from infected cells. M2 proteins with a minor component of the envelop proteins process ion channel activity. M1 proteins which locate underneath the viral lipid membrane form a matrix holding the viral ribonucleoprotein (vRNPs). And these vRNPs are made up of the viral negative stranded RNAs, which are wrapped by NP. Three polymerase PB1, PB2 and PA proteins attach the end of each vRNP to form the RNA polymerase complex [Figure 5.2] [216].

\subsubsection{Virus replication cycle}

In general, IAV replication cycle can be separated into five stages as shown in Figure 5.3. 


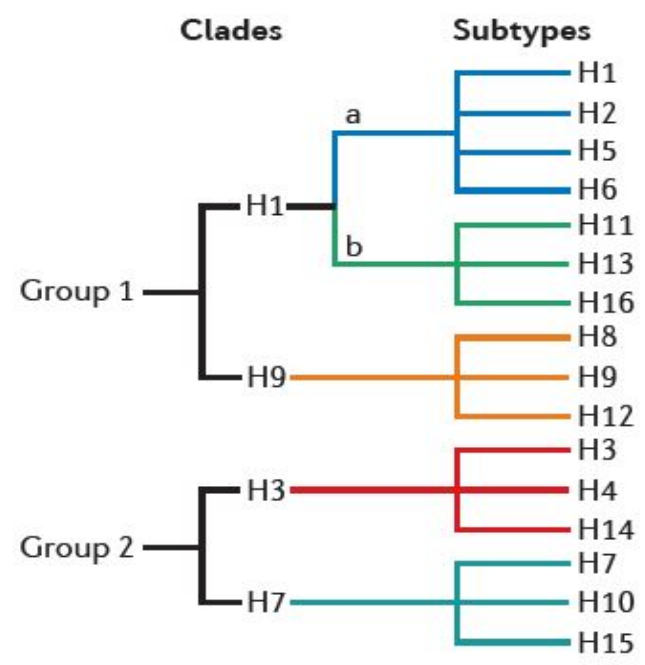

Figure 5.1 Classification of IAV based on HA. 16 subtypes can be summarized into 5 clades and 2 groups in further (adapted from Medina RA et al., 2011)[217].

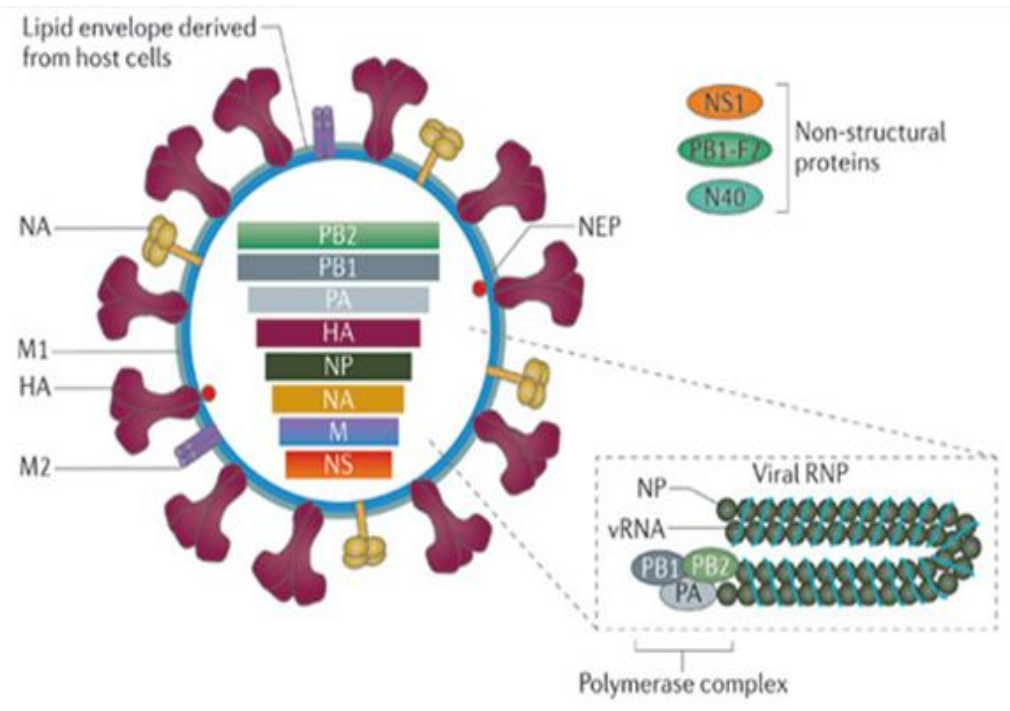

Figure 5.2 IAV genome structure (adapted from Medina RA et al., 2011)[217].

\subsubsection{Entry of virus into the host cell}

HA forms spikes on the viral lipid membrane, and these spikes of HA bind to sialic acid receptors on host cell surface. Two major linkages that are important for HA specificity have been found between sialic acids: $\alpha(2,3)$ and $\alpha(2,6)$. And these two linkages are the determinant in restricting the transmission of IAV in different species. For example, $\alpha(2,6)$ linkage is recognized in viruses from humans while $\alpha(2,3)$ linkage is recognized in those from avians and equines. In addition, both these two linkages are able to be recognized in viruses from swine. 
Virus enters into the cell as an endosome. Low PH in endosome, around 5 to 6 , is able to trigger the fusion of both viral and endosomal membranes. After HA is cleaved by cellular proteases, HA2 fusion peptide is exposed to insert itself into the endosomal membrane, causing the contact between viral membranes and endosomal membranes. Besides, the acidic environment of the endosome also opens up the M2 ion channel, which results in acidification of the inside of the viral core and subsequently un-coats the RNP complex and releases the vRNP from M1 into host cell's cytoplasm [368][385-386].

\subsubsection{Entry of vRNPs into the nucleus}

Since transcription and replication of IAV occur in the nucleus, the vRNP must enter the nucleus after the virus entry into the cytoplasm. The vRNA consists of NP, PA, PB1 and PB2: the NP coats the viral RNA, and the remaining three polymerase proteins bind to the partially complementary ends of the viral RNA. Subsequently, polymerase proteins and nucleoprotein create a distinctive panhandle structure together with the viral RNA.

The width of RNPs is too large to allow direct entry into the nucleus. Thus, they must rely on an active nuclear import mechanism. Nuclear localization signals (NLSs) can mediate specific interaction with cellular nuclear import machinery. After the dissociation with the M1 protein, the RNPs enter the nucleus through the nuclear pore. There are two types of importins: importin $\alpha$ recognizes and binds the NLS on the cargo proteins first and then importin $\beta$ recognizes and bounds to the complex, subsequently the whole complex binds to the fibrils of the nuclear pore complex (NPC) to complete the actual translocation. It should be noted that the two viral proteins, M1 and NS2/NEP are critical for assisting the nuclear export of RNPs [218]. 


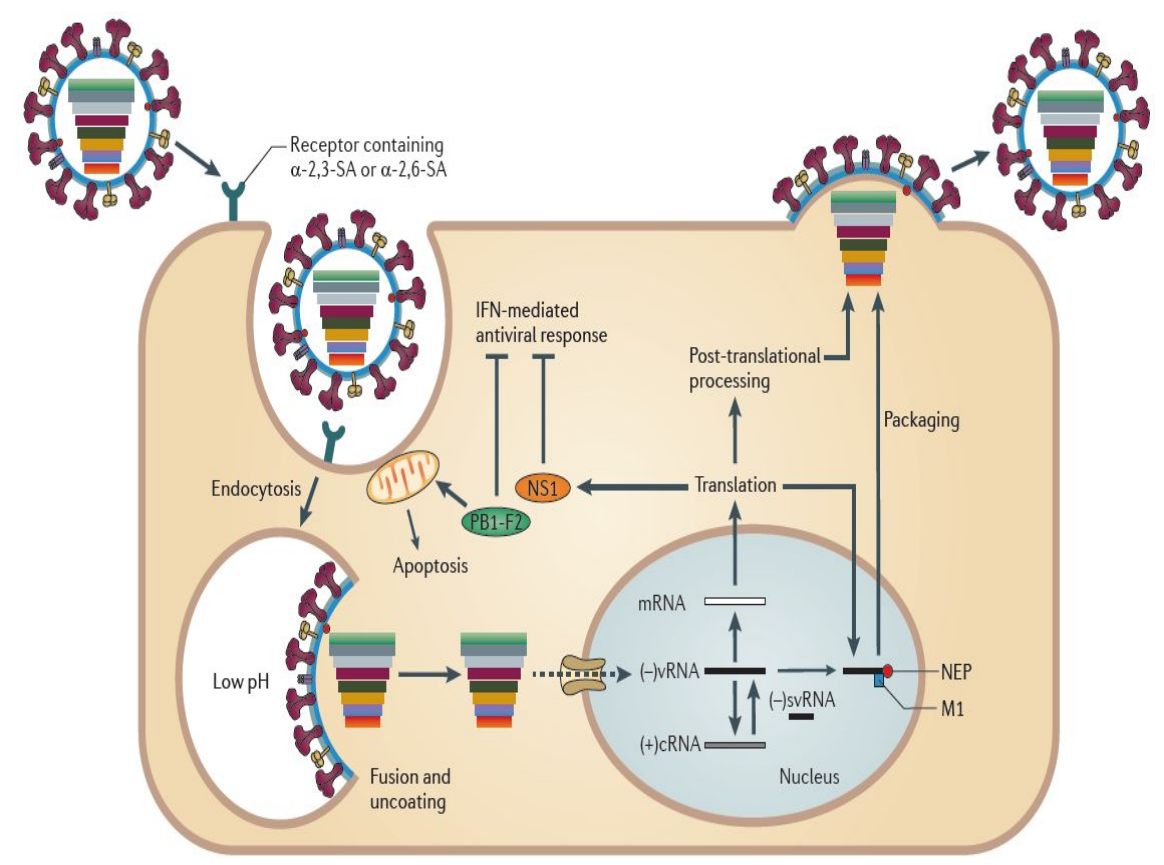

Figure 5.3 Replication cycle of IAV (adapted from Medina RA et al., 2011)[217].

\subsubsection{Transcription and replication of viral genome}

It is known that IAV only encode 11 or 12 proteins which are not enough for own transcription. Hence, IAV have generated sophisticated methods to hijack the transcription machinery in host cells for its own benefits. Because the IAV are negative sense RNA viruses, the negative sense RNA must be converted into the corresponding positive sense RNA first to serve as a template to produce viral RNAs.

As we know, mature mRNAs have a 5' methylated cap and a poly(A) tail. However, the vRNPs do not have either 5' methylated cap or poly(A) tails. Studies have approved the existence of "cap-snatching" mechanism [Figure 5.4]. In the process of "cap-snatching", PB2 from viral RNA polymerase has endonuclease activity. This ability allows PB2 bind to the 5' methylated caps of mRNAs and cleave the mRNAs' 10 to 15 nucleotides 3' to the cap structure [219]. This cleaved host-capped mRNA is then used as a primer for the initiation and transcription of the PB1 protein. Since the PB1 protein binds both the 5'- and 3'- ends of the viral mRNA, the polyadenylation and termination of the vRNAs occur before the 5 ' end is reached.

Among all the eight segments in influenza genome, some of them encode for two proteins. For example, M1 and M2 are encoded by segment 7; 
NS1 and NS2/NEP are encoded by segment 8 . To encode more than one protein, influenza virus effectively uses host cell's splicing machinery again.

The switch from viral mRNAs to template RNAs requires the change from capped RNA-primed initiation to unprimed initiation. This different initiation strategy will prevent termination and polyadenylation at the poly(A) site, which is used during viral mRNA synthesis [220]. The replication of vRNA is fulfilled by two steps: the synthesis of template RNAs and the copying of the template RNAs into vRNAs. NP protein is also necessary to elongate the RNA molecules.

\subsubsection{Export of vRNPs from the nucleus}

Exportins are the proteins which take cargo out of the nucleus. In IAV, chromosome region maintenance protein 1 (CRM1) as regular exportin mediates the export of numerous proteins that carry a nuclear export signal (NES) from the nucleus [221]. The vRNP nuclear export is initiated by forming a vRNP-M1-NS2/NEP complex in the nucleus, with the NES provided by NS2/NEP [222]. The nuclear export signal also will overcome the NLS sequence in the NP and polymerase protein. NS2/NEP protein interacts with CRM1 and CRM1 will associate with RAN-GTP protein to mediate export of the vRNP-M1-NS2/NEP from the nucleus into the cytoplasm through CRM1 mediated pathway [218]. Finally, formed CRM1-RAN-GTP-NS2/NEP-M1vRNP complex will be allowed to export nucleus. After the export action finishes, the complex will dissociate followed by the dephosphorylation of RAN-GTP to RAN-GDP [Figure 5.5].

\subsubsection{Assembly and budding of virus}

After the vRNPs leave the nucleus, the left thing is to form viral particles and leave the cell. The plasma membrane from host cell is used to form IAV particles. When the virus particles bud from the apical side of polarized cells, HA, NA and M2 are translocated into ER through Trans-Golgi networking and transported into the apical plasma membrane. Different kinds of

proteins play different roles in the process: HA is used to initiate the budding process, however, NA can compensate the role of HA when laking of HA; M1 may alter membrane curvature and cause membrane budding; M2 is essential 
and necessary for the completion of virus budding as well as virion release [Figure 5.6] [223]. Packaging of an infectious influenza virus particle will require the incorporation of all eight segments of the viral genome, and an organized packaging method might exist to correctly incorporate eight different segments into the virion, possibly either by RNA interactions or protein-protein interactions [224]. The HA and NA contain terminal sialic acids that make the viruses to clump together and adhere to the cell surface. After that, NA of newly assembled viruses cleaves these sialic acid residues and subsequently releases the virus from the host cells. In this process, the filamentous virion assembly is dependent on the microfilament network, indicating a raft-actin interaction that maintains the necessary organisation of HA-containing lipid rafts for virus budding [225].

\subsubsection{Viral-Host interactions}

The viral infection generally initiates a complex set of interactions between the viruses and their targeted host cells. The specific antiviral signaling is triggered in host cells upon the viral infection. At the meanwhile, viruses have evolved some specific mechanisms to counteract with antiviral responses. Subsequently, these viral-host interactions determine the severity of the disease and the performances of the adaptive immune response [226].

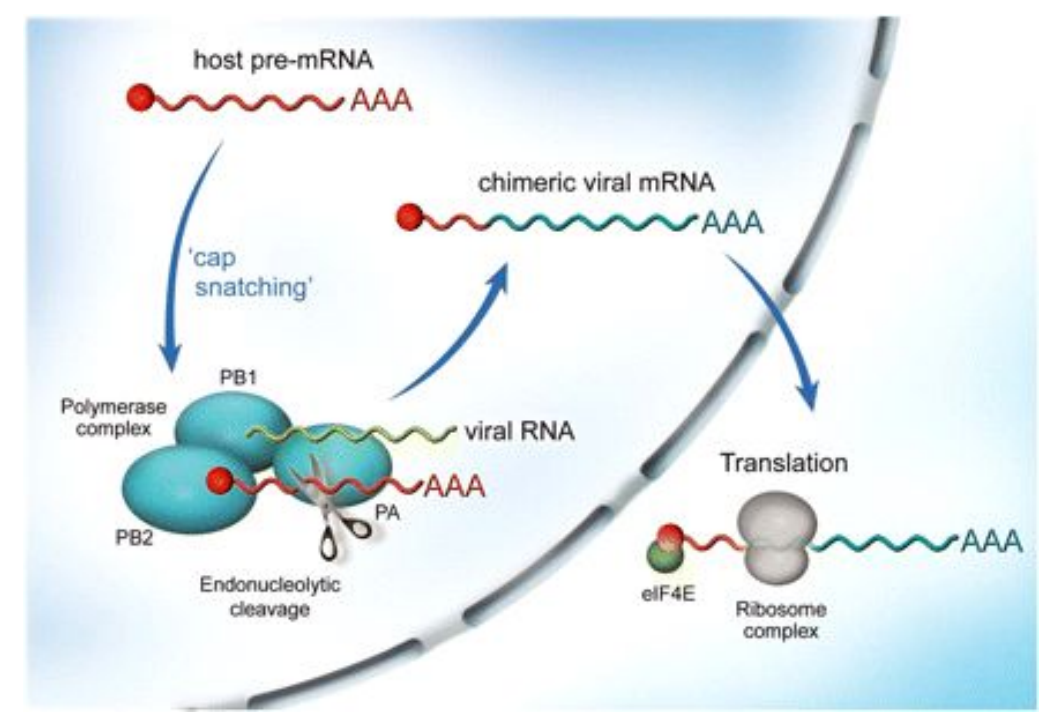

Figure 5.4 Cap-snatching transcription mechanism. Influenza polymerase consisting of PA, PB1 and PB2 locates in the nucleus. During the process of viral transcription, PB2 binds the 5', 7-methylguanosine cap of a host pre-mRNA molecule (red) and cleaves 10-15 nucleotides downstream by the PA endonuclease. This cleaved host-capped mRNA initiates polymerization 
by the PB1 subunit using 5'- and 3'-bound vRNA (green) as template, and thus the capped, polyadenylated, chimeric mRNA molecules are synthesized (red and blue). Finally, these mRNA molecules are exported to the cytoplasm for further translation (adapted from Boivin $\mathrm{S}$ et al., 2010) [219].

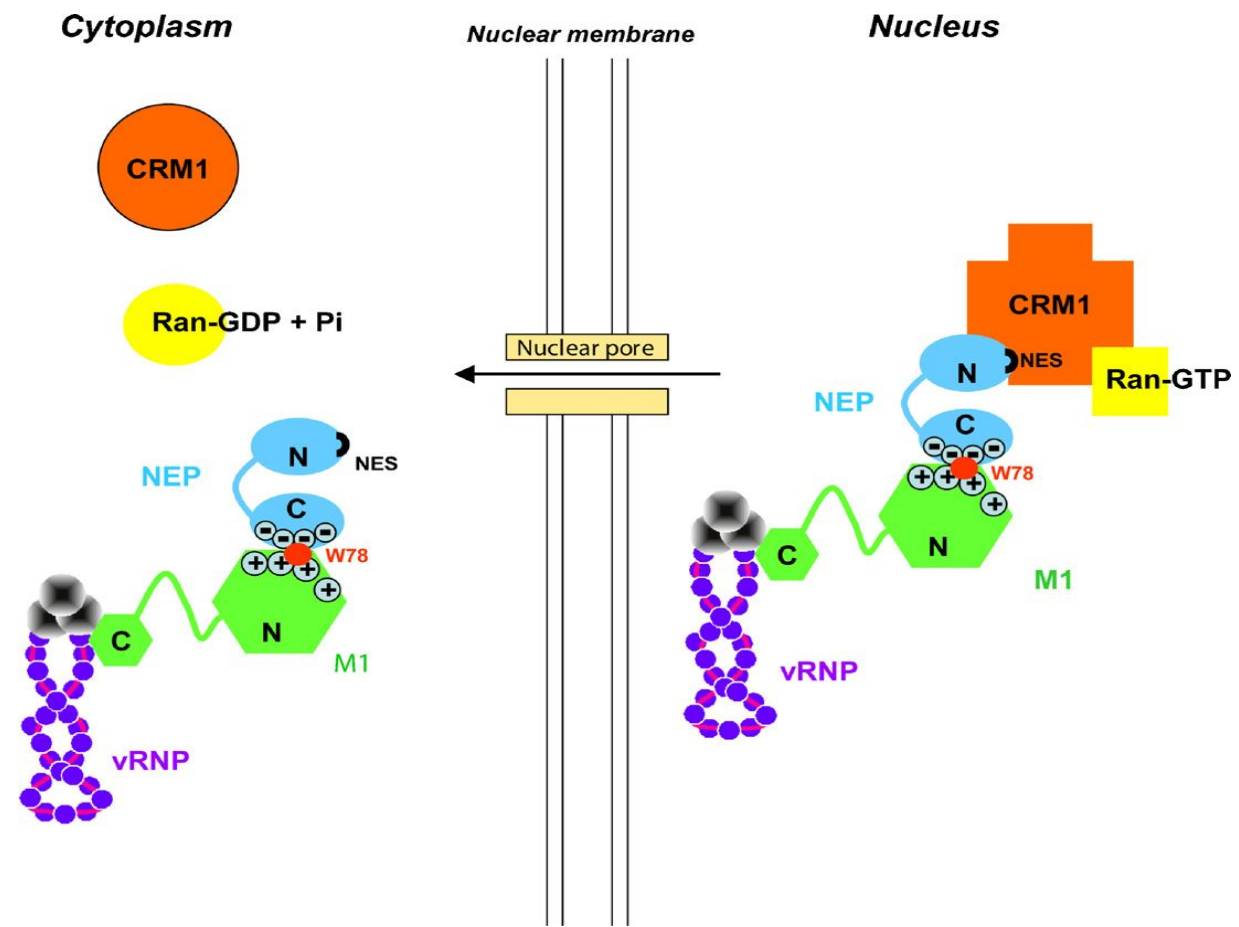

Figure 5.5 Strategy of the newly assembled IAV RNPs exporting from the nucleus to the cytoplasm. There are conformational changes of CRM1 and Ran occurred upon hydrolysis of GTP and subsequent dissociation of the complex from its cargo (adapted from Boulo $\mathrm{S}$ et al., 2007) [218].

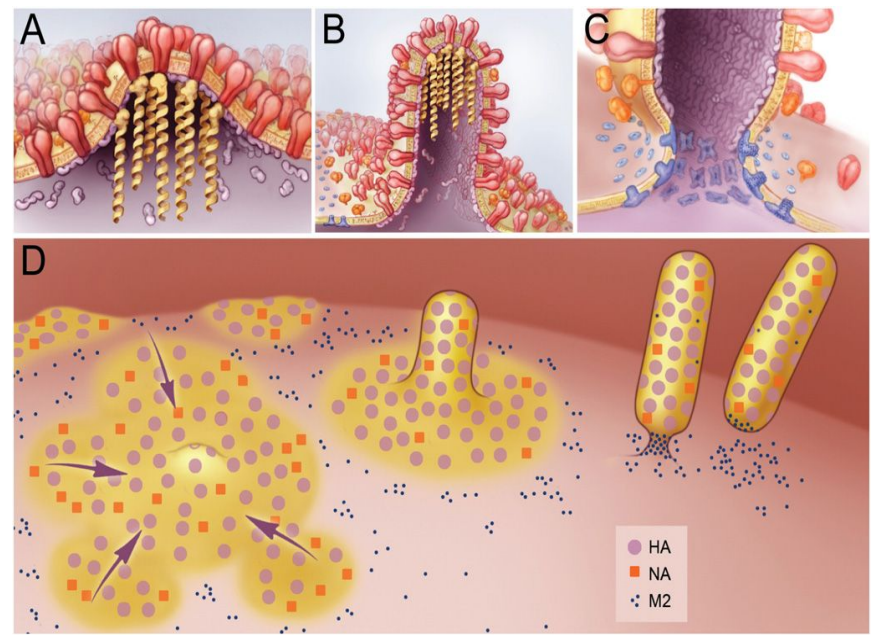

Figure 5.6 Model of IAV budding. A) M1 (shown in purple) binds to the cytoplasmic tails of HA (shown in red) and NA (shown in orange) in order to serve as a docking site for the vRNPs (shown in yellow), which in turn to initiate the virus budding. B) Polymerization of the M1 results in the elongation of the budding virion, and thereby a polarized localization of the vRNPs is formed. C) M2 (shown in blue) is inserted at the lipid phase boundary so as to alter the membrane curvature at the neck of the budding virus and lead to the virus release. D) Global strategy of the budding process of IAV (adapted from Rossman S et al., 2011) [223]. 


\subsubsection{Activation of the innate type I IFN system}

TLRs and RIG-I have been proved to be involved in induction of interferons during IAV infection. TLR1/2/4 locate on the cell surface, while TLR3/7/8/9 have been detected to be expressed in intracellular compartments. During the process of virus entry, IAV fusion the envelope so as to release the segmented genome. In this situation, some virions may expose the viral RNA to TLR3/7/8 present in the host cells, which recognize the single stranded RNA. Before assembly of new virions, the newly synthesized viral RNA segments move to the cytoplasm and are recognized by RIG-I. Thus, TLR signaling by the adaptors TRIF or MyD88 and RIG-I signaling by the adaptor MAVS trigger a signal transduction, which in further activate IRF3/7 and NF- $\kappa$ B factors, and these factors are then translocated into the nucleus to stimulate the synthesis of type I and type III IFN mRNAs [226].

\subsubsection{Type I IFN stimulated gene products}

As described above, IFN $\alpha / \beta$ binds to IFNAR once it is secreted. This action directly stimulates the JAK/STAT pathway, which induces formation of the trimeric transcription factor ISGF3 that consists of STAT1, STAT2 and IRF-9. And this transcription factor subsequently regulates the expression of ISGs, such as Mx1, p56, the 2' - 5' oligoadenylate synthetases (OAS), Viperin (RSAD2) and the protein kinase R (PKR). These ISGs spread widely to fight with the IAV replication [8].

\subsection{Myxovirus Resistance (Mx)}

Mx proteins comprise a small family of GTPases, and their resistance phenotype is specific to the members in the orthomyxovirus family. Gene expression of Mx1 is rapidly induced through the action of virus-induced type I $(\alpha / \beta)$ or type III $(\lambda)$ IFNs upon virus infection. Relevant studies have provided evidences that Mx1 has intrinsic antiviral activity and is the majorly effective molecule which is able to protect infected animals from severe influenza and even death [227].

In humans, an Mx homologue (MxA) is encoded by the human MX1 gene. The human MxA protein also exhibits similar effects to murine Mx1 protein. However, both human MxA protein and murine Mx1 protein have 
different mechanism. Human MxA protein inhibits steps involved in viral replication and transcription, which develops in the later phase of viral replication. Murine Mx1 inhibits in the early viral transcription steps. In spite of the difference in mechanism of Mx in different species the Mx proteins of the two species recognize the same or similar viral target structures. Besides, it has been also demonstrated that different strains of IAV might have different degree of sensitivity against the Mx proteins [228]. In contrast to Mx1, cytoplasmic Mx2 (also known as MxB) showed ineffective against influenza virus [372] and influenzavirus replication was also not affected by the expression of MX2 protein in Vero cells [384].

\subsection{The 2' - 5' oligoadenylate synthetase and RNase $L$}

OAS catalyzes the synthesis of $2^{\prime}-5^{\prime}$ connected oligoadenylates, commonly abbreviated 2-5A. RNase L becomes activated by binding 2-5A oligonucleotides and subsequently degrades viral and cellular RNA [229]. All in all, OAS/RNaeL impairs the efficiency of viral replication through creating an antiviral state within the cells.

\subsection{Viperin (RSAD2)}

Viperin, as an ISG, is induced by IFNs after infections of a broad range of DNA or RNA viruses. Recently, it has been published that Viperin is able to block IAV budding and release [230]. On one hand, Viperin expression affects the formation of lipid raft, which is the site of IAV budding. On the other hand, Viperin interacts with an inhibitor of farnesyl diphosphate synthase (FPPS). FPPS is a one of the crucial enzymes involved in cholesterol metabolism pathway. Experiments proved that reduction of FPPS expression by Viperin inhibited IAV replication and release [231].

\subsection{The protein kinase $R$}

PKR as a dsRNA dependent protein kinase plays an essential role in the antiviral response against IAV infection. PKR has several downstream substrates, such as IRF3 and eukaryotic translation initiation factor 2 alpha subunit (eIF2 $\alpha$ ). Following virus replication, the generation of dsRNA activates the stress-induced MAPK pathway p38/JNK resulting in the AP-1 activation. 
PKR is activated following binding to dsRNA, phosphorylates eIF-2 $\alpha$, and inhibits protein translation. PKR may also associate with the IKK $\beta$ subunit. Viral nucleocapsid (N) and dsRNA activate VAK, a virus activated kinase, leading to C-terminal phosphorylation of IRF-3. IRF-3 activation stimulates target genes such as RANTES, IL-15, and IFNs [] (Servant MJ et al., 2002). Thus, PKR is not directly involved in the activation of IRF3. Activation of PKR also results in the phosphorylation of so that eIF $2 \alpha$ is not able to participate in translation initiation and protein synthesis of viral mRNAs. In addition, PKR is also able to regulated NF- $\mathrm{BB}$ signaling and ISGs [232]. To offset the antiviral role of PKR, IAV have evolved several inhibitory mechanisms to offset the antiviral function of PKR. For example, NS1 of IAV directly binds to PKR so as to prevent its activation; IAV nucleoprotein also exploits Hsp40 to inhibit PKR.

\subsubsection{Viral strategies to counteract to the innate immune responses}

IFN $\alpha / \beta$ expression is a host immune response to IAV infection at an early stage. Facing to the antagonistic response, pathogens have evolved different mechanisms to prevent IFN expression as well as IFN induced signaling.

\subsection{NS1 - a viral antagonist of the innate immune response}

In IAV, the activities of NS1 are not only important for the pathogenicity of IAV but also partially responsible for the ability of IAV to infect multiple animal species [226].

In the first place, NS1 is essential for antagonizing IFN $\alpha / \beta$-dependent responses through limiting IFN $\beta$ production from two respects: pretranscriptional and post-transcriptional limitation of IFN $\beta$ induction. (i) Studies on PR8/NS1 show that it may mediate pre-transcriptional block on IFN $\beta$ induction by forming a complex with RIG-I dependent upon two residues in NS1: Arg-38 and Lys-41; (ii) To prevent the nuclear post-transcriptional processing by targeting to RNA polymerase II is another common strategy for NS1 to limit IFN $\beta$ production in many IAV infected cells [233].

Secondly, NS1 protein can directly inhibit IFN-responsive factors including OAS/RNase L and PKR. Both these two factors are key regulators of 
viral transcription and translation processes, and also take action on host innate defence such as IFN $\beta$ induction and apoptotic response [234]. Since the activation of RNase L is totally dependent on dsRNA activation of 2-5 OAS, the action of NS1 out-competing OAS for interact with dsRNA can lead to inhibition of IFN $\alpha / \beta$ induced OAS/RNase L pathway [235]. Studies have also revealed that NS1 (residues 123-127) binded to linker region in PKR, and thereby prevented a conformational change that is required for release of PKR auto-inhibition [236].

Thirdly, NS1 also interacts with other cellular antiviral factors such as RIG-I. Gack MU et al (2009) described that the NS1 protein interfered with TRIM25 multimerization through binding its CCD [237]. TRIM25 multimerization is required for ubiquitination of RIG-I CARDs, a modification that is necessary for maximizing IFN production. Thus, directly inhibiting the activity of TRIM25 by NS1 enables to suppress RIG-I signal transduction and ultimately IFN- $\beta$ production.

Additionally, NS1 also displays some anti-apoptotic functions. For example, PI3K and its downstream effector, Akt, function on cell growth, proliferation and survival. And it has been proposed that NS1 activated PI3K signaling by binding to its $p 85-\beta$ regulatory subunit [238].

\subsection{The role of PB1-F2}

Besides the NS1, some subtypes of IAV express a second viral nonstructural protein, PB1-F2. This protein is generated from PB1 gene by an alternative open reading frame (ORF) [239]. Recent researches have proved that PB1-F2 is associated with the host immune and cell death responses in IAV infected cells. Dudek SE et al (2011) found that PB1-F2 exhibited IFN $\beta$ antagonistic activities by interfering with the RIG-I RNA-sensory pathway at the level of the adaptor protein MAVS but not inhibiting IKK $\varepsilon$-mediated expression of IFN $\beta$ [240]. Furthermore, PB1-F2 could also induce the apoptosis of BAL immune cells through TNF/FasL-mediated apoptosis signaling pathway [241]. Therefore, a virus that produce the active PB1-F2 protein is considered as a highly pathogenic virus. Moreover, a single amino acid substitution N66S in the PB1-F2 molecule has been reported as a contributing factor for the high virulence and lethality of the virus [242]. Recent studies have also indicated that 
PB1-F2 was able to inhibit NF-אB dependent signaling [377] and it also initiated caspase-3-independent apoptosis under regulation of sulfatide [378].

\subsubsection{Influenza A viruses in different hosts}

\subsubsection{Human}

Currently, there are two HA subtypes (H1 and H3) and two NA subtypes (N1 and N2) that are circulating and transmit efficiently among humans.

\subsection{Host response to H1N1}

H1N1 is one of the IAV subtypes and is currently endemic in human population. The typical seasonal influenza virus infects approximately $5 \%$ of total population with 100,000 deaths annually. The first H1N1 subtype was first successfully isolated by Wilson Smith in 1933, which is named A/WS/33 (H1N1). There was a major influenza pandemic caused by H1N1 influenza virus strain in 1918, commonly known as "Spanish Flu". After the 1918 "Spanish Flu" pandemic, the H1N1 subtype of virus diminished rapidly for almost 50 years, and the pandemic occurred with H2N2 and H3N2 in 1957 and 1968 [243]. Since then, both H3N2 and H1N1 re-circulate in human population. Geiss GK et al (2002) pointed out that a virus containing the 1918 pandemic NS1 gene was able to block the expression of IFN-regulated genes more efficiently than its parental influenza A/WS/33 virus in human lung cells [244], which demonstrate the significant influence by the sequence of the NS1 gene in counteracting to the host antiviral response triggered by virus infection.

\subsection{Host response to $\mathrm{H} 3 \mathrm{~N} 2$}

Currently, influenza A virus $\mathrm{H} 3 \mathrm{~N} 2$ is the important seasonal human influenza that cause higher morbidity and mortality than H1N1. Annually, H3N2 influenza viruses infect 5-15\% of the total population [245]. Since 2002, the antigenic evolution of $\mathrm{H} 3 \mathrm{~N} 2$ viruses has followed from previously dominating the A/Sydney/5/1997-like viruses and A/Fujian/441/2002-like viruses to the $\mathrm{A} / \mathrm{California} / 7 / 2004-$ like viruses and to the A/Wisconsin/67/2005-like strains in a 5 year span [246]. And A/Perth/16/2009 and A/Victoria/361/2011 were responsible for the epidemic in 2011-2012 
season. Global genomic gene expression profiles from the individual subjecting to infection of $\mathrm{H} 3 \mathrm{~N} 2 / \mathrm{Wisconsin}$ have revealed that potential immune response events determined the pathogenicity of influenza viral infection [247]

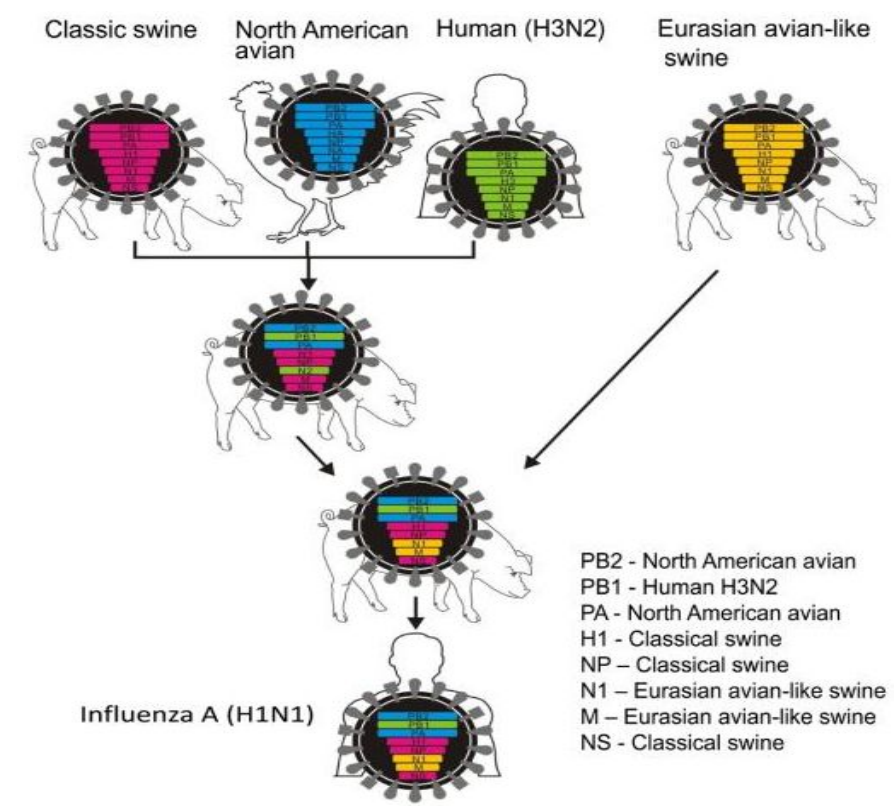

Figure 5.7 The origin of pandemic H1N1 2009 virus (pH1N1) (adapted from Neumann G et al., 2009)[248].

\subsection{Host response to Pandemic 2009 H1N1 Swine influenza virus}

A novel swine-origin influenza A virus initially had an outbreak in Mexico and then spreaded rapidly in the rest of the world. By June 2009, nearly 30,000 cases of $2009 \mathrm{H} 1 \mathrm{~N} 1$ virus (pH1N1) had been confirmed in the worldwide, prompting the WHO to declare the outbreak of the 2009 influenza pandemic [249]. Recent researches indicate that the $\mathrm{pH} 1 \mathrm{~N} 1$ virus arose from a reassortment of triple reassortment: HA, NP and NS from classic swine virus origin; PB2 and PA from the North American avian virus origin; PB1 from human seasonal H3N2; NA and M from Eurasian avian-like swine virus origin [Figure 5.7] [248].

Mukherjee S et al (2011) examined the global host response at a series of time points in A549 cells based on microarray technique [250]. The results demonstrated that $\mathrm{pH} 1 \mathrm{~N} 1$ induced immune response earlier than seasonal H1N1 viruses. However, the host immune responses were suppressed at the late stages of $\mathrm{pH} 1 \mathrm{~N} 1$ infection, with considerable expression decrease of cytokine and other immune genes. In addition, both viruses, especially $\mathrm{pH} 1 \mathrm{~N} 1$, had the property of antagonizing IFN $\beta$. In conclusion, these observations indicated 
greater replication ability of $\mathrm{pH} 1 \mathrm{~N} 1$ in A549 cells when compared to seasonal H1N1. A comprehensive investigation on innate response has indicated that the productions of a scale of cytokines were induced under pH1N1 infection, and it was also found that the IL6 and IL10 production were high in patients under severe pH1N1 infection [251, 252]. Microarray analysis also revealed that infection of $\mathrm{pH} 1 \mathrm{~N} 1$ contributed to a more potent early immune response with higher expression levels of genes relevant to lipid metabolism and cell death when compared to infection of 1918-like classical swine influenza virus in pigs [253].

\subsubsection{Avian}

Wild birds are the natural host for all known subtypes of IAV, and they do not become sick when infected with avian IAV. In general, avian influenza viruses (AIV) are classified into HPAI or LPAI based on their intravenous pathogenicity. LPAI viruses may have been considered of negligible risk, but there is evidence that HPAI might arise from LPAI by mutations. Avian IAV of $\mathrm{H} 7, \mathrm{H} 9$ and $\mathrm{H} 5$ subtypes have been reported for human infection.

\subsection{Host response to H5N1}

Among the most well known HPAI outbreaks was HPAI H5N1. The first outbreak of H5N1 HPAI was reported in Hong Kong in 1997 in poultry. Later, this virus was classified as HPAI because of its high mortality rate (70\%$100 \%$ ) in chickens. It also possess $60 \%$ mortality rate in humans [254]. Although avian $\mathrm{H} 5 \mathrm{~N} 1$ continues to transmission to humans, this virus has not adapted to efficient human-to-human transmission till now.

Zeng $\mathrm{H}$ et al (2007) did a series of experiments to assess the potential virulence of HPAI H5N1 (2004) in human cells [255]. It was observed that $\mathrm{H} 5 \mathrm{~N} 1$ triggered a delayed and weaker IFN $\beta$ response and ISGs production than human H3N2 virus. Consequently, a highly virulence H5N1 virus showed a better ability to attenuate the host IFN response than less virulent human viruses in human respiratory cells. In addition, it has been also proposed that H5N1 virus showed sensitive to the antiviral activity of Mx [370]. 


\subsection{Host response to H9N2}

IAV H9 subtype have been extensively circulated in the worldwide and caused a series of outbreaks in chickens, ducks and pigs after its discovery. In 1999 and 2007, self-limiting mild human infections with LPAI H9N2 were recorded in Hong Kong. And it has been also pointed out that some avian H9 viruses have acquired receptor binding characteristics typical of human strains, which may increase the potentiality of reassortment in both human and swine respiratory tracts.

In human epithelial cells, H9N2/1997 was observed to replicate at a lower rate than $\mathrm{H} 1 \mathrm{~N} 1 / 2002$ and $\mathrm{H} 5 \mathrm{~N} 1 / 2004$. In the early phase, infections of H5N1/2004 and H9N2/1997 viruses generated stronger up-regulated expression of TNF- $\alpha$, RANTES, CXCL-10/IP10 than infection of H1N1/2002. And TNF- $\alpha$, IL-6, IL-8, RANTES and CXCL-10/IP-10 remained at high mRNA expression levels in the late phase of H5N1/2004 and H9N2/1997 infections but not H1N1/2002 infection [256].

\subsubsection{Objective}

As we know, different IAV strains interact with different host cells in various ways. It is interesting to note that some IAV strains are able to infect and replicate well in particular hosts, but others not. This phenomenon suggests that there must be specific interaction patterns to assist or inhibit the virus growth in the different combinations of each virus and each host cell. Avian-tohuman transmission of HPAI viruses are often associated with high fatality rates, whereas associated fatalities due to human transmission of LPAI viruses have not been reported. In addition, AIVs can play a role in the evolution of seasonal influenza virus strains, with unpredictable consequences. LPAI viruses that are circulating in avian populations can lead to the emergence of HPAI viruses [379]. Pathogen-host interactions have been relatively well characterized in laboratory-adapted influenza viruses and in some HPAI virus isolates (e.g. H5N1), but in general our understanding of host interactions during AIV infection is comparatively poor [380].

In this report, we examined the host responses of representative LPAI viruses that were circulating in South-East Asia. These viruses, including H5N2, H5N3 and H9N2 virus subtypes, were isolated from live broiler ducks imported into 
Singapore during routine surveillance, and they were the first LPAI viruses isolated in South-East Asia that were completely characterized at the genetic level. The properties of these viruses were compared with that of the laboratoryadapted human $\mathrm{H} 1 \mathrm{~N} 1 / \mathrm{WS}$ isolate, and $\mathrm{pH} 1 \mathrm{~N} 1$ virus that was isolated from humans in Singapore during the influenza pandemic in 2009. In our study, selected LPAI and two human influenza strains were applied to infected common cell line (A549, CEF or MDCK) so that the pathogen-host interaction during LPAI infection could be well evaluated. All three cell types (A549, CEF and MDCK) are standard cell types used for infection of influenza viruses. MDCK cells allow influenza virus replication and virus tiles are established based on this cell type. A549 as human alveolar basal epithelial cells and CEF as chick embryo fibroblasts are also permissive for avian influenza viruses so that they are often used for infection of influenza viruses. Since A549 are human based cell types and CEF are avian based cell types, selection of these two cell types benefits the analysis of the host proteins interactions in humans and avian.

On this basis, we chose eight IAV strains belonging to five subtypes, and infected them in four types of host cells. Global host gene expression profiles were monitored using microarray platform, and differential expression of interested genes were validated by techniques such as cytokine assays [365]. Then different types of software were applied into further analysis from different perspectives. The aims of this research are as following:

(1). Compare different gene expression performances in different virus-infected cells.

(2). Investigate the expression changes of genes belonging to interesting groupings.

(3). Establish the mechanisms which have been involved to antagonize the immune response upon IAV infection.

(4). Reveal the relationship between the viral replication performances and its corresponding global host gene expression profiles.

\subsection{Experiment workflow}




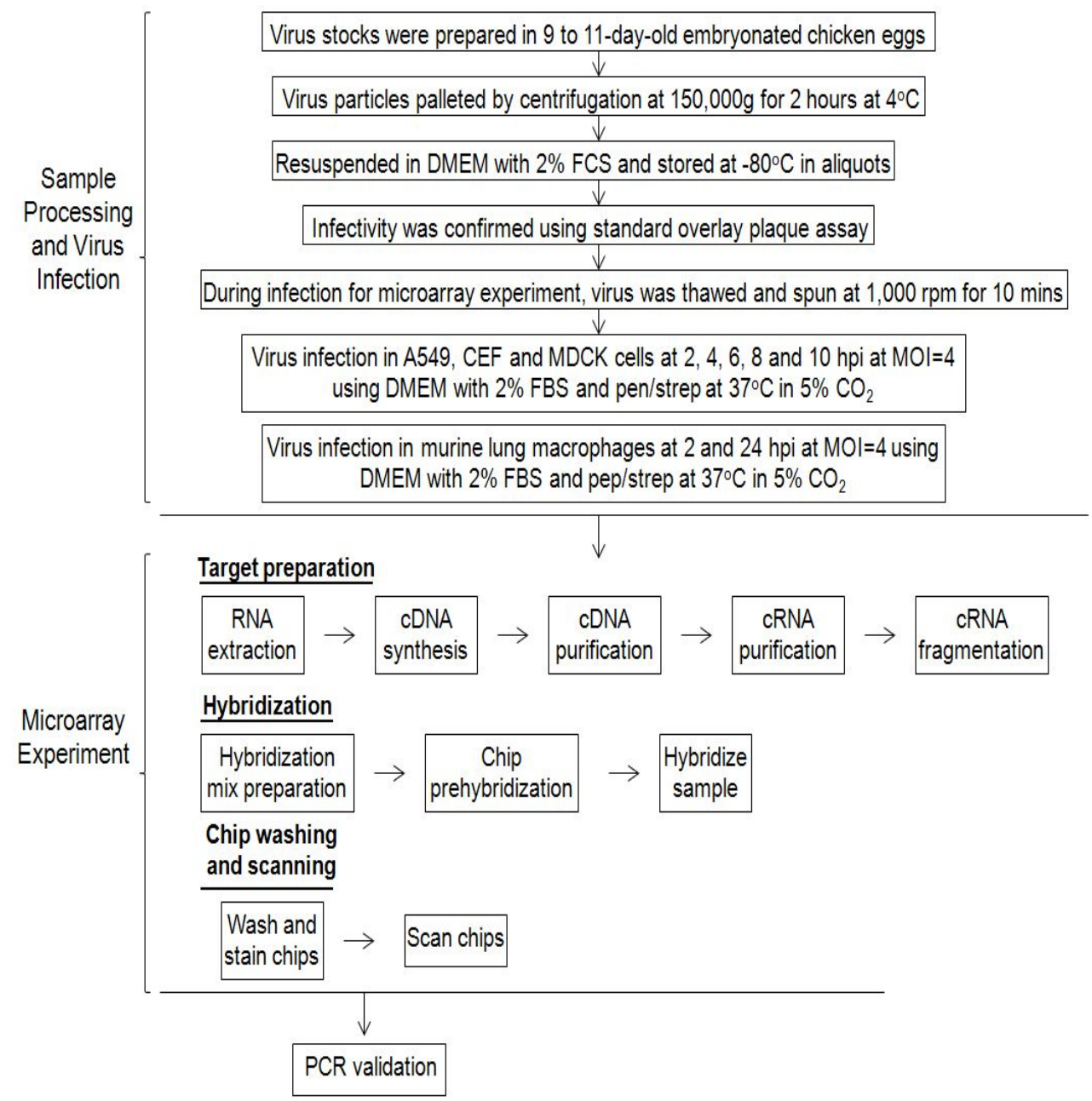

Figure 5.8 Microarray experiment workflow during influenza A strains infections.

\subsection{Result and Discussion}

\subsubsection{Host gene expression in A549 cells}

\subsubsection{Global profiling of gene expression}

\subsection{Heat maps of global gene expression}

Global heat maps showed that the numbers of differentially expressed probe sets increased with the infection time increasing in all these four viruses infected A549 cells. Detailed examination indicated that the genomic gene expression patterns were different upon infections of different influenza A virus strains [Figure 5.9]. At 10 hpi, almost half of the probe sets showed downregulated expression after two human viruses infections, while only a small batch of probe sets showed down-regulated expression after two avian viruses infections. Besides, the number of probe sets with up-regulated expression was lowest in A549 cells after infection of H1N1 when compared to other three strains. 


\subsection{Distribution of differentially expressed probe sets}

Upon different viruses infections, the largest numbers of probe sets exhibiting differential expression changes occurred at $10 \mathrm{hpi}$, therefore we compared the host gene expression performances from different influenza A viruses infections at this time point [Table 5.1]. In A549 cells, H5N3 infection induced highest percentage of probe sets with up-regulated expression, followed by H5N2/F59, pH1N1, H9N2 and H5N2/F118. Whereas, H1N1 infection only resulted in a small number of probe sets with up-regulated expression.

Despite of the lowest percentage of up-regulated probe sets in H1N1 infected A549 cells, almost half of probe sets in H1N1 infected A549 cell showed down-regulated expression. Besides infection of H1N1, infections of other influenza A strains including H5N2/F59, H5N2/F189 and pH1N1 also led to considerable batches of probe sets with the repressed expression. Among three $\mathrm{H} 5 \mathrm{~N} 2$ strains, the numbers of probe sets with up-regulated expression were similar after H5N2/F59 and H5N2/F118 viruses infections, while the numbers of probe sets with down-regulated expression were similar after H5N2/F59 and H5N2/F189 viruses infections.

\subsection{Functional classification}

In terms of biological functions from $\mathrm{GO}$ and KEGG database, differentially expressed probe sets retrieved from different influenza A strains infections at 10 hpi were classified into interested functional families. Three fold regulation scales were also offered for detailed investigation.

After classifying these differentially expressed probe sets into different functional groups, it was clear that the up-regulated probe sets were majorly grouped into "Immune Response", "RNA Binding", "DNA Binding” as well as "Signal Transduction", with probe sets associated with "Immune Response" showing high fold regulations [Figure 5.10]. It was noted that the numbers of up-regulated probe sets belonging to "Immune Response" showed lower after infections of two human strains $(\mathrm{H} 1 \mathrm{~N} 1$ and $\mathrm{pH} 1 \mathrm{~N} 1)$ when compared to other avian strains. And among these avian strains, infections of H5N2/F59 and H9N2 strains strongly induced the expression of the highest numbers of probe sets functionally associated with "Immune Response". Thus, it was assumed 
that host immune response triggered by infections of $\mathrm{H} 1 \mathrm{~N} 1$ and $\mathrm{pH} 1 \mathrm{~N} 1$ might be suppressed to some extent, while the host immune response was stimulated well after infections of other avian strains, especially H5N2/F59 and H9N2. Among the three H5N2 strains, infection of H5N2/F59 induced expression of more probe sets belonging to "Immune Response" and "RNA binding" than infections of other two strains, suggesting strong antiviral activities in H5N2/F59 infected A549 cells.

In H1N1 infected A549 cells, a high portion of down-regulated probe sets was identified. These probe sets were majorly classified in groups such as "RNA Binding", "DNA Binding", "Signal Transduction" and "Transcription Factor", indicating the inhibition of a large scale of cell activities after H1N1 infection in A549 cells. In addition to H1N1, infections of the other human strain, pH1N1, and another two avian strains, H5N2/F59 and H5N2/F189, also resulted in relatively large numbers of probe sets with down-regulated expression, and these probe sets were located in similar functional groups as the down-regulated probe sets retrieved from H1N1 infection. Detailed investigation also indicated that some of the probe sets were inhibited deeply, even with higher than 10-FC, at their expression level after H1N1 infection. However, few probe sets with down-regulated expression higher than 10-FC could be detected after infection of all other strains.

Table 5.1 Differentially expressed probe sets in A549 cells infected with influenza A viruses at $10 \mathrm{hpi}$.

\begin{tabular}{|c|c|c|c|c|c|c|c|c|}
\hline \multirow{2}{*}{ A549 } & \multicolumn{2}{|c|}{ Probe sets $>=2$ fold } & \multicolumn{2}{|c|}{ Probe sets ( $>=3$ fold } & \multicolumn{2}{|c|}{ Probe sets ( $>=5$ fold) } & \multicolumn{2}{|c|}{ Probe sets $>=10$ fold $)$} \\
\hline & Up-regulated & Down-regulated & Up.regulated & Down-regulated & Up.regulated & Down-regulated & Up.regulated & Down-regulated \\
\hline H1N1 & $0.28 \%$ & $46.00 \%$ & $0.21 \%$ & $25.29 \%$ & $0.14 \%$ & $8.40 \%$ & $0.08 \%$ & $1.59 \%$ \\
\hline pH1N1 & $1.44 \%$ & $33.37 \%$ & $0.86 \%$ & $562 \%$ & $0.48 \%$ & $0.58 \%$ & $0.22 \%$ & $0.07 \%$ \\
\hline H5N2/F59 & $1.64 \%$ & $34.87 \%$ & $1.03 \%$ & $11.06 \%$ & $0.63 \%$ & $1.66 \%$ & $0.35 \%$ & $0.14 \%$ \\
\hline H5N2/F118 & $1.39 \%$ & $9.53 \%$ & $0.72 \%$ & $2.50 \%$ & $0.38 \%$ & $0.31 \%$ & $0.25 \%$ & $0.04 \%$ \\
\hline H5N2/F189 & $0.89 \%$ & $32.17 \%$ & $0.61 \%$ & $5.85 \%$ & $0.39 \%$ & $0.34 \%$ & $0.22 \%$ & $0.03 \%$ \\
\hline H5N3 & $2.19 \%$ & $5.07 \%$ & $1.12 \%$ & $1.85 \%$ & $0.42 \%$ & $0.41 \%$ & $0.18 \%$ & $0.06 \%$ \\
\hline H7N1 & $0.74 \%$ & $9.37 \%$ & $0.47 \%$ & $1.10 \%$ & $0.26 \%$ & $0.12 \%$ & $0.15 \%$ & $0.02 \%$ \\
\hline H9N2 & $1.41 \%$ & $2.25 \%$ & $0.84 \%$ & $0.26 \%$ & $0.48 \%$ & $0.04 \%$ & $0.27 \%$ & $0.00 \%$ \\
\hline
\end{tabular}

The global host gene expression profiles were retrieved from microarray analysis with different time points examined. The ratios of differentially expressed probe sets (P-value $\leq 0.05)$ up- or down-regulated with different fold changes ( $\geq 2-\mathrm{FC}, \geq 3-\mathrm{FC}, \geq 5-\mathrm{FC}$ and $\geq 10-\mathrm{FC}$ ) in relative to their corresponding "expressing probe sets" are represented in percentage. The expressing probe sets refer to probe sets detected in the mock-infected corresponding cells. 
H1N1

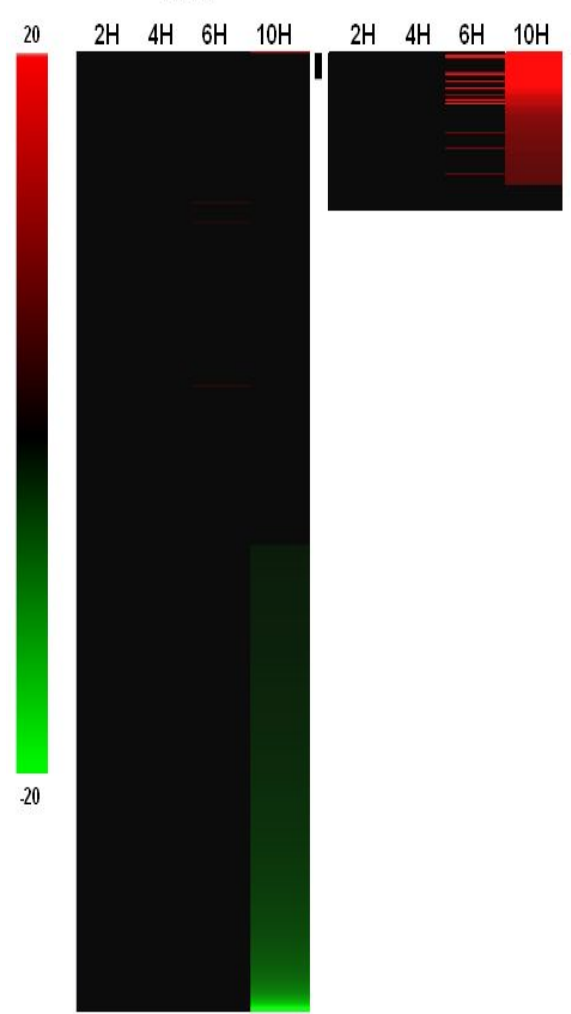

H9N2

$2 \mathrm{H} \quad 4 \mathrm{H} \quad 6 \mathrm{H} \quad 8 \mathrm{H} \quad 10 \mathrm{H} \quad 2 \mathrm{H} \quad 4 \mathrm{H} \quad 6 \mathrm{H} \quad 8 \mathrm{H} \quad 10 \mathrm{H}$

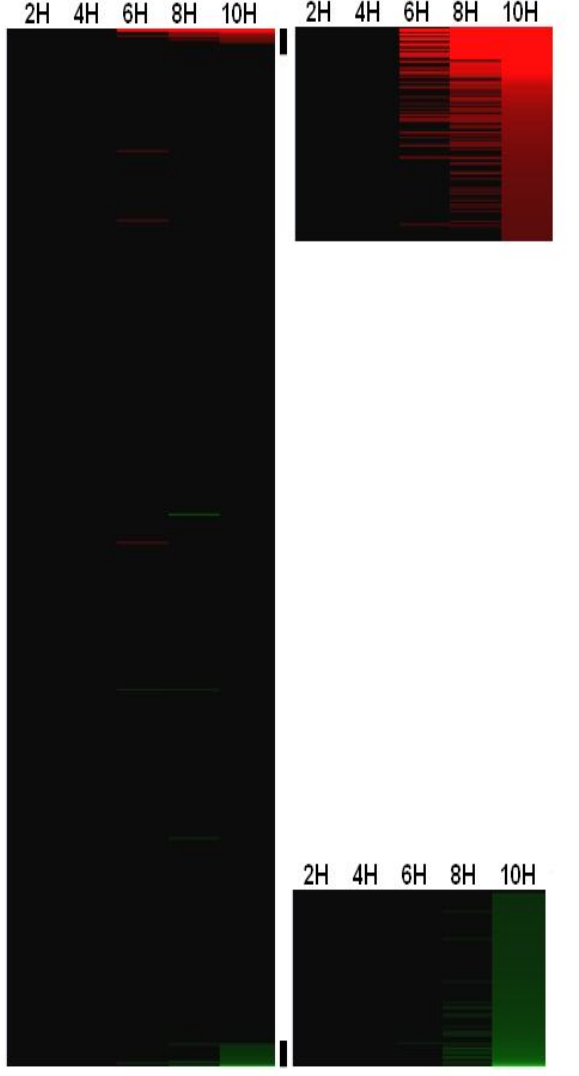

$\mathrm{H} 5 \mathrm{~N} 2 / \mathrm{F} 118$

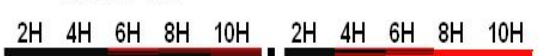

pH1N1

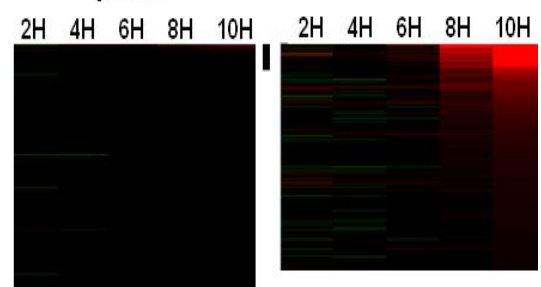

Figure 5.9 Temporal changes in the host cell transcriptome in A549 cells infected by four influenza virus strains. The global host gene expression profiles were retrieved from microarray analysis with different time points examined. The probe sets showing $\geq 2$ fold change (FC) up- or down-regulated in expression are indicated (Pvalue $\leq 0.05$ ). Expression profiles of up-regulated (red), down-regulated (green) and no significant change (black) are shown. 

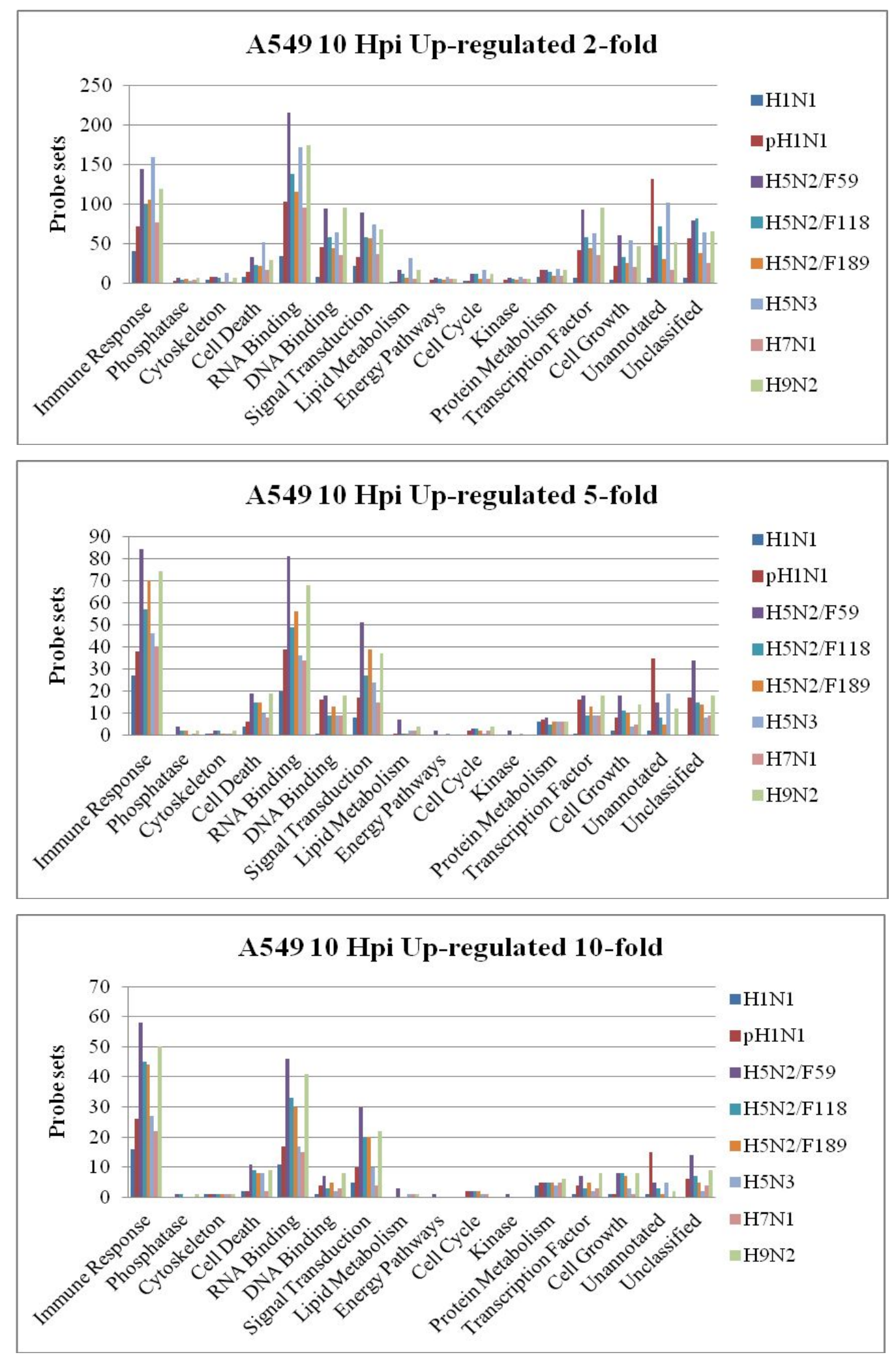

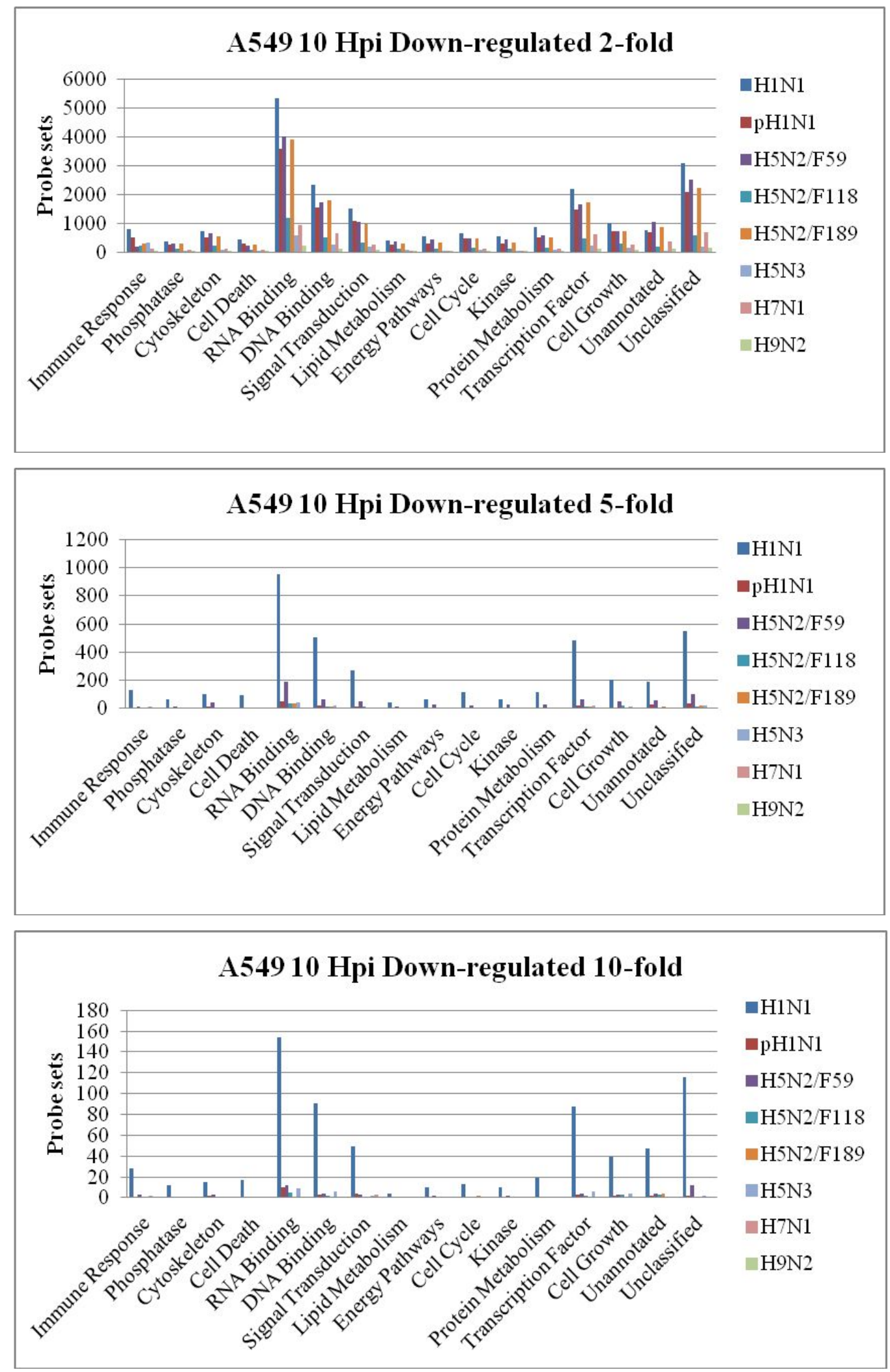

Figure 5.10 Overview of distributions of differentially expressed probe sets into different biological functions in $\mathbf{A 5 4 9}$ cells infected with influenza A viruses. The numbers of probe sets in the different functional families, including non-annotated and unclassified groups, showing up- or down-regulated with different fold changes ( $\geq 2-\mathrm{FC}, \geq 5$-FC and $\geq 10$-FC) in gene expression are presented. 


\subsection{Cluster analysis}

To our knowledge, genes with similar temporal expression trends might have related biological functions and possibly correspond to some critical cellular processes and pathways [91]. So the aim of this cluster analysis is to classify probe sets with similar expression profiles into common biological groups, which is beneficial for further functional analysis. Since only four influenza A strains H1N1, pH1N1, H5N2/F118, H9N2 were examined with multiple time points in A549 cell lines, the probe sets at more than one time point of infection with $\geq 2$-FC ( $\mathrm{P}$-value $\leq 0.05$ ) were clustered into similar gene expression profiles using the Expander version 5.0 software [Figure 5.11-5.14]. Using the same software, the data were further analyzed into genes relating to different functional groups or canonical pathways, and enriched transcription factor were also identified. All enriched functional groups, canonical pathways and transcriptional factors are displayed in Tables 5.2-5.5.

\section{Up-regulated}

Due to the low percentage of up-regulated probe sets in H1N1 A549 $(0.28 \%)$, genes encoded by this batch of probe sets were not able to be identified in cluster analysis. Consequently, we picked out this group of genes and did further enrichment analysis of functional groups, pathways and transcription factors independently. Initial comparison of genes with upregulated expression trend indicated that there was a time lag for gene expression stimulation upon infections of $\mathrm{H} 1 \mathrm{~N} 1$ and $\mathrm{pH} 1 \mathrm{~N} 1$ (started at around 6 hpi) when compared to infections of H5N2/F118 and H9N2 (started at about 2 or 4 hpi).

Parallel comparison has revealed that genes with up-regulated expression in A549 cells infected by all these four influenza A strains shared several significant pathways such as "Cytokine-Cytokine receptor interaction" and "RIG-I- like receptor signaling pathway". However, smaller numbers of genes belonging to these two pathways were detected after infections of H1N1 and $\mathrm{pH} 1 \mathrm{~N} 1$ than those detected after infections of H5N2/F118 and H9N2. This observation indicated that the innate immune response was triggered upon the infections of all these examined influenza A strains in A549 cells, and this 
antiviral response was stronger upon infection of two avian strains than two human strains.

Besides these two pathways, other pathways such as "Jak-STAT signaling pathway" and "Toll-like receptor signaling pathway" were also overrepresented after H5N2/F118 and H9N2 infections. Interestingly, "Toll-like receptor signaling pathway" was also enriched by the down-regulated genes after pH1N1 infection. Toll-like receptors are a type of PRRs in innate immune responses. Opposite regulation of this pathway in different influenza A strains might suggest that some regulations were initiated to interrupt normal human innate response reaction from the early infection stage in pH1N1 infected A549 cells.

"B cell receptor signaling pathway" was another pathway significantly enriched only in genes with up-regulated expression following H9N2 infection. B cell responses play a key role on survival from both primary as well as secondary infection with influenza virus. Recent studies have indicated that innate stimuli such as TLR signals functions as important signals for B cell regulation and type I IFN signals positively regulate influenza virus-specific B cell responses [257]. Hence, significant activation of "B cell receptor signaling pathway" provided evidence that H9N2 infection triggered a series of strong innate inflammatory response in A549 cells.

Besides these canonical pathways, a batch of key functional groups was also enriched in these up-regulated genes after infections with different influenza A strains. The analysis based on the H1N1, pH1N1/478, H5N2/F118 and H9N2 infections revealed that the differentially up-regulated genes share common functional gene groups such as response to virus and nucleic acid binding. In addition, the avian viruses have more functional gene groups in common and these groups are genes involved in immune response, cytokine receptor binding, transition metal ion binding and cell death. In the case of $\mathrm{pH} 1 \mathrm{N1/478}$ infection, the differentially up-regulated genes that were specific to this virus were involved in transcription regulatory activity, RNA metabolic process, transcription from RNA polymerase II promoter and multicellular organismal development. 
Transcription factors, IRF-1, IRF-3 and IRF-7, which are involved in the IFN signaling pathway, were observed to be significantly up-regulated in a temporal manner in A549 cells infected with seasonal influenza virus [258]. In our study, these two potential transcription factors both showed significantly up-regulated expression levels after avian viruses infections. However, only transcription factor $I R F-7$ showed faint elevation in its expression after human viruses infections at specific time points. These observations were consistent with the phenomenon that larger numbers of downstream genes with upregulated expression were detected in A549 cells infected with two avian strains than two human strains. No apparent expression changes of IRF-3 were observed following the infections of four influenza A strains, which could be due to the binding of influenza NS1 to RIG-1, which in turn inhibits the activation of IRF-3 [259].

On the basis of performances of genes with up-regulated expression, these four influenza A strains can be roughly classified into two groups. One group included avian strains, H5N2/F118 and H9N2, and their infections generated strong host immune response with many genes belonging to antiviralrelated pathways showing up-regulated expression; the other group included human strains, $\mathrm{H} 1 \mathrm{~N} 1$ and $\mathrm{pH} 1 \mathrm{~N} 1$, and their infections displayed postponed and weak host antiviral reaction. It was assumed that viruses such as $\mathrm{H} 1 \mathrm{~N} 1$ and pH1N1 might evolve some specific mechanism which counteracts the host genes' immune reaction after virus invasion from an early infection stage.

\section{Down-regulated}

In contrast to the differentially up-regulated genes, larger proportions of genes showed down-regulated expression after two human strains infections, and this allowed more functional groups and canonical pathways to be enriched. Canonical pathways such as "Metabolic pathways", "Cell cycle", "Regulation of actin cytoskeleton", “Adherence junction”, "Lysosome”, "Endocytosis" and "Pathway ways in cancer" were over-represented based on genes with downregulated expression after the two human influenza A strains and H5N2/F118 infections, with a greater number of genes involved in infections of two human strains. These common pathways were generally related to cell regulation or cell interactions, suggesting that infections of these three influenza A strains 
inhibited expression of a batch of genes involved in cell activity regulation in A549 cells. And the limitation of the cell activity might serve to benefit for viral survival or replication in A549 cells.

Besides these pathways, other down-regulated genes detected during H1N1 and pH1N1 infections were involved in other pathways such as "Ubiquitin mediated proteolysis", "Wnt signaling pathway", "Apoptosis" and "RNA degradation". Gene expression repression in these pathways might imply that cell apoptosis and RNA degradation were postponed to facilitate the virus replication in A549 cells.

Common functional gene groups (e.g. genes involved in nitrogen compound metabolic process, regulation of macromolecule metabolic process, nucleic acid binding) were observed between the human and avian strains infections, with a greater number of genes involved in the former infections. Specific to the 2 human viruses, the genes involved in the protein metabolicrelated processes showed a decreasing temporal trend in the expression profiles, and this is the first evidence reported here. Similar changes in differential expression of transcripts involved in protein metabolism have been reported for the HPAI H5N1 in infected CEF cells [260]. This observation was not seen in the avian viruses in our study, suggesting that the human viruses and HPAI share a closer role in protein metabolism involvement than with the LPAI.

A differential decrease in gene expression profile was observed for the genes involved in zinc ion binding and ATP binding for the seasonal human virus H1N1, and faintly detected for pH1N1. In H1N1-infected A549 cells, the temporal trend for the Zinc Finger (ZNF) genes decreased sharply from 6 to 10 hpi, with the most significant down-regulated fold changes detected at the last time point. Among these ZNF genes, the topmost significantly down-regulated genes are listed: ZNF226 $=41.84-F C$, ZNF675 $=40.08-F C$, ZNF764 $=39.84-$ FC, ZNF488 $=36.5$-FC. Our results concurred with that reported in Lee et al., 2010 where other ZNF genes were presented with significant changes (ZBTB3, ZNF175, ZNF383, and ZNF587 genes; ZNF175 and ZNF587 were observed in our study) in primary alveolar epithelial cells infected with a seasonal H1N1 (A/Hong Kong/54/1998) than with pandemic H1N1(A/Hong Kong/415742/2009) [261]. The same study suggested that the ZNF may have a 
role in suppressing viral transcription and this may facilitate more efficient replication of seasonal H1N1 in the host cells as compared to the pH1N1.

In contrast, the temporal trend for ATP binding associated genes remained gradual from early to late infection time points, but the fold changes were significant enough to be detected. Reports have described that ATP is required for influenza virus budding [262] and replication [263], but it is not clear how the ATP binding associated genes relates to the ATP requirement.

A larger number of transcription factors were enriched based on downregulated genes from infections of two human strains than two avian strains in A549 cells. The transcription factors detected in the two human strains and H5N2/F118 were analyzed using the IPA network and these factors were found to be associated with: gene expression, cell death, organismal development, carbohydrate metabolism, small molecule biochemistry and infectious disease. More than $50 \%$ of these transcription factors were observed to be downregulated at their expression level after $\mathrm{H} 1 \mathrm{~N} 1$ infection, whereas almost no expression changes were detected in the A549 cells infected with the remaining three viruses. Furthermore, three of these factors, E2F, ETF and Sp1, were observed to be commonly shared among infections of all four examined influenza A strains, with more down-regulated genes involved in the H1N1/WS infection. Detailed investigation indicated faintly down-regulated expression of ETF was observed only after H1N1/WS and H5N2/F118 infections while faintly down-regulated expression of sp1 was observed only after H1N1/WS and $\mathrm{pH} 1 \mathrm{~N} 1 / 478$ infections.

Altogether, infection of H1N1 contributed to most genes with decreased expression in A549 cells, followed by $\mathrm{pH} 1 \mathrm{~N} 1$ and H5N2/F118. And these regulations might target to create a more beneficial environment for viral survival and replication through interrupting the normal regulation of cell. Compared to these three influenza A strains, H9N2 almost did nothing to combat with the host cells antiviral control. 

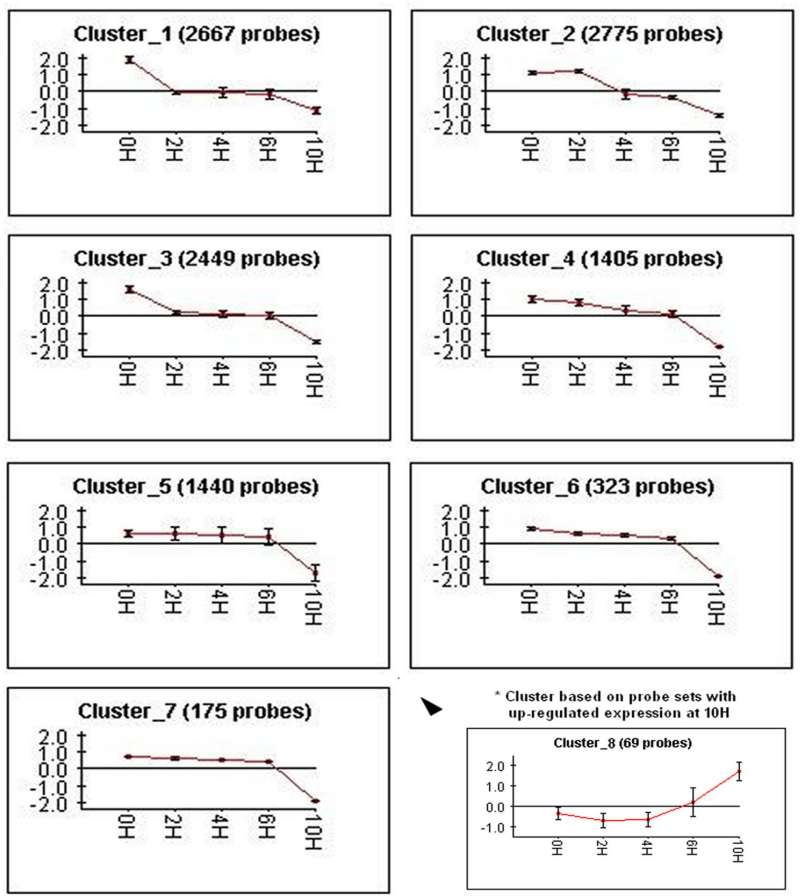

Figure 5.11 Clustering analysis of temporal gene expression profiles in H1N1-infected A549 cells. A549 cells were infected with $\mathrm{H} 1 \mathrm{~N} 1$ at 2, 4, 6, and 10 hpi. Probe sets showing $\geq 2-$ fold changes up- or down-regulated $(\mathrm{P}$-value $\leq 0.05)$ at least over one time point were analyzed with Expander 5 software. X-axis represents post-infection in hours $(\mathrm{H})$, and $\mathrm{Y}$-axis means normalized expression changes of probe sets. The extra cluster included the probe sets showing up-regulated expression at $10 \mathrm{hpi}$. 
Table 5.2 Summary of functional groups, canonical pathways and transcription factors enriched based on differentially expressed genes in A549 cells infected with H1N1.

\begin{tabular}{|c|c|}
\hline Cluster No. & Enriched Transcription Factors (H1N1) \\
\hline \multirow{3}{*}{ Cluster 1} & EIK:431 | E2F:1267 | Nrf-1:699 | ETF:929 | GABP:513 | E2F-1:487 | Sp1:1135 | C-Myc:Max:506 | ZF5:726 | HIC1:715 | Tax/CREB:345 \\
\hline & AHRHIF:444 | ZF5:355 | Egr-1:502 | CREB:302 | Tel-2:230 | MOV0-B:729 | E2F-1:192 | EGR:631 | AP-2:641 | ATF:420 | NF-Y:440 \\
\hline & AP-2:568|AP-2:563 \\
\hline \multirow{3}{*}{ Cluster 2} & ETF:978 | E2F:1235 | Elk-1:402 | GABP:510 | Nrf-1:677 | Sp1:1100 | ATF:440 | NF-Y:469 | E2F-1:468 | C-Myc:Max:499 | ZF5:699 | ZF5:348 \\
\hline & E2F-1:101 | Pax-3:203 | Tel-2:226 | AP-2:622 | Pax-3:260 | Tax/CREB:327 |XBP-1:293 | AHRHIF:431 | E2F-1:181 | ATF4:312 | STAT1:73 \\
\hline & CREB:291 \\
\hline \multirow{2}{*}{ Cluster 3} & ETF:870 | E2F:1111 | Nrf-1:603 | Elk-1:358 | Sp1:1002 | ZF5:649 | AP-2:541 | GABP:416 | AP-2:593 | E2F-1:421 | HIC1:617 | Tel-2:217 \\
\hline & E2F-1:96 | AP-2:504 | AP-2alpha:360 | AhR:259 | E2F:108 | ATF:379 | ZF5:309 | Egr-1:418 \\
\hline Cluster 4 & E2F:637 | ETF:480 | Nrf-1:346 | ZF5:373 | MOV0-B:370 |E2F-1:246 | ATF:223 | Ap1:559 | AHRHIF:233 \\
\hline Cluster 5 & ETF:474 | E2F:598 | ATF:241 | Elk-1:197 | E2F-1:244 | |RF-1:123 | SRY:288 \\
\hline Cluster 6 & E2F:171| AP-2:97| Sp1:150 | Nrf-1:92 \\
\hline Cluster 7 & Sp1:85|ETF: 69 \\
\hline Cluster $8^{*}$ & ISRE:28 | IRF-7:22 | IRF-1:20 \\
\hline Cluster No. & Enriched Canonical Pathways (H1N1) \\
\hline \multirow{2}{*}{ Cluster 1} & Metabolic pathways: 188 | Ubiquitin mediated proteolysis:32 | Progesteron-mediated oocyte maturation:26 | Cell cycle:28 \\
\hline & Insulin signaling pathway:28 | Lysosome:23 | Pancreatic cancer:17 | Chronic myeloid leukemia:17 | Gap junction:19 \\
\hline \multirow{3}{*}{ Cluster 2} & Metabolic pathways:158 | Endocytosis:43 | Ubiquitin mediated proteolysis:34 | Pancreatic cancer:21 | N-Glycan biosynthesis:15 \\
\hline & Pathways in cancer:52 | Progesteron-mediated oocyte maturation:24 | Cell cycle:27 | Apoptosis:21 | Adherens junction:19 | Lyosome:24 \\
\hline & 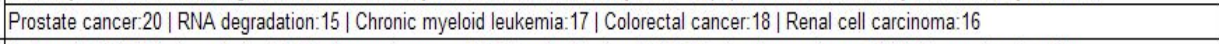 \\
\hline \multirow{3}{*}{ Cluster 3} & Cell cycle:32 | RNA degradation:20 | Pathways in cancer:52 | Focal adhesion:36 | ErbB signaling pathway:21 | Adherens junction:19 \\
\hline & 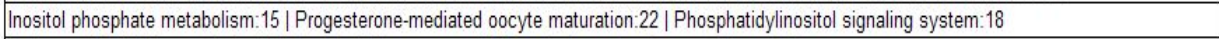 \\
\hline & Aminoacyl-tRNA biosynthesis:12 | Small cell lung cancer:18 | Regulation of actin cytoskeleton:32 \\
\hline \multirow{2}{*}{ Cluster 4} & Pahtways in cancer:31 | Cell cycle:17 | p53 signaling pathway:12 | Ubiquitin mediated proteolysis:17 | TGF-beta signaling pathway \\
\hline & Adherens junction: 12 \\
\hline Cluster 5 & Pathways in cancer:29 \\
\hline Cluster 6 & Cell cycle:8 \\
\hline Cluster $8^{*}$ & Cytokine-cytokine receptor interaction:6 | RIG-like receptor signaling pathway:4 \\
\hline Cluster No. & Enriched Gene Ontology Terms (H1N1) \\
\hline \multirow{4}{*}{ Cluster 1} & Cellular protein metabolic process - G0:0044267:349 | Protein metabolic process - G0:0019538:393 | Macromolecule localization - G0:0033036:195 \\
\hline & Protein transport - G0:0015031:157 | Nucleotide binding - G0:0000166:321 | Protein localization - G0:0008104:168 \\
\hline & \begin{tabular}{|l} 
Nitrogen compound metabolic process - G0:0006807:422 | Cellular localization - G0:0051641:157 | Biosynthetic process - G0:0009058:386 \\
\end{tabular} \\
\hline & Transferase activity - G0:0016740:259 \\
\hline \multirow{4}{*}{ Cluster 2} & Nitrogen compound metabolic process - G0:0006807:470 | Nucleobase, nucleoside, nucleotide and nucleic acid metabolic process - G0:0006139:425 \\
\hline & Cellular biosynthetic process - G0:0044249:420 | Gene expression - G0:0010467:370 | Cellular protein metabolic process - G0:0044267:342 \\
\hline & Nucleotide binding - G0:0000166:324 | Macromolecule biosynthetic process - G0:0009059:343 | RNA metabolic process - G0:0016070:178 \\
\hline & Protein metabolic process - G0:0019538:380 | Protein transport - G0:0015031:151 \\
\hline \multirow{4}{*}{ Cluster 3} & Nucleobase, nucleoside, nucleotide and nucleic acid metabolic process - G0:0006139:385 | Nitrogen compound metabolic process - G0:0006807:414 \\
\hline & 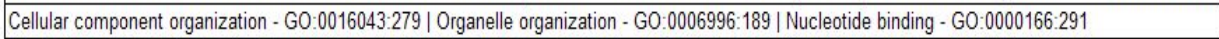 \\
\hline & Nucleic acid binding - G0:0003676:388 | Gene expression - G0:0010467:315 | ATP binding - G0:0005524:211 \\
\hline & RNA metabolic process - G0:0016070:154 | Cellular protein metabolic process - G0:0044267:285 \\
\hline \multirow{4}{*}{ Cluster 4} & Nucleobase, nucleoside, nucleotide and nucleic acid metabolic process - G0:0006139:248 | Nucleic acid binding - G0:0003676:260 \\
\hline & Regulation of cellular metabolic process - G0:0031323:248 | Regulation of macromolecule metabolic process - G0:0060255:239 \\
\hline & Regulation of primary metabolic process - G0:0080090:237 | Regulation of gene expression - G0:0010468:220 | Gene expression - G0:0010467:205 \\
\hline & Zinc ion binding - G0:0008270:192 | Macromolecule biosynthetic process - G0:0009059:185 | Cellular biosynthetic process - G0:0044249:208 \\
\hline \multirow{5}{*}{ Cluster 5} & Nucleobase, nucleoside, nucleotide and nucleic acid metabolic process - G0:0006139:256 | Nucleic acid binding - G0:0003676:276 \\
\hline & Gene expression - G0:0010467:234 | Regulation of macromolecule metabolic process - G0:0060255:250 \\
\hline & 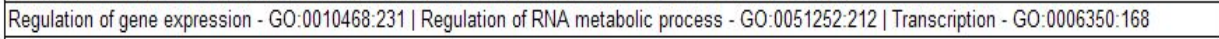 \\
\hline & Cellular biosynthetic process - G0:0044249:245 | Regulation of macromolecule biosynthetic process - G0:0010556:228 \\
\hline & Regularion of cellular metabolic process - G0:0031323:253 \\
\hline \multirow{5}{*}{ Cluster 6} & Transition metal ion binding - G0:0046914:68 | Regulation of cellular metabolic process - G0:0031323:69 \\
\hline & Regulation of macromolecule metabolic process - G0:0060255:66 | Cellular macromolecule biosynthetic process - G0:0034645:55 \\
\hline & Nucleobase, nucleoside, nucleotide and nucleic acid metabolic process - G0:0006139:63 | DNA binding - G0:0003677:53 \\
\hline & 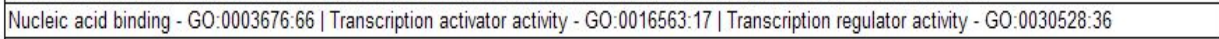 \\
\hline & Cellular macromolecule catabolic process - G0:0044265:24 \\
\hline Cluster 7 & Zinc ion binding - G0:0008270:27 \\
\hline \multirow{2}{*}{ Cluster $8^{*}$} & Response to virus - G0:0009615:10 | Immune response - G0:0006955:14 | Response to stimulus - G0:0050896:21 \\
\hline & Cytokine activity - G0:0005125:5 | Nucleic acid binding - G0:0003676:14 \\
\hline
\end{tabular}

Differentially expressed genes were significantly categorized into different GO terms and canonical pathways. Different transcription factors that can potentially be involved in the regulation of gene expressions are shown for each cluster under Expander 5 software analysis (P-value $\leq 0.05)$. Each functional group, canonical pathway or transcription factor is followed by the number of corresponding genes. * represents the cluster which is based on probe sets with up-regulated expression at $10 \mathrm{hpi}$. 

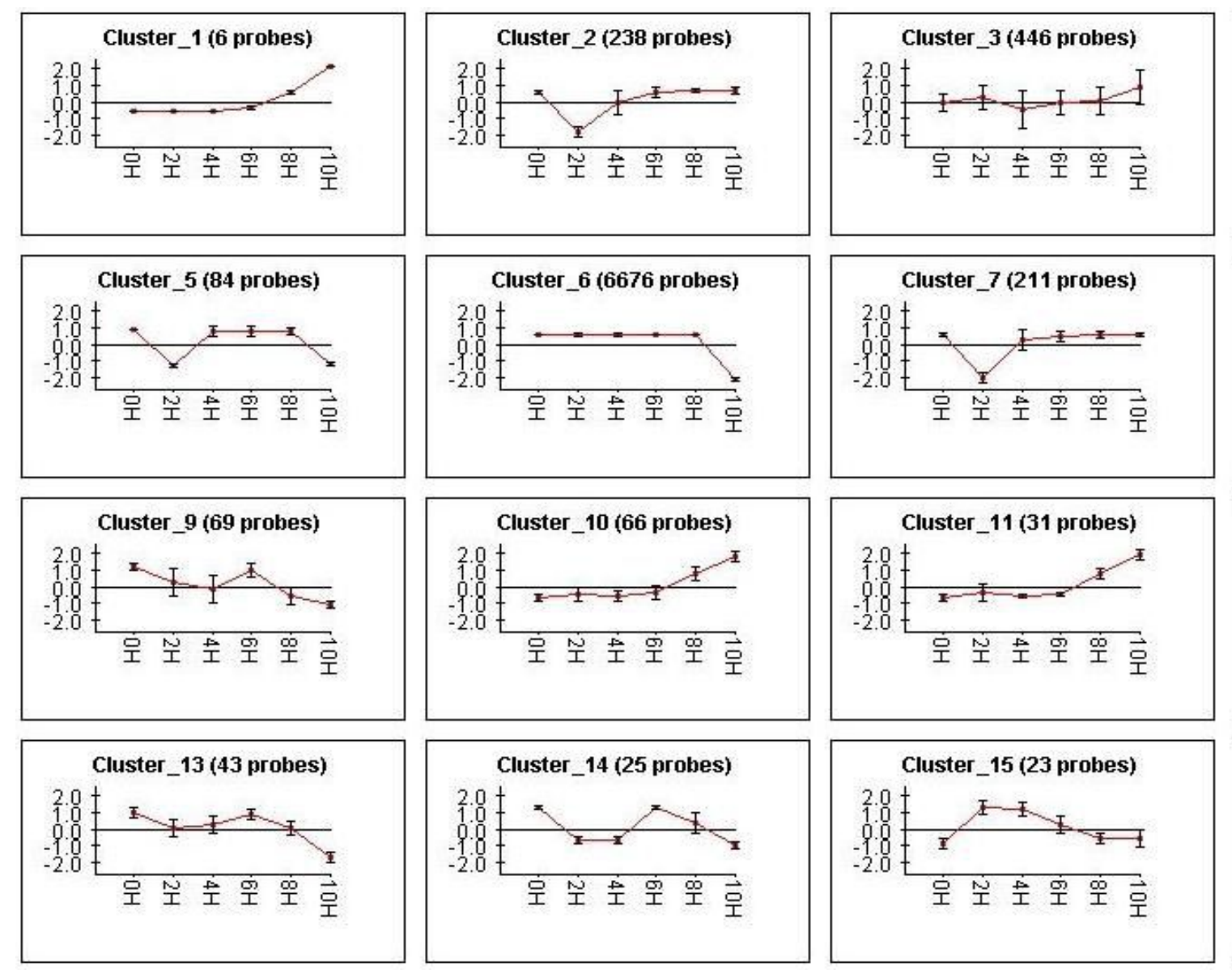
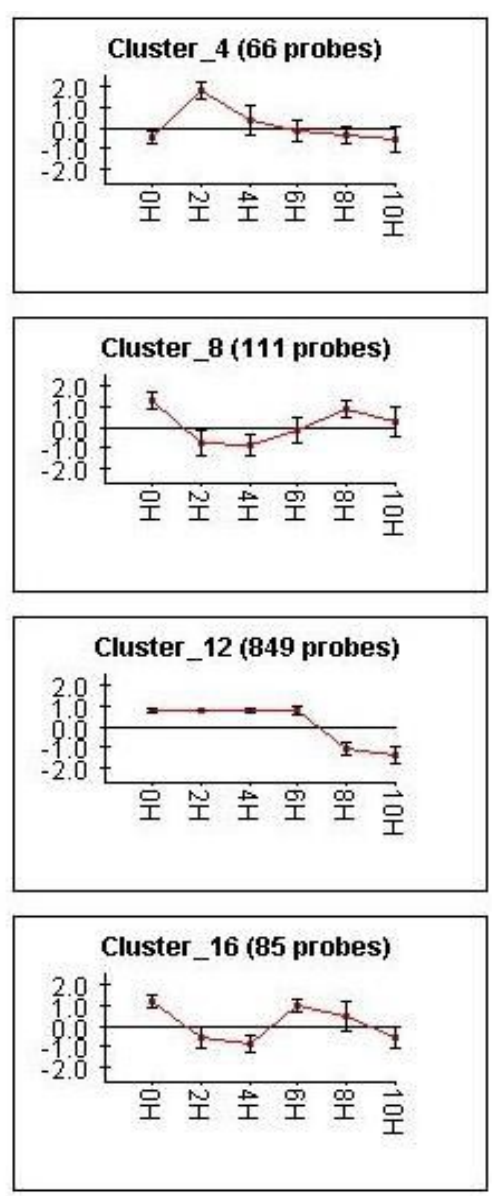

Figure 5.12 Clustering analysis of temporal gene expression profiles in pH1N1-infected A549 cells. A549 cells were infected with pH1N1 at $2,4,6,8$ and 10 hpi. Probe sets showing $\geq 2$-fold changes up- or down-regulated $(\mathrm{P}$-value $\leq 0.05)$ at least over one time point were analyzed with Expander 5 software. $X$-axis represents post-infection in hours $(\mathrm{H})$, and $\mathrm{Y}$-axis means normalized expression changes of probe sets. 
Table 5.3 Summary of functional groups, canonical pathways and transcription factors enriched based on differentially expressed genes in A549 cells infected with pH1N1.

\begin{tabular}{|c|c|}
\hline Cluster №. & Enriched Transcription Factors (pH1N1) \\
\hline Cluster 1 & c-Myb:3|ISRE:4 \\
\hline Cluster 3 & NF-kappaB_(p65):25 \\
\hline \multirow{3}{*}{ Cluster 6} & E2F:2493 | ETF:1961 | Nrf-1:1313 | Sp1:2224 |ZF5:1459 | AP-2:1312 |E2F-1:931 | MOVO-B:1486 | C-Myc:Max:993| EIK-1:712 | AP-2:1159 \\
\hline & AHRHIF:881 | HIC1:1369 | AP-2:1152 | Egr-1:986 | ZF5:700 | GABP:908 | EGR:1236 | AP-2alpha:778 | AhR:562 | HIF-1:1007 | ATF:810 \\
\hline & E2F-1:363 | Tax/CREB:637 | AhR:Arnt:627 | UF1H3EETA:1640 | N-Myc:738 | NF-Y:842 | E2F:213 | Pax-3:491 | Tel-2:407 | E2F-1:170 \\
\hline Cluster 10 & ISRE:12 \\
\hline Cluster 11 & |RF-7:11||ISRE:11||RF-1:7 \\
\hline Cluster 12 & ETF:270 | NFF-1:202 | E2F:329 | Tax/CREB:109 \\
\hline Cluster No. & Enriched Canonical Pathways (pH1N1) \\
\hline Cluster 4 & Toll-like receptor signaling pathway:4 \\
\hline \multirow{7}{*}{ Cluster 6} & Pathways in Cancer: 121 | Ubiquitin mediated proteolysis:63 | Adherens junction:43 | Endocytosis:72 | Cell cycle:52 | Wnt signaling pathway:57 \\
\hline & 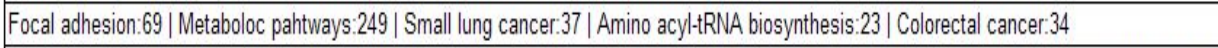 \\
\hline & Progesterone-mediated oocyte maturation:39 | Neurotrophin signaling pathway:44 | MAPK signaling pathway:26 | Insulin signaling pathway : 46 \\
\hline & Tight junction:45 | Chronic myeloid leukenia:30 | TAF-beta signaling pathway:33 | DNA replocation:18 | Pancreatic cancer:28 \\
\hline & Regulation of actin cytoskeleton:61 | Axon guidance:41 | ECM-receptor interaction:30 | Lysosome:37 | GnRH signaling pathway:33 \\
\hline & Apoptosis:30 | Purine metabolism:45 | 0-Glycan biosynthesis:15 | Gap junction:30 | Renal cell carcinoma:25 | ErbB signaling pathway:29 \\
\hline & Lysine degradation:19 | RNA degradation:22 | Endometrial cancer:20 \\
\hline Cluster 9 & Aminoacyl-tRNA biosynthesis:3 \\
\hline Cluster 10 & Cytokine-Cytokine receptor interaction: 5 \\
\hline Cluster No. & Enriched Gene Ontology Terms (pH1N1) \\
\hline \multirow{2}{*}{ Cluster 2} & Transition metal ion binding - G0:0046914:41 | Transcription - G0:0006350:29 | Nitrogen compound metabolic process - G0:0006807:43 \\
\hline & Gene expression - G0:0010467:37 | Cellular biosynthetic process - G0:0044249:41 | Nucleic acid binding - G0;0003676:40 \\
\hline \multirow{4}{*}{ Cluster 3} & Nucleic acid binding - G0:0003676:64 | Regulation of gene expression - G0:0010468:56 | Transcription regulatory activity - G0:0030528:36 \\
\hline & Response to virus - G0:0009615:9 | Response to biotic stimulus - G0:0009607:14 | RNA metabolic process - G0:0016070:24 \\
\hline & 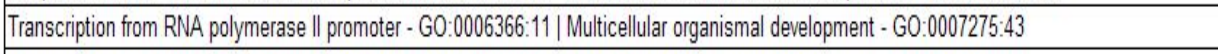 \\
\hline & Organ development - G0:0048513:28 | Multi-organism process - G0:0051704:17 \\
\hline Cluster 4 & Inflammatory response - G0:0006954:5 \\
\hline \multirow{5}{*}{ Cluster 6} & Nucleobase, nucleoside, nucleotide and nucleic acid metabolic process - G0:0006139:772 | Nucleotide binding - G0:0000166:608 \\
\hline & Nitrogen compound metabolic process - G0:0006807:838 | Cellular protein metabolic process - G0:0044267:636 \\
\hline & Protein metabolic process - G0:0019538:719 | Regulation of macromolecule metabolic process - G0:0060255:761 \\
\hline & Nucleic acid binding - G0:0003676:800 | Transition metal ion bindng - G0:0046914:715 | Purine nucleotide binding - G0:0017076:526 \\
\hline & Nucleoside binding - G0:0001882:460 \\
\hline Cluster 7 & Nucleic acid binding - G0:0003676:43 \\
\hline Cluster 8 & Cellular amino acid biosynthetic process - G0:0008652:5 | Biosynthetic process - G0:0009058:23 \\
\hline Cluster 9 & Nucleic acid binding - G0:0003676:21 | Nucleobase, nucleoside, nucleotide and nucleic acid metabolic process - G0:0006139:18 \\
\hline Cluster 10 & Response to biotic stimulus - G0:0009607:7| Receptor binding - G0:0005102:8 \\
\hline Cluster 11 & Immune system process - G0:0002376:7| Response to virus - G0:0009615:4 \\
\hline
\end{tabular}

Differentially expressed genes were significantly categorized into different GO terms and pathways. Different transcription factors that can potentially be involved in the regulation of gene expressions are shown for each cluster under Expander 5 software analysis (P-value $\leq 0.05)$. Each functional group, canonical pathway or transcription factor is followed by the number of corresponding genes. 

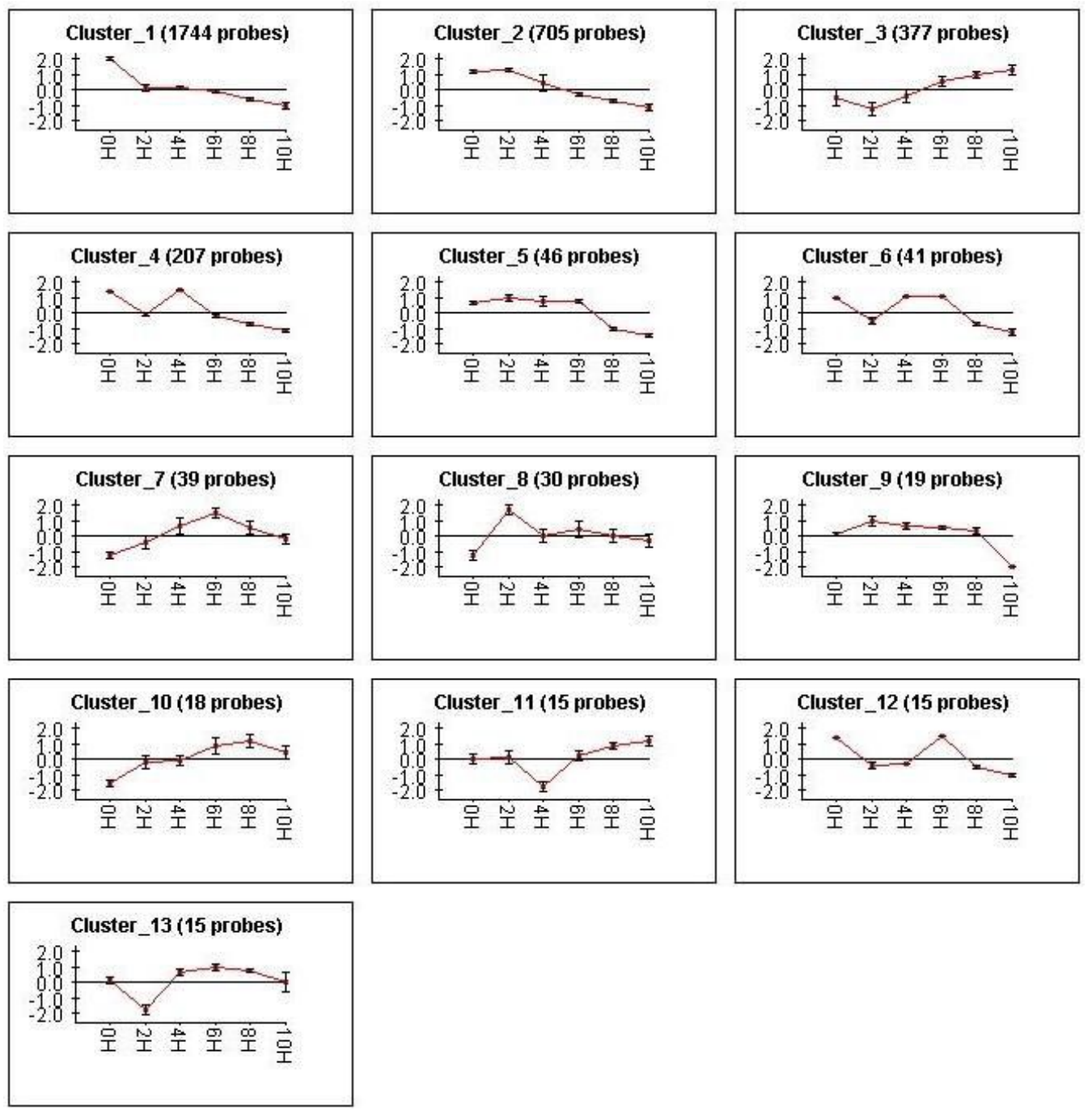

Figure 5.13 Clustering analysis of temporal gene expression profiles in H5N2/F118infected A549 cells. A549 cells were infected with H5N2/F118 at 2, 4, 6, 8 and 10 hpi. Probe sets showing $\geq 2$-fold changes up- or down-regulated ( $\mathrm{P}$-value $\leq 0.05$ ) at least over one time point were analyzed with Expander 5 software. X-axis represents post-infection in hours $(\mathrm{H})$, and Yaxis means normalized expression changes of probe sets. 
Table 5.4 Summary of functional groups, canonical pathways and transcription factors enriched based on differentially expressed genes in A549 cells infected with H5N2/F118.

\begin{tabular}{|c|c|}
\hline Cluster No. & Enriched Transcription Factors (H5N2/F118) \\
\hline \multirow{2}{*}{ Cluster 1} & E2F:751 | ETF:583 | Sp1:701 | NfF-1:421 | E2F-1:306 | AHRHIF:279 | ZF5:434 | Tax/CREB:219 | AP-2:372 | NF-Y:279 | c-Myc:Max:304 \\
\hline & HIF-1:321|HIC1:420 \\
\hline \multirow{2}{*}{ Cluster 2} & E2F:323 | ZF5:201 | ETF:245 | Sp1:290 | NF-Y:140 | HIC1:199 | MOV0-B:198 | AP-2:167 | AP-2alpha:114 | AP-2:184 | NF-1:170 \\
\hline & UF1H3BETA:226 | HNF4:297 \\
\hline Cluster 3 & |RF-7:63 | ISRE:64 | |RF:60 | |RF1:46 \\
\hline Cluster 8 & SRF:3 \\
\hline Cluster No. & Enriched Canonical Pathways (H5N2/F118) \\
\hline \multirow{3}{*}{ Cluster 1} & Metabolic pathways:109 | Lysosome:27 | Endocytosis:31 | Pahtways in cancer:39 | Cell cycle:21 | Citrate cycle (TCA cycle):10 \\
\hline & Progesterone-mediated oocyte maturation:18 | Biosynthesis of unsaturated fatty acids:8 | Steroid biosynthesis:7 \\
\hline & Aminoacyl-tRNA biosynthesis:10 | Pathogenic Escherichia coli infection:11 \\
\hline \multirow{2}{*}{ Cluster 2} & Pathways in cancer:23 | Focal adhesion:17 | Endocytosis: 15 | Cell cycle:12 | Tight junction:12 | Adherens junction:9 \\
\hline & Regulation of actin cytoskeleton:15 | TGF-beta signaling pathway \\
\hline \multirow{2}{*}{ Cluster 3} & Jak-STAT signaling pathway:10 | RIG-Ilike receptor signaling pathway:7 | Toll-like receptor signaling pathway:8 \\
\hline & Cytokine-cytokine receptor interaction: 10 \\
\hline Cluster 4 & Focal adhesion:8 \\
\hline Cluster 5 & p53 signaling pathway:4 | Glioma:3 \\
\hline Cluster No. & Enriched Gene Ontology Terms (H5N2/F118) \\
\hline \multirow{4}{*}{ Cluster 1} & Nucleotide binding - G0:0000166:231 | Purine nucleoside binding - G0:0001883:176 | Cellular component organization - G0:0016043:180 \\
\hline & Localization - G0:0051179:241 | Nitrogen compound metabolic process - G0;0006807:255 | Hydrolase activity - G0:0016787:194 \\
\hline & Cell cycle - G0:0007049:83 | Macromolecule localization - G0:0033036-106 | Protein metabolic process - G0:0019538:217 \\
\hline & Cellular localization - G0:0051641:91 \\
\hline \multirow{4}{*}{ Cluster 2} & Cellular component organization - G0:0016043:100 | Regulation of cellular metabolic process - G0:0031323:135 \\
\hline & Organelle organization - G0:0006996:66 | Developmental process - G0:0032502:109 | DNA binding - G0:0003677:94 \\
\hline & Cell cycle - G0:0007049:45 | Nucleic acid binding - G0:0003676:118 | Cellular biosynthetic process - G0:0044249:107 \\
\hline & Cellular macromolecule biosynthetic process - G0:0034645:90 | Actin binding - G0:0003779:28 \\
\hline \multirow{3}{*}{ Cluster 3} & Response to virus - G0:0009615:23 | Immune response - G0:0006955:31 | Multi-organism process - G0:0051704:29 \\
\hline & Response to stimulus - G0:0050896:58 | Nucleic acid binding - G0:0003676:51 | Defense response - G0:0006952:17 \\
\hline & RNA binding - G0:0003723:18 \\
\hline \multirow{3}{*}{ Cluster 4} & Regulation of macromolecule metabolic process - G0:0060255:46 | Transition metal ion binding - G0:0046914:40 \\
\hline & Nucleic acid binding - G0:0003676:43 | Protein metabolic process - G0:0019538:37 \\
\hline & Cellular macromolecule biosynthetic process - G0:0034645:31 \\
\hline Cluster 5 & Regulation of cellular metabolic process - G0:0031323:14 \\
\hline
\end{tabular}

Differentially expressed genes were significantly categorized into different GO terms and canonical pathways. Different transcription factors that can potentially be involved in the regulation of gene expressions are shown for each cluster under Expander 5 software analysis (P-value $\leq 0.05)$. Each functional group, canonical pathway or transcription factor is followed by the number of corresponding genes. 

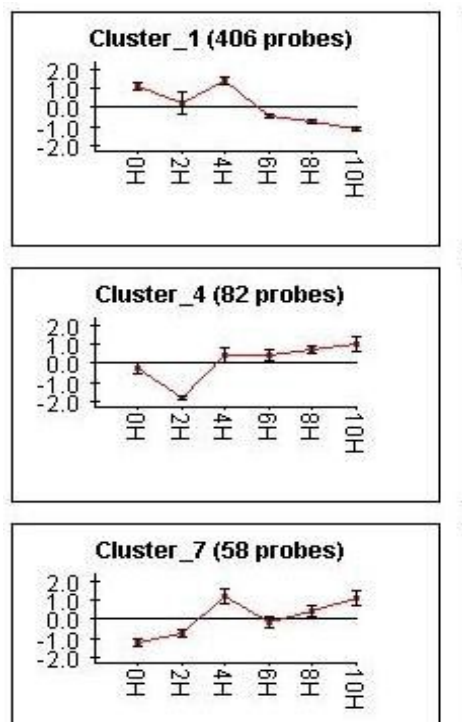
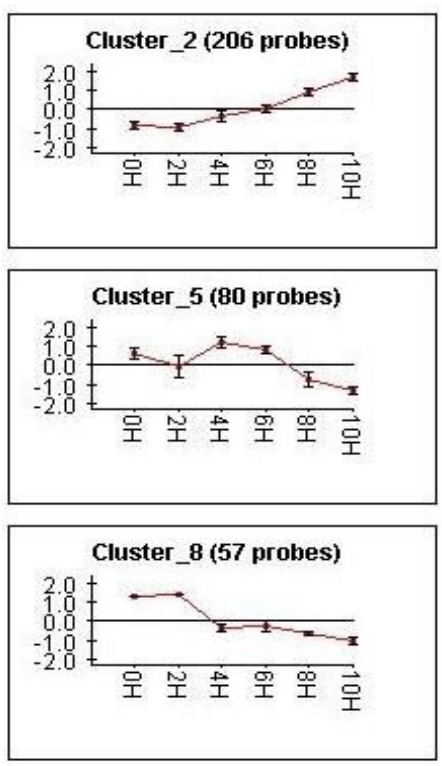
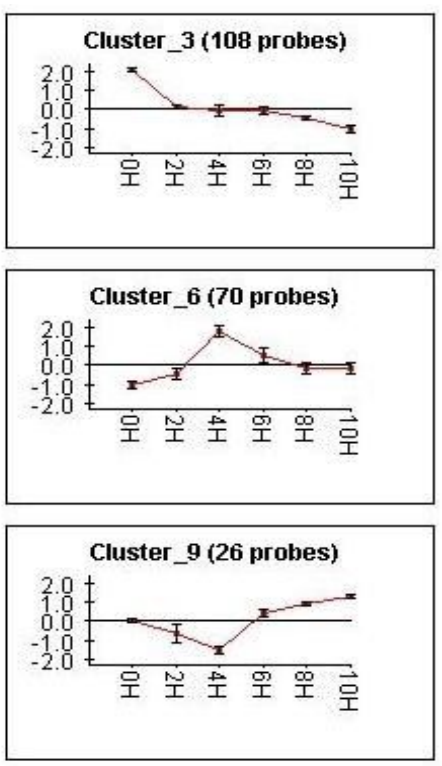

Figure 5.14 Clustering analysis of temporal gene expression profiles in H9N2-infected A549 cells. A549 cells were infected with $\mathrm{H} 9 \mathrm{~N} 2$ at 2, 4, 6, 8 and 10 hpi. Probe sets showing $\geq 2$ fold changes up- or down-regulated $(\mathrm{P}$-value $\leq 0.05)$ at least over one time point were analyzed with Expander 5 software. $\mathrm{X}$-axis represents post-infection in hours $(\mathrm{H})$, and $\mathrm{Y}$-axis means normalized expression changes of probe sets.

Table 5.5 Summary of functional groups, canonical pathways and transcription factors enriched based on differentially expressed genes in A549 cells infected with H9N2.

\begin{tabular}{|c|c|}
\hline Cluster No. & Enriched Transcription Factors (H9N2) \\
\hline Cluster 1 & ETF:123|E2F:161 \\
\hline Cluster 2 & | ISRE:63 | IRF-7:51 | IRF-1:48 | IRF1:36 | NF-kappaB_(p65):16 \\
\hline Cluster 3 & Sp1:54 \\
\hline Cluster 4 & ISRE: 14 | IRF-1:14 \\
\hline Cluster No. & Enriched Canonical Pathways (H9N2) \\
\hline \multirow{2}{*}{ Cluster 2} & Toll-like receptor signaling pathway:9 | RIG-I-like receptor signaling pathway:8 | Jak-STAT signaling pathway:9 \\
\hline & Cytokine-cytokine receptor interaction:9 | B cell receptor signaling pathway: 5 \\
\hline Cluster 3 & Pahtways in cancer: 7 \\
\hline Cluster 8 & MAPK signaling pathway: 6 | Pahtways in cancer: 6 \\
\hline Cluster No. & Enriched Gene Ontology Terms (H9N2) \\
\hline \multirow[t]{4}{*}{ Cluster 1} & Nucleic acid binding - G0;0003676:68 | Gene expression - G0:0010467:53 | Nitrogen compound metabolic process - G0:0006807:62 \\
\hline & Regulation of macromolecule metabolic process - G0:0060255:58 | Anatomical structure deevelopment - G0:0048856:42 \\
\hline & Negative regulation of biological process - G0;0048519:32 | Macromolecule biosynthetic process - G0:0009059:46 \\
\hline & Intracellular transport - G0:0046907:19 | Cytoskeletal protein binding - G0:0008092:16 \\
\hline \multirow{4}{*}{ Cluster 2} & Response to virus - G0:0009615:19 | Multi-organism process - G0:0051704:25 | Response to stimulus - G0:0050896:47 \\
\hline & 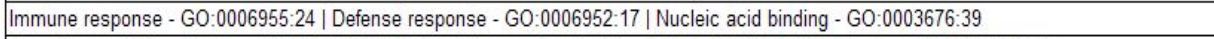 \\
\hline & Transition metal ion binding - G0:0046914:33 | Cytokine receptor binding - G0:0005126:8 | RNA binding - G0:0003723:14 \\
\hline & Cell death - G0:0008219:12 \\
\hline \multirow{3}{*}{ Cluster 3} & Localization - G0:0051179:23 | Nitrogen compound metabolic process - G0:0006807:24 | Nucleoside binding - G0:0001882:16 \\
\hline & Actin filament binding - G0:0051015:4 | RNA splicing, via transesterification reactions with bulged adenosine as nucleophile - G0:0000377:6 \\
\hline & Cellular component organization - G0:0016043:17 | Enzyme linked receptor protein signaling pathway - G0:0007167:7 \\
\hline Cluster 4 & Response to virus - G0:0009615:6 | Immune system process - G0:0002376:10 \\
\hline Cluster 5 & RNA processing - G0:0006396:7 \\
\hline Cluster 8 & Organ development - G0:0048513:13 | Response to stress - G0:0006950:11 \\
\hline Cluster 9 & Response to virus - G0:0009615:4 | Immune system process - GO:0002376:6 \\
\hline
\end{tabular}

Differentially expressed genes were significantly categorized into different GO terms and canonical pathways. Different transcription factors that can potentially be involved in the regulation of gene expressions are shown for each cluster under Expander 5 software analysis (P-value $\leq 0.05$ ). Each functional group, canonical pathway or transcription factor is followed by the number of corresponding genes. 


\subsubsection{Functional groups related to host response}

After IAV infections, many host genes with important functions have been found to show differential expression. For example, antiviral genes encoding for cytokines, which are responsible for local inflammatory reactions as well as systemic effects, were induced in their expression after IAV infections in order to impede virus infection [264]; genes relevant to cell death showed gene expression stimulation as a consequence of activation of host cellular defense mechanism to limit the virus spread by removing infected cells [265]; other genes participating in cell regulation also demonstrated their potential ability to alter their regular expression after virus interruption. Accordingly, we investigated expression levels of a batch of key genes so as to evaluate the host-virus interactions after IAV infections.

\subsection{Immune response}

After IAV infections, the host cells will initiate a series of defense responses to counteract the IAV invasions. In this process, a lot of genes will be activated to play the antiviral roles by the host cells or even inactivated to repress the antiviral response by IAV themselves [266].

In A549 cells, a few important cytokines such as RANTES (CCL5), IL28A and IL29 were detected with elevated expression levels after infections of all eight influenza A strains [Figure 5.15]. Expression levels of other ISGs including MX1, OAS1/2, RASD2, DDX58 (RIG-I), TLR3, IRF-1, IFIT1/2/3, IFI44, GIP2/3, GBP1 were also triggered after IAV infections. Detailed evaluation of these gene expression performances among different influenza $\mathrm{A}$ strains infections revealed that these genes showed relatively faint up-regulation in their expression after H1N1 infection. And this phenomenon demonstrated that IFN pathway was triggered in a weak state following H1N1 infection. Moreover, it was noted that H5N2/F118 infection initiated the up-regulated expression of these genes from an early stage (2hpi), indicating a timely initiation of host antiviral actions in H5N2/F118-infected A549 cells.

There also existed other important genes involved in immune defense such as PKR. PKR (EIF2AK2) is a crucial component of the host innate immune system. It has several downstream substrates, and one of them is the eIF $2 \alpha$, phosphorylation of which by activated PKR renders it incapable of 
participating in translation initiation, therefore leading to translation arrest and inhibition of protein synthesis from viral mRNAs. Moreover, PKR maybe also associated with the IKK $\beta$ subunit of the IKK complex which in turn activate NF- $\mathrm{BB}[232]$.

In our study, this gene was up-regulated with faint expression changes after H9N2, H5N2/F189 and H7N1 viruses infections, which might suggest the activation of this gene in these viruses infected host cells. Whereas, PKR showed down-regulated expression with 3.7-FC after H1N1 infection, which might be caused by the action from cellular inhibitor or viral proteins such as NS1 [232, 235]. Through blocking the activation of PKR, the antiviral actions of this factor would be subsequently inhibited.

Excluding these genes with up-regulated expression, there also existed a batch of antiviral genes with down-regulated expression in A549 cells after infections of these influenza A strains, especially H1N1. These host genes with significantly down-regulated expression following H1N1 infection included IFN- $\alpha$ receptor (IFNAR)-1/2, Interferon responsive gene (IFRG)-15, B-cell CLL/lymphoma 10/9-like (BCL10/9L), B-cell translocation gene 1 (BTG1), influenza virus NS1A binding protein (NS1A-BP), GALNAC4S-6ST, SOCS $5 / 6$.

Among these genes, some of them such as IFNAR1/2 and IFRG15 are related to IFN signaling pathway, and their repressed expression after infections of two human viruses, especially H1N1, could lead to the impairment of the IFN signaling transduction as well as further host antiviral function. As reported, BCL10 is essential to gene expression of A20 which is a target of NF- $\kappa B$, thereby regulating B-cell proliferation mediated by B-cell receptor signaling [267]. And B cells are able to sense these viral antigenic signatures and provide extensive protection against IAV infection [268, 269]. Down-regulated expression of BCL10 in our study indicated the negative control of host cell immune response from $\mathrm{H} 1 \mathrm{~N} 1$ infection. It has been revealed that NS1A-BP plays a role in mediating the splicing-inhibitory effects of NS1 protein through interacting with NS1 [270]. Hence, the down-regulated expression of NS1A-BP might attenuate the splicing-inhibitory effects of NS1 protein. Members in SOCS family are known to be cytokine-inducible negative regulators of 
cytokine signaling, and the potential reason for strongly down-regulated expression of SOCS 5/6 after H1N1 infection still remains to be discovered.

\subsection{Cell death}

It has been reported that infections of IAV influence the cell death regulation in host cells $[271,272]$. Thus we focused on the gene expression performances of genes related to cell death in different influenza A strains infected A549 cells.

At the first glance, H1N1 infection contributed to the relative large number of down-regulated genes while $\mathrm{H} 9 \mathrm{~N} 2$ and $\mathrm{H} 5 \mathrm{~N} 3$ infections led to a small number of down-regulated genes [Figure 5.16]. Further investigation showed that caspase 3 and caspase 8 only showed down-regulated expression after H1N1 virus infection, while caspase 9 showed down-regulated expression after H1N1, pH1N1, H5N2/F59 and H5N2/F189 viruses infections. It was found that Fas showed up-regulated expression after H1N1 infection but downregulated expression after H5N2/F59 infection in A549 cells. Besides, caspase relevant genes such as CFLAR and FADD were identified with a significantly decreasing trend in their expression in A549 cells after infection of two human strains, H1N1 and pH1N1.

It has been reported that XAF1 antagonizes the anticaspase activity of XIAP, which inhibits initiator caspase-9 that is directly involved in the activation of executioner caspase-3 [273]. In our A549 cells, expression of XAF1 was significantly elevated after infections of all influenza A strains except for H1N1, and fold regulation of this gene even reached 242-FC and 244-FC after infections of H9N2 and H5N2/F118, respectively. Accordingly, we assumed that apoptosis signaling might be induced strongly in these influenza A strains (except H1N1) infected A549 cells, particularly in H9N2 and H5N2/F118 infected A549 cells.

TNFSF10 (TRAIL), a cytokine belonging to the TNF ligand family, plays a role in the extrinsic apoptosis pathway. The binding of this protein to its receptors has been shown to trigger the activation of MAPK8/JNK, caspase 8 , and caspase 3, and induce apoptosis in further. In our study, expression of this gene showed high up-regulation after H9N2, H5N2/F118, H5N2/F59 and H5N3 
viruses infections, indicating the induction of cell apoptosis in these viruses infected A549 cells.

MDM2 and MDM4 were down-regulated with significant expression changes in A549 cells following infection of H1N1, followed by pH1N1 and H5N2/F59. Francoz S (2006) has pointed out that Mdm2 and Mdm4 are critical for the regulation of p53 levels and the fine-tuning of p53 transcriptional activity, separately [274]. However, it was strange that no significant expression change of p53 mRNA was detected, indicating the possibility that other mechanisms might exist to offset the impact on p53 from Mdm2 and Mdm4.

In summary, quite a few genes which are responsible for apoptosis signaling were suppressed in their expression in IAV infected A549 cells. Among all these influenza A strains, H1N1 infection caused the expression suppression of these genes with the highest fold changes while H9N2 and H5N2/F118 infections showed weak ability to reduce expression levels of these genes. It has been proposed that expression levels of genes relevant to cell death were stimulated as a consequence of activation of host cellular defense mechanism to limit the virus spread by removing infected cells [265]. Thus, it was hypothesized that the inhibited expression of these cell apoptosis related genes may be beneficial for virus replication and spread in A549 cells especially after H1N1 infection.

\subsection{Genes with remarkable regulations}

In addition to genes which are functionally related to immune response or cell death, a batch of host genes also showed interesting expression changes in different influenza A strains infected A549 cells. Some of these genes such as APOL2/6, PTX3, $\mathrm{CH} 25 \mathrm{H}, \mathrm{ACE} 2$ and CEACAM1 showed up-regulated expression after H9N2 infection [Figure 5.17].

APOL6, a member of the apolipoprotein L gene family, is a proapoptotic BH3-only member of the Bcl-2 family. Previous experiments indicated that over-expression of this gene induced the apoptosis of DLD-1 cells characterized by release of cytochrome c and Smac/DIABLO as well as activation of caspase-9. And during the induction of cell apoptosis, APOL6 interacted with lipid/fatty acid components, suggesting its role in connecting lipid second messengers and cell death [275]. Recent studies have also revealed 
that human APOL6 is one of the downstream targets of interferon- $\gamma$ (IFN $\gamma$ ), which sensitizes atherosclerotic lesion-derived cells (LDCs) to Fas-induced apoptosis [276]. This gene showed up-regulated expression in all our influenza A strains infected A549 cells, with the highest fold regulations detected after infections of H9N2, H5N3 and three H5N2 strains. And these observations might suggest the strong induction of apoptosis occurring in these influenza A strains infected A549 cells but the weak induction of apoptosis occurring in other strains infected A549 cells.

Another gene called long pentraxin PTX3 also showed significant upregulations in its expression after infection of H9N2 but not other influenza A strains. Interestingly, PTX3 is produced in tissues under the control of primary proinflammatory signals, such as IL- $1 \beta$ and TNF $\alpha$. It has been proved that PTX3 specifically binds to dying cells during inflammatory reactions and clear the apoptotic cells [277]. Recent studies focus on the connections of this gene with IAV infections, and it has been found that PTX3 as a new pattern recognition receptor plays a novel antiviral role in early host defense against influenza infections [278]. Furthermore, through expression a single Nglycosylation sequon, both seasonal and pandemic H1N1 are resistant to the antiviral activities of PTX3 [279]. Accordingly, antiviral function of PTX3 might be indeed resisted by other influenza A strains but not H9N2 in A549 cells.

$\mathrm{CH} 25 \mathrm{H}$, cholesterol 25-hydroxylase, plays a critical role in regulating gene expression, lipid metabolism and immune activation. Previous experiments uncovered that TLR3- and TLR4-induced transcription levels of $\mathrm{CH} 25 \mathrm{H}$ relied on the TRIF-mediated production of type I IFNs and required signaling through the IFNR and JAK/STAT1 pathway [280]. Strikingly, this gene was up-regulated with 21.8-FC after H9N2 infection. Since previous observations implied that H9N2 infection induced obvious IFN signaling, elevated expression changes of $\mathrm{CH} 25 \mathrm{H}$ might provide more evidence that this gene was indeed functionally associated with IFN pathway. In addition, $\mathrm{CH} 25 \mathrm{H}$ has also been proved to be a cholesterol related gene which has an established role in the regulation of cholesterol homeostasis, thus it was assumed that cholesterol regulation might also be involved in the host cell antiviral functions after H9N2 infection in A549 cells [281]. 
Besides these genes with up-regulated expression, some genes with down-regulated expression were also found out to be specific to H1N1 infection. These genes included MGA, ELL2, GJC1, TMEM177, GIGYF2, GLT8D3, CCDC12 and a group of genes belonging to zinc finger family.

In H1N1 infected A549 cells, the temporal expression trend of the Zinc Finger (ZNF) genes decreased sharply from 6 to 10 hpi as described above, with the most significant down-regulation $\geq 10$-FC detected at the last time point. Our results concurred with that reported in Lee et al., 2010 where ZNF genes were presented with more significant expression changes in primary alveolar epithelial cells infected with a seasonal H1N1 (A/Hong Kong/54/1998) than with pandemic H1N1 (A/Hong Kong/415742/2009) [261]. The same study suggested that the ZNF may have a role in suppressing viral transcription and this may facilitate more efficient replication of seasonal H1N1 in the host cells as compared to $\mathrm{pH} 1 \mathrm{~N} 1$. Accordingly, down-regulated expression levels of many zinc finger proteins in $\mathrm{H} 1 \mathrm{~N} 1$ infected A549 cells might indicate good adaption of this influenza A strain in A549 cells. Down-regulated expressions of these ZNF genes were also detected after infections of other influenza A strains such as pH1N1, H5N2/F59, H5N2/F189 and H7N1 with a relative low fold changes, indicating relative weak adaption of these influenza A strains in A549 cells.

MGA as a novel Max-interacting protein is not only able to interact to bHLHZip domain, but also conceive a T-domain DNA-binding motif [282]. Studies indicated that heterodimers formed by Max and other genes MYC and MGA target the genes that are involved in cell cycle progression [283]. Subsequently, down-regulated expression of this gene with 27.3-FC after H1N1 infection might implicate the negative control on the target genes associated with cell cycle.

ELL2 is a member of ELL family of RNA polymerase II elongation factor. Together with ELL, it can increase the catalytic rate of RNA polymerase II transcription on catalyzing the transcription of DNA to synthesize precursors of mRNA and most snRNA and microRNA [284]. In H1N1 infected A549 cells, both ELL2 and its positive regulator EAF1/2 were significantly down-regulated at their expression level, denoting negative influence on transcription activities of RNA polymerase II. 
Other genes which are relevant to cell gap junction or adherence junction also showed repressed expression in H1N1 infected A549 cells. Among these factors, GJC1 is a component of gap junctions which are composed of arrays of intercellular channels while CD44 functions as a cellsurface glycoprotein that is involved in cell-cell interactions, cell adhesion and migration.

\subsubsection{Regulations of gene expression in canonical pathways}

According to recent researches, regulations of some cellular signaling pathways are altered following IAV infection: JAK-STAT pathway, NF$\mathrm{kB} / \mathrm{IKK}$ pathway, MAPKs signaling pathway, PI3K/Akt pathway and so on. These pathways involved in the host antiviral response are important for viral entry, viral replication, viral propagation and apoptosis. IAV manipulates the molecular function of signaling molecules for efficient viral pathogenesis [285]. 


\begin{tabular}{|c|c|}
\hline 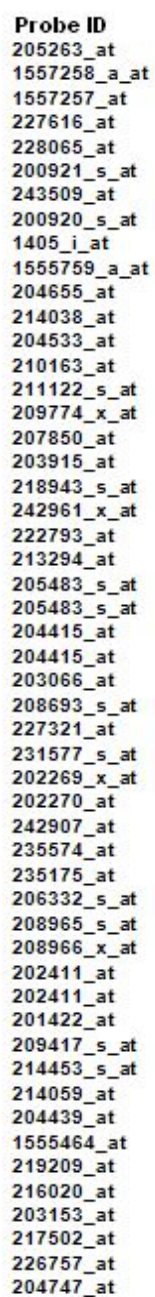 & 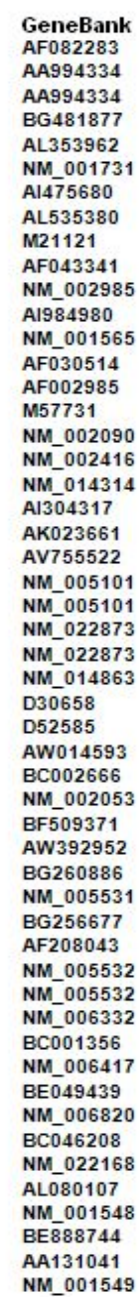 \\
\hline
\end{tabular}

Gene Title B-cell CLLlymphoma 10

B-cell CLLIlymphoma 10

B-cell CLL/ymphoma 9-like

B-cell translocation gene 1 , anti-proliferative

B-cell translocation gene 1, anti-proliferative

B-cell translocation gene 1 , anti-proliferative

chemokine $(C-C$ motin) ligand 5

chemokine $C C \mathrm{C}$ motif ligand

chemokine (C-X-C motiff) ligand 11

chemokne $(C-x-C$ moth) ligand 11

chemokine ( $\mathrm{C}-\mathrm{X}-\mathrm{C}$ motif) ligand 3

chemokine ( $\mathrm{C}-\mathrm{X}-\mathrm{C}$ motif) ligand 9

DEAD (Asp-Glu-Ala-Asp) box polypeptide 58

DEAD(Asp-GIUAlaAsp) box polypeptide 58

eukaryotic translation initiation factor 2-alpha kinase 2

interferon, alpha-inducible protein (clone IFI-15K)

interferon, alpha-inducible protein (clone IFI-15K

interferon, alpha-inducible protein (clone IFI-6-16)

Bcell RAG as sociated protein

glycyl-tRNA synthetase

opposite strand transcription unit to STAG3

guanylate binding protein 1 , interferon-inducible, $67 \mathrm{kD}$

guanylate binding protein, intefreron-inducible, $67 \mathrm{kD}$

guanylate binding protein 4

guanylate binding protein 4

intereron, gamma-inducible protein 16

interferon, gamma-inducible protein 16

interferon, al pha-inducible protein 27

interferon, alpha-inducible protein 27

interferon, gamma-inducible protein 30

interferon-induced protein 35

Interferon-induced protein 44

interferon-induced protein 44-like

interferon induced with helicase $\mathrm{C}$ domain 1

interon

interferon-induced protein with tetratricopeptide repeats 1

interferon-induced protein with tetratricopeptide repeats 2

interferon-induced protein with tetratricopeptide repeats 2
interferon-induced protein with tetratricopeptide repeats 3

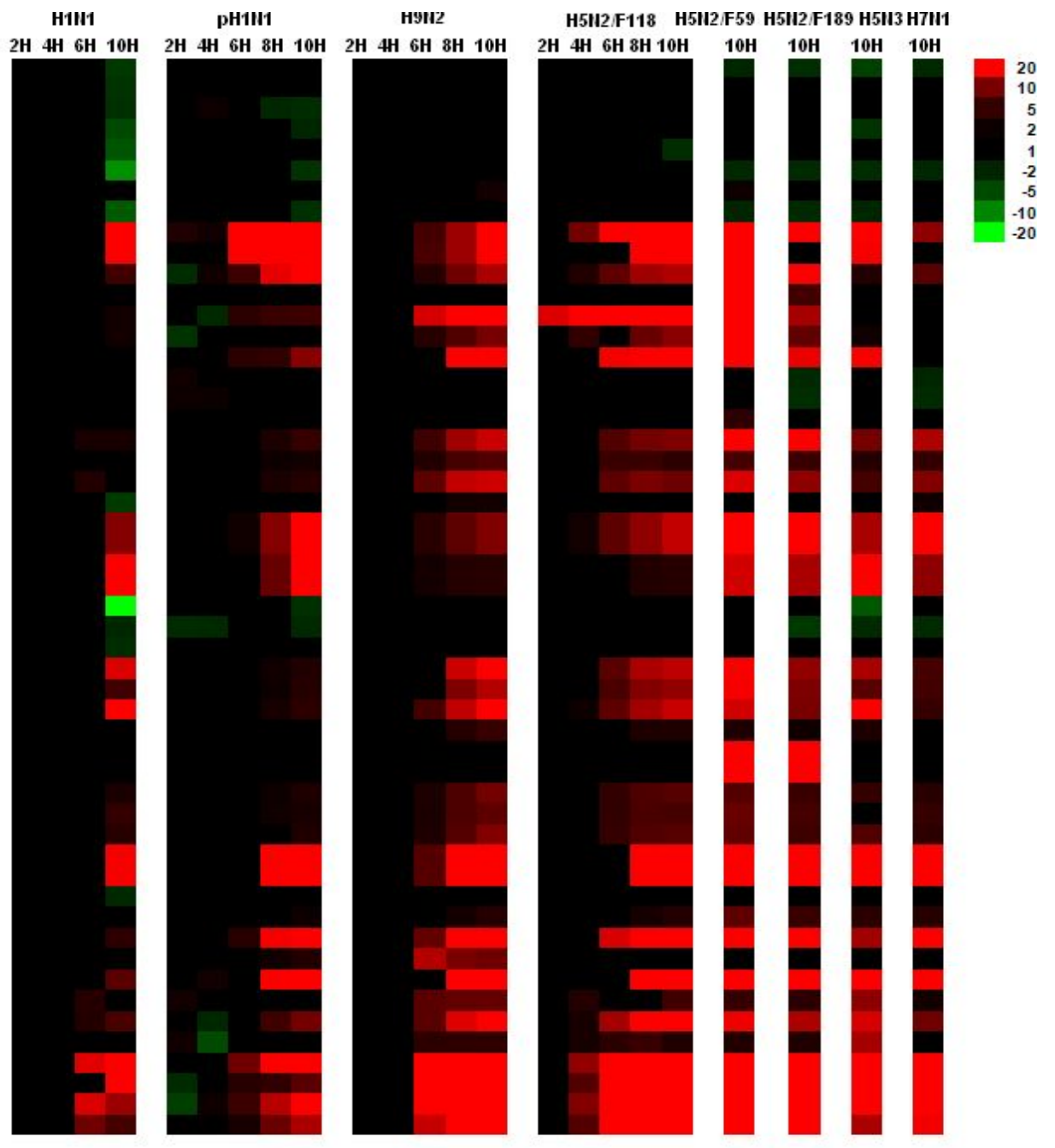




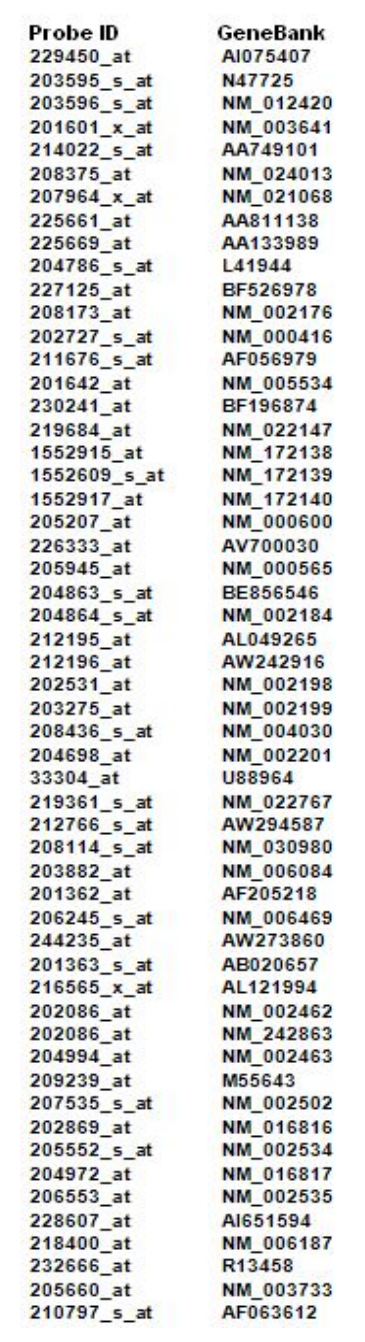

Gene Title

interferon-induced protein with tetratricopeptide repeats interferon-induced protein with tetratricopeptide repeats 5
interferon-induced protein with tetratricopeptide repeats 5 interferon induced transmembrane protein $1(9-27)$ interferon, alpha 1

interferon (alpha, beta and omega) receptor 1
interferon (al pha, beta and omega) receptor 1 interferon (alpha, beta and omega) receptor Interferon (alpha, beta and omeal receptor 2 interferon, beta 1 , fibroblast interferon gamma receptor 1 interferon gamma receptor 2 (interferon gamma transducer 1 )
Interferon responsive gene 15 $28 \mathrm{kD}$ interferon responsive protein interleukin $28 \mathrm{~A}$ (interferon, lambda 2) interleukin 29 (interferon, lambda interleukin 6 (interferon, beta 2) interleukin 6 receptor

interleukin 6 signal transducer (gp130, oncostan $M$ receptor) interleukin 6 signal transducer (gp130, oncostatin M receptor) Interleukin 6 signal transducer (gp130, oncostatin M receptor) Interleukin 6 signal transducer (gp 130, oncostatin $M$ receptor) interferon regulatory factor 2

interferon regulatory factor 7

interferon stimulated exonuclease gene $20 \mathrm{kDa}$

\section{interferon stimulated exonuclease gene $20 \mathrm{kDa}$-like}

interferon stimulated exonuclease gene $20 \mathrm{kDa}-$-like
interferon stimulated exonuclease gene $20 \mathrm{kDa}$-like

interferon-stimulated transcription factor 3 , gamma $48 \mathrm{kD}$

influenza virus NS1A binding protein

influenza virus NS1A binding protein

influenza virus NS1A binding protein

myxovirus (influenza virtses

myxovirus (influenza virus) resistance 1

myxovirus (influenza virus) resistance 2 (mouse)

nuclear factor of kappa light polypeptide gene enhancer in B-cells

2', $5^{\prime}$-oligoadenylate synthetase $1,40 / 46 \mathrm{kDa}$

$2^{2}-5$-oligoadenylate synthetase $2,69 / 71 \mathrm{kDa}$

$2 \cdot-5$ '-oligoadenylate synthetase 2,69/71k $\mathrm{ka}$

$2 '-5$ '-oligoadenylate synthetase $3,100 \mathrm{kDa}$

$2 '-5$ '-oligoadenylate synthetase $3,100 \mathrm{kDa}$

2 '-5'-oligoadenylate synthetase-like
$2^{2}-5$-oligoadenylate synthetase-like

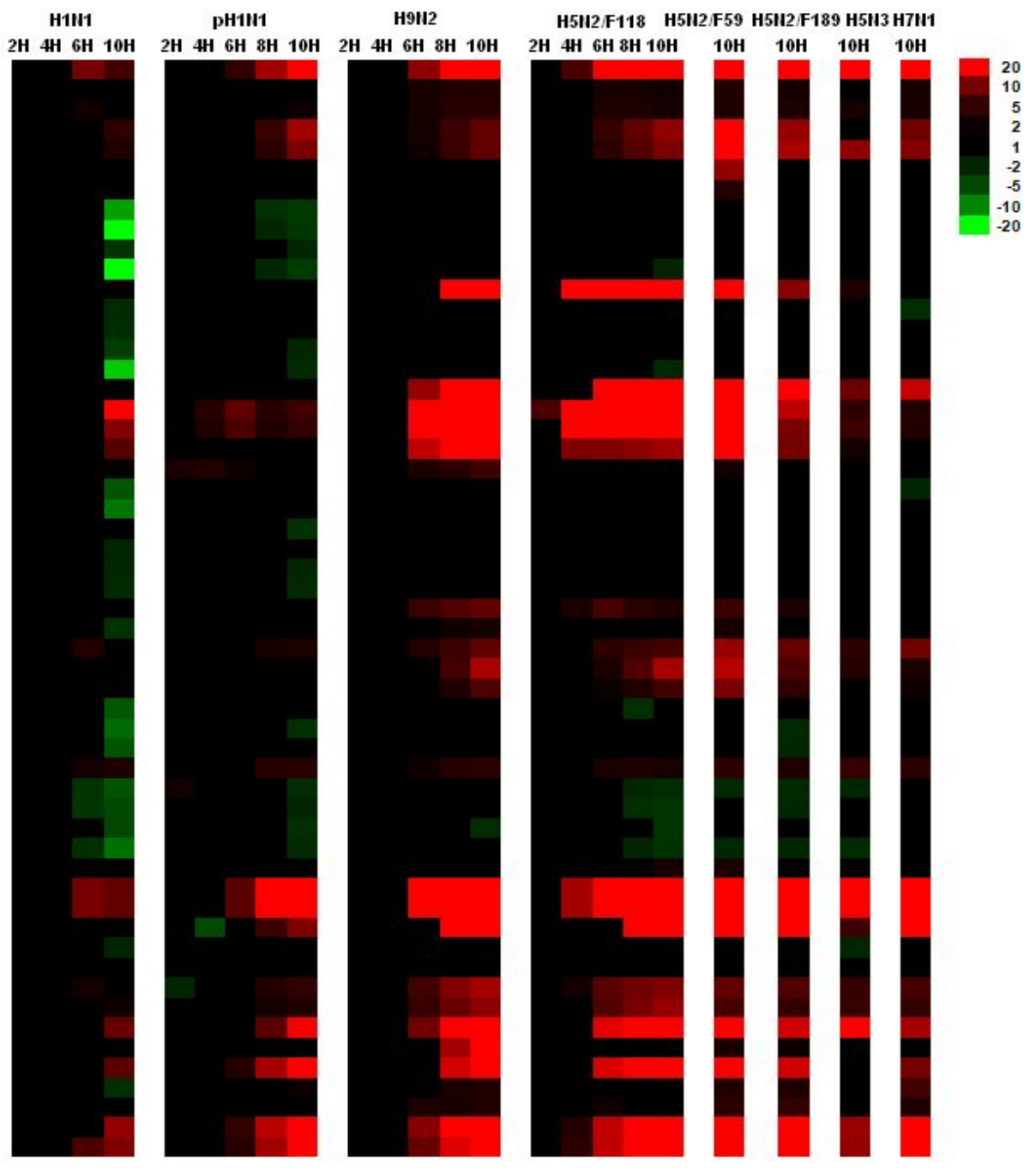




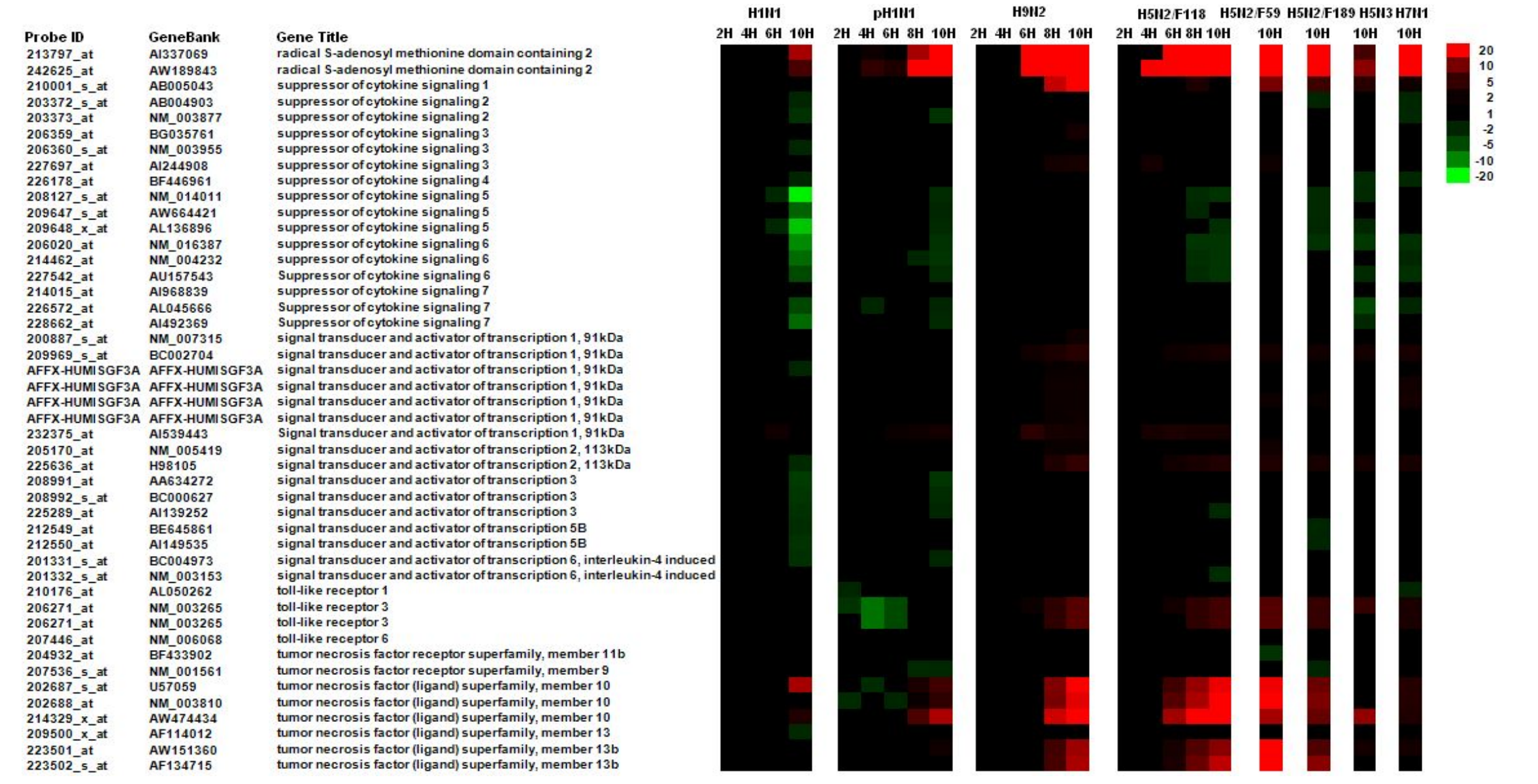

Figure 5.15 Expression of probe sets involved in immune response in influenza A viruses-infected A549 cells. Eight influenza A strains were infected with different examined cells, and the expression of probe sets related to immune response was examined by microarray analysis at different time points as shown. The data is represented by heat map analysis showing up-regulated (red), down-regulated (green) or no changes (black) in expression, and the FC range is indicated (P-value $\leq 0.05)$. 


\begin{tabular}{|c|c|}
\hline 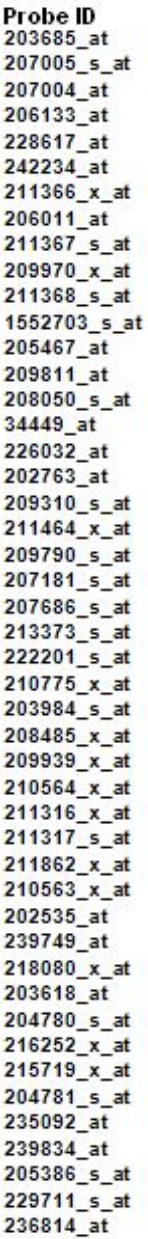 & 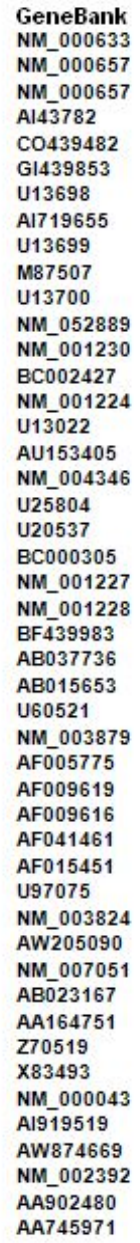 \\
\hline
\end{tabular}

Gene Title

B-cell CUلlymphoma 2

B-cell CLLIymphoma 2

XIAP as sociated factor-1

XIAP as sociated factor-1

caspase 1 , apoptosis-related cysteine peptidase caspase 1, apoptosis relatedcysteine peptidase caspase 1 , poptosis related cysteine peptidase caspase 1 , apoptosis-related cysteine peptidase caspase 1 , apoptosis-related cysteine peptidase caspase 10, apoptosis-related cysteine peptidas caspase 2 , apoptosis-related cysteine peptidase caspase 2 , apoptosis-related cysteine peptidase azpase 2 apoptosis selated cysteine peptidase caspase 3 apoptosis-related cysteine peptidase caspase 4, apoptosis-related cysteine peptidase caspase 6 , apoptosis-related cysteine peptidase caspase 6, apoptosis-related cysteine peptidase caspase 7 , apoptosis-related cysteine peptidase caspase 8 , apoptosis -related cysteine peptidase CASP8 as sociated protein 2

caspase 9 , apoptosis related cysteine peptidase caspase 9 , apoptosis-related cysteine peptidase CASP8 and FADD-like apoptosis regulator CASPP and FADD-like apoptosis regulator CASP8 and FADD-like apoptosis regulator CASP8 and FADD-like apoptosis regulator CASP8 and FADD-like apoptosis regulator CASP8 and FADD-like apoptosis regulator Fas (TNFRSF6)-associated via death domain Fas (TNFRSF6) as sociated factor 1 Fas (TNFRSF6) associated factor 1 Fas (TNF receptor superfamily, member 6)
Fas (TNF receptor superfamily, member 6) Fas (TNF receptor superfamily, member 6 ) Fas (TNF receptor superfamily, member Cytokine induced apoptosis inhibitor 1

Cytokine induced apoptosis inhibitor 1

Mdm2, transformed 373 cell double minute 2, p53 binding protein Mdm2, transformed 3 T3 cell double minute 2, p53 binding protein
Mdm4, transformed 3 T3 cell double minute 2, p53 binding protein
H1III

$2 \mathrm{H} 4 \mathrm{H} 6 \mathrm{H} 10 \mathrm{H}$

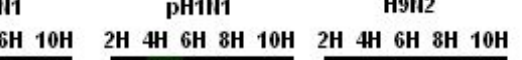

H9112

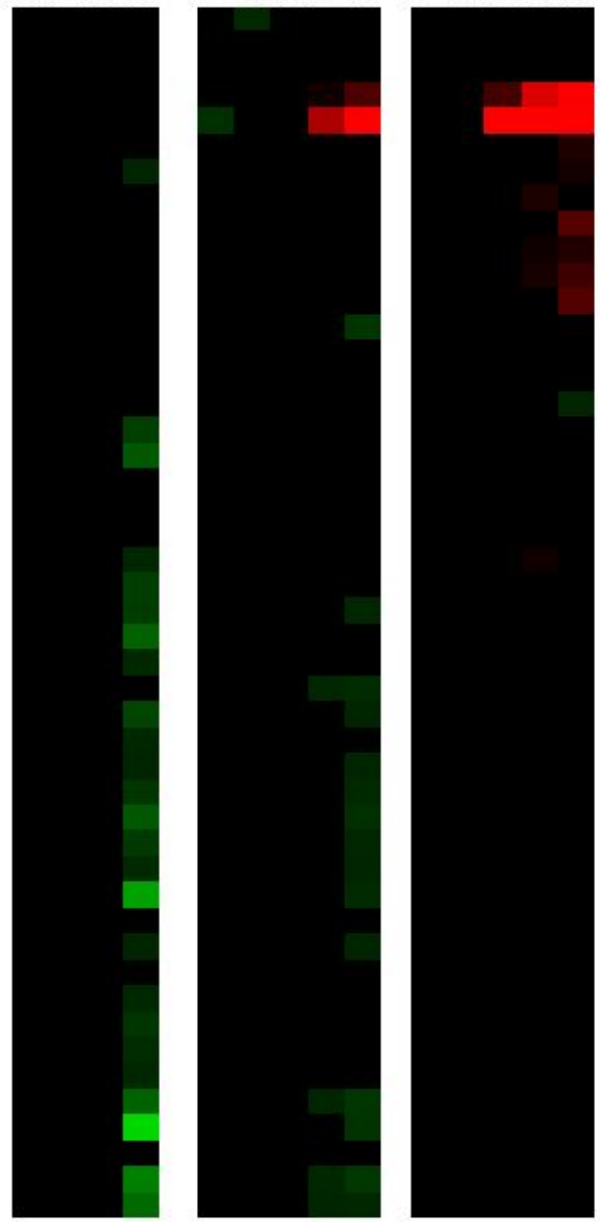

$4 \mathrm{H}$ (H)

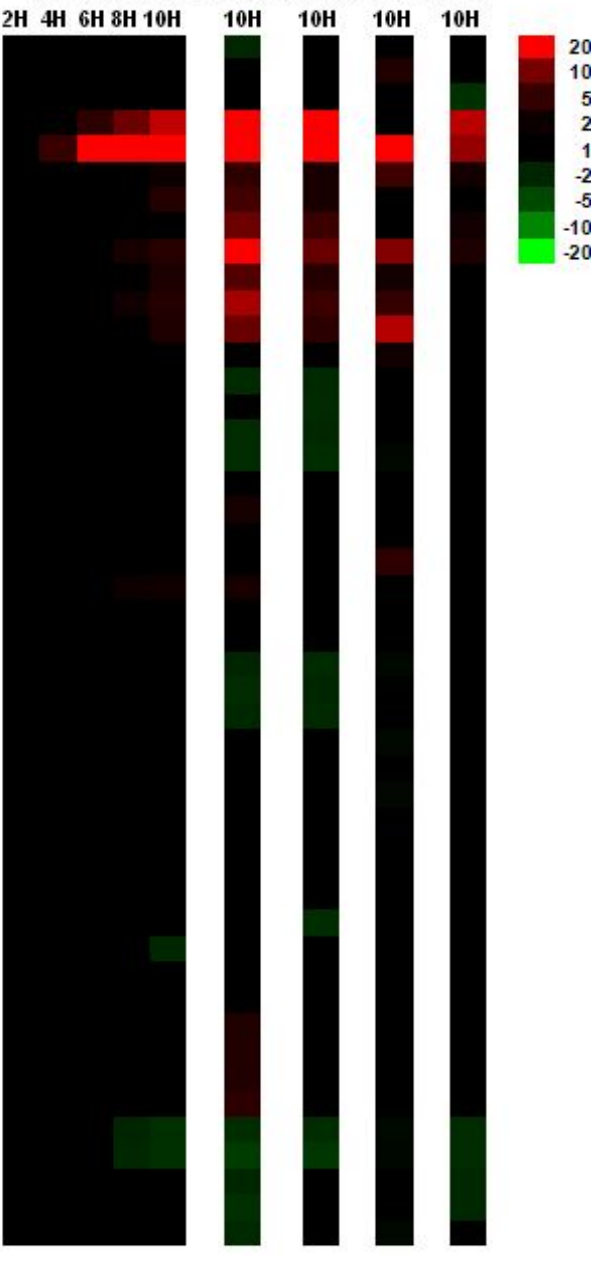



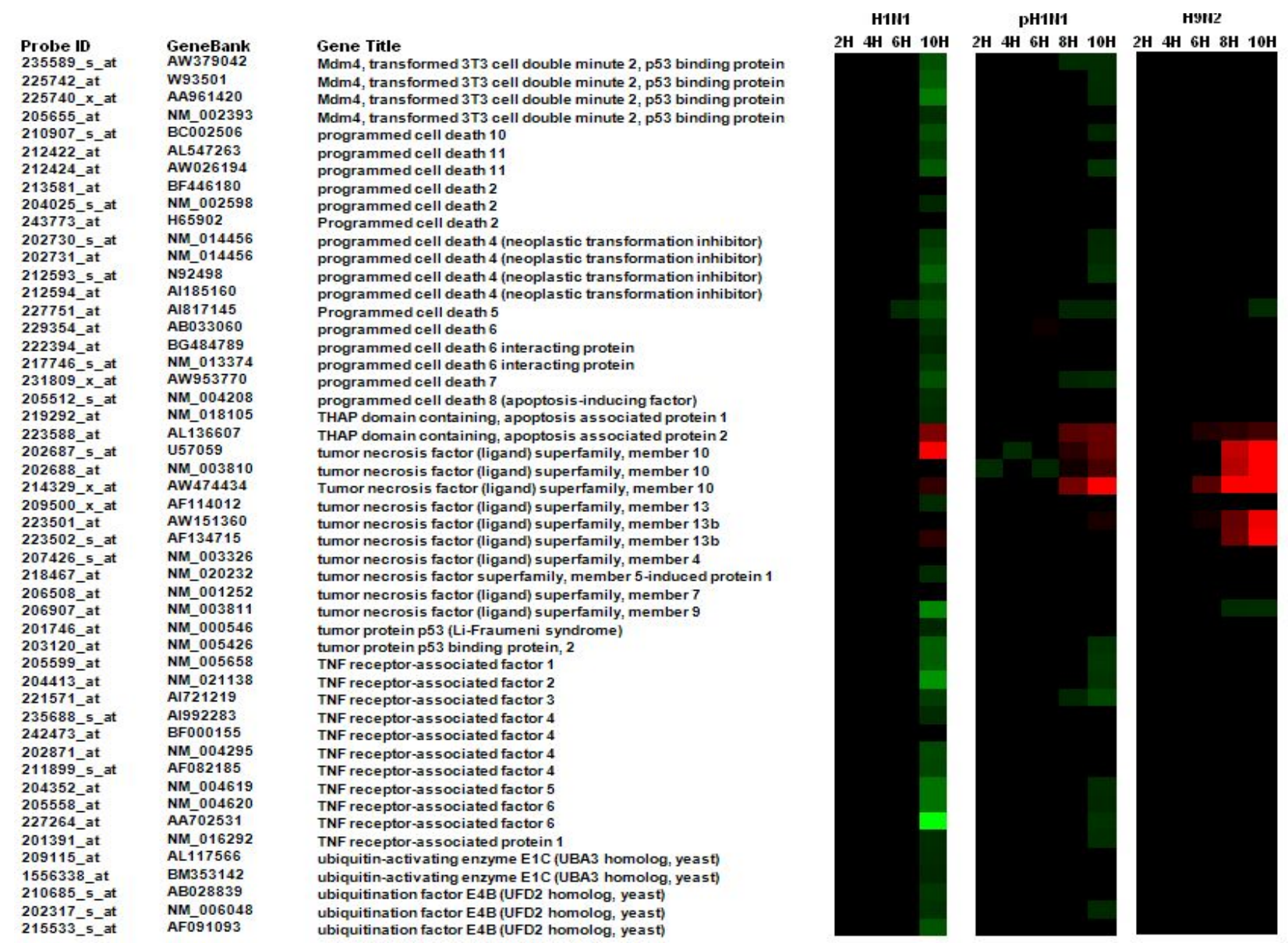

H5H2F118 H5H2F59 H5H2F189 H5H3 H7H11

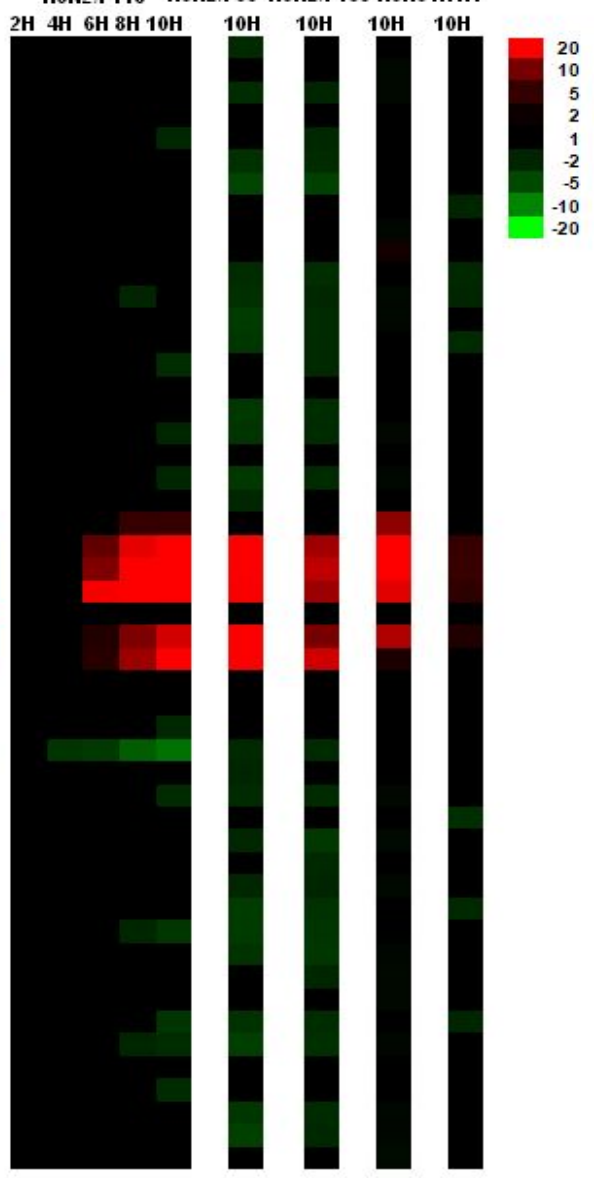

Figure 5.16 Expression of probe sets involved in cell death in influenza A viruses-infected A549 cells. Eight influenza A strains were infected with different examined cells, and the expression of probe sets related to cell death was examined by microarray analysis at different time points as shown. The data is represented by heat map analysis showing up-regulated (red), down-regulated (green) or no changes (black) in expression, and the $\mathrm{FC}$ range is indicated ( $\mathrm{p} \leq 0.05$ ). 

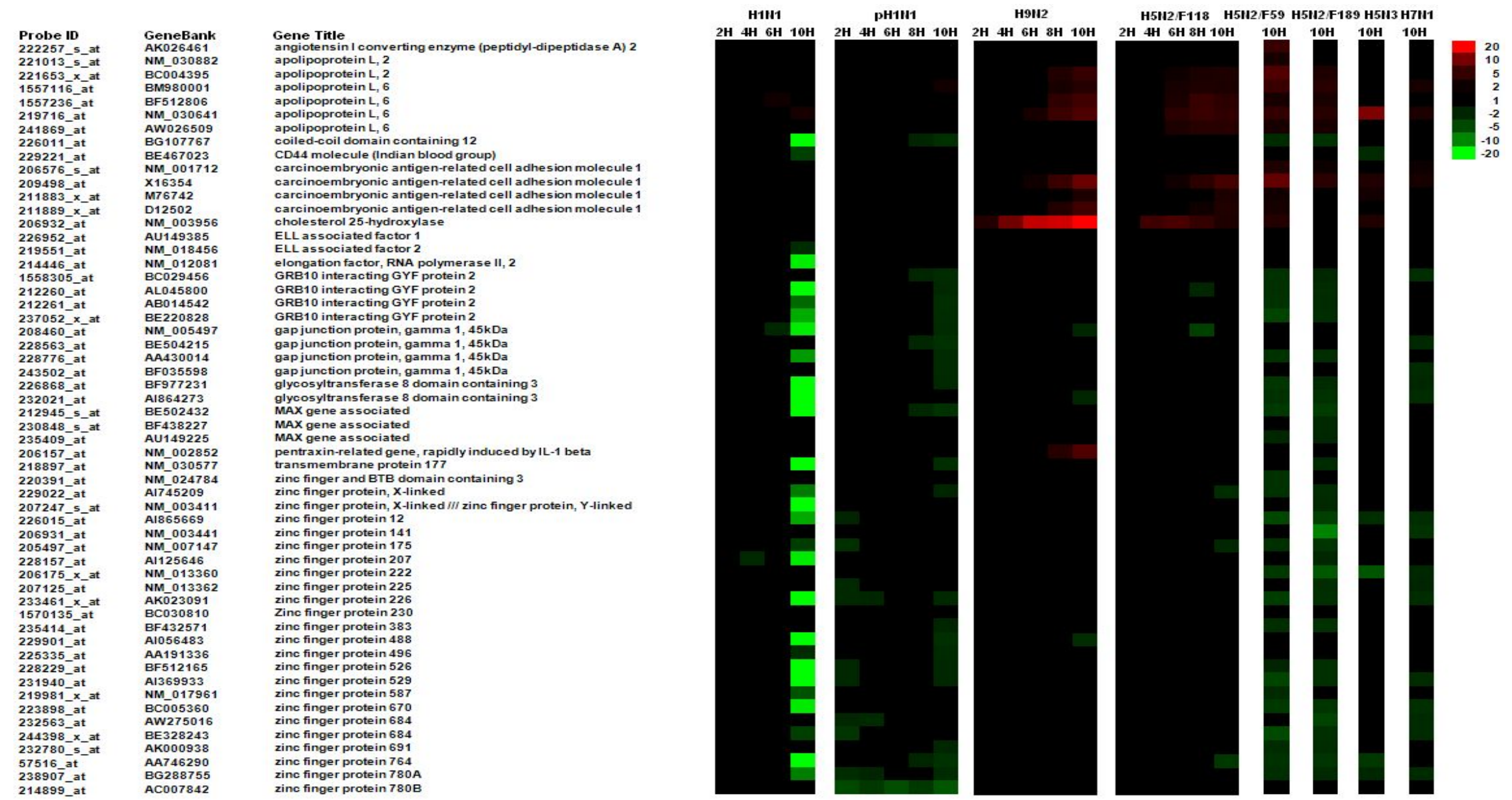

Figure 5.17 The probe sets with topmost expression changes in influenza A viruses-infected A549 cells. Eight influenza A strains were infected with different examined cells, and the global host gene expression was examined by microarray analysis at different time points. The probe sets with topmost expression changes were listed. The data is represented by heat map analysis showing up-regulated (red), down-regulated (green) or no changes (black) in expression, and the FC range is indicated ( $\mathrm{p} \leq 0.05$ ). 


\subsection{Interferon signaling pathway}

At the early stage of IAV infection, IFN signaling pathway, especially type I, is activated. After that, signals will be transferred into the corresponding receptors, and multiple cytokines inside the nucleus will be triggered. On this basis, a series of immune responses will be initiated to reject the virus invasion [226].

Different gene expression profiles in different influenza A strains infected A549 cells revealed that IFN type I signaling was obviously triggered after infections of all avian viruses. And among the avian viruses infections, different gene expression patterns were detected in the pathway. For example, expression of IFN $\alpha / \beta$ and a few downstream genes were initiated at 4 hpi after H5N2/F118 infection but initiated at 6 hpi after H9N2 infection. So it was concluded that H5N2/F118 infection initiated the TNF type I pathway as well as following immune reactions at an earlier stage when compared to H9N2 infection.

Expression of IFN $\alpha / \beta$ in IFN type I signaling transduction were repressed around $8 \mathrm{hpi}$, and expression of a series of downstream molecules were subsequently repressed after infections of two human viruses, particularly H1N1. The comparison of gene expression in this pathway between infections of two human viruses showed that IFNAR1/2 were down-regulated with a more prominent fold change after H1N1 infection, and expression of STAT1 and STAT2 were only down-regulated in H1N1 infected A549 cells. These observations indicated that interferon signaling pathway was inhibited to a more significant extent after H1N1 infection than pH1N1 infection.

Mechanism of controlling IFN type I mediated antiviral response has become a hot study topic these years, and researchers have found competitive interactions between virus and receptors like RIG-I to repress the IFN type I pathway [259]. In our study, specific influenza A strains such as H1N1 and pH1N1 might indeed evolve some new mechanisms to limit the production of IFN $\alpha / \beta$ and STAT1/2 from an early stage at the transcriptional level.

\subsection{NF-кB activation by viruses}

Nuclear Factor- $\kappa \mathrm{B}$ pathway plays a vital role in mediating inflammation, immune response, proliferation and apoptosis. Based on its key role, influenza 
virus has evolved strategies to direct antiviral NF- $\mathrm{B}$ activity into an IFNsuppressive and apoptosis-promoting function. During productive virus infection, NF- $\mathrm{B}$ regulates expression of a number of genes, including the antiviral cytokine IFN $\beta$, the proapoptotic factors TRAIL, Fas and FasL and the suppressor of cytokine signaling SOCS-3. While IFN $\beta$ primarily exerts antiviral functions by inducing an innate antiviral gene expression program, the simultaneous vRNA-induced expression of SOCS-3 limits this response. Furthermore, TRAIL and FasL induce caspase activation, and active caspases allow an enhanced release of vRNP complexes from the nucleus [Figure 5.18] [23].

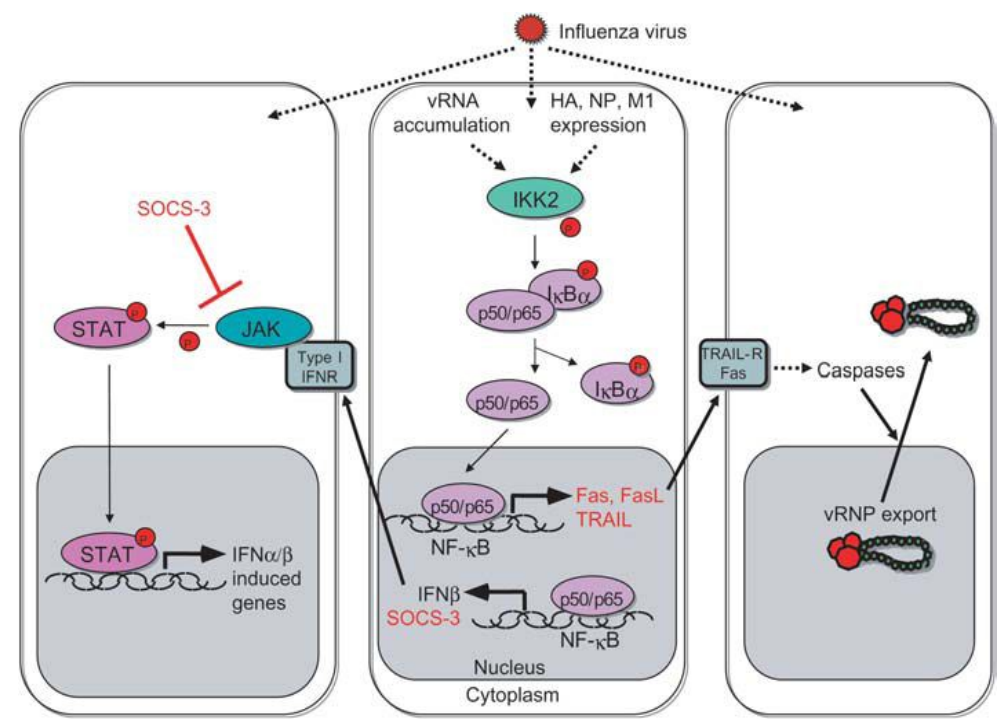

Figure 5.18 Two virus supportive functions of the IKK/NF- $\kappa$ B signaling module in influenza virus infected cells (adapted Ludwig $\mathrm{S}$ et al., 2008)[23].

Observation of NF- $\mathrm{BB}$ pathway in $\mathrm{H} 1 \mathrm{~N} 1$ and $\mathrm{pH} 1 \mathrm{~N} 1$ infected A549 cells illustrated that majority of genes participating in this pathway showed down-regulated expression from 8 to 10 hpi. Through shutting down this pathway, migration of NF- $\kappa \mathrm{B}$ into nucleus would diminish, subsequently relevant antiviral and anti-apoptotic activities mediated by this pathway were all inactivated.

In contrary to infections of $\mathrm{H} 1 \mathrm{~N} 1$ and $\mathrm{pH} 1 \mathrm{~N} 1$, no gene was found differentially down-regulated obviously at its expression level in H9N2 infected A549 cells. In this case, host cells were able to activate this pathway normally and relevant functions were induced to fight with H9N2 invasion. As to A549 cells infected with other influenza A strains, part of the genes showed down- 
regulated expression while others did not show any significant expression change.

\subsection{Role of MAPK signaling in pathogenesis in influenza}

MAPK cascades are involved in the conversation of various extracellular signals into cellular responses as diverse as immune response, cell death and proliferation. Nowadays, studies have proved that this pathway plays important roles in proinflammatory during IAV infection [286, 287].

Till now, studies show that IAV infection activates all these 4 members of MAPK family in order to promote vRNP traffic and virus production. P38 MAPK activation regulates the expression of RANTES production and chemokines after IAV infection [288]. Experiments showed that p38 was able to induce TNF cytokine in H5N1-infected cells. Besides, inhibition of p38 decreased the virus titer, which suggested that IAV infection induced TNF in a p38-dependent manner and p38 MAPK activation was essential for virus replication. Similar to $\mathrm{p} 38$, JNK is also associated with the expression of RANTES. Ludwig S et al (2001) have observed that stimulation of AP1dependent gene expression strongly correlated with activation of JNK, which maybe contributed by viral RNA accumulation during replication [289]. Block of JNK signaling of the cascade resulted in not only inhibition of virus-induced JNK activation but also repression of AP-1 activity as well as impairing transactivation of IFN $\beta$ promoter, thereby increasing virus products. Consequently, it is concluded that IAV induced activation of JNK and AP-1 is part of the innate antiviral response. As to Raf/MEK/ERK pathway, Pleschka S et al (2001) did experiments to show that cells infected with IAV led to upregulate this signaling pathway [290]. Inhibition of this pathway can cause the impairment of the virus growth and decreasing of the RNP nucleus export, however, it will not influence the viral RNA and protein synthesis. Additionally, ERK $1 / 2$ and ERK are vital for the expression of pro-inflammatory like IL1 $\beta$, IL6 , TNF- $\alpha$, etc. Recent experiment found that HA accumulation in the membrane and its tight association with lipid-raft domains triggered activation of the MAPK cascade and induced RNP export, which proved again that Raf/MEK/ERK pathway was tight related to IAV replication [291]. 
Through investigating the details of this pathway in different influenza A strains infected A549 cells, we found that caspase 3 was strongly downregulated at its expression level after H1N1 infection so that the caspase 3 mediated cell death could be attenuated.

Although expressions of JNK or even MKK 4/7 were down-regulated following infections of viruses excluding H9N2, RANTES and IP10 showed up-regulated expression after infections of most of the influenza A strains. Among them, H5N2/F118 infection triggered their expression elevation from 2 hpi while H9N2 infection initiated their expression elevation from 6 hpi. Although RANTES was up-regulated with 3.4-FC in its expression at 2 hpi, expression levels of RANTES and IP10 were apparently inhibited around 4 hpi and then induced again from 6 hpi after pH1N1 infection. And H1N1 infection contributed to the remarkable up-regulation of these two chemokines in their expression around $10 \mathrm{hpi}$. It has been reported by $\mathrm{Qu} \mathrm{B}$ et al (2012) that RANTES and IP10 might be the crucial contributors to pro-inflammatory responses in H9N2-infected intestinal epithelia [292]. Thus, significant and early up-regulation of RANTES and IP-10 at their expression level in H5N2/F118 infected A549 cells might implicate strong and timely proinflammatory responses triggered by these two genes.

\subsection{Role of PI3K/Akt signaling in pathogenesis in influenza virus}

$\mathrm{PI} 3 \mathrm{~K}$ and its downstream effector Akt/protein kinase B (PKB) have been identified as IAV induced signaling mediators recently, and full activation of Akt/PKB requires phosphorylation at Thr308 and Ser473 [8]. PI3K/Akt pathway plays different functions on IAV infection. Initially, Lu X et al (2011) found that PI3K/Akt pathway was involved in CXCL-10 promoter activity upon IAV infection [293] and Chiou WF et al (2011) mentioned that IRF3 and NF$\kappa \mathrm{B}$ might also involve in this procedure [294]. Furthermore, experiments suggested that block of PI3K/Akt activation lead to a reduction in virus yield [295] and PI3K/Akt pathway is induced by NS1 protein to support effective IAV replication and propagation [296]. Besides, PI3K is also activated by RIGI to be essential for complete IRF-3 activation and consequent induction of the type I interferon response [297]. 
In our study, the expression inhibition of PI3K p110 resulted in the suppression of GSK3 $\beta$ and Caspase 9 at their transcriptional level in A549 cells infected with H1N1, pH1N1, H5N2/F59 and H5N2/F189. These observations indicated that the antiviral role of PI3K might be inhibited and this apopsotis signaling might be impaired in these viruses infected A549 cells.

\subsection{Role of Wnt/GSK-3ß signaling in pathogenesis in influenza virus}

WNT family members increase IFN production in IAV-infected cells. Genes in this pathway globally showed in a inhibited status at their expression level in A549 cells after infections of all influenza A strains except H5N3 and H9N2. However, down-regulated expression of IFN was only detected after H1N1 and pH1N1 infections. In this situation, gene expression levels in IFN signaling pathway do not always correlate with gene expression levels in Wnt/GSK-3 $\beta$ pathway, indicating that other mechanisms might also contribute to IFN production.

Shapira SD et al (2009) have observed that viral proteins interact with NF- $\mathrm{B}$, apoptosis and WNT pathways primarily through NS1 and also through two viral polymerase subunits PB1 and PB2 [298]. On the basis of this observation, the inhibitory gene expression in the Wnt/GSK-3 $\beta$ might be due to the negative interactions from these influenza proteins.

\subsection{Cell cycle: G1/S and G2/M checkpoints regulation}

The cell cycle consists of four distinct phases. During G1 phase (growth phase), various enzymes that are mainly required for DNA replication in S phase are synthesized at a marked rate. Next, the cell continues to grow and significant biosynthesis occurs in G2 phase, and the production of microtubules is mainly involved in this phase. During final $\mathrm{M}$ phase, cell growth stops and the cell is division into two daughter cells. The duration of the phases of the cell cycle is variable in different cell lines. For a typical rapidly proliferating human cell with a total cycle time of 24 hours, M phases is about 1 hour; G1 is about 11 hours; S phase is completed within 8 hours; and G2 is about 4 hours.

The cell cycle progression is accomplished by a series of control points. A major regulatory point which occurs late in G1 and controls progression from G1 to $\mathrm{S}$ is called G1/S checkpoint. This G1/S checkpoint stall the cell cycle 
until repairs are made or target the cell for destruction via apoptosis in the case that the repairs cannot be made. The other G2/M checkpoint senses unreplicated DNA, which generates a signal to stall the cell cycle and therefore prevents the initiation of M phase before completion of S phase [299].

The increased expression of cyclin D allows its interaction with CDK4/6 by competing with $\mathrm{CDK}$ inhibitor 16 for binding, and thereby overcomes checkpoint G1/S. Once the active CDK4/6-cyclin D complexes form, they phosphorylate $\mathrm{Rb}$, which relieves the inhibition of the transcription factor E2F. $\mathrm{E} 2 \mathrm{~F}$ is then able to induce cyclin $\mathrm{E}$ expression, and cyclin $\mathrm{E}$ interacts with CDK2 to allow for G1-S phase transition.

The CDKs that are associated with checkpoint G2/M are activated by phosphorylation of the CDK by the action of a Mitosis Promoting Factor (MPF), which is related to cyclin B-Cdc2 kinase complex. The molecular nature of this checkpoint involves $\mathrm{Cdc} 25$, which under favorable conditions removes the inhibitory phosphates present within the MPF complex.

Observation from A549 global host gene profiles showed that quite a lot of genes involved in the G1/S and G2/M checkpoint regulation showed downregulated expression after H1N1, pH1N1, H5N2/F59 and H5N2/F189 viruses infections. On the contrary, few genes showed down-regulated expression after H9N2 infection. For G1/S checkpoint regulation pathway, it seems that all key factors such as cyclin D, CDK4/6, phosphate-Rb, cyclin E, CDK2 except E2F were down-regulated at their expression level and the whole network was shut down at 10 hpi in H1N1-infected A549 at 10 hpi. Infections of pH1N1, H5N2/F59 and H5N2/F189 also caused expression suppression of most genes. In the contrary, none of them has significant expression change in H9N2infected A549 cells. According to previous knowledge, shutting down of this pathway in H1N1, pH1N1, H5N2/F59 and H5N2/F189-infected A549 cells might result in the arrest of cell cycle in G1 stage.

When it comes to the G2/M DNA damage checkpoint regulation, key molecules such as cyclin B, Cdc2, Cdc25 all showed down-regulated expression at mRNA level in H1N1, pH1N1, H5N2/F59 and H5N2/F189infected A549 cells. However, none of them has significant expression change in H9N2-infected A549 cells. 
Other researchers also did a series of experiments to uncover the cell cycle regulation after IAV infection. In the experiments, A549 cells were infected with $\mathrm{A} / \mathrm{WS} / 33$ and then assayed for expression of cellular proteins at 8hpi, 12hpi and 24 hpi, using corresponding antibodies. Experimental results revealed that key molecules mentioned above including cyclin E, cyclin D, phosphorylated $\mathrm{Rb}$ were down-regulated at the protein level and cell cycle analysis by flow cytometry displayed that the percentage of cells in G1 phase of the cell cycle is much higher in IAV infected cells than the normal percentage in MOCK cells. Consequently, it is concluded that IAV replication induces cell cycle arrest in G0/G1 phase [300]. When compared to our microarray data which detected at mRNA level, cyclin E, cyclin D, phosphorylated Rb were also down-regulated in our H1N1 infected A549 cell, which was absolutely consistent with previous experiments. The only difference was the expression change of $\mathrm{p} 21$, which was tested with up-regulated expression by $\mathrm{He} \mathrm{Y}$ et al (2010) but showed down-regulated expression in our A549 cells after H1N1 infection. Anyway, this finding roughly supported our assumption that cell cycle were arrest in our H1N1 virus infected A549 cell to some extent.

\subsubsection{Host gene expression in CEF cells}

\subsubsection{Global profiling of gene expression}

\subsection{Heat maps of global gene expression}

In influenza A strains infected with CEF cells, the numbers of differentially expressed probe sets increased with the infection time increasing following infections of all H1N1, H9N2 and H5N2/F118 viruses. And the largest number of probe sets with up-regulated expression and the lowest number of probe sets with down-regulated expression were both detected after H1N1 infection at 10 hpi [Figure 5.19]. It was interesting that infection of H5N2/F118 triggered an earlier expression repression of some probe sets in their expression compared to other two strains.

\subsection{Distribution of differentially expressed probe sets}

In CEF cells, infections of H1N1, H9N2 and H5N2/F118 induced high portions of genes with up-regulated expression [Table 5.6]. However, portions of up-regulated probe sets were low after infections of other influenza A viruses, 
especially H7N1. In addition, infections of H5N2/F118 and H5N2/F189 caused more probe sets with down-regulated expression than infections of other influenza A strains in CEF cells. Among the three H5N2 strains, H5N2/F118 infection resulted in the highest portion of probe sets with either up- or downregulated expression.

Table 5.6 Differentially expressed probe sets in CEF cells infected with influenza A viruses at 10 hpi.

\begin{tabular}{|c|c|c|c|c|c|c|c|c|}
\hline \multirow{2}{*}{ CEF } & \multicolumn{2}{|c|}{ Probe sets $>=2$ fold) } & \multicolumn{2}{c|}{ Probe sets $>=3$ fold } & \multicolumn{2}{c|}{ Probe sets $>=5$ fold } & \multicolumn{2}{c|}{ Probe sets $>=10$ fold } \\
\cline { 2 - 9 } & Up-regulated & Down-regulated & Up-regulated & Down-regulated & Up-regulated & Down-regulated & Up-regulated & Down-regulated \\
\hline H1N1 & $1.47 \%$ & $6.89 \%$ & $0.98 \%$ & $1.54 \%$ & $0.55 \%$ & $0.44 \%$ & $0.33 \%$ & $0.04 \%$ \\
\hline H5N2/F59 & $0.49 \%$ & $6.92 \%$ & $0.37 \%$ & $1.15 \%$ & $0.25 \%$ & $0.10 \%$ & $0.21 \%$ & $0.01 \%$ \\
\hline H5N2/F118 & $1.46 \%$ & $22.82 \%$ & $0.94 \%$ & $10.27 \%$ & $0.51 \%$ & $2.06 \%$ & $0.14 \%$ & $0.27 \%$ \\
\hline H5N2F 189 & $0.43 \%$ & $18.47 \%$ & $0.26 \%$ & $1.79 \%$ & $0.17 \%$ & $0.16 \%$ & $0.09 \%$ & $0.02 \%$ \\
\hline H5N3 & $0.89 \%$ & $10.45 \%$ & $0.58 \%$ & $2.52 \%$ & $0.33 \%$ & $0.55 \%$ & $0.18 \%$ & $0.04 \%$ \\
\hline H7N1 & $0.28 \%$ & $10.18 \%$ & $0.10 \%$ & $1.71 \%$ & $0.05 \%$ & $0.12 \%$ & $0.05 \%$ & $0.03 \%$ \\
\hline H9N2 & $1.23 \%$ & $12.85 \%$ & $0.73 \%$ & $3.09 \%$ & $0.29 \%$ & $0.86 \%$ & $0.12 \%$ & $0.13 \%$ \\
\hline
\end{tabular}

The global host gene expression profiles were retrieved from microarray analysis with different time points examined. The ratios of differentially expressed probe sets ( $\mathrm{P}$-value $\leq 0.05$ ) up- or down-regulated with different fold changes ( $\geq 2-F C, \geq 3-F C, \geq 5-F C$ and $\geq 10-F C$ ) in relative to their corresponding "expressing probe sets" are represented in percentage. The expressing probe sets refer to probe sets detected in the mock-infected corresponding cells. 
H1N1

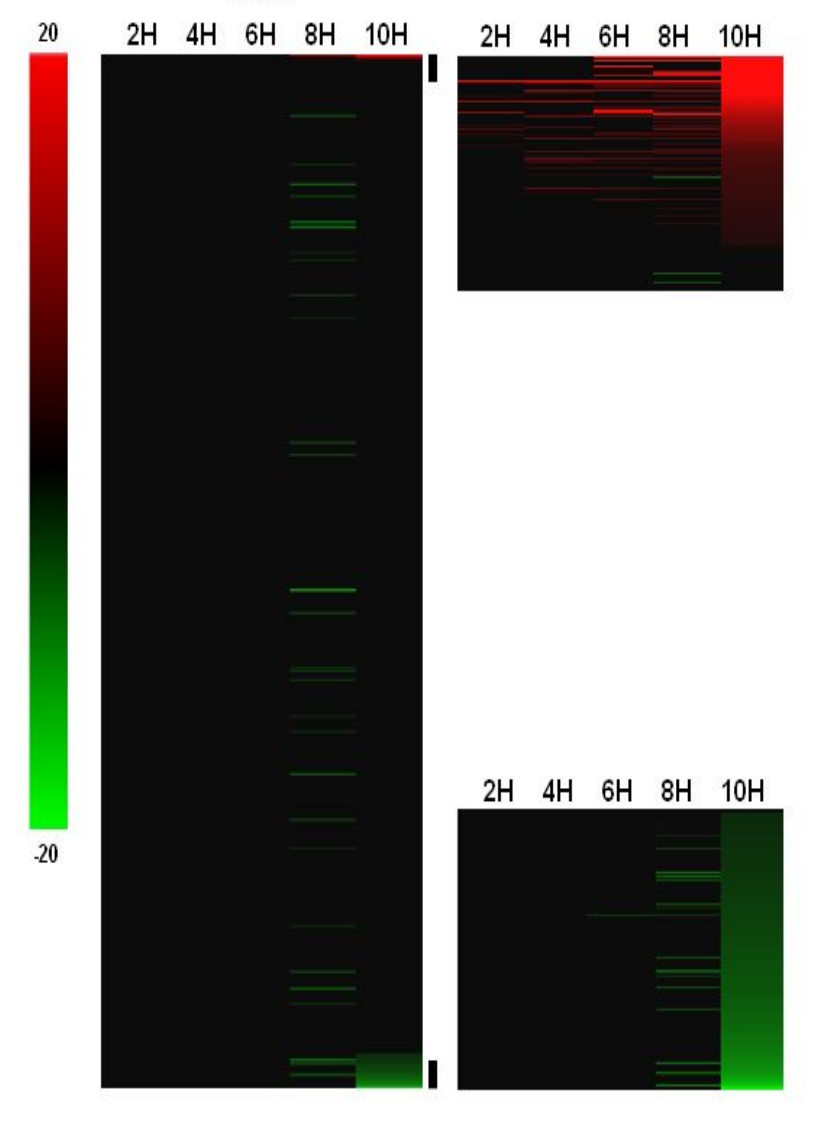

H9N2

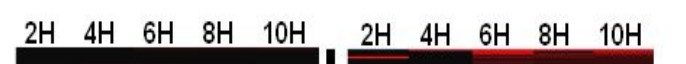

H5N2/F118

$\begin{array}{lllllllllllllllll}2 \mathrm{H} & 4 \mathrm{H} & 6 \mathrm{H} & 8 \mathrm{H} & 10 \mathrm{H} & 2 \mathrm{H} & 4 \mathrm{H} & 6 \mathrm{H} & 8 \mathrm{H} & 10\end{array}$

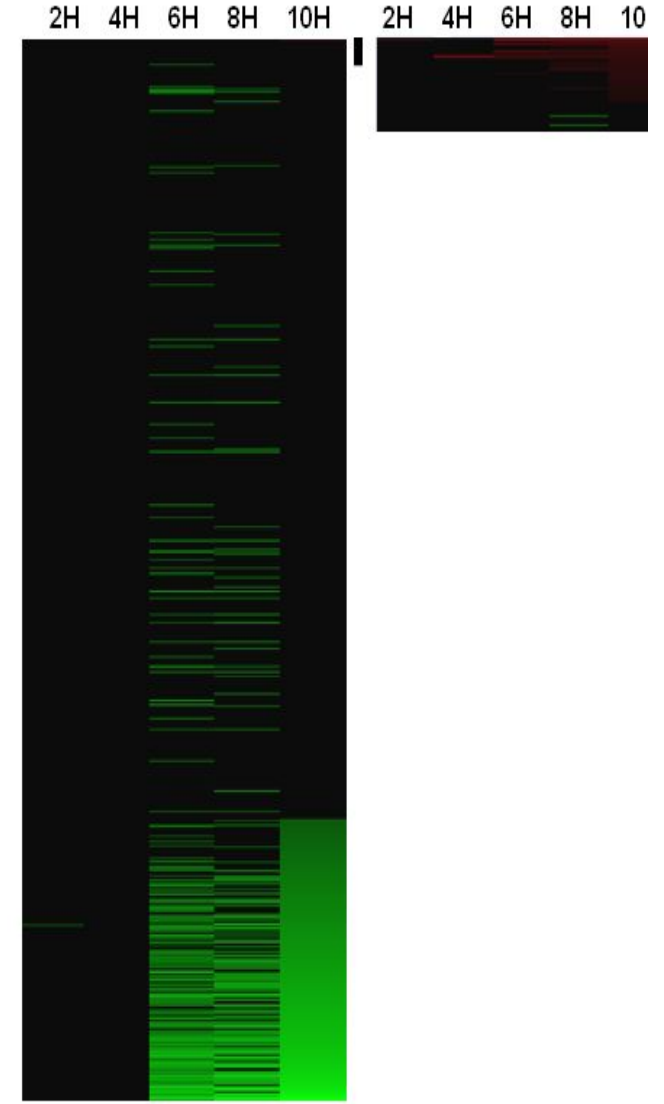

Figure 5.19 Temporal changes in the host cell transcriptome in CEF cells infected by four influenza virus strains. The global host gene expression profiles were retrieved from microarray analysis with different time points examined. The probe sets showing $\geq 2$ fold change (FC) up- or down-regulated in expression are indicated (Pvalue $\leq 0.05$ ). Expression profiles of up-regulated (red), down-regulated (green) and no significant change (black) are shown. 


\subsection{Functional classification}

Excluding the "Un-annotated" and "Un-classified" probe sets, the remaining probe sets with up-regulated expression were majorly associated with "Immune Response", "RNA Binding", "DNA Binding" and "Transcription Factor" [Figure 5.20]. In contrast with H1N1 infected A549 cells, H1N1 infected CEF cells showed stronger immune response than other influenza A strains infected CEF cells. Besides, infections of H5N2/F59, H5N3 and H9N2 also led to relative big numbers of immune response associated probe sets with up-regulated expression. When the cutoff was raised to $10-\mathrm{FC}$, some of the probe sets still showed significant up-regulation in their expression after infections of H1N1, H5N2/F59 and H5N3, especially H1N1. In summary, the host immune response was strongly activated in H1N1, H5N2/F59 and H5N3infected CEF cells when compared to other strains infected CEF cells.

The largest number of probe sets with down-regulated expression was detected in CEF cells after infection of H5N2/F118, and some of these probe sets showed high fold regulation with $\geq 5$-FC. Among them, a big batch of these probe sets played roles in "RNA Binding" and "DNA Binding". Compared to H5N2/F118, infections of another two H5N2 subtype strains, H5N2/F59 and H5N2/F189, only inhibited the expression of hundreds of probe sets, and the corresponding fold changes majorly located between 2-FC and 3-FC. The high percentages of differentially expressed probe sets belonging to 'Un-annotated' and "Un-classified" is majorly due to the incomplete annotation for probe sets in Chicken Genome.

\subsection{Cluster analysis}

Before clustering analysis, comparison of these three groups of data suggested that the numbers of probe sets with up-regulated expression were low and similar among three influenza A strains. However, the numbers of those with down-regulated expression were high but different among these three strains, with H5N2/F118 infection contributing to largest number of downregulated probe sets. In the cluster analysis process, the probe sets with differential expression after H1N1, H5N2/F118 and H9N2 infections were separated into two groups to do cluster analysis so as to better separate the probe sets into different clusters 
As described, the number of up-regulated probe sets was much lower than the number of down-regulated ones, particularly in H5N2/F118 and H9N2 infected CEF cells, therefore those up-regulated probe sets weren't capable to be picked out in these two groups of data. Although those probe sets showing up-regulated expression at 10 hpi after H5N2/F118 or H9N2 infection were clustered into additional groups, functional annotation of which indicated that no gene ontology term was able to be significantly enriched. In H1N1 infected CEF cells, probe sets with up-regulated expression were able to be clustered into Cluster I-11, with only a function group called "NAD + ADP ribosyltransferase activity" significantly enriched in only 4 genes [Figure 5.21] [Table 5.7].

Another Cluster II-6 generated from H1N1 infected CEF cells showed interesting trend in that the expression of probe sets dropped first, followed by a suddenly increase at $6 \mathrm{hpi}$ and another decrease at $8 \mathrm{hpi}$. Gene ontology terms including "lipid localization" and "cytokine activity" were significantly enriched in genes belonging to this cluster. Accordingly, immune response and cell membrane activities may be triggered around 6 hpi first however repressed at later infection stage after $\mathrm{H} 1 \mathrm{~N} 1$ infection.

A batch of functional groups identified in down-regulated genes were common to three influenza A strains infections in CEF cells. These groups included "nucleotide binding", "protein binding" and "cellular metabolic process".

Besides these GO terms, functional groups such as "metal ion binding", "localization", “ATP binding”, "response to stress" and "helicase activity" were significantly enriched in genes with down-regulated expression after two avian strains infections [Figure 5.22] [Table 5.8].

A function named "regulation of cell-substrate adhesion" was significantly enriched in Cluster I-5 after H9N2 infection. Expression trend of this cluster fell down from 2 hpi to 4 hpi, and then there was a sudden increase occurred from 4 hpi and another decrease occurred from 6 hpi. Expression levels of these probe sets were fluctuated, and it was hypothesized that some special cell regulation might happen to count for the short gene expression stimulation in the middle of the examined infection period. 
Infection of H5N2/F118 led to most genes with down-regulated expression in CEF cells. Subsequently, much more functional groups such as "post-translational protein modification", "hydrolase activity", “organ development", "Zinc ion binding", "cell cycle", "ligase activity", "cellular localization", "transferase activity", "phosphorylation" and "transmembrane receptor protein kinase activity" were enriched in these down-regulated genes [Figure 5.23] [Table 5.9]. Inhibitory expression of these genes participating in different and important biological functions suggested that a lot of host cell activities were impaired due to the H5N2/F118 invasion. And impairment of normal expression of these key molecules during H5N2/F118 infection might be a solution evolved by the H5N2/F118 virus that aimed to create a beneficial environment for its survival and replication.

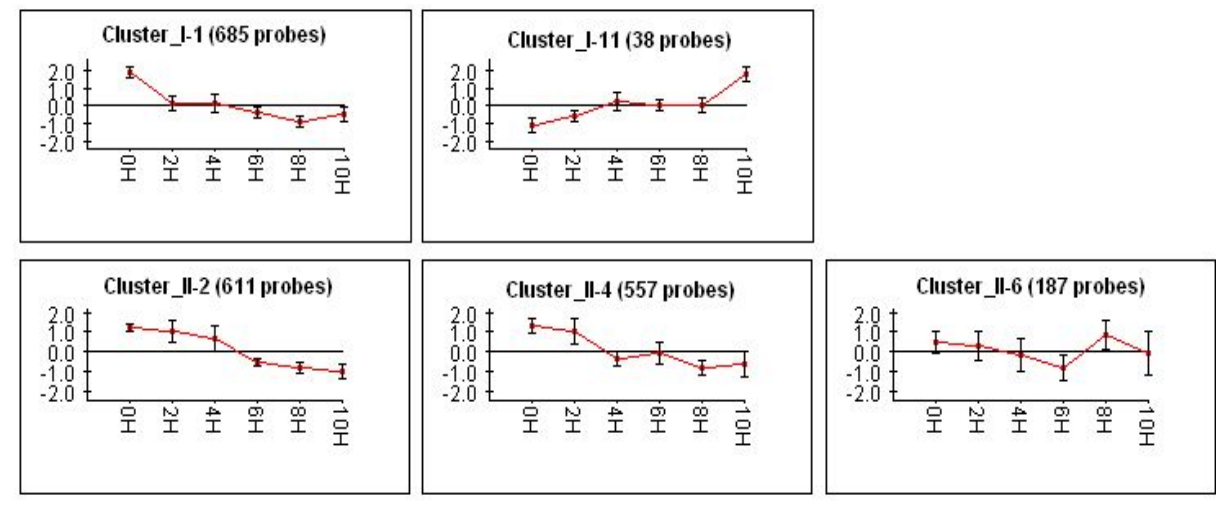

Figure 5.21 Clustering analysis of temporal gene expression profiles in H1N1-infected CEF cells. CEF cells were infected with $\mathrm{H} 1 \mathrm{~N} 1$ at 2, 4, 6, 8 and 10 hpi. Probe sets showing $\geq 2-$ fold changes up- or down-regulated $(\mathrm{P}$-value $\leq 0.05)$ at least over one time point were analyzed with Expander 5 software. X-axis represents post-infection in hours $(\mathrm{H})$, and $\mathrm{Y}$-axis means normalized expression changes of probe sets.
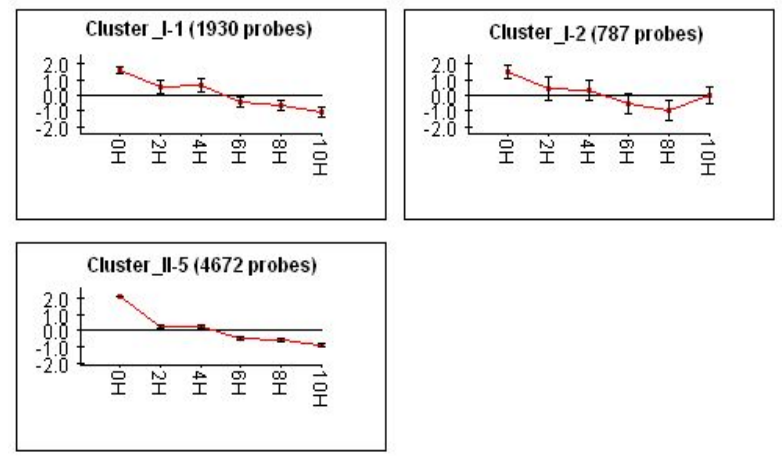

Figure 5.22 Clustering analysis of temporal gene expression profiles in H5N2/F118infected CEF cells. CEF cells were infected with H5N2/F118 at 2, 4, 6, 8 and 10 hpi. Probe sets showing $\geq 2$-fold changes up- or down-regulated $(\mathrm{P}$-value $\leq 0.05)$ at least over one time point were analyzed with Expander 5 software. X-axis represents post-infection in hours $(\mathrm{H})$, and $\mathrm{Y}$ axis means normalized expression changes of probe sets. 

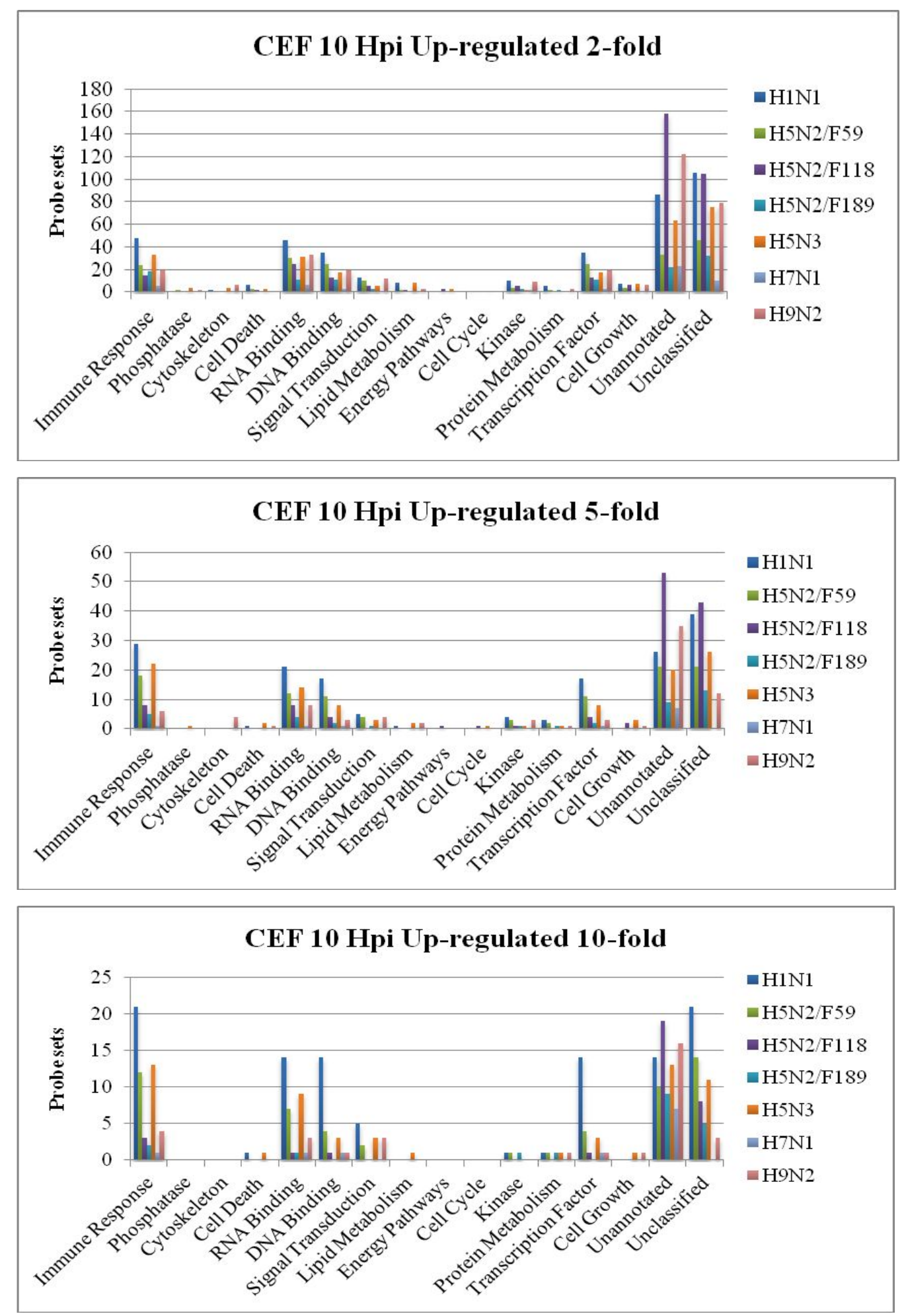

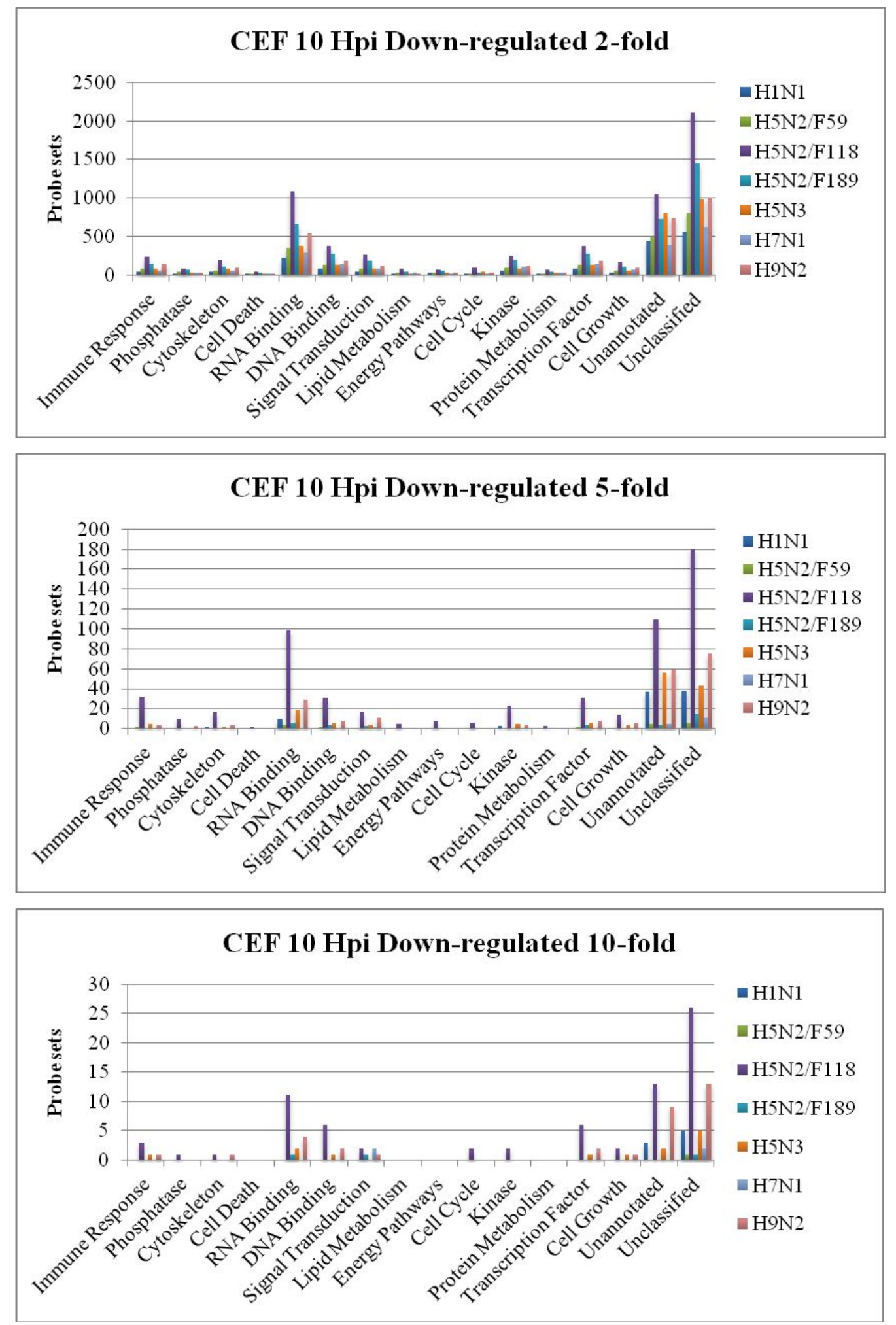

Figure 5.20 Overview of distributions of differentially expressed probe sets into different biological functions in CEF cells infected with influenza A viruses. The numbers of probe sets in the different functional families, including non-annotated and unclassified groups, showing up- or down-regulated with different fold changes ( $\geq 2-\mathrm{FC}, \geq 5$-FC and $\geq 10$-FC) in gene expression are presented. 
Table 5.7 Summary of functional groups enriched based on differentially expressed genes in CEF cells infected with H1N1.

\begin{tabular}{|l|l|}
\hline Cluster No. & \multicolumn{1}{|c|}{ Enriched Gene Ontology Terms (CEF H1N1) } \\
\hline Cluster I-1 & purine ribonucleotide binding - G0:0032555:51 \\
\hline Cluster I-11* & NAD + ADP-ribosyltransferase activity - G0:0003950:4 \\
\hline Cluster II-2 & nucleotide binding - G0:0000166:64 | protein binding - G0:0005515:100 | cellular metabolic process - G0:0044237:81 \\
\hline Cluster II-4 & primary metabolic process - G0:0044238:82 | RNA binding - G0:0003723:17 | cellular metabolic process - G0:0044237:74 \\
\hline Cluster II-6 & lipid localization - G0:0010876:5 | cytokine activity - G0:0005125:5 \\
\hline
\end{tabular}

Differentially expressed genes were significantly categorized into different GO terms under Expander 5 software analysis ( $\mathrm{P}$-value $\leq 0.05$ ). Each functional group is followed by the number of corresponding genes. * represents the up-regulated trend of the corresponding cluster.

Table 5.8 Summary of functional groups enriched based on differentially expressed genes in CEF cells infected with $\mathrm{H5N2/F118.}$

\begin{tabular}{|c|c|}
\hline Cluster No. & Enriched Gene Ontology Terms (CEF H5N2/F118) \\
\hline \multirow{8}{*}{ Cluster I-1 } & ATP binding - G0:0005524:121 | protein binding - G0:0005515:267 | regulation of signal transduction - G0:0009966:45 \\
\hline & phosphate metabolic process - G0:0006796:69 | nucleoside-triphosphatase activity - G0:0017111:57 | protein kinase activity - G0:0004672:52 \\
\hline & cellular component organization - G0:0016043:71 | post-translational protein modification - G0:0043687:71 | hydrolase activity - G0:0016787:127 \\
\hline & 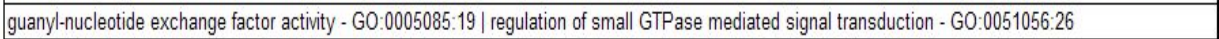 \\
\hline & regulation of biological process - G0:0050789:232 | organ development - G0:0048513:62 | helicase activity - G0:0004386:20 \\
\hline & anatomical structure morphogenesis - G0:0009653:45 | cation binding - G0:0043169:162 | tissue morphogenesis - G0:0048729:17 \\
\hline & transmembrane receptor protein kinase activity - G0:0019199:16 | regulation of Rho protein signal transduction - G0:0035023:13 \\
\hline & nucleic acid binding - G0:0003676:139 | zinc ion binding - G0:0008270:98 | chordate embryomic development - G0:0043009:24 \\
\hline Cluster $1-2$ & cation binding - G0:0043169:80 \\
\hline \multirow{12}{*}{ Cluster II-5 } & protein binding - G0:0005515:619 | nucleotide binding - G0:0000166:360 | nucleoside binding - G0:0001882:276 | metabolic process - G0:0008152:660 \\
\hline & macromolecule metabolic process - G0:0043170:448 | cellular metabolic process - G0:0044237:506 | primary metabolic process - G0:0044238:543 \\
\hline & cellular macromolecular metabolic process - G0:0044260:385 | nucleobase, nucleoside, nucleotide and nucleic acid metabolic process - G0:0006139:194 \\
\hline & nitrogen compound metbolic process - G0:0006807:222 | RNA metabolic process - G0:0016070:86 | nucleic acid binding - G0:0003676:336 \\
\hline & transition metal ion binding - G0:0046914:273 | cation binding - G0:0043169:374 | macromolecule localization - G0:0033036:94 \\
\hline & cellular component organization - G0:0016043:152 | hydrolase activity - G0:0016787:281 | cell cycle - G0:0007049:53 | ligase activity - G0:0016874:59 \\
\hline & ncRNA metabolic process - G0:134660:38 | cellular localization - G0:0051641:76 | mucleoside-triphosphatase activity - G0:0017111:109 \\
\hline & catabolic process - G0:0009056:143 | biosynthetic process - G0:0009085:184 | gene expression - G0:0010467:103 | mitotic cell cycle - G0:0000278:26 \\
\hline & cellular catabolic proces - G0:0044248:82 | transferase activity - G0:0016740:216 | celllar response to stress - G0:0033554:49 \\
\hline & response to DNA damige stimulus - G0:0006974:44 | helicase activity - G0:0004386:35 | ATPase activity, coupled - G0:0042623:50 \\
\hline & phosphorylation - G0:0016310:112 | vesicle-mediated transport - G0:0016192:47 | cellular macromolecule catabolic process - G0:0044265:60 \\
\hline & modification-dependent macromolecule catabolic process - G0:0043632:42 \\
\hline
\end{tabular}

Differentially expressed genes were significantly categorized into different GO terms under Expander 5 software analysis (P-value $\leq 0.05$ ). Each functional group is followed by the number of corresponding genes.

Table 5.9 Summary of functional groups enriched based on differentially expressed genes in CEF cells infected with H9N2.

\begin{tabular}{|l|l|}
\hline Cluster No. & \multicolumn{1}{|c|}{ Enriched Gene Ontology Terms (CEF H9N2) } \\
\hline \multirow{4}{*}{ Cluster I-1 } & ATP binding - G0:0005524:54 | protein binding - G0:0005515:107 | helicase activity - G0:0004386:14 | ATPase activity - G0:0016887:11 \\
\cline { 2 - 3 } & nucleoside-triphosphatase activity - G0:0017111:29 | organ morphogenesis - G0:0009887:20 | tube development - G0:0035295:11 \\
\cline { 2 - 3 } & cellular macromolecule metabolic process - G0:0044260:65 | regulation of signal transduction - G0:0009966:21 | angiogenesis - G0:0009966:21 \\
\cline { 2 - 3 } & biopolymer metabolic process - G0:0043283:69 \\
\hline Cluster I-2 & cellular macromolecule metabolic process - G0:0044260:46 | centrosome organization - G0:0051297:4 \\
\hline Cluster I-5 & regulation of cell-substrate adhesion- G0:0010810:5 \\
\hline \multirow{3}{*}{ Cluster II-1 } & protein binding - G0:0005515:180 | adenyl ribonucleotide binding - G0:0032559:8 | multicellular organismal process - G0:0032501:76 \\
\cline { 2 - 3 } & metal ion binding - G0:0046872:104 | developmental process - G0:0032501:68 | transition metal ion binding - G0:0046914:90 \\
\cline { 2 - 3 } & extracellular matrix structure constituent - G0:0005201:8 | nucleic acid binding - G0:0003676:104 | localization - G0:0051179:89 \\
\hline \multirow{2}{*}{ Cluster II-3 } & protein binding - G0:0005515:87 | response to stress - G0:0006950:23 | ATP binding - G0:0005524:39 | developmental process - G0:0032502:34 \\
\hline
\end{tabular}

Differentially expressed genes were significantly categorized into different GO terms under Expander 5 software analysis (P-value $\leq 0.05$ ). Each functional group is followed by the number of corresponding genes. 

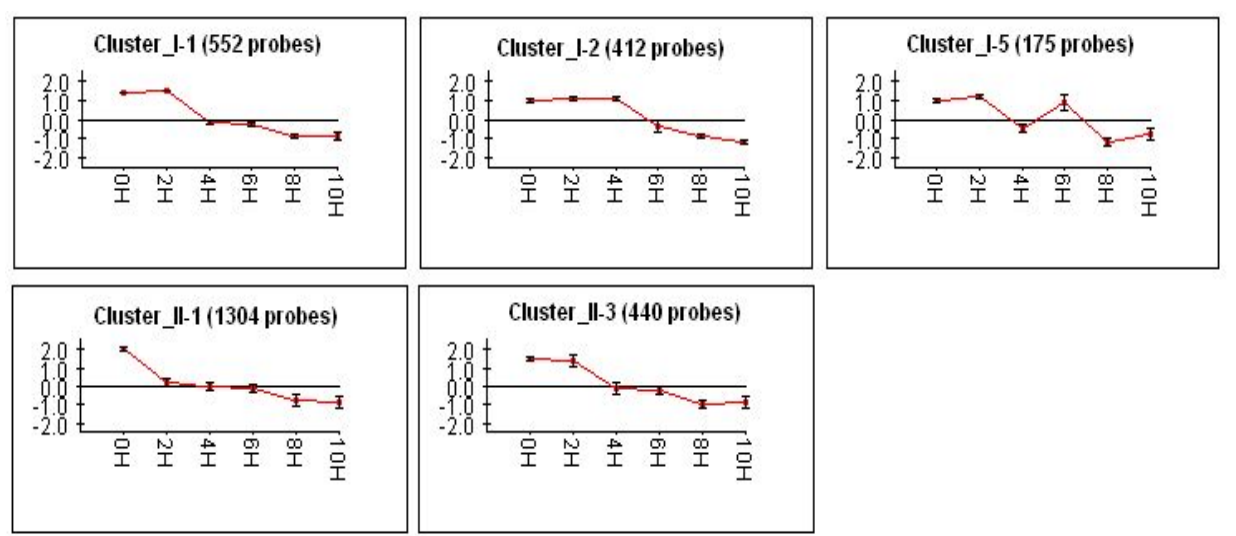

Figure 5.23 Clustering analysis of temporal gene expression profiles in H9N2-infected CEF cells. CEF cells were infected with H9N2 at 2, 4, 6, 8 and 10 hpi. Probe sets showing $\geq 2-$ fold changes up- or down-regulated $(\mathrm{P}$-value $\leq 0.05)$ at least over one time point were analyzed with Expander 5 software. $\mathrm{X}$-axis represents post-infection in hours $(\mathrm{H})$, and $\mathrm{Y}$-axis means normalized expression changes of probe sets.

\subsubsection{Functional groups related to host response}

\subsection{Immune response}

In CEF cells, a batch of IFN related genes including IFIH1, IFIT-5, IRF1/7, TLR3, IFI35, STAT4, MX and OASL showed strong elevation at their expression level after infections of H1N1, H9N2, H5N2/F59, H5N2/189 and H5N3 [Figure 5.24]. Altered expression of these genes with high-level fold changes indicated that $\mathrm{CEF}$ cells strongly initiated a series of immune reactions in response to the invasion of these influenza A strains. Furthermore, data derived from infections of $\mathrm{H} 1 \mathrm{~N} 1$ and $\mathrm{H} 9 \mathrm{~N} 2$ also demonstrated that these genes were activated from a very early infection phase (around 2 or 4 hpi), indicating the timely inflammatory response in these infected CEF cells.

Although there were not so many genes with quite significant expression changes detected in H5N2/F118-infected CEF cells, some key factors such as IFIT-5, OASL, IFIH and IRF7 were identified with up-regulated expression, with the most significant fold changes identified at 4 hpi. This observation might imply that some mechanism was triggered to attenuate antiviral actions after 4hpi in H5N2/F118 infected CEF cells.

Besides these genes with up-regulated expression, infection of H5N2/F118 also resulted in many genes with down-regulated expression in CEF cells. Under investigation, these genes generally showed significant downregulation at their expression level from 6hpi, which was consistent with the 
decreasing expression trend of up-regulated genes from 6 hpi mentioned previously.

\subsection{Cell death}

A group of programmed cell death genes, PDCD 2/4/5/6/7/8/10/11, all showed down-regulated expression in H1N1, H5N2/F59, H5N2/F189-infected CEF cells. Whereas, only some of them identified in pH1N1 and H7N1 infected-CEF cells showed down-regulated expression and few identified in H9N2, H5N2/F118 and H5N3-infected CEF cells showed down-regulated expression [Figure 5.25].

In H5N2/F118-infected CEF cells, many apoptosis-related genes were down-regulated in their expression from an early time points (6 hpi). Besides these genes with early down-regulated expression, Fas, TNFSF10 and caspase 6/9 showed differentially down-regulated expression in H5N2/F118 infected CEF cells at late infection stage.

Another two factors that are associated with p53 are USP7 and AMID. USP7, known as ubiquitin carboxyl-terminal hydrolase 7, direct antagonizes Mdm2 to protect p53 from Mdm2-mediated degradation [301]. And AMID (PRG3) was reported to be a p53-downstream gene involved in tumorigenesis by Wu M et al (2004) [302]. In H5N2/F118-infected CEF cells, both these two

genes showed down-regulated expression. Accordingly, it was assumed that p53 might be inactivated to some extent even if expression of p53 showed no differential change in our H5N2/F118-infected CEF cells. In addition, infections of H1N1 and H5N2/F59 led to the lowest number of apoptosis related genes with down-regulated expression in CEF cells.

\subsection{Genes with remarkable regulations}

In CEF cells, some genes with key functions including ZC3HAV1, IPO7, GPR151, GNG13 and ZNFX1 showed up-regulated expression [Figure 5.26]. ZC3HAV1 (ZAP/PAPR13) that encodes a CCCH-type zinc finger protein was commonly up-regulated in several influenza A strains infected A549 cells, with the most significant expression change 30.2-FC detected after H1N1 infection. Previously researchers have reported that this interferonstimulated gene restricted the replication of retroviruses, alphaviruses, and 
filoviruses, and viral induction of it occurred under the direct control of IRF3 [303]. When it came to our study in CEF cells, no significant expression change of IRF3 could be detected, but expression of IRF1 and IRF7 showed in a consistent pattern with ZC3HAV1. This phenomenon might be caused by diverse controlling in different viruses, and it was assumed that IAV might evolve alternative control pathway dependent of IRF1/7, taking the place of IRF3.

Zaitseva L et al (2009) uncovered that IPO7 (IMP7) facilitated nuclear trafficking of DNA and HIV-1 exploited IMP7 to maximize nuclear import of its DNA genome [304]. Besides, other groups also mentioned that IMP might play an impact on HIV-1's replication performance [305]. Although there is no concrete evidence proving that this protein is involved in IAV's nuclear import activity, the obvious up-regulated expression of this gene from 4 to $10 \mathrm{hpi}$ in H5N2/F118-infected CEF cells indicated that it might have a potentiality to make some important function in IAV nuclear transport action or even IAV viral replication.

G protein-coupled receptor 151 (GPR151) also showed common upregulation at its expression level in $\mathrm{H} 1 \mathrm{~N} 1, \mathrm{H} 5 \mathrm{~N} 2 / \mathrm{F} 118$ and H9N2 infected-CEF cells. Previous report has revealed that $\mathrm{G}$ protein and protein kinase signaling regulated IAV budding in MDCK cells [306]. Hence, the differential expression might implicate the exertion of corresponding GPR151 roles in these influenza A strains infected CEF cells.

In addition to these genes with up-regulated expression, some genes showing down-regulated expression were also identified with important functions. Transmembrane gene TMEM170 was found down-regulated at its expression level in H5N2/F118, H9N2 and H5N3-infected CEF cells. Moreover, a batch of ZNF genes also showed down-regulated expression in different influenza A strains infected CEF cells, and these ZNF genes might be responsible for some regulations in these influenza A strains infected CEF cells. 


\subsubsection{Host gene expression in MDCK cells}

\subsubsection{Global profiling of gene expression}

\subsection{Heat maps of global gene expression}

In MDCK cells, the largest numbers of differentially expressed probe sets, up- or down-regulated, were detected after H1N1 infection. Compared to H9N2, H5N2/F118 infection resulted in repressed expression of relative high number of probe sets from early infection stage [Figure 5.27].

\subsection{Distribution of differentially expressed probe sets}

There were also more probe sets with up-regulated expression detected in MDCK cells infected with H9N2, H1N1 and H5N2/F118 than other influenza A strains [Table 5.10]. Percentages of probe sets with down-regulated expression were considerable after infections of all influenza A strains excluding H9N2. Moreover, some probe sets even showed down-regulated expression with high-level fold changes $\geq 10-\mathrm{FC}$, particularly in $\mathrm{H} 1 \mathrm{~N} 1$, H5N2/F118 and H7N1-infected MDCK cells. Among three H5N2 strains, infection of H5N2/F118 also caused the highest portion of probe sets with both up- and down-regulated expression. Besides, H5N2/F189 infection also generated big batch of probe sets with down-regulated expression.

Table 5.10 Differentially expressed probe sets in MDCK cells infected with influenza A viruses at $10 \mathrm{hpi}$.

\begin{tabular}{|c|c|c|c|c|c|c|c|c|}
\hline \multirow{2}{*}{ MDCK } & \multicolumn{2}{|c|}{ Probe sets (>=2 fold) } & \multicolumn{2}{c|}{ Probe sets $>=3$ fold } & \multicolumn{2}{c|}{ Probe sets (>=5 fold) } & \multicolumn{2}{c|}{ Probe sets $>=10$ fold $)$} \\
\cline { 2 - 9 } & Up.regulated & Down.regulated & Up.regulated & Down-regulated & Up.regulated & Down-regulated & Up.regulated & Down.regulated \\
\hline H1N1 & $1.31 \%$ & $49.90 \%$ & $0.74 \%$ & $30.04 \%$ & $0.35 \%$ & $12.50 \%$ & $0.14 \%$ & $3.31 \%$ \\
\hline H5N2F59 & $0.74 \%$ & $16.12 \%$ & $0.37 \%$ & $4.27 \%$ & $0.21 \%$ & $0.80 \%$ & $0.09 \%$ & $0.08 \%$ \\
\hline H5N2F118 & $1.44 \%$ & $33.42 \%$ & $0.97 \%$ & $20.99 \%$ & $0.50 \%$ & $8.13 \%$ & $0.20 \%$ & $2.14 \%$ \\
\hline H5N2F189 & $0.17 \%$ & $32.43 \%$ & $0.00 \%$ & $12.38 \%$ & $0.05 \%$ & $3.31 \%$ & $0.02 \%$ & $0.55 \%$ \\
\hline H5N3 & $0.45 \%$ & $28.28 \%$ & $0.17 \%$ & $9.06 \%$ & $0.07 \%$ & $1.87 \%$ & $0.03 \%$ & $0.30 \%$ \\
\hline H7N1 & $0.32 \%$ & $28.30 \%$ & $0.16 \%$ & $14.21 \%$ & $0.07 \%$ & $6.25 \%$ & $0.03 \%$ & $1.83 \%$ \\
\hline H9N2 & $2.33 \%$ & $6.28 \%$ & $1.34 \%$ & $2.22 \%$ & $0.72 \%$ & $0.71 \%$ & $0.22 \%$ & $0.10 \%$ \\
\hline
\end{tabular}

The global host gene expression profiles were retrieved from microarray analysis with different time points examined. The ratios of differentially expressed probe sets ( $\mathrm{P}$-value $\leq 0.05)$ up- or down-regulated with different fold changes ( $\geq 2-\mathrm{FC}, \geq 3-\mathrm{FC}, \geq 5-\mathrm{FC}$ and $\geq 10-\mathrm{FC}$ ) in relative to their corresponding "expressing probe sets" are represented in percentage. The expressing probe sets refer to probe sets detected in the mock-infected corresponding cells. 


\subsection{Functional classification}

Figure 5.28 suggested that some probe sets belonging to "Immune Response" were induced at their expression level in MDCK cells after influenza A strains infections, especially H9N2, H1N1 and H5N2/F59. And among these probe sets, some of those from H1N1 and H5N2/F59 infections showed quite high fold changes. In the contrary, only a couple of probe sets with increased expression were observed related to "Immune Response" in H5N3 and H7N1infected MDCK cells, indicating weaker host antiviral action. Regarding to another two H5N2 strains, infection of H5N2/F118 also stimulate expression of a small batch of probe sets, while few probe sets were observed to be stimulated at their expression level after infection of H5N2/F189.

Down-regulated probe sets in influenza A strains infected MDCK cells mostly had a relationship with "RNA Binding", "DNA Binding" and "Transcription Factor". Furthermore, dozens of probe sets located in groups such as "RNA binding" and "DNA Binding" were inhibited in their expression with quite significant fold regulation at $\geq 10$-FC in $\mathrm{H} 1 \mathrm{~N} 1, \mathrm{H} 5 \mathrm{~N} 2 / \mathrm{F} 118$, H5N2/F189, H5N3 and H7N1-infected MDCK cells. And among these upregulated probe sets from different influenza A strains infected-MDCK cells, the expression of some probe sets from infections of H1N1 and H5N2/F118 showed quite high fold change level. In contrary to the situation happened in H5N2/F118 and H5N2/189-infected MDCK cells, infection of H5N2/F59 only induced the expression of a relative small number of probe sets in MDCK cells. The high percentages of differentially expressed probe sets belonging to 'Unannotated' and "Un-classified" is majorly due to the incomplete annotation for probe sets in Canine Genome 2.0 Array.

\subsubsection{Functional groups related to host response}

\subsection{Immune response}

In MDCK cells, expression levels of IFN type I stimulated genes OAS1, MX1 and RASD2 were all up-regulated after H1N1 infection and selectively up-regulated after other influenza A strains infections [Figure 5.29]. Cytokines, CCL5 and CXCL10, were also detected with significantly up-regulated expression after infections of all influenza A strains except H5N3. 


\subsection{Cell death}

Expression profiles of cell death relevant genes in MDCK cells implicated that most genes with down-regulated expression were detected after $\mathrm{H} 1 \mathrm{~N} 1$ infection and least genes with down-regulated expression were detected after H9N2 infection [Figure 5.30]. Key genes such as caspase 2/3/8, Fas and TNF were all down-regulated at their expression level in H1N1 infected MDCK cells, indicating the inhibition of cell apoptotic activities.

\subsection{Genes with remarkable regulations}

Parallel observation of gene expression in IAV infected MDCK cells revealed that several genes related to transmembrane and zinc finger were significantly up-regulated at their expression level [Figure 5.31]. Among them, expression of TMEM106A, TMEM55A, TMEM30C were up-regulated after infections of H5N2/F59, H5N2/F118 and H9N2 separately; expression of ZNF391/662 was induced after infection of H5N2/F118. In addition, lipid and adhesion associated genes such as $\mathrm{CH} 25 \mathrm{H}$, ALCAM were also detected with up-regulated expression in H5N2/F118 and H5N3 infected MDCK cells.

Moreover, there also existed quite a lot of genes with suppressed expression and these genes were majorly relevant to zinc finger, transmembrane, G protein, cell cycle and cholesterol. These genes included GPR98 for H5N2/F59 infection, GPR56 for H5N2/F118 and H1N1 infection, GPR110 for H1N1 infection and so on. 


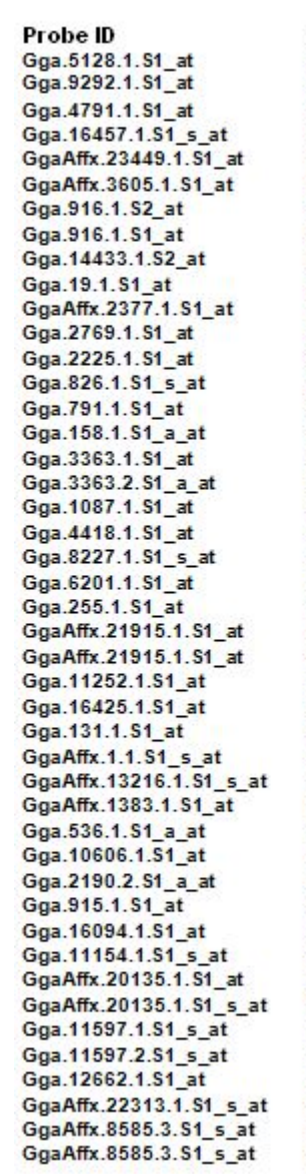

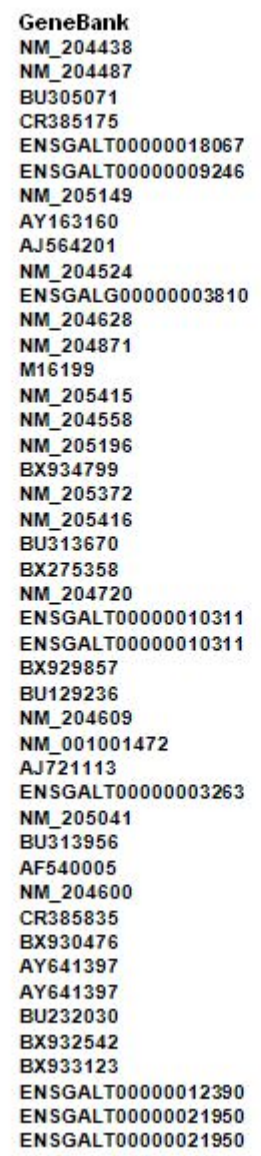

Gene Title

chemokine (C-C motif) ligand 20

H912 H5H2F118 H5H2,F59 H512/F189 H5H3 H7II1

政 2

interferon induced with helicase $\mathrm{C}$ domain 1

interferon beta

interferon gamma

interleukin 12B

interleukin 1 , beta

(interferon, lambda

, oncostatin M receptor

interferon regulatory factor 10

interferon regulatory factor 2

interferon regulatory factor 7

interferon regulatory factor 8

putative ISG12-1 protein

putative ISG12-2 protei

similar to Interferon-induced protein with tetratricopeptide repeats 5 (IFIT-5) (C) A19 precursor (CC19)

Mx protein
nuclear factor of kappa light polypeptide gene enhancer in B-cells inhibitor, alph

2-5-oligoadenylate synthetase-like
suppres sor of cytokine signaling 1

suppressor of cytokine signaling

suppressor cloyine signaling 3

signal transducer and activator of transcription 3 (acute-phase response factor) signal transducer and activator of transcription 3 (acute-phase response factor) signal transducer and activator of transcription 4

Signal transducer and actvator oftranscription 4

Signal transducer and activator of transcription 4

GgaAffx.8585.3.S1_5_at ENSGALT00000021950 toll-like receptor

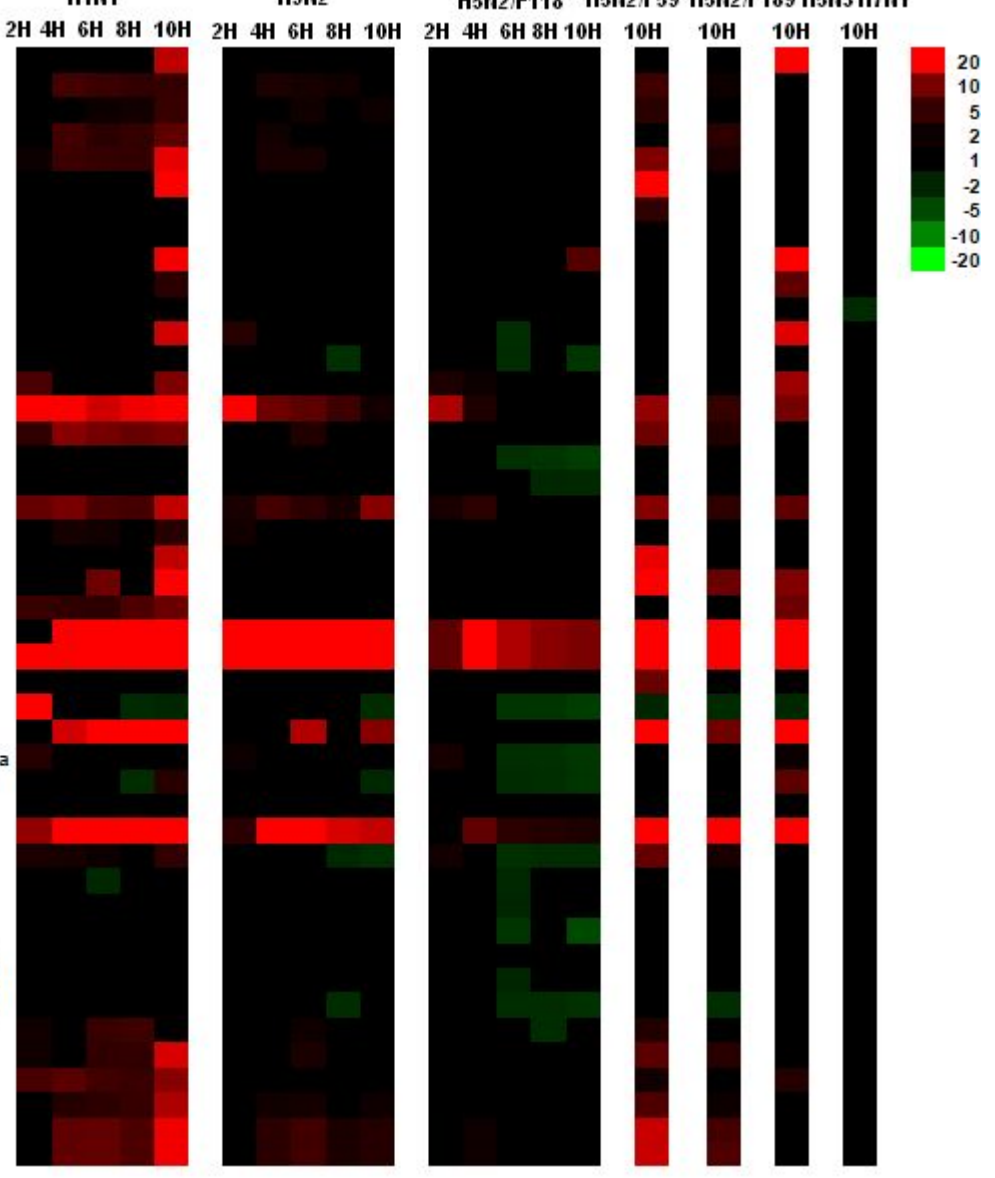

Figure 5.24 Expression of probe sets involved in immune response in influenza A viruses-infected CEF cells. Eight influenza A strains were infected with different examined cells, and the expression of probe sets related to immune response was examined by microarray analysis at different time points as shown. The data is represented by heat map analysis showing up-regulated (red), down-regulated (green) or no changes (black) in expression, and the FC range is indicated (P-value $\leq 0.05$ ). 


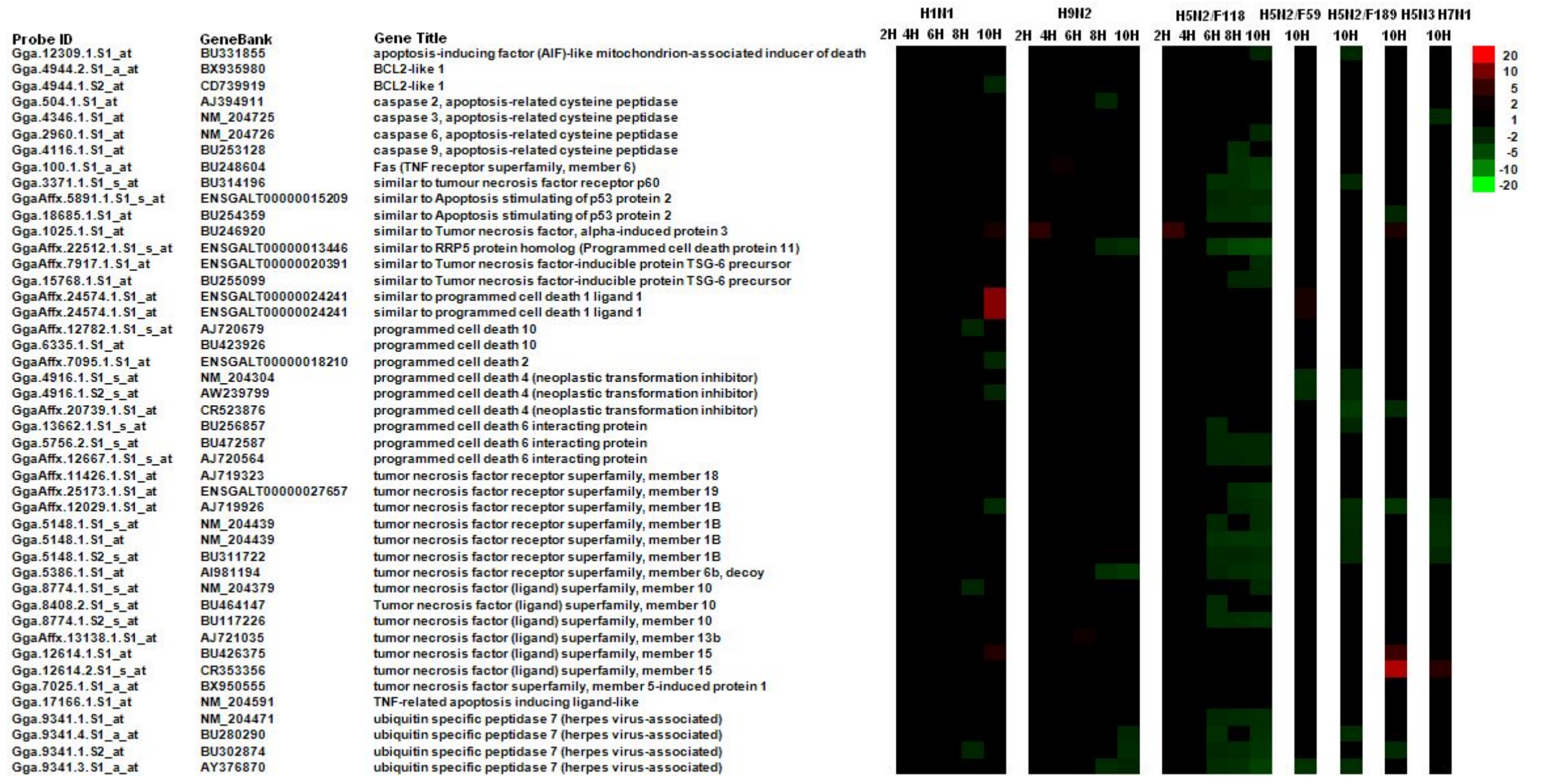

Figure 5.25 Expression of probe sets involved in cell death in influenza A viruses-infected CEF cells. Eight influenza A strains were infected with different examined cells, and the expression of probe sets related to cell death was examined by microarray analysis at different time points as shown. The data is represented by heat map analysis showing up-regulated (red), down-regulated (green) or no changes (black) in expression, and the FC range is indicated (p $\leq 0.05$ ). 


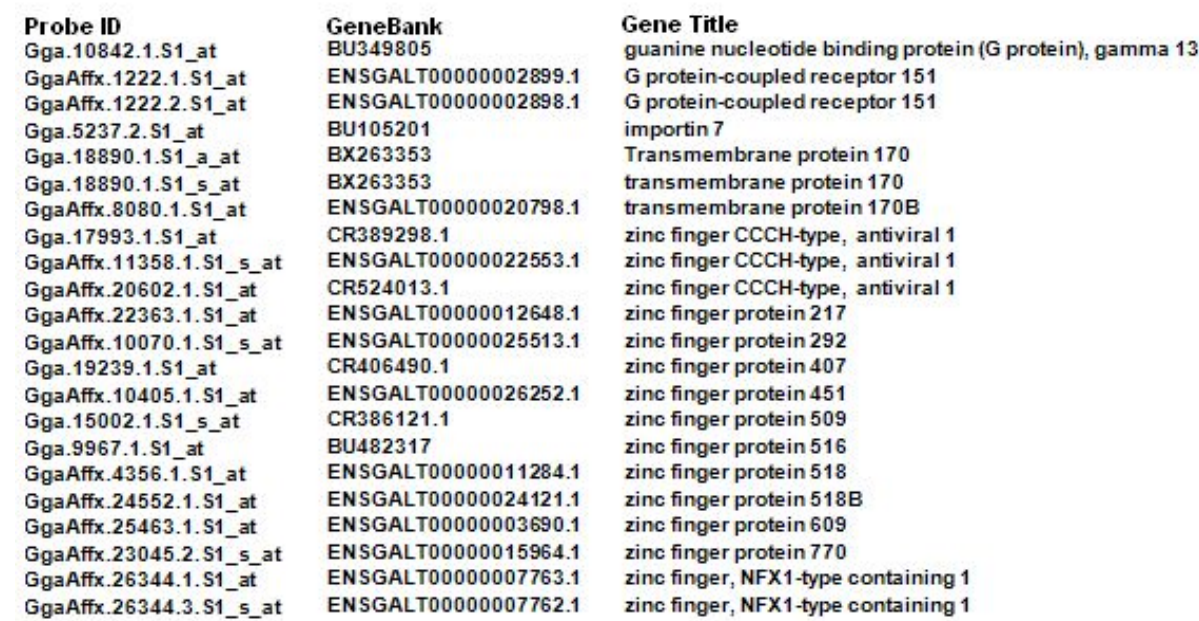

Probe ID

Gga.10842.1.S1_at GgaAffx.1222.1.S1_a Ga Gga.18890.1.S1 Gga.18890.1.S1_s_at GgaAffx.8080.1.S1_at Gga.17993.1.S1_at GgaAffx.11358.1. S1_s_a GgaAffx.20602.1. S1_a GgaAffx.22363.1.S1_at GgaAffx. 10070.1.S1_. GgaAffx.10405.1.S1 Gga.15002.1.S1_s_at Gga.9967.1.S1_at GgaAffx.4356.1.S1_at GgaAffx. 24552.1. S1_a GgaAffx.25463.1.S1_at GgaAffx.23045.2.51_s GgaAffx 26344.3.S1 5 ENSGALT00000002898. NSGALT00000020798. CR389298.

CNGALT00000022553.1 EN524013. CR386121.1

BU482317

ENSGALT00000011284 ENSGALT00000024121 ENSGALTO0000015964. ENSGALT00000007763. ENSGALT00000007762.

Gene Title

oleotide binding protein ( $G$ protein), gamma 13

protein-coupled receptor 15

mportin 7

Gransmembrane protein 170

transmembrane protein 170

ransmembrane protein $170 \mathrm{~B}$

zinc finger $\mathrm{CCCH}$-type, antiviral 1

zinc finger CCCH-type, antiviral

inc finger CCCH-type, antiviral

zinc finger protein 292

zinc finger protein 407

zinc finger protein 45

zinc finger protein 509

zinc finger protein 516

zinc finger protein 518

zinc finger protein $518 \mathrm{~B}$

zinc finger protein 609

zinc finger, NFX1 - type containing 1

zinc finger, NFX1-type containing 1

H1II1

H9112

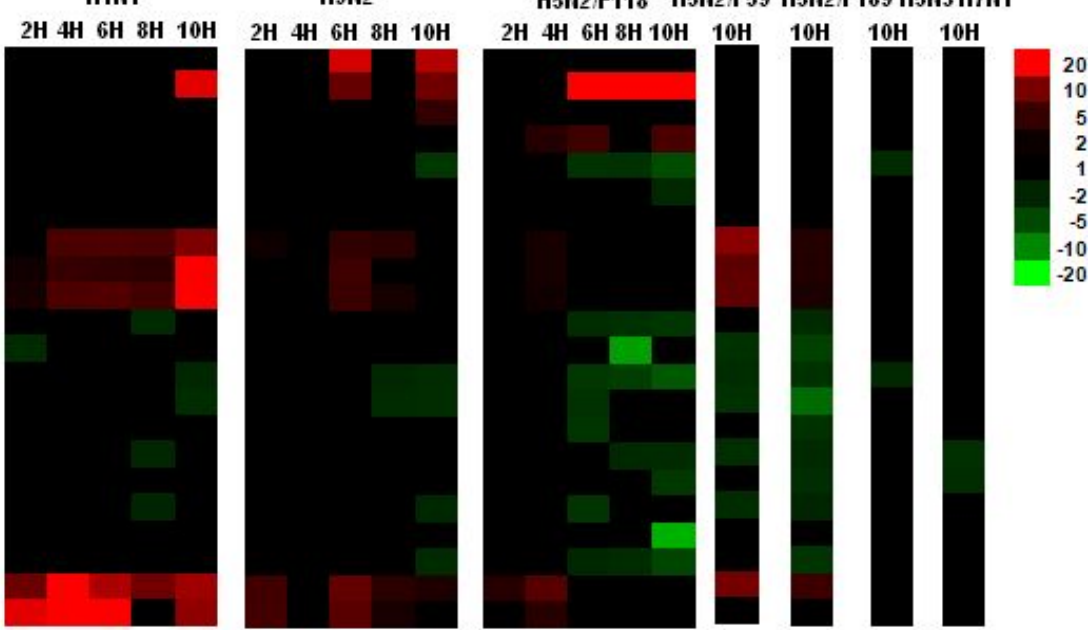

Figure 5.26 The probe sets with topmost expression changes in influenza A viruses-infected CEF cells. Eight influenza A strains were infected with different examined cells, and the global host gene expression was examined by microarray analysis at different time points. The probe sets with topmost expression changes were listed. The data is represented by heat map analysis showing up-regulated (red), down-regulated (green) or no changes (black) in expression, and the FC range is indicated ( $\mathrm{p} \leq 0.05$ ). 

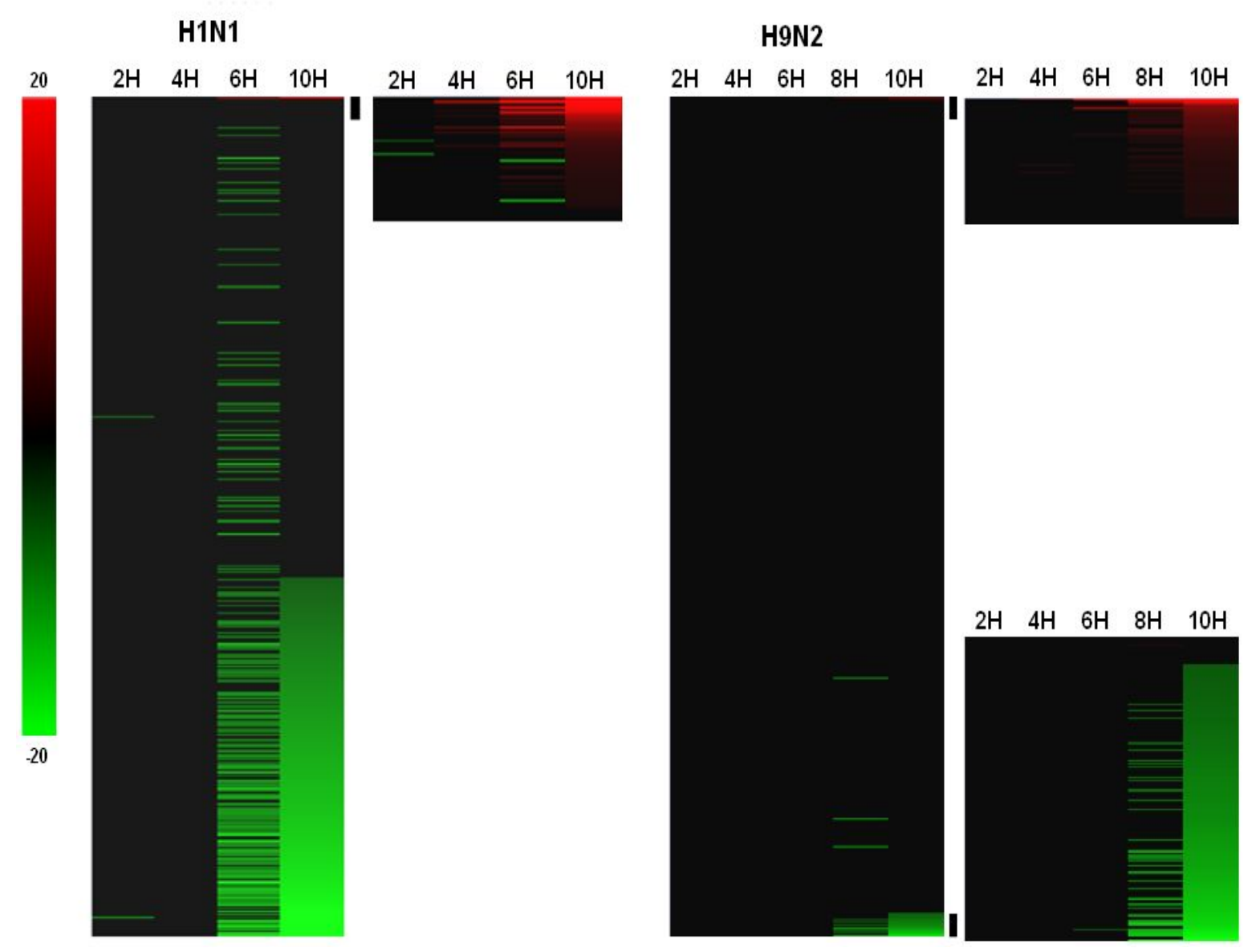

\section{H5N2/F118}

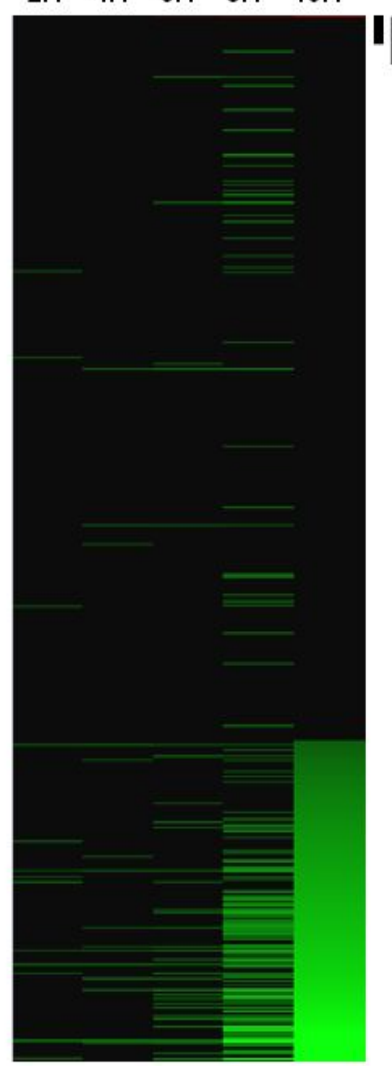

Figure 5.27 Temporal changes in the host cell transcriptome in MDCK cells infected by four influenza virus strains. The global host gene expression profiles were retrieved from microarray analysis with different time points examined. The probe sets showing $\geq 2$ fold change (FC) up- or down-regulated in expression are indicated (Pvalue $\leq 0.05$ ). Expression profiles of up-regulated (red), down-regulated (green) and no significant change (black) are shown. 

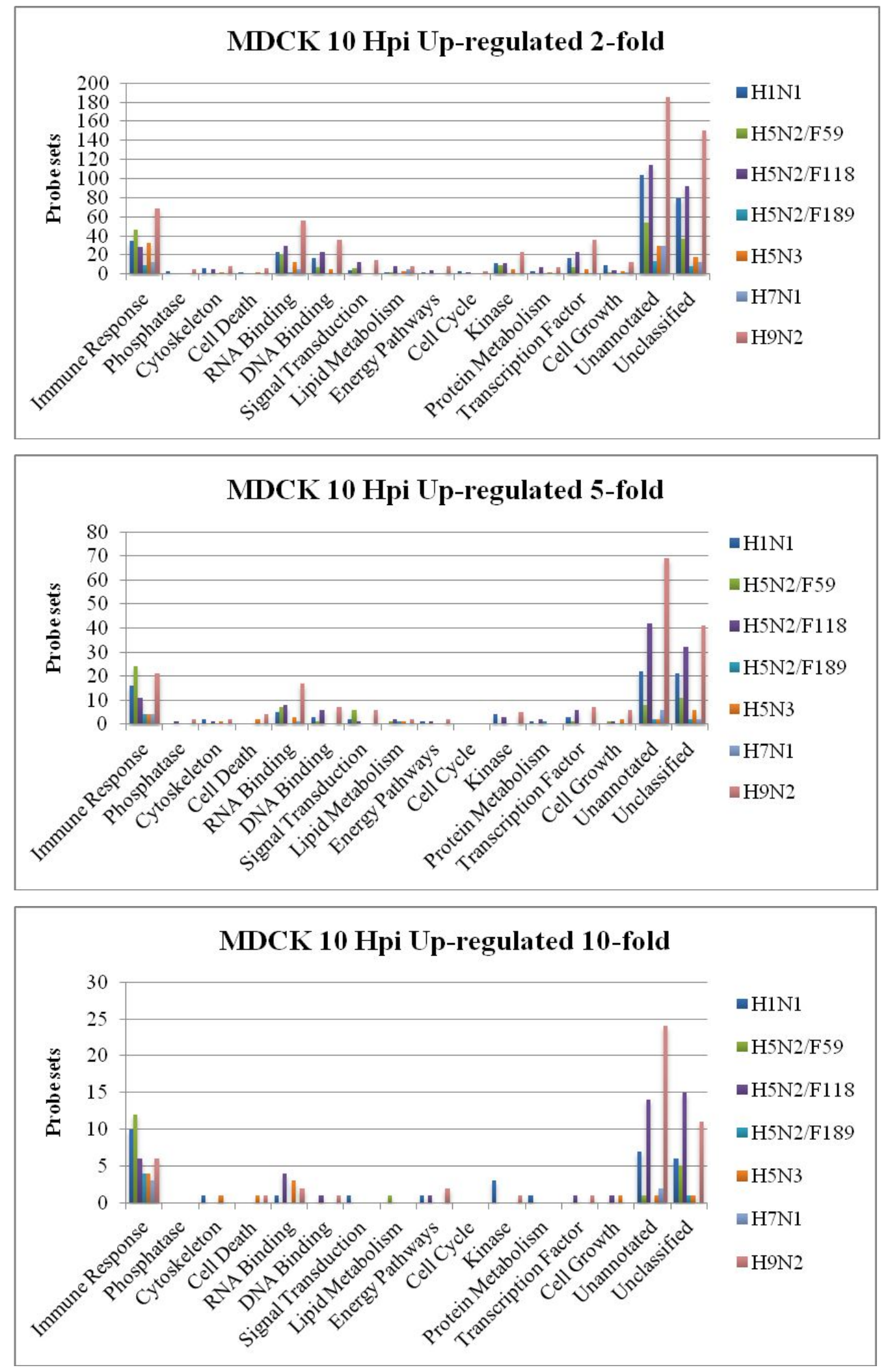

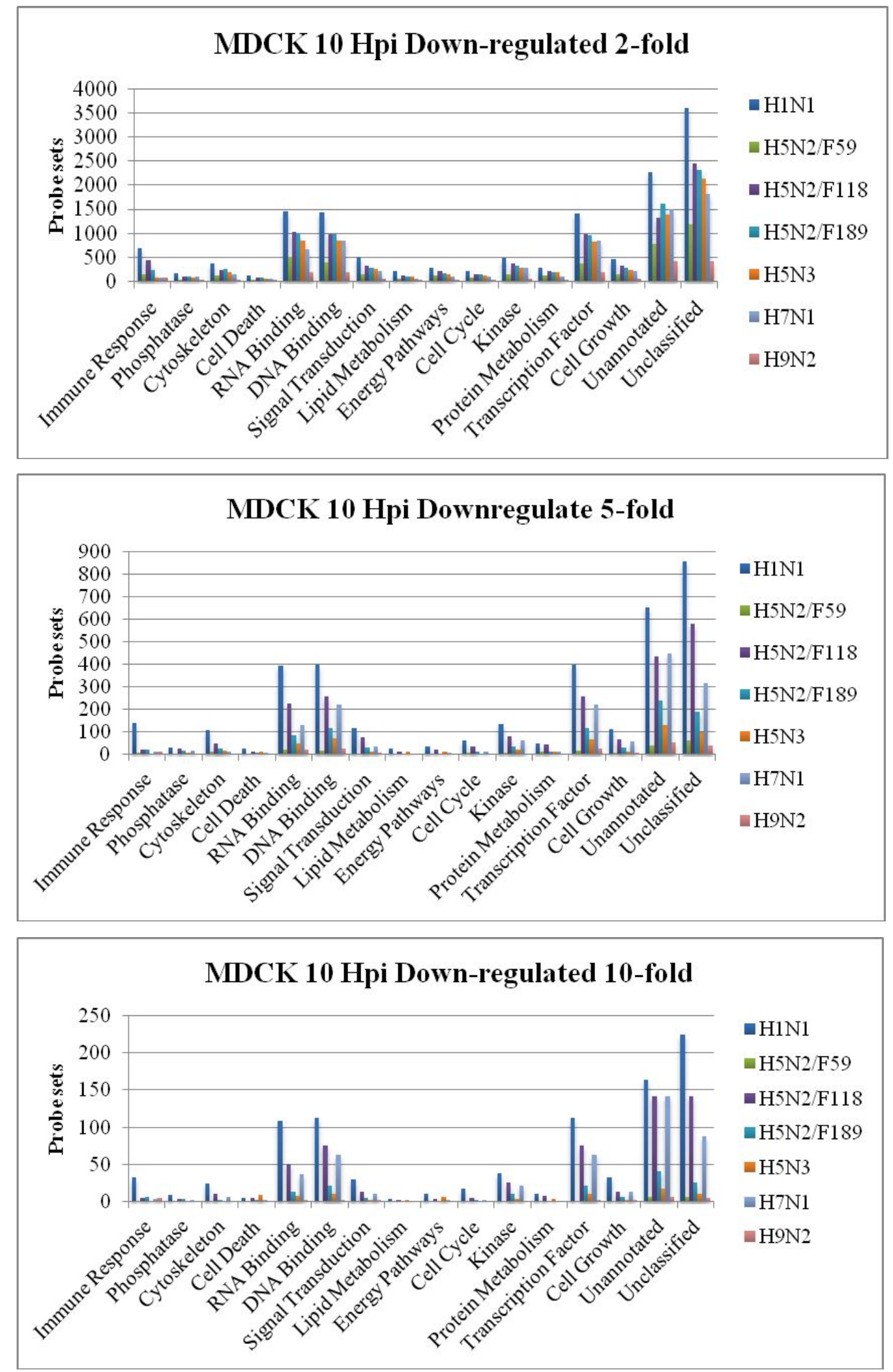

Figure 5.28 Overview of distributions of differentially expressed probe sets into different biological functions in MDCK cells infected with influenza A viruses. The numbers of probe sets in the different functional families, including non-annotated and unclassified groups, showing up- or down-regulated with different fold changes ( $\geq 2-\mathrm{FC}, \geq 5$-FC and $\geq 10$-FC) in gene expression are presented. 


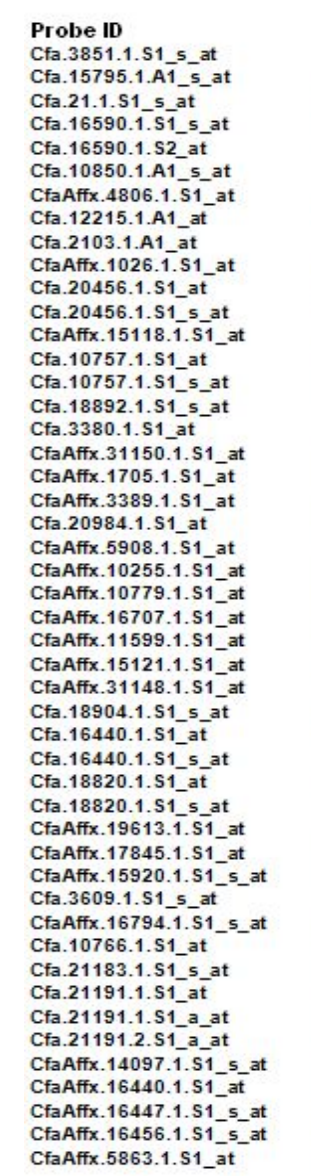

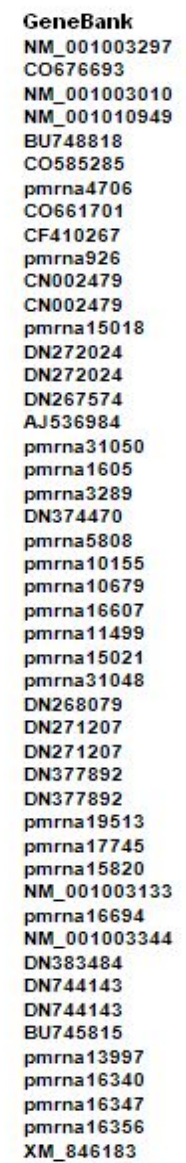

Gene Title

chemokine (C-C motif) ligand 2
chemokine (C-C motiff ligand

chemokine (C-C motif) ligand 5

chemokine (C- $X$ - $C$ motit) ligand 10

chemokine (C-X-C motif ligand 10
suppres sor of cytokine signaling 5

suppres sor of cytokine signaling 5

similar to

Suppres sor of cytokine signaling 6 ;
suppres sor of cytokine signaling

similar to interferon induced $6-16$ protein isoform a

similar to interferon induced 6-16 protein is oform a

interferon-induced proteinwith teratricopeptide repeats 2

ISG15 ubiquitin-like modifier

interferon-induced

interferon-induced protein 44-like

similar tointededpotin 44 ine

similar tol Interferon beta precursor (IFN-beta) (Fibroblast interferon)

interferon-related developmental regulator 1

interferon-related developmental regulator 1

similar to Inteferon regulatory factor 7 (IRF 7 )

interferon-induced protein with tetratricopeptide repeats 5

intericts

interferon-ind

interferon-induced protein 35

interferon-induced protein 35

interferon-induced protein 35

myxovirus (influenza virus) resistance 1 , interferon-inducible prote in p78 myxovirus (intuenza virus) resistance 2 (mouse)

nuclear factor of

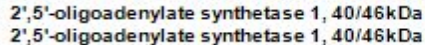

$2^{\prime}, 5$ '-oligoadenylate synthetase $1,40 / 46 \mathrm{kDa}$
$2^{\prime}, 5^{\prime}$-oligoadenylate synthetase $1,40 / 46 \mathrm{kDa}$

2,5 -oligoadenylate synthetase $1,40 / 46 \mathrm{kD}$

2 2'-5'-oligoadenylate synthetase $2,69 / 4 k$
$2^{2}-5$-oligoadenylate synthetase-like

2'-5'-oligoadenylate synthetase-like

radical S-adenosyl methion
H1II1

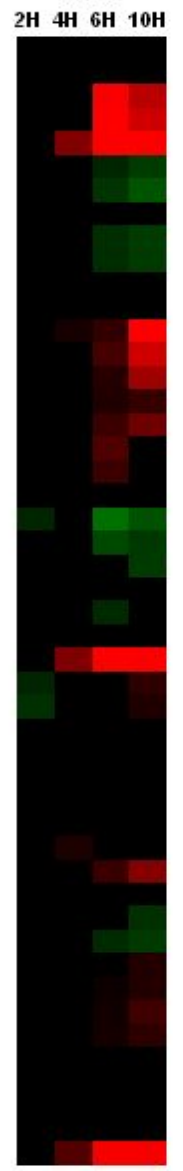

H9112

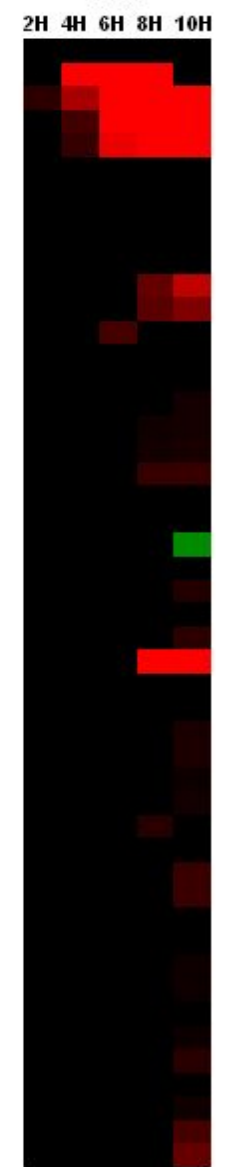

H5H2F118 H5H2/F59 H5H2/F189 H5H3 H7H1

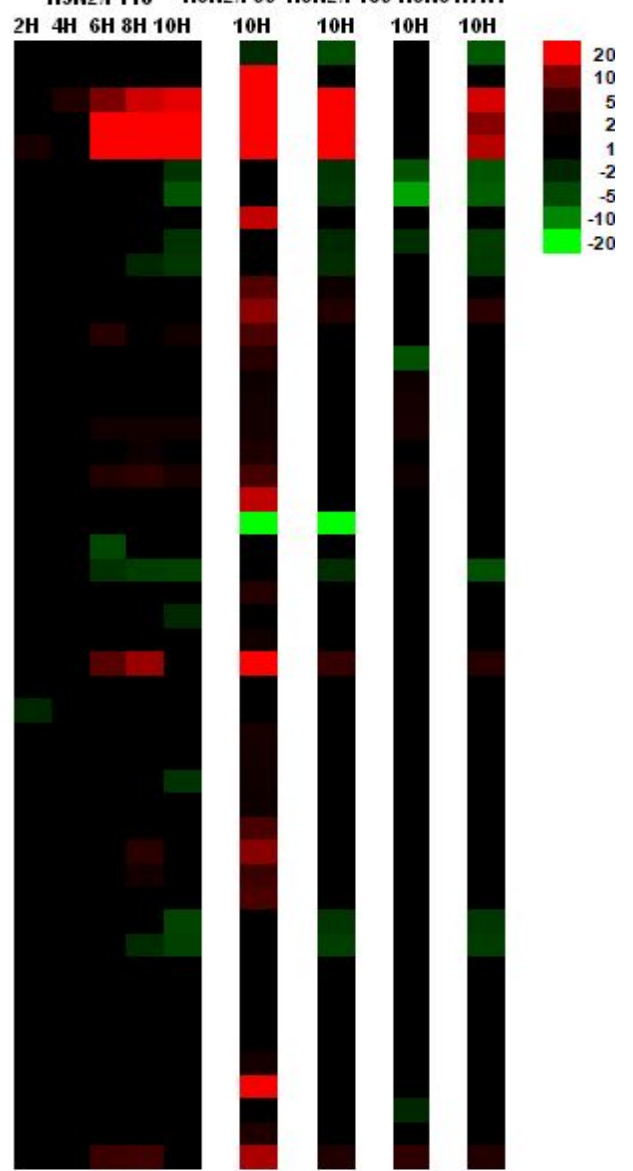

Figure 5.29 The expression of probe sets involved in immune response in influenza A viruses-infected MDCK cells. Eight influenza A strains were infected with different examined cells, and the expression of probe sets related to immune response was examined by microarray analysis at different time points as shown. The data is represented by heat map analysis showing up-regulated (red), down-regulated (green) or no changes (black) in expression, and the FC range is indicated (P-value $\leq 0.05$ ). 
Probe ID

Cfa.110.1.S1_5_at

CfaAffx.6401.1.s1_s_at

Cfa.12400.1.A1_at

Ca.6ffx.12454.1. S1 s at

CfaAffx.12454.1.S1

CfaAffx.7087.1.S1 s at

Cfa.8198.1.S1_at

CfaAffx.8021.1.S1_s_at
CfaAffx.8022.1.S1_at

CfaAffx.16255.1.S1_s_at

CfaAfix.16319.1.S1_at

CfaAffx 16895 . 1 s. 51 at

CfaAffx.16895.1.S1_s_at

Cfa.2490.1.A1_5_at

CfaAffx.28795.1.S1_s_a

CfaAffx. 27855.1.S1_at

Cfa. 3417.1 .51 at

CfaAffx.24073.1.S1_at

Cfa.17926.1.S1_s_at

Cfa.2830.1.S1_5_at

Cfa.18114.1.S1_at

Cfa.18114.1.S1_s_at

CfaAffx.18869.1.s1_at

CfaAffx.18864.1.S1_s_at

CfaAffx.18878.1.51_5_at

Cfa.7342.2.A1_at

CfaAffx.28514.1.S1_at

CfaAffx.4087.1.S1_

Cra.17439.1.S1

Cfa.691.1.A1_at

CfaAffx 23380.1151

Cfa.3498.1.S1_at

CfaAffx.25562.1.S1_s_at
CraAffx.27855.1.S1_s_
GeneBank

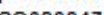

CO715709

pmrna1235

CX005610

pmrna6987

0595574

pmrna7921

pinnay22

pmrna16155

DN320720
pmrna16795

pmrna16795

CF410501

pmrna21174

pmrna28695

pmrna27755

pmrna27755

A.J537250

pmrna23973

DN394512

DN746382

DN376899

DN376899

pmrna18769

pmrna18764

pmrna18778

CF408972

pmrna28414

pmrna 3987

DN748333

CF

pmrna1618

DN413932
Gene Title

H1II1

Beell CLLilymphoma 2

coptidase

apoptosis-related cysteine peptidase

programmed cell death 2

programmed cell death 6 interacting protein;

programmed cell death 6 interacting protein;

programmed cell death 11

programmed cell death 4 (neoplastic transformation inhibitor);

(neoplastic transformation inhibitor)

programmed cell death 4 (neoplastic transformation inhibitor);

cell division cycle and apoptosis regulator 1

mor necrosis factor superfamily, memer 5 -induced protein

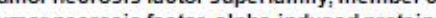

(ancer

Fas (TNF receptor superfamily, member 6 )

programmed cell death 10 ;

programmed cell death 10 ;

CASP8 and FADDIke apoptosis regulator;

CASP8 and FADD-like apoptosis regulator

AD-like apoptosis regulator;

Caspase 8 , apoptosis-related cysteine peptidas

tumor necrosis tactor, alpha-induced protein 1 (endothelial); prot 1 (endothelia): mmed cell death 7

nor necrosis factor (TNF superfamily, member 2)

mor necrosis factor receptor superfamily, member $1 A$

tumor protein p53 (Li-Fraumeni syndrome) н911

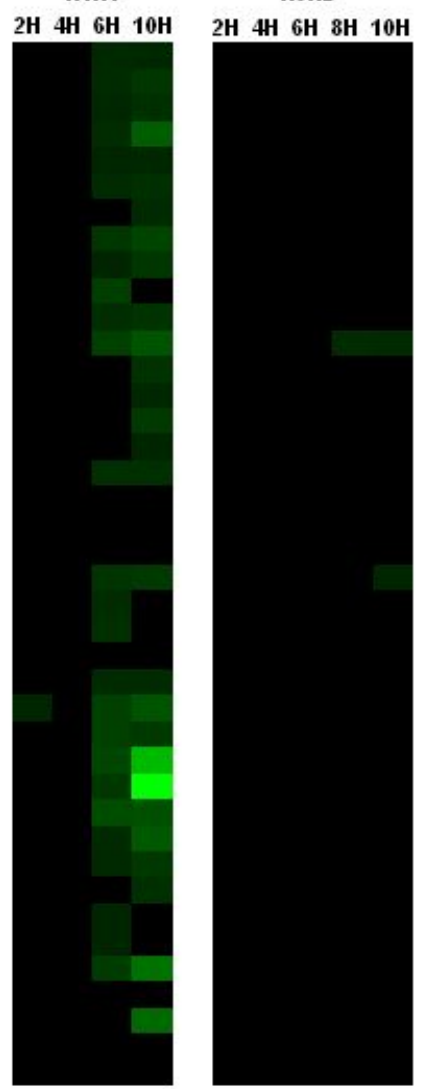

H5H2F118 H5H2/F59 H5H2/F189 H5H3 H7HI

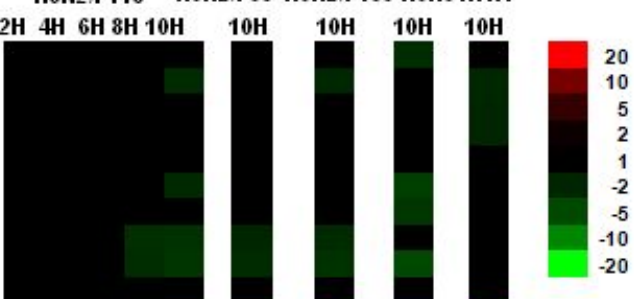

(1) examined cells, and the expression of probe sets related to cell death was examined by microarray analysis at different time points as shown. The data is represented by heat map analysis showing up-regulated (red), down-regulated (green) or no changes (black) in expression, and the FC range is indicated ( $\mathrm{p} \leq 0.05$ ). 


\section{Probe ID}

Pfa.3742.1.S1_5_at

Cfa.16245.1.S1_at

Cfa.16245.1.S1-s_at

CfaAffx.12272.1.S1_s_at

CfaAffx.13734.1.S1_s_a

Cfa.11349.1.A1_at

CfaAffx.29398.1. 1

CfaAffx.29399.1.S1_s_a

Cfa.1994.1.A1_at

Cfa 16947.1A1_S1_5_at

Cfa.14406.1.A1_at

Cfa.21520.1.S1_s_at

Cfa.7073.2.A1_a_at

CfaAffx.4026.1.S1_at

CfaAffx.8936.1.S1_at

CraAffx. 13764.1.51_5_

CraAffx. 13502.1.S1_at

Cfa.20631.1.S1_at

Cfa.21522.2.S1_s_at

Cfa.11398.1.A1_at

Cfa.17863.1.S1_s_at

Cfa.17863.2.51_s_at

CfaAffx. 22470.1.51_at

Ca.2ffx.14570.1.s1

Cfa.15200.1.S1 at

CfaAffx.868.1.s1 a

CfaAffx.7641.1. 1 1_at

CfaAffx.8989.1.S1_5_at
DN357326

pmrna 12172

pmrna13634

CO668650

Co683252

pmrna29298

pmrna29299
CF409920

pmrna8376

DN751348

DN422685

CO650099

pmrna392

pmrna13402

DN350566

CF408018

DN745718

Pmrna14470

BU749737

Pmrna768
Gene Title

activated

cyclin B3

cyclin G2

cyclin G2

cyclin L2

cyclin L2

cyclinL2

cyclin L2

cyclin T2

olesterol 25-hydroxylas

farnesyl-diphos phate farnesyltransferase

tarnesyl-diphos hate farnesyltransferase 1

(2)

G protein-coupled receptor 110

Gproteinco

$G$ protein-coupled receptor 87

$G$ protein-coupled receptor, family $C$, group 5 , member $C$

$G$ protein-coupled receptor, family C, group 5, member C

3-hydroxy-3-methylglutaryl-Coenzyme A synthase 1 (soluble)

3-hydroxy-3-methylglutaryl-Coenzyme $A$ synthase 1 (soluble

transmembrane protein $106 \mathrm{~A}$

brane protein $30 \mathrm{C}$

transmembrane protein $55 \mathrm{~A}$

zinc finger protein 391

zinc finger protein 662
H1II1

2H $4 \mathrm{H} 6 \mathrm{H} \mathrm{10H}$

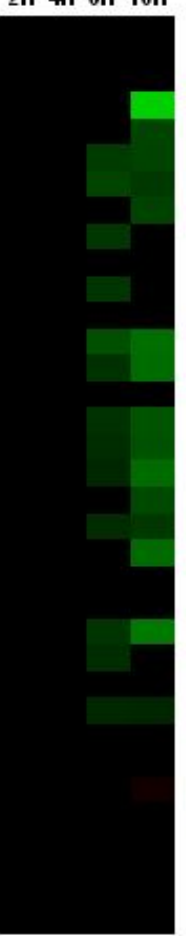

H9I12

$2 \mathrm{H} 4 \mathrm{H} 6 \mathrm{H} 8 \mathrm{H} 10 \mathrm{H}$

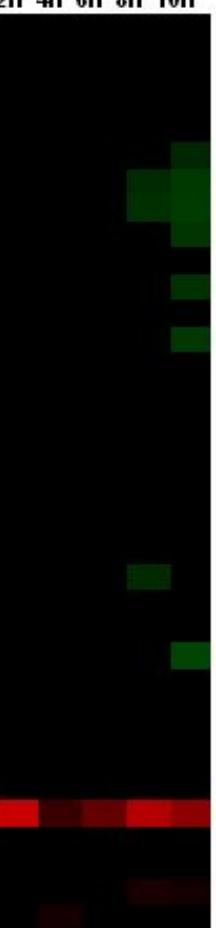

H5H2,F118 H5H12/F59 H5H2F189 H5H3 H7II $2 \mathrm{H}^{4} \quad 6 \mathrm{H}^{2} 8 \mathrm{H}_{10 \mathrm{H}} \quad 10 \mathrm{H} \quad 10 \mathrm{H} \quad 10 \mathrm{H} \quad 10 \mathrm{H}$

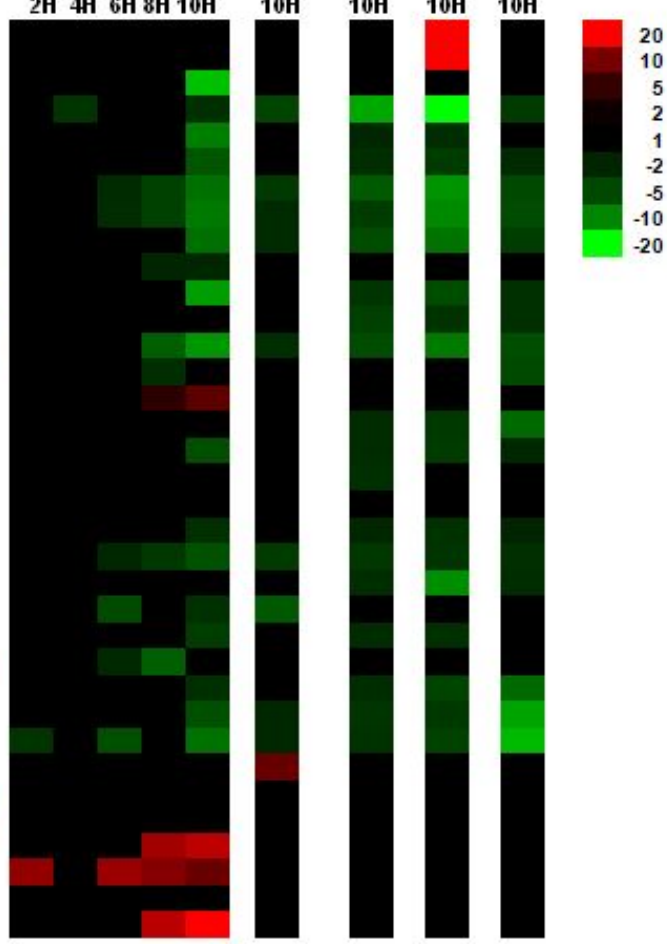

Figure 5.31 The probe sets with topmost expression changes in influenza A viruses-infected MDCK cells. Eight influenza A strains were infected with different examined cells, and the global host gene expression was examined by microarray analysis at different time points. The probe sets with topmost expression changes were listed. The data is represented by heat map analysis showing up-regulated (red), down-regulated (green) or no changes (black) in expression, and the FC range is indicated $(\mathrm{p} \leq 0.05)$. 


\subsubsection{Host gene expression in macrophages}

\subsubsection{Global profiling of gene expression}

\subsection{Heat maps of global gene expression}

The numbers of differentially expressed genes in $\mathrm{H} 1 \mathrm{~N} 1$ and H5N2/F118-infected macrophages both showed decreasing from 2 hpi to 24 hpi. And among these differentially expressed genes, it was interesting that larger numbers of genes with down-regulated expression were detected at 2 hpi while larger numbers of genes with up-regulated expression were detected at 24 hpi after infections of both H1N1 and H5N2/F118.

In the macrophages infected with H5N3, the expression profiles at 24 hpi indicated more up-regulated genes were detected when compared to those detected in macrophages infected with H1N1 and H5N2/F118 at 24 hpi. This phenomenon could suggest that a larger batch of antiviral genes were activated during H5N3 infection than $\mathrm{H} 1 \mathrm{~N} 1$ and $\mathrm{H} 5 \mathrm{~N} 2 / \mathrm{F} 118$ infections in macrophages [Figure 5.32].

\subsection{Distribution of differential expressed probe sets}

In macrophages with infection of $\mathrm{H} 1 \mathrm{~N} 1$, a large number of genes were detected with down-regulated expression at $\geq 2-F C$ at 2 hpi. However, this number decreased steeply at later infection period, with only few genes with down-regulated expression left at 24 hpi [Table 5.11]. In the contrary to the down-regulated genes, only a small number of genes with the up-regulated expression at $\geq 2$-FC were detected at $2 \mathrm{hpi}$, and this number almost remained no change with the infection time increasing.

Infection of H5N2/F118 in macrophages resulted in larger numbers of genes with up- or down-regulated expression at $\geq 2$-FC at 2 hpi when compared to 24 hpi. This phenomenon indicated the early but short-term host gene response upon H5N2/F118 infection in macrophages.

In $\mathrm{H} 5 \mathrm{~N} 3$ infected macrophages, only the gene expression profile at 24 hpi was investigated. It was noted that more differentially expressed genes (upor down-regulated) were identified after infection of $\mathrm{H} 5 \mathrm{~N} 3$ than those identified after infections of $\mathrm{H} 1 \mathrm{~N} 1$ and $\mathrm{H} 5 \mathrm{~N} 2 / \mathrm{F} 118$ at 24hpi, indicating activated gene regulation activities occurred in $\mathrm{H} 5 \mathrm{~N} 3$-infected macrophages at late infection stage. Moreover, some of the genes with elevated expression after infection of 
H5N3 showed high-level fold regulation at $\geq 5$-FC, which might indicate strong antiviral regulation in these infected macrophages.

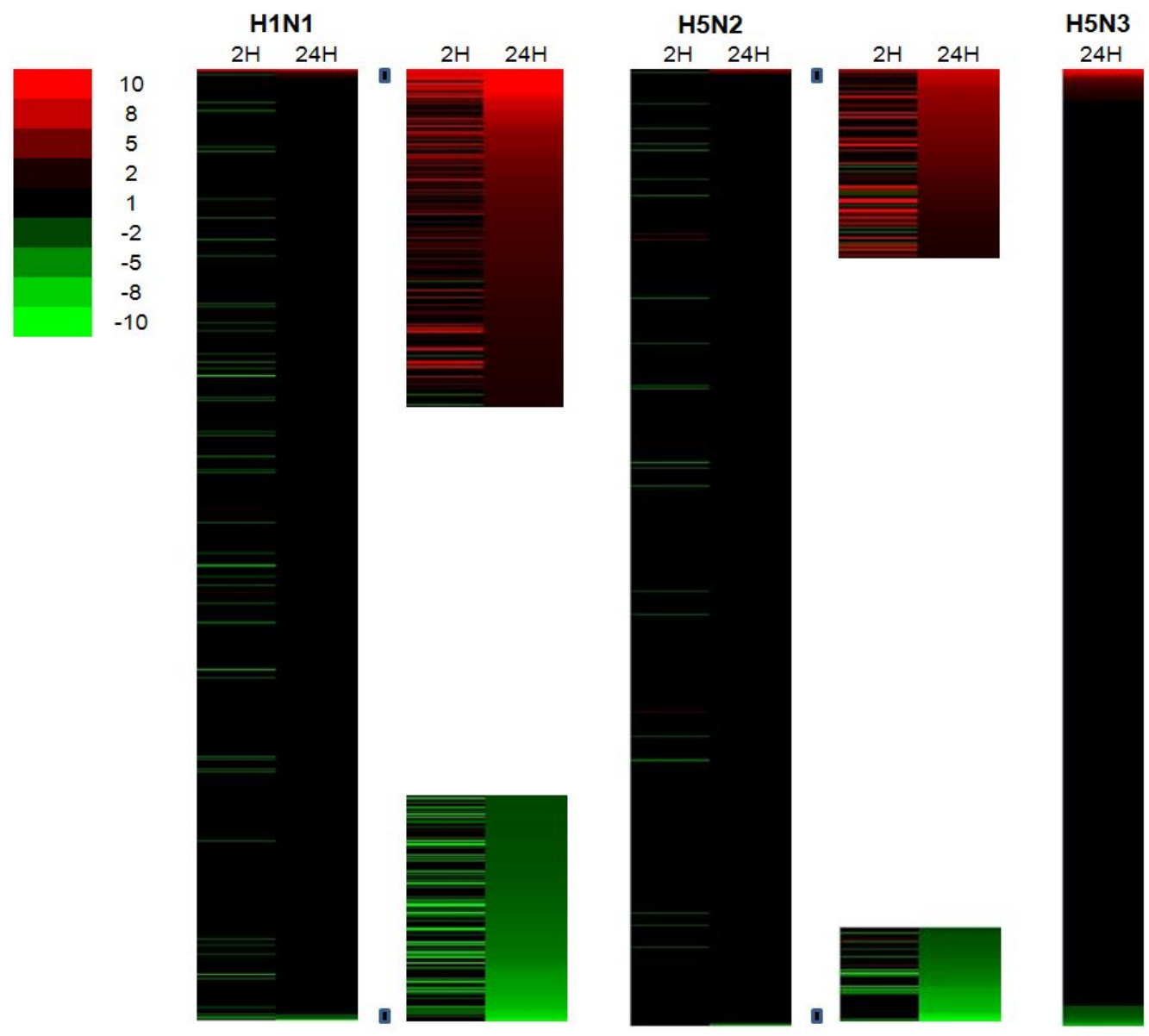

Figure 5.32 Temporal changes in the host cell transcriptome in macrophages infected with influenza A strains. The global host gene expression profiles were retrieved from microarray analysis with different time points examined. The genes showing $\geq 2$ fold change (FC) up- or down-regulated in expression are indicated (P-value $\leq 0.05)$. Expression profiles of up-regulated (red), down-regulated (green) and no significant change (black) are shown.

Table 5.11 Differentially expressed genes in macrophages infected with influenza A strains.

\begin{tabular}{|c|c|c|c|c|c|c|}
\hline \multirow{2}{*}{ Macrophages } & \multicolumn{2}{|c|}{ Genes ( $\geq 2 \cdot F C)$} & \multicolumn{2}{|c|}{ Genes ( $\geq 3-\mathrm{FC}$ ) } & \multicolumn{2}{|c|}{ Genes ( $\geq 5 \cdot \mathrm{FC}$ ) } \\
\hline & Up-regulated & Down-regulated & Up-regulated & Down-regulated & Up-regulated & Down-regulated \\
\hline H1N1 2H & $0.92 \%$ & $5.65 \%$ & $0.52 \%$ & $1.50 \%$ & $0.26 \%$ & $0.56 \%$ \\
\hline H1N1 24H & $0.90 \%$ & $0.65 \%$ & $0.56 \%$ & $0.33 \%$ & $0.24 \%$ & $0.15 \%$ \\
\hline $\mathrm{H} 5 \mathrm{~N} 2 / \mathrm{F} 1182 \mathrm{H}$ & $1.19 \%$ & $2.82 \%$ & $0.60 \%$ & $0.80 \%$ & $0.29 \%$ & $0.22 \%$ \\
\hline $\mathrm{H} 5 \mathrm{~N} 2 / \mathrm{F} 11824 \mathrm{H}$ & $0.51 \%$ & $0.24 \%$ & $0.36 \%$ & $0.17 \%$ & $0.19 \%$ & $0.09 \%$ \\
\hline $\mathrm{H} 5 \mathrm{~N} 324 \mathrm{H}$ & $3.27 \%$ & $1.99 \%$ & $1.83 \%$ & $0.87 \%$ & $0.94 \%$ & $0.25 \%$ \\
\hline
\end{tabular}

The global host gene expression profiles were retrieved from microarray analysis with different time points examined. The ratios of differentially expressed genes (P-value $\leq 0.05)$ up- or downregulated with different fold changes $(\geq 2-\mathrm{FC}, \geq 3-\mathrm{FC}$ and $\geq 5-\mathrm{FC})$ in relative to their corresponding "expressing genes" are represented in percentage. The expressing genes refer to genes detected in the mock-infected macrophages. 


\subsection{Functional classification}

In macrophages infected with three influenza A strains, most of the genes with up-regulated expression were detected after infection of H5N3 at 24 hpi, followed by infections of H1N1 and H5N2/F118 at 2 hpi [Figure 5.33]. These genes were majorly function-related to "Signaling Transduction", "Antiviral" and "Cytokine", "Cell Death", "DNA Transcription Factor", "Kinase" and "RNA Binding". Compared to situations detected in the early phase of virus infections, infections of H1N1 and H5N2/F118 induced expression of relative low number of genes at late stage, 24 hpi. And this phenomenon might indicate the host immune response became weaken during the late infection phase. Moreover, some of the antiviral and signal transduction associated genes detected after H5N3 infection at 24 hpi also showed upregulated expression with high-level fold changes.
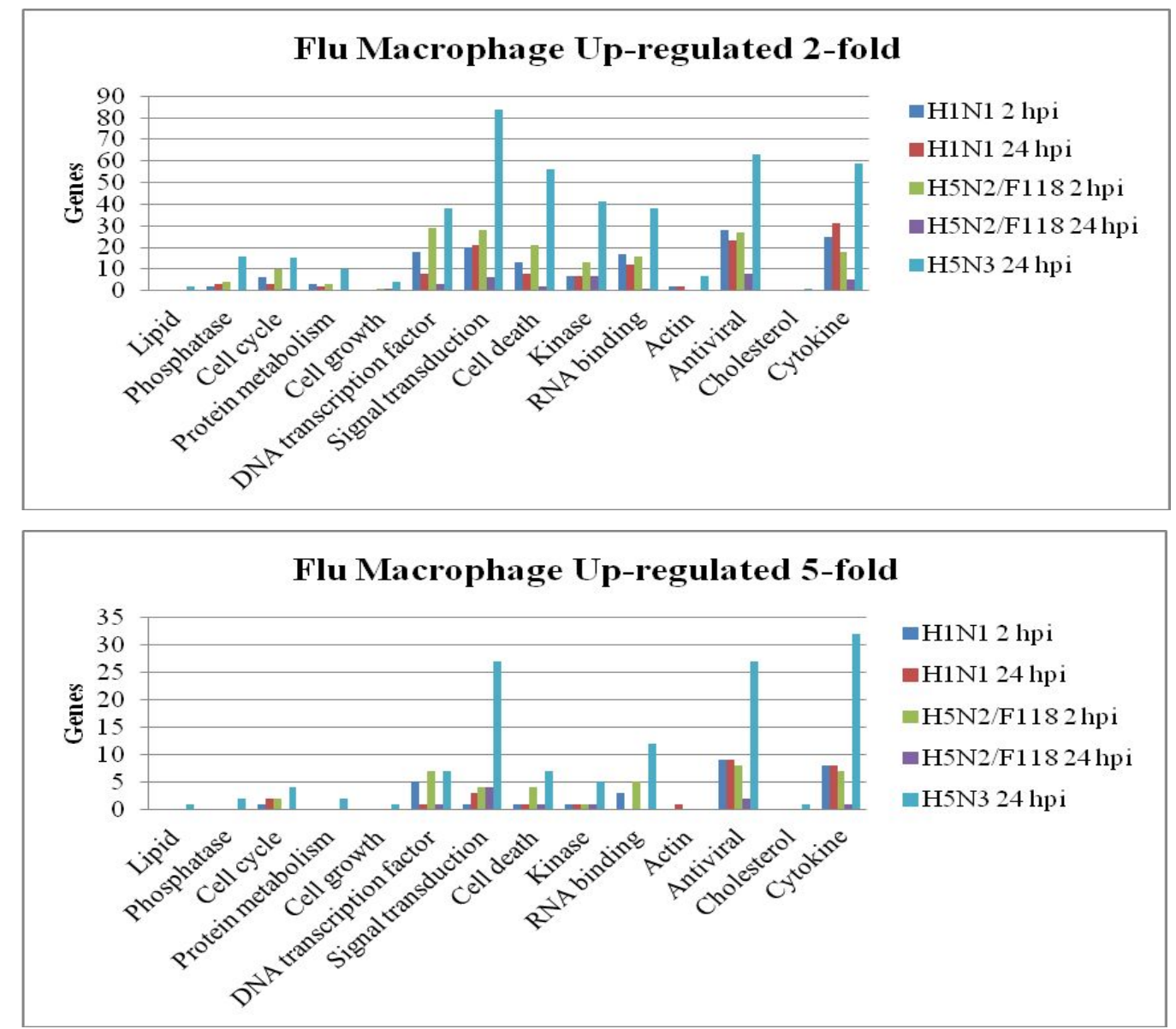

For the genes with down-regulated expression, those detected after infections of H1N1 and H5N2/F118 at 2 hpi were majorly related to "DNA Transcriptional Factor”, "Signal Transduction”, "Kinase”, "RNA Binding” and 
"Cell Death", while those detected after infections of H5N3 at 24 hpi were functionally associated with "DNA Transcription Factor", "Signal Transduction", "Kinase", "RNA Binding", "Cell Cycle", "Cell Death" and "Antiviral".
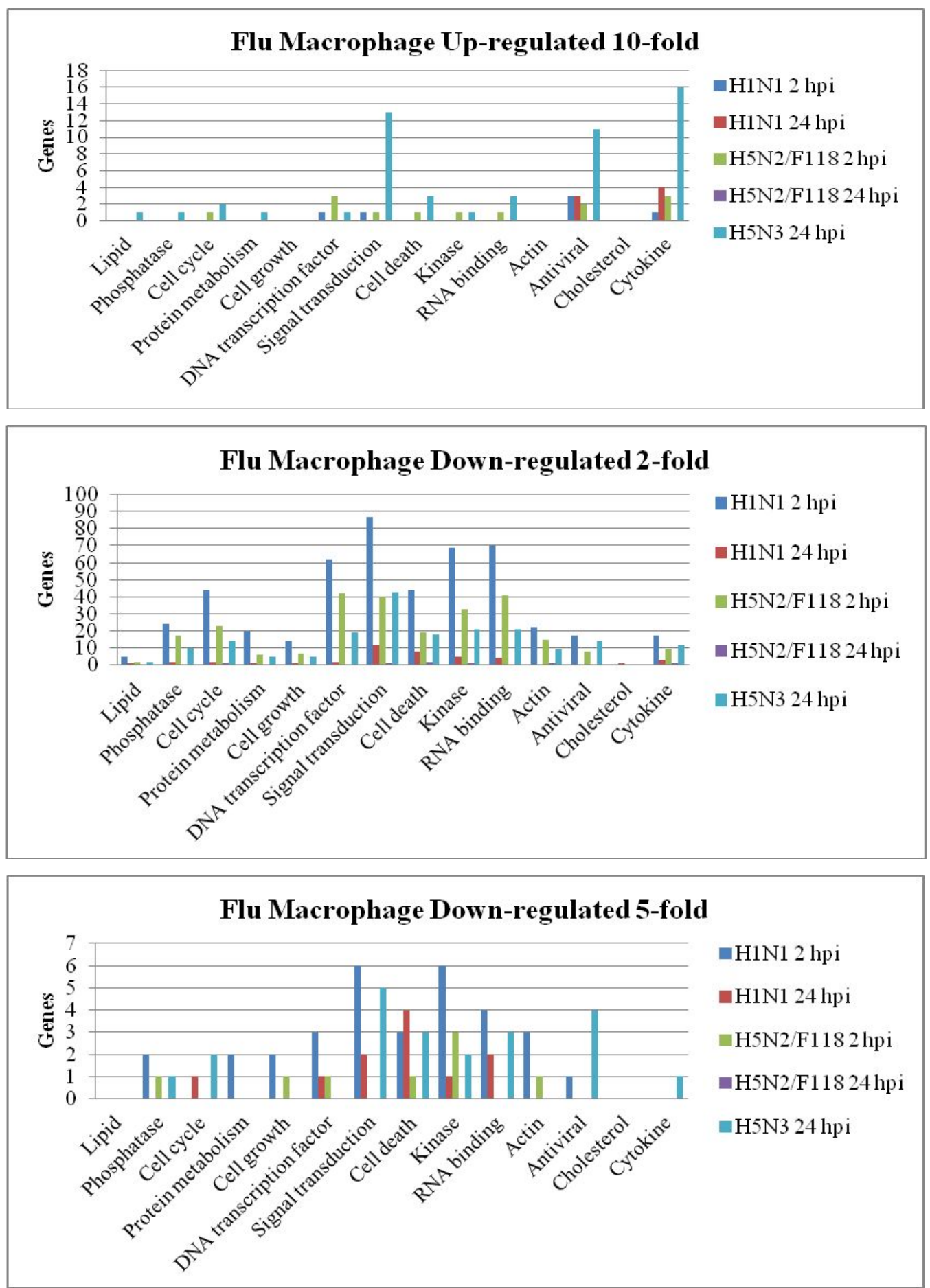

Figure 5.33 Overview of distributions of differentially expressed genes into different biological functions in macrophages infected with influenza $A$ strains. The numbers of genes in the different functional families, showing up-regulated or down-regulated with different fold changes $(\geq 2-F C, \geq 5-F C$ and $\geq 10-F C)$ in gene expression are presented. 


\subsection{Core analysis in macrophages}

Subjecting to H1N1 infection at $2 \mathrm{hpi}$, networks such as "Molecular Transport", "Cell cycle, DNA replication" and "Cellular Assembly and Organization" were highly scored. Biological functions such as "Inflammatory Response", "Cancer", "Cellular Growth and Proliferation", "Cellular Movement", "Cell death", "Cell cycle”, "Tissue Development” were enriched with hundreds of molecules included for each. And "RhoA Signaling", "Actin Cytoskeleton Signaling", "ILK Signaling" and "Role of Macrophages, Fibroblasts and Endothelial Cells in Rheumatoid Arthritis" were listed as top canonical pathways as well [Figure 5.34-5.37]. Enrichment of these key functions and pathways might indicate the activation of antiviral actions at a very early infection stage. With the infection time increasing, gene numbers in these biological functions went down, which implied the gradual elimination of cell regulations in response to the $\mathrm{H} 1 \mathrm{~N} 1$ infection.

In H5N2/F118 infected macrophages, differentially expressed genes were represented by top networks such as "Cell-To-Cell signaling and interaction", "Cell Growth and Proliferation", "Cellular Assembly and Organization", "'Cellular Movement" and top biological functions such as "Cancer", "Inflammatory Response", "Cell Cycle", "Cell Death" and "Tissue Development" at 2 hpi. And pathways named "Role of Pattern Recognition Receptors in Recognition of Bacteria and Viruses", "Activation of IRF by Cytosolic Pattern Recognition Receptors" and "Role of JAK2 in Hormone-like Cytokine Signaling" were enriched as top canonical pathways at 2 hpi [Figure 5.38-5.39]. At $24 \mathrm{hpi}$, although inflammatory response related functions and pathways were still listed, the numbers of molecules belonging to these groups became lower, which was similar to the situation happened in H1N1 infected macrophages [Figure 5.40-5.41]. This phenomenon might implicate the weaker host cell response occurred at 24 hpi when compared to 2 hpi in H5N2/F118 infected macrophages.

With regards to $\mathrm{H} 5 \mathrm{~N} 3$ infected macrophages, the functional groups and pathways relevant to immune response were identified based on differentially expressed genes at 24 hpi [Figure 5.42-5.43]. And almost all of these functional groups were enriched by hundreds of molecules, implicatinging strong immune defense. 
H1N1 Macrophage 2 hpi

\section{Top Networks}

ID Associated Network Functions $\quad$ Score

Molecular Transport, Developmental Disorder, Genetic Disorder 47

Cell Cycle, DNA Replication, Recombination, and Repair, Cell Morphology

Cellular Assembly and Organization, Skeletal and Muscular System Development and Function, Cellular Development

Skeletal and Muscular System Development and Function, Tissue Morphology, Cardiovascular System Development and Function 33

Connective Tissue Disorders, Genetic Disorder, Dermatological Diseases and Conditions

Diseases and Disorders

\begin{tabular}{lll} 
Name & p-value & \# Molecules \\
\hline Cancer & $1.80 \mathrm{E}-09-4.00 \mathrm{E}-03$ & 344 \\
Gastrointestinal Disease & $1.19 \mathrm{E}-07-4.00 \mathrm{E}-03$ & 154 \\
Reproductive System Disease & $1.52 \mathrm{E}-07-2.83 \mathrm{E}-03$ & 182 \\
Organismal Injury and Abnormalities & $2.99 \mathrm{E}-07-2.48 \mathrm{E}-03$ & 74 \\
Inflammatory Response & $2.69 \mathrm{E}-06-3.67 \mathrm{E}-03$ & 150
\end{tabular}

Molecular and Cellular Functions

Name $\quad$ p-value $\quad$ \# Molecules

$\begin{array}{lrr}\text { Cellular Growth and Proliferation } & 1.95 \mathrm{E}-10-4.11 \mathrm{E}-03 & 272\end{array}$

Cellular Movement $\quad 2.94 \mathrm{E}-08-4.13 \mathrm{E}-03 \quad 193$

Cell Death $\quad 4.65 \mathrm{E}-07-4.22 \mathrm{E}-03 \quad 291$

Cellular Development $\quad 5.39 \mathrm{E}-07-4.22 \mathrm{E}-03 \quad 241$

$\begin{array}{lrr}\text { Cell Cycle } & 2.23 \mathrm{E}-06-4.22 \mathrm{E}-03 & 124\end{array}$

Physiological System Development and Function

Name $\quad p$-value $\quad$ \# Molecules

Skeletal and Muscular System Development and Function $4.38 \mathrm{E}-11-4.22 \mathrm{E}-03 \quad 168$

Tissue Development $\quad 4.38 \mathrm{E}-11-3.87 \mathrm{E}-03 \quad 259$

Cardiovascular System Development and Function $\quad 6.48 \mathrm{E}-08-3.87 \mathrm{E}-03 \quad 132$

Organismal Development $\quad 4.16 \mathrm{E}-07-3.87 \mathrm{E}-03 \quad 179$

Hematological System Development and Function $\quad 2.03 \mathrm{E}-06-4.22 \mathrm{E}-03 \quad 158$

Top Canonical Pathways

\begin{tabular}{lll} 
Name & p-value & Ratio \\
\hline RhoA Signaling & $1.67 \mathrm{E}-05$ & $20 / 114(0.175)$ \\
Caveolar-mediated Endocytosis Signaling & $3.34 \mathrm{E}-05$ & $15 / 85(0.176)$ \\
Actin Cytoskeleton Signaling & $1.35 \mathrm{E}-04$ & $28 / 238(0.118)$ \\
ILK Signaling & $2.95 \mathrm{E}-04$ & $24 / 193(0.124)$ \\
Role of Macrophages, Fibroblasts and Endothelial Cells in Rheumatoid Arthritis & $4.65 \mathrm{E}-04$ & $34 / 336(0.101)$
\end{tabular}

Figure 5.34 Summary of top functional groups enriched in differentially expressed genes in H1N1-infected with macrophages at 2 hpi. Differentially expressed genes detected at different time points were significantly categorized into different networks, functions and pathways under the analysis of Ingenuity Pathway Analysis (IPA) version 2012 (P-value $\leq 0.05$ ). 
A

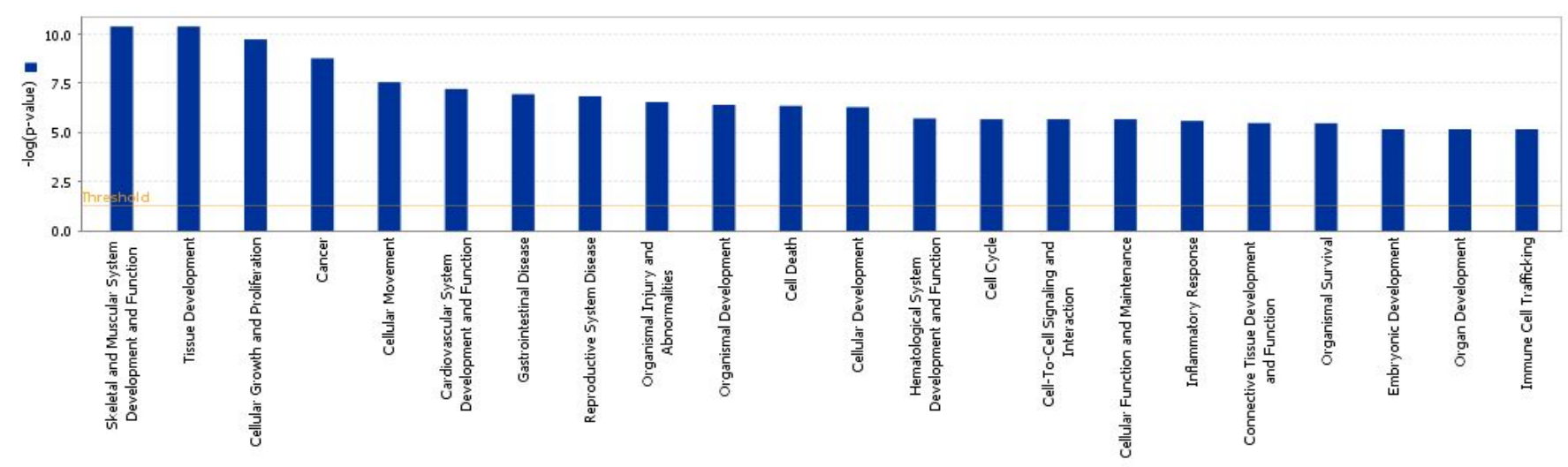

B

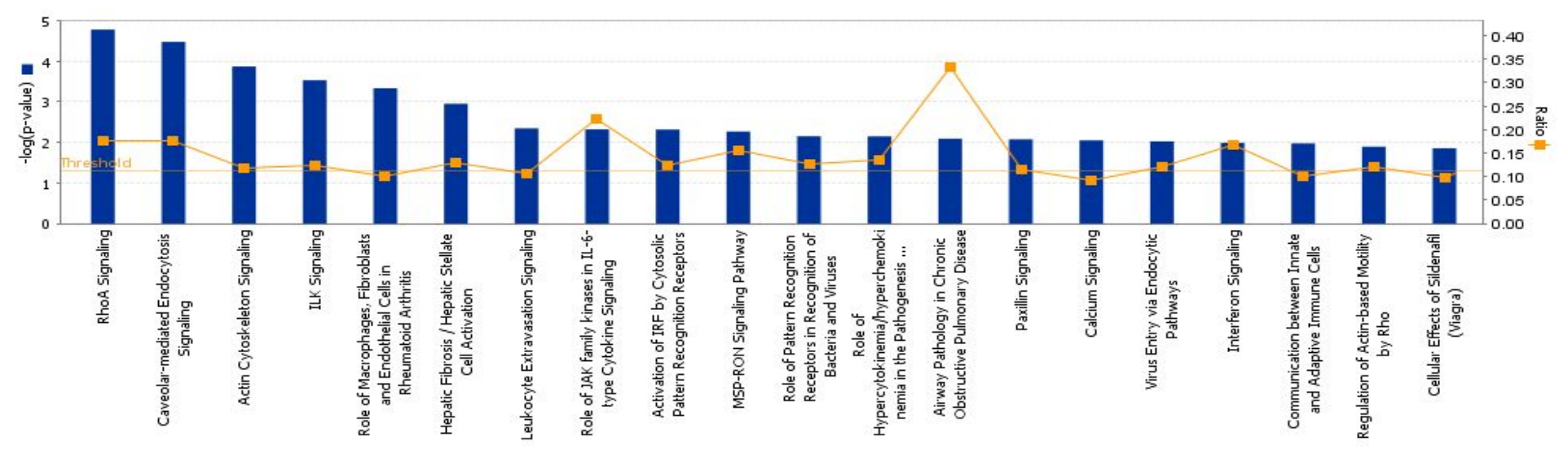

Figure 5.35 Top 20 (A) biological functions and (B) canonical pathways significantly enriched in differentially expressed genes from H1N1-infected macrophages at 2 hpi (P-value $\leq \mathbf{0 . 0 5})$. Threshold was set at p-value $=0.05$ and indicated as $-\log$ (p-value) on the $\mathrm{Y}$-axis and the $\mathrm{X}$-axis shows the terms of each biological function or canonical pathway. The orange boxes indicate the ratio (Ratio) of the number of genes with differential expression changes and the total number of genes in the respective canonical pathway. 
ID Associated Network Functions

Score

Infectious Disease, Antimicrobial Response, Inflammatory Response

Dermatological Diseases and Conditions, Genetic Disorder, Antigen Presentation

Cell-To-Cell Signaling and Interaction, Cellular Growth and Proliferation, Hematological System Development and Function

46

Cell-To-Cell Signaling and Interaction, Hematological System Development and Function, Immune Cell Trafficking

41

5 Cellular Movement, Cellular Assembly and Organization, Cellular Compromise

Diseases and Disorders

\begin{tabular}{lcc} 
Name & p-value & \# Molecules \\
\hline Dermatological Diseases and Conditions & $3.60 \mathrm{E}-17-3.93 \mathrm{E}-03$ & 47 \\
Genetic Disorder & $3.60 \mathrm{E}-17-3.70 \mathrm{E}-03$ & 65 \\
Infectious Disease & $2.55 \mathrm{E}-15-2.85 \mathrm{E}-03$ & 48 \\
Inflammatory Response & $2.83 \mathrm{E}-15-3.95 \mathrm{E}-03$ & 66 \\
Antimicrobial Response & $9.34 \mathrm{E}-10-3.95 \mathrm{E}-03$ & 19
\end{tabular}

Molecular and Cellular Functions

\begin{tabular}{lcc} 
Name & p-value & \# Molecules \\
\hline Cellular Movement & $1.66 \mathrm{E}-10-3.95 \mathrm{E}-03$ & 60 \\
Cell-To-Cell Signaling and Interaction & $4.01 \mathrm{E}-10-3.95 \mathrm{E}-03$ & 56 \\
Antigen Presentation & $1.19 \mathrm{E}-09-3.34 \mathrm{E}-03$ & 36 \\
Cellular Growth and Proliferation & $1.42 \mathrm{E}-07-3.86 \mathrm{E}-03$ & 74 \\
Cell Signaling & $1.46 \mathrm{E}-07-1.92 \mathrm{E}-03$ & 37
\end{tabular}

Physiological System Development and Function

\begin{tabular}{lcc} 
Name & p-value & \# Molecules \\
\hline Hematological System Development and Function & $1.66 \mathrm{E}-10-3.95 \mathrm{E}-03$ & 58 \\
Immune Cell Trafficking & $1.66 \mathrm{E}-10-3.95 \mathrm{E}-03$ & 40 \\
Tissue Development & $1.86 \mathrm{E}-07-3.72 \mathrm{E}-03$ & 50 \\
Tissue Morphology & $7.24 \mathrm{E}-07-3.75 \mathrm{E}-03$ & 42 \\
Cell-mediated Immune Response & $1.09 \mathrm{E}-05-3.95 \mathrm{E}-03$ & 9
\end{tabular}

Top Canonical Pathways

\begin{tabular}{lll} 
Name & p-value & Ratio \\
\hline Activation of IRF by Cytosolic Pattern Recognition Receptors & $8.09 \mathrm{E}-11$ & $11 / 72(0.153)$ \\
Interferon Signaling & $4.62 \mathrm{E}-09$ & $8 / 36(0.222)$ \\
Role of Pattern Recognition Receptors in Recognition of Bacteria and Viruses & $6.45 \mathrm{E}-08$ & $10 / 87(0.115)$ \\
Role of RIG1-like Receptors in Antiviral Innate Immunity & $5.68 \mathrm{E}-05$ & $5 / 49(0.102)$ \\
Role of IL-17F in Allergic Inflammatory Airway Diseases & $1.6 \mathrm{E}-04$ & $5 / 46(0.109)$
\end{tabular}

Figure 5.36 Summary of top functional groups enriched in differentially expressed genes in H1N1-infected with macrophages at 24 hpi. Differentially expressed genes detected at different time points were significantly categorized into different networks, functions and pathways under the analysis of Ingenuity Pathway Analysis (IPA) version 2012 (P-value $\leq 0.05$ ). 
A

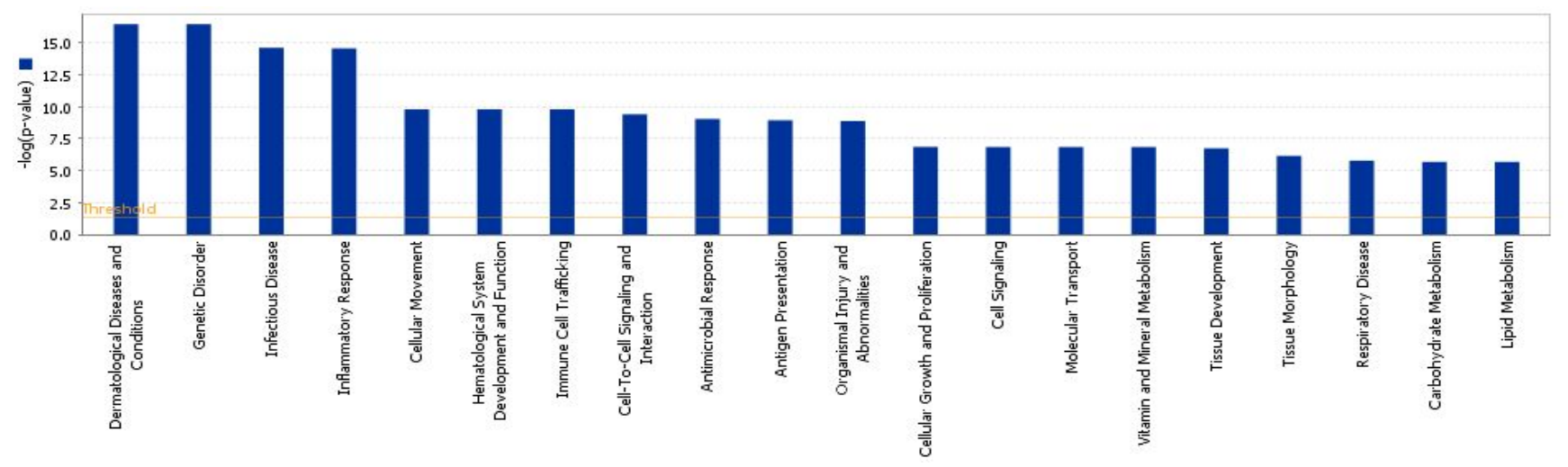

B

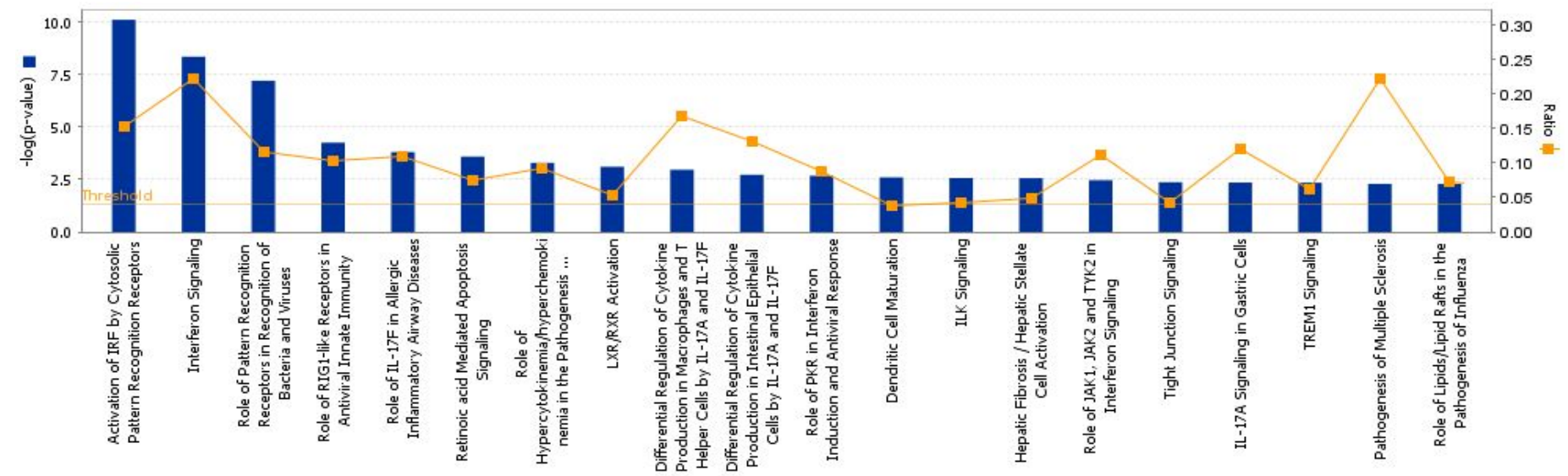

Figure 5.37 Top 20 (A) biological functions and (B) canonical pathways significantly enriched in differentially expressed genes from H1N1-infected macrophages at $24 \mathbf{~ h p i ~ ( P - v a l u e ~} \leq \mathbf{0 . 0 5})$. Threshold was set at $\mathrm{p}$-value $=0.05$ and indicated as $-\log$ ( $\mathrm{p}$-value) on the $\mathrm{Y}$-axis and the $\mathrm{X}$-axis shows the terms of each biological function or canonical pathway. The orange boxes indicate the ratio (Ratio) of the number of genes with differential expression changes and the total number of genes in the respective canonical pathway. 
ID Associated Network Functions

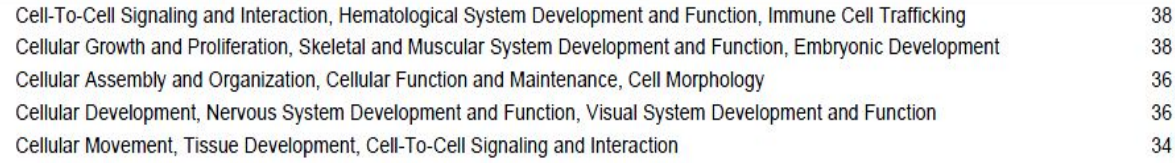

Diseases and Disorders

\begin{tabular}{lll} 
Name & p-value & \# Molecules \\
\hline Cancer & $4.97 \mathrm{E}-17-2.72 \mathrm{E}-03$ & 266 \\
Reproductive System Disease & $1.10 \mathrm{E}-09-1.41 \mathrm{E}-04$ & 156 \\
Inflammatory Response & $1.26 \mathrm{E}-09-3.04 \mathrm{E}-03$ & 115 \\
Dermatological Diseases and Conditions & $1.28 \mathrm{E}-09-2.05 \mathrm{E}-03$ & 75 \\
Genetic Disorder & $1.28 \mathrm{E}-09-2.05 \mathrm{E}-03$ & 113
\end{tabular}

Molecular and Cellular Functions

Name p-value 3.87E-13 - 3.30E-03 \# Molecules

Cellular Movement

E-03 146

$3.77 \mathrm{E}-10-2.72 \mathrm{E}-03$

4.30E-10 - 3.24E-03

Cell Cycle

4. $85 \mathrm{E}-10-2.99 \mathrm{E}-03$

219

Cell Death

$4.85 \mathrm{E}-10-2.99 \mathrm{E}-03$
$1.38 \mathrm{E}-09-3.28 \mathrm{E}-03$

96

Cellular Development

210

187

Physiological System Development and Function

Name

Organismal Development

p-value 1.19E-11 - 3.28E-03 146

2.38E-11 - 3.28E-03

$4.41 \mathrm{E}-09-3.28 \mathrm{E}-03$

$1.22 \mathrm{E}-08-3.18 \mathrm{E}-03$

Tissue Development

$1.22 \mathrm{E}-08-3.18 \mathrm{E}-03$
$2.21 \mathrm{E}-08-2.92 \mathrm{E}-03$

Connective Tissue Development and Function

110

117

\section{Top Canonical Pathways}

Name

Role of Pattern Recognition Receptors in Recognition of Bacteria and Viruses

p-value

Ratio

ILK Signaling

Caveolar-mediated Endocytosis Signaling

Activation of IRF by Cytosolic Pattern Recognition Receptors

6.67E-06 $13 / 87(0.149)$

Role of JAK2 in Hormone-like Cytokine Signaling

7.15E-05

$19 / 193(0.098)$

$11 / 85(0.129)$

1.79E-04 $\quad 9 / 72(0.125)$

2.67E-04 $7 / 37(0.189)$

Figure 5.38 Summary of top functional groups enriched in differentially expressed genes in H5N2/F118-infected with macrophages at 2 hpi. Differentially expressed genes detected at different time points were significantly categorized into different networks, functions and pathways under the analysis of Ingenuity Pathway Analysis (IPA) version 2012 (P-value $\leq 0.05$ ). 
A

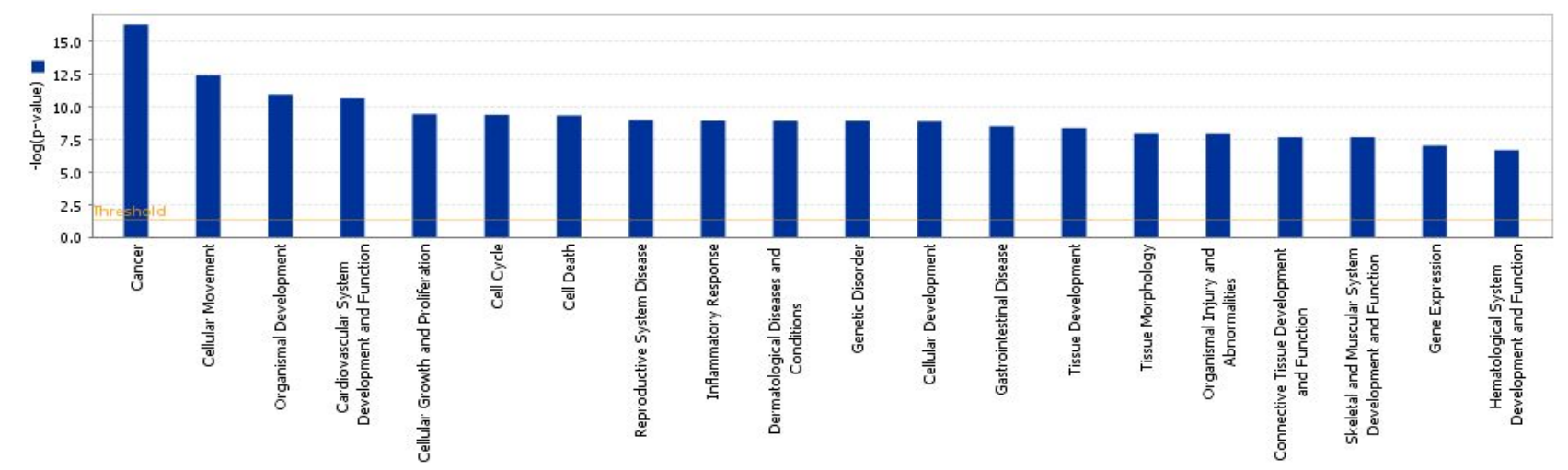

B

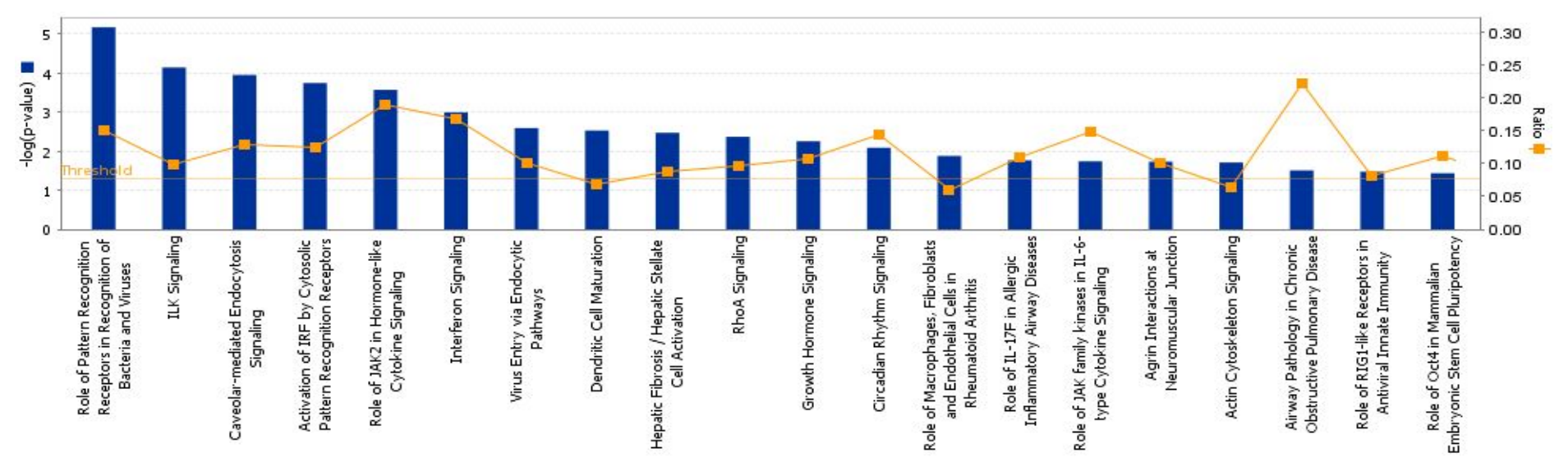

Figure 5.39 Top 20 (A) biological functions and (B) canonical pathways significantly enriched in differentially expressed genes from H5N2/F118-infected macrophages at 2 hpi $(\mathbf{P}$-value $\leq \mathbf{0 . 0 5})$. Threshold was set at $\mathrm{p}$-value $=0.05$ and indicated as $-\log (\mathrm{p}$-value) on the $\mathrm{Y}$-axis and the $\mathrm{X}$-axis shows the terms of each biological function or canonical pathway. The orange boxes indicate the ratio (Ratio) of the number of genes with differential expression changes and the total number of genes in the respective canonical pathway. 
H5N2/F118 Macrophage 24 hpi

\section{Top Networks}

ID Associated Network Functions $\quad$ Score

1 Inflammatory Response, Cell-To-Cell Signaling and Interaction, Antigen Presentation 40

2 Cell Signaling, Small Molecule Biochemistry, Cellular Compromise 29

3 Infectious Disease, Cellular Compromise, Gastrointestinal Disease $\quad 29$

4 Cellular Movement, Immune Cell Trafficking, Hematological System Development and Function 20

5 Infectious Disease, Cell-To-Cell Signaling and Interaction, Connective Tissue Development and Function 17

\section{Top Bio Functions}

\section{Diseases and Disorders}

Name

Auditory Disease

Genetic Disorder

Neurological Disease

Antimicrobial Response

Inflammatory Response

p-value $6.18 \mathrm{E}-05-2.65 \mathrm{E}-02$

$6.18 \mathrm{E}-05-4.59 \mathrm{E}-02$

$6.18 \mathrm{E}-05-2.65 \mathrm{E}-02$

$9.91 \mathrm{E}-05-2.65 \mathrm{E}-02$

$9.91 \mathrm{E}-05-4.75 \mathrm{E}-02$
\# Molecules

3

5

22

Molecular and Cellular Functions

Name

Cellular Movement

p-value 1.19E-04 - 4.75E-02

$2.65 \mathrm{E}-04-4.97 \mathrm{E}-02$

$2.65 \mathrm{E}-04-4.59 \mathrm{E}-02$

$2.65 \mathrm{E}-04-3.95 \mathrm{E}-02$

Cellular Assembly and Organization

3

Protein Folding

$2.65 \mathrm{E}-04-2.65 \mathrm{E}-04 \quad 2$

Physiological System Development and Function

Name

Hematological System Development and Function

p-value

Immune Cell Trafficking

Endocrine System Development and Function

Hematopoiesis

Lymphoid Tissue Structure and Development

$\begin{array}{cl}\text { p-Value } & \text { \# Molecules } \\ 1.19 \mathrm{E}-04-4.75 \mathrm{E}-02 & 13 \\ 1.19 \mathrm{E}-04-4.75 \mathrm{E}-02 & 11 \\ 2.65 \mathrm{E}-04-1.99 \mathrm{E}-02 & 3 \\ 1.21 \mathrm{E}-03-3.95 \mathrm{E}-02 & 6 \\ 1.21 \mathrm{E}-03-3.95 \mathrm{E}-02 & 4\end{array}$

Top Canonical Pathways

Name

p-value Ratio

Activation of IRF by Cytosolic Pattern Recognition Receptors

3.49E-04

$4 / 72(0.056)$

Interferon Signaling

9.44E-04 $3 / 36(0.083)$

Role of Lipids/Lipid Rafts in the Pathogenesis of Influenza

$5.23 \mathrm{E}-02 \quad 1 / 28(0.036)$

Differential Regulation of Cytokine Production in Macrophages and T Helper Cells by IL-17A and IL- $\quad 9.59 \mathrm{E}-02 \quad 1 / 18(0.056)$

$17 \mathrm{~F}$

Cardiomyocyte Differentiation via BMP Receptors

$1.08 \mathrm{E}-01 \quad 1 / 20(0.05)$

Figure 5.40 Summary of top functional groups enriched in differentially expressed genes in H5N2/F118-infected with macrophages at $24 \mathrm{hpi}$. Differentially expressed genes detected at different time points were significantly categorized into different networks, functions and pathways under the analysis of Ingenuity Pathway Analysis (IPA) version 2012 (P-value $\leq 0.05$ ). 
A

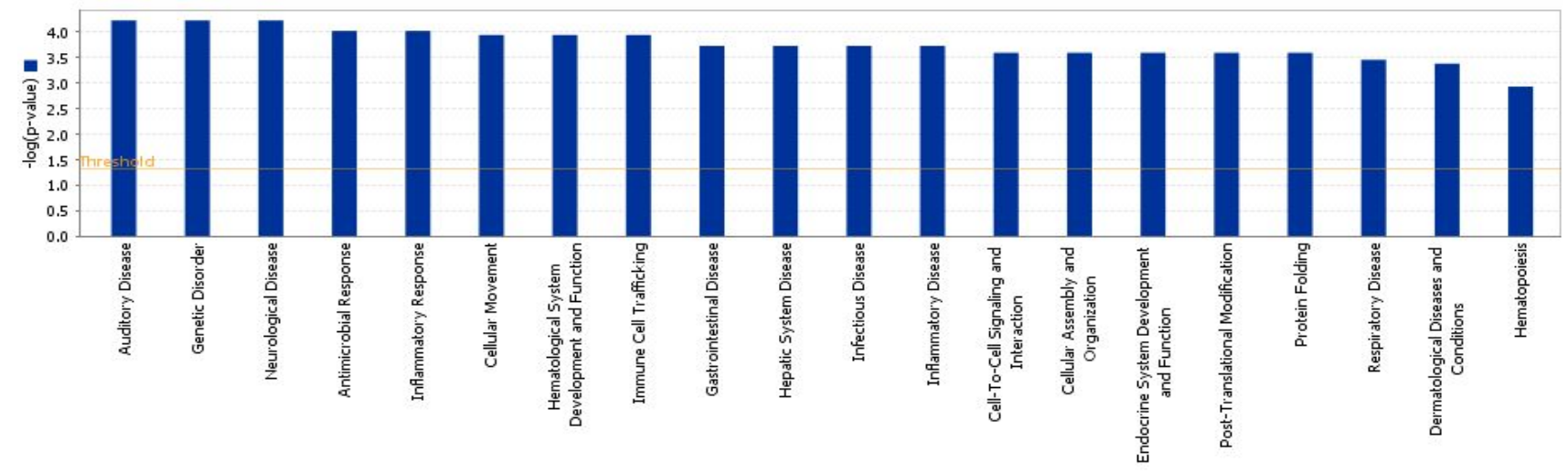

B

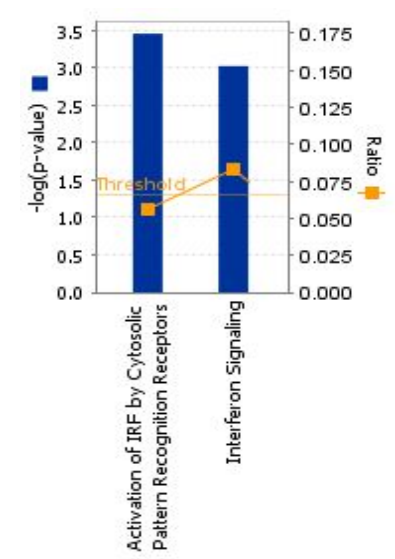

Figure 5.41 Top 20 (A) biological functions and (B) canonical pathways significantly enriched in differentially expressed genes from H5N2/F118-infected macrophages at $24 \mathbf{~ h p i ~ ( P - v a l u e ~} \leq \mathbf{0 . 0 5})$. Threshold was set at $\mathrm{p}$-value $=0.05$ and indicated as $-\log$ ( $\mathrm{p}$-value) on the $\mathrm{Y}$-axis and the $\mathrm{X}$-axis shows the terms of each biological function or canonical pathway. The orange boxes indicate the ratio (Ratio) of the number of genes with differential expression changes and the total number of genes in the respective canonical pathway. 
H5N3 Macrophage 24 hpi

Top Networks

ID Associated Network Functions

1 Lipid Metabolism, Small Molecule Biochemistry, Gene Expression

Score

Cell Death, Inflammatory Response, Hematological System Development and Function

44

Cell-To-Cell Signaling and Interaction, Cellular Movement, Cellular Growth and Proliferation

Antigen Presentation, Cell-To-Cell Signaling and Interaction, Hematological System Development and Function

Dermatological Diseases and Conditions, Genetic Disorder, Antimicrobial Response

33

Top Bio Functions

Diseases and Disorders

Name

p-value

4.50E-94 - 7.20E-10

$1.60 \mathrm{E}-37-9.65 \mathrm{E}-10$

$6.51 \mathrm{E}-35-3.84 \mathrm{E}-10$

4.12E-32 - 1.90E-10

Immunological Disease

4.12E-32 - $1.90 \mathrm{E}-10$

\# Molecules

Connective Tissue Disorders

- 221

Inflammatory Disease

372

278

329

Molecular and Cellular Functions

\begin{tabular}{lcc} 
Name & p-value & \# Molecules \\
\hline Cellular Movement & $6.28 \mathrm{E}-61-6.96 \mathrm{E}-10$ & 257 \\
Cell-To-Cell Signaling and Interaction & $9.37 \mathrm{E}-50-7.20 \mathrm{E}-10$ & 257 \\
Cellular Growth and Proliferation & $2.51 \mathrm{E}-43-9.63 \mathrm{E}-10$ & 333 \\
Cell Death & $1.36 \mathrm{E}-39-6.13 \mathrm{E}-10$ & 341 \\
Antigen Presentation & $2.56 \mathrm{E}-33-7.20 \mathrm{E}-10$ & 169
\end{tabular}

Physiological System Development and Function

\begin{tabular}{lcc} 
Name & p-value & \# Molecules \\
\hline Immune Cell Trafficking & $6.28 \mathrm{E}-61-6.96 \mathrm{E}-10$ & 231 \\
Hematological System Development and Function & $2.62 \mathrm{E}-57-9.63 \mathrm{E}-10$ & 310 \\
Tissue Morphology & $3.06 \mathrm{E}-45-3.82 \mathrm{E}-10$ & 203 \\
Tissue Development & $2.76 \mathrm{E}-38-5.50 \mathrm{E}-10$ & 294 \\
Organismal Survival & $3.26 \mathrm{E}-35-1.49 \mathrm{E}-17$ & 187
\end{tabular}

Top Canonical Pathways

Name $\quad$ p-value Ratio

Dendritic Cell Maturation $\quad 6.15 \mathrm{E}-21 \quad 6.43 \mathrm{E}-17$

$\begin{array}{llr}\text { Communication between Innate and Adaptive Immune Cells } & 3.43 \mathrm{E}-17 & 26 / 109(0.239)\end{array}$

Role of Pattern Recognition Receptors in Recognition of Bacteria and Viruses $\quad 3.76 \mathrm{E}-17 \quad 3.727 / 87$ (0.31)

$\begin{array}{lll}\text { Hepatic Fibrosis / Hepatic Stellate Cell Activation } & 3.12 \mathrm{E}-16 & 35 / 147(0.238)\end{array}$

Activation of IRF by Cytosolic Pattern Recognition Receptors $\quad 8.09 \mathrm{E}-15 \quad 215 \quad(0.292)$

Figure 5.42 Summary of top functional groups enriched in differentially expressed genes in H5N3-infected with macrophages at $24 \mathrm{hpi}$. Differentially expressed genes detected at different time points were significantly categorized into different networks, functions and pathways under the analysis of Ingenuity Pathway Analysis (IPA) version 2012 analysis (Pvalue $\leq 0.05)$. 
A

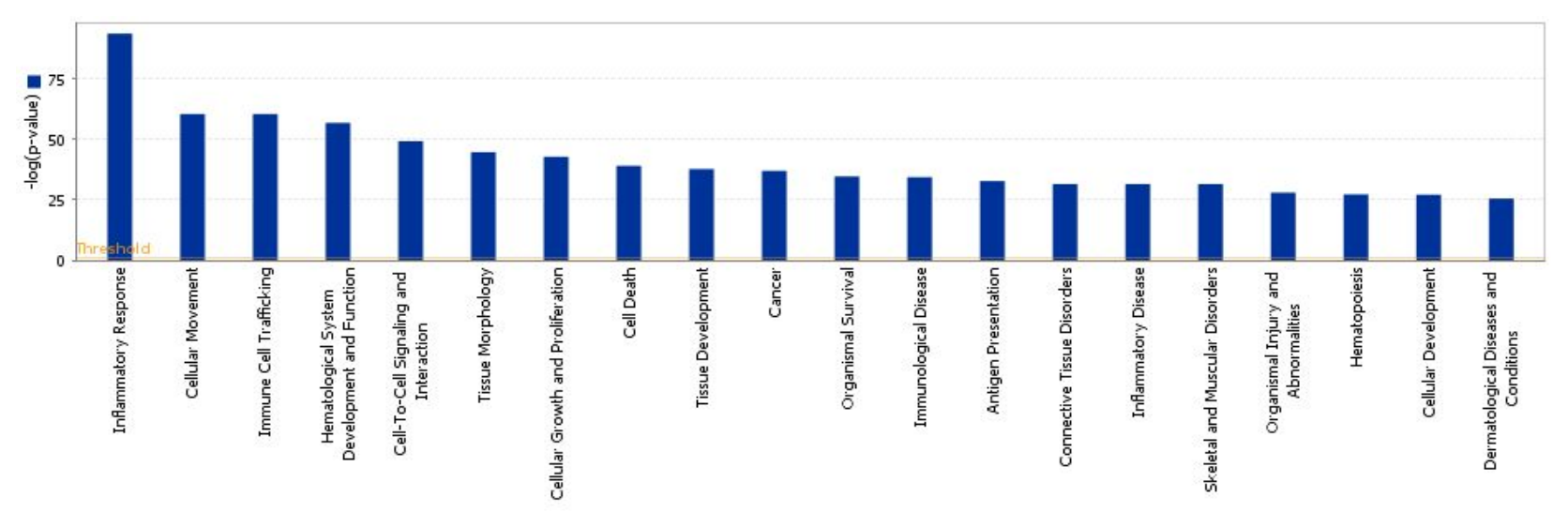

B

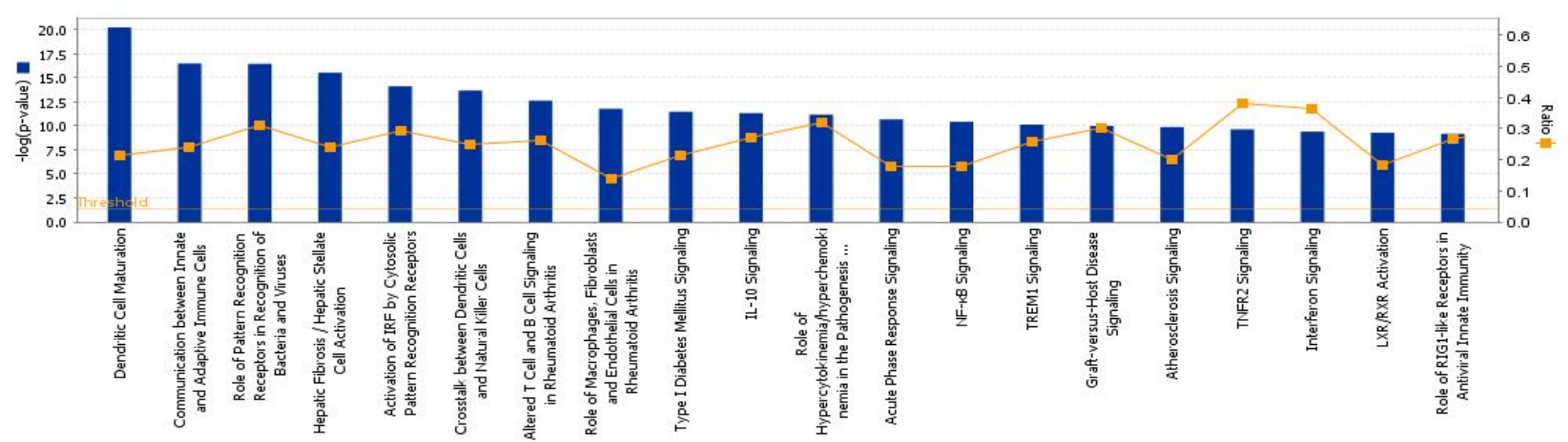

Figure 5.43 Top 20 (A) biological functions and (B) canonical pathways significantly enriched in differentially expressed genes from H5N3-infected macrophages at 24 hpi (P-value $\leq \mathbf{0 . 0 5})$. Threshold was set at $\mathrm{p}$-value $=0.05$ and indicated as $-\log$ ( $\mathrm{p}$-value) on the $\mathrm{Y}$-axis and the $\mathrm{X}$-axis shows the terms of each biological function or canonical pathway. The orange boxes indicate the ratio (Ratio) of the number of genes with differential expression changes and the total number of genes in the respective canonical pathway. 


\subsubsection{Regulations of gene expression in canonical pathways}

\subsection{Interferon signaling}

In macrophages following infections of influenza $A$ viruses, the activation of IFN signaling pathway was observed. Among three influenza A strains, H5N3 infection globally initiated the significant up-regulation of gene expression levels in IFN pathway (particularly type I) at 24 hpi, followed by H1N1 infection. These up-regulated genes included JAK2, STAT1/2, OAS1, IFIT3, IRF9 and so on. H5N2/F118 infection also caused expression induction of several genes in IFN pathway at $2 \mathrm{hpi}$, but few expression changes were identified at 24 hpi. And we assumed that some mechanism might potentially play a role to suppress the activation of IFN signaling pathway at the later stage of H5N2/F118 infection in macrophages.

\subsection{NF-кB signaling}

In H5N3-infected macrophages, many genes involved in NF- $\mathrm{B}$ signaling pathway showed up-regulated expression. A20, TNF $\alpha$ and CD40 also showed strong induction at their expression level, which might be responsible for the activation and inhibition of NF- $\kappa \mathrm{B}$ signaling pathway. It was surprising that genes in NF- $\mathrm{BB}$ signaling pathway almost showed no expression changes in both H5N2/F118 and H1N1-infected macrophages.

\subsection{Toll-like receptor signaling}

$\mathrm{H} 5 \mathrm{~N} 3$ infection in macrophages resulted in the similar gene expression pattern between at 2 and 24hpi, with expression levels of genes including TLR2, CD14, MYD88, NF- $\kappa$ B and p38 MAPK all showing elevated. Gene expression performance of TLR signaling pathway in after infections of H1N1 and H5N2/F118 showed different when compared to the performance after infection of H5N3. H5N2/F118 infection caused faint down-regulation of TLR1 and p38 MAPK in their expression at $2 \mathrm{hpi}$, with no expression change detected at 24 hpi. Whereas, H1N1 infection resulted in faint down-regulation expression of TLR1, TLR4 and p38 MAPK at 2 hpi, with exception of TLR2 and PKR showing faint up-regulation expression at $24 \mathrm{hpi}$. 


\subsection{Antigen presentation pathway}

The situation was a bit complicated in H5N3 infected macrophages. Although some factors in MHC I- $\alpha$ group showed up-regulated expression but some showed down-regulated expression, and the up-regulated expression of downstream genes made the hypothesis convincible that MHC I antigen presentation was activated. Furthermore, MHC II- $\alpha$ antigen presentation pathway also displayed in an activated state, suggesting high activation of macrophages after H5N3 infection at 24 hpi.

The status of antigen presentation pathway in H1N1 infected macrophages was similar to in H5N2/F118 infected macrophages. MHC I- $\alpha$ was detected with repressed expression during H5N2/F118 infection and both MHC I- $\alpha$ and MHC II- $\beta$ were identified with repressed expression during H1N1 infection. And when it comes to $24 \mathrm{hpi}$, genes in both antigen presentation pathways showed no expression changes.

\subsection{Conclusion}

Although the associated fatalities due to human transmission of LPAI viruses have not been reported, the LPAI viruses have the potentiality to develop into HPAI viruses, transmission of which are often associated with high fatality rates. In this project, the host response to the low LAPI H5N2, H9N2, H5N3 and H7N1 were investigated in A549, CEF and MDCK cells based on microarray platform. And host response to another human influenza strains H1N1 and pH1N1 were also involved in corresponding cell types for parallel comparison. Besides, the host response to low LAPI H5N2 and H5N3 as well as human strain H1N1 were also examined and compared in mouse macrophages.

Global evaluation of probe sets with differential expression suggest that host genes performed differently even if the different cell types were infected with the same influenza A strain. After infection of H1N1, half of the probe sets in A549 and MDCK cells showed down-regulated expression while only a small portion of probe sets showed down-regulated expression in CEF cells. In host cells infected with H9N2, more probe sets with down-regulated expression and less probe sets with up-regulated expression were observed in CEF cells compared to A549 and MDCK cells. In addition, the global gene expression 
profiles detected in H5N2/F118 infected CEF and MDCK cells were far away from the one detected in H5N2/F118 infected A549 cells. And compared to situations happed in CEF and MDCK cells, smaller fraction of probe sets with decreased expression and larger fraction of probe sets with increased expression were identified after H5N2/F118 infection in A549 cells.

Further investigation at $10 \mathrm{hpi}$ also indicated the global gene expression profiles were also different upon infections of different influenza A strains in specific cell types. Among these different strains, infections of LPAI H5N3, H5N2/F59, H5N2/F118 as well as H9N2 and infection of human influenza A strain $\mathrm{pH} 1 \mathrm{~N} 1$ caused the largest numbers of probe sets with up-regulated expression at 10 hpi in A549 cells. Whereas, infections of two human influenza A strains, especially H1N1, and two LPAI H5N2/F59 and H5N2/F189 led to the largest numbers of probe sets with down-regulated expression at $10 \mathrm{hpi}$. In CEF cells, infection of human strain H1N1 resulted in the highest number of probe sets with up-regulated expression but lowest number of those with downregulated expression at $10 \mathrm{hpi}$. The distribution of differentially expressed genes showed different in CEF cells after infections of different LAPI viruses, even three H5N2 strains. In MDCK cells, infection of human strain H1N1 led to not only the largest number of probe sets with down-regulated expression but also relative big number of probe sets with up-regulated expression at $10 \mathrm{hpi}$. Results from macrophages suggested that $\mathrm{H} 5 \mathrm{~N} 3$ infection contributed to larger number of differentially expression probe sets, either up- or down-regulated, when compared to H1N1 and H5N2/F118 infections at 24 hpi.

By assigning these probe sets encoded genes to certain biological processes or pathways, it was noted that functional classifications such as "Immune Response", "DNA Binding", "RNA Binding" and "Signal Transduction" were over-represented based on up-regulated probe sets in A549 cells infected with LPAI virus, especially H5N2/F118 and H9N2. And those probe sets classified into "Immune response" showed high fold changes at their expression level. Down-regulated probe sets were majorly grouped into functions such as "DNA Binding", "RNA Binding", "Transcription factor" and "Signal Transduction", particularly in H1N1 infected A549 cells. In macrophages, H5N3 infection induced expression of relative high number of probe sets related to "Signal Transduction" and "Antiviral" at 24 hpi, while 
H1N1 infection repressed expression of considerable big number of probe sets associated with "Signal Transduction", "Cell Cycle", "Cell Death" and "Kinase" at 2 hpi. Since the annotation of probe sets in Chicken Genome and Canine Genome 2.0 Array is not complete, only part of these probe sets were classified into listed groups.

Detailed investigation also displayed that common functional groups such as response to virus and nucleic acid binding were enriched in genes with up-regulated expression following infections of two avian strains (H5N2/F118 and H9N2) and one human strain pH1N1/478. Moreover, the avian viruses had more functional groups in common with each other and these genes were involved in immune response, cytokine receptor binding, transition metal ion binding and cell death. In contrast to the differentially up-regulated genes, common functional gene groupings (e.g. genes involved in nitrogen compound metabolic process, regulation of macromolecule metabolic process, nucleic acid binding) were observed based on down-regulatd genes between the human and avian virus infections, with a greater number of genes involved in the former infections. Specific to the 2 human viruses, the genes involved in the protein metabolic-related processes, zinc ion binding and ATP binding showed a decreasing temporal trend in the expression profiles, especially after H1N1 infection. In CEF cells, genes with down-regulated expression were also enriched in pathways with various cellular functions after infections of three influenza A strains, especially H5N2/F118. In macrophages, similar functional groups that are related to inflammatory reponse were detected following infection of all three influenza A strains. However, larger number of molecules belonged to these functions following H5N3 infection at 24 hpi, indicating the stronger antiviral actions at the late infection stage.

Besides these well-known functional groups, other pathways such as "Cytokine-Cytokine receptor interaction" and "RIG-I like receptor signaling pathway" were commonly enriched in genes with up-regulated expression from both two avian strains (H5N2/F118 and H9N2) and two human strains (H1N1 and pH1N1) infected A549 cells, while other pathways such as "Jak-STAT signaling" and "Toll-like receptor signaling pathway" were found only be enriched in genes with up-regulated expression from the two avian strains infected A549 cells. These canonical pathways play important role in innate 
immune response, over-representation of them in genes with up-regulated expression indicated that the antiviral response were efficiently triggered in infected host cells, especially after avian strains infections. Further analysis revealed that pathways involving different cell activities such as "Cell cycle", "Metabolic" and "Endocytosis" were enriched in genes with down-regulated expression in human strains infected A549 cells, suggesting the inhibition of normal cell regulations in various aspects.

Furthermore, a couple of potential transcriptional factors were also significantly enriched based on differentially expressed genes in infected A549 cells. For example, transcription factors such as IRF-1, IRF-3 and IRF-7 were found to be enriched in probe sets with up-regulated expression from A549 cells infected with two avian strains H5N2/F118 and H9N2 and human strain pH1N1; transcription factors such as E2F, ETF and Sp1 were identified to be enriched in probe sets with down-regulated expression from A549 cells that were majorly infected with two human strains and avian strain H5N2/F118.

Pathways analysis revealed that the signaling transduction in immune response was initiated in A549 cells infected with several avian strains such as H5N2/F118 and H9N2. Whereas, the signaling transduction in immune response pathways was impaired to some extend in A549 cells infected with two human strains, in particular H1N1. As to the gene expression performances in infected A549 cells, the key genes participating in cell cycle checkpoint regulation were found to be inhibited in their expression particularly following infections of human strains, which was consistent with previous findings. All together, these mechanisms such as the impairment of the inflammatory response and arrest of cell cycle in infected host A549 cells after infections of two human strains might be a solution to benefit the viral replication.

Recently, host responses of different influenza A strains in different cell lines were also investigated by other groups. Gerlach RL et al (2013) did microarray studies on well-differentiated normal human bronchial epithelial cells upon infection of a H1N1 seasonal isolate (A/BN/59/07) and two H1N1 pandemic isolates: fatal one $(\mathrm{A} / \mathrm{KY} / 180 / 10)$ and nonfatal one $(\mathrm{A} / \mathrm{KY} / 136 / 09)$ [383]. Analysis on the data indicated that cells infected with $\mathrm{A} / \mathrm{KY} / 180 / 10$ showed a greater difference in gene expression levels when compared to another two isolates. Besides, many genes from the early innate immune 
response pathways showed common stimulated expression to infections of these three isolates. However, infections of $\mathrm{pH} 1 \mathrm{~N} 1 / 478$ and $\mathrm{H} 1 \mathrm{~N} 1 / \mathrm{WS}$ resulted in lower expression level of ISGs and larger number of genes with down-regulated expression when compared to observations from this study. In another report, the virus-host interactions at 24hpi in human bronchial airway epithelial cells infected with H1N1 (A/PR/8/34) virus were defined through quantifying host protein alteration. Their findings demonstrated that proteins functionally related to protein metabolism, purine biosynthesis, cytoskeleton and carbohydrate showed elevated expression [390]. Comparably, although genes associated with these functions also showing up-regulated expression were observed in A549 cells after infection of two human strains, the numbers are quite limited. These differences might be partly caused by the different host cells and the postinfection stage. 


\section{Chapter VI. Poxviruses}

\subsection{Introduction}

The Poxviridae, as a large family of viruses, infect a wide range of vertebrates and invertebrate animals. Poxviruses are of serious concern due to their high pathogenicity and wide distribution. The best studied poxviruses include variola virus, vaccinia virus, cowpox virus and monkeypox virus. These poxviruses all belong to Orthopoxvirus genus and become considerable concern for public health and biodefense [307, 308]. Global eradication of smallpox was achieved decades ago initiated by the WHO, and the eradication used vaccinia virus as a live vaccine [309].

Poxviruses are different from other animal viruses in several respects. First, many enzymes encoded by poxviruses are necessary for either macromolecular precursor pool regulation or biosynthetic processes. Second, the complex morphogenesis of poxviruses is involved in the composition of virus-specific membranes and inclusion bodies. Lastly, the genomes of poxviruses are able to encode many proteins that interact with host factors at cellular or systemic levels.

\subsubsection{Virus structure}

Poxviruses constitute a large family of enveloped DNA viruses, with large size genomes from 150 to $300 \mathrm{kbp}$ that encode 200 or more open reading frames. The functions of these encoding proteins include synthesis of viral RNA/DNA, assembly of virion and modulation of host immune defenses [310].

There are two unique forms of infectious particles: intracellular mature virions (IMV) and extracellular enveloped virions (EEV). Compared to IMVs, EEVs have one additional membrane. Specifically, IMVs consist of the viral core containing the dsDNA genome encased in a proteinaceous core with around 60 viral proteins, and one lipid bilayer containing around 25 viral proteins while EVs consist of an MV-like particle surrounded by a second viral membrane containing cellular and at least six unique viral proteins. From the functional perspective, IMVs are the more abundantly produced infectious form and are thought to mediate host-to-host transmission, while EEVs disseminate virus in the infected host and protect against immune defenses [311]. 


\subsubsection{Virus replication cycle}

\subsubsection{Virus Entry}

Entry of enveloped viruses can be divided into three steps: virus attachment, fusion activation, and membrane fusion. Initial study on entry of vaccinia virus has depicted that IMV entry into host cells by phagocytosis first and then IMV fuse with the plasma membrane. Carter GC et al (2005) have also discovered that the effect of soluble GAGs on IMV infectivity is cell typespecific [312]. In addition, reinvestigation by electron and confocal microscopy has demonstrated that IMV enter into cells by fusion of its single membrane with the plasma membrane. And this fused membrane is then flattened into the plane of the cell surface, with a naked core released into the cytoplasm. As to EEVs, disruption of the EEV wrapper within endosomes has been suggested and after that, IMVs membrane is exposed. And then IMVs' entry follows the same steps as described above [310]. In this process, studies revealed that two viral proteins $\mathrm{A} 28$ and $\mathrm{H} 2$ were each required for cell entry and cell-cell fusion [313].

\subsubsection{DNA release from the core to cytoplasm}

Following attachment to cell surfaces and fusion with the plasma membrane, DNA-containing core is delivered into the cytoplasm. After penetration, cores can bind to the microtubules (MTs) and then reach the site of disassembly and DNA-release with the assistance of MTs. Under these apparent core movements, early proteins synthesis that is necessary for core uncoating is moving forward. Poxvirus cores enable to harbor the viral DNA-dependent RNA polymerase and transcription factors necessary for gene expression at early stage. Previous researches have proposed that core must contain pores that allow the release of the early mRNAs into the cytoplasm but avoid the entry of DNAse into the core. After these early mRNAs release from the core, they travel along MTs to the location where they become anchored into translation competent complexes. In this process, L4R as a core-associated protein may help mediate the binding and translation of early mRNAs to or along MTs. After synthesis of at least 100 early proteins, the parental DNA is finally released into cytoplasm from the core. During this process, some other viral factors excluding early proteins are also associated with the DNA release. 
These factors include B1R kinase, I3L, H5R and E8R. These key factors including early proteins may be required to uncoat the core so as to mediate DNA release, and they also serve to anchor-release DNA to the ER in order to protect it against degradation before DNA replication in next step.

\subsubsection{DNA replication}

Released DNA is tightly associated with rER by a complex of early proteins and the DNA replication occurs in ER-enwrapped cytoplasmic sites. Upon the initiation of replication, ER cisternae are recruited to and enclose the replication sites in order to create a cytosolic subcompartment that facilitates viral DNA synthesis. These new synthesized mRNAs move away from their site of synthesis for future efficient assembly.

Along with DNA replication, intermediate and late genes are transcribed. The expression of intermediate genes that encode specific transcription factors of late gene is triggered by the intermediate transcription factors. The late genes primarily include structural proteins required for progeny virion assembly and enzymes needed for early gene expression during the next round of infection. Two factors, E8R and A40R, are indispensable in this process.

\subsubsection{Virus assembly}

Virus assembly is generally initiated from 5 hpi. Crescent-shaped membrane appears on the sites of DNA replication, accompanying with the breakdown of the rER envelop. More than 80 viral genes are involved in the assembly of MV. After integral viral membrane proteins made in the ER, they are transported to viral factories along with ER derived lipid to be assembled into crescents which contain a lipid bilayer and the membrane proteins, scaffolded on a honeycomb structure composed of the D13 protein. Crescent formation is controlled by phosphorylation. The crescents become into immature virions (IVs) accompanied by encapsidation of the genome. Metamorphosis to IMVs is accompanied by loss of the D13 scaffold, proteolysis, as well as further addition of membrane proteins and movement of particles outside of factories. IMV acquire Golgi derived membranes to become wrapped virions (WVs), which is a intermediate state between IMVs and EEVs. In the last stage, WVs are exocytosed through the plasma membrane to become 
EEVs [314]. In addition to the viral D13 scaffold protein, a series of known viral proteins are also necessary for the poxvirus assembly process. For instance, several membrane (A14 and A17) and nonmembrane (A11, F10, G5, and H5) viral proteins are required for crescent formation; A9 protein is necessary at a later stage of morphogenesis [308].

\subsubsection{Viral-Host interactions}

Host cells will initiate a series of innate and adaptive immune response pathways in react to the invasion of poxvirus. The details include detection of the pathogen, induction of the cytokine responses, establishment of the antiviral state and so on. In the meanwhile, poxviruses, as a large type of dsDNA evolves extremely sophisticated mechanisms for evading the immune system [11].

\subsubsection{Blockade of interferon response}

Poxvirus evolves several strategies to counteract the interferon response: (1) Montanuy I et al (2011) has proposed that GAGs mediated retention of the poxvirus type I interferon binding protein so as to locally block interferon antiviral responses [316]. In their experiment, they treated variola virus and monkeypox virus as experimental targets and discovered the interaction between soluble viral IFN type I receptor potential and GAGs. However, there is a big structural variation existing within GAGs due to their composition, linkage, or modification. In their case, site-directed mutagenesis of poxvirus basic residues in the first Ig domain of the protein rendered IFN $\alpha / \beta$ unable to interact with the cell surface; (2) Meng X et al (2012) recently has proved that K1L and C7L antagonized type I IFNs and IRF1-induced antiviral activities, and this function of C7L was observed evolutionally conserved in all poxviruses [317]; (3) Poxvirus-encoded IFN- $\gamma$ binding protein has been reported to be able to dampen the host immune response although they were not required for virus replication in vitro [318]; (4) Experiment targeting to vaccinia virus also indicated that B18R competitively bound to and inhibited a broad range of type I IFNs so that the induction of anti-viral response was blocked [319]; (5) Besides these above descriptions, a series of vaccinia virus-encoding 
proteins was proved to suppress the IFN pathways from different ways [Figure $6.1]$.

\subsubsection{Suppression of cytokine signaling}

During virus infection, many cytokines are produced by the host system to counter the effect of the viral presence. In the meanwhile, different immune evasion mechanisms also were initiated by poxviruses to lower these cytokines' expression levels. To date, a few studies coming from different research groups have already provided evidences for interactions between cytokines and poxviruses. For instance, Smith VP et al (2000) have discovered that poxviruses secreted and expressed inhibitors of cytokines such as soluble IL18-binding protein that was expressed in all lister virus, ectromelia and cowpox virus infected KG-1 cells [320]; Another group has reported that ectromelia virus encoded a homologue of CD30, which was capable to block the binding of $\mathrm{CD} 30 \mathrm{~L}$ to its receptor and induce reverse signaling in cells expressing CD30L.

Moreover, the viral $\mathrm{CD} 30$ has been observed to abrogate $\mathrm{T}$ cell proliferation, therefore it blocked type 1 cytokine-mediated $\mathrm{T}$ cell responses [322]; An ectromelia virus protein called E163 has been also identified to interact with chemokines so as to influence the chemokines activities. Interaction of certain chemokines with GAGs is crucial for the correct function of the chemokines network. However, E163 protein has also been proved to have high affinity to interact with GAGs binding domain of those chemokines, thus E163 indirectly blocked the chemokine-GAGs interaction and further influenced the chemokines mediated immune response [323, 324]. 


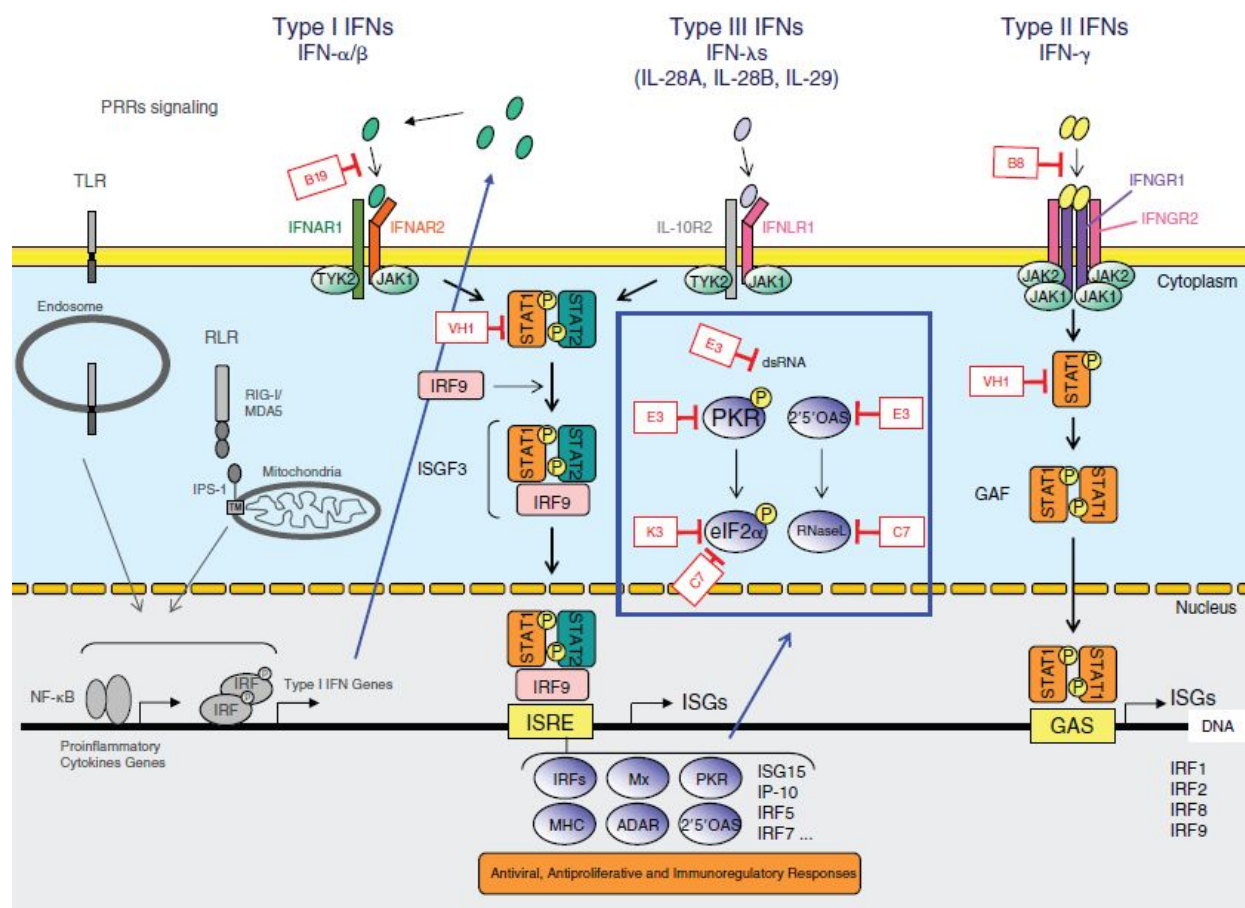

Figure 6.1 Interplay between IFN-signaling pathways and vaccinia virus proteins (adapted from Perdiguero B et al., 2009) [321]. Vaccinia virus encodes IFN receptors B8 and B19 which block the binding of IFNs to their cell surface receptors. The E3L gene encodes a protein that is capable to inhibit PKR activation and block IFN responses. The E3 blocks the IFN-induced 2'5'-OAS antiviral pathway. An eIF2 $\alpha$ homolog encoded by K3L t interferes with PKR. Through dephosphorylation of STAT1, the VH1 phosphatase also intercepts the IFN signaling pathway.

\subsubsection{Inhibition of TNF-induced responses}

It has been well known that poxviruses encode a variety of proteins that interfere the activation of NF- $\mathrm{KB}$ in order to further impair the TNF-induced responses. In one strategy, many poxviruses express soluble viral receptors or cytokine binding proteins which can intercept cellular ligand-receptor interactions, thus blocking the signaling that lead to NF- $\kappa \mathrm{B}$ activation. Relative proteins include $\mathrm{T} 2$ that is expressed as a secreted glycoprotein for binding and inhibiting TNF detected in myxoma virus infected rabbit host, cytokine response modifier B, C, D and E which act as soluble vTNFRs to intercept TNF ligand-receptor interaction in cowpox virus with other orthologs also found in other poxviruses, CD30 that acts as a receptor of CD153, and so on [325]. Another strategy adopted is to express intracellular factors to regulate signaling pathways leading to NF- $\kappa \mathrm{B}$ activation. For instance, vaccinia protein A46R has been reported to target the host TIR adaptors myeloid differentiation factor (MyD88) and TRIF, thereby interfered with downstream activation of NF- $\kappa \mathrm{B}$, MAP kinase and IFN- $\beta$ [326]. Moreover, another Ankyrin repeat (ANK) NF- $\kappa B$ 
inhibitor also has been discovered to be encoded by cowpox virus. Detailed

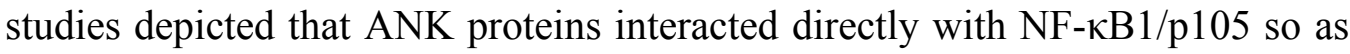
to inhibit NF- $\mathrm{B}$ signaling pathway [327]. And perhaps most surprising of all, MC159 protein from molluscum contagiosum poxvirus just has been proved to have an ability to interact with the IкB kinase complex in order to inhibit NF$\kappa \mathrm{B}$ activation [328].

\subsubsection{T-cell evasion by repression of MHC I expression}

CD8+ T-cell-mediated responses function on the control of infection of intracellular pathogens. T-cells will become activated once their T-cell receptor recognizes an antigen-derived peptide presented by MHC I. Thus, in order to avoid presentation of viral peptides and kill the infected cells via CD8+ T-cell many viruses interfere with the MHC I presentation pathway in different ways such as MHC I expression, proteasomal protein degradation, TAP-mediated peptides transportation, MHC I peptide complex assembly or trafficking [329].

Results obtained from Byun M et al (2009) indicated that cowpox virus inhibited expression of MHC class I by dissociation of MHC class I from TAP [330]. Another report pointed out cowpox virus interfered with CD8+ responses in another way. In the experiment, the cowpox virus was found to inhibit the intracellular transport of MHC I at the early infection stage. This mechanism was able to completely inhibit MHC I exit from the endoplasmic reticulum, independent of viral replication [331].

\subsubsection{Blockade of host cell apoptosis}

Induction of apoptosis is essential for elimination of pathogen infection. In reaction to this pressure initiated from host system, viruses such as poxviruses have evolved some mechanisms by which the cell apoptosis is blocked, and thereby the successful replication and dissemination are guaranteed.

Caspases activation and apoptotic death can be triggered by both extrinsic and intrinsic signals [332]. Stewart TL et al (2005) reported a finding that vaccinia virus encoding F1L, a new member of the tail-anchored protein family, localized to mitochondria during virus infection, leading to inhibit cell apoptosis and enhance virus survival [333]. In another experiment, a poxvirus- 
encoded protein called M13L-PYD was identified. This protein was proved to colocalize and interact with a cellular PYD protein, ASC-1, as a mean to modulate caspase- 1 activity and process IL-1 $\beta$ and IL-18, in further inhibit host inflammatory and apoptotic responses to infection [334]. Other interesting finding demonstrated the presence of a TNFR-like T2 protein in all poxviruses, and this $\mathrm{T} 2$ protein physically associated with and colocalized with human TNFRs so that inhibited cellular TNFR1-induced cell death [335].

\subsubsection{Poxviruses infections in different host cells}

Several reports have been published to uncover the viral strategy and host response in different cells following infections of different types of poxviruses. For example, Turner PC et al (2002) summarized that different immune modulators expressed by poxviruses function on suppressing the host response to infections [336]. Besides, they described that multiple modulators were able to target the same pathways at different steps. A high-density microarray was applied to analyze the host response in NYVAC infected Hela cells. The result suggested that expression of apoptotic genes and NF- $\mathrm{BB}$ responsive genes were stimulated. At the meanwhile, vaccinia K1L gene played a role to inhibit the $\mathrm{NF}-\kappa \mathrm{B}$ activation [337]. It was also discovered that ectromelia virus encoded a protein homologous to the ectodomain of the IFN- $\gamma$ receptor 1 , and this protein enabled to bind IFN- $\gamma$ and subsequently dampened the host immune response to virus infection in host cells [318].

\subsubsection{Objective}

As the largest known DNA viruses, members of the Poxviridae family infect both vertebrate and invertebrate animals. Orthopoxvirus, as a genus of Poxviridae, includes many virus species such as variola, monkeypox, vaccinia and cowpox viruses. Infection of these viruses results in febrile illnesses associated with vesicular rash in humans and animals. The most notorious member is variola virus that caused the outbreak of the disease called smallpox with about 500 million deaths during the 1900s. And until 1980, WHO announced that the world was finally free of it [338]. Till now, although studies on the complex interactions between host cells and poxviruses are among well understood, the functions of critical genes which contribute to poxvirus biology still remains to be enriched. 
Since we can't use the actual smallpox virus, we focused our researches on another three types of poxviruses. Cowpox virus is the closest relative to smallpox virus and is a good surrogate virus [281]. It got its name from the distribution of the disease when dairymaids touched the udders of infected cows. Cowpox is similar to but much milder than the highly contagious and often deadly smallpox disease. It resembles mild smallpox, and was the basis of the first smallpox vaccines. When the patient recovers from cowpox, the person is immune to smallpox. Lister virus is one subtype of vaccinia virus, and vaccinia virus is the vaccine for smallpox virus and as the vaccine gives side effects. Thus it is worthy while investigating the host response. Ectromelia virus is able to cause fatal mousepox. It is the only poxvirus to cause disease naturally in mice. In our experiment, mousepox is used to compare with other poxviruses as RAW cells originated from mice and mousepox is a good control to study whether RAW cells can be used as a study model [282].

In our study, three types of poxviruses were infected with A549 and mouse RAW cells at specific time points. The corresponding transciptomic profiles were examined using microarray platform. Different types of software were employed into further analysis. The aims of this research are as following:

(1). Investigate the viral-host interactions in poxviruses infected A549 cells.

(2). Investigate the viral-host interactions in poxviruses infected mouse RAW cells.

(3). Compare the different gene expression performances after infections of different types of poxviruses in different host cells.

\subsection{Experiment workflow}




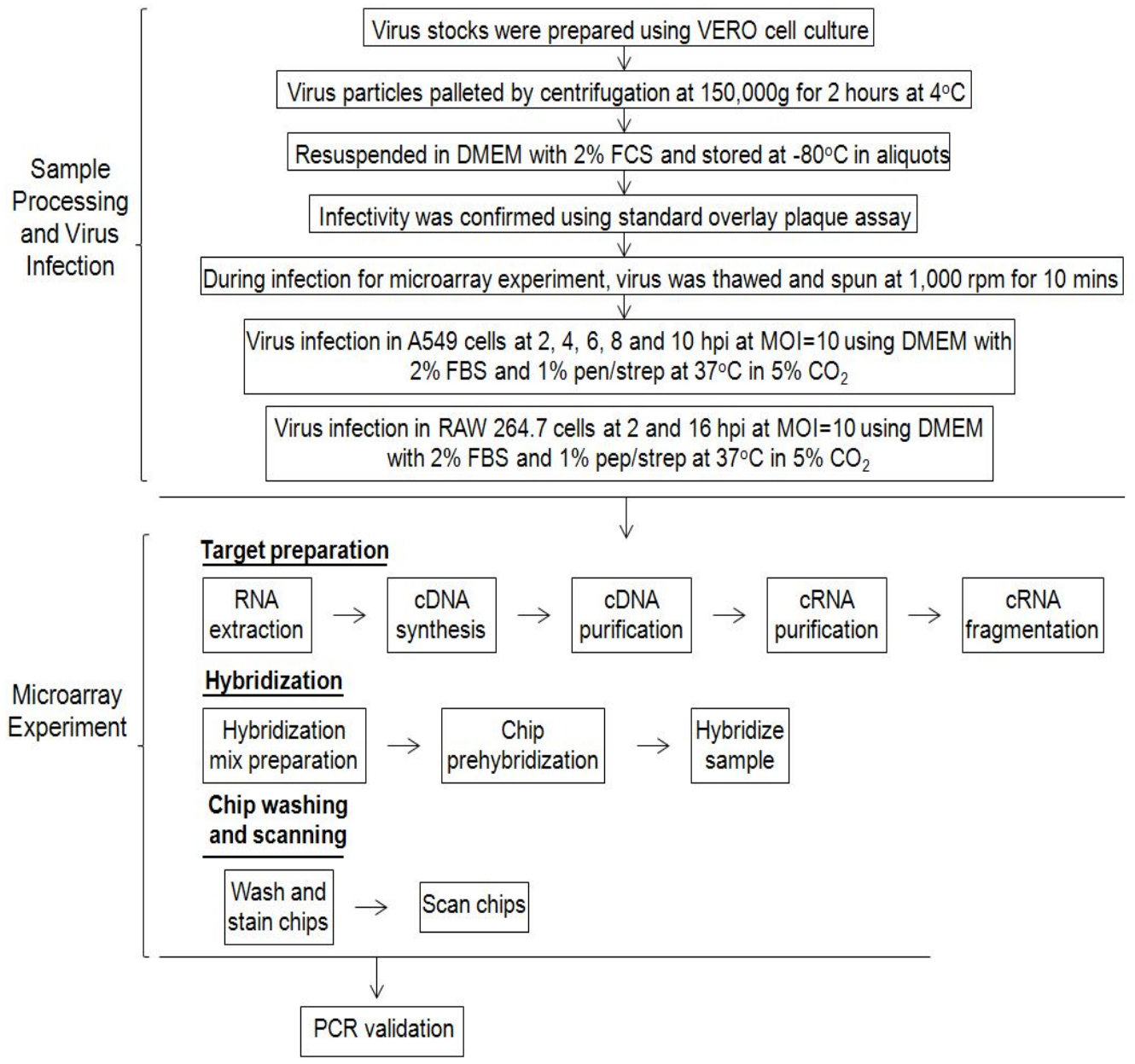

Figure 6.2 Microarray experimental workflow during different types of poxviruses infections.

\subsection{Result and Discussion}

\subsubsection{Global Profiling of gene expression}

\subsubsection{Heat maps of global gene expression}

The global transcriptional profiles in two types of poxviruses infected A549 cells illustrated that most of the differentially expressed probe sets showed down-regulated expression changes. Compared to lister virus, infection of cowpox virus led to less probe sets with up-regulated expression but more probe sets with down-regulated expression.

The numbers of probe sets with up-regulated expression showed in an increasing trend with the infection time increasing in two types of poxviruses infected A549 cells. However, the situation for probe sets with down-regulated expression was complicated. In cowpox virus-infected A549 cells, considerable numbers of probe sets with down-regulated expression was observed from 4 hpi, with the largest number detected around 8 hpi. At the meanwhile, a sharp 
increase of numbers of probe sets with down-regulated expression was observed from 4 hpi to $6 \mathrm{hpi}$, with the largest numbers detected at $6 \mathrm{hpi}$ [Figure $6.3]$.

Based on the global transcriptional profiles, the numbers of differentially expressed probes sets were increased with the infection time increasing in poxviruses infected mouse RAW cells [Figure 6.4]. Among these differentially expressed probe sets, most of the probe sets showed downregulated changes after infections of three types of poxviruses in infected mouse RAW cells, which was similar to the observation in infected A549 cells. Detailed investigation illustrated that infection of ectromelia virus was responsible for the largest number of probe sets with elevated expression while infection of lister virus was responsible the largest number of probe sets with inhibited expression at 16 hpi.

\subsubsection{Distribution of differentially expressed probe sets}

In cowpox virus-infected A549 cells, $0.44 \%$ percentage of probe sets showed up-regulated at their expression level at 2 hpi. However, this number was suddenly reduced to $0.31 \%$ at 4 hpi. After $4 \mathrm{hpi}$, the number of upregulated probe sets became increased again and reached the highest level at 10 hpi.

Regarding the probe sets with down-regulated expression, the sudden increase of the percentage was observed from 2 hpi to 4 hpi, indicating that some repression regulations might be strongly stimulated in this interphase. The highest percentage of probe sets with down-regulated expression was detected at $8 \mathrm{hpi}$, and the relative high percentage was still able to be detected even if the cut-off was raised to $10-\mathrm{FC}$.

In lister virus-infected A549 cells, the percentage of probe sets with upregulated expression showed high at the beginning of infection ( 2 hpi), however, this percentage dropped at $4 \mathrm{hpi}$. Another round of expression stimulation of a big batch of probe sets was detected from $8 \mathrm{hpi}$. This trend of percentages of probe sets with up-regulated expression observed after infection of lister virus was concordant with the trend observed after infection of cowpox virus, but the detailed percentages from lister virus infection were much higher than those from cowpox virus infection across the whole infection period. 
Further observations demonstrated that the highest percentage of probe sets with down-regulated expression was detected at 6 hpi after infection of lister virus, with the increasing of the percentage occurred majorly between 4 hpi and 6 hpi in infected A549 cells. As compared to cowpox virus, the percentages of probe sets with down-regulated expression were lower across the whole infection course after infection of lister virus than cowpox virus no matter based on any fold change cutoff [Table 6.1].

Global investigation on expression alterations of probe sets in mouse RAW cells suggested that infections of cowpox virus and ectromelia virus induced highest percentages of probe sets with up-regulated expression at $2 \mathrm{hpi}$ and 16 hpi separately. And when the fold regulation cutoff was raised to 10-FC, only a small batch of probe sets with up-regulated expression could be detected at $16 \mathrm{hpi}$ after the infection of ectromelia virus.

In contrast, infection of lister virus caused highest percentages of probe sets with down-regulated expression at both time points. Few probe sets were detected with down-regulated expression $\geq 10$-FC at 2 hpi after infections of all these three types of poxviruses, while considerable numbers of those with down-regulated expression $\geq 10$-FC were identified at 16 hpi after infection of lister virus, followed by ectromelia virus [Table 6.2].

\subsubsection{Functional classification}

In cowpox virus-infected A549 cells, the probe sets with up-regulated expression were majorly enriched in "RNA Binding", "DNA Binding" and "Transcription Factor" across the whole infection period [Figure 6.5]. In addition, functional terms including "Immune response", "Cytoskeleton", "Signal Transduction" and "Cell Growth" were also over-represented. Compared to other time points, the numbers of up-regulated probe sets involved in these functional groups were higher at 10 hpi than other time points.

Functional groups significantly over-represented by the probe sets with down-regulated expression were "RNA Binding”, followed by "DNA Binding", "Signaling Transduction" and "Transcription Factor" in cowpox virus-infected A549 cells. Among the five time points, down-regulated probe sets related to these functions showed highest fold regulation at $8 \mathrm{hpi}$, followed by those at 10 hpi. 
When the cutoff was set at 2-FC or 5-FC, majority of probe sets with up-regulated expression in lister virus infected-A549 cells located in functional groups such as "RNA Binding", "DNA Binding" and "Transcription Factor". And the numbers of up-regulated probe sets located in these groups reached highest at 10 hpi [Figure 6.6]. However, similar numbers of up-regulated probe sets were functional associated with "RNA Binding" and "DNA Binding" at 4, 6,8 and 10 hpi when the cutoff setting was raised to $10-\mathrm{FC}$, indicating that the related genes with remarkable expression elevation was initiated at 4 hpi and sustained up to $10 \mathrm{hpi}$. Another interesting finding was that the larger number of probe sets relevant to "Cytoskeleton" were detected at 4 hpi compared to other time points, which might suggest active lipid membrane activities at this time point.

When it turned to the probe sets with down-regulated expression in lister virus infected-A549 cells, most of them were prominently associated with "RNA Binding" no matter of the fold change cutoff setting. Under detailed investigation, the highest number of down-regulated probe sets related to "RNA Binding" was detected at 4 hpi under 2-fold cutoff while the highest number of down-regulated probe sets related to "RNA Binding" was detected at 8 hpi under 5- or 10-fold cutoff. This observation might suggest that the moderate and extensive suppression of gene expression occurred from 4 hpi but strong and specific suppression action exerted at later infection stage ( 8 hpi).

Similar to the situation happened in poxviruses-infected A549 cells, few probe sets were detected with up-regulated expression at 2 hpi in poxvirusesinfected mouse RAW cells. Among three different types of poxviruses, infection of cowpox induced expression of most probe sets, and this batch of probe sets was majorly functional related to "Protein Metabolism", "DNA Transcription Factor", "Cell Death" and "RNA Binding”. In addition, almost no probe set with up-regulated fold change $\geq 5$-FC was able to be picked out [Figure 6.7].

With the infection time increasing up to $16 \mathrm{hpi}$, infection of ectromelia virus stimulated expression of larger number of probe sets than cowpox and lister virus. Detailed classification of these up-regulated probe sets demonstrated that they majorly belonged to "DNA Transcription Factor" and "RNA Binding" [Figure 6.8]. Moreover, some up-regulated probe sets 
belonging to "Cell Cycle", "Cell Death" and "Kinase" showed high-level fold regulation at $\geq 10$-FC, indicating the actively regulatory activities at cellular level at the late stage of poxviruses infections.

Although lister virus infection almost didn't stimulate expression of any probe sets related to our listed functional groups, its infection resulted in most probe sets with down-regulated expression in infected mouse RAW cells. These down-regulated probe sets were majorly classified into "DNA Transcription Factor", "RNA Binding", "Cell Death" and "Signal Transduction”, followed by "Cell Cycle" and "Kinase". Compared to lister virus, invasion of ectromelia virus contributed to relative less probe sets with down-regulated expression at $\geq$ 2-FC and invasion of cowpox virus led to least probe sets with down-regulated expression in corresponding infected mouse RAW cells.

Consistent with the performances at $2 \mathrm{hpi}$, infection of lister virus also generated most probe sets with down-regulated expression at $16 \mathrm{hpi}$. Although infection of cowpox virus caused down-regulated expression of larger number of probe sets than infection of ectromelia virus based on 2-FC cutoff, more probe sets showed significantly down-regulated expression after infection of extromelia virus but not cowpox virus when the cutoff was raised to 5-FC. "DNA Binding" and other common functional terms were over-represented by down-regulated probe sets in these three types of poxviruses infected mouse RAW cells.

Table 6.1 Differentially expressed probe sets in A549 cells infected with poxviruses at different time points.

\begin{tabular}{|c|c|c|c|c|c|c|c|c|}
\hline \multirow{2}{*}{ A549 } & \multicolumn{2}{|c|}{ Probe sets (>=2 fold) } & \multicolumn{2}{|c|}{ Probe sets $>=3$ fold } & \multicolumn{2}{|c|}{ Probe sets $>=5$ fold } & \multicolumn{2}{|c|}{ Probe sets $(>=10$ folda) } \\
\hline & Up.regulated & Down-regulated & Up.regulated & Down-regulated & Up-regulated & Down-regulatec & Up.regulated & Down.regulatec \\
\hline Cowpox 2 hpi & $0.44 \%$ & $4.68 \%$ & $0.12 \%$ & $0.61 \%$ & $0.02 \%$ & $0.15 \%$ & $0.00 \%$ & $0.03 \%$ \\
\hline Cowpox 4 hpi & $0.31 \%$ & $44.75 \%$ & $0.17 \%$ & $16.39 \%$ & $0.11 \%$ & $2.43 \%$ & $0.05 \%$ & $0.22 \%$ \\
\hline Cowpox 6 hpi & $0.34 \%$ & $62.76 \%$ & $0.20 \%$ & $45.07 \%$ & $0.14 \%$ & $17.92 \%$ & $0.08 \%$ & $2.46 \%$ \\
\hline Cowpox 8hpi & $0.45 \%$ & $65.50 \%$ & $0.25 \%$ & $56.05 \%$ & $0.14 \%$ & $35.85 \%$ & $0.08 \%$ & $11.09 \%$ \\
\hline Cowpox $10 \mathrm{hpi}$ & $1.01 \%$ & $57.02 \%$ & $0.50 \%$ & $43.84 \%$ & $0.22 \%$ & $25.36 \%$ & $0.10 \%$ & $7.98 \%$ \\
\hline Lister 2 hpi & $1.48 \%$ & $1.26 \%$ & $0.29 \%$ & $0.29 \%$ & $0.07 \%$ & $0.09 \%$ & $0.00 \%$ & $0.01 \%$ \\
\hline Lister 4 hpi & $0.61 \%$ & $11.99 \%$ & $0.26 \%$ & $2.29 \%$ & $0.18 \%$ & $0.33 \%$ & $0.11 \%$ & $0.05 \%$ \\
\hline Lister 6 hpi & $0.71 \%$ & $56.06 \%$ & $0.28 \%$ & $34.30 \%$ & $0.19 \%$ & $9.82 \%$ & $0.10 \%$ & $1.26 \%$ \\
\hline Lister 8hpi & $6.16 \%$ & $45.31 \%$ & $2.60 \%$ & $32.02 \%$ & $0.70 \%$ & $15.66 \%$ & $0.21 \%$ & $3.89 \%$ \\
\hline Lister 10 hpi & $10.61 \%$ & $35.39 \%$ & $5.63 \%$ & $23.25 \%$ & $1.71 \%$ & $11.32 \%$ & $0.30 \%$ & $3.07 \%$ \\
\hline
\end{tabular}


Table 6.2 Differentially expressed probe sets in RAW cells infected with poxviruses at different time points.

\begin{tabular}{|c|c|c|c|c|c|c|c|c|}
\hline \multirow{2}{*}{ RAW cells } & \multicolumn{2}{|c|}{ Probe sets $>=2$ foldd } & \multicolumn{2}{|c|}{ Probe sets $>=3$ fold } & \multicolumn{2}{|c|}{ Probe sets $>=5$ folda) } & \multicolumn{2}{|c|}{ Probe setts $>=10$ fold } \\
\hline & \begin{tabular}{|l|} 
Up.regulated \\
\end{tabular} & Down-regulated & Up.regulated & Down-regulated & Up.regulated & Down-regulated & Up.regulated & Down-regulated \\
\hline Cowpox 2 hpi & $0.42 \%$ & $0.75 \%$ & $0.03 \%$ & $0.19 \%$ & $0.00 \%$ & $0.06 \%$ & $0.00 \%$ & $0.01 \%$ \\
\hline Cowpox $16 \mathrm{hpi}$ & $1.55 \%$ & $36.14 \%$ & $0.58 \%$ & $20.18 \%$ & $0.17 \%$ & $5.94 \%$ & $0.05 \%$ & $0.56 \%$ \\
\hline Ectromelia 2 & 0. & 1.8 & $0.02 \%$ & 0 & $0.01 \%$ & $0.05 \%$ & 0.00 & $0.01 \%$ \\
\hline Ectromeli & $6.36^{\circ}$ & $28.37 \%$ & $2.75 \%$ & $19.58 \%$ & $0.71 \%$ & $10.65 \%$ & $0.12 \%$ & $3.33 \%$ \\
\hline Liste & $0.01 \%$ & $2.22 \%$ & $0.00 \%$ & $0.22 \%$ & $0.00 \%$ & $0.01 \%$ & $0.00 \%$ & $0.00 \%$ \\
\hline Lister $16 \mathrm{hpi}$ & $2.09 \%$ & $45.86 \%$ & $0.67 \%$ & $34.09 \%$ & $0.14 \%$ & $19.43 \%$ & $0.03 \%$ & $6.13 \%$ \\
\hline
\end{tabular}

\section{Cowpox}

$2 \mathrm{H} 4 \mathrm{H} 6 \mathrm{H} 8 \mathrm{H} \quad 10 \mathrm{H}$

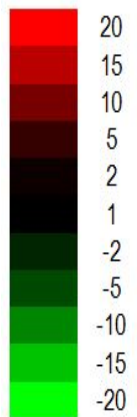

\section{Lister}

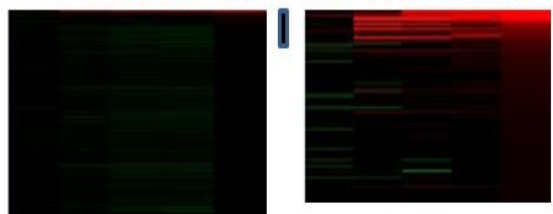

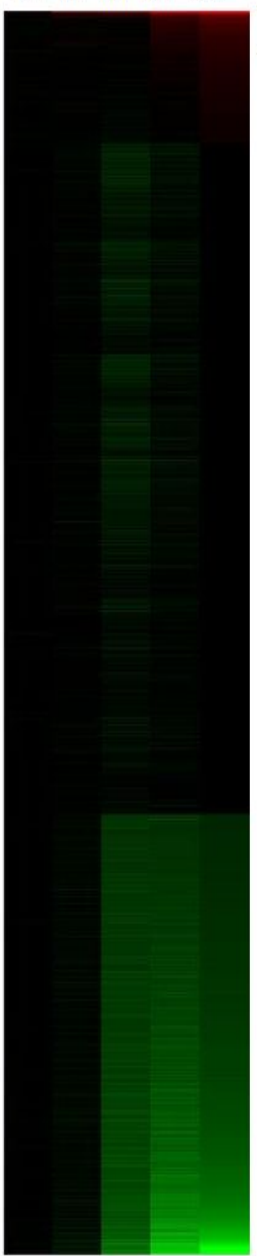

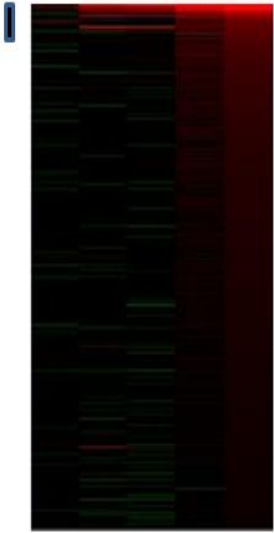

Figure 6.3 Temporal changes in the host cell transcriptome in A549 cells infected by two types of poxviruses. The global host gene expression profiles were retrieved from microarray analysis with different time points examined. The probe sets showing $\geq 2$ fold change (FC) upor down-regulated in expression are indicated (P-value $\leq 0.05)$. Expression profiles of upregulated (red), down-regulated (green) and no significant change (black) are shown. 


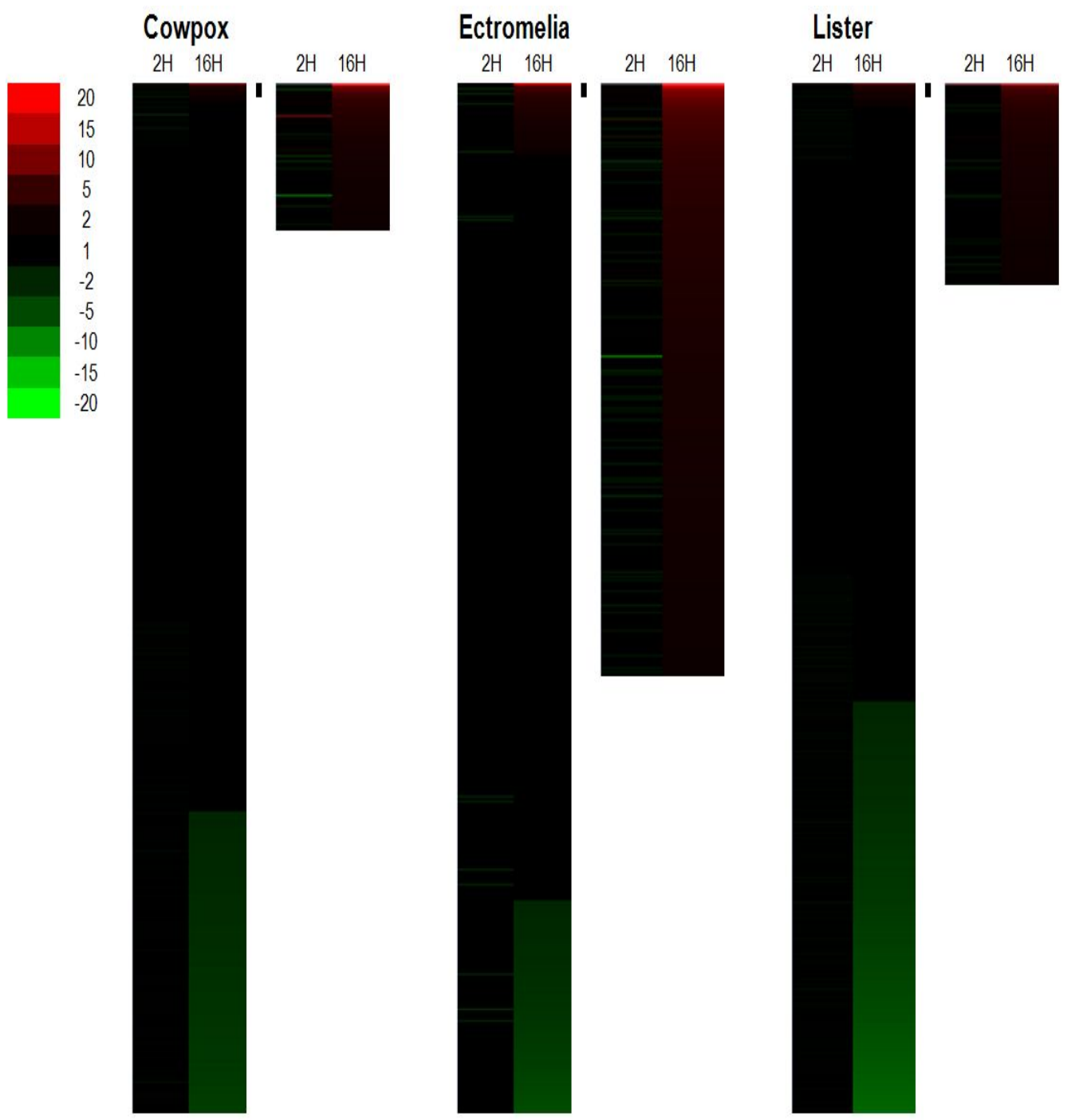

Figure 6.4 Temporal changes in the host cell transcriptome in RAW cells infected by three types of poxviruses. The global host gene expression profiles were retrieved from microarray analysis with different time points examined. The probe sets showing $\geq 2$ fold change (FC) upor down-regulated in expression are indicated (P-value $\leq 0.05)$. Expression profiles of upregulated (red), down-regulated (green) and no significant change (black) are shown. 

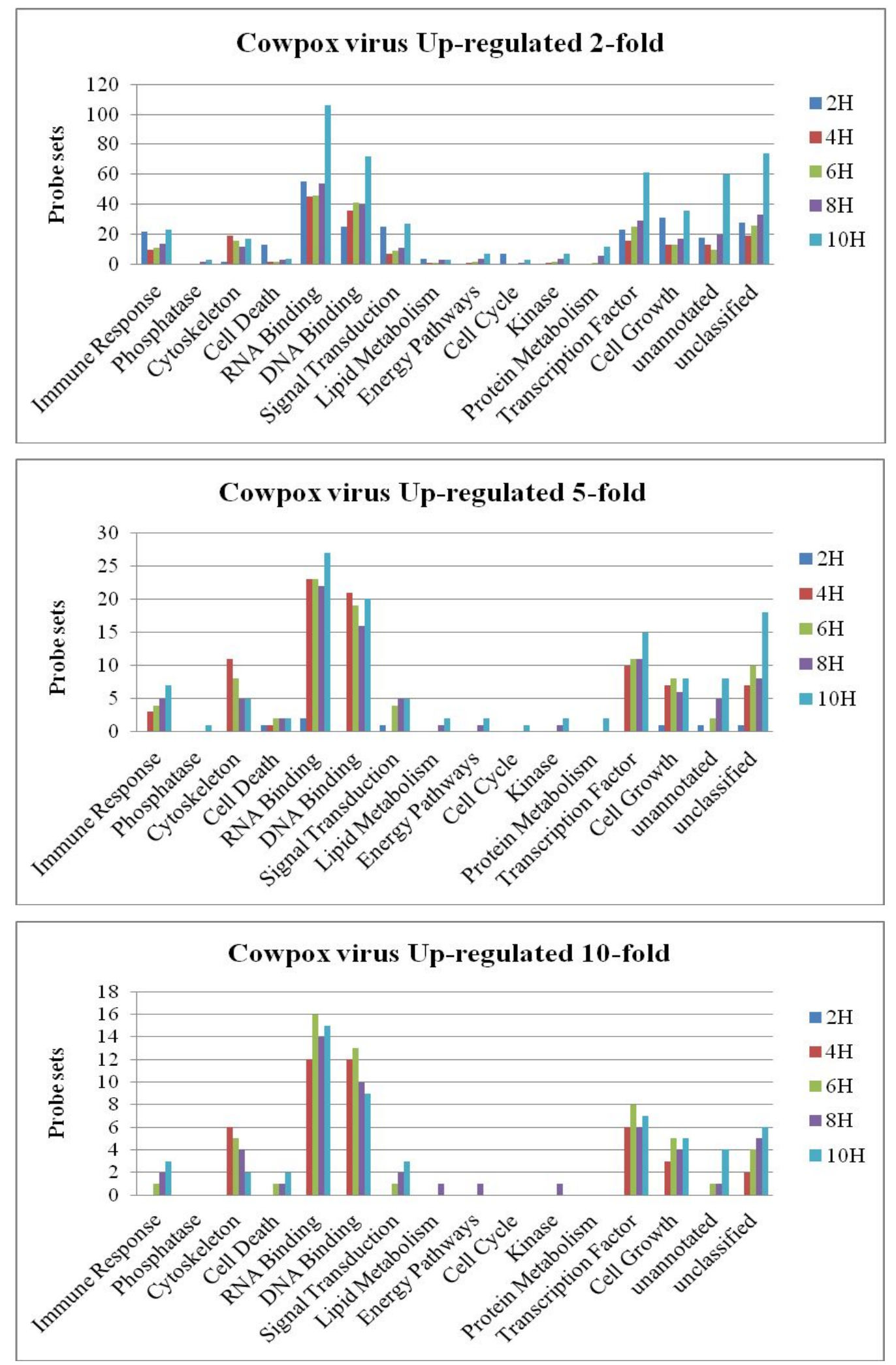

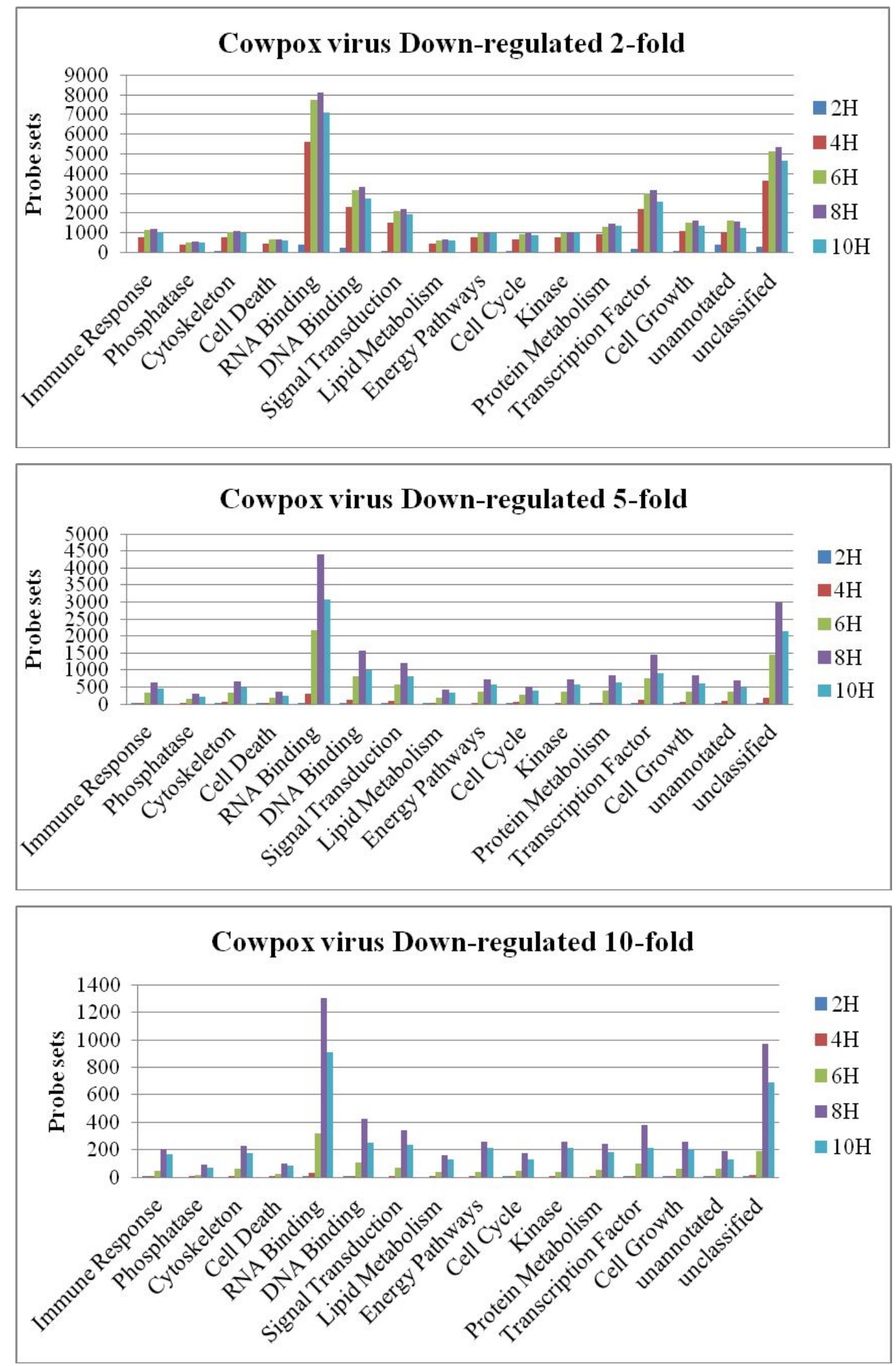

Figure 6.5 Overview of distributions of differentially expressed probe sets into different biological functions in $\mathbf{A 5 4 9}$ cells infected with cowpox virus. The numbers of probe sets in the different functional families, including non-annotated and unclassified groups, showing upregulated or down-regulated with different fold changes $(\geq 2-F C, \geq 5-F C$ and $\geq 10-F C)$ in gene expression are presented. 

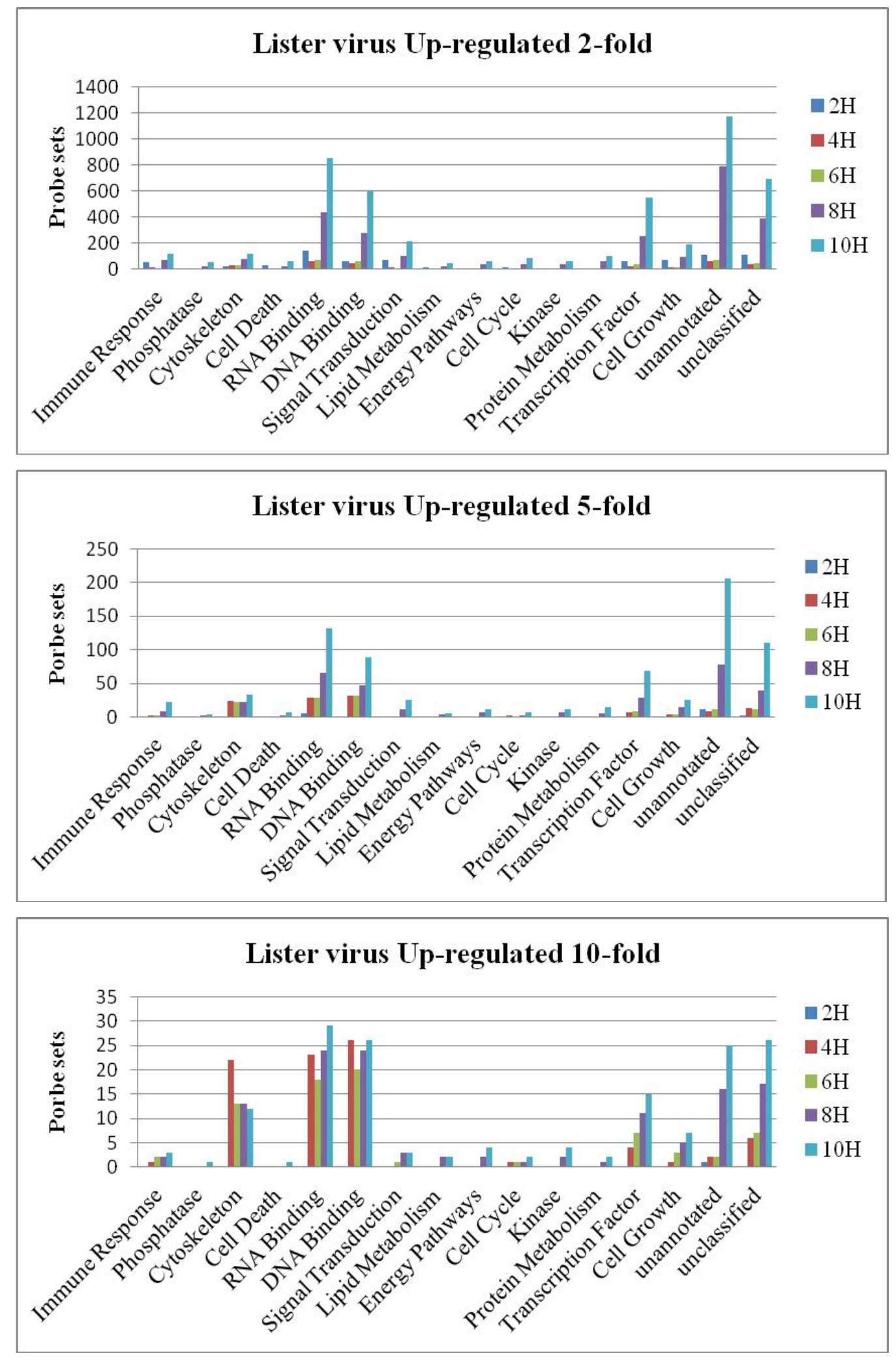

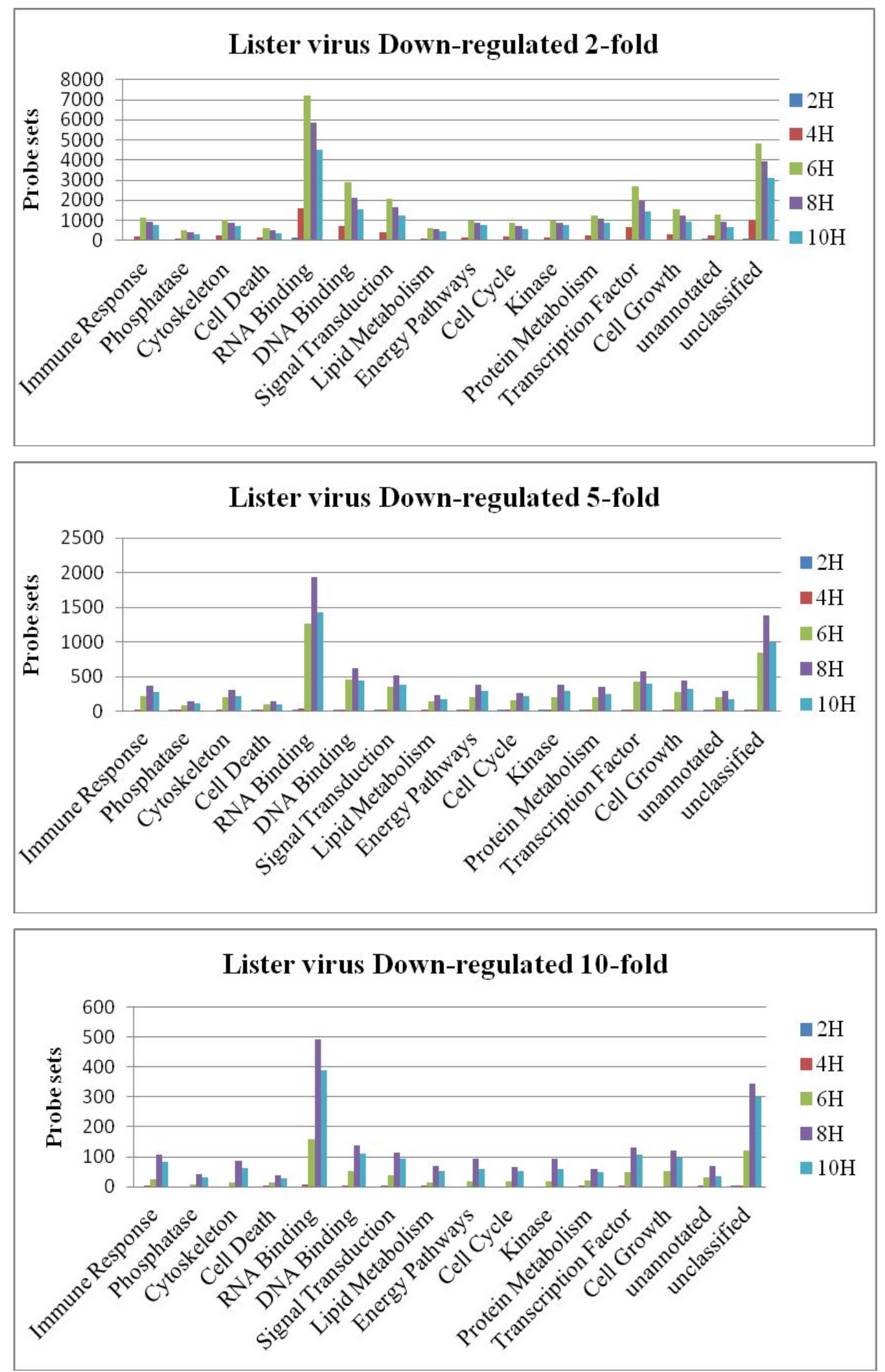

Figure 6.6 Overview of distributions of differentially expressed probe sets into different biological functions in A549 cells infected with lister virus. The numbers of probe sets in the different functional families, including non-annotated and unclassified groups, showing upregulated or down-regulated with different fold changes ( $\geq 2-F C, \geq 5-F C$ and $\geq 10-F C)$ in gene expression are presented. 

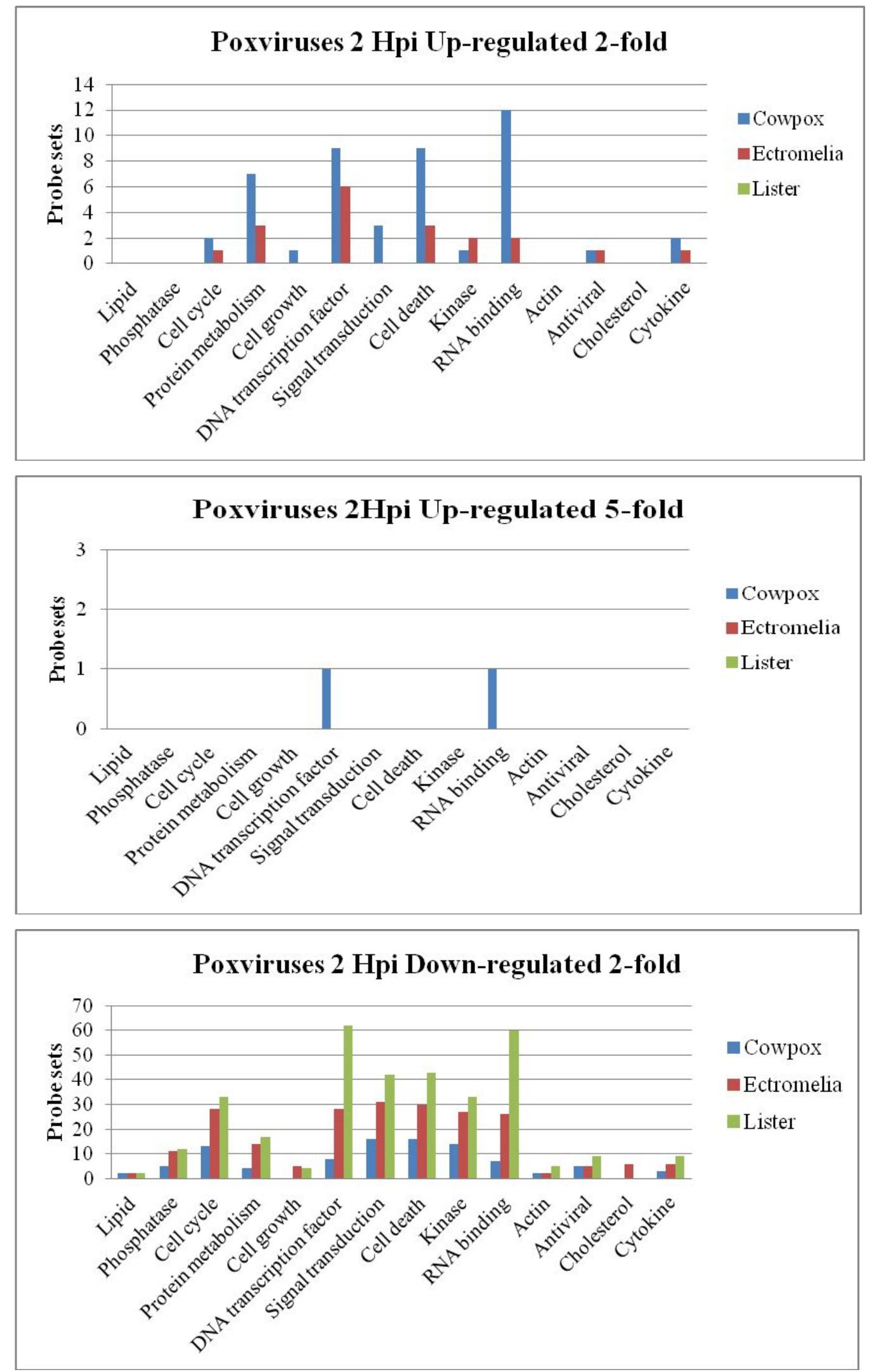


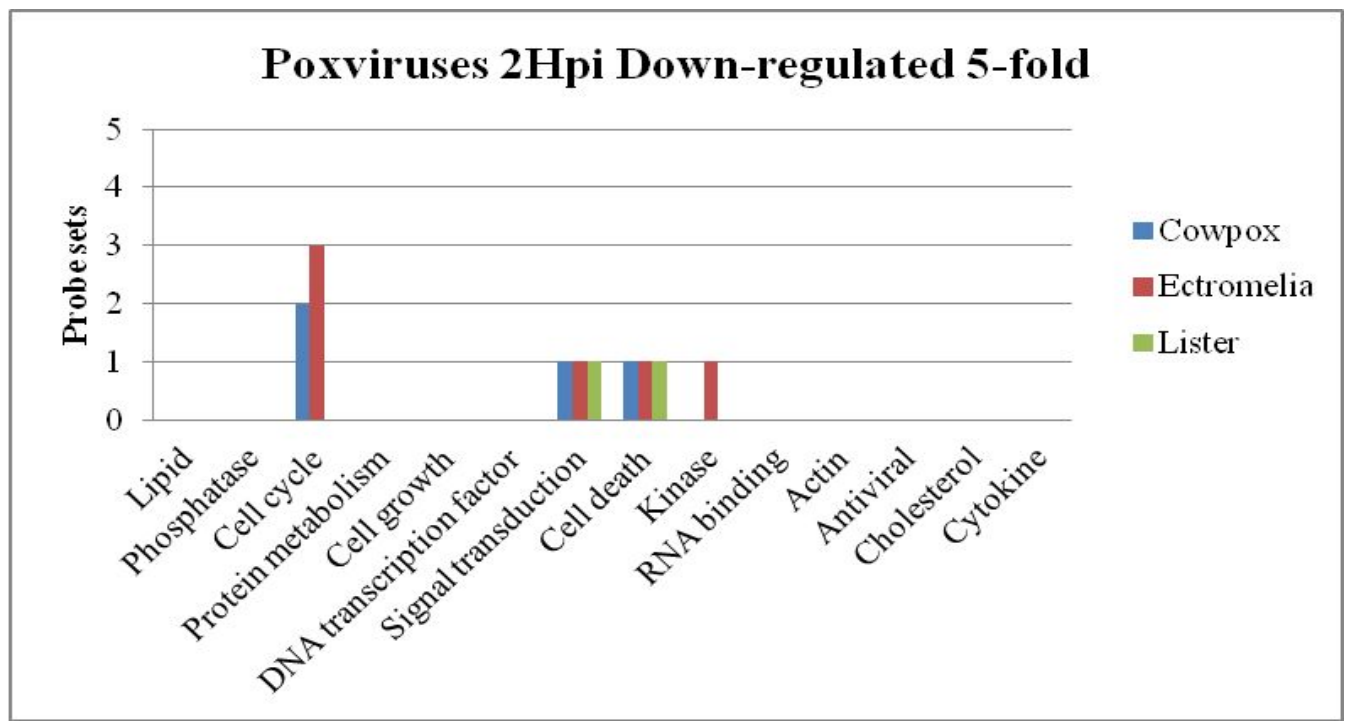

Figure 6.7 Overview of distributions of differentially expressed probe sets into different biological functions in RAW cells infected with three types of poxviruses at $\mathbf{2}$ hpi. The numbers of probe sets in the different functional families, including non-annotated and unclassified groups, showing up-regulated or down-regulated with different fold changes $(\geq 2$ $\mathrm{FC}$ and $\geq 5$-FC) in gene expression are presented. 

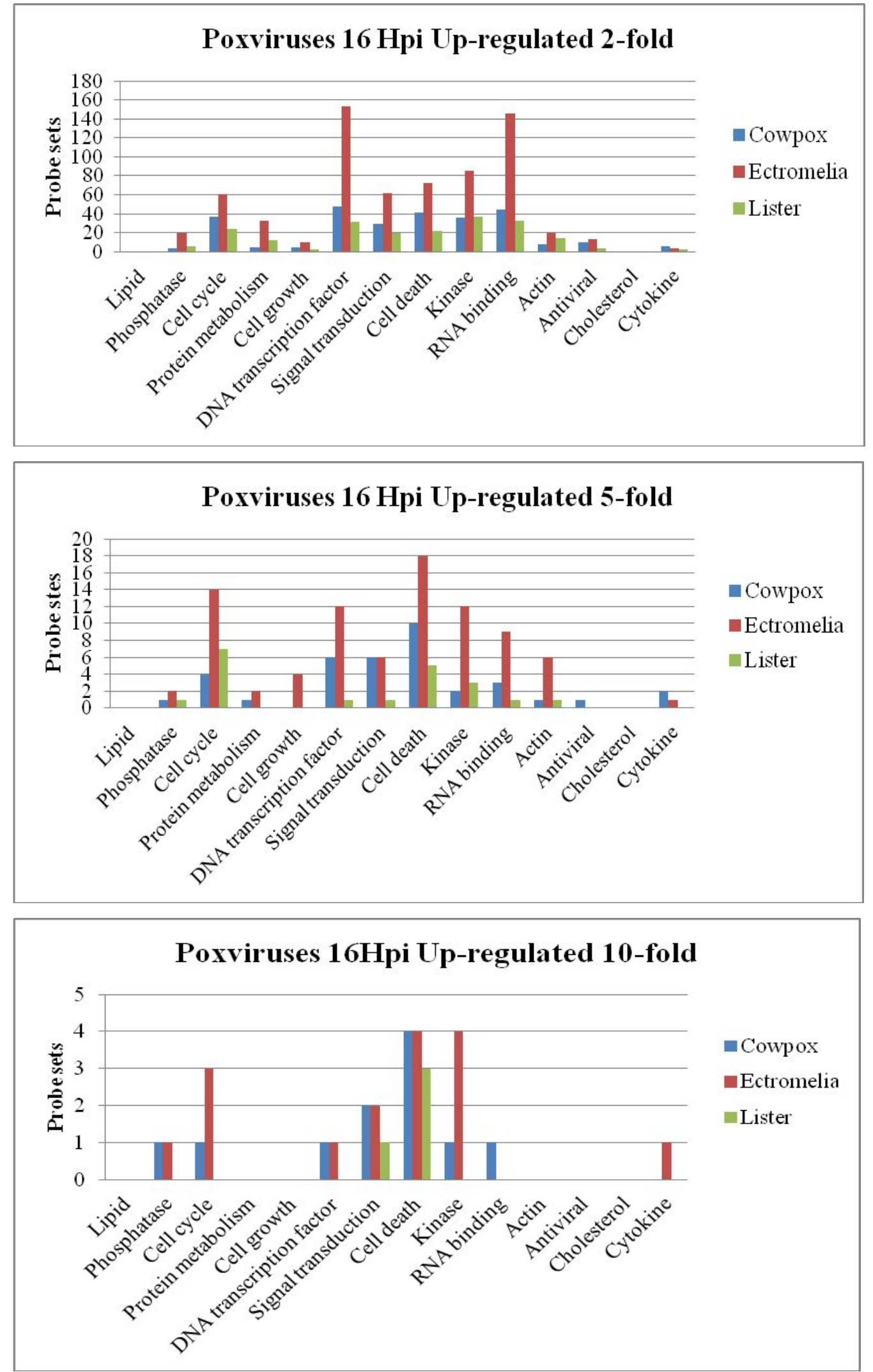

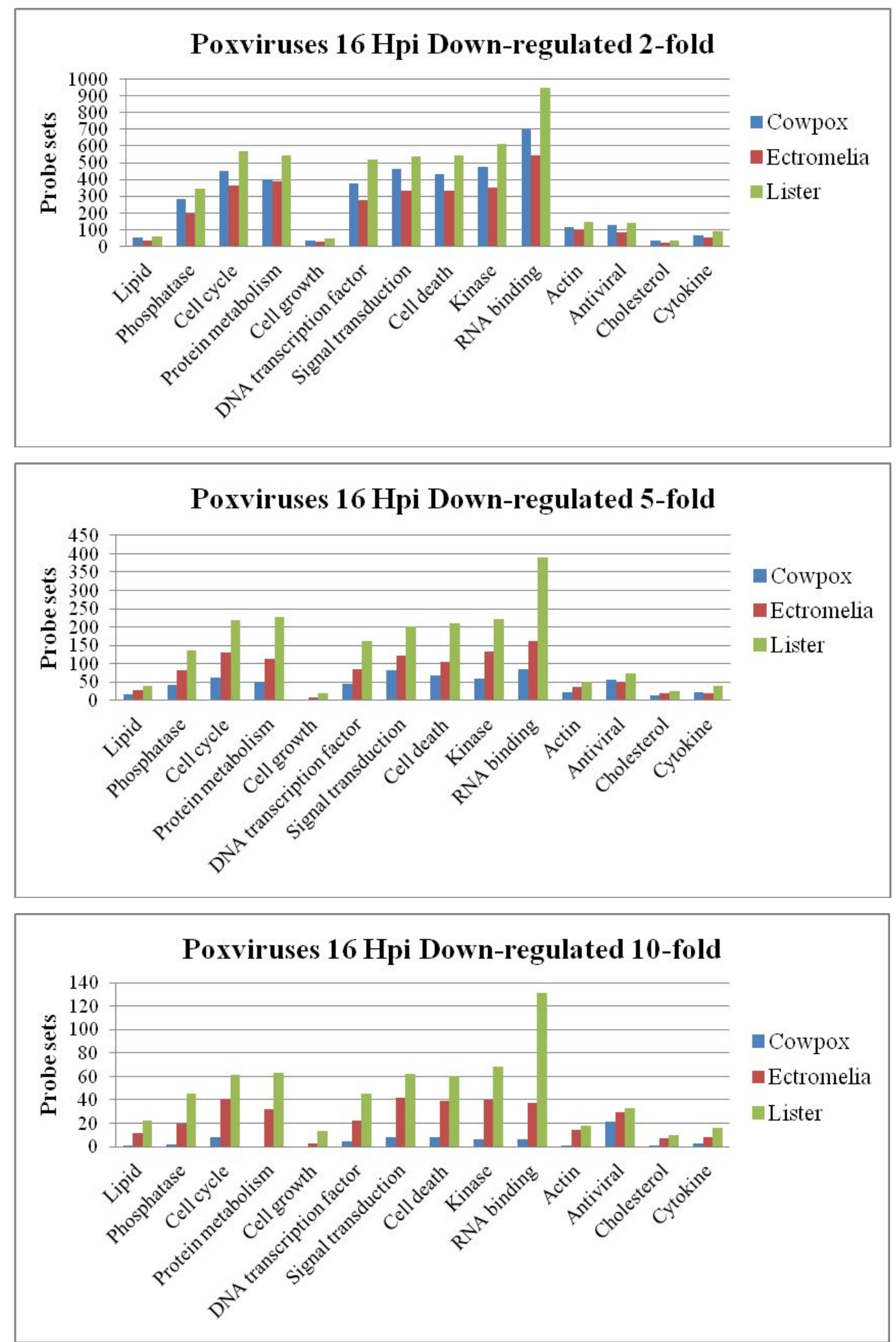

Figure 6.8 Overview of distributions of differentially expressed probe sets into different biological functions in RAW cells infected with three types of poxviruses at 16 hpi. The numbers of probe sets in the different functional families, including non-annotated and unclassified groups, showing up-regulated or down-regulated with different fold changes $(\geq 2$ $\mathrm{FC}, \geq 5$-FC and $\geq 10-\mathrm{FC}$ ) in gene expression are presented. 


\subsubsection{Cluster analysis in A549 cells}

To our knowledge, genes with similar temporal expression trends might have related biological functions and possibly correspond to some critical cellular processes and pathways [91]. So the aim of this cluster analysis is to classify probe sets with similar expression profiles into common biological groups, which is beneficial for further functional analysis. In our study, A549 cells were infected with cowpox and lister virus, and their transcriptomes were analyzed at 2, 4, 6, 8 and 10 hpi. Probe sets with $\geq 2$-FC (P-value $\leq 0.05$ ) upregulated at least at $10 \mathrm{hpi}$ and those with $\geq 10$-FC (P-value $\leq 0.05$ ) downregulated at least at $10 \mathrm{hpi}$ in cowpox or lister virus infected A549 cells were separately clustered into similar gene expression profiles using the Expander version 5.0 software [Figure 6.9-6.12]. Using the same software, the data were further analyzed into genes relating to different functional groups or canonical pathways, and enriched transcription factor, chromosome locations and microRNAs were also identified. All enriched functional groups, canonical pathways, transcriptional factors, chromosome locations and microRNAs are displayed in Table 6.3-6.6.

Pathway analysis revealed that the pathway called "Systemic lupus erythematosus" was found to be commonly enriched in genes with up-regulated expression at least at 10 hpi in cowpox and lister virus infected A549 cells, with more genes following lister virus infection involved in this pathway than those following cowpox virus infection. Systemic lupus erythematosus (SLE) is a chronic autoimmune disease that can affect almost any organ system, and molecules in this pathway majorly play a role on mediating a systemic inflammatory response. Through detailed investigation on genes involved in this pathway, we found that almost all these genes from both cowpox virus and lister virus infected A549 cells encoded histone proteins.

Alkhalil A et al (2010) has depicted the similar situation happened in monkeypox virus infected MK2 cells [339]. In those infected host cells, all the core histone genes excluding HIST3H2A exhibited up-regulated expression at both examined 3 and 7 hpi. However, unlike these histone genes with increasing expression trend, major transcription regulators of histones expression such as CITED2, NCOA3, CREB1, YY1 and HDAC2 showed increasing suppression at their transcriptional level across the whole infection 
course. Similarly, many enzymes that control modifications of histones and chromatin organization dynamics such as FBXO11, PRMT3, MYST2, MYCBP2, and RARS2 also showed steady down-regulation in their mRNA level. As we know, histones in eukaryotic cell nucleic play an essential function as chief protein components of chromatin to package and order the DNA into structural units called nucleosomes. Besides, poxviruses are double-stranded DNA mammalian viruses with large genomes reaching few hundreds of micrometers in length. Thus, Alkhalil A made a hypothesis that host cell histones played a role on viral DNA compaction and nucleosome formation, and up-regulated expression of these histones following infection of poxviruses might be a potential implication of viral replication. With regards to transcription regulator and enzymes with down-regulated expression, they proposed that these observations implied part of host response actions including chromatin-mediated silencing of the viral genome and activation of DNA damage, or part of the viral strategies to take over its host.

In our study, up-regulated expression levels of histones after infections of cowpox and lister virus were consistent with their observations after infection of monkeypox virus. Consequently, if the hypothesis mentioned above is correct, more histone genes with up-regulated expression in lister virusinfected A549 cells could potentially indicate better virus replication than in cowpox-virus infected A549 cells. Furthermore, most of the histones related enzymes and transcription regulators also showed down-regulated expression from 4 hpi, with deeper repression detected after infection of cowpox virus than lister virus. This observation which was consistent with previous reports supported the previous finding in further.

A transcription factor called SRF was enriched in genes with upregulated expression in cowpox virus-infected A549 cells. The protein encoded by this gene binds to the promoter region of serum response element in order to regulate the activity of many immediate-early genes, and thereby plays roles on the regulations of cell cycle, cell growth, cell differentiation and cell death. Over-representation of this transcription factors based on up-regulated genes might suggest the potential abnormal regulation on cell growth and apoptosis in infected host cells. In addition, transcription factors including NF-Y, ETF and POU1F1were only enriched in up-regulated genes after infection of lister virus. 
Among these factors, only ETF showed faint down-regulation in its expression in lister virus infected A549 cells. It has been reported that transcription factor ETF played a role in mediating expressions of genes related to cell proliferation [340], the inconsistent gene expression performances of ETF and downstream genes might implicate more complex regulation network.

A batch of gene ontology terms was enriched in genes with up-regulated expression from both cowpox and lister virus-infected A549 cells. These functional terms included "nucleic acid binding", "gene expression", "zinc ion binding", "DNA binding" and "regulation of cellular metabolic process". Besides these common functional terms, other interesting functional groups were also enriched specific to the infection of lister virus. These unique gene ontology terms were "regulation of cellular biosynthetic process", "transition metal ion binding", "macromolecule biosynthetic process", "cellular biopolymer catabolic process", "cellular biopolymer biosynthetic process", "negative regulation of cellular process", "negative regulation of transcription", "regulation of transcription, DNA-dependent" and so on. Over-representative of these pathways suggested that there might exist some mechanism regulations relevant to macromolecules, cellular biopolymer, as well as some metal ion transition upon infection of lister virus in A549 cells.

In addition, a series of potentially regulatory microRNAs were also picked out to bind with the up-regulated genes during cowpox virus infection. And around 70 genes with elevated expression in lister virus infected A549 cells were also predicted to locate at two common chromosome sites (chromosome $2 \mathrm{p}$ and chromosome $14 \mathrm{q}$ ). 

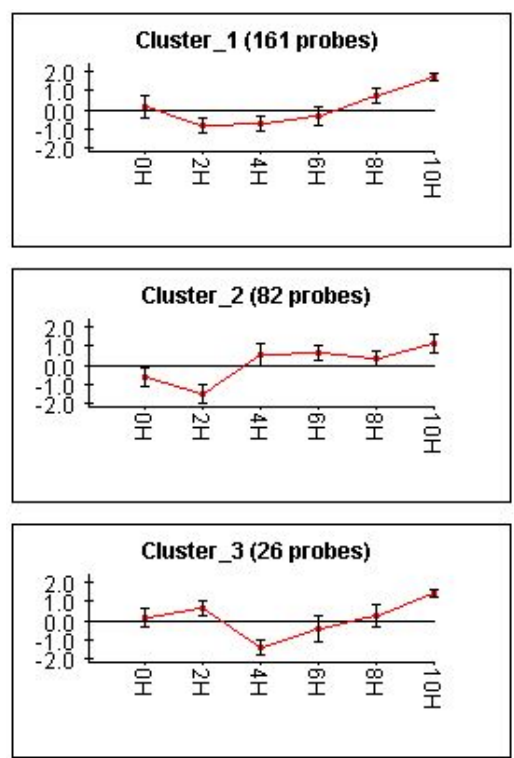

Figure 6.9 Clustering analysis of temporal gene expression profiles in cowpox virusinfected $\mathbf{A 5 4 9}$ cells. A549 cells were infected with cowpox virus at 2, 4, 6, 8 and 10 hpi. Probe sets showing $\geq 2$-fold changes up-regulated (P-value $\leq 0.05$ ) at least at 10 hpi were analyzed with Expander 5 software. $\mathrm{X}$-axis represents post-infection in hours $(\mathrm{H})$, and Y-axis means normalized expression changes of probe sets.

Table 6.3 Summary of functional groups, canonical pathways, transcription factors and microRNAs enriched based on up-regulated genes in A549 cells infected with cowpox virus.

\begin{tabular}{|l|c|}
\hline \multicolumn{2}{|c|}{ Cowpox Group I } \\
\hline Cluster No. & Enriched Transcription Factors \\
\hline Cluster 2 & M00186[SRF]:8 | M00152[SRF]:7 \\
\hline Cluster N0. & \multicolumn{1}{|c|}{ Enriched Canonical Pathways } \\
\hline Cluster 2 & Systemic lupus erythematosus:7 \\
\hline Cluster No. & \multicolumn{2}{|c|}{ Enriched Gene Ontology Terms } \\
\hline \multirow{2}{*}{ Cluster 1} & nucleic acid binding - G0:0003676:31 | regulation of macromolecule metabolic process - G0:0060255:29 | gene expression - G0:0010467:25 \\
\cline { 2 - 3 } & zinc ion binding - G0:0008270:23 | nitrogen compound metabolic process - G0:0006807:27 | transcription factor activity - G0:0003700:13 \\
\hline \multirow{2}{*}{ Cluster 2} & transcription factor activity - G0:0003700:16 | regulation of cellular metabolic process - G0:0031323:26 | DNA binding - G0:0032502:19 \\
\cline { 2 - 3 } & brain development - G0:0007420:5 \\
\hline Cluster N0. & \\
\hline Cluster 3 & mir-21/590-5p:3 | mir-183:3 | mir-320/320abcd:3 | mir-590/590-3p:3 \\
\hline
\end{tabular}

Genes with up-regulated expression were significantly categorized into different functional groups and canonical pathways. Different transcription factors and microRNAs that can potentially be involved in the regulation of gene expressions are shown for each cluster under Expander 5 software analysis (P-value $\leq 0.05$ ). Each functional group, canonical pathway, transcription factor or microRNA is followed by the number of corresponding genes. 

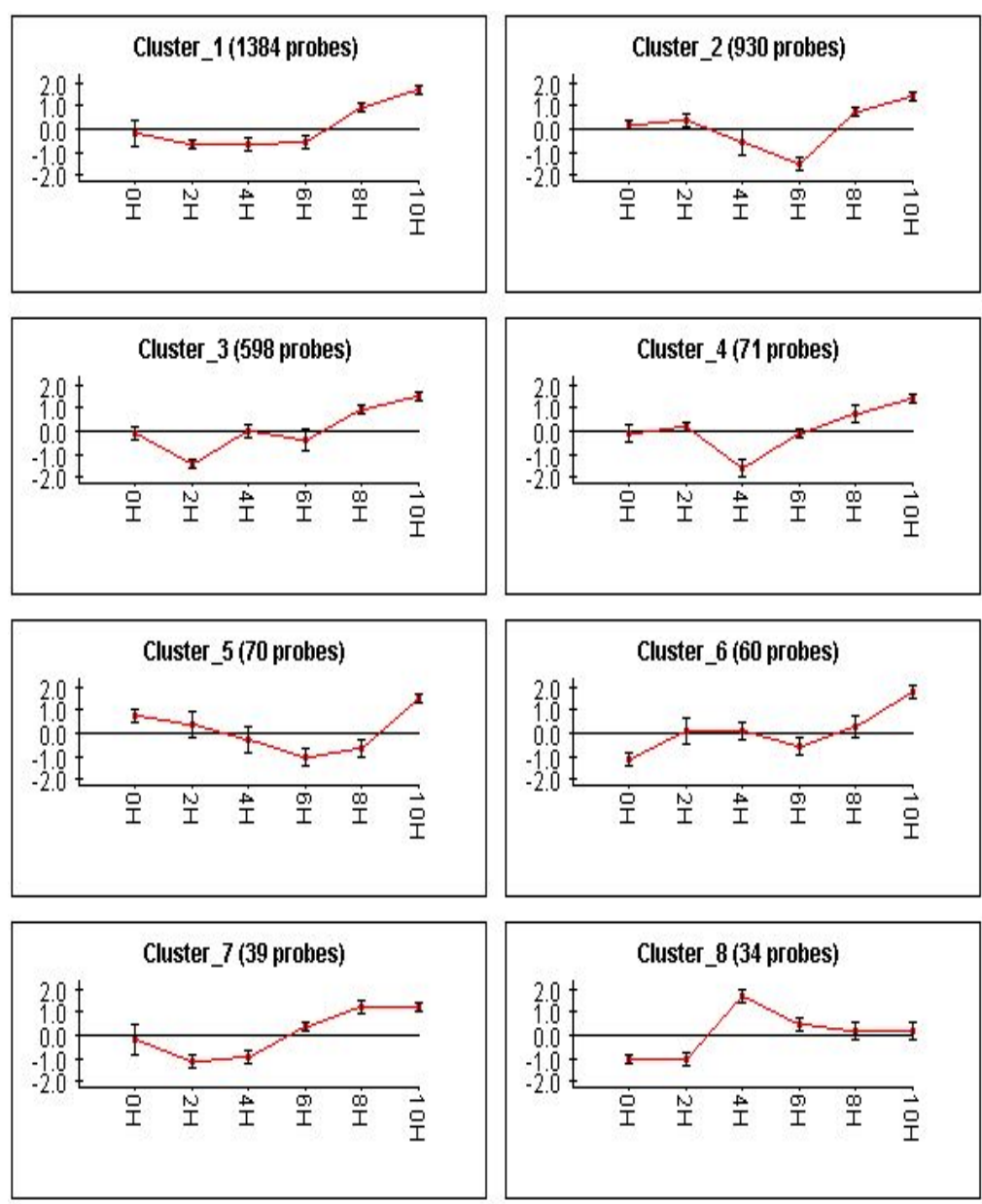

Figure 6.10 Clustering analysis of temporal gene expression profiles in lister virus-infected A549 cells. A549 cells were infected with cowpox virus at 2, 4, 6, 8 and 10 hpi. Probe sets showing $\geq 2$-fold changes up-regulated (P-value $\leq 0.05$ ) at least at $10 \mathrm{hpi}$ were analyzed with Expander 5 software. X-axis represents post-infection in hours $(\mathrm{H})$, and Y-axis means normalized expression changes of probe sets. 
Table 6.4 Summary of functional groups, canonical pathways, transcription factors and chromosome locations enriched based on up-regulated genes in A549 cells infected with lister virus.

\begin{tabular}{|c|c|}
\hline \multicolumn{2}{|r|}{ Lister Group I } \\
\hline Cluster №. & Enriched Transcription Factors \\
\hline Cluster 1 & M00287[NF-Y]:145 \\
\hline Cluster 2 & M00695[ETF]:210 \\
\hline Cluster 7 & M00744[POU1F1]:9 \\
\hline Cluster №. & Enriched Canonical Pathways \\
\hline Cluster 8 & Systemic lupus erythematosus:26 \\
\hline Cluster No. & Enriched Gene Ontology Terms \\
\hline \multirow{7}{*}{ Cluster 1} & nucleic acid binding - G0:0003676:208 | regulation of gene expression - G0:0010468:179 | regulation of RNA metabolic process - G0:0051252:166 \\
\hline & gene expression - G0:0010467:169 | regulation of macromolecule biosynthetic process - G0:0010556:176 \\
\hline & 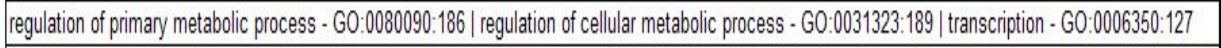 \\
\hline & Nnucleobases, nucleoside, nucleotide and mucleic acid metabolic process - G0:0006139:171 | cellular biopolymer biosynthetic process - G0:0034961:142 \\
\hline & zinc ion binding - G0:0008270:148 | RNA binding - G0:0003723:50 | RNA splicing - G0:0008380:27 | nucleotide binding - G0:0000166:94 \\
\hline & modification-dependent macromolecule catabolic process - G0:0043632:34 | chromatin modification - G0:0016568:18 \\
\hline & cellular protein metabolic process - G0"0044267:86 \\
\hline \multirow{6}{*}{ Cluster 2} & regulation of cellular metabolic process - G0:0031323:142 | regulation of cellular biosynthetic process - G0:0031326:130 \\
\hline & regulation of nucleobase, nucleoside, nucleotide and nucleic acid metabolic process - G0:0019219:126 | nucleic acid binding - G0:0003676:134 \\
\hline & DNA binding - G0:0003677:106 | zinc ion binding - G0:0008270:104 | transition metal ion binding - G0:0046914:116 | gene expression - G0:0010467:95 \\
\hline & nucleobse, nucleoside, nucleotide and nucleic acid metabolic process - G0:0006139:103 | transcription regulator activity - G0:0030528:64 \\
\hline & macromolecule biosynthetic process - G0:0009059:88 | cellular biopolymer catabolic process - G0:0034962:31 \\
\hline & negative regulation of transcription - G0:0016481:20 | nucleotide binding - G0:0000166:68 | negative regulation of cellular process - G0:0048523:45 \\
\hline \multirow{3}{*}{ Cluster3 } & gene expression - G0:0010467:84 | zinc ion binding - G0:0008270:80 | nucleic acid binding - G0:0003676:95 \\
\hline & nucleobase, nucleoside, nucleotide and nucleic acid metabolic process - G0:0006139:88 | DNA binding - G0:0003677:75 | transcription - G0:0006350:62 \\
\hline & cellular biopolymer biosynthetic process - G0:0034961:71 | regulation of transcription, DNA-dependent - G0:0006355:74 \\
\hline Cluster №. & Enriched Chromosome Locations \\
\hline Cluster 1 & chromosome 2p:34 | chromosome 14q:35 \\
\hline
\end{tabular}

Genes with up-regulated expression were significantly categorized into different functional groups and canonical pathways. Different transcription factors and micRNAs that can potentially be involved in the regulation of gene expressions are shown for each cluster under Expander 5 software analysis (P-value $\leq 0.05)$. Each functional group, canonical pathway, transcription factor or microRNA is followed by the number of corresponding genes.

As shown in Table 6.5 and Table 6.6, a common transcription factor named sp1 was found to be enriched in genes with down-regulated expression in both two types of poxviruses infected A549 cells. In addition to this common factor, other factors such as $\mathrm{Nrf}-1$ and GABP were also listed to potentially regulate the down-regulated genes during cowpox virus infection, while E2F was significantly identified to potentially regulate those down-regulated genes during lister virus infection.

Pathways including "Metabolic pathways", "Oxidative phosphorylation", "Glutathione metabolism", "Glycolysis", "Purine metabolism" were commonly enriched in genes with down-regulated expression upon the infections of both two types of poxviruses, with more 
down-regulated genes in these pathways detected after infection of cowpox virus. Unique to lister virus infection, "DNA replication" pathway was also significantly enriched in down-regulated genes in corresponding A549 cells, which might be caused by the antiviral action exerted by host cells to limit the viral replication. Similarly, several other pathways such as "TCA cycle", "Lysosome", "Cell cycle" were specifically enriched in down-regulated genes in cowpox virus-infected A549 cells.
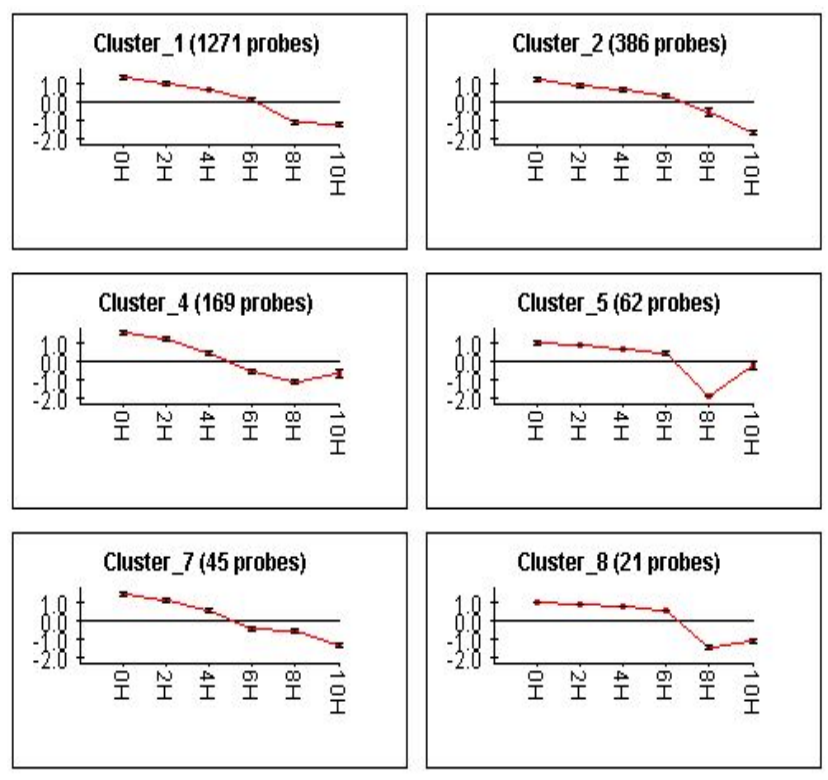
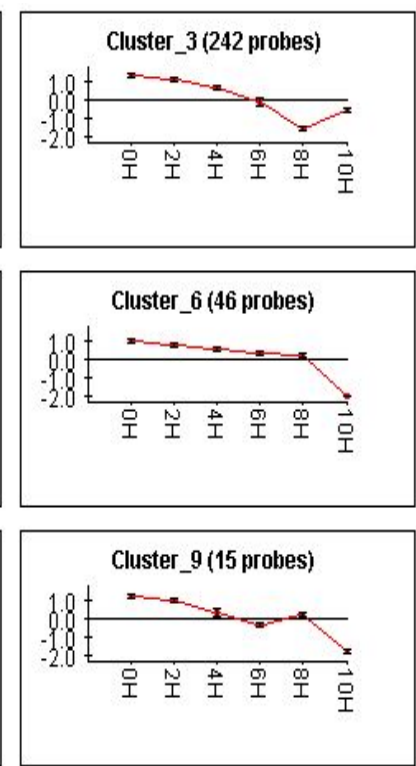

Figure 6.11 Clustering analysis of temporal gene expression profiles in cowpox virusinfected $\mathbf{A 5 4 9}$ cells. A549 cells were infected with cowpox virus at 2, 4, 6, 8 and 10 hpi. Probe sets showing $\geq 10$-fold changes down-regulated ( $\mathrm{P}$-value $\leq 0.05$ ) at least at 10 hpi were analyzed with Expander 5 software. X-axis represents post-infection in hours $(\mathrm{H})$, and Y-axis means normalized expression changes of probe sets.

Quite a lot of GO terms were over-represented based on down-regulated genes in infected A549 cells after infections of both poxviruses, especially after infection of cowpox virus. Among these enriched terms, biological functions associated with "oxidation reduction", "nitrogen compound metabolic process", "hydrolase activity", 'cell metabolism", "cell cycle”, 'cellular catabolic process and organelle organization" were commonly over-represented after infections of both two types of poxviruses. Besides, biological functions such as "generation of precursor metabolites and energy", "establishment of localization", "transferase activity and structural molecule activity" were enriched based on down-regulated genes specifically in cowpox virus-infected A549 cells. 
Table 6.5 Summary of functional groups, canonical pathways, transcription factors, chromosome locations and microRNAs enriched based on down-regulated genes in A549 cells infected with cowpox virus.

\begin{tabular}{|c|c|}
\hline & Cowpox Group II \\
\hline Cluster No. & Enriched Transcription Factors \\
\hline Cluster 1 & |M00652[NF-1]:308 | M00341[GABP]:230 | M00196[Sp1]:509 \\
\hline Cluster No. & Enriched Canonical Pathways \\
\hline \multirow{3}{*}{ Cluster 1} & Metabolic pathways:137 | Huntington's disease:39 | Oxidative phosphorylation:30 | Parkinson's disease:30 | Alzheime's disease:33 \\
\hline & Glutathione metabolism:15 | Pentose phosphate pathway:10 | Cardiac muscle contraction:13 | Pathogenic Escherichia coli infection:11 \\
\hline & Glycolysis / Gluconeogenesis:11 | Citrate cycle (TCA cycle):18 | Purine metabolism:18 | Lysosome:14 \\
\hline Cluster 2 & Metabolic pathways:41 \\
\hline Cluster 3 & Metabolic pathways:38 | Drug metabolism -other enzymes:5 | Cell cycle:7 \\
\hline Cluster No. & Enriched Gene Ontology Terms \\
\hline \multirow{22}{*}{ Cluster 1} & oxidoreductase activity - G0:0016491:103 | oxidation reduction - G0:0055114:69 | generation of precursor metabolites and energy - G0:0006091:52 \\
\hline & heterocycle metabolic process - G0:0046483:40 | cellular catabolic process - G0:0044248:87 | electron transport chian - G0:0022900:28 \\
\hline & |localization - G0:0051179:175 | establishment of localization - G0:0051234;157 | oxidative phosphorylation - G0:0006119:22 \\
\hline & catabolic process - G0:0009056:111 | nucleoside phosphate metabolic process - G0:0006753:33 | hydrolase activity - G0:0016787:145 \\
\hline & oxidoreductase activity, acting on NADH or NADHP - G0:0016651:21 | respiratory chain - G0:0070469:20 | cellular respiration - G0:0045333:22 \\
\hline & organic acid metabolic process - G0:0006082:52 | biosynthetic process - G0:0009058:173 | cofactor metabolic process - G0:0051186:28 \\
\hline & cell redox homemstasis - G0:0045454;17 | nitrogen compound metabolic process - G0:0006807:174 | protein metabolic process - G0:0019538:155 \\
\hline & isomerase activity - G0:0016853;23 | oxidoreductase activity, acting on the $\mathrm{CH}-\mathrm{OH}$ group of donors, NAD or NADP as acceptor - G0:0016616 \\
\hline & cellular nitrogen compound metabolic process - G0:0034641:29 | cellular amino acid and derivative metabolic process - G0:0006519:36 \\
\hline & inorganic cation transmembrane transporter activity - G0:0022890:24 | cellular carbohydrate metabolic process - G0:0044262:33 \\
\hline & response to oxidative stress - G0:0006979:19 | ribonucleotide metabolic process - G0:0009259:17 | carbohydrate metabolic process - G0:0005975:46 \\
\hline & cellular homeostasis - G0:0019725:36 | regulation of biological quality - G0:0065008:73 | translation - G0:0006412:34 \\
\hline & transferase activity, transferring alkyl or aryl (other than methy) groups - G0:0016765:13 | cellular compound organization - G0:0016043:107 \\
\hline & $\mid$ acid binding - G0:0003779:32 | organelle organization - G0:0006996:69 | methionine metabolic process - G0:0006555:6 | cofactor binding - G0:0048037:25 \\
\hline & Water-soluble vitamin metabolic process - G0:0006767:12 | intracellular transport - G0:0046907:46 | monosaccharide catabolic process - G0:0046365:14 \\
\hline & water-soluble vitamin biosynthetic process - G0:0042364:8 | lyase activity - G0:0016829:20 | response to stress - G0:0006950:79 \\
\hline & transferase activity - G0:0016740:97 | structural molecule activity - G0:0005198:49 | regulation of protein metabolic process - G0:0051246:33 \\
\hline & glucose metabolic process - G0:0006006:16 | lipid metabolic process - G0:0006629:53 | lipid binding 0 G0:0008289:37 \\
\hline & sulfur metabolic process - G0:0006790:14 | regulation of programmed cell death - G0:0043067:42 | ligase activity - G0:0016874:32 \\
\hline & cell cycle - G0:0007049:45 | protein maturation - G0:0051604:14 | DNA metabolic process - G0:0006259:35 \\
\hline & calcium- dependent phospholipid binding - G0:0005544:7 | DNA replication - G0:0006260:19 | antioxidant activity - G0;0016209:10 \\
\hline & celllar macromolecule localization - G0:0070727:32 \\
\hline \multirow{2}{*}{ Cluster 2} & oxidation reduction - G0:0055114:26 | cellular ketone metabolic process - G0:0042180:19 \\
\hline & nucleobase, nucleoside and nucleotide metabolic process - G0:0055086:12 I protein amino acid N-linked glycosylation - G0:0006487:6 \\
\hline \multirow{4}{*}{ Cluster 3} & cellular component organization - G0:0016043:45 | macromolecular complex assembly - G0:0065003:16 | oxidoreductase activity - G0:0016491:21 \\
\hline & nuclear division - G0:0000280:11 | cell division - G0:0051301:12 | catabolic process - G0:0009056:30 | protein tetramerization - G0:0051262:5 \\
\hline & lipid metabolic process - G0:0006629:19 | regulation of biological quality - G0:0065008:22 | protein metabolic process - G0;0019538:40 \\
\hline & protein domain specific binding - G0:0019904:9 \\
\hline \multirow{2}{*}{ Cluster 4} & Nnuclear division - G0:0000280:11 | organelle organization 0 G0:0006996:23 | cell cycle - G0:0007049:16 \\
\hline & cytoskeleton organization - G0:0007010:12 | ATP binding - G0:0005524:23 | microtubule cytoskeleton organization - G0:0000226:7 \\
\hline Cluster 7 & cofactor binding - G0;0048037:7 \\
\hline Cluster No. & Enriched Chromosome Locations \\
\hline Cluster 1 & chromosome $13 q 32: 9 /$ chromosome $2: 81$ \\
\hline Cluster 3 & chromosome 2:26 \\
\hline Cluster No. & Enriched microRNAs \\
\hline Cluster 5 & mir-374/374ab:31 mir-543:3 \\
\hline Cluster 6 & mir-22:5| mir-530:4 \\
\hline Cluster 7 & mir-31:3 \\
\hline
\end{tabular}

Genes with down-regulated expression were significantly categorized into different functional groups and canonical pathways. Different transcription factors and microRNAs that can potentially be involved in the regulation of gene expressions are shown for each cluster under Expander 5 software analysis (P-value $\leq 0.05)$. Each functional group, canonical pathway, transcription factor or microRNA is followed by the number of corresponding genes. 
Excluding enrichments of these transcription factors and pathways, mir128 also displayed the potential possibility to bind and regulate three downregulated genes in lister virus infected A549 cells, while other microRNAs like mir-374/374as, mir-543, mir-22, mir-530 and mir-31 were also picked out in genes with deceased expression during cowpox virus infection. Meanwhile, some chromosome locations especially chromosome 2 were detected to be the targeting location for over one hundred genes with down-regulated expression.

Table 6.6 Summary of functional groups, canonical pathways, transcription factors and microRNAs enriched based on down-regulated genes in A549 cells infected with lister virus.

\begin{tabular}{|c|c|}
\hline \multicolumn{2}{|r|}{ Lister Group II } \\
\hline Cluster No. & Enriched Transcription Factors \\
\hline Cluster 1 & MO0803[E2F]:238 | M00196[Sp1]:217 \\
\hline Cluster No. & Enriched Canonical Pathways \\
\hline \multirow{2}{*}{ Cluster 1} & Metabolic pathways:60 | Glutathione metabolism :9 | Glycolysis / Gluconeogenesis:8 | Purine metabolism:12 | DNA replication:6 \\
\hline & Oxidative phosphorylation: 10 \\
\hline Cluster 2 & Metabolic pathways:26 | DNA replication:5 \\
\hline Cluster No. & Enriched Gene Ontology Terms \\
\hline \multirow{12}{*}{ Cluster 1} & oxidation reduction - G0:0055114:47 | generarion of precursor metabolites and energy - G0:0006091:24 | cellular ketone metabolic process - G0:0042180:27 \\
\hline & electron transport chain - G0:0022900:13 | organic acid metabolic process - G0:0006082:26 | cellular amino acid derivative metabolic process - G0:0006575:13 \\
\hline & cellular amino acid and derivative metabolic process - G0:0006519:20 | oxidoreductase activity, acting on CH-OH group of donors - G0:0016614:12 \\
\hline & cellular nitrogen compound metabolic process - G0:0034641:16 | lipid metabolic process - G0:0006629 | cellular carbohydrate catabolic process - G0:0044275:9 \\
\hline & hydrolase activity - G0:0016787:62 I cell cycle process - G0:0022402:22 | cellular carbohydrate metabolic process - G0:0044262:17 \\
\hline & cell cycle process - G0:0022402:22 | biosynthetic process - G0:0009058:74 | nitrogen compound metabolic process - G0;0006807:76 \\
\hline & intrsmolecular oxidoreductase activity - G0:0016860:7 | steroid binding - G0:0005496:-11 | organelle organization - G0:0006996:34 \\
\hline & nucleobase, nucleoside and nucleotide metabolic process - G0:0055086:14 | localization - G0:0051179:69 | single-stranded DNA binding - G0:0003697:7 \\
\hline & cellular catabolic process - G0:0044248:32 |DNA metabolic process - G0:0006259:20 | heterocycle metabolic process - G0:0046483:14 \\
\hline & cofactor binding - G0:0048037:13 | organelle fission - G0:0048285:12 | structual molecule activity - G0:0005198:25 | response to stress - G0:0006950:38 \\
\hline & vitamin metabolic process - G0:0006766:8 | transferase activity - G0:0016740:46 | coenzyme metabolic process - G0:0006732:10 \\
\hline & positive regulation of biological process - G0:0048518:37 | cellular aromatic compound metabolic process - G0:0006725:9 \\
\hline Cluster 2 & oxidoreductase activity - G0:0016491:21 | DNA replication - 60:0006260:9 \\
\hline Cluster 3 & alcohol catabolic process - G0:0046164:4 \\
\hline Cluster 5 & negative regulation of cellular protein metabolic process - G0:0032269:4 \\
\hline Cluster No. & Enriched micRNAs \\
\hline Cluster 1 & mir-128:3 \\
\hline
\end{tabular}

Genes with down-regulated expression were significantly categorized into different functional groups and canonical pathways. Different transcription factors and microRNAs that can potentially be involved in the regulation of gene expressions are shown for each cluster under Expander 5 software analysis (P-value $\leq 0.05$ ). Each functional group, canonical pathway, transcription factor or microRNA is followed by the number of corresponding genes. 

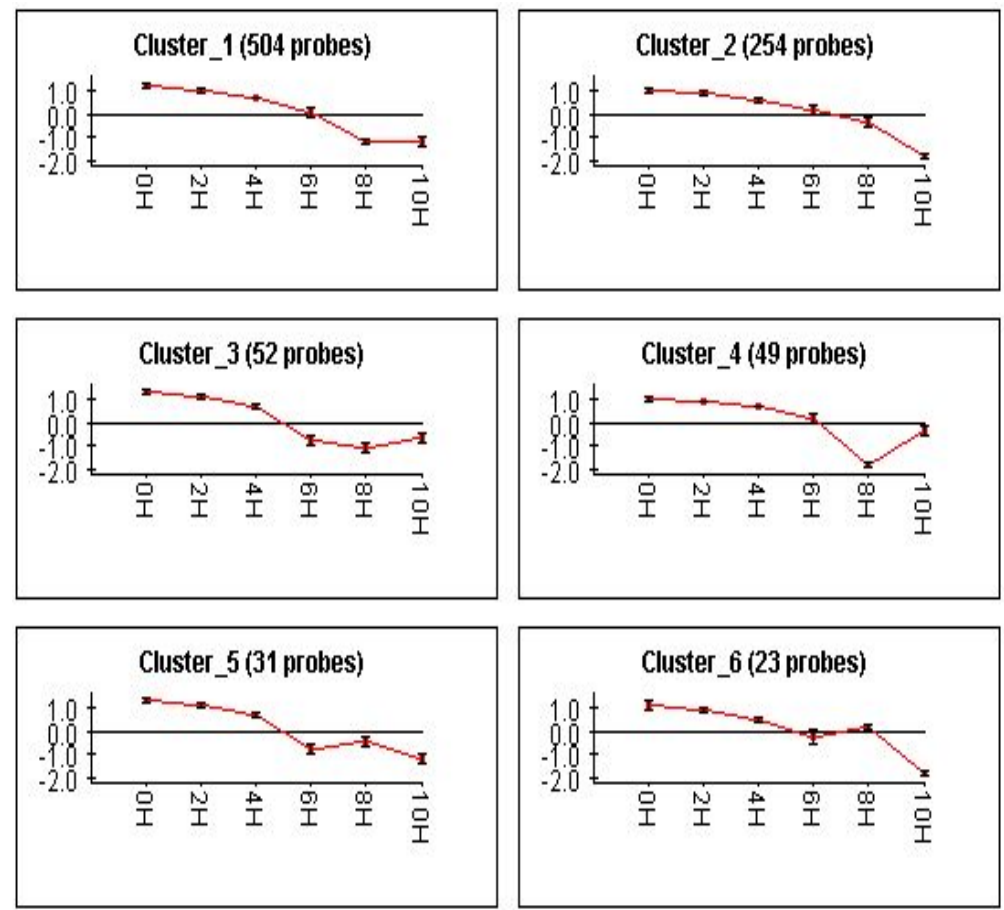

Figure 6.12 Clustering analysis of temporal gene expression profiles in lister virus-infected A549 cells. A549 cells were infected with cowpox virus at 2, 4, 6, 8 and 10 hpi. Probe sets showing $\geq 10$-fold changes down-regulated (P-value $\leq 0.05$ ) at least at 10 hpi were analyzed with Expander 5 software. X-axis represents post-infection in hours $(\mathrm{H})$, and Y-axis means normalized expression changes of probe sets.

\subsubsection{Core analysis in mouse RAW cells}

At early time point, several shared functions were identified based on differentially expressed genes in all these three types of poxviruses infected mouse RAW cells, and these top biological functions were "Immunological Disease", "Cellular Growth and Proliferation", "Cell Cycle", "Cell Death" and "Connective Tissue Development and Function" [Figure 6.13-6.24]. Significant enrichment of these common functions indicated that the immune reaction and cell growth regulation were initiated at the early stage of poxviruses infections. Of particular interest, the number of molecules belonging to "Gene expression", "Cell Cycle", "Cell death" and "Cellular Growth and Proliferation" showed highest upon the infection of lister virus. This phenomenon indicated that the most significant regulation of cell activities occurred in mouse RAW cells, infected with lister virus.

When it came to canonical pathways, pathways such as "Glycosis/Gluconegenesis" and "Frutose and Mannose Metabolism" were common in ectromelia and cowpox virus infected mouse RAW cells. However, the pathways over-represented in lister virus infected mouse RAW cells were 
associated with cell cycle checkpoint control. Subsequently, enrichment of different pathways indicated that more regulations of energy metabolism occurred after infections of ectromelia and cowpox virus while more controls of cell cycle occurred after infection of lister virus in mouse RAW cells. Furthermore, network of "Lipid metabolism, Molecular Transport, Small Molecule Biochemistry" was found to be unique to cowpox virus infection, which might also implicate the potential regulation of membrane activities.

Among top five potential transcription factors, four common ones including STAT4, HIF1A, EPAS1 and ATF4 were shared by infections of cowpox and ectromelia virus in mouse RAW cells at 2 hpi. Among these four factors, STAT4 is essential for mediating responses to IL12 in lymphocytes, and regulating the differentiation of $\mathrm{T}$ helper cells [341], while HIF1A and EPAS1 are both transcription regulators related to oxygen level. Enrichment of these transcription factors based on differentially expressed genes might implicate the differential regulation on some cytokines' activities as well as oxygen level in these two types of poxviruses infected mouse RAW cells.

Top five transcription regulators over-presented in differentially expressed genes from lister virus infected mouse RAW cells at 2 hpi were DACH1, MNT, SIN3B, TP53 and ELK1. TP53 is a famous factor that is responsible for the control of cell cycle and cell death. Its enrichment was concordant with the previous enrichment of functional groups including cell death and cell cycle [342], and thereby this observation supported the hypothesis that cell cycle and cell death regulation were triggered at the initial step in lister virus infected mouse RAW cells.

At $16 \mathrm{hpi}$, many common features were shared in response to infections of these three types of poxviruses in mouse RAW cells. Functions including "Infectious Disease", "RNA Post-Transcription Modification", "Cell cycle", "Embryonic Development", “Connetive Tissue Development and Function", "Hematological System Development and Function" were all shared by these three types of poxviruses. Furthermore, four common pathways such as "Oxidative Phosphorylation", "Mitochondrial Dysfunction", "Protein Ubiquitination Pathway" and "Ubiquinone Biosynthesis" were also enriched based on the differentially expressed genes after infections of these poxviruses, 
suggesting existing regulation of oxygen, energy and apoptosis in infected mouse RAW cells.

It was striking that "Cell death" was listed as one of the topmost five molecular and cellular functions in ectromelia virus infected mouse RAW cells with 1085 genes included, with a sharp increase of 94 molecules from 2 hpi. Thus observation might suggest the initiation of numerous mechanisms by which ectromelia virus inhibited apoptosis thereby modulating cell life [332].

Apart from these pathways and functional groups, a couple of transcription factors were also shared by infections of these three types of poxviruses. Among them, E2F4 and MYC are famous transcription factors that play a crucial role in the control of cell cycle [343]. Enrichment of these two factors implied the special regulated state of cell cycle.

\subsubsection{Selection of common genes modulated by infections of different poxviruses in mouse RAW cells}

As described above, hundreds or thousands of probes were up- or downregulated in these three types of poxviruses infected mouse RAW cells. To further investigate the underlying common regulation mechanisms among these poxviruses, we constructed venn diagrams in order to select out the probe sets with common expression alteration [Figure 6.25-6.26].

Since only small number of probe sets with stimulated expression was identified in mouse RAW cells after infections at $2 \mathrm{hpi}$, no probe set was finally detected with common stimulation in its expression in all these three poxviruses infected mouse RAW cells. Twenty probe sets with up-regulated expression $(\geq 2$-FC\& $<5$-FC) were observed upon infections of cowpox and ectromelia virus, which might represent the existence of some common host reactions upon these two viruses infection at early infection stage.

With more up-regulated probe sets detected at $16 \mathrm{hpi}, 65$ probe sets were found to be commonly up-regulated $(\geq 2-\mathrm{FC})$ in these three types of poxviruses infected mouse RAW cells. This batch of up-regulated probe sets played functions on "Cell Cycle", "Cell Death", "Kinase”, "RNA Binding” and "Actin", and common expression elevation of these gene implied that infected mouse RAW cells fighted with infections of poxviruses through initiating similar mechanisms. 
Among these probe sets, the probe sets encoding Lats2 and Hspalb were of particular interest as their fold regulations were at $\geq 5$-FC in all three types of poxviruses infected mouse RAW cells. Lats2, large tumor suppressor 2, is an AGC kinase of the NDR family of kinases which inhibits cell growth at the G1/S transition by down-regulating cyclin E/CDK2 kinase activity. The corresponding probe sets was elevated with 7.6-FC under lister virus infection, 5.6-FC under cowpox virus infection and 20.18-FC under ectromelia infection at 16 hpi. High-level fold regulation of this probe sets, especially in ectromelia infected mouse RAW cells, might be treated as an indication that the cell cycle was arrested at $\mathrm{G} 1 / \mathrm{S}$ checkpoint in reaction to these poxviruses infections.

The other gene Hspalb is a member of heat shock protein 70 family, which is involved in the ubiquitin-proteasome pathway through interaction with the AU-rich element RNA-binding protein 1. The corresponding probe set was up-regulated with around 200-FC and 50-FC upon lister and ectromelia virus infections. Outstanding expression elevation of this probe set implicated the potential induction of cell death activities, which might be responsible to impede the virus replication in mouse RAW host cells. Up-regulated expression of this gene with 15-FC after cowpox virus infection also implicated moderate apoptosis regulation in the corresponding host cells.

At 2 hpi, only 10 probe sets $(6.1 \%$ for $163,3.0 \%$ for 385 and $2.1 \%$ for 478) were commonly down-regulated with $\geq 2$-FC in mouse RAW cells after infections of three types of poxviruses. And this indicated that the negative control of gene expression at the early infection stage was different from each other among three types of poxviruses infections in infected mouse RAW cells.

Different from the situation happened at 2 hpi, 4539 probe sets $(57.9 \%$ for $7845,75.2 \%$ for 6034 and $45.9 \%$ for 9880 ) were identified with common down-regulation $(\geq 2$-FC) in their expression in three poxviruses infected mouse RAW cells. What's more, 40 probe sets showed down-regulated expression with fold regulation $\geq 10$-FC after infections of all these three types of poxviruses. Functional annotation suggested that these 4539 probe sets were functional associated with "RNA binding", "Protein metabolism", "Kinase", “Cell Cycle”, “Cell Death", "Signal transduction”, "DNA transcription factor" and "Phosphatase", while those 40 probe sets were closely related to “Antiviral", "Cell Death", "Cell Cycle" and "Kinase". 
These 40 commonly down-regulated probe sets with high-level fold changes encoded genes including Ifitm3/6, Cadm1, Clec4e/5a, Fabp5 and so on. Among them, genes such as Mpeg1, H2-K1, and Msr1 are associated with macrophage function. Besides, other transmembrane gene such as Tm6sf1 also showed repressed expression in these poxviruses infected mouse RAW cells. 
ID Associated Network Functions Score

Lipid Metabolism, Molecular Transport, Small Molecule Biochemistry
Gene Expression, Cellular Function and Maintenance, Cellular Growth and Proliferation
Gene Expression, Cell-To-Cell Signaling and Interaction, Cellular Development
Cardiovascular System Development and Function, Cellular Movement, Organismal Development
Post-Translational Modification, Cellular Response to Therapeutics, Connective Tissue Development and Function

Diseases and Disorders

Name

Renal and Urological Disease

p-value

3.04E-05- $1.25 \mathrm{E}-02$

$6.47 \mathrm{E}-05-1.25 \mathrm{E}-02$

$6.47 \mathrm{E}-05-1.25 \mathrm{E}-02$

Immunological Disease

Cardiovascular Disease

$8.45 \mathrm{E}-05-1.25 \mathrm{E}-02$

Inflammatory Response

$1.03 \mathrm{E}-04-1.25 \mathrm{E}-02$

\# Molecules

Molecular and Cellular Functions

Name

\section{Cellular Development}

Cellular Growth and Proliferation

Cellular Function and Maintenance

Cell Death

Cell Cycle

\begin{tabular}{cl} 
p-value & \# Molecules \\
\hline $2.00 \mathrm{E}-07-1.25 \mathrm{E}-02$ & 47 \\
$4.16 \mathrm{E}-05-1.25 \mathrm{E}-02$ & 61 \\
$4.46 \mathrm{E}-05-1.25 \mathrm{E}-02$ & 36 \\
$5.48 \mathrm{E}-05-1.25 \mathrm{E}-02$ & 58 \\
$6.47 \mathrm{E}-05-1.25 \mathrm{E}-02$ & 11
\end{tabular}

Physiological System Development and Function

Name p-value 28

76

17

29

12

Connective Tissue Development and Function

Tumor Morphology

Skeletal and Muscular System Development and Function

Cardiovascular System Development and Function 2.00E-07 - 1.25E-02

$1.90 \mathrm{E}-05-1.25 \mathrm{E}-02$

4.16E- $05-1.25 \mathrm{E}-02$

$4.89 \mathrm{E}-05-1.25 \mathrm{E}-02$

4.89E-05 - $1.25 \mathrm{E}-02$

Organismal Development

\section{Top Canonical Pathways}

Name

Glycolysis/Gluconeogenesis

Starch and Sucrose Metabolism

Pentose Phosphate Pathway

Inhibition of Angiogenesis by TSP1

Fructose and Mannose Metabolism

\begin{tabular}{cl} 
p-value & Ratio \\
\hline $2.3 \mathrm{E}-03$ & $5 / 130(0.038)$ \\
$3.99 \mathrm{E}-03$ & $4 / 166(0.024)$ \\
$6.08 \mathrm{E}-03$ & $3 / 80(0.038)$ \\
$7.29 \mathrm{E}-03$ & $3 / 39(0.077)$ \\
$1.75 \mathrm{E}-02$ & $3 / 134(0.022)$
\end{tabular}

\section{Top Transcription Factors}

\begin{tabular}{lcc} 
Transcription Regulator & p-value of overlap & $\begin{array}{l}\text { Predicted } \\
\text { Activation } \\
\text { State }\end{array}$ \\
\hline STAT4 & & Inhibited \\
HIF1A & $3.80 \mathrm{E}-16$ & Inhibited \\
EPAS1 & $7.58 \mathrm{E}-11$ & Inhibited \\
ATF4 & $1.54 \mathrm{E}-08$ & \\
SP3 & $1.63 \mathrm{E}-07$ &
\end{tabular}

Figure 6.13 Summary of top functional networks of differentially expressed genes in cowpox virus-infected mouse RAW cells at 2 hpi. Differentially expressed genes were significantly categorized into different networks, functions and pathways under the analysis of Ingenuity Pathway Analysis (IPA) version 2012 (P-value $\leq 0.05$ ). 
A

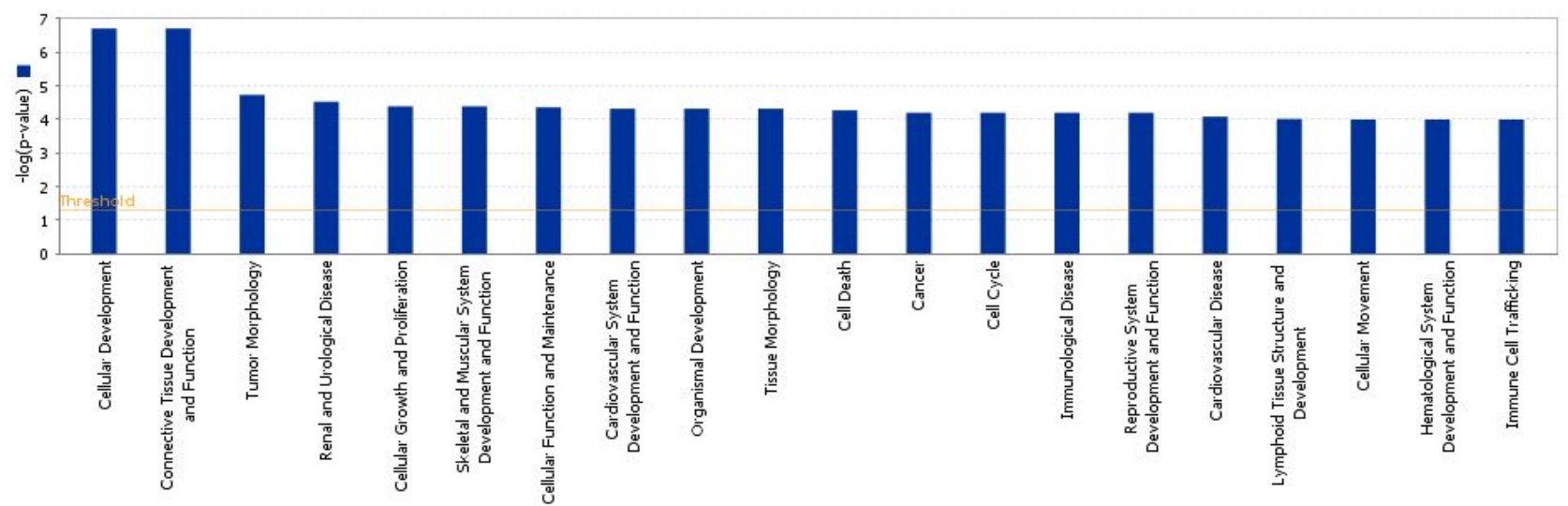

B

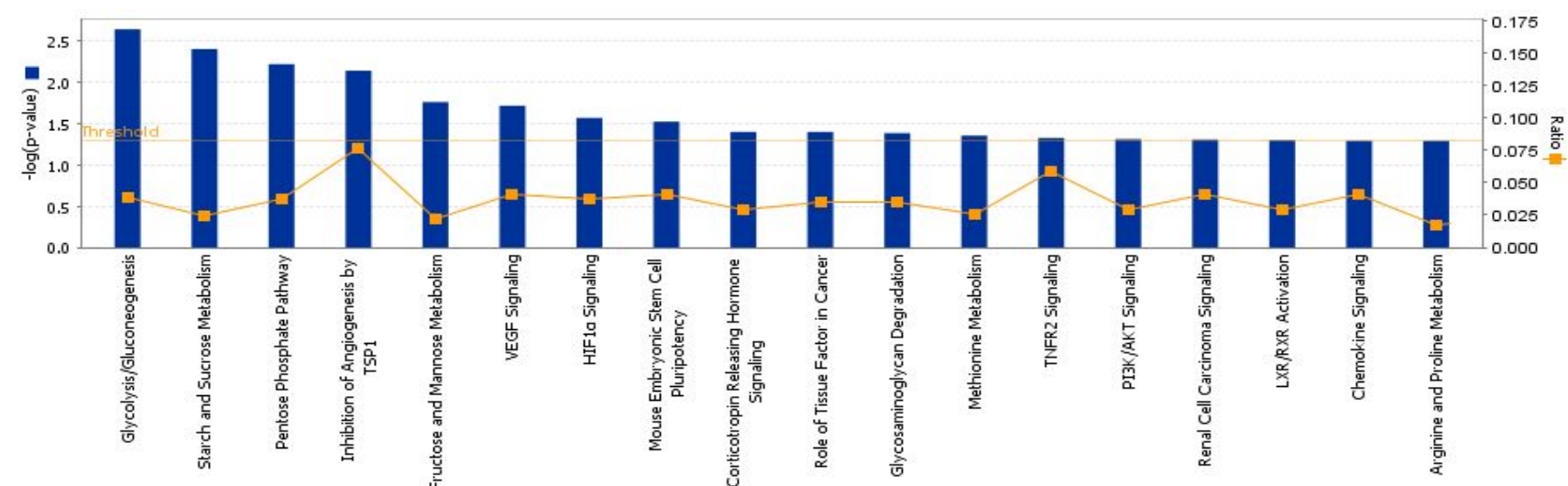

Figure 6.14 Top 20 (A) biological functions and (B) canonical pathways significantly enriched in differentially expressed genes from cowpox virus-infected mouse RAW cells at $\mathbf{2}$ hpi (P-value $\leq \mathbf{0 . 0 5})$. Threshold was set at $\mathrm{p}$-value $=0.05$ and indicated as $-\log$ (p-value) on the $\mathrm{Y}$-axis and the $\mathrm{X}$-axis shows the terms of each biological function or canonical pathway. The orange boxes indicate the ratio (Ratio) of the number of genes with differential expression changes and the total number of genes in the respective canonical pathway. 


\section{Top Networks}

ID Associated Network Functions

Score

$\begin{array}{ll}\text { Genetic Disorder, Metabolic Disease, Developmental Disorder } & 28 \\ \text { Cellular Assembly and Organization, Cell Cycle, Molecular Transport } & 28 \\ \text { Hematological Disease, Immunological Disease, Small Molecule Biochemistry } & 28 \\ \text { Cellular Compromise, Cell Cycle, Cellular Assembly and Organization } & 28 \\ \text { Protein Degradation, Protein Synthesis, RNA Post-Transcriptional Modification } & 28\end{array}$

\section{Top Bio Functions}

Diseases and Disorders

Name p-value \# Molecules

Infectious Disease $\quad 9.92 \mathrm{E}-14-7.31 \mathrm{E}-03 \quad 690$

Cardiovascular Disease $\quad 6.77 \mathrm{E}-10-2.71 \mathrm{E}-03 \quad 31$

Genetic Disorder $\quad 6.77 \mathrm{E}-10-8.77 \mathrm{E}-03 \quad 519$

Metabolic Disease $\quad 6.77 \mathrm{E}-10-5.80 \mathrm{E}-03 \quad 230$

$\begin{array}{lrr}\text { Neurological Disease } & 6.77 \mathrm{E}-10-5.80 \mathrm{E}-03 & 64\end{array}$

Molecular and Cellular Functions

Name

Cell Cycle

RNA Post-Transcriptional Modification

Cellular Assembly and Organization

DNA Replication, Recombination, and Repair

Cellular Movement

$\begin{array}{cc}\text { p-value } & \text { \# } \\ \text { 7.67E-18 - 8.77E-03 } & 703 \\ 1.29 \mathrm{E}-13-5.71 \mathrm{E}-03 & 17 \\ 1.43 \mathrm{E}-12-8.77 \mathrm{E}-03 & 70 \\ 1.43 \mathrm{E}-12-6.00 \mathrm{E}-03 & 57 \\ 3.78 \mathrm{E}-12-8.77 \mathrm{E}-03 & 91\end{array}$

Physiological System Development and Function

Name p-value $1.32 \mathrm{E}-05-8.77 \mathrm{E}-03$ 1.37E-05- $-7.04 \mathrm{E}-03$ $9.26 \mathrm{E}-05-5.89 \mathrm{E}-03$ $5.71 \mathrm{E}-04-6.53 \mathrm{E}-03$ $5.71 \mathrm{E}-04-6.53 \mathrm{E}-03$

Connective Tissue Development and Function

Hematological System Development and Function

Embryonic Development

Lymphoid Tissue Structure and Development

Organ Morphology

28

28

28

\section{Top Canonical Pathways}

Name

Oxidative Phosphorylation

Mitochondrial Dysfunction

Ubiquinone Biosynthesis

Protein Ubiquitination Pathway

Hereditary Breast Cancer Signaling

p-value Ratio

1.24E-27 96/159 $(0.604)$

1.7E-21 91/174 (0.523)

6.33E-15 $46 / 110(0.418$

2.55E-10 $133 / 269$

3.42E-10 70/127 $(0.551)$

\section{Top Transcription Factors}

\begin{tabular}{lcc} 
Transcription Regulator & p-value of overlap & $\begin{array}{c}\text { Predicted } \\
\text { Activation } \\
\text { State }\end{array}$ \\
\hline HNF4A & $3.88 E-31$ & \\
MYC & 1.56 E-19 & Inhibited \\
E2F4 & $5.30 E-19$ & \\
XBP1 (includes EG:140614) & $3.89 E-13$ & \\
POU4F1 & $6.26 \mathrm{E}-13$ &
\end{tabular}

Figure 6.15 Summary of top functional networks of differentially expressed genes in cowpox virus-infected mouse RAW cells at 16 hpi. Differentially expressed genes were significantly categorized into different networks, functions and pathways under the analysis of Ingenuity Pathway Analysis (IPA) version 2012 (P-value $\leq 0.05$ ). 
A

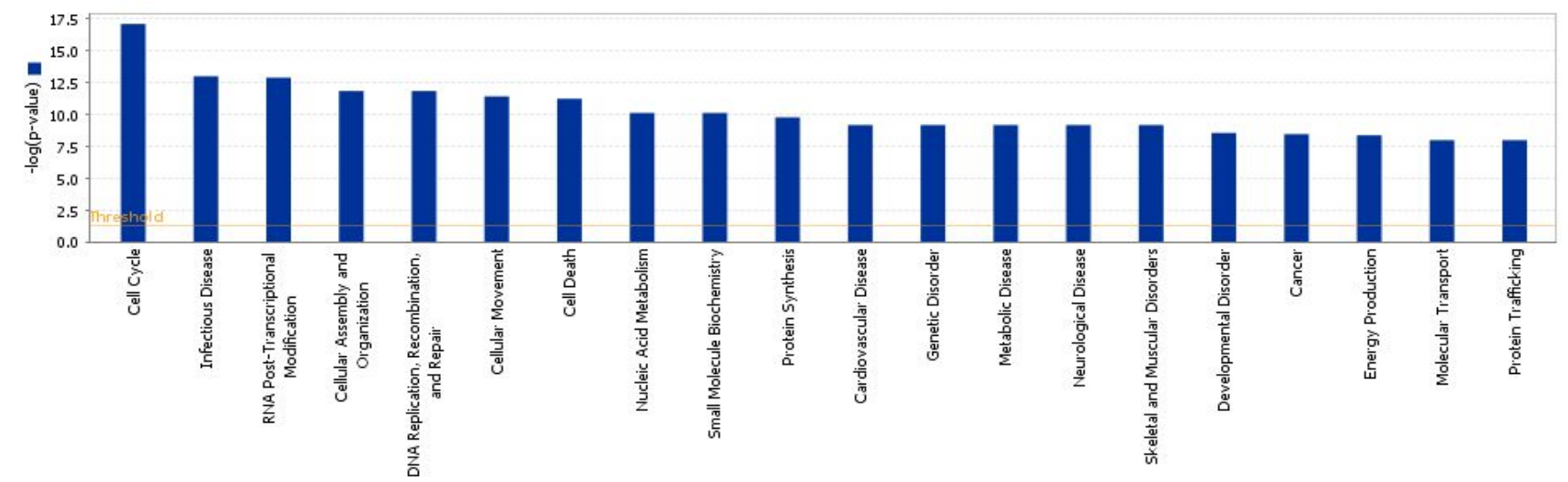

B

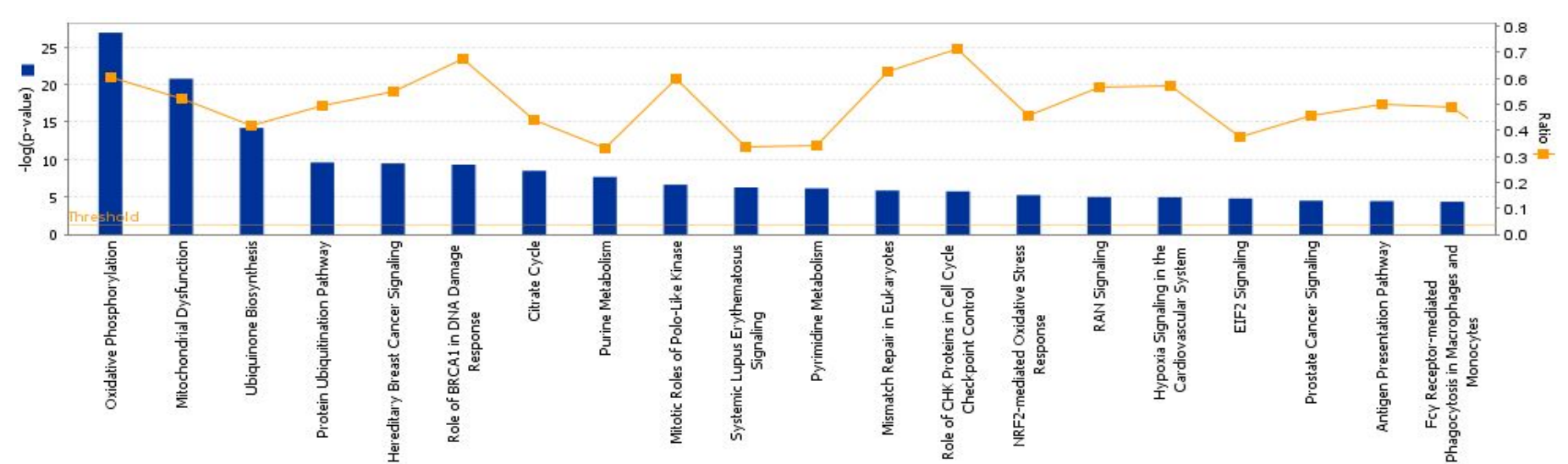

Figure 6.16 Top 20 (A) biological functions and (B) canonical pathways significantly enriched in differentially expressed genes from cowpox virus-infected mouse RAW cells at 16 hpi (P-value $\leq \mathbf{0 . 0 5})$. Threshold was set at $\mathrm{p}$-value $=0.05$ and indicated as $-\log$ (p-value) on the $\mathrm{Y}$-axis and the $\mathrm{X}$-axis shows the terms of each biological function or canonical pathway. The orange boxes indicate the ratio (Ratio) of the number of genes with differential expression changes and the total number of genes in the respective canonical pathway. 
ID Associated Network Functions $\quad$ Score

1 Gene Expression, Molecular Transport, RNA Trafficking 43

2 Gene Expression, Cellular Development, Connective Tissue Development and Function 41

3 Post-Translational Modification, Connective Tissue Disorders, Immunological Disease 40

4 Cancer, Cellular Development, Organ Development 54

Genetic Disorder, Neurological Disease, Connective Tissue Disorders 34

Top Bio Functions

Diseases and Disorders

Name

p-value \# Molecules

Cardiovascular Disease

$8.86 \mathrm{E}-05-6.60 \mathrm{E}-03 \quad 6$

$8.86 \mathrm{E}-05-1.31 \mathrm{E}-02 \quad 10$

Organismal Injury and Abnormalities

$2.50 \mathrm{E}-04-1.75 \mathrm{E}-02$

Connective Tissue Disorders

$2.50 \mathrm{E}-04-2.64 \mathrm{E}-02 \quad 30$

Immunological Disease

Inflammatory Disease

$2.50 \mathrm{E}-04-2.64 \mathrm{E}-02$

Molecular and Cellular Functions

Name

Gene Expression

p-value 3.17E-13-1.94E-02 130

1.34E-06 - 2.64E-02 72

Cell Cycle

$3.61 \mathrm{E}-06-2.64 \mathrm{E}-02 \quad 124$

Cellular Growth and Proliferation

$1.30 \mathrm{E}-05-2.64 \mathrm{E}-02 \quad 119$

Cell Death

$5.43 \mathrm{E}-05-2.64 \mathrm{E}-02 \quad 13$

Physiological System Development and Function

Name

Hepatic System Development and Function

p-value $3.61 \mathrm{E}-06-2.43 \mathrm{E}-02 \quad 24$

$4.82 \mathrm{E}-06-2.45 \mathrm{E}-02 \quad 67$

Hematological System Development and Function

$4.82 \mathrm{E}-06-2.44 \mathrm{E}-02$

Skeletal and Muscular System Development and Function

$5.43 \mathrm{E}-05-2.64 \mathrm{E}-02$

Connective Tissue Development and Function

$9.94 \mathrm{E}-05-2.64 \mathrm{E}-02$

\section{Top Canonical Pathways}

Name

IL-17A Signaling in Fibroblasts

p-value

Cell Cycle: G1/S Checkpoint Regulation

Telomere Extension by Telomerase

Role of CHK Proteins in Cell Cycle Checkpoint Control

Cell Cycle Regulation by BTG Family Proteins

$6 / 65(0.092)$

6.57E-03 $\quad 3 / 17(0.176)$

1.07E-02 $\quad 4 / 35(0.114)$

1.19E-02 $\quad 4 / 36(0.111)$

Top Transcription Factors

\begin{tabular}{|c|c|c|}
\hline Transcription Regulator & $p$-value of overlap & $\begin{array}{l}\text { Predicted } \\
\text { Activation } \\
\text { State }\end{array}$ \\
\hline$\overline{\mathrm{DACH} 1}$ & $8.51 \mathrm{E}-08$ & \\
\hline MNT & $1.12 \mathrm{E}-05$ & \\
\hline SIN3B & $1.34 \mathrm{E}-05$ & \\
\hline TP53 (includes EG:22059) & 2.04E-05 & \\
\hline ELK1 & 2.33E-05 & \\
\hline
\end{tabular}

Figure 6.17 Summary of top functional networks of differentially expressed genes in lister virus-infected mouse RAW cells at 2 hpi. Differentially expressed genes were significantly categorized into different networks, functions and pathways under the analysis of Ingenuity Pathway Analysis (IPA) version 2012 (P-value $\leq 0.05$ ). 
A

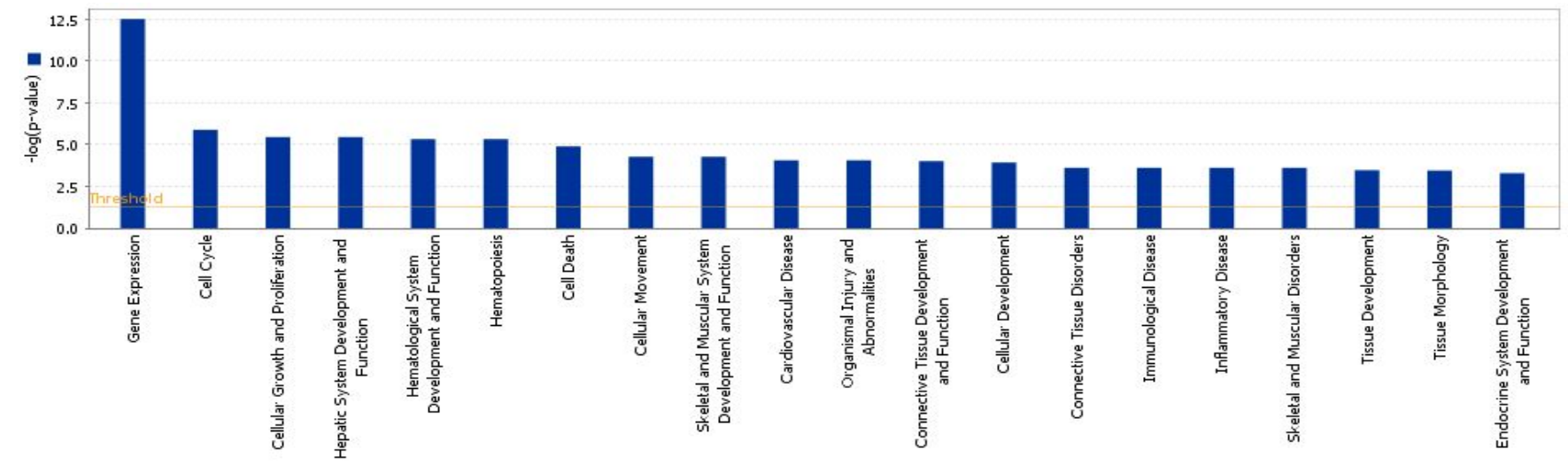

B

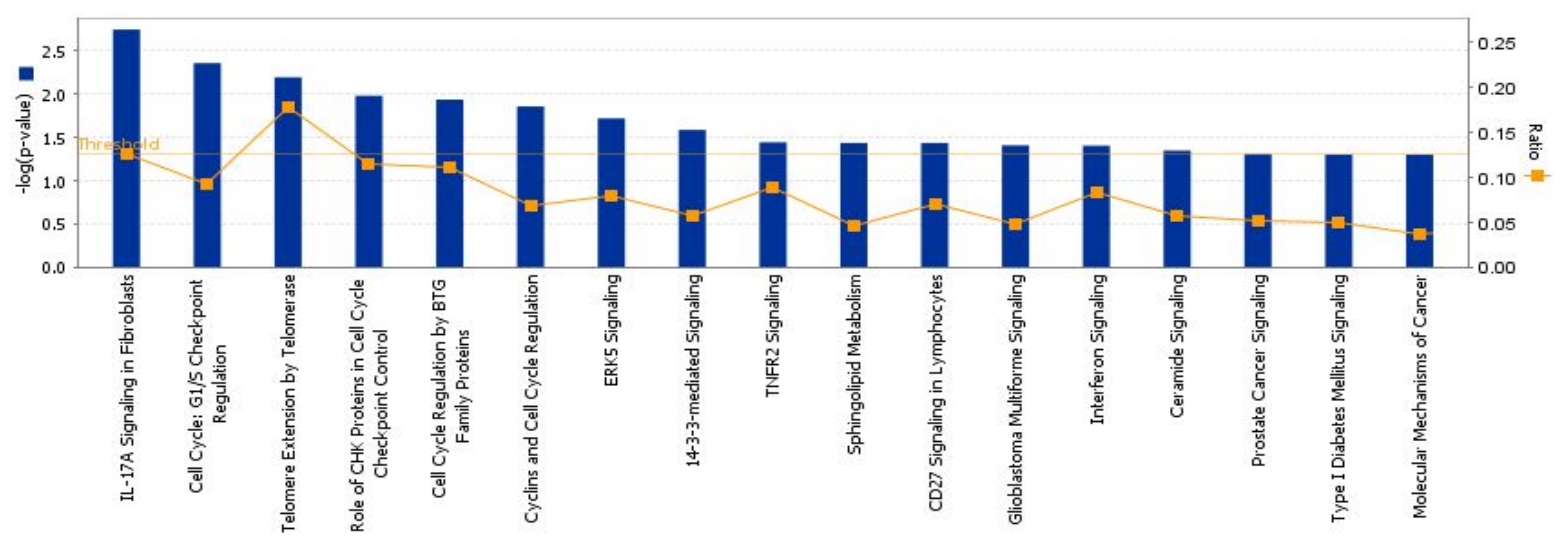

Figure 6.18 Top 20 (A) biological functions and (B) canonical pathways significantly enriched in differentially expressed genes from lister virus-infected mouse RAW cells at $\mathbf{2}$ hpi $(\mathbf{P}$-value $\leq \mathbf{0 . 0 5})$. Threshold was set at $\mathrm{p}$-value $=0.05$ and indicated as $-\log (\mathrm{p}$-value) on the $\mathrm{Y}$-axis and the $\mathrm{X}$-axis shows the terms of each biological function or canonical pathway. The orange boxes indicate the ratio (Ratio) of the number of genes with differential expression changes and the total number of genes in the respective canonical pathway. 


\section{Top Networks}

ID Associated Network Functions $\quad$ Score

1 Infectious Disease, DNA Replication, Recombination, and Repair, Molecular Transport 26

2 Carbohydrate Metabolism, Cardiac Dilation, Cardiovascular System Development and Function 26

RNA Post-Transcriptional Modification, Dermatological Diseases and Conditions, Infectious Disease 26

Cell Cycle, Cellular Assembly and Organization, Genetic Disorder 26

Genetic Disorder, Renal and Urological Disease, Ophthalmic Disease

Top Bio Functions

Diseases and Disorders

Name

Infectious Disease

p-value

5.11E-15-8.04E-03 814

$2.74 \mathrm{E}-13-1.87 \mathrm{E}-02 \quad 207$

Organismal Injury and Abnormalities

Dermatological Diseases and Conditions

$2.74 \mathrm{E}-13-1.87 \mathrm{E}-02$
$6.54 \mathrm{E}-13-6.54 \mathrm{E}-13$

$1.64 \mathrm{E}-12-1.52 \mathrm{E}-02-277$

Renal and Urological Disease

$2.00 \mathrm{E}-08-1.22 \mathrm{E}-02 \quad 3$

\# Molecules

Cardiovascular Disease

Molecular and Cellular Functions

Name

RNA Post-Transcriptional Modification

p-value

Cell Cycle

Molecular Transport

Protein Trafficking

9.57E-24 - 6.75E-03

$4.03 \mathrm{E}-19-1.82 \mathrm{E}-02$

$2.48 \mathrm{E}-14-1.27 \mathrm{E}-02$

218

$2.48 \mathrm{E}-14-4.92 \mathrm{E}-03 \quad 230$

Gene Expression

$3.67 \mathrm{E}-14-1.19 \mathrm{E}-02$

Physiological System Development and Function

Name p-value 4.58E-07 - 1.66E- 02 4.58E-07- $-4.92 \mathrm{E}-03$ 8.27E- $05-4.33 \mathrm{E}-04$

Tissue Development

Organismal Development

Hematological System Development and Function

1.55E-04 - 1.80E-02

$1.96 \mathrm{E}-04-1.82 \mathrm{E}-02$

Connective Tissue Development and Function

60
92

201

236

\section{Top Canonical Pathways}

Name

Oxidative Phosphorylation

Mitochondrial Dysfunction

EIF2 Signaling

Protein Ubiquitination Pathway

3.29E-22 $97 / 159(0.61)$

6.87E-19 95/174 (0.546)

8.04E-15 108/205

Ubiquinone Biosynthesis

4.24E-14 $\quad 159 / 269$

$6.51 \mathrm{E}-11 \quad 45 / 110(0.409)$

Top Transcription Factors

Transcription Regulator

-value of overlap Predicted

Activation

\begin{tabular}{llc} 
& & Activation \\
\hline HNF4A & $1.14 \mathrm{E}-44$ & \\
MYC & $4.06 \mathrm{E}-24$ & Inhibited \\
E2F4 & $2.10 \mathrm{E}-21$ & \\
ISL1 & $1.56 \mathrm{E}-14$ & \\
POU4F1 & $3.32 \mathrm{E}-14$ &
\end{tabular}

Figure 6.19 Summary of top functional networks of differentially expressed genes in lister virus-infected mouse RAW cells at 16 hpi. Differentially expressed genes were significantly categorized into different networks, functions and pathways under the analysis of Ingenuity Pathway Analysis (IPA) version 2012 (P-value $\leq 0.05$ ). 
A

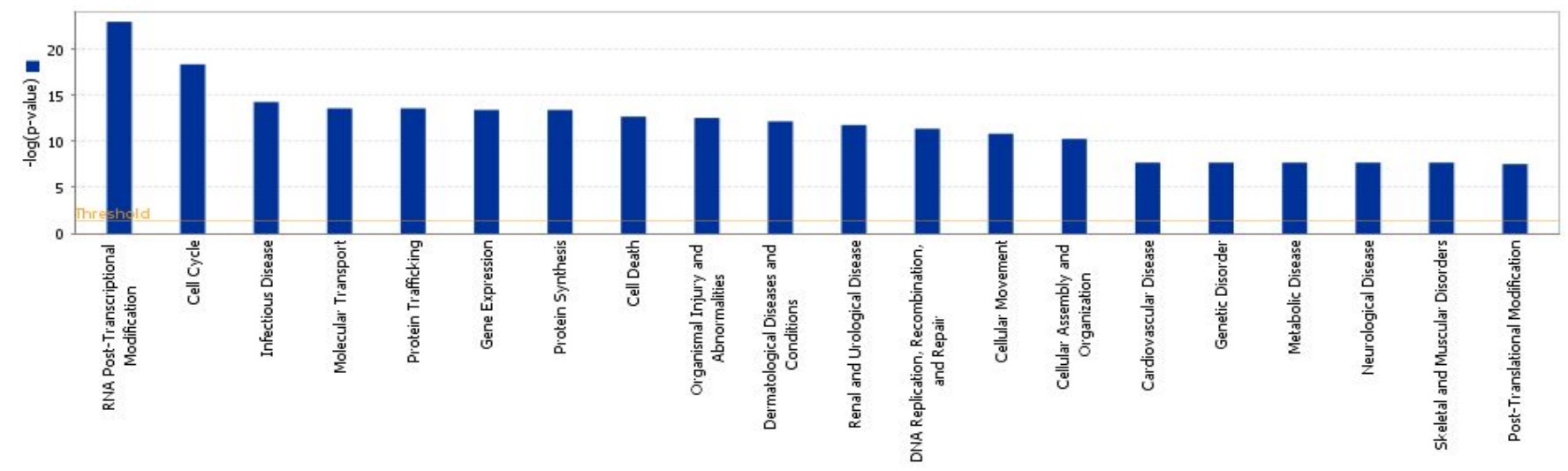

$\mathrm{B}$

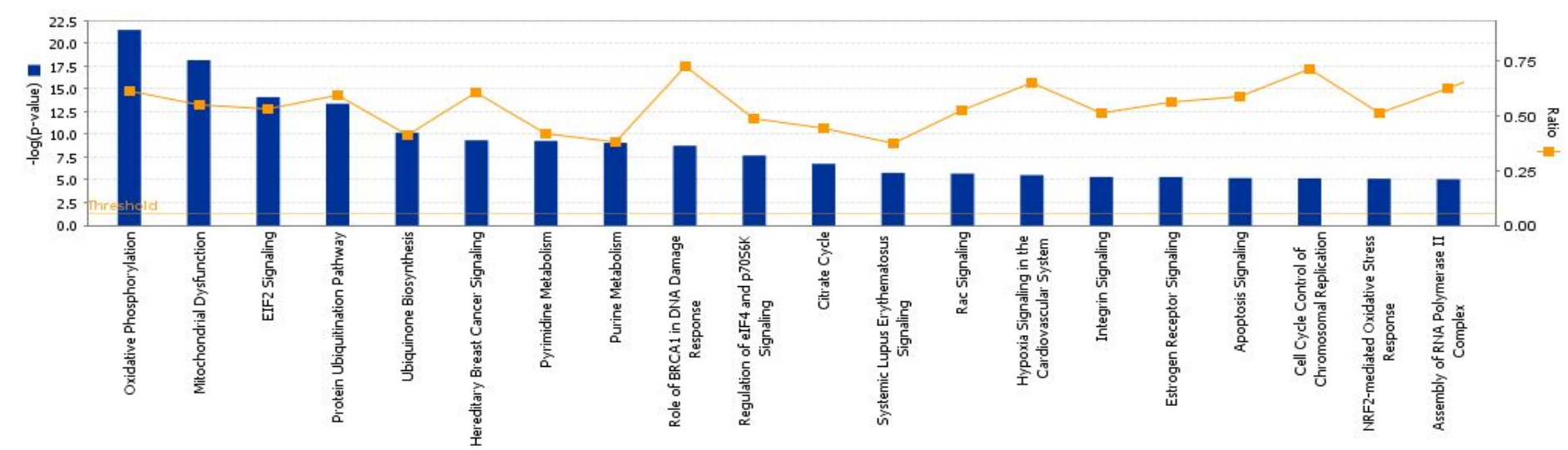

Figure 6.20 Top 20 (A) biological functions and (B) canonical pathways significantly enriched in differentially expressed genes from lister virus-infected mouse RAW cells at 16 hpi (P-value $\leq \mathbf{0 . 0 5})$. Threshold was set at $p$-value $=0.05$ and indicated as $-\log$ (p-value) on the $Y$-axis and the X-axis shows the terms of each biological function or canonical pathway. The orange boxes indicate the ratio (Ratio) of the number of genes with differential expression changes and the total number of genes in the respective canonical pathway. 
ID Associated Network Functions

Score

Cell Morphology, Cellular Function and Maintenance, Cell Death
Hematological System Development and Function, Hematopoiesis, Tissue Morphology
Cell Cycle, Cancer, Reproductive System Development and Function
Cellular Development, Cellular Growth and Proliferation, Cancer
Cellular Development, Cellular Growth and Proliferation, Hematological System Development and Function

Cellular Development, Cellular Growth and Proliferation, Hematological System Development and Function

38

35

32
32

\section{Top Bio Functions}

Diseases and Disorders

Name

p-value \# Molecules

Immunological Disease $1.38 \mathrm{E}-06-2.02 \mathrm{E}-02$

2.54E-06 - 2.01E-02

1.22E-04 - 2.02E-02

$1.22 \mathrm{E}-04-2.02 \mathrm{E}-02$

Endocrine System Disorders

Hematological Disease

$1.55 \mathrm{E}-04-2.02 \mathrm{E}-02$

Ophthalmic Disease

Molecular and Cellular Functions

Name

Cell Death

p-value

Cellular Development

Cell Cycle

Cellular Growth and Proliferation

Cellular Function and Maintenance

p-value
$2.43 \mathrm{E}-07-2.02 \mathrm{E}-02$
$3.25 \mathrm{E}-07-2.02 \mathrm{E}-02$
$1.60 \mathrm{E}-06-2.02 \mathrm{E}-02$
$4.17 \mathrm{E}-05-2.02 \mathrm{E}-02$
$1.24 \mathrm{E}-04-2.02 \mathrm{E}-02$

Physiological System Development and Function

Name

Connective Tissue Development and Function

p-value

Embryonic Development

Endocrine System Development and Function

Organ Development

Organismal Development

3.25E-07 - 2.02E-02

$4.80 \mathrm{E}-06-202 \mathrm{E}-02$

$4.80 \mathrm{E}-06-1.70 \mathrm{E}-03$

$4.80 \mathrm{E}-06-2.02 \mathrm{E}-02$

4. $80 \mathrm{E}-06-2.02 \mathrm{E}-02$

\# Molecules

33

10

22

28

Top Canonical Pathways

Name

Fructose and Mannose Metabolism

p-value

Ratio

Biosynthesis of Steroids

Aminosugars Metabolism

Glycolysis/Gluconeogenesis

TNFR2 Signaling

2.53E-05 7/134 (0.052)

8.25E-03 $3 / 122(0.025)$

$1.26 \mathrm{E}-02 \quad 5 / 120(0.042)$

$1.67 \mathrm{E}-02 \quad 5 / 130(0.038)$

$1.83 \mathrm{E}-02 \quad 3 / 34(0.088)$

\section{Top Transcription Factors}

\begin{tabular}{|c|c|c|}
\hline Transcription Regulator & p-value of overlap & $\begin{array}{l}\text { Predicted } \\
\text { Activation } \\
\text { State } \\
\end{array}$ \\
\hline STAT4 & $5.53 \mathrm{E}-10$ & \\
\hline HIF1A & $5.85 \mathrm{E}-08$ & \\
\hline ATF4 & $3.02 \mathrm{E}-07$ & \\
\hline EPAS1 & 3.85E-06 & \\
\hline FOXO4 & 3.43E-05 & \\
\hline
\end{tabular}

Figure 6.21 Summary of top functional networks of differentially expressed genes in ectromelia virus-infected mouse RAW cells at 2 hpi. Differentially expressed genes were significantly categorized into different networks, functions and pathways under the analysis of Ingenuity Pathway Analysis (IPA) version 2012 (P-value $\leq 0.05$ ). 
A

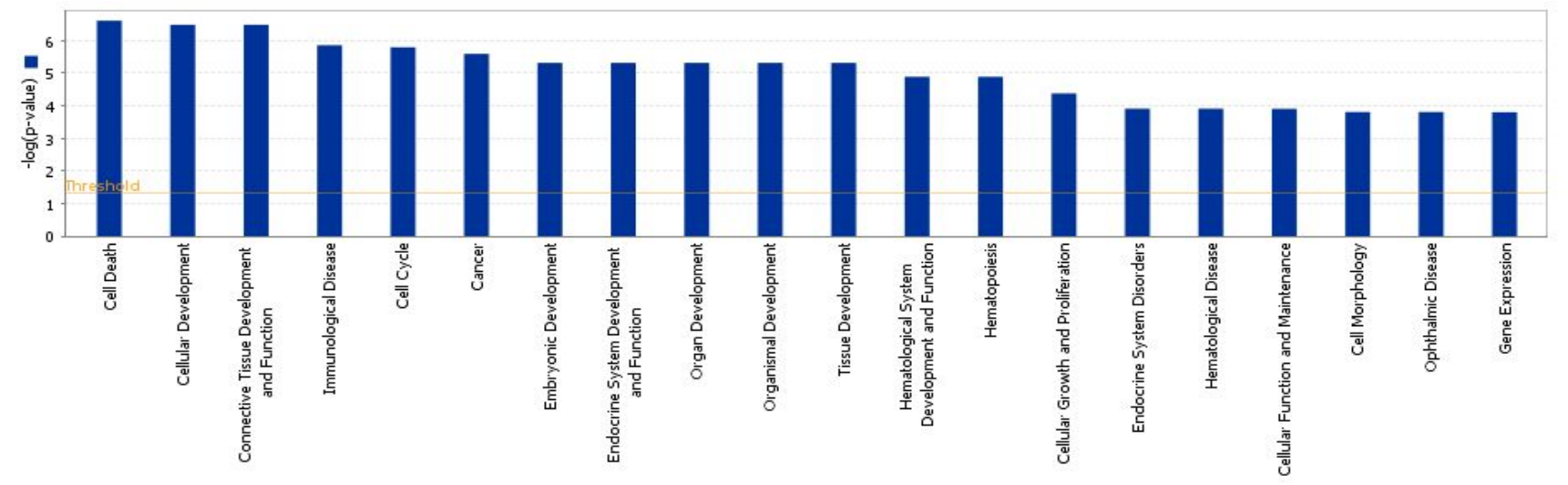

B

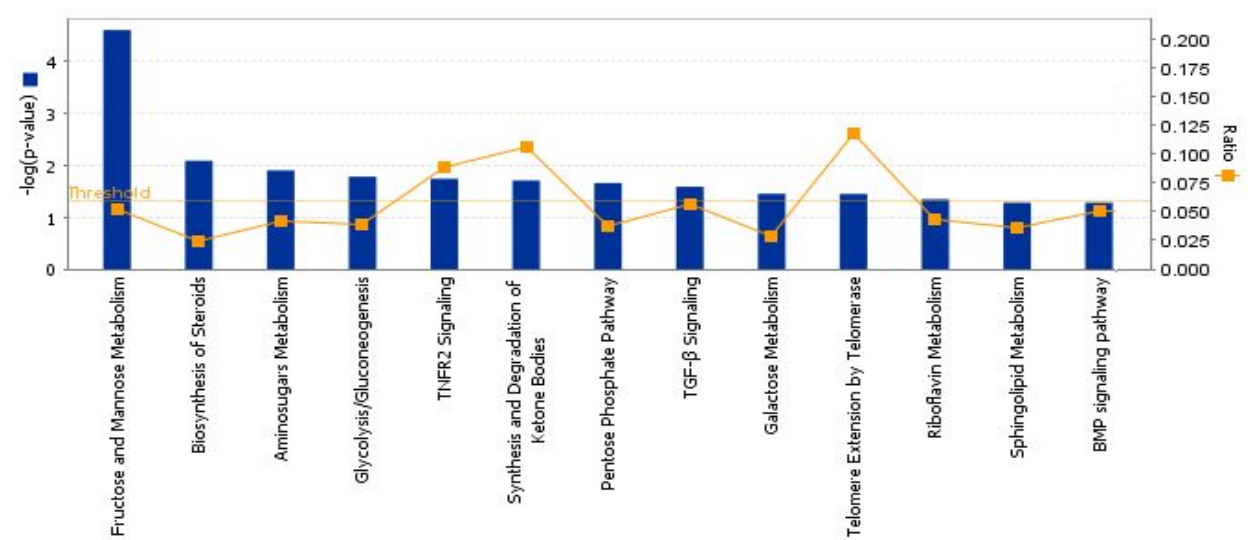

Figure 6.22 Top 20 (A) biological functions and (B) canonical pathways significantly enriched in differentially expressed genes from ectromelia virus-infected mouse RAW cells at 2 hpi (P-value $\leq \mathbf{0 . 0 5})$. Threshold was set at $\mathrm{p}$-value $=0.05$ and indicated as $-\log (\mathrm{p}$-value) on the $\mathrm{Y}$-axis and the $\mathrm{X}$-axis shows the terms of each biological function or canonical pathway. The orange boxes indicate the ratio (Ratio) of the number of genes with differential expression changes and the total number of genes in the respective canonical pathway. 


\section{Top Networks}

ID Associated Network Functions $\quad$ Score

1 Protein Degradation, Protein Synthesis, Cellular Assembly and Organization 30

2 Carbohydrate Metabolism, Small Molecule Biochemistry, Developmental Disorder 30

Lipid Metabolism, Nucleic Acid Metabolism, Small Molecule Biochemistry 30

$\begin{array}{ll}\text { Genetic Disorder, Metabolic Disease, Hematological Disease } & 30\end{array}$

Molecular Transport, RNA Trafficking, RNA Damage and Repair 28

\section{Top Bio Functions}

Diseases and Disorders

Name

p-value \# Molecules

Dermatological Diseases and Conditions

9.44E-15-1.39E-02

Organismal Injury and Abnormalities

$1.07 \mathrm{E}-13-870 \mathrm{E}-03$

$1.07 \mathrm{E}-13-9.87 \mathrm{E}-03$

$3.34 \mathrm{E}-13-9.97 \mathrm{E}-03$

188

Renal and Urological Disease

Cancer

$1.21 \mathrm{E}-06-1.36 \mathrm{E}-02 \quad 1193$

Molecular and Cellular Functions

Name

\begin{tabular}{lll} 
Name & p-value & \# Molecules \\
\hline RNA Post-Transcriptional Modification & $2.24 \mathrm{E}-17-1.39 \mathrm{E}-02$ & 191 \\
Cell Cycle & $1.16 \mathrm{E}-14-1.39 \mathrm{E}-02$ & 663 \\
Protein Synthesis & $2.60 \mathrm{E}-13-1.14 \mathrm{E}-02$ & 402 \\
Gene Expression & $3.65 \mathrm{E}-12-1.35 \mathrm{E}-02$ & 162 \\
Cell Death & $1.92 \mathrm{E}-11-1.39 \mathrm{E}-02$ & 1085
\end{tabular}

Physiological System Development and Function

Name p-value $8.21 \mathrm{E}-06-1.39 \mathrm{E}-02$

$3.55 \mathrm{E}-05-1.25 \mathrm{E}-02$

$1.89 \mathrm{E}-04-1.39 \mathrm{E}-02$

$2.00 \mathrm{E}-04-1.39 \mathrm{E}-02$

Connective Tissue Development and Function

Hematological System Development and Function

$3.45 \mathrm{E}-04-1.82 \mathrm{E}-03$

Organismal Development

260

103

79

\section{Top Canonical Pathways}

Name

p-value

3.76E-21 85/159(0.535)

Mitochondrial Dysfunction

Protein Ubiquitination Pathway

$5.51 \mathrm{E}-17 \quad 81 / 174(0.466)$

5.04E-15 136/269

Ubiquinone Biosynthesis

$1.14 \mathrm{E}-12 \quad 42 / 110(0.382)$

EIF2 Signaling

1.1E-11 87/205 (0.424)

Top Transcription Factors

\begin{tabular}{lcc} 
Transcription Regulator & p-value of overlap & $\begin{array}{c}\text { Predicted } \\
\text { Activation } \\
\text { State }\end{array}$ \\
\hline HNF4A & $2.35 E-23$ & \\
E2F4 & $5.64 E-20$ & Inhibited \\
MYC & $1.91 E-18$ & \\
E2F1 & $1.21 E-13$ & \\
POU4F1 & $1.62 \mathrm{E}-12$ &
\end{tabular}

Figure 6.23 Summary of top functional networks of differentially expressed genes in ectromelia virus-infected mouse RAW cells at $16 \mathrm{hpi}$. Differentially expressed genes were significantly categorized into different networks, functions and pathways under the analysis of Ingenuity Pathway Analysis (IPA) version 2012 (P-value $\leq 0.05$ ). 
A

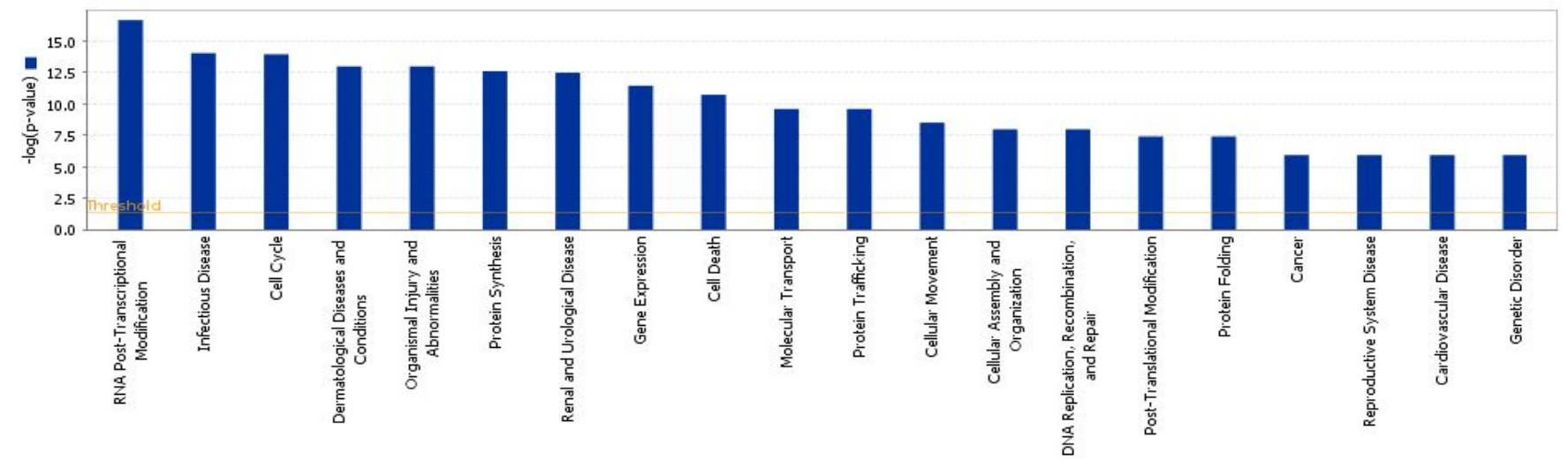

$\mathrm{B}$

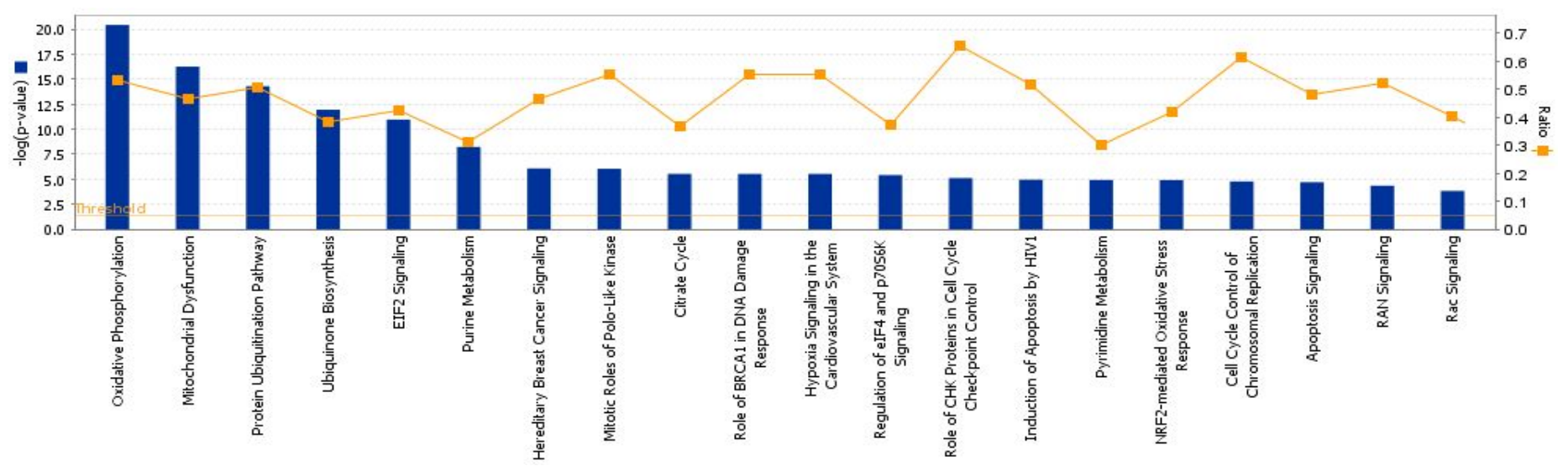

Figure 6.24 Top 20 (A) biological functions and (B) canonical pathways significantly enriched in differentially expressed genes from ectromelia virus-infected mouse RAW cells at $16 \mathbf{~ h p i ~ ( P - v a l u e ~} \leq \mathbf{0 . 0 5})$. Threshold was set at $\mathrm{p}$-value $=0.05$ and indicated as $-\log (\mathrm{p}$-value) on the $\mathrm{Y}$-axis and the $\mathrm{X}$-axis shows the terms of each biological function or canonical pathway. The orange boxes indicate the ratio (Ratio) of the number of genes with differential expression changes and the total number of genes in the respective canonical pathway. 
Poxviruses 2H Up-regulated 2-fold

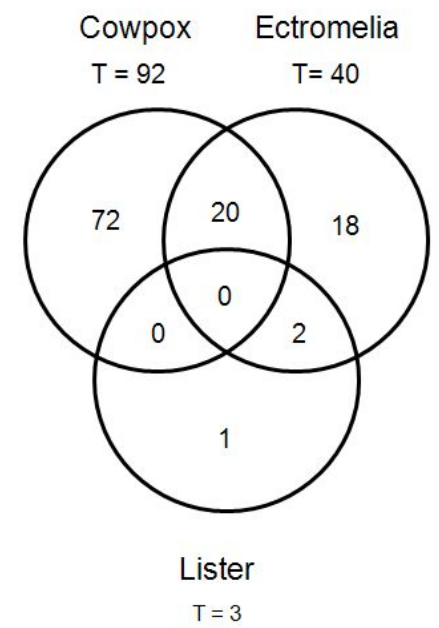

Poxviruses 2H Up-regulated 5-fold

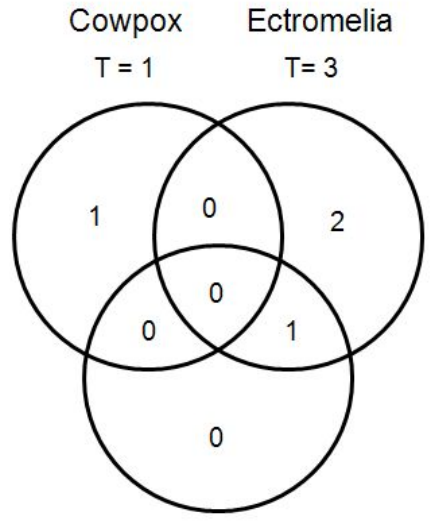

Lister

$\mathrm{T}=1$

Poxviruses 2H Up-regulated 10-fold

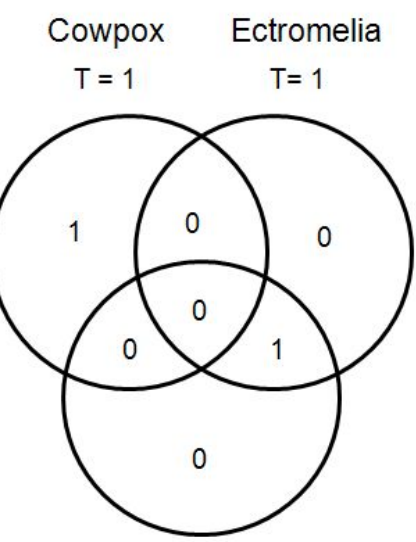

Lister

$T=1$
Poxviruses 16H Up-regulated 2-fold

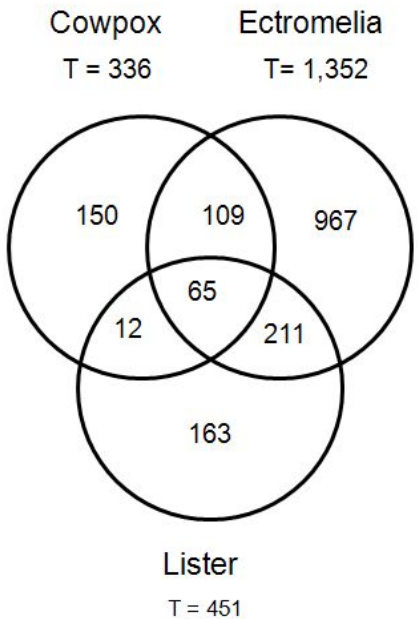

Poxviruses 16H Up-regulated 5-fold

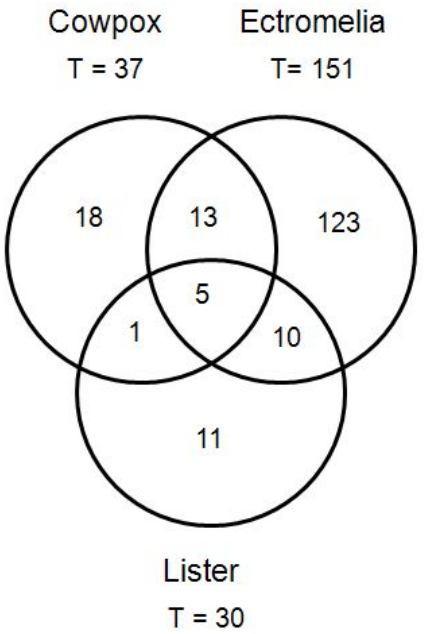

Poxviruses 16H Up-regulated 10-fold

$$
\text { Cowpox Ectromelia }
$$$$
T=11 \quad T=26
$$

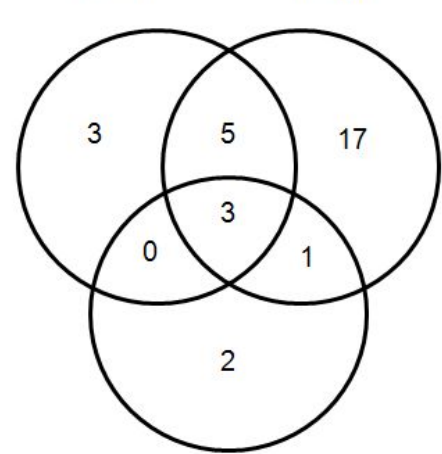

Lister

$T=6$

Figure 6.25 Venn diagram of differentially up-regulated probe sets between three poxviruses infections. A549 cells were infected with cowpox, ectromelia and lister viruses and significantly up-regulated transcripts $(\mathrm{P}$-value $\leq 0.05)$ were overlapped for comparison. Two infection time points including 2 and $16 \mathrm{hpi}$ are shown and the results are based on fold changes at $\geq 2$-FC, $\geq 5$-FC and $\geq 10$-FC. $T$ represents the total number of probe sets with up-regulated expression. 
Poxviruses 2H Down-regulated 2-fold

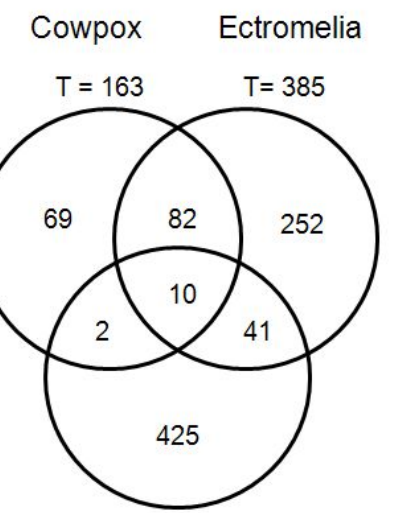

Lister

$\mathrm{T}=478$

Poxviruses 2H Down-regulated 5-fold

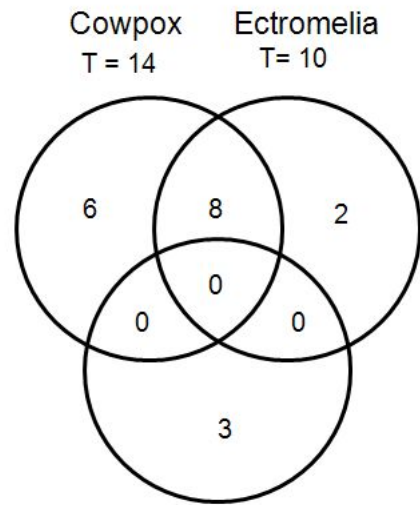

Lister

$T=3$

Poxviruses 2H Down-regulated 10-fold

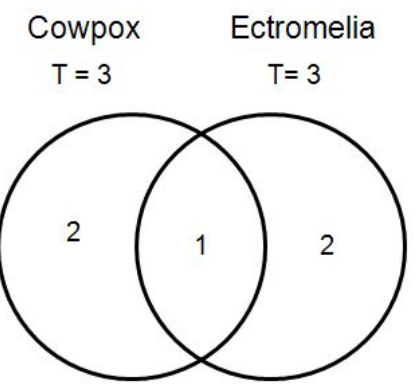

Poxviruses 16H Down-regulated 2-fold

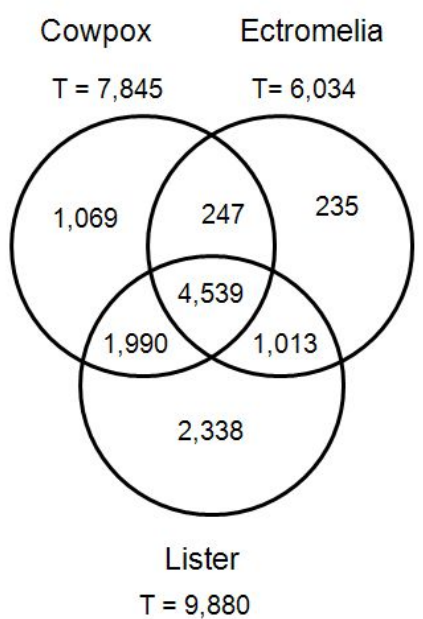

Poxviruses 16H Down-regulated 5-fold

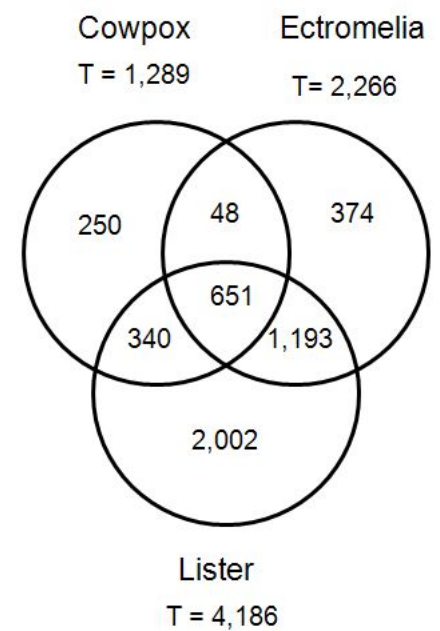

Poxviruses 16H Down-regulated 10-fold

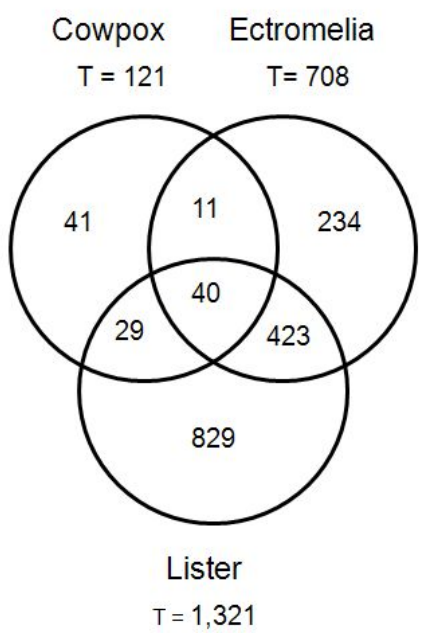

Figure 6.26 Venn diagram of differentially down-regulated probe sets between three poxviruses infections. A549 cells were infected with cowpox, ectromelia and lister viruses and significantly down-regulated transcripts $(\mathrm{P}$-value $\leq 0.05)$ were overlapped for comparison. Two infection time points including 2 and 16 hpi are shown and the results are based on fold changes at $\geq 2$-FC, $\geq 5$-FC and $\geq 10$-FC. $T$ represents the total number of probe sets with downregulated expression. 


\subsubsection{Regulations of gene expression in canonical pathways}

\subsubsection{Interferon signaling}

As described earlier, interferon signaling transduction will be initiated upon the poxvirus infection to induce the production of downstream cytokines. At the meanwhile, poxviruses have also evolved a few strategies to impair the interferon responses which are capable to inhibit virus replication and limit their viral spread [321, 344].

In cowpox virus-infected A549 cells, no expression change was detected across the whole interferon signaling pathway at 2 hpi. However, a big batch of genes with down-regulated expression was suddenly detected at $4 \mathrm{hpi}$, and this number showed increasing with the infection time increasing up to $10 \mathrm{hpi}$. These down-regulated genes included not only the interferon receptors and JAK/STAT but also the downstream ISGs. In addition, the genes in interferon signaling pathways from lister virus-infected A549 cells also exhibited similar expression performances as those from cowpox virus-infected A549 cells, including more and more genes with down-regulated expression identified from 2 to10 hpi.

Thus, the host interferon signaling pathways showed impaired in both poxviruses infected A549 cells, and stronger down-regulation of gene expression at the global level from cowpox virus infection implicated more stringent interruption of interferon signaling transduction in corresponding A549 cells.

In mouse RAW cells, cowpox infection contributed to no gene expression change at the early time point, while it resulted in down-regulated expression of many key genes at late time point. These genes with downregulated expression included IFNAR2, IFN $\gamma R \beta$, JAK1, STAT1, OAS1 and so on, indicating that cowpox virus indeed counteract with the interferon response and impair the corresponding immune response to some extent.

When it turned to ectromelia virus infected mouse RAW cells, some of genes such as IFNAR2 and IFI35 showed down-regulated expression, while other key factors in the pathway such as IFN $\gamma \mathrm{R} \alpha$, JAK1/2, STAT1/2 showed no expression changes at $16 \mathrm{hpi}$. In addition, IFNAR1 as the type I IFN receptor expressed in an elevated state with 13.6-FC, which might imply the potential initiation of this type I IFN signaling pathway. Altogether, it was assumed that 
both host antivirus response and viral adaptive regulation were triggered and combated with each other, leading to the final gene expression performance of interferon pathway at 16 hpi in ectromelia virus-infected mouse RAW cells as described above.

Infection of lister virus also resulted in the inhibitory status of the interferon signaling pathway at 16 hpi with receptors, transduction factors and several downstream genes showing down-regulated gene expression. Global observation suggested that the gene expression performance in interferon signaling pathway detected after lister virus infection was similar to the gene expression performance detected after cowpox virus infection but not extromelia virus infection.

\subsubsection{Toll-like receptor signaling}

TLRs are a class of proteins that are crucial in the innate immune response to pathogens. Through recognizing and responding to pathogens associated molecular pattern, genes encoding for pro-inflammatory cytokines, chemokines and co-stimulatory molecules are then transcriptional activated. Besides, TLR signaling enables to result in the activation of NF- $\kappa \mathrm{B}$ which is a key transcription factor for proinflammatory genes including TNF and type I IFN. It has been reported that protein N1L and A52R encoded by poxviruses were able to inhibit the multiple TRL pathways to NF- $\kappa B$, subsequently evading the host immune response $[345,346]$.

In cowpox virus-infected A549 cells, few genes with altered gene expression changes in toll-like receptor signaling pathway were identified at 2 hpi. At 4 hpi, many genes with down-regulated expression were detected, and more and more genes with down-regulated expression in this pathway were detected with infection time increasing. At 10 hpi, TLR1, TLR6, TLR3, MYD88 as well as downstream recognition receptors P38 MAPK and NF- $\kappa B$ were all inhibited in their expression. Moreover, another interesting phenomenon was that gene c-Fos was induced in its expression across the whole infection period except 2 hpi.

Similarly, no significant gene expression changes were detected in tolllike receptor signaling pathway in lister virus-infected A549 cells at 2hpi except c-Fos with 3.08-FC and c-Jun with 3.85-FC. From 4 hpi, more and more factors 
in this pathway became to show reduction in their expressions. At $8 \mathrm{hpi}$, the repression state of this pathway reached the most significant with genes including TLR1/3/6, TIRAP, MYD88, TOLLIP, NF-кB and p38 MAPK all showing down-regulated expression. It was interesting that the repressed state of toll-like receptor signaling became a bit moderate at 10 hpi as compared to 8 hpi. In addition, the gene expression of c-Fos climbed up across the whole infection time course, and reached maximum with 62.7-FC at 10 hpi.

Overall, the global gene expressions in toll-like receptor signaling pathway were indeed impaired in two types of poxviruses infected A549 cells, and this observation might be attributed to the interference of the host antiviral activities from poxviruses.

In mouse RAW cells, cowpox virus infection resulted in similar gene expression performance of toll-like receptor signaling as in A549 cells, with few altered gene expression changes occurred at early infection time point but a big batch of alter gene expression changes detected at the late time point. However, it was surprising that TLR2, TLR3, TLR4 and TLR7 showed downregulated expression in mouse RAW cells, which was different from TLR1, TLR3 and TLR6 that showed down-regulated expression in A549 cells. And this finding might implicate the different signal transduction pathway in different cell types. In addition, c-Fos still showed elevated expression with 9.01-FC in cowpox virus-infected mouse RAW cells at 16 hpi.

When it comes to the infection of ectromelia virus, the gene expression performance in toll-like receptor signaling pathway was a bit different from the performance under infection of cowpox virus, with a smaller number of genes showing down-regulated expression detected at $16 \mathrm{hpi}$.

Genes with down-regulated expression in toll-like receptor signaling pathway was able to be detected from 2 hpi in lister virus-infected mouse RAW cells. And most of genes even including c-Fos in this pathway showed downregulated expression at $16 \mathrm{hpi}$.

\subsubsection{Apoptosis signaling}

Specialized immune cells function on removing virus-infected cells through the induction of apoptosis. All apoptosis signaling pathways converge on a common machinery of cell destruction that is activated by a family of 
caspases that enable to cleave proteins at aspartate residues. And these caspase activation and apoptotic death can be triggered by both extrinsic and intrinsic signals. Different publications have proved that different molecules were synthesized by poxvirus to inhibit the apoptosis as well as the processing of pro-inflammatory cytokines, thereby benefit the virus replication [332, 347].

In apoptosis signaling pathway, infection of cowpox virus in A549 cells only induced the expression of TNFR and Bfl-1 at 2hpi, and majority of genes in this pathway including caspase2/3/8/9/10, p53, BAX, Bcl-2 suddenly showed down-regulated expression around 4hpi. With the infection time increasing, the down-regulated expression of these genes became more and more significant, and the deepest down-regulated state was detected around 8 hpi.

Compared to cowpox virus infection, lister virus infection caused similar gene expression status in apoptosis signaling pathway, with majority of genes in this pathway showing down-regulated expression.

Observation on gene expression performances in apoptosis signaling pathway in these two types of poxviruses infected A549 cells provided evidence to prove that poxviruses initiated some mechanisms to impair the apoptosis signaling in infected host cells.

At the early time point, almost no altered gene expression changes in apoptosis signaling pathway were observed in all these three types of poxviruses infected mouse RAW cells. At the late time point, cowpox virus infection resulted in a group of genes with moderate down-regulation at their expression level, while ectromelia and lister virus infections led to a group of genes with outstanding down-regulation at their expression level.

\subsubsection{Cell cycle: G1/S checkpoint regulation}

Yoo $\mathrm{N}$ et al (2008) did a series of experiments to understand cell cycle control mechanism in vaccinia virus infected human tumor cells [348]. Finally, they provided evidences to support that the cell progression regulation was correlated with the inactivation of p53 and $\mathrm{Rb}$, which were further associated with the RNA polymerase III transcription factor B subunits, TBP and Brf1 respectively. In the same report, they also proposed that infection of vaccinia virus induced the expression of $\mathrm{Mdm} 2$ as well as its translocation into the nucleus, thereby leading to a disruption of $\mathrm{p} 53$. At the meanwhile, infection of 
vaccinia virus also reduced the expression of cyclin-dependent kinase 4 and 6 in order to enhance the level of hypophosphorylated $\mathrm{Rb}$, that appeared to be largely sequestered into a complex with Brf1 resulting in the blockage of $\mathrm{Rb}$ function and repression of E2F1 transactivation. Subsequently, control of these cell progression related key factors led to a moderately higher proportion of cells in the S and G2 phases, thereby contributing to the efficient replication of the virus in rapidly growing cells.

Lister virus infection also resulted in a suddenly gene expression suppression in this network around 4 hpi and this inhibitory regulation of gene expressions became more and more significant across the whole infection time course. In general, gene expression performances in this network from both cowpox and lister viruses infected A549 cells coincide with the experimental observation in previous report, indicating a premise of efficient poxvirus replication.

Gene expression performances of G1/S checkpoint regulation network in poxviruses infected mouse RAW cells were distinct from the performances in poxviruses infected A549 cells. In mouse RAW cells infected with all three types of poxviruses, no gene expression changes were detected at 2 hpi. At 16 hpi, the gene expression profile in mouse RAW cells infected with cowpox virus was a bit complicated. Gene expression of c-Myc which was reported to be a positive regulator of G1-specific cyclin-dependent kinases (CDKs) was elevated with 5-FC, while the targeting genes showed down-regulated expression. In ectromelia virus infected mouse RAW cells, more genes including MDM2, p21 showed elevated expression when compared to cowpox virus infected mouse RAW cells. Other genes such as CDK4/6, Cyclin D/E still showed in a repressed expression status. Besides, infection of lister virus resulted in a batch of genes with faintly down-regulated expression in mouse RAW cells.

\subsubsection{Antigen presentation pathway}

Two types of MHC groups participate in antigen presentation processes. MHC class I participate in the intracellular antigens' presentation which are produced mainly by viruses replicating within a host cell, while MHC class II attend extracellular antigens' presentation coming from exogenous pathogens. 
Previous publications have suggested that molecules encoded by vaccinia virus enabled to inhibit CD1d-mediated antigen presentation to natural killer $\mathrm{T}$ cells [349].

In mouse RAW cells, expression of MHC I- $\alpha$ was faintly downregulated after infections of cowpox and lister virus and few gene expression changes were able to be detected after ectromelia virus infection at $2 \mathrm{hpi}$. At 16 hpi, expression of all MHC I- $\alpha / \beta$ and MHC II- $\alpha / \beta$ were strongly repressed in cowpox virus-infected mouse RAW cells. Whereas, only some of them were inhibited at their expression level after ectromelia and lister virus infections, with MHC I- $\alpha / \beta$ down-regulated after ectromelia virus infection and MHC I- $\alpha / \beta$ as well as MHC II- $\alpha$ down-regulated after lister virus infection. Altogether, the gene expression in antigen presentation pathways detected from infections of different types of poxviruses indicated that all these examined poxviruses exerted some mechanisms to inhibit the antigen presentation in infected mouse RAW cells. Besides, down-regulation of different members in MHC groups at their expression level after infections of different types of poxviruses indicated the different signal inhibitory routes in these infected mouse RAW cells.

\subsection{Conclusion}

As we know, few studies have been reported to aim to describe the host response towards infections of cowpox virus, lister virus and ectromelia virus in a parallel way in A549 cells or mouse RAW cells. In our study, we made the attempt to compare the transcriptional expression profiles in cowpox and lister virus-infected A549 cells as well as the profiles in cowpox, lister and ectromelia virus-infected mouse RAW cells, respectively. Furthermore, genomic expression profiles in different cell types that were infected with the same type of poxvirus were also able to be compared.

Global evaluation indicated that the temporal distribution trend of numbers of probe sets with up- or down-regulated expression were similar among infections of different types of poxviruses either in A549 cells or mouse RAW cells. However, the detailed numbers of differently expressed probe sets were apparently different upon the infections of different types of poxviruses. For example, infection of lister virus resulted in more probe sets with upregulated expression while infection of cowpox virus resulted in more probe 
sets with down-regulated expression in A549 cells; infection of extromelia virus led to the largest number of probe sets with up-regulated expression at $16 \mathrm{hp}$ while infection of lister virus led to the largest number of probe sets with downregulated expression at both 2 and 16 hpi in mouse RAW cells.

By assigning these probe sets encoded genes to certain biological processes or pathways, it was noted that "DNA Binding", "RNA Binding" and "Transcription Factor" were over-represented and shared by those differentially expressed probe sets in A549 cells infected with two types of poxviruses. However, functional classifications of "Cell Cycle", "Cell Death", "Kinase" and "RNA Binding" were commonly enriched by those differentially expressed probe sets in mouse RAW cells infected with three types of poxviruses.

Besides these well-known functional groups, pathways associated with metabolism such as "Metabolic pathway" and "Glycolysis/ Gluconeogenesis" were enriched based on genes with down-regulated expression in lister and cowpox virus-infected A549 cells. And other canonical pathways such as "Immunological Disease", “Glycosis/Gluconegenesis", "RNA PostTranscription Modification", "Oxidative Phosphorylation", "Mitochondrial Dysfunction" and "Ubiquitin Biosynthese" were also enriched based on other differentially expressed genes in mouse RAW cells. These observations might indicate the regulations on a wide range of host cell machinaries in poxviruses infected mouse RAW cells.

In addition, a batch of histone genes and related transcription factors showed up-regulated expression in A549 cells after infections of poxviruses, especially lister virus. This observation was consistent with previous finding and might support the positive function of histone genes on virus replication. Furthermore, a couple of potential transcriptional factors were also significantly enriched based on differentially expressed genes.

Pathways analysis revealed the impairment of signaling transduction in immune response pathways in A549 cells infected with two types of poxviruses, especially cowpox virus. The complicated gene expression performances in immune response related pathways were detected in infected mouse RAW cells, and it was assumed that part of the significant gene expression might be due to the host antivirus response, while part of the significant gene expression might be caused by the viral adaptive regulation. In A549 cells, the inhibited 
expression of genes belonging to apoptosis signaling pathway at the late infection stage indicated that virus might evolve some mechanism to postpone the cell death, and thereby benefit the virus replication. Besides, it was also assumed that the infections of cowpox and lister virus contributed the cell cycle arrest so that to benefit efficient virus replication. In the antigen presentation pathway, different gene expression patterns in mouse RAW cells infected with three different types of poxviruses might imply the different signal transduction route in different infected cells.

Parallel studies performed by Bartel S et al., (2011) targeted to uncover the proteome of HEK293 cells in the late phase of virus (vaccinia virus IHD-W strain) replication [374]. And the analysis highlighted the significant regulation of several cellular function groups, such as apoptosis modulation, regulation of cellular gene expression and the regulation of energy metabolism. And these findings overlapped with our results. In another report, HeLa cells were infected by cowpox, monkeypox and vaccinia virus, and microarray technique was applied to investigate the modulation at transcriptional levels. The target was to to identify mechanisms that are either common to orthopoxvirus infection or specific to certain orthopoxvirus species [382]. Their results demonstrated that majority of host genes remained unaffected after virus infection. In our data, although a small number of genes with up-regulated expression was observed, there existed a large number of genes with down-regulated expression, which is different from Bourquain's observation. 


\section{Chapter VII. Conclusion and Future Work}

To deeply understand the host responses to infections of different viruses, we compared the genomic gene expression profiles in different virusesinfected the same cell types. And the results suggested that the same type of host cells took different actions to fight with the infections of different viruses. In the meanwhile, different viruses also evolved different strategies to counter host antiviral responses.

In macrophages, the genomic gene expression profiles upon RSV infection were examined at 4 and 24 hpi. Besides, the genomic gene expression profiles upon three influenza A strains infections were also examined, with $\mathrm{H} 1 \mathrm{~N} 1$ and H5N2/F118 investigated at 2 and $24 \mathrm{hpi}$ as well as H5N3 investigated at 24 hpi. Parallel comparison of these genomic profiles in infected macrophages demonstrated that infection of RSV contributed to larger numbers of genes with up-regulated expression than infections of influenza A strains across the whole infection stage. And among the three influenza A strains, H5N3 infection resulted in larger number of genes with differential expression, either up- or down-regulated, than H5N2/F118 and H1N1 infections at 24 hpi. This observation might indicate that the prominent host gene expression regulations happened in mouse macrophages infected with RSV, followed by H5N3.

Functional annotation of these differentially expressed genes in infected mouse macrophages suggested that these genes with significantly up-regulated expression were associated with "Cytokine", "Antiviral", "Cell death" and "RNA binding" while those genes with down-regulated expression were associated with "Cell cycle", "Kinase" and "Signal transduction". Detailed information also demonstrated that a bacth cytokines showing up-regulated expression with high fold changes (even $\geq 10$-FC) were detetected in RSV and H5N3 viruses infected mouse macrophages, suggesting that the strong immune defenses happened in these cells. Further investigations also revealed that a bigger batch of genes in RSV and H5N3-infected macrophages were calssified into functions such as "Infllammatory response" and "Immunological disease" as well as pathways such as "Dentritic cell maturation", "Communication between innate and adaptive immune cells" and "Role of pattern recognition 
receptors in recognition of bacteria and viruses". And these findings supported that a stronger activated antiviral state was detected after infections of RSV and H5N3 when compared to infections of H1N1 and H5N2/F118.

Analysis of key canonical pathway such as interferon signaling, antigen presentation and apoptosis signaling also provided details of the host responses in different viruses infected mouse macrophages. Among both RSV and three influenza A strains, RSV infection contributed to strongest innate immune response and macrophage activation, followed by $\mathrm{H} 5 \mathrm{~N} 3$ infection. Whereas, H1N1 and H5N2/F118 infections contributed to relatively faint innate immune responses and weak macrophage activations, and H5N2/F118 infection was responsible for stronger expression elevation of several ISGs at early time stage. Moreover, differential expression of genes related to either apoptosis or antiapoptosis were both detected in only RSV-infected mouse macrophages, suggesting bi-directional regulations in apoptosis signaling pathways. And this situation was not observed in three influenza A strains infected mouse marcophages.

Besides the host responses investigated in mouse macrophages, A459 cells were also infected with not only different influenza A strains but also different types of poxviruses. Global investigation indicated the big differences existed in genomic gene expression profiles between infections of influenza A strains and infections of poxviruses. Among eight examined influenza A stains, only infections of two human strains (H1N1 and pH1N1) and two H5N2 strains (H5N2/F59 and H5N2/F189) contributed to large numbers of probe sets with down-regulate expression. Further examination also indicated that a big sharp increase of the numbers of those probe sets with down-regulated expression was observed between 8 and 10 hpi after H1N1 infection. However, infections of both cowpox and lister virus inhibited the expression of bigger batches of the probe sets than infections of indluenza A strains. Furthermore, this large scale of expression inhibitory regulations was initiated from very early infection stage. Accordingly, these observations were far away from the situations observed during infections of influenza A strains.

Further functional analysis also showed the different host gene expression performances detected in A549 cells following infections of influenza A viruses and poxviruses. A functional group called "Immune 
response" was only prominently enriched based on the up-regulated genes from infections of influenza A viruses. And considerable numbers of genes showed quite significant fold regulation with $\geq 10$-FC especially after infections of H5N2/F59, H5N2/F118, H5N2/F189 and H9N2, indicating strong activation of immune defense system in corresponding A549 cells. Although a part of genes was also stimulated at their expression level in A549 cells at $10 \mathrm{hpi}$ after infection of poxviruses, in particular lister virus. Besides "RNA binding" and "DNA binding", these genes with elevated expression were majorly associated with "Cytoskeleton", indicting active membrane activities.

Although the functional groups such as "RNA Binding", "DNA Binding", "Signal Transduction" and "Transcription Factor" were commonly enriched by gene with down-regulated expression in both influenza A viruses and poxviruses-infcted A549 cells, these genes exert different spefic function during virus replication process. For example, down-regulated expression of zinc finger related genes were beneficial to the efficient virus replication in A549 cells after infections with influenza A strains, especially two human strains. Whereas, down-regulated expression of histone genes and related transcription factors might facilitate the viral DNA compaction of poxviruses in infected A549 cells.

Comparison of pathway analysis results also revealed different features of host response upon infections of influenza A viruses and poxviruses in A549 cells. For example, interferon signaling pathways were almost activated at the first beginning of infections coming from different influenza A strains while it showed no apparent activated status upon infections of two types of poxviruses at 2 hpi, but with deeper and deeper repressed status detected with the infection time increasing. However, the phenomenon that several key genes involved in cell cycle checkpoint showed inhibited expression during infections of poxviruses was also observed during infections of several influenza A strains such as H1N1 and pH1N1. And expression inhibition of these key factors at their transcriptional level aimed to retard the cell growth progression and in further create a better environment for effective virus replication in both influenza A strains and poxviruses-infected A549 cells.

Regarding to the big scale of genes with significantly down-regulated expression, especially detected in H1N1 infected-A549 cells, a number of 
biological function groups was enriched. Since cell apoptosis itself implies the degeneration of a wide range of cellular functions, we should put more attention on whether the virus infection directly or indirectly caused reduced gene expression in our further analysis.

Furthermore, the half-life of messenger RNA (mRNA) in the cytoplasm should be also carefully considered. Since all mRNAs will eventually be translated into an amino acid chain to exert cellular functions, mRNAs concentration not only depends on the transcription rates but also degradation rates. And balancing of these two rates is under complicated regulation in different cellular conditions [373]. During infection of influenza viruses, the mRNAs in the host cells are also degraded under the cap snatching process. In the replication cycle of poxviruses, only a subset of viral genes is transcribed initially, and early gene products are responsible for activating the intermediate genes, which then activate the late genes. Independence from the host cells' own transcription machinery allows virus to shut down the cell nucleus in order to occupy all the metabolic resources [392]. In our further analysis, we will make efforts to investigation the detailed cellular regulation of mRNAs' dynamic concentration during virus infection. At the meanwhile, the regulation of corresponding protein level should be also examined for parallel comparison with mRNA level. Besides, the expression levels of microRNAs in different viruses- infected different cell types are also under investigation in our lab. Since microRNAs have been proved to control the mRNA degradation, detailed understanding of microRNAs in their expression level will be also beneficial for deep understanding the half-life of mRNAs during virus infection.

In our future experiments, more experiments based on techniques such as RT-PCR and cytokine assays will be performed to validate the information retrieved from microarray paltform. In terms of these validation data, the further functional analysis will be more reliable. In addtion, more important virus strains such as H7N9 will be involved in our researches. After virus infection in specific cell types, the genomic gene expression profiles will be examined using microarray platform. After that, the functional and pathway analysis will also be performed based on different bioinformatics software.

RNA interference is a process in which small non-coding RNAs slicence the expression of a sequence-homologous target RNA [350]. Since its 
recovery, RNA interference has become an important research tools in functional genome analysis. Small interfering RNAs (siRNA) as one major type of small non-coding RNAs have been widely applied in building the screen for functional investigation of target genes [351].

Based on their characteristics, different groups applied genome-wide RNA interference screening to interpret virus-host interactions as well as identify the drug targets. In 20120, Karlas A et al (2010) constructed a genomewide RNA interference screen based on siRNAs and aimed to identify host cell factors involved in the influenza virus infection cycle in human cells [352]. Finally, 278 human host cell genes involved in influenza A virus replication were reported, and potential targets for novel antiviral stratigies were proposed. Thus, in our future experiment, RNA interference screen will be designed based on siRNA and function of interested cellular and viral genes will be investigated comprehensively.

Another type of small non-codeing RNAs which are involved in RNA interference is microRNAs which are a large family of $\sim 22$ nucleotide noncoding RNAs. Viruses have an intricate interaction with the host cell, and combating viral infection by targeting unique viral peoteins and pathways is an important topic in host antiviral system. Recently, more and more researches have discovered that different cellular microRNAs function on RNA interference process in the defense against viral infection. In the contrary, viruses also encode their own microRNAs in order to silence cellular pathways, and thereby benefit its viral propagation and infection [353-355].

Detailed experiments performed by Li Y et al (2010) have revealed that an array of microRNAs, including miR-200a and miR-223, showed differential expression in reaction to influenza virus infection in mouse [356]. Moreover, expression of predicted cellular target mRNAs was inversely correlated with the expression of these microRNAs. And gene ontology analysis also revealed that these mRNAs were associated with immune response and cell death pathways, which play critical roles in virulence. Bakre A et al (2012) also found that RSV infection in A549 cells induced the expression of five microRNAs and repressed the expression of two microRNAs, and these microRNAs may target several cell cycle genes. Besides, it was also proposed that RSV G protein was involved in induction of let-7 miRNA expression, and sebsequently regulated 
corresponding host genes to modulate virus replication during RSV infection [357]. 


\section{Reference}

1. Hurk SvDL-vd, Watkiss ER: Pathogenesis of respiratory syncytial virus. Current Opinion in Virology 2012, 2:300-305.

2. Kuiken T, Riteau B, Fouchier RA, Rimmelzwaan GF: Pathogenesis of influenza virus infections: the good, the bad and the ugly. Current Opinion in Virology 2012, 2(3):276-286.

3. Racloz V, Ramsey $\mathrm{R}$, Tong $\mathrm{S}, \mathrm{Hu} \mathrm{W}$ : Surveillance of dengue fever virus: a review of epidemiological models and early warning systems. PLOS Neglected Tropical Diseases 2012, 6(5):e1648.

4. McGoldrick C: HIV and employment. Occup Med (Lond) 2012, 62(4):242-253.

5. Acheson NH: Fundamentals of Molecular Virology - 2nd edition. 2011.

6. Bowie AG, Haga IR: The role of Toll-like receptors in the host response to viruses. Mol Immunol 2005, 42(8):859-867.

7. Galiana-Arnoux D, Imler JL: Toll-like receptors and innate antiviral immunity. Tissue Antigens 2006, 67(4):267-276.

8. Ehrhardt C, Seyer R, Hrincius ER, Eierhoff T, Wolff T, Ludwig S: Interplay between influenza A virus and the innate immune signaling. Microbes Infect 2010, 12(1):8187.

9. Carty M, Bowie AG: Recent insights into the role of Toll-like receptors in viral infection. Clin Exp Immunol 2010, 161(3):397-406.

10. Katze MG, He Y, Gale M, Jr.: Viruses and interferon: a fight for supremacy. Nat Rev Immunol 2002, 2(9):675-687.

11. Alzhanova D, Fruh K: Modulation of the host immune response by cowpox virus. Microbes and Infection 2010, 12(12-13):900-909.

12. Bonjardim CA: Interferons (IFNs) are key cytokines in both innate and adaptive antiviral immune responses--and viruses counteract IFN action. Microbes Infect 2005, 7(3):569-578.

13. Tisoncik JR, Korth MJ, Simmons CP, Farrar J, Martin TR, Katze MG: Into the eye of the cytokine storm. Microbiol Mol Biol Rev 2012, 76(1):16-32.

14. Benedict CA: Viruses and the TNF-related cytokines, an evolving battle. Cytokine Growth Factor Rev 2003, 14(3-4):349-357.

15. Dinarello CA: Immunological and inflammatory functions of the interleukin-1 family. Annu Rev Immunol 2009, 27:519-550.

16. Culley FJ, Pennycook AM, Tregoning JS, Dodd JS, Walzl G, Wells TN, Hussell T, Openshaw PJ: Role of CCL5 (RANTES) in viral lung disease. J Virol 2006, 80(16):8151-8157.

17. Kalvakolanu DV: Virus interception of cytokine-regulated pathways. Trends Microbiol 1999, 7(4):166-171.

18. Everett H, McFadden G: Apoptosis: an innate immune response to virus infection. Trends Microbiol 1999, 7(4):160-165.

19. Mata JF, Silveira VS, Mateo EC, Cortez MA, Queiroz RG, Yunes JA, Lee ML, Toledo SR, Petrilli AS, Brandalise SR et al: Low mRNA expression of the apoptosis-related genes CASP3, CASP8, and FAS is associated with low induction treatment response in childhood acute lymphoblastic leukemia (ALL). Pediatr Blood Cancer 2010, 55(1):100-107.

20. Rawlings JS, Rosler KM, Harrison DA: The JAK/STAT signaling pathway. $J$ Cell Sci 2004, 117(Pt 8):1281-1283.

21. Pauli EK, Schmolke M, Wolff T, Viemann D, Roth J, Bode JG, Ludwig S: Influenza A virus inhibits type I IFN signaling via NF-kappaB-dependent induction of SOCS-3 expression. PLoS Pathog 2008, 4(11):e1000196.

22. Levy DE, Garcia-Sastre A: The virus battles: IFN induction of the antiviral state and mechanisms of viral evasion. Cytokine Growth Factor Rev 2001, 12(2-3):143156.

23. Ludwig S, Planz O: Influenza viruses and the NF-kappaB signaling pathway towards a novel concept of antiviral therapy. Biol Chem 2008, 389(10):1307-1312.

24. Zhang YL, Dong C: MAP kinases in immune responses. Cell Mol Immunol 2005, 2(1):20-27. 
25. Fukushi S, Tani H, Yoshikawa T, Saijo M, Morikawa S: Serological assays based on recombinant viral proteins for the diagnosis of arenavirus hemorrhagic fevers.

Viruses 2012, 4(10):2097-2114.

26. Deschaght P, Schelstraete P, Santiago GLdS, Simaey LV, Haerynck F, daele SV, Wachter ED, Malfroot A, Lebecque P, Knoop C et al: Comparison of culture and qPCR for the detection of Pseudomonas aeruginosa in not chronically infected cystic fibrosis patients. BMC Microbiology 2010, 10(245).

27. Wang D, Urisman A, Liu Y-T, Springer M, Ksiazek TG, Erdman DD, Mardis ER, Hickenbotham M, Magrini $\mathrm{V}$, Eldred $\mathrm{J}$ et al: Viral discovery and sequence recovery using DNA microarrays. Plos Biology 2003, 1(2).

28. Ejima M, Kadoi K, Honda A: Influenza virus infection induces cellular Ebp1 gene expression. Genes Cells 2011, 16(9):927-937.

29. Kanagaraj AP, Verma D, Daniell H: Expression of dengue-3 premembrane and envelope polyprotein in lettuce chloroplasts. Plant Mol Biol 2011, 76(3-5):323-333.

30. Muraki Y, Furukawa T, Kohno Y, Matsuzaki Y, Takashita E, Sugawara K, Hongo S: Influenza C virus NS1 protein upregulates the splicing of viral mRNAs. J Virol 2010, 84(4):1957-1966.

31. Shan L, Yang HC, Rabi SA, Bravo HC, Shroff NS, Irizarry RA, Zhang H, Margolick JB, Siliciano JD, Siliciano RF: Influence of host gene transcription level and orientation on HIV-1 latency in a primary-cell model. $J$ Virol 2011, 85(11):53845393.

32. $\mathrm{Wu} \mathrm{Y,} \mathrm{Peng} \mathrm{C,} \mathrm{Xu} \mathrm{L,} \mathrm{Zheng} \mathrm{X,} \mathrm{Liao} \mathrm{M,} \mathrm{Yan} \mathrm{Y,} \mathrm{Jin} \mathrm{Y,} \mathrm{Zhou} \mathrm{J:} \mathrm{Proteome} \mathrm{dynamics}$ in primary target organ of infectious bursal disease virus. Proteomics 2012, 12(11):1844-1859.

33. Mous K, Jennes W, De Roo A, Pintelon I, Kestens L, Van Ostade X: Intracellular detection of differential APOBEC3G, TRIM5alpha, and LEDGF/p75 protein expression in peripheral blood by flow cytometry. $J$ Immunol Methods 2011, 372(12):52-64.

34. Ramsay G: DNA chips: state-of-the art. Nat Biotechnol 1998, 16(1):40-44.

35. Tefferi A, Bolander ME, Ansell SM, Wieben ED, Spelsberg TC: Primer on medical genomics. Part III: Microarray experiments and data analysis. Mayo Clin Proc 2002, 77(9):927-940.

36. Dorrell N, Hinchliffe SJ, Wren BW: Comparative phylogenomics of pathogenic bacteria by microarray analysis. Curr Opin Microbiol 2005, 8(5):620-626.

37. Sturdevant DE, Virtaneva K, Martens C, Bozinov D, Ogundare O, Castro N, Kanakabandi K, Beare PA, Omsland A, Carlson JH et al: Host-microbe interaction systems biology: lifecycle transcriptomics and comparative genomics. Future Microbiol 2010, 5(2):205-219.

38. Haagmans BL, Andeweg AC, Osterhaus AD: The application of genomics to emerging zoonotic viral diseases. PLoS Pathog 2009, 5(10):e1000557.

39. Lu DD, Chen SH, Zhang SM, Zhang ML, Zhang W, Bo XC, Wang SQ: Screening of specific antigens for SARS clinical diagnosis using a protein microarray. Analyst 2005, 130(4):474-482.

40. Davies DH, Wyatt LS, Newman FK, Earl PL, Chun S, Hernandez JE, Molina DM, Hirst S, Moss B, Frey SE et al: Antibody profiling by proteome microarray reveals the immunogenicity of the attenuated smallpox vaccine modified vaccinia virus ankara is comparable to that of Dryvax. J Virol 2008, 82(2):652-663.

41. Stevens J, Blixt O, Paulson JC, Wilson IA: Glycan microarray technologies: tools to survey host specificity of influenza viruses. Nat Rev Microbiol 2006, 4(11):857-864.

42. Uttamchandani M, Neo JL, Ong BN, Moochhala S: Applications of microarrays in pathogen detection and biodefence. Trends in Biotechnology 2009, 27(1):53-61.

43. Paliy O, Agans R: Application of phylogenetic microarrays to interrogation of human microbiota. FEMS Microbiol Ecol 2012, 79(1):2-11.

44. Novelli A, Grati FR, Ballarati L, Bernardini L, Bizzoco D, Camurri L, Casalone R, Cardarelli L, Cavalli $\mathrm{P}$, Ciccone $\mathrm{R}$ et al: Microarray application in prenatal diagnosis: a position statement from the cytogenetics working group of the Italian Society of Human Genetics (SIGU), November 2011. Ultrasound Obstet Gynecol 2012, 39(4):384-388. 
45. Sreenivasulu N, Sunkar R, Wobus U, Strickert M: Array platforms and bioinformatics tools for the analysis of plant transcriptome in response to abiotic stress. Methods Mol Biol 2010, 639:71-93.

46. Palacios G, Quan PL, Jabado OJ, Conlan S, Hirschberg DL, Liu Y, Zhai J, Renwick N, Hui J, Hegyi $\mathrm{H}$ et al: Panmicrobial oligonucleotide array for diagnosis of infectious diseases. Emerg Infect Dis 2007, 13(1):73-81.

47. Sengupta S, Onodera K, Lai A, Melcher U: Molecular detection and identification of influenza viruses by oligonucleotide microarray hybridization. J Clin Microbiol 2003, 41(10):4542-4550.

48. Wang D, Coscoy L, Zylberberg M, Avila PC, Boushey HA, Ganem D, DeRisi JL: Microarray-based detection and genotyping of viral pathogens. Proc Natl Acad Sci U S A 2002, 99(24):15687-15692.

49. Wong CW, Heng CL, Wan Yee L, Soh SW, Kartasasmita CB, Simoes EA, Hibberd ML, Sung WK, Miller LD: Optimization and clinical validation of a pathogen detection microarray. Genome Biol 2007, 8(5):R93.

50. Chou CC, Lee TT, Chen CH, Hsiao HY, Lin YL, Ho MS, Yang PC, Peck K: Design of microarray probes for virus identification and detection of emerging viruses at the genus level. BMC Bioinformatics 2006, 7:232.

51. Snyder JC, Bateson MM, Lavin M, Young MJ: Use of Cellular CRISPR (Clusters of Regularly Interspaced Short Palindromic Repeats) Spacer-Based Microarrays for Detection of Viruses in Environmental Samples. Applied and Environmental Microbiology 2010, 76(21):7251-7258.

52. Kang X, Qin C, Li Y, Liu H, Lin F, Li Y, Li J, Zhu Q, Yang Y: Improvement of the Specificity of a Pan-Viral Microarray by Using Genus-Specific Oligonucleotides and Reduction of Interference by Host Genomes. Journal of Medical Virology 2011, 83:1624-1630.

53. Gardner SN, Jaing CJ, McLoughlin KS, Slezak TR: A microbial detection array (MDA) for viral and bacterial detection. BMC Genomics 2010, 11(668).

54. Erlandsson L, Rosenstierne MW, McLoughlin K, Jaing C, Fomsgaard A: The Microbial Detection Array Combined with Random Phi29-Amplification Used as a Diagnostic Tool for Virus Detection in Clinical Samples. Plos One 2011, 6(8).

55. Lockhart DJ, Winzeler EA: Genomics, gene expression and DNA arrays. Nature 2000, 405:827-836.

56. Krijnse-Locker J: Cellular virology. Cell Microbiol 2005, 7(9):1213-1215.

57. DeFilippis V, Raggo C, Moses A, Fruh K: Functional genomics in virology and antiviral drug discovery. Trends in Biotechnology 2003, 21(10):452-457.

58. Raaben M, Whitley P, Bouwmeester D, Setterquist RA, Rottier PJ, de Haan CA: Improved microarray gene expression profiling of virus-infected cells after removal of viral RNA. BMC Genomics 2008, 9:221.

59. Katze MG, Fornek JL, Palermo RE, Walters KA, Korth MJ: Innate immune modulation by RNA viruses: emerging insights from functional genomics. Nat Rev Immunol 2008, 8(8):644-654.

60. Russell GC, Benavides J, Grant DM, Todd H, Thomson J, Puri V, Nath M, Haig DM: Host gene expression changes in cattle infected with Alcelaphine herpesvirus 1. Virus Res 2012, 169(1):246-254.

61. Munoz-Erazo L, Natoli R, Provis JM, Madigan MC, King NJ: Microarray analysis of gene expression in West Nile virus-infected human retinal pigment epithelium. Mol Vis 2012, 18:730-743.

62. Lee J, Bottje WG, Kong BW: Genome-wide host responses against infectious laryngotracheitis virus vaccine infection in chicken embryo lung cells. $B M C$ Genomics 2012, 13:143.

63. Palermo RE, Patterson LJ, Aicher LD, Korth MJ, Robert-Guroff M, Katze MG: Genomic analysis reveals pre- and postchallenge differences in a rhesus macaque AIDS vaccine trial: insights into mechanisms of vaccine efficacy. $J$ Virol 2011, 85(2):1099-1116.

64. Cho WK, Yu J, Lee KM, Son M, Min K, Lee YW, Kim KH: Genome-wide expression profiling shows transcriptional reprogramming in Fusarium graminearum by Fusarium graminearum virus 1-DK21 infection. BMC Genomics 2012, 13:173. 
65. Shair KH, Raab-Traub N: Transcriptome changes induced by Epstein-Barr virus LMP1 and LMP2A in transgenic lymphocytes and lymphoma. MBio 2012, 3(5).

66. Zhang H, Li Y, Huang X, Zheng C: Global transcriptional analysis of model of persistent FMDV infection reveals critical role of host cells in persistence. Vet Microbiol 2013, 162(2-4):321-329.

67. Yan Q: Bioinformatics databases and tools in virology research: an overview. In Silico Biol 2008, 8(2):71-85.

68. Vogel C, Marcotte EM: Insights into the regulation of protein abundance from proteomic and transcriptomic analyses. Nature Reviews Genetics 2012, 13(4):227232.

69. Cox J, Mann M: Is Proteomics the New Genomics? Cell 2007, 130:395-398.

70. Pareek CS, Smoczynski R, Tretyn A: Sequencing technologies and genome sequencing. $J$ Appl Genet 2011, 52(4):413-435.

71. Zheng J, Sugrue RJ, Tang K: Mass spectrometry based proteomic studies on viruses and hosts--a review. Anal Chim Acta 2011, 702(2):149-159.

72. Radford AD, Chapman D, Dixon L, Chantrey J, Darby AC, Hall N: Application of next-generation sequencing technologies in virology. J Gen Virol 2012, 93(Pt 9):1853-1868.

73. Wojcik J, Schachter V: Proteomic databases and software on the web. Brief Bioinform 2000, 1(3):250-259.

74. Bao S, Jiang R, Kwan W, Wang B, Ma X, Song YQ: Evaluation of next-generation sequencing software in mapping and assembly. J Hum Genet 2011, 56(6):406-414.

75. Stein LD: An introduction to the informatics of "next-generation" sequencing. Curr Protoc Bioinformatics 2011, Chapter 11:Unit 1111.

76. Hecker M, Lorenz P, Steinbeck F, Hong L, Riemekasten G, Li Y, Zettl UK, Thiesen $\mathrm{HJ}$ : Computational analysis of high-density peptide microarray data with application from systemic sclerosis to multiple sclerosis. Autoimmun Rev 2012, 11(3):180-190.

77. McLoughlin KS: Microarrays for Pathogen Detection and Analysis. Briefings in Functional Genomics 2011, 10(6):342-353.

78. Urisman A, Fischer KF, Chiu CY, Kistler AL, Beck S, Wang D, DeRisi JL: E-Predict: a computational strategy for species identification based on observed DNA microarray hybridization patterns. Genome Biology 2005, 6(9).

79. Watson M, Dukes J, Abu-Median A-B, King DP, Britton P: DetectiV: visualization, normalization and significance testing for pathogen-detection microarray data. Genome Biol 2007, 8(9).

80. Allred AF, Wu G, Wulan T, Fischer KF, Holbrook MR, Tesh RB, Wang D: VIPR: A probabilistic algorithm for analysis of microbial detection microarrays. $B M C$ Bioinformatics 2010, 11(384).

81. Allred AF, Renshaw H, Weaver S, Tesh RB, Wang D: VIPR HMM: a hidden Markov model for detecting recombination with microbial detection microarrays. Bioinformatics 2012, 28(22):2922-2929.

82. Rehrauer H, Schönmann S, Eberl L, Schlapbach R: PhyloDetect: a likelihood-based strategy for detecting microorganisms with diagnostic microarrays. Bioinformatics 2008, 24(16).

83. Gentleman RC, Carey VJ, Bates DM, Bolstad B, Dettling M, Dudoit S, Ellis B, Gautier L, Ge Y, Gentry J et al: Bioconductor: open software development for computational biology and bioinformatics. Genome Biol 2004, 5(10).

84. Smyth GK: Limma: linear models for microarray data. In Bioinformatics and Computational Biology Solutions using R and Bioconductor. Springer 2005:397420.

85. Schuldt B, Lin Q, Muller FJ, Loring J: Basic approaches to gene expression analysis of stem cells by microarrays. Methods Mol Biol 2011, 767:269-282.

86. Selvaraj S, Natarajan J: Microarray data analysis and mining tools. Bioinformation 2011, 6(3):95-99.

87. Kanehisa M, Goto S, Hattori M, Aoki-Kinoshita KF, Itoh M, Kawashima S, Katayama T, Araki M, Hirakawa M: From genomics to chemical genomics: new developments in KEGG. Nucleic Acids Res 2006, 34(Database issue):D354-357.

88. Latendresse M, Paley S, Karp PD: Browsing metabolic and regulatory networks with BioCyc. Methods Mol Biol 2012, 804:197-216. 
89. Szabo PM, Tamasi V, Molnar V, Andrasfalvy M, Tombol Z, Farkas R, Kovesdi K, Patocs A, Toth M, Szalai C et al: Meta-analysis of adrenocortical tumour genomics data: novel pathogenic pathways revealed. Oncogene 2010, 29(21):3163-3172.

90. Yona G, Dirks W, Rahman S: Comparing algorithms for clustering of expression data: how to assess gene clusters. Methods Mol Biol 2009, 541:479-509.

91. Burgess ST, Greer A, Frew D, Wells B, Marr EJ, Nisbet AJ, Huntley JF: Transcriptomic analysis of circulating leukocytes reveals novel aspects of the host systemic inflammatory response to sheep scab mites. Plos One 2012, 7(8):e42778.

92. Jay JJ, Eblen JD, Zhang Y, Benson M, Perkins AD, Saxton AM, Voy BH, Chesler EJ, Langston MA: A systematic comparison of genome-scale clustering algorithms. BMC Bioinformatics 2012, 13 Suppl 10:S7.

93. Ruan J, Dean AK, Zhang W: A general co-expression network-based approach to gene expression analysis: comparison and applications. BMC Syst Biol 2010, 4:8.

94. Zare H, Kaveh M, Khodursky A: Inferring a transcriptional regulatory network from gene expression data using nonlinear manifold embedding. Plos One 2011, 6(8): 21969.

95. Lieber D, Haas J: Viruses and microRNAs: a toolbox for systematic analysis. Wiley Interdiscip Rev RNA 2011, 2(6):787-801.

96. Scaria V, Hariharan M, Maiti S, Pillai B, Brahmachari SK: Host-virus interaction: a new role for microRNAs. Retrovirology 2006, 3:68.

97. Veksler-Lublinsky I, Shemer-Avni Y, Meiri E, Bentwich Z, Kedem K, Ziv-Ukelson M: Finding quasi-modules of human and viral miRNAs: a case study of human cytomegalovirus (HCMV). BMC Bioinformatics 2012, 13:322.

98. Ladunga I: An overview of the computational analyses and discovery of transcription factor binding sites. Methods Mol Biol 2010, 674:1-22.

99. Burgess ST, McNeilly TN, Watkins CA, Nisbet AJ, Huntley JF: Host transcription factors in the immediate pro-inflammatory response to the parasitic mite Psoroptes ovis. Plos One 2011, 6(9):e24402.

100. Ho Sui SJ, Fulton DL, Arenillas DJ, Kwon AT, Wasserman WW: oPOSSUM: integrated tools for analysis of regulatory motif over-representation. Nucleic Acids Res 2007, 35(Web Server issue):W245-252.

101. Elkon R, Linhart C, Sharan R, Shamir R, Shiloh Y: Genome-wide in silico identification of transcriptional regulators controlling the cell cycle in human cells. Genome Res 2003, 13(5):773-780.

102. Hertzberg L, Izraeli S, Domany E: STOP: searching for transcription factor motifs using gene expression. Bioinformatics 2007, 23(14):1737-1743.

103. Sharan R, Maron-Katz A, Shamir R: CLICK and EXPANDER: a system for clustering and visualizing gene expression data. BIOINFORMATICS 2003, 19(14):1787-1799.

104. Shamir R, Maron-Katz A, Tanay A, Linhart C, Steinfeld I, Sharan R, Shiloh Y, Elkon $\mathrm{R}$ : EXPANDER - an integrative program suite for microarray data analysis. BMC Bioinformatics 2005, 6(232).

105. Griffiths S, Lau J: The influence of SARS on perceptions of risk and reality. $J$ Public Health (Oxf) 2009, 31(4):466-467.

106. JY S: Another nightmare after SARS: knowledge perceptions of and overcoming strategies for H1N1 influenza among chronic renal disease patients in Hong Kong. Qual Health Res 2010, 20(7):893-904.

107. Tay J, Ng YF, Cutter JL, James L: Influenza A (H1N1-2009) pandemic in Singapore--public health control measures implemented and lessons learnt. Ann Acad Med Singapore 2010, 39(4):313-312.

108. Yoo SM, Lee SY: Diagnosis of pathogens using DNA microarray. Recent Patents on Biotechnology 2008, 2(2):124-129.

109. Vora GJ, Meador CE, Anderson GP, Taitt CR: Comparison of detection and signal amplification methods for DNA microarrays. Molecular and Cellular Probes 2008, 22(5-6):294-300.

110. Yozwiak NL, Skewes-Cox P, Stenglein MD, Balmaseda A, Harris E, DeRisi JL: Virus Identification in Unknown Tropical Febrile Illness Cases Using Deep Sequencing. PLOS Neglected Tropical Diseases 2012, 6(2).

111. Huguenin A, Moutte L, Renois F, Leveque N, Talmud D, Abely M, Nguyen Y, Carrat F, Andreoletti L: Broad Respiratory Virus Detection in Infants Hospitalized for 
Bronchiolitis by Use of a Multiplex RT-PCR DNA Microarray System. Journal of Medical Virology 2012, 84:979-985.

112. Bozdech Z, Zhu J, Joachimiak MP, Cohen FE, Pulliam B, DeRisi JL: Expression profiling of the schizont and trophozoite stages of Plasmodium falciparum with a long-oligonucleotide microarray. Genome Biol 2003, 4(2).

113. Quackenbush J: Microarray data normalization and transformation. Nature Genetics 2002, 32:496-501.

114. Nguyen V-A, Lió P: Measuring similarity between gene expression profiles: a Bayesian approach. BMC Genomics 2009, 10.

115. Goldstein H, Harron $\mathrm{K}$, AngieWade: The analysis of record-linked data using multiple imputation with data value priors. Statistics in Medicine 2012, 31:34813493.

116. Wu Y, Wang $\mathrm{X}$ : Optimal weight in estimating and comparing areas under the receiver operating characteristic curve using longitudinal data. Biometrical Journal 2011, 53(5):764-778.

117. Yao J, Chang C, Salmi ML, Hung YS, Loraine A, Roux SJ: Genome-scale cluster analysis of replicated microarrays using shrinkage correlation coefficient. $B M C$ Bioinformatics 2008, 9(288).

118. Fujita A, Sato JR, Demasi MA, Sogayar MC, Ferreira CE, Miyano S: Comparing Pearson, Spearman and Hoeffding's D measure for gene expression association analysis. J Bioinform Comput Biol 2009, 7(4):663-684.

119. MacKay DJ: Baysian Inference and Sampling Theory. Cambridge University Press 2003.

120. Zhao JX, Foulkes AS, George EI: Exploratory Bayesian Model Selection for Serial Genetics Data. Biometrics 2005, 61(2):591-599.

121. Cianci C, Meanwell N, Krystal M: Antiviral activity and molecular mechanism of an orally active respiratory syncytial virus fusion inhibitor. $J$ Antimicrob Chemother 2005, 55(3):289-292.

122. Collins PL, Melero JA: Progress in understanding and controlling respiratory syncytial virus: still crazy after all these years. Virus Res 2011, 162(1-2):80-99.

123. Munday DC, Emmott E, Surtees R, Lardeau CH, Wu W, Duprex WP, Dove BK, Barr JN, Hiscox JA: Quantitative proteomic analysis of A549 cells infected with human respiratory syncytial virus. Mol Cell Proteomics 2010, 9(11):2438-2459.

124. McLellan JS, Yang Y, Graham BS, Kwong PD: Structure of respiratory syncytial virus fusion glycoprotein in the postfusion conformation reveals preservation of neutralizing epitopes. J Virol 2011, 85(15):7788-7796.

125. Sugrue RJ: Interactions between respiratory syncytial virus and the host cell: opportunities for antivirus strategies? Expert Rev Mol Med 2006, 8(21):1-17.

126. Feldman SA, Crim RL, Audet SA, Beeler JA: Human respiratory syncytial virus surface glycoproteins F, G and SH form an oligomeric complex. Arch Virol 2001, 146(12):2369-2383.

127. Brown G, Rixon HW, Steel J, McDonald TP, Pitt AR, Graham S, Sugrue RJ: Evidence for an association between heat shock protein 70 and the respiratory syncytial virus polymerase complex within lipid-raft membranes during virus infection. Virology 2005, 338(1):69-80.

128. Hallak LK, Spillmann D, Collins PL, Peeples ME: Glycosaminoglycan sulfation requirements for respiratory syncytial virus infection. J Virol 2000, 74(22):1050810513.

129. Cowton VM, McGivern DR, Fearns R: Unravelling the complexities of respiratory syncytial virus RNA synthesis. J Gen Virol 2006, 87(Pt 7):1805-1821.

130. Harrison MS, Sakaguchi T, Schmitt AP: Paramyxovirus assembly and budding: building particles that transmit infections. Int J Biochem Cell Biol 2010, 42(9):1416-1429.

131. Oshansky CM, Zhang W, Moore E, Tripp RA: The host response and molecular pathogenesis associated with respiratory syncytial virus infection. Future Microbiol 2009, 4(3):279-297.

132. Batonick M, Wertz GW: Requirements for Human Respiratory Syncytial Virus Glycoproteins in Assembly and Egress from Infected Cells. Adv Virol 2011, 2011. 
133. Kallewaard NL, Bowen AL, Crowe JE, Jr.: Cooperativity of actin and microtubule elements during replication of respiratory syncytial virus. Virology 2005, 331(1):73-81.

134. Gower TL, Peeples ME, Collins PL, Graham BS: RhoA is activated during respiratory syncytial virus infection. Virology 2001, 283(2):188-196.

135. Krzyzaniak MA, Zumstein MT, Gerez JA, Picotti P, Helenius A: Host cell entry of respiratory syncytial virus involves macropinocytosis followed by proteolytic activation of the F protein. PLoS Pathog 2013, 9(4):e1003309.

136. Elliott J, Lynch OT, Suessmuth Y, Qian P, Boyd CR, Burrows JF, Buick R, Stevenson NJ, Touzelet $\mathrm{O}$, Gadina $\mathrm{M}$ et al: Respiratory syncytial virus NS1 protein degrades STAT2 by using the Elongin-Cullin E3 ligase. J Virol 2007, 81(7):3428-3436.

137. Moore EC, Barber J, Tripp RA: Respiratory syncytial virus (RSV) attachment and nonstructural proteins modify the type I interferon response associated with suppressor of cytokine signaling (SOCS) proteins and IFN-stimulated gene-15 (ISG15). Virol J 2008, 5:116.

138. Harris J, Werling D: Binding and entry of respiratory syncytial virus into host cells and initiation of the innate immune response. Cell Microbiol 2003, 5(10):671-680.

139. Lo MS, Brazas RM, Holtzman MJ: Respiratory syncytial virus nonstructural proteins NS1 and NS2 mediate inhibition of Stat 2 expression and alpha/beta interferon responsiveness. $J$ Virol 2005, 79(14):9315-9319.

140. Bueno SM, Gonzalez PA, Riedel CA, Carreno LJ, Vasquez AE, Kalergis AM: Local cytokine response upon respiratory syncytial virus infection. Immunol Lett 2011, 136(2):122-129.

141. Domachowske JB, Bonville CA, Mortelliti AJ, Colella CB, Kim U, Rosenberg HF: Respiratory syncytial virus infection induces expression of the anti-apoptosis gene IEX-1L in human respiratory epithelial cells. J Infect Dis 2000, 181(3):824-830.

142. Thomas KW, Monick MM, Staber JM, Yarovinsky T, Carter AB, Hunninghake GW: Respiratory syncytial virus inhibits apoptosis and induces NF-kappa B activity through a phosphatidylinositol 3-kinase-dependent pathway. J Biol Chem 2002 , 277(1):492-501.

143. Spann KM, Tran KC, Chi B, Rabin RL, Collins PL: Suppression of the induction of alpha, beta, and lambda interferons by the NS1 and NS2 proteins of human respiratory syncytial virus in human epithelial cells and macrophages [corrected]. $J$ Virol 2004, 78(8):4363-4369.

144. Ling Z, Tran KC, Teng MN: Human respiratory syncytial virus nonstructural protein NS2 antagonizes the activation of beta interferon transcription by interacting with RIG-I. $J$ Virol 2009, 83(8):3734-3742.

145. Ramaswamy M, Shi L, Varga SM, Barik S, Behlke MA, Look DC: Respiratory syncytial virus nonstructural protein 2 specifically inhibits type I interferon signal transduction. Virology 2006, 344(2):328-339.

146. Swedan S, Musiyenko A, Barik S: Respiratory syncytial virus nonstructural proteins decrease levels of multiple members of the cellular interferon pathways. J Virol 2009, 83(19):9682-9693.

147. Bitko V, Shulyayeva O, Mazumder B, Musiyenko A, Ramaswamy M, Look DC, Barik $\mathrm{S}$ : Nonstructural proteins of respiratory syncytial virus suppress premature apoptosis by an NF-kappaB-dependent, interferon-independent mechanism and facilitate virus growth. J Virol 2007, 81(4):1786-1795.

148. Oshansky CM, Barber JP, Crabtree J, Tripp RA: Respiratory syncytial virus F and G proteins induce interleukin 1alpha, $\mathrm{CC}$, and $\mathrm{CXC}$ chemokine responses by normal human bronchoepithelial cells. J Infect Dis 2010, 201(8):1201-1207.

149. Zhang W, Choi Y, Haynes LM, Harcourt JL, Anderson LJ, Jones LP, Tripp RA: Vaccination to induce antibodies blocking the CX3C-CX3CR1 interaction of respiratory syncytial virus $G$ protein reduces pulmonary inflammation and virus replication in mice. $J$ Virol 2010, 84(2):1148-1157.

150. Graham BS, Johnson TR, Peebles RS: Immune-mediated disease pathogenesis in respiratory syncytial virus infection. Immunopharmacology 2000, 48(3):237-247.

151. Hornsleth A, Loland L, Larsen LB: Cytokines and chemokines in respiratory secretion and severity of disease in infants with respiratory syncytial virus (RSV) infection. J Clin Virol 2001, 21(2):163-170. 
152. Tripp RA, Oshansky C, Alvarez R: Cytokines and respiratory syncytial virus infection. Proc Am Thorac Soc 2005, 2(2):147-149.

153. John AE, Berlin AA, Lukacs NW: Respiratory syncytial virus-induced

CCL5/RANTES contributes to exacerbation of allergic airway inflammation. Eur J Immunol 2003, 33(6):1677-1685.

154. Durbin JE, Johnson TR, Durbin RK, Mertz SE, Morotti RA, Peebles RS, Graham BS:

The role of IFN in respiratory syncytial virus pathogenesis. $J$ Immunol 2002, 168(6):2944-2952.

155. Ternette N, Wright C, Kramer HB, Altun M, Kessler BM: Label-free quantitative proteomics reveals regulation of interferon-induced protein with tetratricopeptide repeats 3 (IFIT3) and 5'-3'-exoribonuclease 2 (XRN2) during respiratory syncytial virus infection. Virol $J 2011,8(1): 442$.

156. Yeo DS, Chan R, Brown G, Ying L, Sutejo R, Aitken J, Tan BH, Wenk MR, Sugrue RJ: Evidence that selective changes in the lipid composition of raft-membranes occur during respiratory syncytial virus infection. Virology 2009, 386(1):168-182.

157. Classen A, Lloberas J, Celada A: Macrophage activation: classical versus alternative. Methods Mol Biol 2009, 531:29-43.

158. Shirey KA, Pletneva LM, Puche AC, Keegan AD, Prince GA, Blanco JC, Vogel SN: Control of RSV-induced lung injury by alternatively activated macrophages is IL-4R alpha-, TLR4-, and IFN-beta-dependent. Mucosal Immunol 2010, 3(3):291300.

159. Senft AP, Taylor RH, Lei W, Campbell SA, Tipper JL, Martinez MJ, Witt TL, Clay CC, Harrod KS: Respiratory syncytial virus impairs macrophage IFN-alpha/betaand IFN-gamma-stimulated transcription by distinct mechanisms. Am J Respir Cell Mol Biol 2010, 42(4):404-414.

160. Zhao DC, Yan T, Li L, You S, Zhang C: Respiratory syncytial virus inhibits interferon-alpha-inducible signaling in macrophage-like U937 cells. J Infect 2007, 54(4):393-398.

161. Nakamura-Lopez Y, Villegas-Sepulveda N, Sarmiento-Silva RE, Gomez B: Intrinsic apoptotic pathway is subverted in mouse macrophages persistently infected by RSV. Virus Res 2011, 158(1-2):98-107.

162. Kaan PM, Hegele RG: Interaction between respiratory syncytial virus and particulate matter in guinea pig alveolar macrophages. Am J Respir Cell Mol Biol 2003, 28(6):697-704.

163. Chai J, Tarnawski AS: Serum response factor: discovery, biochemistry, biological roles and implications for tissue injury healing. J Physiol Pharmacol 2002, 53(2):147-157.

164. Gilmore AP, Owens TW, Foster FM, Lindsay J: How adhesion signals reach a mitochondrial conclusion--ECM regulation of apoptosis. Curr Opin Cell Biol 2009, 21(5):654-661.

165. Spencer VA, Xu R, Bissell MJ: Gene expression in the third dimension: the ECMnucleus connection. J Mammary Gland Biol Neoplasia 2010, 15(1):65-71.

166. Lotz MT, Peebles RS, Jr.: Mechanisms of respiratory syncytial virus modulation of airway immune responses. Curr Allergy Asthma Rep 2012, 12(5):380-387.

167. Groskreutz DJ, Monick MM, Yarovinsky TO, Powers LS, Quelle DE, Varga SM, Look DC, Hunninghake GW: Respiratory syncytial virus decreases p53 protein to prolong survival of airway epithelial cells. J Immunol 2007, 179(5):2741-2747.

168. Thomas LH, Wickremasinghe MI, Sharland M, Friedland JS: Synergistic upregulation of interleukin-8 secretion from pulmonary epithelial cells by direct and monocyte-dependent effects of respiratory syncytial virus infection. $J$ Virol 2000, 74(18):8425-8433.

169. Kotenko SV: IFN-lambdas. Curr Opin Immunol 2011, 23(5):583-590.

170. Vesely PW, Staber PB, Hoefler G, Kenner L: Translational regulation mechanisms of AP-1 proteins. Mutat Res 2009, 682(1):7-12.

171. George KS, Wu S: Lipid raft: A floating island of death or survival. Toxicol Appl Pharmacol 2012, 259(3):311-319.

172. Chang TH, Segovia J, Sabbah A, Mgbemena V, Bose S: Cholesterol-rich lipid rafts are required for release of infectious human respiratory syncytial virus particles. Virology 2012, 422(2):205-213. 
173. Boukhvalova MS, Prince GA, Blanco JC: Inactivation of respiratory syncytial virus by zinc finger reactive compounds. Virol J 2010, 7:20.

174. Singh D, McCann KL, Imani F: MAPK and heat shock protein 27 activation are associated with respiratory syncytial virus induction of human bronchial epithelial monolayer disruption. Am J Physiol Lung Cell Mol Physiol 2007, 293(2):L436-445.

175. Neefjes J, Jongsma ML, Paul P, Bakke O: Towards a systems understanding of MHC class I and MHC class II antigen presentation. Nat Rev Immunol 2011, 11(12):823-836.

176. Suttles J, Stout RD: Macrophage CD40 signaling: a pivotal regulator of disease protection and pathogenesis. Semin Immunol 2009, 21(5):257-264.

177. Blander JM: Phagocytosis and antigen presentation: a partnership initiated by Toll-like receptors. Ann Rheum Dis 2008, 67 Suppl 3:iii44-49.

178. Miyashita M, Oshiumi H, Matsumoto M, Seya T: DDX60, a DEXD/H box helicase, is a novel antiviral factor promoting RIG-I-like receptor-mediated signaling. $\mathrm{Mol}$ Cell Biol 2011, 31(18):3802-3819.

179. Vince JE, Pantaki D, Feltham R, Mace PD, Cordier SM, Schmukle AC, Davidson AJ, Callus BA, Wong WW, Gentle IE et al: TRAF2 must bind to cellular inhibitors of apoptosis for tumor necrosis factor (tnf) to efficiently activate $n f-\{k a p p a\} b$ and to prevent tnf-induced apoptosis. $J$ Biol Chem 2009, 284(51):35906-35915.

180. Wang Z, Choi MK, Ban T, Yanai H, Negishi H, Lu Y, Tamura T, Takaoka A, Nishikura K, Taniguchi T: Regulation of innate immune responses by DAI (DLM1/ZBP1) and other DNA-sensing molecules. Proc Natl Acad Sci U S A 2008, 105(14):5477-5482.

181. DeFilippis VR, Alvarado D, Sali T, Rothenburg S, Fruh K: Human cytomegalovirus induces the interferon response via the DNA sensor ZBP1. J Virol 2010, 84(1):585598.

182. Flo TH, Smith KD, Sato S, Rodriguez DJ, Holmes MA, Strong RK, Akira S, Aderem A: Lipocalin 2 mediates an innate immune response to bacterial infection by sequestrating iron. Nature 2004, 432(7019):917-921.

183. Schroder WA, Le TT, Major L, Street S, Gardner J, Lambley E, Markey K, MacDonald KP, Fish RJ, Thomas R et al: A physiological function of inflammationassociated SerpinB2 is regulation of adaptive immunity. J Immunol 2010, 184(5):2663-2670.

184. Geserick P, Kaiser F, Klemm U, Kaufmann SH, Zerrahn J: Modulation of T cell development and activation by novel members of the Schlafen (slfn) gene family harbouring an RNA helicase-like motif. Int Immunol 2004, 16(10):1535-1548.

185. Brady G, Boggan L, Bowie A, O'Neill LA: Schlafen-1 causes a cell cycle arrest by inhibiting induction of cyclin D1. J Biol Chem 2005, 280(35):30723-30734.

186. Katsoulidis E, Carayol N, Woodard J, Konieczna I, Majchrzak-Kita B, Jordan A, Sassano A, Eklund EA, Fish EN, Platanias LC: Role of Schlafen 2 (SLFN2) in the generation of interferon alpha-induced growth inhibitory responses. $\mathrm{J}$ Biol Chem 2009, 284(37):25051-25064.

187. Sohn WJ, Kim D, Lee KW, Kim MS, Kwon S, Lee Y, Kim DS, Kwon HJ: Novel transcriptional regulation of the schlafen-2 gene in macrophages in response to TLR-triggered stimulation. Mol Immunol 2007, 44(13):3273-3282.

188. Ivan FX, Rajapakse JC, Welsch RE, Rozen SG, Narasaraju T, Xiong GM, Engelward $\mathrm{BP}$, Chow VT: Differential pulmonary transcriptomic profiles in murine lungs infected with low and highly virulent influenza H3N2 viruses reveal dysregulation of TREM1 signaling, cytokines, and chemokines. Funct Integr Genomics 2012, 12(1):105-117.

189. Lattin JE, Schroder K, Su AI, Walker JR, Zhang J, Wiltshire T, Saijo K, Glass CK, Hume DA, Kellie S et al: Expression analysis of G Protein-Coupled Receptors in mouse macrophages. Immunome Res 2008, 4:5.

190. Lesinski GB, Zimmerer JM, Kreiner M, Trefry J, Bill MA, Young GS, Becknell B, Carson WE, 3rd: Modulation of SOCS protein expression influences the interferon responsiveness of human melanoma cells. BMC Cancer 2010, 10:142.

191. Idel S, Dansky HM, Breslow JL: A20, a regulator of NFkappaB, maps to an atherosclerosis locus and differs between parental sensitive $\mathrm{C57BL} / 6 \mathrm{~J}$ and resistant FVB/N strains. Proc Natl Acad Sci U S A 2003, 100(24):14235-14240. 
192. Gelbmann CM, Leeb SN, Vogl D, Maendel M, Herfarth H, Scholmerich J, Falk W, Rogler G: Inducible CD40 expression mediates NFkappaB activation and cytokine secretion in human colonic fibroblasts. Gut 2003, 52(10):1448-1456.

193. Akira S, Takeda K: Toll-like receptor signalling. Nat Rev Immunol 2004, 4(7):499511.

194. Moynagh PN: TLR signalling and activation of IRFs: revisiting old friends from the NF-kappaB pathway. Trends Immunol 2005, 26(9):469-476.

195. Marchant D, Singhera GK, Utokaparch S, Hackett TL, Boyd JH, Luo Z, Si X, Dorscheid DR, McManus BM, Hegele RG: Toll-like receptor 4-mediated activation of p38 mitogen-activated protein kinase is a determinant of respiratory virus entry and tropism. $J$ Virol 2010, 84(21):11359-11373.

196. Murawski MR, Bowen GN, Cerny AM, Anderson LJ, Haynes LM, Tripp RA, KurtJones EA, Finberg RW: Respiratory syncytial virus activates innate immunity through Toll-like receptor 2. J Virol 2009, 83(3):1492-1500.

197. Xie XH, Law HK, Wang LJ, Li X, Yang XQ, Liu EM: Lipopolysaccharide induces IL-6 production in respiratory syncytial virus-infected airway epithelial cells through the toll-like receptor 4 signaling pathway. Pediatr Res 2009, 65(2):156-162.

198. Gagro A, Tominac M, Krsulovic-Hresic V, Bace A, Matic M, Drazenovic V, MlinaricGalinovic G, Kosor E, Gotovac K, Bolanca I et al: Increased Toll-like receptor 4 expression in infants with respiratory syncytial virus bronchiolitis. Clin Exp Immunol 2004, 135(2):267-272.

199. Strasser A, O'Connor L, Dixit VM: Apoptosis signaling. Annu Rev Biochem 2000, 69:217-245.

200. Coleman CM, Plant K, Newton S, Hobson L, Whyte MK, Everard ML: The AntiApoptotic Effect of Respiratory Syncytial Virus on Human Peripheral Blood Neutrophils is Mediated by a Monocyte Derived Soluble Factor. Open Virol J 2011, 5:114-123.

201. Kotelkin A, Prikhod'ko EA, Cohen JI, Collins PL, Bukreyev A: Respiratory syncytial virus infection sensitizes cells to apoptosis mediated by tumor necrosis factorrelated apoptosis-inducing ligand. $J$ Virol 2003, 77(17):9156-9172.

202. Gibbs JD, Ornoff DM, Igo HA, Zeng JY, Imani F: Cell cycle arrest by transforming growth factor beta1 enhances replication of respiratory syncytial virus in lung epithelial cells. $J$ Virol 2009, 83(23):12424-12431.

203. Russell GC, Stewart JP, Haig DM: Malignant catarrhal fever: a review. Vet J 2009, 179(3):324-335.

204. Molinari NA, Ortega-Sanchez IR, Messonnier ML, Thompson WW, Wortley PM, Weintraub E, Bridges CB: The annual impact of seasonal influenza in the US: measuring disease burden and costs. Vaccine 2007, 25(27):5086-5096.

205. Guan Y, Vijaykrishna D, Bahl J, Zhu H, Wang J, Smith GJ: The emergence of pandemic influenza viruses. Protein Cell 2010, 1(1):9-13.

206. Patterson KD, Pyle GF: The geography and mortality of the 1918 influenza pandemic. Bull Hist Med 1991, 65(1):4-21.

207. Michaelis M, Doerr HW, Cinatl J, Jr.: An influenza A H1N1 virus revival pandemic H1N1/09 virus. Infection 2009, 37(5):381-389.

208. Lewis DB: Avian flu to human influenza. Annu Rev Med 2006, 57:139-154.

209. Hoffmann E, Stech J, Leneva I, Krauss S, Scholtissek C, Chin PS, Peiris M, Shortridge $\mathrm{KF}$, Webster RG: Characterization of the influenza $A$ virus gene pool in avian species in southern China: was H6N1 a derivative or a precursor of H5N1? J Virol 2000, 74(14):6309-6315.

210. de Wit E, Fouchier RA: Emerging influenza. J Clin Virol 2008, 41(1):1-6.

211. Spackman E: A brief introduction to the avian influenza virus. Methods Mol Biol 2008, 436:1-6.

212. Xi X, Fang Q, Gu Q, Du B: Avian influenza A (H7N9) infections: Intensivists as virus hunters in the new century. J Crit Care 2013.

213. Wong CK, Zhu H, Li OT, Leung YH, Chan MC, Guan Y, Peiris JS, Poon LL: Molecular Detection of Human H7N9 Influenza A Virus Causing Outbreaks in China. Clin Chem 2013, 59(7):1062-1067.

214. Gao R, Cao B, Hu Y, Feng Z, Wang D, Hu W, Chen J, Jie Z, Qiu H, Xu K et al: Human infection with a novel avian-origin influenza A (H7N9) virus. $N$ Engl J Med 2013, 368(20):1888-1897. 
215. Tang RB, Chen HL: An overview of the recent outbreaks of the avian-origin influenza A (H7N9) virus in the human. J Chin Med Assoc 2013, 76(5):245-248.

216. Samji T: Influenza A: understanding the viral life cycle. Yale J Biol Med 2009, 82(4):153-159.

217. Medina RA, Garcia-Sastre A: Influenza A viruses: new research developments. Nat Rev Microbiol 2011, 9(8):590-603.

218. Boulo S, Akarsu H, Ruigrok RW, Baudin F: Nuclear traffic of influenza virus proteins and ribonucleoprotein complexes. Virus Res 2007, 124(1-2):12-21.

219. Boivin S, Cusack S, Ruigrok RW, Hart DJ: Influenza A virus polymerase: structural insights into replication and host adaptation mechanisms. $\mathrm{J}$ Biol Chem 2010, 285(37):28411-28417.

220. Deng T, Vreede FT, Brownlee GG: Different de novo initiation strategies are used by influenza virus RNA polymerase on its CRNA and viral RNA promoters during viral RNA replication. $J$ Virol 2006, 80(5):2337-2348.

221. Yu M, Liu X, Cao S, Zhao Z, Zhang K, Xie Q, Chen C, Gao S, Bi Y, Sun L et al: Identification and characterization of three novel nuclear export signals in the influenza A virus nucleoprotein. J Virol 2012, 86(9):4970-4980.

222. Akarsu H, Burmeister WP, Petosa C, Petit I, Muller CW, Ruigrok RW, Baudin F: Crystal structure of the M1 protein-binding domain of the influenza $A$ virus nuclear export protein (NEP/NS2). Embo J 2003, 22(18):4646-4655.

223. Rossman JS, Lamb RA: Influenza virus assembly and budding. Virology 2011, 411(2):229-236.

224. Leser GP, Lamb RA: Influenza virus assembly and budding in raft-derived microdomains: a quantitative analysis of the surface distribution of $\mathrm{HA}, \mathrm{NA}$ and M2 proteins. Virology 2005, 342(2):215-227.

225. Simpson-Holley M, Ellis D, Fisher D, Elton D, McCauley J, Digard P: A functional link between the actin cytoskeleton and lipid rafts during budding of filamentous influenza virions. Virology 2002, 301(2):212-225.

226. Garcia-Sastre A: Antiviral response in pandemic influenza viruses. Emerg Infect Dis 2006, 12(1):44-47.

227. Haller O, Staeheli $\mathrm{P}$, Kochs G: Interferon-induced Mx proteins in antiviral host defense. Biochimie 2007, 89(6-7):812-818.

228. Dittmann J, Stertz S, Grimm D, Steel J, Garcia-Sastre A, Haller O, Kochs G: Influenza $A$ virus strains differ in sensitivity to the antiviral action of Mx-GTPase. J Virol 2008, 82(7):3624-3631.

229. Samuel CE: Antiviral actions of interferons. Clin Microbiol Rev 2001, 14(4):778809 , table of contents.

230. Fitzgerald KA: The interferon inducible gene: Viperin. J Interferon Cytokine Res 2011, 31(1):131-135.

231. Wang X, Hinson ER, Cresswell P: The interferon-inducible protein viperin inhibits influenza virus release by perturbing lipid rafts. Cell Host Microbe 2007, 2(2):96105.

232. Sharma K, Tripathi S, Ranjan P, Kumar P, Garten R, Deyde V, Katz JM, Cox NJ, Lal $\mathrm{RB}$, Sambhara $\mathrm{S}$ et al: Influenza A virus nucleoprotein exploits Hsp40 to inhibit PKR activation. Plos One 2011, 6(6):e20215.

233. Hale BG, Randall RE, Ortin J, Jackson D: The multifunctional NS1 protein of influenza A viruses. J Gen Virol 2008, 89(Pt 10):2359-2376.

234. Wolff T, Ludwig S: Influenza viruses control the vertebrate type I interferon system: factors, mechanisms, and consequences. J Interferon Cytokine Res 2009, 29(9):549-557.

235. Min JY, Li S, Sen GC, Krug RM: A site on the influenza A virus NS1 protein mediates both inhibition of PKR activation and temporal regulation of viral RNA synthesis. Virology 2007, 363(1):236-243.

236. Li S, Min JY, Krug RM, Sen GC: Binding of the influenza A virus NS1 protein to PKR mediates the inhibition of its activation by either PACT or double-stranded RNA. Virology 2006, 349(1):13-21.

237. Gack MU, Albrecht RA, Urano T, Inn KS, Huang IC, Carnero E, Farzan M, Inoue S, Jung JU, Garcia-Sastre A: Influenza A virus NS1 targets the ubiquitin ligase TRIM25 to evade recognition by the host viral RNA sensor RIG-I. Cell Host Microbe 2009, 5(5):439-449. 
238. Jackson D, Killip MJ, Galloway CS, Russell RJ, Randall RE: Loss of function of the influenza $A$ virus NS1 protein promotes apoptosis but this is not due to a failure to activate phosphatidylinositol 3-kinase (PI3K). Virology 2010, 396(1):94-105.

239. Payungporn S, Panjaworayan N, Makkoch J, Poovorawan Y: Molecular characteristics of the human pandemic influenza A virus (H1N1). Acta Virol 2010, 54(3):155-163.

240. Dudek SE, Wixler L, Nordhoff C, Nordmann A, Anhlan D, Wixler V, Ludwig S: The influenza virus PB1-F2 protein has interferon antagonistic activity. Biol Chem 2011, 392(12):1135-1144.

241. Le Goffic R, Leymarie O, Chevalier C, Rebours E, Da Costa B, Vidic J, Descamps D, Sallenave JM, Rauch M, Samson $\mathrm{M}$ et al: Transcriptomic analysis of host immune and cell death responses associated with the influenza A virus PB1-F2 protein. PLoS Pathog 2011, 7(8):e1002202.

242. Conenello GM, Zamarin D, Perrone LA, Tumpey T, Palese P: A single mutation in the PB1-F2 of H5N1 (HK/97) and 1918 influenza A viruses contributes to increased virulence. PLoS Pathog 2007, 3(10):1414-1421.

243. Cox NJ, Subbarao K: Global epidemiology of influenza: past and present. Annu Rev Med 2000, 51:407-421.

244. Geiss GK, Salvatore M, Tumpey TM, Carter VS, Wang X, Basler CF, Taubenberger JK, Bumgarner RE, Palese $\mathrm{P}$, Katze MG et al: Cellular transcriptional profiling in influenza A virus-infected lung epithelial cells: the role of the nonstructural NS1 protein in the evasion of the host innate defense and its potential contribution to pandemic influenza. Proc Natl Acad Sci U S A 2002, 99(16):10736-10741.

245. Viboud C, Miller M, Olson D, Osterholm M, Simonsen L: Preliminary Estimates of Mortality and Years of Life Lost Associated with the 2009 A/H1N1 Pandemic in the US and Comparison with Past Influenza Seasons. PLoS Curr 2010, 2:RRN1153.

246. Russell CA, Jones TC, Barr IG, Cox NJ, Garten RJ, Gregory V, Gust ID, Hampson AW, Hay AJ, Hurt AC et al: The global circulation of seasonal influenza A (H3N2) viruses. Science 2008, 320(5874):340-346.

247. Huang Y, Zaas AK, Rao A, Dobigeon N, Woolf PJ, Veldman T, Oien NC, McClain MT, Varkey JB, Nicholson B et al: Temporal dynamics of host molecular responses differentiate symptomatic and asymptomatic influenza a infection. PLoS Genet 2011, 7(8):e1002234.

248. Neumann G, Noda T, Kawaoka Y: Emergence and pandemic potential of swineorigin H1N1 influenza virus. Nature 2009, 459(7249):931-939.

249. Christman MC, Kedwaii A, Xu J, Donis RO, Lu G: Pandemic (H1N1) 2009 virus revisited: an evolutionary retrospective. Infect Genet Evol 2011, 11(5):803-811.

250. Mukherjee S, Vipat VC, Mishra AC, Pawar SD, Chakrabarti AK: Pandemic (H1N1) 2009 influenza virus induces weaker host immune responses in vitro: a possible mechanism of high transmissibility. Virol $J$ 2011, 8:140.

251. Yu X, Zhang X, Zhao B, Wang J, Zhu Z, Teng Z, Shao J, Shen J, Gao Y, Yuan Z et al: Intensive cytokine induction in pandemic H1N1 influenza virus infection accompanied by robust production of IL-10 and IL-6. Plos One 2011, 6(12):e28680.

252. Paquette SG, Banner D, Zhao Z, Fang Y, Huang SS, Leomicronn AJ, Ng DC, Almansa $\mathrm{R}$, Martin-Loeches I, Ramirez $\mathrm{P}$ et al: Interleukin-6 is a potential biomarker for severe pandemic H1N1 influenza A infection. Plos One 2012, 7(6):e38214.

253. Ma W, Belisle SE, Mosier D, Li X, Stigger-Rosser E, Liu Q, Qiao C, Elder J, Webby $\mathrm{R}$, Katze MG et al: 2009 pandemic H1N1 influenza virus causes disease and upregulation of genes related to inflammatory and immune responses, cell death, and lipid metabolism in pigs. $J$ Virol 2011, 85(22):11626-11637.

254. Gambotto A, Barratt-Boyes SM, de Jong MD, Neumann G, Kawaoka Y: Human infection with highly pathogenic H5N1 influenza virus. Lancet 2008, 371(9622):1464-1475

255. Zeng H, Goldsmith C, Thawatsupha P, Chittaganpitch M, Waicharoen S, Zaki S, Tumpey TM, Katz JM: Highly pathogenic avian influenza H5N1 viruses elicit an attenuated type $\mathrm{i}$ interferon response in polarized human bronchial epithelial cells. J Virol 2007, 81(22):12439-12449. 
256. Lam WY, Yeung AC, Chu IM, Chan PK: Profiles of cytokine and chemokine gene expression in human pulmonary epithelial cells induced by human and avian influenza viruses. Virol $J$ 2010, 7:344.

257. Chang WL, Coro ES, Rau FC, Xiao Y, Erle DJ, Baumgarth N: Influenza virus infection causes global respiratory tract $B$ cell response modulation via innate immune signals. J Immunol 2007, 178(3):1457-1467.

258. Veckman V, Osterlund P, Fagerlund R, Melen K, Matikainen S, Julkunen I: TNFalpha and IFN-alpha enhance influenza-A-virus-induced chemokine gene expression in human A549 lung epithelial cells. Virology 2006, 345(1):96-104.

259. Mibayashi M, Martinez-Sobrido L, Loo YM, Cardenas WB, Gale M, Jr., Garcia-Sastre A: Inhibition of retinoic acid-inducible gene I-mediated induction of beta interferon by the NS1 protein of influenza A virus. J Virol 2007, 81(2):514-524.

260. Sarmento L, Afonso CL, Estevez C, Wasilenko J, Pantin-Jackwood M: Differential host gene expression in cells infected with highly pathogenic $\mathrm{H5N} 1$ avian influenza viruses. Vet Immunol Immunopathol 2008, 125(3-4):291-302.

261. Lee SM, Chan RW, Gardy JL, Lo C-k, Sihoe AD, Kang SS, Cheung TK, Guan Y, Chan MC, Hancock RE et al: RESEARCH Open Access Systems-level comparison of host responses induced by pandemic and seasonal influenza A H1N1 viruses in primary human type I-like alveolar epithelial cells in vitro. Respiratory Research 2010, 11(47).

262. Hui EK, Nayak DP: Role of ATP in influenza virus budding. Virology 2001, 290(2):329-341.

263. Klumpp K, Ford MJ, Ruigrok RW: Variation in ATP requirement during influenza virus transcription. J Gen Virol 1998, 79 ( Pt 5):1033-1045.

264. Ramirez-Martinez G, Cruz-Lagunas A, Jimenez-Alvarez L, Espinosa E, OrtizQuintero B, Santos-Mendoza T, Herrera MT, Canche-Pool E, Mendoza C, Banales JL et al: Seasonal and pandemic influenza H1N1 viruses induce differential expression of SOCS-1 and RIG-I genes and cytokine/chemokine production in macrophages. Cytokine 2013, 62(1):151-159.

265. Herold S, Ludwig S, Pleschka S, Wolff T: Apoptosis signaling in influenza virus propagation, innate host defense, and lung injury. $J$ Leukoc Biol 2012, 92(1):75-82.

266. Kreijtz JH, Fouchier RA, Rimmelzwaan GF: Immune responses to influenza virus infection. Virus Res 2011, 162(1-2):19-30.

267. Xu W, Xue L, Sun Y, Henry A, Battle JM, Micault M, Morris SW: Bcl10 is an essential regulator for A20 gene expression. J Physiol Biochem 2013.

268. Takahashi Y, Onodera T, Kobayashi K, Kurosaki T: Primary and secondary B-cell responses to pulmonary virus infection. Infect Disord Drug Targets 2012, 12(3):232-240.

269. Fang Y, Banner D, Kelvin AA, Huang SS, Paige CJ, Corfe SA, Kane KP, Bleackley $\mathrm{RC}$, Rowe $\mathrm{T}$, Leon AJ et al: Seasonal H1N1 influenza virus infection induces crossprotective pandemic H1N1 virus immunity through a CD8-independent, B celldependent mechanism. $J$ Virol 2012, 86(4):2229-2238.

270. Wolff T, O'Neill RE, Palese P: NS1-Binding protein (NS1-BP): a novel human protein that interacts with the influenza A virus nonstructural NS1 protein is relocalized in the nuclei of infected cells. $J$ Virol 1998, 72(9):7170-7180.

271. Tran AT, Cortens JP, Du Q, Wilkins JA, Coombs KM: Influenza virus induces apoptosis via BAD-mediated mitochondrial dysregulation. J Virol 2013, 87(2):1049-1060.

272. Tripathi S, Batra J, Cao W, Sharma K, Patel JR, Ranjan P, Kumar A, Katz JM, Cox NJ, Lal RB et al: Influenza A virus nucleoprotein induces apoptosis in human airway epithelial cells: implications of a novel interaction between nucleoprotein and host protein Clusterin. Cell Death Dis 2013, 4:e562.

273. Li P, Nijhawan D, Wang X: Mitochondrial activation of apoptosis. Cell 2004, S116:S57-S59.

274. Francoz S, Froment P, Bogaerts S, De Clercq S, Maetens M, Doumont G, Bellefroid E, Marine JC: Mdm4 and Mdm2 cooperate to inhibit p53 activity in proliferating and quiescent cells in vivo. Proc Natl Acad Sci U S A 2006, 103(9):3232-3237.

275. Liu Z, Lu H, Jiang Z, Pastuszyn A, Hu CA: Apolipoprotein 16, a novel proapoptotic Bcl-2 homology 3-only protein, induces mitochondria-mediated apoptosis in cancer cells. Mol Cancer Res 2005, 3(1):21-31. 
276. Zhaorigetu S, Yang Z, Toma I, McCaffrey TA, Hu CA: Apolipoprotein L6, induced in atherosclerotic lesions, promotes apoptosis and blocks Beclin 1-dependent autophagy in atherosclerotic cells. $J$ Biol Chem 2011, 286(31):27389-27398.

277. Rovere P, Peri G, Fazzini F, Bottazzi B, Doni A, Bondanza A, Zimmermann VS, Garlanda C, Fascio U, Sabbadini MG et al: The long pentraxin PTX3 binds to apoptotic cells and regulates their clearance by antigen-presenting dendritic cells. Blood 2000, 96(13):4300-4306.

278. Reading PC, Bozza S, Gilbertson B, Tate M, Moretti S, Job ER, Crouch EC, Brooks AG, Brown LE, Bottazzi B et al: Antiviral activity of the long chain pentraxin PTX3 against influenza viruses. J Immunol 2008, 180(5):3391-3398.

279. Job ER, Deng YM, Tate MD, Bottazzi B, Crouch EC, Dean MM, Mantovani A, Brooks AG, Reading PC: Pandemic H1N1 influenza A viruses are resistant to the antiviral activities of innate immune proteins of the collectin and pentraxin superfamilies. J Immunol 2010, 185(7):4284-4291.

280. Park K, Scott AL: Cholesterol 25-hydroxylase production by dendritic cells and macrophages is regulated by type I interferons. J Leukoc Biol 2010, 88(6):10811087.

281. Diczfalusy U, Olofsson KE, Carlsson AM, Gong M, Golenbock DT, Rooyackers O, Flaring U, Bjorkbacka H: Marked upregulation of cholesterol 25-hydroxylase expression by lipopolysaccharide. J Lipid Res 2009, 50(11):2258-2264.

282. Hurlin PJ, Steingrimsson E, Copeland NG, Jenkins NA, Eisenman RN: Mga, a dualspecificity transcription factor that interacts with Max and contains a T-domain DNA-binding motif. Embo $J$ 1999, 18(24):7019-7028.

283. Grandori C, Cowley SM, James LP, Eisenman RN: The Myc/Max/Mad network and the transcriptional control of cell behavior. Annu Rev Cell Dev Biol 2000, 16:653699.

284. Shilatifard A, Duan DR, Haque D, Florence C, Schubach WH, Conaway JW, Conaway RC: ELL2, a new member of an ELL family of RNA polymerase II elongation factors. Proc Natl Acad Sci U S A 1997, 94(8):3639-3643.

285. Gaur P, Munjhal A, Lal SK: Influenza virus and cell signaling pathways. Med Sci Monit 2011, 17(6):RA148-154.

286. Xing Z, Cardona CJ, Anunciacion J, Adams S, Dao N: Roles of the ERK MAPK in the regulation of proinflammatory and apoptotic responses in chicken macrophages infected with H9N2 avian influenza virus. J Gen Virol 2010, 91(Pt 2):343-351.

287. Hui KP, Lee SM, Cheung CY, Ng IH, Poon LL, Guan Y, Ip NY, Lau AS, Peiris JS: Induction of proinflammatory cytokines in primary human macrophages by influenza A virus (H5N1) is selectively regulated by IFN regulatory factor 3 and p38 MAPK. J Immunol 2009, 182(2):1088-1098.

288. Kujime K, Hashimoto S, Gon Y, Shimizu K, Horie T: p38 mitogen-activated protein kinase and c-jun-NH2-terminal kinase regulate RANTES production by influenza virus-infected human bronchial epithelial cells. J Immunol 2000, 164(6):3222-3228.

289. Ludwig S, Ehrhardt C, Neumeier ER, Kracht M, Rapp UR, Pleschka S: Influenza virus-induced AP-1-dependent gene expression requires activation of the JNK signaling pathway. J Biol Chem 2001, 276(24):10990-10998.

290. Pleschka S, Wolff T, Ehrhardt C, Hobom G, Planz O, Rapp UR, Ludwig S: Influenza virus propagation is impaired by inhibition of the Raf/MEK/ERK signalling cascade. Nat Cell Biol 2001, 3(3):301-305.

291. Marjuki H, Alam MI, Ehrhardt C, Wagner R, Planz O, Klenk HD, Ludwig S, Pleschka $\mathrm{S}$ : Membrane accumulation of influenza A virus hemagglutinin triggers nuclear export of the viral genome via protein kinase Calpha-mediated activation of ERK signaling. J Biol Chem 2006, 281(24):16707-16715.

292. Qu B, Li X, Gao W, Sun W, Jin Y, Cardona CJ, Xing Z: Human intestinal epithelial cells are susceptible to influenza virus subtype H9N2. Virus Res 2012, 163(1):151159.

293. Lu X, Masic A, Liu Q, Zhou Y: Regulation of influenza A virus induced CXCL-10 gene expression requires PI3K/Akt pathway and IRF3 transcription factor. $\mathrm{Mol}$ Immunol 2011, 48(12-13):1417-1423.

294. Chiou WF, Chen CC, Wei BL: 8-Prenylkaempferol Suppresses Influenza A VirusInduced RANTES Production in A549 Cells via Blocking PI3K-Mediated 
Transcriptional Activation of NF-kappaB and IRF3. Evid Based Complement Alternat Med 2011, 2011:920828.

295. Shin YK, Liu Q, Tikoo SK, Babiuk LA, Zhou Y: Effect of the phosphatidylinositol 3-kinase/Akt pathway on influenza A virus propagation. J Gen Virol 2007, 88(Pt 3):942-950.

296. Pei D, Dai J, Kuang Y, Wang H, Ren L, Shao J, Zuo B, Li S, Jiang Z, Li M: Effect of influenza A virus non-structural protein 1(NS1) on a mouse model of diabetes mellitus induced by Streptozotocin. Biochem Biophys Res Commun 2012, 419(1):120-125.

297. Hrincius ER, Dierkes R, Anhlan D, Wixler V, Ludwig S, Ehrhardt C:

Phosphatidylinositol-3-kinase (PI3K) is activated by influenza virus vRNA via the pathogen pattern receptor Rig-I to promote efficient type I interferon production Cell Microbiol 2011, 13(12):1907-1919.

298. Shapira SD, Gat-Viks I, Shum BO, Dricot A, de Grace MM, Wu L, Gupta PB, Hao T, Silver SJ, Root DE et al: A physical and regulatory map of host-influenza interactions reveals pathways in H1N1 infection. Cell 2009, 139(7):1255-1267.

299. GM C: The cell: a molecular approach. 2000.

300. He Y, Xu K, Keiner B, Zhou J, Czudai V, Li T, Chen Z, Liu J, Klenk HD, Shu YL et al: Influenza A virus replication induces cell cycle arrest in G0/G1 phase. $J$ Virol 2010, 84(24):12832-12840.

301. Li M, Chen D, Shiloh A, Luo J, Nikolaev AY, Qin J, Gu W: Deubiquitination of p53 by HAUSP is an important pathway for p53 stabilization. Nature 2002, 416(6881):648-653.

302. $\mathrm{Wu} \mathrm{M,} \mathrm{Xu} \mathrm{LG,} \mathrm{Su} \mathrm{T,} \mathrm{Tian} \mathrm{Y,} \mathrm{Zhai} \mathrm{Z,} \mathrm{Shu} \mathrm{HB:} \mathrm{AMID} \mathrm{is} \mathrm{a} \mathrm{p53-inducible} \mathrm{gene}$ downregulated in tumors. Oncogene 2004, 23(40):6815-6819.

303. Wang N, Dong Q, Li J, Jangra RK, Fan M, Brasier AR, Lemon SM, Pfeffer LM, Li K: Viral induction of the zinc finger antiviral protein is IRF3-dependent but NFkappaB-independent. J Biol Chem 2010, 285(9):6080-6090.

304. Zaitseva L, Cherepanov P, Leyens L, Wilson SJ, Rasaiyaah J, Fassati A: HIV-1 exploits importin 7 to maximize nuclear import of its DNA genome. Retrovirology 2009, 6:11.

305. Ao Z, Huang G, Yao H, Xu Z, Labine M, Cochrane AW, Yao X: Interaction of human immunodeficiency virus type 1 integrase with cellular nuclear import receptor importin 7 and its impact on viral replication. J Biol Chem 2007, 282(18):13456-13467.

306. Hui EK, Nayak DP: Role of $\mathbf{G}$ protein and protein kinase signalling in influenza virus budding in MDCK cells. J Gen Virol 2002, 83(Pt 12):3055-3066.

307. Moss B: Poxvirus cell entry: how many proteins does it take? Viruses 2012, 4(5):688-707.

308. Laliberte JP, Moss B: Lipid membranes in poxvirus replication. Viruses 2010, 2(4):972-986.

309. Barry M, van Buuren N, Burles K, Mottet K, Wang Q, Teale A: Poxvirus exploitation of the ubiquitin-proteasome system. Viruses 2010, 2(10):2356-2380.

310. Moss B: Poxvirus entry and membrane fusion. Virology 2006, 344(1):48-54.

311. Schmidt FI, Bleck CK, Mercer J: Poxvirus host cell entry. Current Opinion in Virology 2012, 2(1):20-27.

312. Carter GC, Law M, Hollinshead M, Smith GL: Entry of the vaccinia virus intracellular mature virion and its interactions with glycosaminoglycans. $J$ Gen Virol 2005, 86(Pt 5):1279-1290.

313. Senkevich TG, Moss B: Vaccinia virus $\mathbf{H 2}$ protein is an essential component of a complex involved in virus entry and cell-cell fusion. $J$ Virol 2005, 79(8):4744-4754.

314. Condit RC, Moussatche N, Traktman P: In a nutshell: structure and assembly of the vaccinia virion. Adv Virus Res 2006, 66:31-124.

315. Schramm B, Locker JK: Cytoplasmic organization of POXvirus DNA replication. Traffic 2005, 6(10):839-846.

316. Montanuy I, Alejo A, Alcami A: Glycosaminoglycans mediate retention of the poxvirus type I interferon binding protein at the cell surface to locally block interferon antiviral responses. Faseb Journal 2011, 25(6):1960-1971. 
317. Meng XZ, Schoggins J, Rose L, Cao JX, Ploss A, Rice CM, Xiang Y: C7L Family of Poxvirus Host Range Genes Inhibits Antiviral Activities Induced by Type I Interferons and Interferon Regulatory Factor 1. J Virol 2012, 86(8):4538-4547.

318. Sakala IG, Chaudhri G, Buller RM, Nuara AA, Bai H, Chen N, Karupiah G: Poxvirusencoded gamma interferon binding protein dampens the host immune response to infection. J Virol 2007, 81(7):3346-3353.

319. Waibler Z, Anzaghe M, Frenz T, Schwantes A, Poehlmann C, Ludwig H, PalomoOtero M, Alcami A, Sutter G, Kalinke U: Vaccinia Virus-Mediated Inhibition of Type I Interferon Responses Is a Multifactorial Process Involving the Soluble Type I Interferon Receptor B18 and Intracellular Components. J Virol 2009, 83(4):1563-1571.

320. Smith SA, Mullin NP, Parkinson J, Shchelkunov SN, Totmenin AV, Loparev VN, Srisatjaluk R, Reynolds DN, Keeling KL, Justus DE et al: Conserved surfaceexposed K/R-X-K/R motifs and net positive charge on poxvirus complement control proteins serve as putative heparin binding sites and contribute to inhibition of molecular interactions with human endothelial cells: a novel mechanism for evasion of host defense. J Virol 2000, 74(12):5659-5666.

321. Perdiguero B, Esteban M: The Interferon System and Vaccinia Virus Evasion Mechanisms. J Interf Cytok Res 2009, 29(9):581-598.

322. Saraiva M, Smith P, Fallon PG, Alcami A: Inhibition of type 1 cytokine-mediated inflammation by a soluble CD30 homologue encoded by ectromelia (mousepox) virus. Journal of Experimental Medicine 2002, 196(6):829-839.

323. Ruiz-Arguello MB, Smith VP, Campanella GSV, Baleux F, Arenzana-Seisdedos F, Luster AD, Alcami A: An ectromelia virus protein that interacts with chemokines through their glycosaminoglycan binding domain. J Virol 2008, 82(2):917-926.

324. Boomker JM, de Leij LFMH, The TH, Harmsen MC: Viral chemokine-modulatory proteins: tools and targets. Cytokine Growth Factor Rev 2005, 16(1):91-103.

325. Mohamed MR, Rahman MM, Rice A, Moyer RW, Werden SJ, McFadden G: Cowpox Virus Expresses a Novel Ankyrin Repeat NF-kappa B Inhibitor That Controls Inflammatory Cell Influx into Virus-Infected Tissues and Is Critical for Virus Pathogenesis. J Virol 2009, 83(18):9223-9236.

326. Stack J, Haga IR, Schroder M, Bartlett NW, Maloney G, Reading PC, Fitzgerald KA, Smith GL, Bowie AG: Vaccinia virus protein Toll-like-interleukin-1 A46R targets multiple receptor adaptors and contributes to virulence. Journal of Experimental Medicine 2005, 201(6):1007-1018.

327. Chang SJ, Hsiao JC, Sonnberg S, Chiang CT, Yang MH, Tzou DL, Mercer AA, Chang W: Poxvirus Host Range Protein CP77 Contains an F-Box-Like Domain That Is Necessary To Suppress NF-kappa B Activation by Tumor Necrosis Factor Alpha but Is Independent of Its Host Range Function. J Virol 2009, 83(9):4140-4152.

328. Randall CMH, Jokela JA, Shisler JL: The MC159 Protein from the Molluscum Contagiosum Poxvirus Inhibits NF-kappa B Activation by Interacting with the I kappa B Kinase Complex. J Immunol 2012, 188(5):2371-2379.

329. Hansen TH, Bouvier M: MHC class I antigen presentation: learning from viral evasion strategies. Nature Reviews Immunology 2009, 9(7):503-513.

330. Byun M, Verweij MC, Pickup DJ, Wiertz EJHJ, Hansen TH, Yokoyama WM: Two Mechanistically Distinct Immune Evasion Proteins of Cowpox Virus Combine to Avoid Antiviral CD8 T Cells. Cell Host Microbe 2009, 6(5):422-432.

331. Dasgupta A, Hammarlund E, Slifka MK, Fruh K: Cowpox virus evades CTL recognition and inhibits the intracellular transport of MHC class I molecules. $J$ Immunol 2007, 178(3):1654-1661.

332. Taylor JM, Barry M: Near death experiences: poxvirus regulation of apoptotic death. Virology 2006, 344(1):139-150.

333. Stewart TL, Wasilenko ST, Barry M: Vaccinia virus F1L protein is a tail-anchored protein that functions at the mitochondria to inhibit apoptosis. J Virol 2005, 79(2):1084-1098.

334. Johnston JB, Barrett JW, Nazarian SH, Goodwin M, Ricciuto D, Wang G, McFadden G: A poxvirus-encoded pyrin domain protein interacts with ASC-1 to inhibit host inflammatory and apoptotic responses to infection. Immunity 2005, 23(6):587-598.

335. Sedger LM, Osvath SR, Xu XM, Li G, Chan FK, Barrett JW, McFadden G: Poxvirus tumor necrosis factor receptor (TNFR)-like $T 2$ proteins contain a conserved 
preligand assembly domain that inhibits cellular TNFR1-induced cell death. $J$ Virol 2006, 80(18):9300-9309.

336. Turner PC, Moyer RW: Poxvirus immune modulators: functional insights from animal models. Virus Res 2002, 88(1-2):35-53.

337. Guerra S, Lopez-Fernandez LA, Pascual-Montano A, Najera JL, Zaballos A, Esteban $M$ : Host response to the attenuated poxvirus vector NYVAC: upregulation of apoptotic genes and NF-kappaB-responsive genes in infected HeLa cells. $J$ Virol 2007, 80(2):985-998.

338. Mahalingam S, Damon IK, Lidbury BA: 25 years since the eradication of smallpox: why poxvirus research is still relevant. Trends Immunol 2004, 25(12):636-639.

339. Alkhalil A, Hammamieh R, Hardick J, Ichou MA, Jett M, Ibrahim S: Gene expression profiling of monkeypox virus-infected cells reveals novel interfaces for host-virus interactions. Virol $J$ 2010, 7:173.

340. Zellmer S, Schmidt-Heck W, Godoy P, Weng H, Meyer C, Lehmann T, Sparna T, Schormann W, Hammad S, Kreutz C et al: Transcription factors ETF, E2F, and SP1 are involved in cytokine-independent proliferation of murine hepatocytes. Hepatology 2010, 52(6):2127-2136.

341. Kaplan MH: STAT4: a critical regulator of inflammation in vivo. Immunol Res 2005, 31(3):231-242.

342. Vousden KH, Prives C: Blinded by the Light: The Growing Complexity of p53. Cell 2009, 137(3):413-431.

343. Barrera G, Pizzimenti S, Dianzani MU: 4-hydroxynonenal and regulation of cell cycle: effects on the pRb/E2F pathway. Free Radic Biol Med 2004, 37(5):597-606.

344. Haig DM: Poxvirus interference with the host cytokine response. Vet Immunol Immunopathol 1998, 63(1-2):149-156.

345. Harte MT, Haga IR, Maloney G, Gray P, Reading PC, Bartlett NW, Smith GL, Bowie A, O'Neill LAJ: The poxvirus protein A52R targets toll-like receptor signaling complexes to suppress host defense. Journal of Experimental Medicine 2003, 197(3):343-351.

346. DiPerna G, Stack J, Bowie AG, Boyd A, Kotwal G, Zhang Z, Arvikar S, Latz E, Fitzgerald KA, Marshall WL: Poxvirus protein N1L targets the I-kappaB kinase complex, inhibits signaling to NF-kappaB by the tumor necrosis factor superfamily of receptors, and inhibits NF-kappaB and IRF3 signaling by toll-like receptors. J Biol Chem 2004, 279(35):36570-36578.

347. Everett H, McFadden G: Poxviruses and apoptosis: a time to die. Curr Opin Microbiol 2002, 5(4):395-402.

348. Yoo NK, Pyo CW, Kim Y, Ahn BY, Choi SY: Vaccinia virus-mediated cell cycle alteration involves inactivation of tumour suppressors associated with Brf1 and TBP. Cell Microbiol 2008, 10(3):583-592.

349. Webb TJ, Litavecz RA, Khan MA, Du W, Gervay-Hague J, Renukaradhya GJ, Brutkiewicz RR: Inhibition of CD1d1-mediated antigen presentation by the vaccinia virus B1R and H5R molecules. Eur J Immunol 2006, 36(10):2595-2600.

350. Jeang KT: RNAi in the regulation of mammalian viral infections. BMC Biol 2012, 10:58.

351. Chen M, Du Q, Zhang HY, Wang X, Liang Z: High-throughput screening using siRNA (RNAi) libraries. Expert Rev Mol Diagn 2007, 7(3):281-291.

352. Karlas A, Machuy N, Shin Y, Pleissner KP, Artarini A, Heuer D, Becker D, Khalil H, Ogilvie LA, Hess $\mathrm{S}$ et al: Genome-wide RNAi screen identifies human host factors crucial for influenza virus replication. Nature 2010, 463(7282):818-822.

353. Grundhoff A, Sullivan CS: Virus-encoded microRNAs. Virology 2011, 411(2):325343.

354. Ghosh Z, Mallick B, Chakrabarti J: Cellular versus viral microRNAs in host-virus interaction. Nucleic Acids Res 2009, 37(4):1035-1048.

355. Umbach JL, Cullen BR: The role of RNAi and microRNAs in animal virus replication and antiviral immunity. Genes Dev 2009, 23(10):1151-1164.

356. Li Y, Chan EY, Li J, Ni C, Peng X, Rosenzweig E, Tumpey TM, Katze MG: MicroRNA expression and virulence in pandemic influenza virus-infected mice. $J$ Virol 2010, 84(6):3023-3032. 
357. Bakre A, Mitchell P, Coleman JK, Jones LP, Saavedra G, Teng M, Tompkins SM, Tripp RA: Respiratory syncytial virus modifies microRNAs regulating host genes that affect virus replication. J Gen Virol 2012, 93(Pt 11):2346-2356.

358. Yeo DS, Ng SH, Liaw CW, Ng LM, Wee EJ: Molecular characterization of low pathogenic avian influenza viruses, isolated from food products imported into Singapore. Vet Microbiol 2009, 138: 304-317.

359. Kaptein JS and Nayak DP: Complete nucleotide sequence of the polymerase 3 gene of human influenza virus A/WS/33. J Virol. 1982, 42(1): 55-63.

360. Akerlind B: Respiratory Syncytial Virus: Heterogeneity of Subgroup B Strains. $J$ Gen Virol 1988, 69: 2145-2154.

361. Downie AW: A study of the lesions produced experimentally by cowpox virus. The Journal of Pathology and Bacteriology 1939, 48(2): 361-379.

362 Bhatt PN, Jacoby RO, Gras L: Mousepox in inbred mice innately resistant or susceptible to lethal infection with ectromelia virus. IV. Studies with the Moscow strain. Arch Virol 1988, 100(3-4): 221-30.

363. Marennikova SS, Ladnyj ID, Ogorodinikova ZI, Shelukhina EM, Maltseva NN: Identification and study of a poxvirus isolated from wild rodents in Turkmenia. Arch Virol 1978, 56(1-2):7-14.

364. Ravi LI, Li L, Sutejo R, Chen H, Wong PS, Tan BH, Sugrue RJ: A systems-based approach to analyse the host response in murine lung macrophages challenged with respiratory syncytial virus. BMC Genomics 2013, 14:190.

365. Sutejo R, Yeo DS, Myaing MZ, Chen H, Xia J, Ko D, Cheung PCF, Tan BH, Sugrue $\mathrm{RJ}$ : Activation of type I and III interferon signalling pathways occurs in lung epithelial cells infected with low pathogenic avian influenza viruses. PLOS ONE 2012, 7(3): e33732.

366. Tong S, Li Y, Rivailler P, Conrardy C, Castillo DAA, Chen LM, Recuenco S, Ellison JA, Davis CT et al: A distinct lineage of influenza A virus from bats. Proc Natl Acad Sci 2012, 109(11):4269-4274.

367. $\mathrm{Li} \mathrm{C}, \mathrm{Bu} \mathrm{Z}$, Chen $\mathrm{H}$ : Avian influenza vaccins against H5N1 'bird flu'. Trends in Biotechnology 2014.

368. Edinger TO, Pohl MO, Stertz S: Entry of influenza A virus: host factors and antiviral targets. Journal of General Virology 2014, 95(2):263-277.

369. Wise HM, Hutchinson EC, Jagger BW, Stuart AD, Kang ZH, Robb N, Schwartzman LM, Kash JC, Fodor E, Firth AE, Gog JR, Taubenberger JK, Digard P: Identification of a Novel Splice Variant Form of the Influenza A Virus M2 Ion Channel with an Antigenically Distinct Ectodomain . PLoS Pathog 2012, 8(11).

370. Horby P, Nguyen NY, Dunstan SJ, Baillie JK: The role of host genetics in susceptibility to influenza: a systematic review. PLOS ONE 2013, 7(3):E33180.

371. Diepen A, Brand HK, Sama I, Lambooy LHJ, Heuvel LP, Well L, Huynen M, Osterhaus ADME, Andeweg AC, Hermans PWM: Quantitative proteome profiling of respiratory virus-infected lung epithelial cells. Journal of Proteomics 2010, 73(9):1680-1693.

372. Goujon C, Moncorge O, Bauby H, Doyle T, Ward CC, Schaller T, Hue S, Barclay WS, Schulz R, Malim MH: Human MX2 is an interferon-induced post-entry inhibitor of HIV-1infection. Nature 2013, s02.

373. Perez-Ortin JE, Alepuz P, Chavez S, Choder M: Eukaryotic mRNA decay: methodologies, pathways and links to other stages of gene expression. J. Mol. Biol. 2013, 425:3750-3775.

374. Bartel S, Doellinger J, Darsow K, Bourquain D, Buchholz R, Nitsche A, Lange HA: Proteime analysis of vaccinia virus IHD-W-infected HEK 293 cells with 2dimensional gel electrophoresis and MALDI-PSD-TOF MS of on solid phase support N-terminally sulfonated peptides. Virology Journal 2011, 8(380).

375. Mosquera RA, Stark JM, Atkins CL, Colasurdo GN, Chevalier J, Samuels CL, Pacheco $\mathrm{SS}$ : Functional and immune response to respiratory syncytial virus replication in aged BALB/c mice: a search for genes determining disease severity. Experimental Lung Research 2014, 40:40-49.

376. Hsieh YC: Influenza pandemics: past, present and future. Journal of the Formosan Medical Association 2006, 105(1):1-6.

377. Reis AL, McCauley JW: The influenza virus protein PB1-F2 interacts with IKK $\boldsymbol{\beta}$ and modulates NF-кB signaling. PLoS ONE 2013, 8(5): e63852. 
378. Takahashi T, Takaguchi M, Kawakami T, Suzuki T: Sulfatide regulates caspase-3independent apoptosis of influenza A virus through viral PB1-F2 protein. PLoS ONE 2013, 8(4):e61092.

379. Soda K, Cheng MC, Yoshida H, Endo M, Lee SH, Okamatsu M, Sakoda Y, Wang CH, Kida H: A low pathogenic H5N2 influenza virus isolated in Taiwan acquired high pathogenicity by consecutive passages in chickens. J. Vet, Med, Sci 2011, 73(6): 767-772.

380. Monne I, Fusaro A, Nelson M, Bonfanti L, Mulatti P, Hughes J, Murcia PR, Schivo A, Valastro V, Moreno A, Holmes EC, Cattoli G: Emergence of a highly pathogenic avian influenza virus from a low pathogenic progenitor. J. Virol. 2014, 10(1128).

381. Rusnock A: Catching cowpox: the early spread of smallpox vaccination, 1798-1810. Bulletin of the History of Medicin 2009, 83(1):17-36.

382. Bourquain D, Dabrowski PW, Nitsche A: Comparison of host cell gene expression in cowpox, monkeypox or vaccinia virus-infected cells reveals viru specific regulation of immune response genes. Virology Journal 2013, 10(61).

383. Gerlach RL, Camp JV, Chu YK, Jonsson CB: Early host responses of seasonal and pandemic influenza $A$ viruses in primary well-differentiated human lung epithelial cells. PLoS One 2013, 8(11):e78912.

384. Jin HK, Yoshimatsu K, Takada A, Ogino M, Asano A, Arikawa J, Watanabe T: Mouse Mx2 protein inhibits hantavirus but not influenza virus replication. Arch. Virol. 2001, 146:41-49.

385. Cohen M, Zhang XQ, Senaati HP, Chen HW, Varki NM, Schooley RT, Gagneux P: Influenza A penetrates host mucus by cleaving sialic acid with neuraminidase. Virol. J. 2013, 10:321.

386. Ni F, Chen X, Shen J, Wang Q: Structural insights into the membrane fusion mechanism mediated by influenza virus hemagglutinin. Biochemistry 2014, 53:846-854.

387. Blasius AL, Beutler B: Intracellular toll-like receptors. Cell 2010, 305.

388. Jagger BW, Wise HM, Kash JC, Walters KA, Wills NM, Xiao YL, Dunfee RL, Schwartzman LM, Ozinsky A, Bell GL, Dalton RM, Lo A, Efstathiou S, Atkins JF, Firth AE, Taubenberger JK, Digard P: An overlapping protein-coding region in influenza A virus segment 3 modulates the host response. Science 2012, 337(199).

389. Fauquet CM, Mayo MA, Maniloff J, Desselberger U, Ball LA: Virus taxonomy: eighth report of the international committee on taxonomy of viruses. Virology Division International Union of Microbiological Societies 2005.

390. Kroeler AL, Ezzati P, Coombs KM, Halayko AJ: Influenza A infection of primary human airway epithelial cells up-regulates proteins related to purine metabolism and ubiquitin-related signaling. Journal of Proteome research 2013, 12:3139-3151.

391. Ravi LI, Li L, Wong PS, Sutejo R, Tan BH, Sugrue RJ: Lavastatin treatment mitigates the pro-inflammatory cytokine response in repespiratory syncytial virus infected macrophage cells. Antiviral Research 2013, 98(2):332-343.

392. Dimmock NJ, Easton AJ, Leppard KN: Introduction to modern virology. 2007.

393. Servant MJ, Grandvaux N, Hiscott J: Multiple signaling pathways leading to the activation of interferon regulatory factor 3. Biochem Pharmacol 2002, 64: 985-992.

394. Earl PL, Cooper N, Wyatt LS, Moss B, Carroll MW: Preparation of cell cultures and vaccinia virus stocks. Culture Protocols in Protein Science 1998, 5.12.1-5.12.2.

395. Guerra S, Lopez-Fernandez LA, Pascual-Montano A, Munoz M, Harshman K, Esteban $\mathrm{M}$ : Cellular gene expression survey of vaccinia virus infection of human Hela cells. Journal of Virology 2003, 77(11):6493.

396. Kilcher S, Schmidt FI, Schneider C, Kopf M, Helenius A, Mercer J: siRNA screen of early poxvirus genes identifies the AAA+ ATPase D5 as the virus genomeuncoating factor. Cell Host \& Microbe 2014, 15:103-112. 


\section{Appendix}

\section{Origin of virus strains}

\begin{tabular}{|c|c|c|}
\hline Name of Strain & Subtype & Origin \\
\hline RSV A2 & A & $\begin{array}{l}\text { This RSV A2 strain was originally isolated in Australia in } 1961 \text { (Taxon: } \\
\qquad 11259 \text { ) (Akerlind B et al ., 1988). }\end{array}$ \\
\hline A/WS/33 & H1N1 & $\begin{array}{l}\text { The laboratory-adapted A/WSN/1933 (H1N1/WSN) was isolated in } \\
\text { throat washings from an influenza patient by Wilson and Smith in } 1933 \\
\text { (Taxon: 381518) (Hit AL and Nayak DP, 1982). }\end{array}$ \\
\hline $\mathrm{A} /$ Singapore $/ 478 / 2009$ & H1N1 & $\begin{array}{l}\text { This virus was obtained from DSO national laboratories, and it was } \\
\text { isolated from an pandemic influenza patient in singapore (Taxon: 872268) } \\
\text { (Sutejo R et al ., 2012). }\end{array}$ \\
\hline ADuck/Malaysia/01 & H9N2 & \multirow{5}{*}{$\begin{array}{l}\text { These viruses were obtained from the Agri-Food and Veterinary } \\
\text { Authority of Singapore (AVA), and they were isolated as part of the } \\
\text { routine surveillance for AIVs in avian species imported from Malaysia } \\
\text { (Taxon: } 882942 / 341207 / 500383 / 500382 / 144182 \text { ) (Yeo DS et al., } \\
\text { 2009). }\end{array}$} \\
\hline A/Duck/Malaysis/F118/08/2004 & $\mathrm{H} 5 \mathrm{~N} 2$ & \\
\hline A/Duck/Malaysia/F59/04/1998 & $\mathrm{H} 5 \mathrm{~N} 2$ & \\
\hline A/Duck/Malaysia/F189/07/2004 & $\mathrm{H} 5 \mathrm{~N} 2$ & \\
\hline ADDuck/Malaysia/F119/3/1997 & $\mathrm{H} 5 \mathrm{~N} 3$ & \\
\hline A/fairybluebird/Singapore/F92/09/94 & $\mathrm{H} 7 \mathrm{~N} 1$ & $\begin{array}{l}\text { This virus was obtained from the Agri-Food and Veterinary Authority of } \\
\text { Singapore (AVA), and it was isolated from fairy blue bird in singapore } \\
\text { (Taxon: 121432). }\end{array}$ \\
\hline Brighton strain & Cowpox & $\begin{array}{l}\text { It was isolated in lesions on hands of milker in England in } 1937 \text { (Taxon: } \\
\text { 265872) (Downie AW, 1939). }\end{array}$ \\
\hline Lister strain & Vaccinia & (Taxon: 10252) \\
\hline Moscow strain & Ectromelia & $\begin{array}{l}\text { It was isolated in white rats and Felidae in the Moscow Zoo (Taxon: } \\
\text { 265874) (Bhatt PN et al., 1988). }\end{array}$ \\
\hline
\end{tabular}

\section{Consideration of contamination}

Contamination is always the major consideration during the experiment of virus infection. For example, mycoplasmas can interfere with virtually every parameter measured in cell cultures during routine cultivation or in experimental investigation. Under this consideration, we performed the virus infection experiment across a series of time points, and the gene expression profiles from multiple time points showed consistent. Moreover, cell and viruses were routinely check for mycoplasmas mainly using commercial available mycoplasmas kits in our experiment to make sure that the cells were out of contamination. And virus preparations were based on multiplex PCR, electron microscope (transmission electron microscope and scanning electron microscope) for direct visualization, immunological detection. In terms of these techniques, the cells became taken over by the virus and viruses replicated with variable rates at different infection stages were observed. 


\section{Consideration of reproducibility}

Reproducibility of results was also under our carful consideration. Firstly, since the microarray experiment is expensive, the performances of experiments were limited to some extent. However, we tried our best to design and carry on the experiments carefully: more than one viral preparation was conducted, and different validation methods including biochemical validation and $\mathrm{qPCR}$ using different viral preparation were involved. Besides, the statistical analysis based on Benjamini \& Hochberg False Discovery method or one-way analysis of variance (ANOVA) was performed with a P-value cutoff of $\leq 0.05$ to determine significance of differential gene expression during virus infection. For some specific strains such as $\mathrm{A} / \mathrm{WS} / 33 / \mathrm{H} 1 \mathrm{~N} 1$, the investigation of gene expression profiles was also performed by another person in another batch of our study, and the results from two different batches of experiments still showed highly consistent, strongly indicating the reproducibility of the results from our experiments. In summary, our study was limited based on the expensive cost of microarray experiments, however, the common features were observed in different viruses and the gene expression trends also showed consistent for individual genes over the whole infection time course.

\section{Consideration of results that a gene transcript showed down-regulated expression at a time point but less down-regulated expression at a later time point}

To make sure to get the precise information in our study, investigation of different gene expression profiles was based on three independent experiments. Only genes that were flagged as present in all three replicates (mock- or virus-infected), and passed the threshold of fold change ( $\geqslant 2$ up- or down-regulated) between virus- and mock-infected samples in all triplicate microarray experiments were selected for further statistical evaluation. The following statistical analysis based on Benjamini \& Hochberg False Discovery method or one-way analysis of variance (ANOVA) was performed with a Pvalue cutoff of $\leq 0.05$ to determine significantly expressed genes during virus infection. The observations such a gene transcript with more down-regulated expression an early time point but less down-regulation at a later time point 
must pass threshold of fold change and P-value. Further investigation involved validation based on qPCR method.

\section{The productivity of the infection of RSV during the whole investigated infection time course for each of the cell types}

The transcriptomic profiles were investigated from 4hpi to $15 \mathrm{hpi}$ in RSV-infected Hep2 cells, and this time course is suffice time to allow formation of progeny virus, but is prior to the cell damage that occurs later in the replication cycle (e.g. following syncytia formation) that could cause indirect changes in the host cell expression profile. Specifically, we could discern the formation of the virus filaments and inclusion bodies followed over the time course (from 8hpi) of a single cycle of infection [164].

In macrophages, similar vRNA levels at between 2.5 and 20 hpi were detected suggesting low levels of vRNA synthesis. The virus infectivity recovered in macrophages indicated virus titres of $2 \times 10^{1} \mathrm{pfu} / \mathrm{ml}$, and this low level of virus titres was likely due to residual virus from the input virus inoculum. Similarly, only sporadic stained cells were detected using the tissue culture supernatant (TCS) of RSV-infected macrophages. Collectively, these data indicated that RSV infection results in the formation of virus antigen and the production of inclusion bodies, efficient infectious virus particle production does not occur [356].

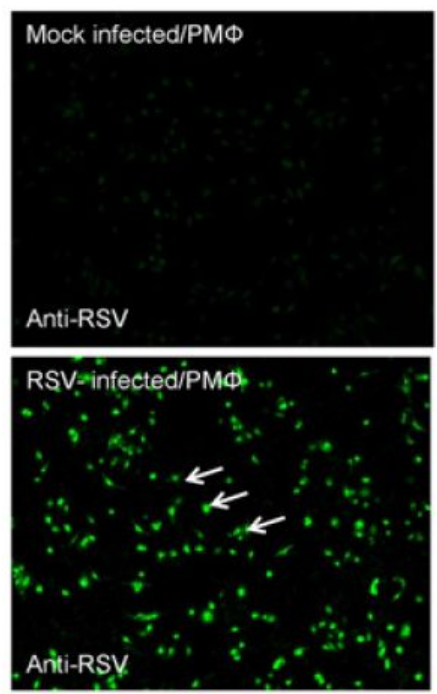

Macrophage cells were mock-infected or RSV-infected, and at 24 hrs post-infection (hpi) stained with anti-RSV and examined using fluorescence microscopy (objective x 10) (highlighted by white arrows) [364]. 

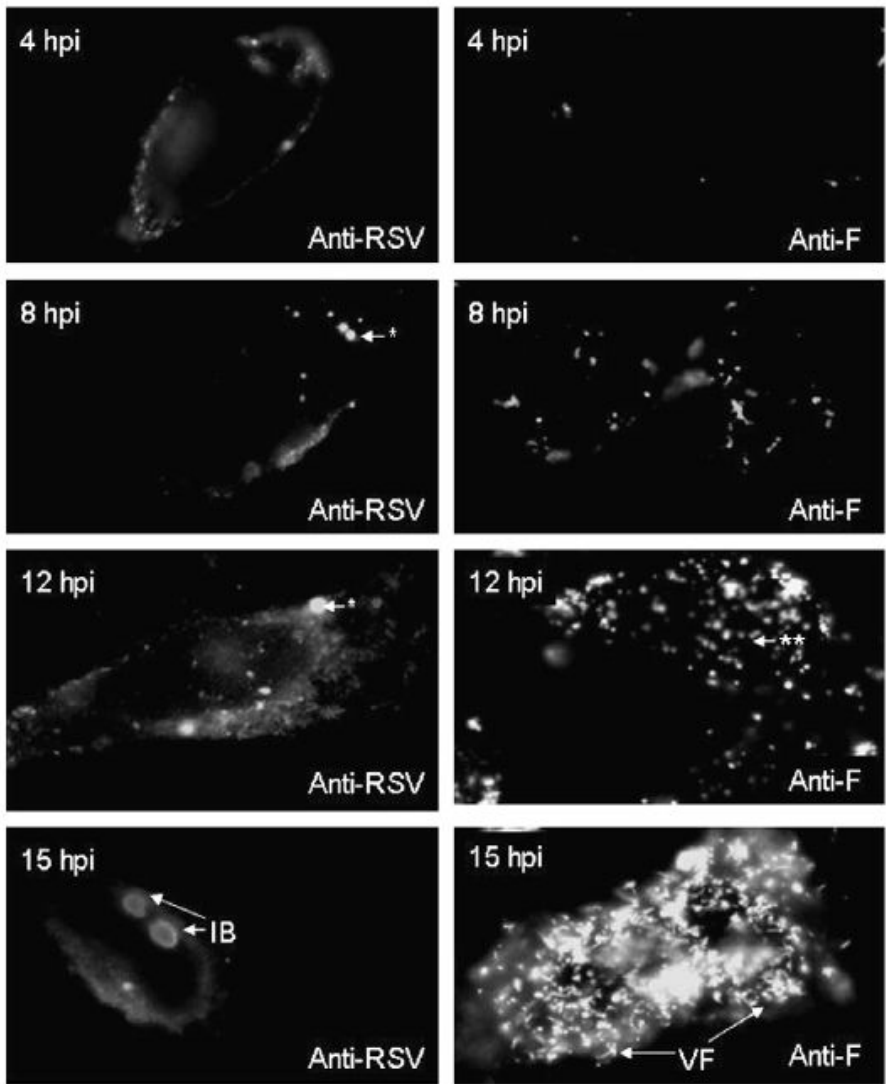

The formation of virus structures coincides with specific changes in the host-gene expression profile. HEp 2 cells were infected with RSV and at the times indicated, the cells were fixed, stained using anti-RSV or anti-F, and examined by confocal microscopy. The inclusion bodies (IB) and virus filaments (VF) are highlighted. The early formation of inclusion bodies $(*)$ and F protein staining $(* *)$ are highlighted [356].
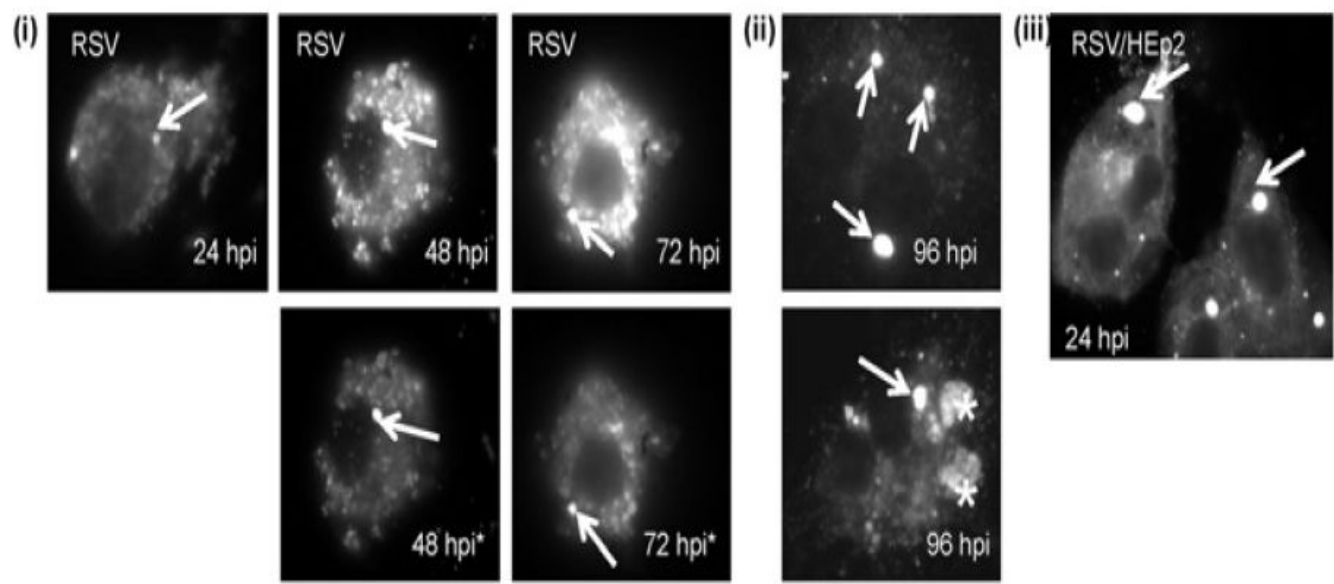

RSVinfected macrophage cells were stained with anti-RSV and examined by fluorescence microscopy at (i) $24 \mathrm{hpi}, 48 \mathrm{hpi}$ and $72 \mathrm{hpi}$ and (ii) $96 \mathrm{hpi}$. The stained cells were examined using either the same camera exposure time or at a reduced exposure time (highlighted by *) to enable the inclusion bodies (IB) to be viewed (at magnification x20). Inclusion bodies are highlighted (white arrow) and a more diffuse anti-RSV staining pattern is highlighted (*). (iii) RSV-infected HEp2 cells stained using anti-RSV at 24 hpi. The IBs are highlighted (white arrow) [364]. 


\section{Relationship between the time of the analyses and different steps of virus replication cycle in RSV study}

There are two major structural features for RSV observed by electron microscopy: the virus filaments (VFs) and the inclusion bodies (IBs). VFs form on the surface of the infected cells where the virus structural proteins interact to form mature virus particles, while IBs exist in the infected cells where the virus polymerase-associated proteins and virus-specific RNA accumulate.

As described before, the transcriptomic profiles were investigated from 4hpi to 15hpi in RSV-infected Hep2 cells. From 8hpi, it was observed that the virus filaments and inclusion bodies were formed with an increasing numbers, and the quite a lot of virus filaments accumulated at 15hpi. Data from microarray study indicated that the productions of cytokines and chemokines were increased quite early (from 4hpi), indicating that the innate response was activated at the first beginning of virus replication cycle, at least before the observation of obvious virus filaments and inclusion bodies. Compared to the response detected at $4 \mathrm{hpi}$, stronger induction of pro-inflammatory response has been observed from $8 \mathrm{hpi}$ and sustained over the late infection stage. This phenomenon might indicate that the antiviral actions were fully taken to prevent the further assembly and release in host cells. At the meanwhile, several mechanisms including inhibiting the expression of type I IFN, delaying host apoptosis and alteration of lipid raft have also been exerted by RSV in order to interfere with the activated immune defense and benefit the further viral replication [156].

In our RSV-infected macrophages, strong immune response was observed from the early infection stage (4hpi) to the late infection stage (24hpi). At the meanwhile, similar vRNA levels at between 2.5 and 20 hpi were also detected. Thus, it might be assumed that sustained activation of genes involved in innate and acquired immune response was responsible for the low respiratory tract infection. In addition, other data also indicated that RSV infection results in the formation of virus antigen and the production of inclusion bodies, efficient infectious virus particle production does not occur [364]. 

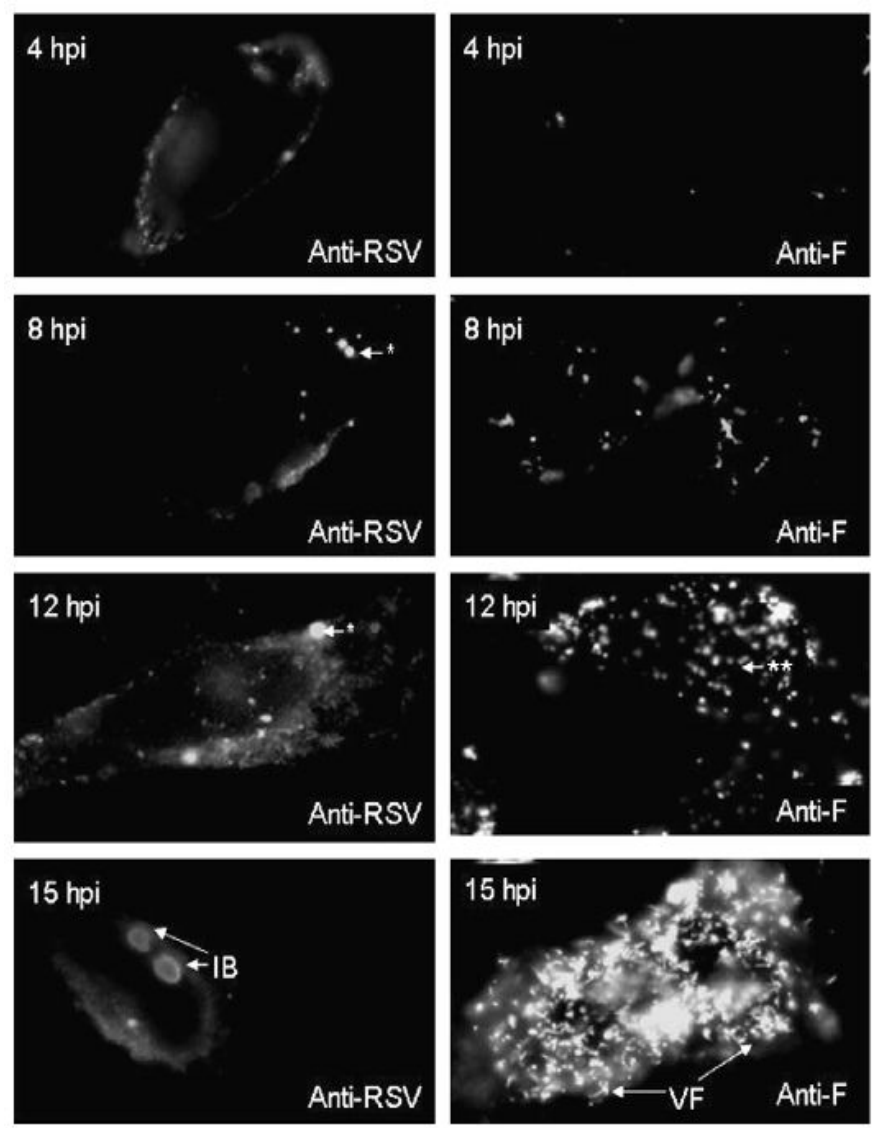

The formation of virus structures coincides with specific changes in the host-gene expression profile. HEp 2 cells were infected with RSV and at the times indicated, the cells were fixed, stained using anti-RSV or anti-F, and examined by confocal microscopy. The inclusion bodies (IB) and virus filaments (VF) are highlighted. The early formation of inclusion bodies (*) and F protein staining $(* *)$ are highlighted [156].
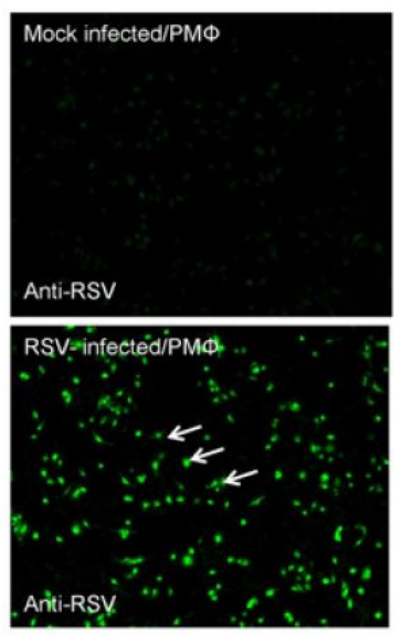

Macrophage cells were mock-infected or RSV-infected, and at 24 hrs post-infection (hpi) stained with anti-RSV and examined using fluorescence microscopy (objective x 10) (highlighted by white arrows) [364]. 

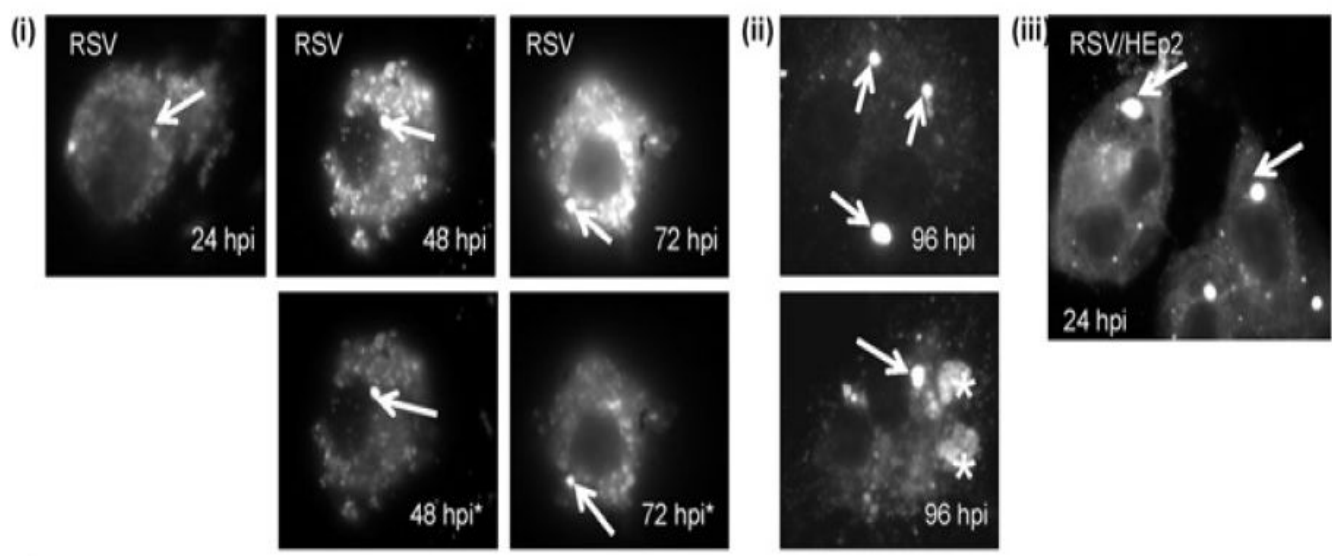

RSVinfected macrophage cells were stained with anti-RSV and examined by fluorescence microscopy at (i) $24 \mathrm{hpi}, 48 \mathrm{hpi}$ and $72 \mathrm{hpi}$ and (ii) $96 \mathrm{hpi}$. The stained cells were examined using either the same camera exposure time or at a reduced exposure time (highlighted by *) to enable the inclusion bodies (IB) to be viewed (at magnification $\mathrm{x} 20$ ). Inclusion bodies are highlighted (white arrow) and a more diffuse anti-RSV staining pattern is highlighted (*). (iii) RSV-infected HEp2 cells stained using anti-RSV at 24 hpi. The IBs are highlighted (white arrow) [364].

\section{Selection of multiplicity of infection in our influenza viruses study}

In our study, if the multiplicity was estimated for each cell type, it was likely that the cells would be challenged with different numbers of virus particles. In terms of this, all the influenza virus tiles were established using MDCK cells, and the multiplicity for different cell types was estimate using MDCK cells. In all cases, a multiplicity of infection (MOI) of 4 was used throughout this Flu study (Sutejo et al., 2012). Thus, infectivity of different virus strains were determined based on equal amount of virus added into different cell lines, which was beneficial for further comparable analysis. Besides, we have used IFA method and confirmed that all the cells were infected. After the infection, the levels of vRNA were measure for different virus strains in different types of different cells.

\section{The infectivity of each of the viruses for each of the cell types in our influenza viruses study}

The replication kinetics was established for the H1N1/WSN, H5N2/F118, H5N3 and H9N2 viruses in each cell type by performing RNA quantification at $1 \mathrm{hr}$ intervals up to $10 \mathrm{hpi}$, and the $\mathrm{M}$ gene universal diagnostic primer was used in qPCR analysis to measure the vRNA levels. A gradual increase in the vRNA levels up to $10 \mathrm{hpi}$ was generally observed following 
virus infection. And $\mathrm{pH} 1 \mathrm{~N} 1 / 478$ replicated less efficiently when compared to other strains [365].

In A549 cells, the vRNA level at 10hpi after infection of H5N3 virus was approximately 10-fold higher than that measured in after infection of H5N2/F118 virus, and approximately 100-fold higher than that after infection of H1N1/WSN virus. In H9N2 virus-infected A549 cells the vRNA levels reached a plateau after $1 \mathrm{hr}$ of infection. The H9N2 virus showed the lowest levels of vRNA synthesis in A549 cells, exhibiting a 10,000-fold reduction in vRNA levels compared to that in H5N2/F118 virus-infected cells. In MDCK cells the vRNA levels in both H1N1/WSN and H5N3 virus-infected cells were comparable, being approximately 10-fold and 100-fold higher than that observed in H5N2/F118 and H9N2 virus-infected cells respectively. In CEF cells, the vRNA levels measured after infection of $\mathrm{H} 5 \mathrm{~N} 3$ virus were approximately 10-fold higher than infection of other viruses, and the vRNA levels measured at 10 hpi in H5N2/F118, H9N2 or H1N1/WSN virus-infected CEF cells were similar. A comparison of the vRNA levels of all AIVs used in this study in each of the three cell types at $10 \mathrm{hpi}$ indicated that the H5N2 viruses behaved similarly. The RNA levels in different cells after infection of H7N1 were also measured by others, and another manuscript including the detailed information is under preparation.
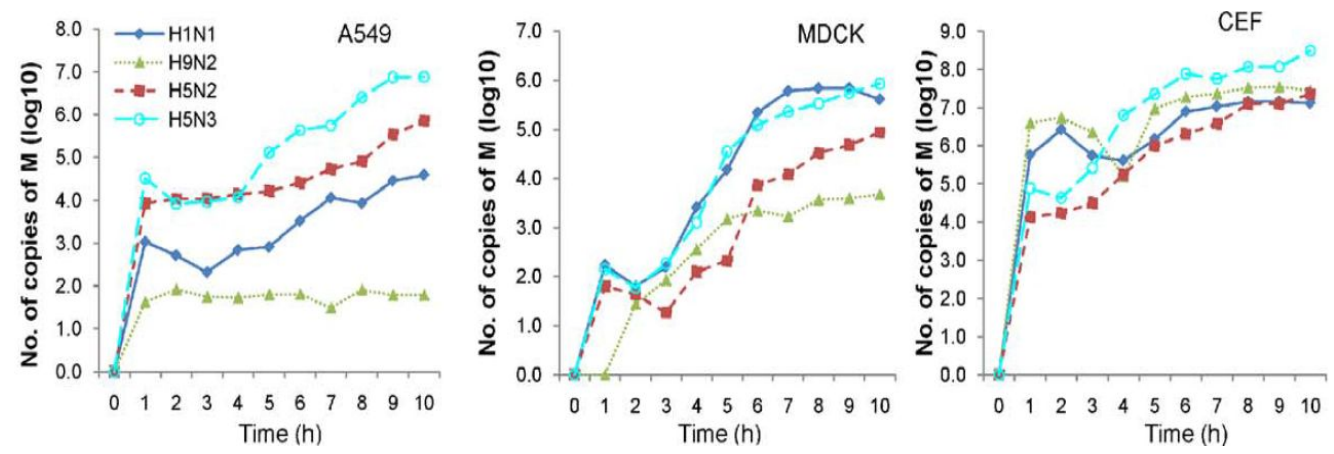

A549, MDCK, and CEF cells were infected either with the H1N1/WSN (a), H9N2 (m), $\mathrm{H} 5 \mathrm{~N} 2 / \mathrm{F} 118(\&)$ or $\mathrm{H} 5 \mathrm{~N} 3(\#)$ viruses using an $\mathrm{MOI}=4$ and incubated at $37^{\circ} \mathrm{C}$. At hourly intervals post infection the cells were harvested and the vRNA levels quantified using qPCR. Each value at a specific time point represents the mean of triplicate measurements $(p, 0.05)$. The data presented are a representative data set from one of two independent experiments [365]. 

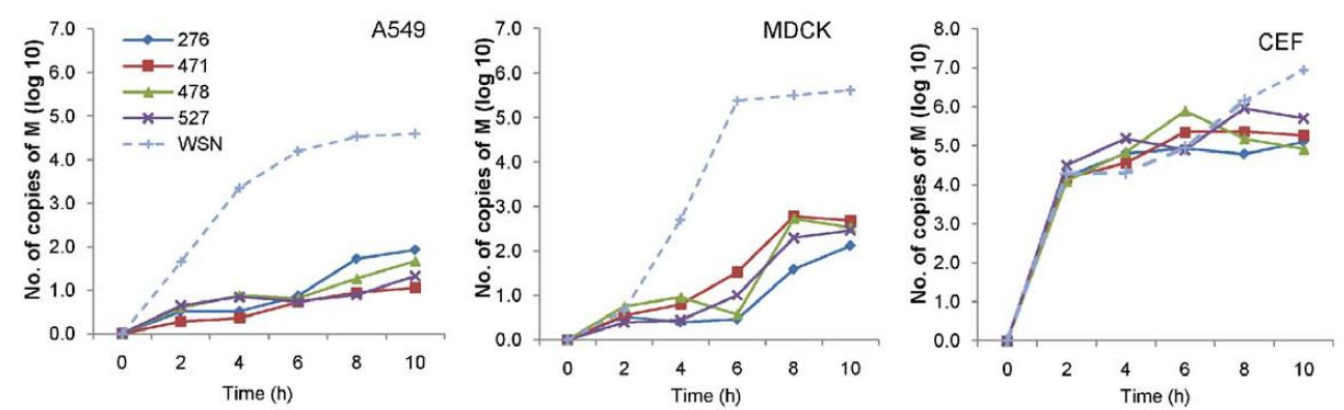

Cells were infected with either the pH1N1/276 (a), pH1N1/471 (\&), pH1N1/478 (m), $\mathrm{pH} 1 \mathrm{~N} 1 / 527(\mathrm{X})$ or H1N1/WSN $(+)$ viruses, and at hourly intervals post infection the cells were harvested and the vRNA levels quantified using qPCR. Each value at a specific time point represents the mean of triplicate measurements $(\mathrm{p}, 0.05)$. The data presented are a representative data set from one of two independent experiments [365].

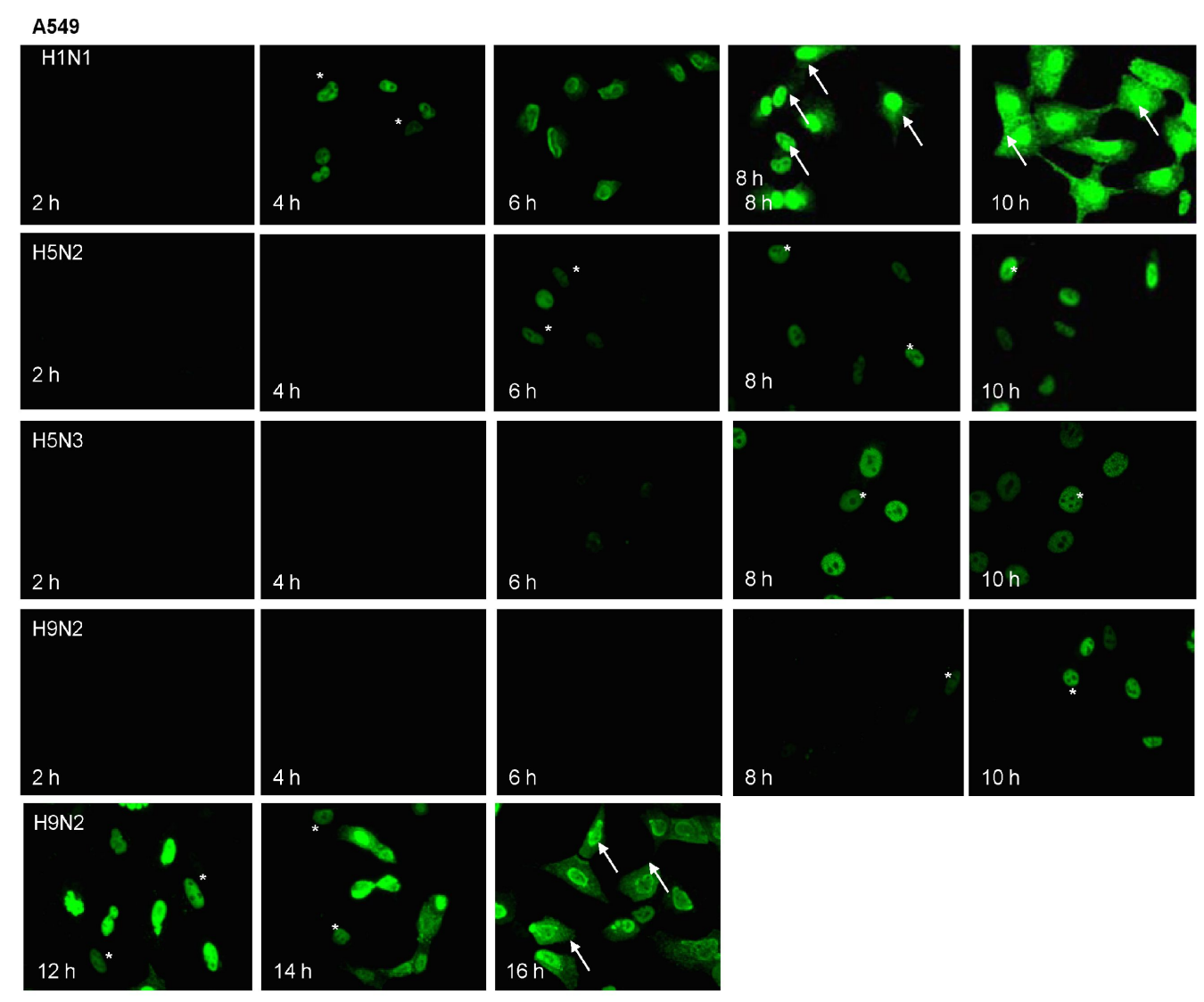

Analysis of the RNP nuclear export in AIVinfected MDCK and CEF cells. The cells were infected with either the H1N1/WS, H9N2, H5N2/F118 or H5N3 viruses using an $\mathrm{MOI}=4$, and at specific times post infection the cells were fixed and labelled using anti-NP and goat antimouse conjugated to Alexa555. The stained cells were visualised using a Nikon Eclipse 80i Microscope at 620 magnification with appropriate machine settings [365].

The NP-stained nuclei (*) and cells (white arrow) are indicated. 


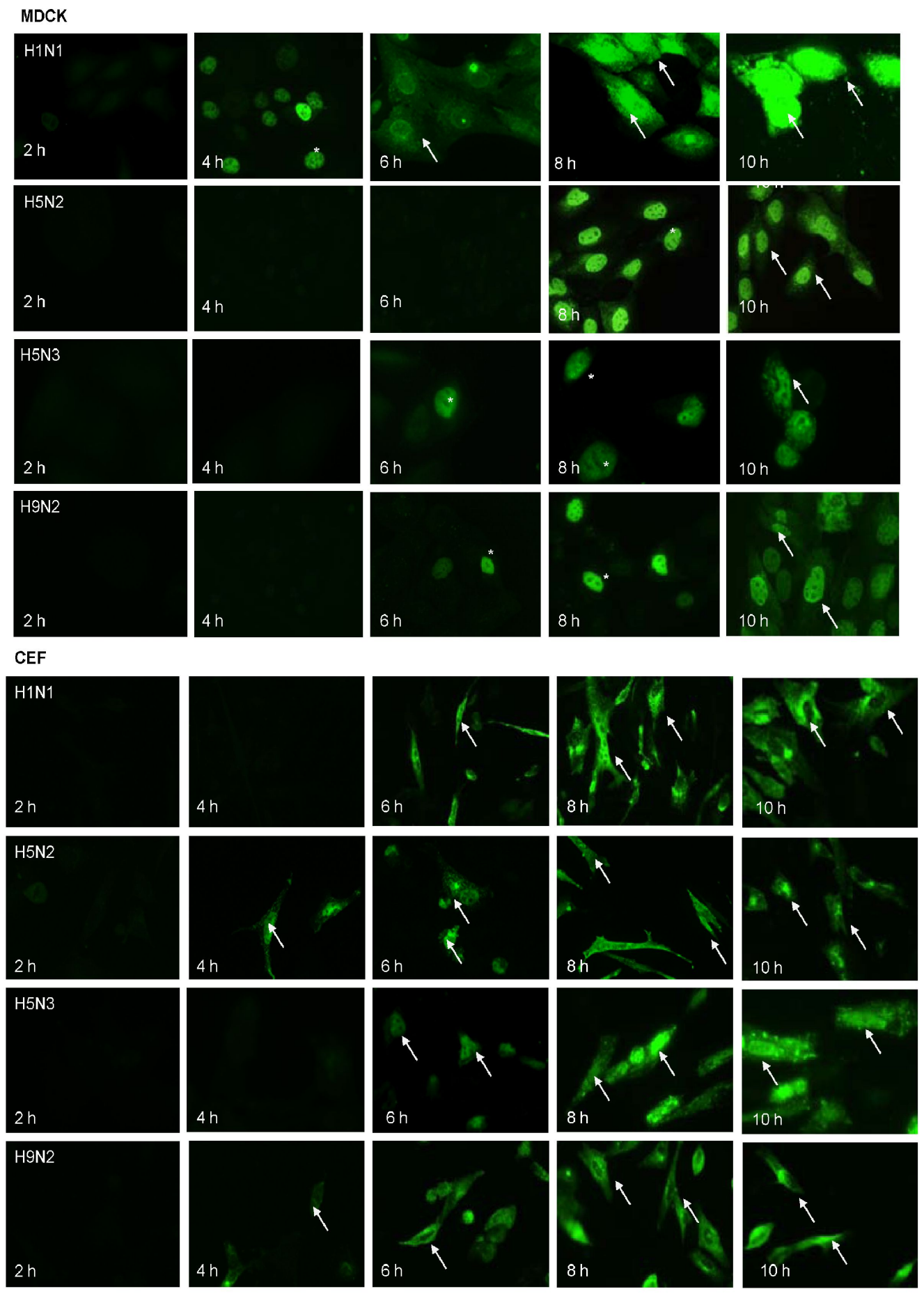

Analysis of the RNP nuclear export in AIVinfected A549 cells. A549 cells were infected with either the H1N1/WS, H9N2, H5N2/F118 or H5N3 viruses using an MOI=4. At specific times post infection the cells were fixed and labelled using anti-NP and goat anti-mouse conjugated to Alexa555. The stained cells were visualised using a Nikon Eclipse 80i Microscope at620 magnification with appropriate machine settings [365].

The NP-stained nuclei (*) and cells (white arrow) are indicated.

\section{Selection of multiplicity of infection in our poxviruses study}

Three types of poxviruses plagues were transfected into Vero E6 cells and they ranged from an estimated $1 * 105$ to $1 * 107$ pfu. The infectivity of all 
three types of poxviruses in mouse RAW or A549 cells were traced using IFA with polyclonal antibody. The time points of infections were carried out at 2, 4, 6, 8, 10 hpi and overnight with MOI of 1, 3, 5 and 10. The results for mouse RAW cells indicated that infections with MOI of 5 at 2 hpi and overnight (16 hpi) were productive to measure the host response. In the case of A549 cells, an MOI of 3 was enough to indicate a productive infection at 2, 4, 6, 8 and 10 hpi.

\section{Correlation between results from microarray study and the stage of virus infection in our poxviruses study}

Growth curves for each virus in different cell types were also performed over the same infection time course. The virus titers were measured in Vero E6 cells and replications of viral RNA were performed by others in our lab. Since the manuscript is still under preparation, the detailed data is not accessible. However, our virus infection results corresponded to the results from other parallel experiments by other researchers [337][382][395][396]. In these studies, different steps of virus replication cycle such as early transcription (genes coding for immunomodulatory proteins, enzymes, and replication and transcription factors are transcribed and translated immediately upon core particle entry into the cytoplasm of the cell), late transcription (genes coding for structural proteins, enzymes, and transcription factors are transcribed and translated), virus assembly and release were observed.

In mouse RAW cells, we performed the experiment at 2 hpi which is equivalent to the early transcription and overnight infection (16 hpi) which is equivalent to the late transcription. In the phase of early transcription, only quite small numbers of genes showed differential expression, while relative large scale of genes showed differential expression during the phase of viral late transcription. Among these differentially expressed genes, genes with upregulated expression majorly function on transcription, RNA binding and cell death especially after infection of ectromelia virus, while genes with downregulated expression majorly function on RNA binding, kinase, cell death and cell cycle especially after infection of lister virus. These observations indicated the weak host-viral interactions at the early stage of viral transcription but the strong interactions at the late stage of viral transcription. 
In A549 cells, the time course from 2 hpi to 10 hpi was chosen to allow enough time for establishment of virus infection and progression to late viral gene expression but also to carefully avoid the risk of cell lysis and RNA degradation after completion of the first replication cycle. And gene expression profiles investigated at five time points from 2 hpi to $10 \mathrm{hpi}$ uncovered the host mRNA expression from early and intermediate to late viral transcription detailedly. For both cowpox and lister virus, the largest number of genes showed down-regulated in their expression around $8 \mathrm{hpi}$, the process from intermediate viral transcription to late viral transcription. And pathways related to metabolic, glycolysis, cell death and cell cycle were significantly enriched in these genes with down-regulated expression, suggesting that the strongest interactions between poxvirus and A549 host cells occurred in this key infection period.

\section{Perl script for data pre-processing before E-Predict analysis}

Script1:

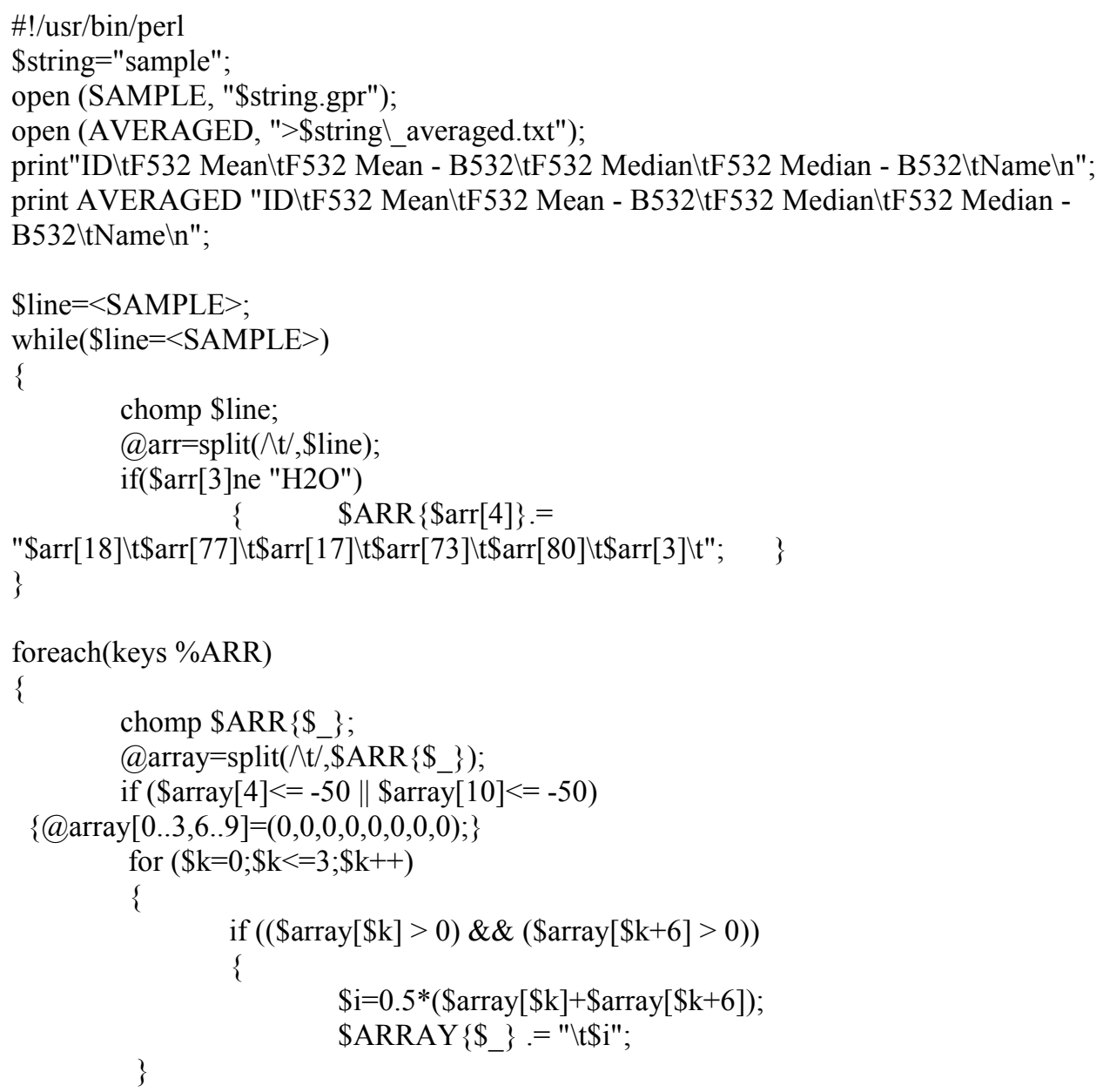




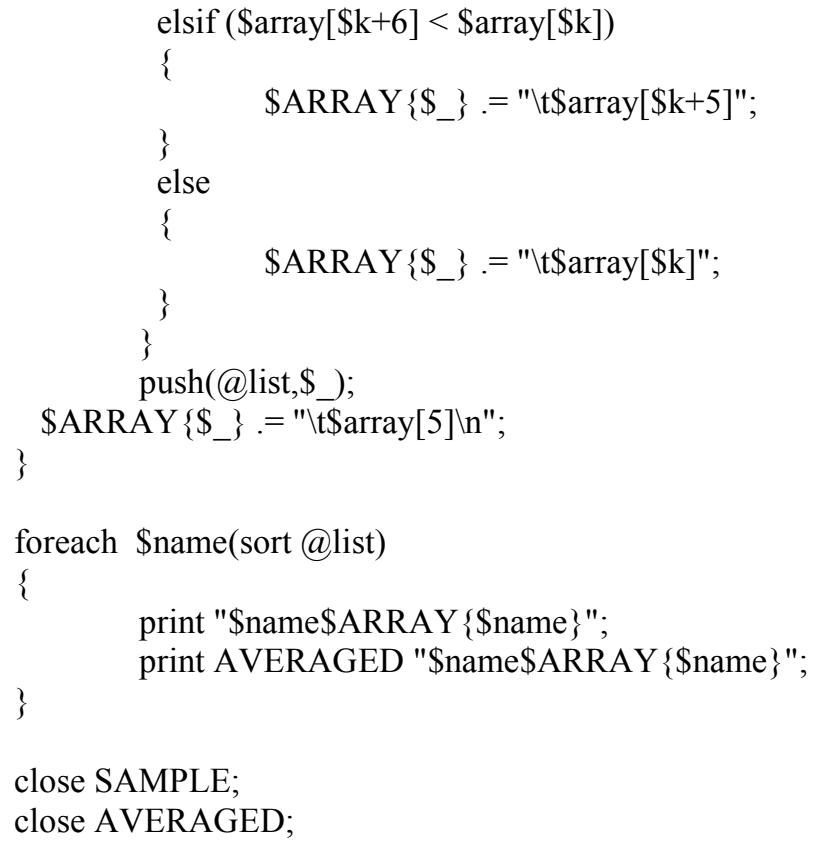

Script2:

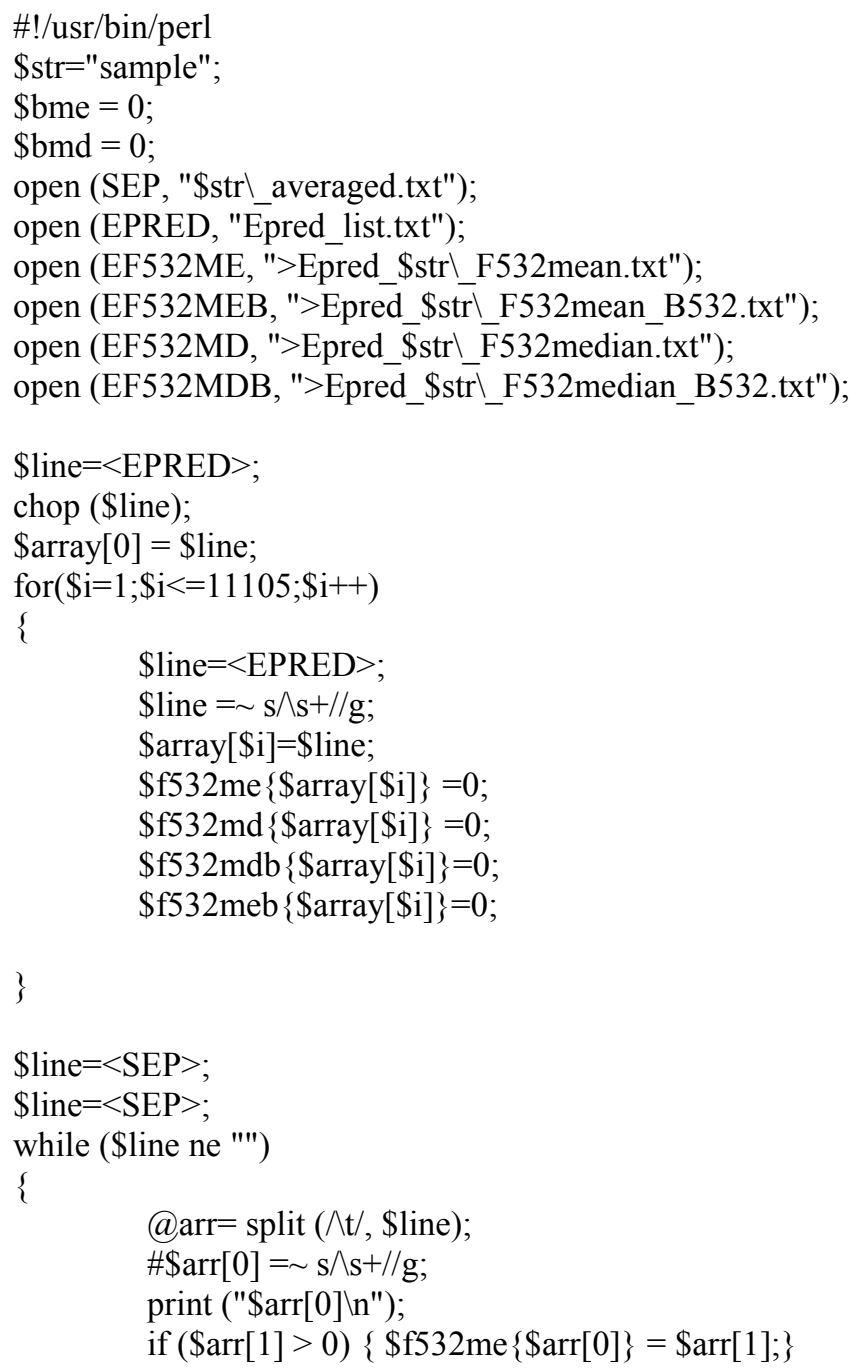




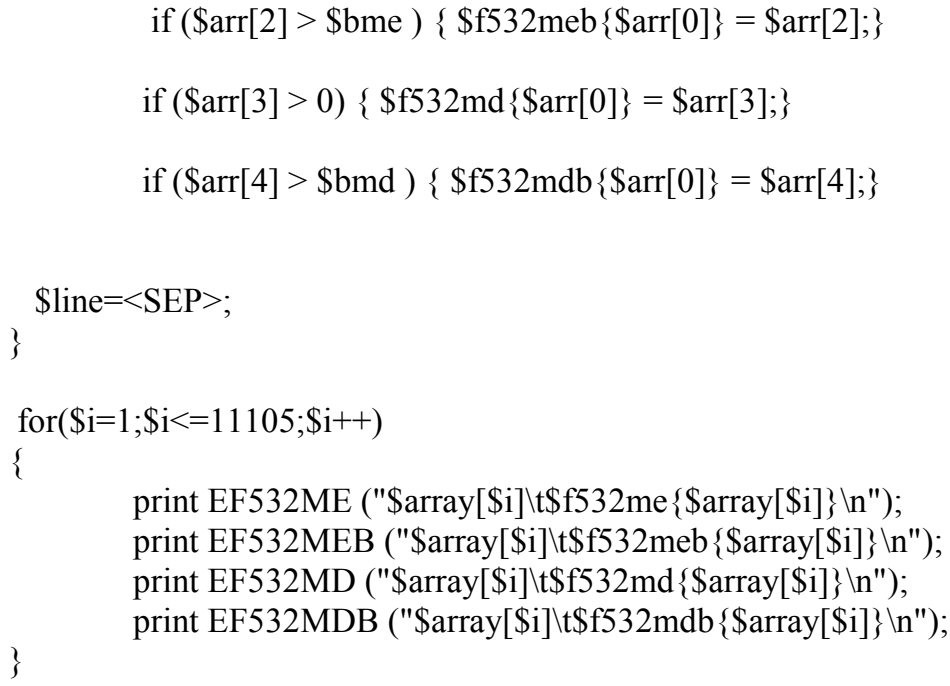

Script3:

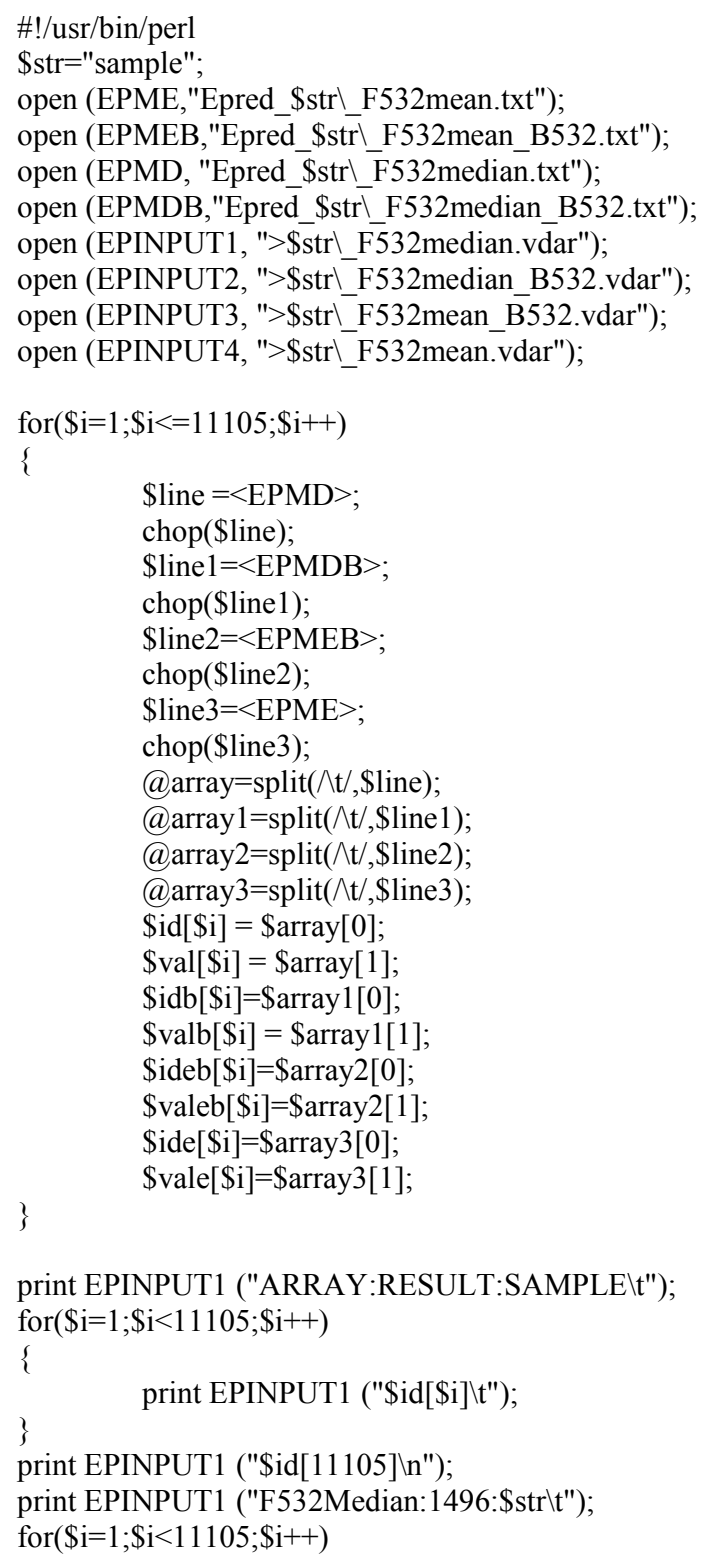




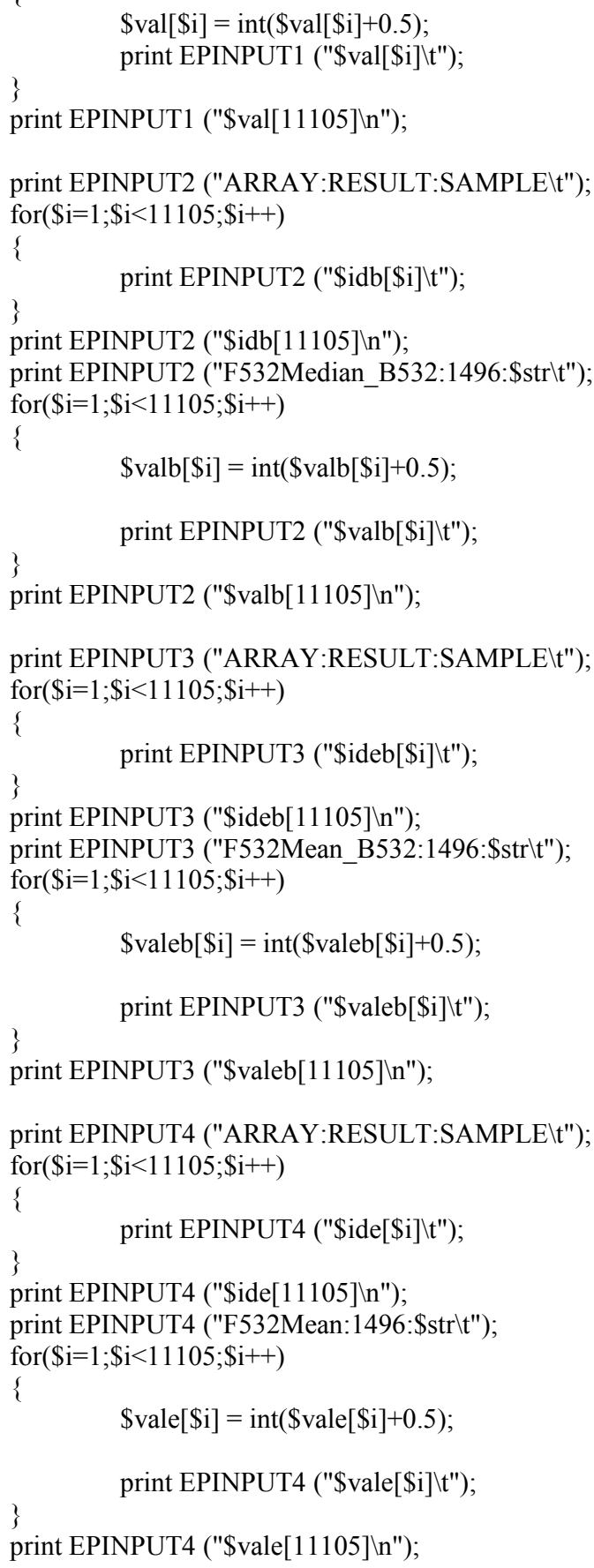

\section{Perl script for functional classification}

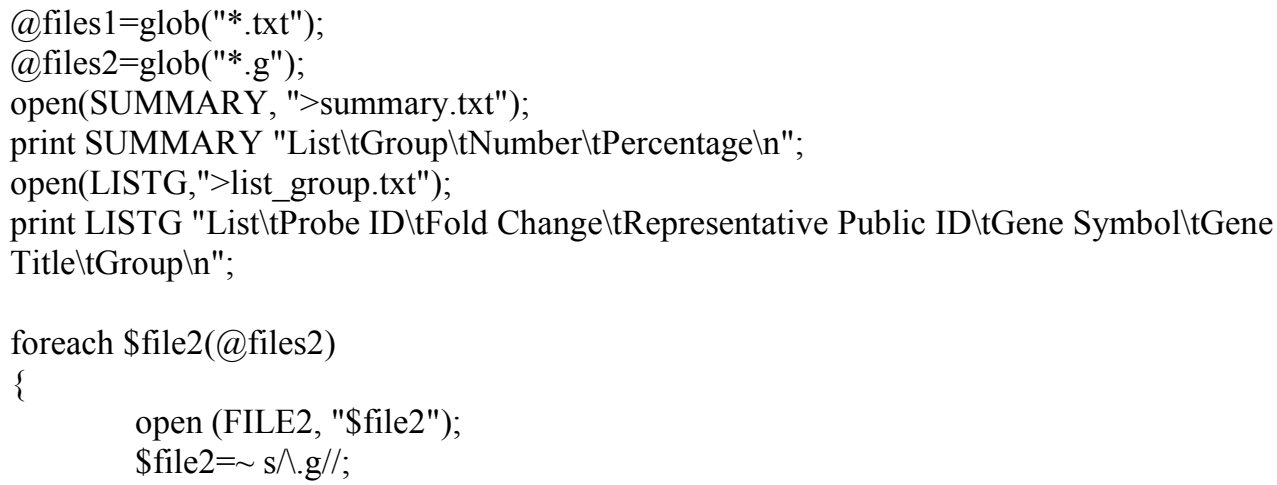




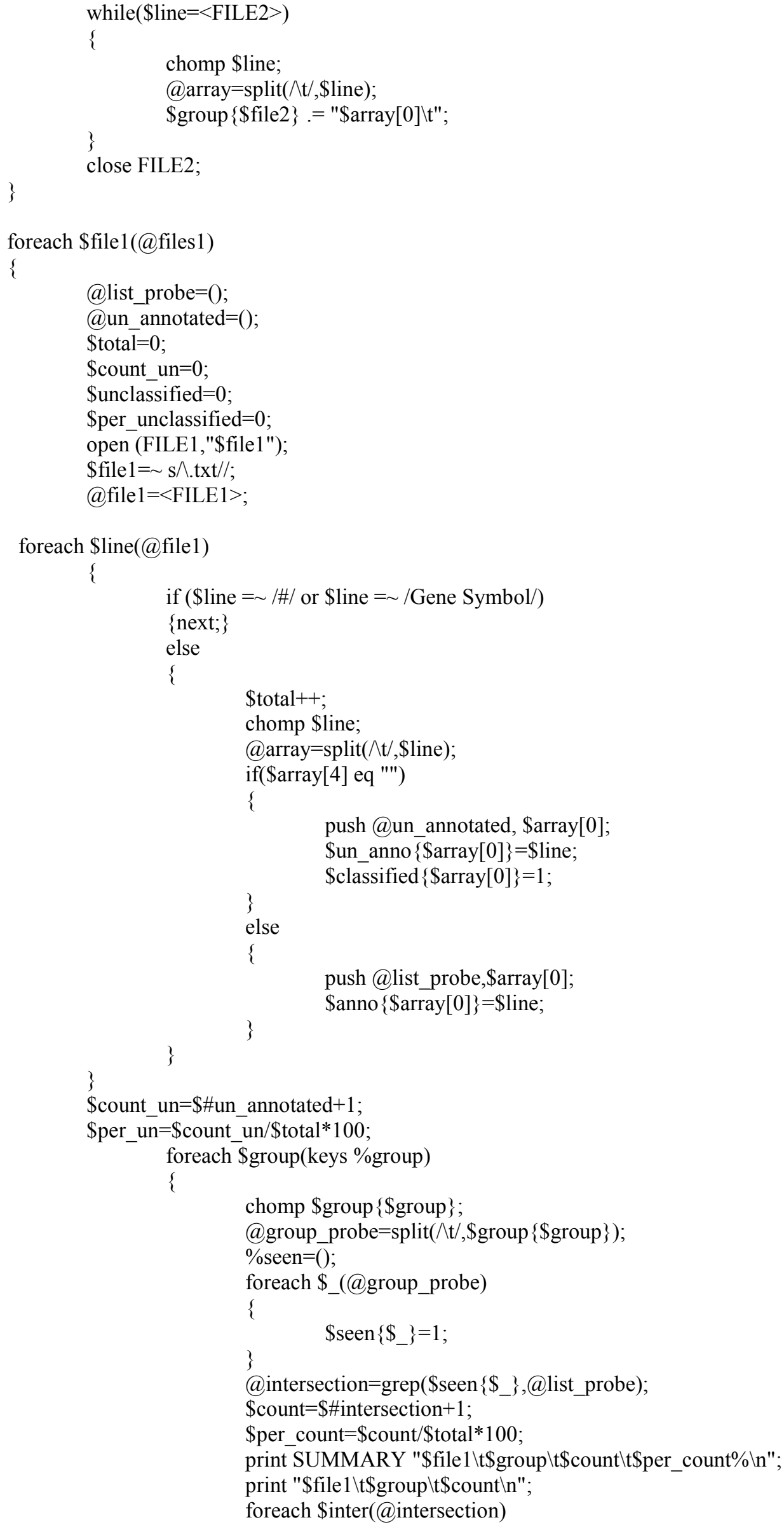




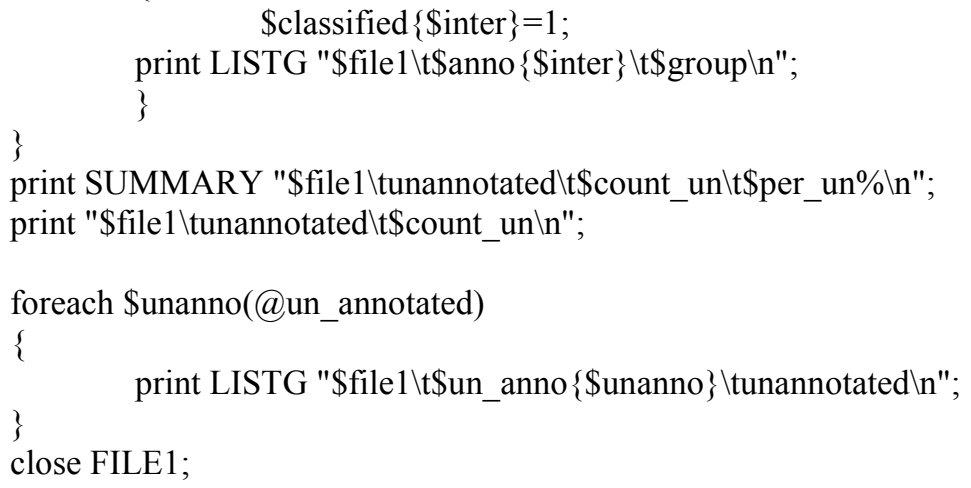

\section{Perl script for venndiagram}

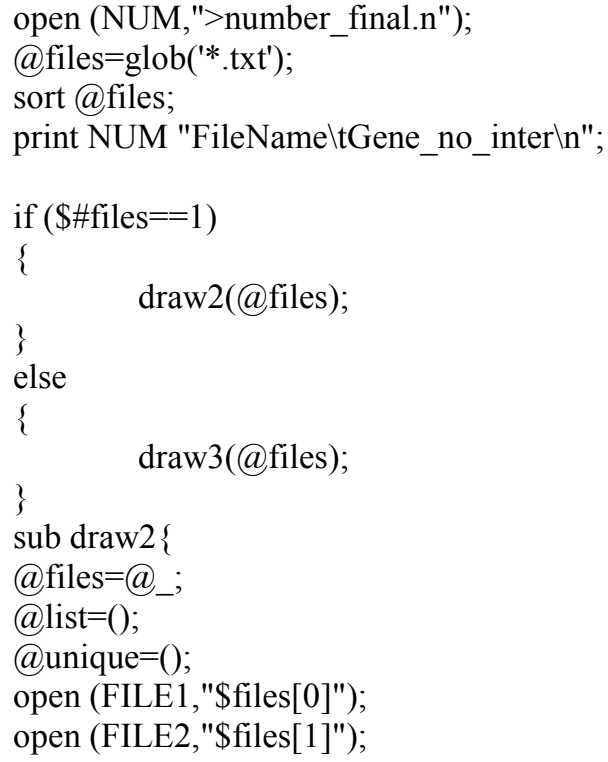




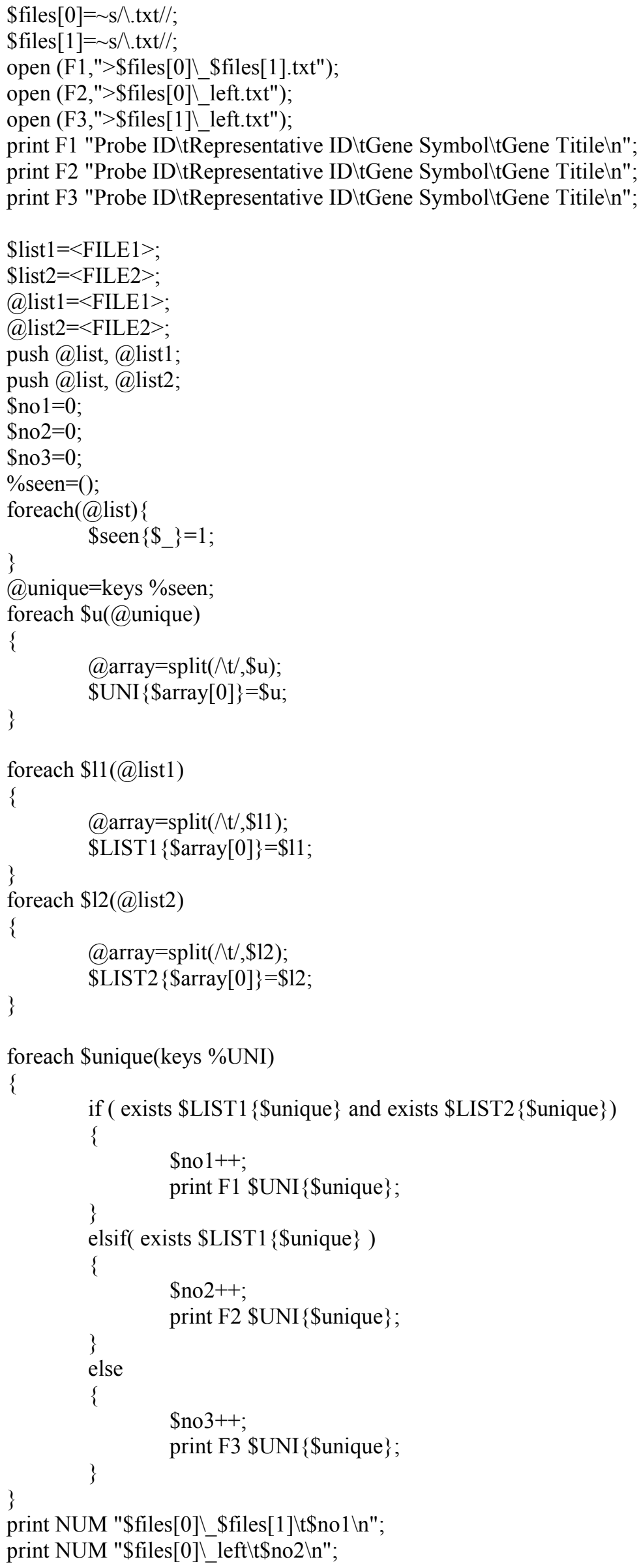




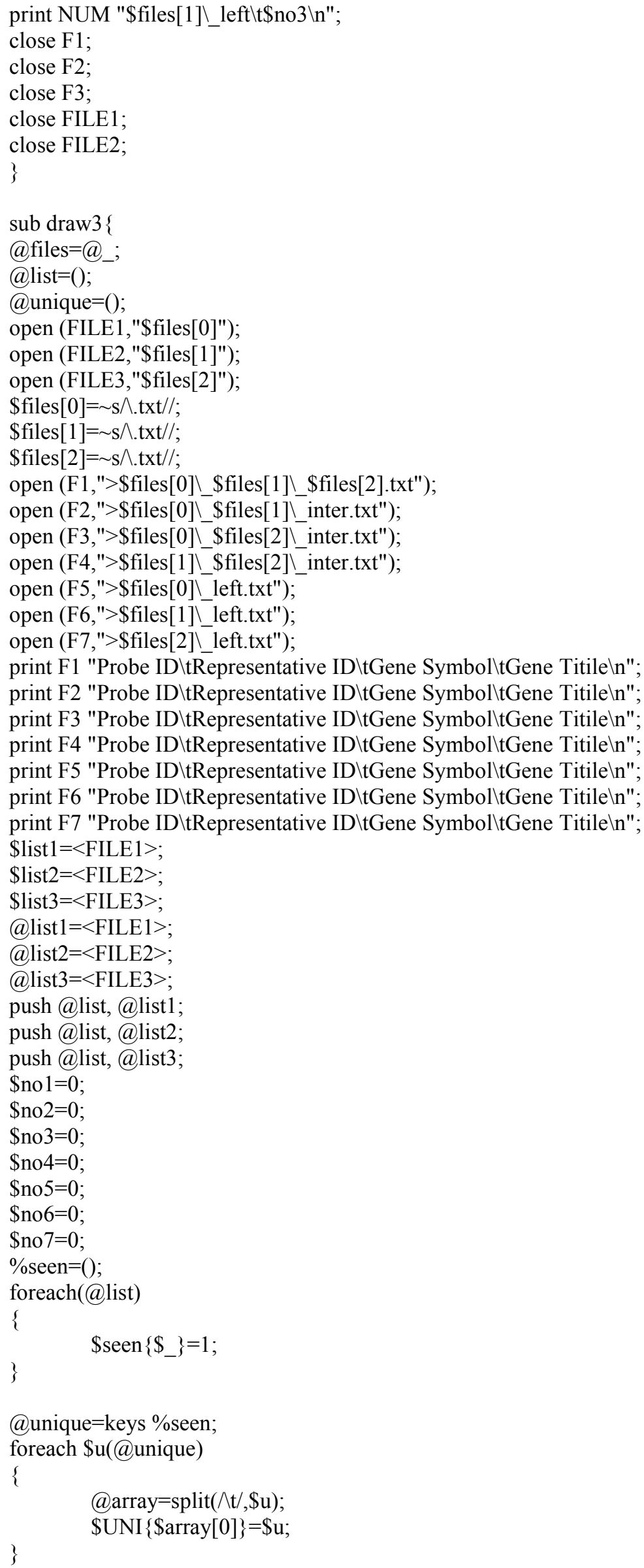




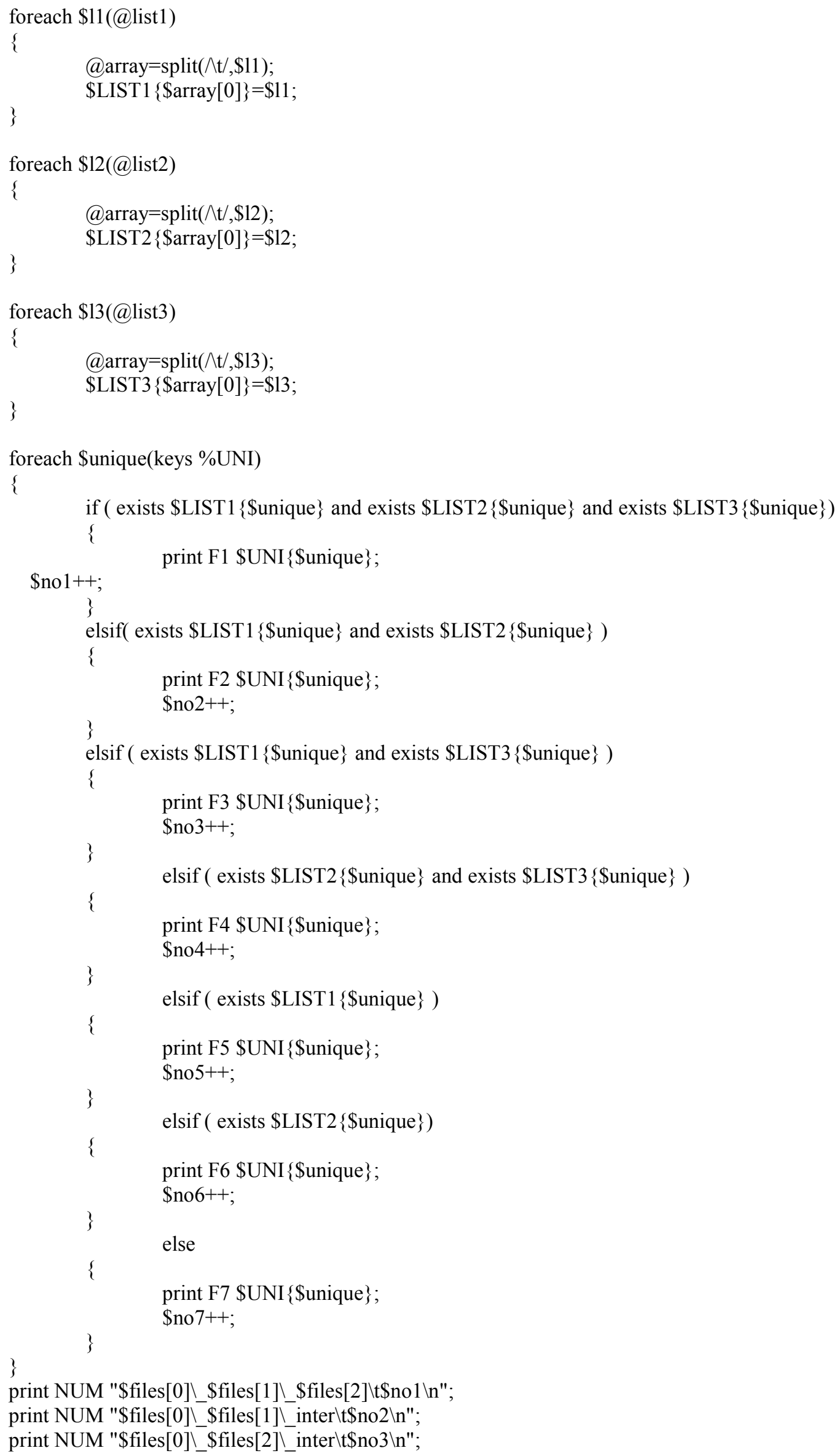


print NUM "\$files[1]\\$files[2]\inter|t\$no4\n"; print NUM "\$files[0]\lefttt\$no5\n";

print NUM "\$files[1]\_lefttt\$no6 \n";

print NUM "\$files[2]\_lefttt\$no7\n";

close $\mathrm{F} 1$;

close F2;

close F3;

close F4;

close F5;

close F6;

close F7;

close FILE1;

close FILE2;

close FILE3;

\} 


\section{Activation of Type I and III Interferon Signalling Pathways Occurs in Lung Epithelial Cells Infected with Low Pathogenic Avian Influenza Viruses}

Richard Sutejo', Dawn S. Yeo ${ }^{1,2}$, Myint Zu Myaing', Chen Hui ', Jiajia Xia', Debbie Ko', Peter C. F. Cheung', Boon-Huan Tan', Richard J. Sugrue ${ }^{1 *}$

1 Division of Nolecular and Cell Biology, School of Biological Sciences, Nanyang Technological University, Singapore, Singapore, 2 Detection and Disgnostics Laboratory, DSO National Labos tories, Singapore, Singapore

\section{Abstract}

The host response to the low pathogenic avian influenza (LPAD) H5N2, H5N3 and H9N2 viruses were examined in A549, MDCK, and CEF cells using a systems-based approach. The H5N2 and H5N3 vinuses replicated effidently in A549 and MDCK cells, while the H9N2 virus replicated least efficiently in these cell types. However, all LPAI viruses exhibited similar and higher replication efficiencies in CEF cells. A comparison of the host responses of these viruses and the H1N1MSN virus and low passage pH1N1 clinical isolates was performed in $A 549$ cells. The $H 9 N 2$ and H5N2 virus subtypes exhibited a robust induction of Type I and Type III interferon (IFN) expression, sustained STAT1 activation from between 3 and $6 \mathrm{hpi}$, which correlated with large increases in IFN-stimulated gene (ISG) expression by $10 \mathrm{hpi}$. In contrast, cells infected with the pH1N1 or $\mathrm{H} 1 \mathrm{~N} 1$ /WSN virus showed only small inceases in Type III IFN signalling, low levels of ISG expression, and down-regulated expression of the IFN type I receptor. JNK activation and increased expression of the pro-apoptotic XAF 1 protein was observed in A549 cells infected with all viruses except the H1N1/WSN virus, while MAPK p38 activation was only observed in cells infected with the pH1N1 and the HS virus subtypes. No IFN expression and low ISG expression levels were generally observed in CEF cells infected with either AN, while increased IFN and ISG expression was observed in response to the HIN1/WSN infection. These data suggest differences in the replication characteristics and antivirus signalling responses both among the different LPAl viruses, and between these viruses and the H1N 1 viruses examined. These virus-specific differences in host cell signalling highlight the importance of examining the host response to avian influenza viruses that have not been extensively adapted to mammalian tissue culture.

Citation: Sutejo R, Yeo DS, Myaing MZ, Hui C, Xis I, et al (2012) Activafon of Type I and wil Interferon Signalling Pattiways Occus in Lung Epithelial Cellis Infected

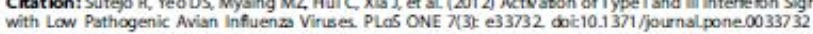

Editor: Karen L Mossman Mclvoster University, Cansh

Received November 24, 2011; Accepted February 16, 2012; Published March 21, 2012

Copyright: $\$ 2012$ Sutejo ef al. This is an openaccess artide distributed under the terms of the Crestive Commons Atribution Licrse, which permits uresticted use, distribution and reproduction in any medium, provided the ofiginal author and source are credited.

Funding: The authors acknowledge the Defence Science and Technology Agency and National Medical Research Coundil of Singapore for funding this curnert study. The funders had no rok in study design, data collection and analysis, decision to publish, or preparation of the manuscrip.

Competing Int arests: The authars have declared that no competing interests exist

- Email: rjougneil ntu.edusg

\section{Introduction}

Avian influenza viruses (AIV) are maintained in feral aquatic bird populations, which are thought to be the reservoir for the influenza A viruses that infect all other animal species [1]. Although AIV infection of domestic poultry is of economic importance, non-avian hosts, induding humans can be infected $[2,3,4]$. Avian-to-human transmission of high pathogenic avian influenza (HPAI) viruses (eg. $\mathrm{H} 5 \mathrm{~N} 1$ ) are often associated with high fatality rates, whereas associated fatalities due to human transmission of low pathogenic avian influenza (LPAI) viruses have not been reported. Poultry workers in China and Japan have tested seropositive for avian $\mathrm{H} .5$ and $\mathrm{H} 9$ suggesting prior infection [5,6], and $\mathrm{H} 9 \mathrm{~N} 2$ infection in humans only results in mild influenza-like-illness [2]. In addition, AIVs can play a role in the evolution of seasonal influenza virus strains, with unpredictable consequences [7,8]. Current AIV surveillance programs place a particular emphasis on $\mathrm{H} 5$ and $\mathrm{H}$ ? subtypes, since gradual introduction of mutations into the vRNA of LPAI viruses that are circulating in avian populations can lead to the emergence of HPAI viruses $[9,10,11]$.
Pathogen-host interactions have been relatively well characterised in laboratory-adapted influenza viruses and in some HPA virus isolates (c.g. $\mathrm{H} 5 \mathrm{~N} 1$ ), but in general our understanding of hos interactions during AIV infection is comparatively poor. Although current animal model systems can provide useful information about the pathology of specific influenza virus isolates, they (e. mice) are not naturally infected with influenea viruses, and they respond to the virus infection in an age-dependant manner $[12,13]$. In general these viruses need to be adapted to their new host, and during the process of species adaptation inheren biological properties of these viruses can be lost or modified. Cell culture systems that are permissive for LPAI virus infection can provide an additional useful complementary experimental ap proach to analyse the fundamental biological properties of nonmammalian adapted LPAI virus isolates that would otherwise grow poorly in mammalian hosts. Many of these permissive cell types (e.g. A549) retain complete signalling networks that are related to the innate host response to infection [e.g. interferon (IFN)], and this can be used to examine the host response to AIV infection. Furthermore, it is expected that these cell types retai 


\title{
A systems-based approach to analyse the host response in murine lung macrophages challenged with respiratory syncytial virus
}

Laxmi lyer Ravi ${ }^{1}$, Liang Li ${ }^{1,2}$, Richard Sutejo ${ }^{1}$, Hui Chen ${ }^{1}$, Pui San Wong ${ }^{3}$, Boon Huan Tan ${ }^{3}$ and Richard J Sugrue ${ }^{1,2^{*}}$

\begin{abstract}
Background: Respiratory syncytial virus (RSV) is an important cause of lower respiratory tract infection in young children. The degree of disease severity is determined by the host response to infection. Lung macrophages play an important early role in the host response to infection and we have used a systems-based approach to examine the host response in RSV-infected lung-derived macrophage cells.

Results: Lung macrophage cells could be efficiently infected (>95\%) with RSV in vitro, and the expression of several virus structural proteins could be detected. Although we failed to detect significant levels of virus particle production, virus antigen could be detected up until 96 hours post-infection (hpi). Microarray analysis indicated that 20,086 annotated genes were expressed in the macrophage cells, and RSV infection induced an 8.996 and $11.3 \%$ change in the global gene transcriptome at $4 \mathrm{hpi}$ and $24 \mathrm{hpi}$ respectively. Genes showing up-regulated expression were more numerous and exhibited higher changes in expression compared to genes showing down-regulated expression. Based on gene ontology, genes with cytokine, antiviral, cell death, and signal transduction functions showed the highest increases in expression, while signalling transduction, RNA binding and protein kinase genes showed the greatest reduction in expression levels. Analysis of the global gene expression profile using pathway enrichment analysis confirmed that up-regulated expression of pathways related to pathogen recognition, interferon signalling and antigen presentation occurred in the lung macrophage cells challenged with RSV. Conclusion: Our data provided a comprehensive analysis of RSV-induced gene expression changes in lung macrophages. Although virus gene expression was detected, our data was consistent with an abortive infection and this correlated with the activation of several antivirus signalling pathways such as interferon type I signalling and cell death signalling. RSV infection induced a relatively large increase in pro-inflammatory cytokine expression, however the maintenance of this pro-inflammatory response was not dependent on the production of infectious virus partides. The sustained pro-inflammatory response even in the absence of a productive infection suggests that drugs that control the pro-inflammatory response may be useful in the treatment of patients with severe RSV infection.
\end{abstract}

Keywords: Respiratory syncytial virus, Macrophage transcriptome, Host response, Interferon, Cytokine induction

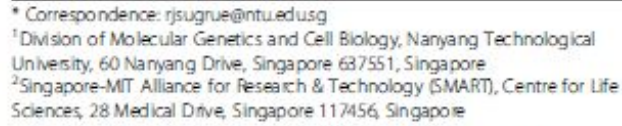

